\title{
ASSESSMENT OF RESEARCH NEEDS FOR ADVANCED FUEL CELLS \\ BY THE
}

\section{DOE ADVANCED FUEL CELL WORKING GROUP (AFCWG)}

November 1985

\author{
Prepared by \\ S. S. Penner, Chairman of DOE/AFCWG \\ Energy Center and Department of Applied \\ Mechanics and Engineering Sciences \\ University of California, San Diego \\ La Jolla, California 92093 \\ Under Contract No. DE-AC01-84ER30060 \\ Prepared for
U.S. Department of Energy \\ Office of Energy Research \\ Office of Program Analysis \\ Washington, D.C. 20545 \\ Under Contract No. DE-AC01-84ER30060
}




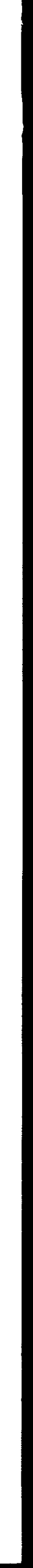




\section{DISCLAIMER}

This report was prepared as an account of work sponsored by an agency of the United States Government. Neither the United States Government nor any agency thereof, nor any of their employees, make any warranty, express or implied, or assumes any legal liability or responsibility for the accuracy, completeness, or usefulness of any information, apparatus, product, or process disclosed, or represents that its use would not infringe privately owned rights. Reference herein to any specific commercial product, process, or service by trade name, trademark, manufacturer, or otherwise does not necessarily constitute or imply its endorsement, recommendation, or favoring by the United States Government or any agency thereof. The views and opinions of authors expressed herein do not necessarily state or reflect those of the United States Government or any agency thereof. 


\section{DISCLAIMER}

Portions of this document may be illegible in electronic image products. Images are produced from the best available original document. 
The DoE Advanced Fuel Cell Working Group (AFCWG) was formed and asked to perform a scientific evaluation of the current status of fuel cells, with emphasis on identification of long-range research that may have a significant impact on the practical utilization of fuel cells in a variety of applications. The AFCWG held six meetings at locations throughout the country where fuel cell research and development are in progress, for presentations by experts on the status of fuel cell research and development efforts, as well as for inputs on research needs. Subsequent discussions by the AFCWG have resulted in the identification of priority research areas that should be explored over the long term in order to advance the design and performance of fuel cells of all types.

Surveys describing the salient features of individual fuel cell types are presented in Chapters 2 to 6 and include elaborations of long-term research needs relating to the expeditious introduction of improved fuel cells.

The Introduction and the Summary (Chapter 1) were prepared by AFCWG. They were repeatedly revised in response to comments and

- criticism. The present version represents the closest approach to a concensus that we were able to reach, which should not be interpreted to mean that each member of AFCWG endorses every $s$ tatement and every unexpressed deletion. The Introduction and Summary always represent a majority view and, occasionally, a unanimous judgment.

Chapters 2 to 6 provide background information and carry the names of identified author 8 . The identified authors of Chapters 2 to 6 , rather than $A F C W G$ as a whole, bear full responsibility for the scientific and technical contents of these chapters. 
University Members

Professor J.0'M. Bockris

Department of Chemistry

Texas A \& M University

College Station, Texas 77840

409-845-4947 or 409-845-5335

Professor J. Robert Selman

Department of Chemical Engineering

Armour College of Engineering

Illinois Institute of Technology, IIT Center Chicago, Illinois 60616

312-567-3037

Professor S.S. Penner, AFCWG Chairman

Director, Energy. Center, B-010

Professor David Shores

University of California, San Diego

Department of Metallurgical Engineering

La Jolla, California 92093

151 Amundson Hall, Univ. of Minnesota

421 Washington Ave., SE

619-452-4284

Minneapol is, Minnesota 55455

Office: 612-373-4183, Home: 612-721-7530

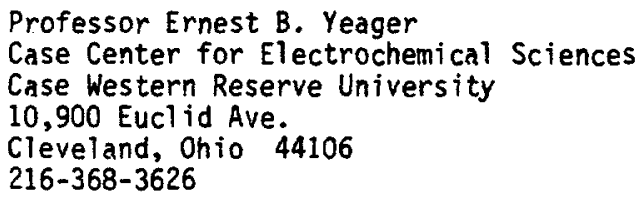

Industry and Research Organization Members

Dr. John Appleby, Project Manager

(Mr. E.A. Gill is, Alternate Member)

Advanced Fuel Cell Technology

Electric Power Research Institute

P.0. Box 10412

Palo Alto, California 94303

415-855-2543

Or. Bernard S. Baker, President

(Dr. Hans Maru, Alternate Member)

Energy Research Corporation

3 Great Pasture Road

Danbury, Connecticut 06810

203-792-1460

Dr. Jack T. Brown

Manager, Materials and Conversion Sys tems Department

Westinghouse Electric Corporation

R\&D Center

1310 Beulah Road

Pittsburgh, Pennsylvania 15235

412-256-1950

Dr. Diane T. Honie

(Dr. John J. Cuttica, Al ternate Member)

Residential/Comercial Energy

Sys tems Research

Gas Research Institute

8600 West Bryn Mawr Ave.

Chicago, Illinois 60631

312-399-8100
Mr. William E. Houghtby

Power Systems Division

United Technologies Corporation

P.0. Box 109

South Windsor, Connecticut 06074

$203-727-2200$

Dr. A. Kaufman, Res. Mgr. for Fuel Cells

(Dr. L. Michael Quick, Alternate Member)

Engel hard Corporation

Menlo Park

Edison, New Jersey 08818

$201-32 I-5286$

Dr. L.G. Marianowski, Associate Director

Energy Conversion and Storage Research

Institute of Gas Technology

3424 South State Street

Chicago, Illinois 60616

$312-567-3650$

Dr. Halina Wroblowa, Principal Res. Scientist

Ford Motor Company

Advanced Components and Energy Systems Dept.

P.0. Box 2053

Dearborn, Michigan 48121-2053

313-337-5052 
Dr. John Ackerman

Manager, Electric Chemical Research CMT /205

Argonne National Laboratory

9700 South Cass Avenue

Argonne, IL 60439

Commander

U.S. Army Belvoir R\&D Center

ATTN: STRBE-EC (Dr. J.A. Joebstl)

Fort Belvoir, VA. 22060

Mr. F. Don Freeburn,

DoE Project Manager

General Engineer

ER-33, GTN

U.S. Department of Energy

Washington, D.C. 20545

Dr. C. Lowell Miller

Acting Director of Advanced Energy

Conversion Systems

FE-22, GTN

U.S. Department of Energy

Washington, O.C. 20545

Mr. John E. Sholes

Chief, Fuel Cells Project Branch Coal Projects Management Division Morgantown Energy Technology Center P.0. Box 880

Morgantown, WV 26505

Dr. Marvin Warshay

Manager, Fuel Cells Project Office

M.S. $500-203$

NASA/Lewis Research Center

Cleveland, $\mathrm{OH} 44135$
Dr. Ken Rogers

Program Director of the Kinetics, Catalys is and Reaction Engineering Program

Chemical and Process Division

Room 1126

National Science Foundation

1800 G Street, NW

Washington, D.C. 20550

Dr. Albert Landgrebe

(Alternate: Dr. Stanley Ruby)

Office of Energy Storage

CE-141, FORSTL

U.S. Department of Energy

Washington, D.C. 20585

Alternates

$\mathrm{Mr}$. Robert Rader

Director, Research and

Technical Assessment Division

ER-33, GTN

U.S. Department of Energy

Washington, D.C. 20545

Mr. Graham Hagey

(Alternate: Mr. Charles Pax, 301-353-2832)

Office of Advanced Energy Conversion Systems

FE-22, GTN

U.S. Department of Energy

Washington, D.C. 20545

Or. John Wilson

Director, Coal Projects

Management Division

Morgantown Energy Technology Center

P.0. Box 880

Morgantown, WV 26505

Dr. Henry Sione

Director, Space Technology

M.S. 3-5

NASA/Lewis Research Center

Cleveland, $\mathrm{OH} \quad 44135$ 


\section{INTRODUCTION}

This is the final report covering work perfomed under Contract No. DE-AC01-84ER30060 with the U.S. Department of Energy. It was prepared for the Office of Program Analys is (OPA) with in the Department's Office of Energy Research. OPA provides independent, objective analyses of the Department's technical needs and opportunities. A function of this activity includes assessing the adequacy of the long-range (technology base) research that supports the Department's research and development (R\&D) programs. This report deals with an assessment of the long-range research needs for advanced fuel cells.

The Principal Investigator of this study was S. S. Penner, Director, Energy Center; and Professor of Engineering Physics, University of California/ San Diego. He was assisted by the DOE Advanced Fuel Celi Working Group (AFCWG). AFCWG members are experts on fuel cell R\&D and were selected from academic institutions, industry, and not-for-profit organizations. Experts from the government, National Laboratories, and DOE Energy Technology Centers served as observers and resource personnel at AFCWG meetings.

The original AFCWG work statement is reproduced in Appendix A. It served as a basis for the development of the ideas presented in this report and was refined and explicated as the result of progressive discussions involving both AFCWG members and the ex officio members who represented the Department of Energy and other federal agencies.

The Summary (Chapter 1) contains our principal findings and recommendations. It is followed by surveys describing the salient features of fuel cells, including elaborations concerning the identification of long-range research needs (Chapters 2 to 6 ).

Cost evaluations and potential market penetration of new fuel-cell technologies have formed integral components of our deliberations and references to these problem areas wll be found in connection with the discussions of individual fuel cells.

Our research recommendations over a wide spectrum of activities and emphasize fundamental science and understanding rather than cell design and development. They have not been constructed to satisfy the primary goal of optimizing a particular cell design or configuration. Adequate long-range, stable support for research on materials science, fundamental electrochemistry, etc. may aid commercial implementation of the right technologies over the long term and may also be of value in the definition and identification of new or different fuelcell designs that merit investigation and development.

The members of AFCWG acknowledge with thanks the advice and assistance provided by many individuals in government, industry and the universities. The following individuals, among others, have contributed to our discussions, evaluations and final recommendations: C. Antoine (NASA Lewis Research Center), R. Barta (GE), J. E. Bauerle (Westinghouse), M. J. Brand (Engelhard), E. J. Cairns (LBL), W. Feduska (Westinghouse), D. Fee (ANL), F. Gme indl (DOE/METC), D. Q. Hoover (Westinghouse), J. Huber (DOE/METC), C. D. I acovangelo (GE), $H$. Isaacs (BNL), A. O. Isenberg (Westinghouse), B. King (NASA Lewis Research Center), K. Kordesch (U. of Graz, Austria), A. K. Kush (ERC), R. M. Latanision (MIT), S. K. Lau (Westinghouse), A. Leonida (ERC), G. Liu (Dow Chemical Co.), 0. Lindstrom (Volvo, Inc., Stockholm, Sweden), R. Meredith (DOE/CE/ESR), L. Paetsch (ERC), D. Pierce (ANL), E. Pigeand (ERC), C. A. Reiser (UTC), R. Rosey (Westinghouse), P. Ross (LBL), R. J. Ruka (Westinghouse), D. W. Sheibley (NASA Lewis Research Center), M. Simnad (USCD), P. Singh (ERC), S. C. Singhal (Westinghouse), S. Srinivasan (Inst. of Hydrogen Systems, Ontario, Canada), J. Taylor (Physical Sciences, Inc.), J. Werth (Engelhard), G. W. Wiener (Westinghouse), E. R. Williams (UCSD), and C. M. Zeh (DOE/METC). 
The productive use of energy with emphasis on electrical energy during the past one hundred years has been a major factor in the improvement of the quality of life for people living in the industrialized world. For a long time, until the early nineteen seventies, energy use was facilitated by its very low cost. In the past decade, however, there has been worldwide a rapid rise in the real price of energy. This increase has manifested itself in the industrialized world in lower growth rates and a small but perceptible decrease in the quality of life as people are forced to use a larger portion of their income to finance basic primary and secondary energy requirements. In some countries of the Third World, huge and politically hazardous debt accounts are consequence of energy costs.

To return to the pre-70s era with respect to energy, in terms of how much time a person must work to buy a kWh or BTU, may not be possible. Clearly, however, we can and should seek to obtain greater output from existing energy inputs. Reasonable measures to aid conservation have been taken in order to reduce input requirements to the energy system. It is now necessary to improve the basic energy structure itself. This goal can be achieved by the development of entirely new technologies, which are intrinsically more efficient and of lower cost than currently used energy-utilization methods. Implementation of this goal is especially important in the generation of electricity.

A very attractive technique for reducing the amount of energy required to produce a specific amount of electricity involves the use of fuel cells (FCs). Furthermore, the use of FCs offers the potential of lowering the cost of electricity. Reducing required energy inputs and lowering the cost of electricity are related but separate issues. Both are important. Reducing the required energy inputs decreases the need for energy imports and also the amount of domestic GNP required for new energy production or utilization. These advantages, in turn, improve the balance of payments and free resources for other uses. Reducing the cost of electricity will benefit consumers and their utility suppliers. Japan, a country which imports virtually all of its energy and is very cost-conscious in its manufactured exports, has realized the impact of both of these factors and has launched a major national FC program.

The potential benefits of $F C$ development and use are the result of unique FC properties. The FC is an electrochemical device which produces electricity directly from the galvanic oxidation (combustion) of a fuel. The usual steps involving primary conversions to heat and mechanical energy are omitted. The theoretical FC efficiencies are not limited by the Carnot cycle and may be very high. Actually achieved efficiencies in electricity production with FCs exceed those of conventional methods for power generation. For example, FCs using natural gas today produce electrical energy at a conversion efficiency of $40 \%$ and will in the future have efficiences up to $65 \%$. Coal-fueled FC systems with comparable FC technology are expected to reach the 35 to $60 \%$ efficiency range. The U.S. average for electric power generation is currently about $33 \%$. For a new system, a doubling of fuel efficiency will reduce the required primary energy inputs correspondingly, whether imported or domestic fuel supplies are used.

A second important characteristic of the FC is the relative independence of power-plant efficiecy on power-plant size. Thus, a 500-kWe power plant may have the same efficiency as a $500-M W_{e}$ power plant. This fact has a very large impact on utility purchase of FCs. In the sixties and seventies, electric utilities in the U.S. and el sewhere began to purchase power-piant units of very large size. One thousand $M W_{e}$ coal and nuclear plants were designed and built or almost built. Unfortunately, nuclear power plants in the U.S. have required 10 to 15 years for construction and the ir costs have often escalated substantially. Moreover, because of the long required lead times, planning to meet future needs has been very difficult. With the FC, smaller units of a standardized modular construction can be added to the grid in a short period of time without sacrificing efficiency. This fact greatly reduces utility financing problems and thus directly improves the financial well-being of the rate payer, who now will only pay for the amount of generation equipment actually needed by his utility. As al ready noted, because of the high FC efficiency, the cost of electricity is expected to be less than for competing systems, regardless of FC system size. 
Because FCs can be made in a variety of sizes, they may be placed at different locations on the grid system, thus allowing the utilities, in some instances, to reduce transmission costs. This advantage is particularly important in congested urban centers, where needed transmission and distribution facilities are expensive to install. Since FC systems operate efficiently at part load, their use may be tailored to actual requirements. Furthermore, FC systems are environmentally higily acceptable. Acid emissions and the resulting air pollution are reduced by several orders of magnitude compared with conventional fossil-fuel-fired generators. Because of this desirable property, FCs may be located anywhere.

The siting advantages provide the opportunity to locate FCs near points of use and, therefore, allow utilization of the waste heat produced by the FCs for such desirable purposes as space heating, water heating or absorption cooling. This last feature has led to interest by U.S. gas utilities and consideration of the use of FCs in conjunction with their extensive gas-distribution systems. The combined use of electricity and heat may result in fuel-utilization systems with overall energy efficiencies of $\sim 90 \%$. Point-of-use FC systems are al so attractive for industrial cogeneration.

Estimates made of the cost of electricity suggest that natural-gasfueled FC systems could produce electricity for about $6 \$ / \mathrm{kW}_{\mathrm{e}} \mathrm{h}$, which equals about half of the interest costs alone for many new nuclear plants.

The desirable flexibility in constructing cost-effective, dispersed power plants will also increase the nation's security in the event of war.

In the succeeding Chapters 1 to 5 , we present overviews on FCs in general and on each of five distinct $F C$ systems that are currently available or under development. There are a number of competing technical approaches and some of the FCs that are at relatively early stages of development offer the best future prospects for higher efficiency and lower system costs. This is the normal sequence of development as technology evolves and is improved. To bring the new FCs to successful commercialization will require coordinated efforts of government, industry, utilities, and universities. Achieving this goal will provide the U.S. with a valuable product for internal use and for export. The potential, long-term capital value of FC equipment sales is very large ( $\$ 10$ billion per year) and therefore merits U.S. attention. 


\section{SUMMARY OF RESEARCH RECOMMENDATIONS}

\section{S-I. Advantages of Fuel Cells}

Compared with other electricity-generating systems that are in current use, fuel cells (FCs) offer the following potential advantages: substantially higher conversion efficiency of fuel energy to electricity, modular construction, high efficiency at part load, minimal siting restrictions, potential for cogeneration, and much lower production of pollutants (including acid-rain precursors). The anticipated results of effective fuel-cell commercialization will be reduced fuel and capital costs, cleaner environments, and hence lower costs to users of electricity.

\section{S-II. Commercialization Schedules}

The current (early 1985) approach to commercial development and relative funding requirements for fuel cells are summarized in Fig. S-1. Phosphoric acid fuel cells (PAFCs) are seen to be within a few years of commercialization for both utility and on-site applications, whereas the molten carbonate fuel cells (MCFCs) and solid oxide fuel cells (SOFCs) could become available 7 to 9 years later. Commercial applications of alkaline fuel cells (AFCs) and solid-polymer-electrolyte fuel cells (SPEFCs) could follow PAFCs by 10-14 years if a decision is made for development.

We estimate the desirable level of basic supporting research for FCs to be $10 \%$ of total $R \& D$ effort after development work begins, which is in accord with industry norms for hightechnology, high-risk programs. Prior to initiation of development for commercialization, a critical level of effort must be supported that will depend on FC-type and on the perceived urgency for introducing an alternative or complementary technology into the market.

\section{S-III. Research Priorities for Selected $F C_{B}$}

Research priorities are summarized for five selected FCs. Supporting documentation for these recommendations will be found in Chapters 2 to 6 , respectively.

\section{Acid Fuel Cells}

The phosphoric acid fuel cell (PAFC) is the most mature FC (see Fig. S-1) in terms of technological advancement and readiness for commercialization in near - and medium-term applications. PAFCs have been under development for about $20 \mathrm{y}$, and it is estimated that the total investment to date from all sources is $400-500$ million dollars. The PAFC was selected for development as the most viable acid FC type because of its superior and unique stability characteristics and despite its inherently poor ionic properties. The major driving force for its dominant position has been the widespread view in the U.S. that it alone among the lower temperature FCs shows relative tolerance for reformed hydrocarbon (HC) fuels (steam raised in the $\mathrm{FC}$ is used for reforming, $\mathrm{CO}$ is removed by a shift reaction, and rejection of $\mathrm{CO}_{2}$ occurs naturally by acid).

Significant improvements in the performance, cost, and durability of PAFCs have been realized during their development. The promise of continued improvement with important commercial implications exists to this day. Improvements have involved all aspects of PAFC development, from basic electrochemistry to overall system optimization. Crucial accomplishments in the emergence of PAFCs as a commercially acceptable power system have involved the qualification and exploitation of carbon materials as the backbone of the fuel-cell stack, reduction of electrocatalyst platinum (Pt) loadings by more than an order of magnitude with the substitution of highly-dispersed, carbon-supported catalysts for the Pt-black types used previously, and elevation of the operating temperature by $60-80^{\circ} \mathrm{C}$ to about $200^{\circ} \mathrm{C}$, which has resulted in significant augmentation of cell and overall system efficiencies. For larger PAFCs of the type directed toward electric utility applications, the development of pressurized systems has further improved efficiency and, hence, economic attractiveness.

Despite the specified significant advances in PAFC technology, the incentive for ongoing and further improvements is great. PAFCs are now projected to establish a significant niche in the electric-and gas-utility markets and other application areas by providing benefits in terms of fuel savings, environmental impacts, and packaging and siting logistics. However, the total market penetration for PAFCs will be dictated by hard economic decisions, and further technological advances are likely to have a major effect on the economic attractiveness of the PAFC relative to available competing systems. For electric utility application, it has been estimated at EPRI that an FC efficiency improvement of $10 \%$ will increase market penetration from about $6-7 \%$ to about $16 \%$. Efficiency improvements of this magnitude have actually been achieved 


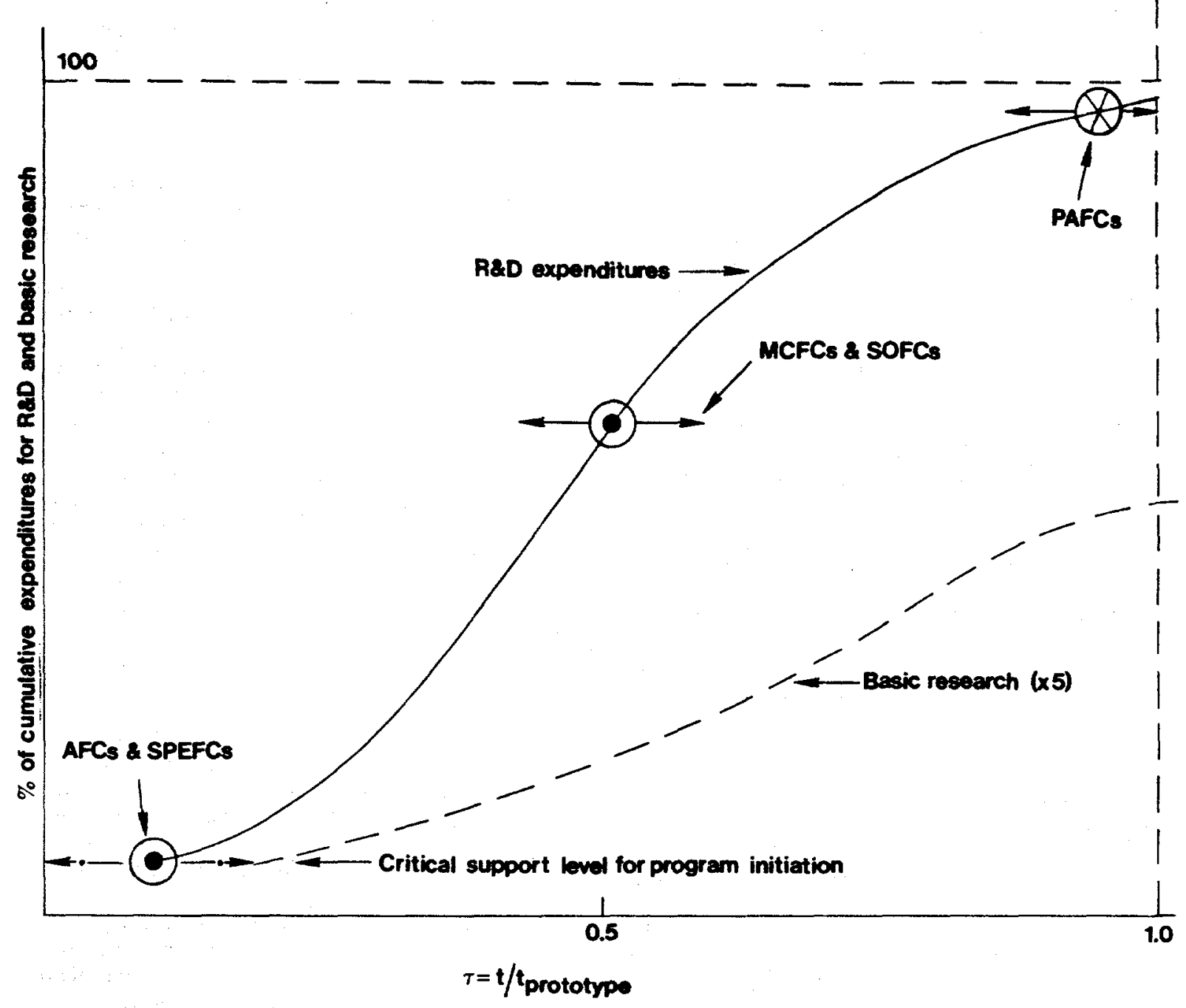

Fig. S-1. Percentages of cumulative costs are plotted vs. fractional time $\tau$ required for FC development (solid curve); for PAFCs, tprototype development $\sim 16 \mathrm{y}$. Also shown are our estimates at $10 \%$ of total $R \& D$ costs for needed basic research (dotted curve); in order to facilitate visual display, the scale for basic research has been augmented by a factor of five relative to the total $R \& D$ scale. Prior to development for commercialization, research programs of critical size (dotdash curve) are needed to support alternative or complementary FC developments; $\mathrm{PAFC} s=$ phosphoric acid $F C_{s} ; \mathrm{MCFCs}=$ molten carbonate $F C_{s}$; SOFCs = solid-oxide FCs; AFCs = alkaline FCs; SPEFCs = solid-polymer electrolyte FCs. Horizontal arrows indicate uncertainties in time. 
during the past 3-4 years. These same technological advancements will also solidify the markets reached with PAFCs during the early years of sales, while costs reflect a condition far up on the technology-learning curve. This period of time is of critical importance in establishing commercial viability for PAFCs. It could perhaps also be crucial to the sustained development effort of next-generation FCs if these show promise of operating at higher efficiencies than PAFCs. There are substantial incentive to identify research areas with aignificant potential impact. The following important research areas are recommended for preferred consideration.

\section{A. Electrocatalysts (Priority I) ${ }^{\dagger}$}

Improvement in cathode-catalyst activity will lead to increased cell efficiency, without negatively impacting the remaining FC system in any way. At is sue are inherently poor electrode kinetics at the PAFC cathode and the lack of under standing of the oxygen-reduction mechanisms that determine the efficiency of this process. It should be noted that some Pt-alloy catalysts have recently yielded significantly improved cathode activity over that of Pt-only catalysts, but there is no consensus on the mechanisms that cause the changes. The incentive for this work is a substantial reduction in cathode overpotential, which is large $(\sim 0.4 \mathrm{~V})$ and the dominant source of voltage 108s. Studies to elucidate the nature of the oxygen-reduction mechanism should continue to be encouraged, together with novel catalyst aystems, including transition-metal macrocycles. Syatems which eliminate the need for precious metals are desirable, but only if they offer substantially equivalent performance while reducing net costs. For the anode, catalyst systems are needed with greatly improved toler ance to carbon monoxide (CO) and sulfur (S) compounds.

The long-term stability of the carbon-based catalyst-support material used in PAFCB at current cathode potentials, temperatures, and pressures is a matter of concern. A significant improvement in cell efficiency resulting from higher cathode activity will exacerbate this problem, as will improvements resulting from operations at increased temperatures or pressures. It is therefore recommended that parallel research be pursued to develop improved cathodesupport materials.

\section{B. Electrolytes (Priority I)}

Although phosphoric acid (PA) has clearly been the acid electrolyte of choice because it has acceptable stability, volatility, compatibility, and capillary properties, its electrode kinetics properties are poor. Studies with fluorinated sulfonic acid electrolytes have demonstrated subotantially improved electrode kinetic activity over that exhibited by PA. The potential improvements are of the same order as those sought by developing advanced cathode catalysts. However, no acceptable alternative electrolytes have thus far been found that offer oignificant improvements while retaining the other requi site electrolyte properties.

Some synthetic acid systems have been identified as having promise of showing superior electrode kinetic properties with acceptable stability characteristics; the latter are presumed to exist because of structural analogies with PA. It is recommended that these and other potentially ouperior acid systems receive attention for the purpose of developing improved acid cells.

\section{Electrode Structures (Priority I)}

Current PAFC technology employs a teflon-bonded catalyst layer, which allows catalyst particles to be wetted by the electrolyte while sustaining gas-filled macropores for internal transport of reactant species. Mathematical modelling of catalyst-layer function suggests that, under typical cell-operating conditions, a large fraction of the active-metal crystallites may be underutilized. Although there have been some tentative indications that geometrical alteratione can at least temporarily improve performance, the potential for significant improvements appears to lie with materials alterations. For example, bifunctional polymers incorporated into the electrode structure can be visualized as being both wetproofing and electron-conducting agents; similarly, proton-conducting polymer can be employed in a dual-electrolyte configuration. These modification. would affect the nature and the intimacy of the catalyat/electrolyte/wetproofing-agent morphology and interactions, with the potential for significant improvements in catalyst utilization and, hence, efficiency. It is recommended that promising new materials approaches to electrode structure, as selected on the basis of supportive modelling studies, be supported in an advanced $R \& D$ program.

An important program need is a usable theoretical approach to the theory of the diatribution of electrochemical activity in porous electrodes, with special reference to the relations between such activity and the detailed structuring of pores. The aim of this work should be the formation of practical design equations.

'Priorities have been ranked only within cell types and not across cell groups. For a given cell type, priority I is more urgent than priority II. 


\section{Direct HC Oxidation (Priority I)}

Since the earliest FC developments, it has been recognized that a direct HC anode (i.e., one not requiring external reforming of the fuel). would be very attractive because it would improve system efficiency, reduce system cost, and reduce size. However, in order for these features to be realized, it is necessary that $F C$ anode performance is not substantially inferior to anode performance with reformed fuel. Unfortunately, there has been little success to date in this development effort. Most of the past work has focused on methanol as fuel, and the potentials at realistic current densities have been at least $100.200 \mathrm{mV}$ lower than needed to compete effectively with indirect HC systems. Nevertheless, the potential attractiveness of direct oxidation remains as a strong incentive for further research. It is recommended that credible new approaches to direct HC oxidation be supported.

\section{Alkaline Fuel Cells (AFCs)}

Alkaline fuel cell (AFC) technology was developed in the early 19608 for the NASA space program. AFCs powered all of the life-support systems in the Apollo spacecraft. The development of AFCs for terrestrial uses is less advanced than that of PAFCs and the high-temperature FC systems because of electrolyte sensitivity to $\mathrm{CO}_{2}$. There has recently been only a very small effort on AFCs in the US and Japan and limited work in Europe; however, during the sixties, there was a substantial worldwide effort. Terrestrial applications of AFCs lag a decade or more behind PAFCs. AFCs should properly be viewed as alternative and complementary systems that are near the beginning of the FC development cycle.

Operation of $A F C s$ requires the use of pure $\mathrm{H}_{2}$ or cost-effective removal of $\mathrm{CO}_{2}$ from $\mathrm{HC}$ fuels. Research on hydrogen storage and on economically acceptable methods for $\mathrm{CO}_{2}$ removal is of concurrent importance with the design of improved AFCs.

High energy efficiency achieved with $F C_{s}$ will be an incentive for their future use as automotive power plants. Any major penetration into the transportation market requires elimination of Pt-group metals as electrocatalysts. AFCs offer the best prospects among the low-temperature FC systems of finding substitutes for noble metals. In addition, $A F C_{B}$ have the following advantages over PAFCs: (a) higher power capability and potentially higher energy efficiency because of higher rates of oxygen reduction; (b) lower operating temperature and hence better materials tolerance; (c) better performance, which offers the possibility of electric vehicle (EV) operation without hybridization with a battery and a faster start-up time.

\section{A. Alter native Electrocatalysts (Priority I)}

Work should be done to find non-noble metals and their alloys, single and mixed metal oxides, macrocycles, perhaps bio-oriented catalysts, and catalysts for peroxide decomposition.

These studies should include examinations of carbonate-bicarbonate-hydrate metals and solid polymer electrolytes. The studies should be aimed at finding systems that reject $\mathrm{CO}_{2}$ and $\mathrm{H}_{2} \mathrm{O}$ and systems with lower $\mathrm{pH}$, which provide better tolerance for catalysts such as macrocycles that have long-term stability in electrolytes with $\mathrm{pH} \sim 10$. Finally, systems should be sought which have acceptable stability for operations at temperatures somewhat higher than $80^{\circ} \mathrm{C}$. These studies should include examinations of systems to remove $\mathrm{CO}_{2}$ from $\mathrm{CO}_{2}-\mathrm{H}_{2}$ mixtures.

Kinetic and mechanistic studies should be aimed at finding materials to catalyze $\mathrm{O}_{2}$ reduction, mainly via a 4-electron path and with particular stress on the important characteristic of lowering the Tafel slope, in addition to increasing the exchange-current density. Good performance is sought, as a function of time, under conditions of continuous and intermittent operation. Studies are needed of synergistic catalyst-support effects.

Special attention should be given to a model interpretation of the key coefficient $(a)$, which determines the Tafel slope and thus the effect of polarization on rate and efficiency.

\section{B. Basic Electrode Structures (Priority I)}

Understanding is needed of presently-used electrode structures and innovations are required to improve the performance of these structures. Studies are needed of wetting properties leading to optimization of electrolyte distribution. Electrode modelling should be done and directed at the development of design equations.

\section{Materials Research (Priority I)}

Stability of cell materials is one of the main limitations of present AFCs. Lifetimes must be extended to $40,000 \mathrm{~h}$. Work should be done to obtain modified, doped carbons with improved characteristics. New materials such as electronic conducting polymers need to be considered, particularly for bipolar cell types. 


\section{Cell Design (Priority II)}

Improvements in cell design should be aimed at development of bipolar systems, minimization of ohmic losses, heat and water management, and evaluation of circulatory electrolyte Bystems.

New, low-cost, stable supports are needed for the more expensive catalysts; studies should include work on carbons, carbides, nitrides, borides, and oxides as catalyst supports.

\section{E. Hydrogen Storage}

Because of stress laid on the difficulty of removing $\mathrm{CO}_{2}$ from mixtures arising from the reforming of $\mathrm{HCs}_{3}$, the use of $\mathrm{AFCs}$ has often been associated with the availability, on a massive scale, of pure $\mathrm{H}_{2}$. This fuel may become available in the future from the use of nuclear electricity in off-peak periods or from bydroelectric power, as at present in Canada, or, according to Japanese predictions, in 4-8 years from solar cells. In view of these possibilities, continued work on hydrogen storage is recommended; without $\mathrm{H}_{2}$, AFCs may not be practical. However, nearly all AFCWG members believe that funds other than the restricted resources allocated for FC development should be used to support research on hydrogen storage. Among possible hydrogen-containing fuels for transportation applications with AFCs is liquid $\mathrm{NH}_{3}$.

\section{Solid Polymer Electrolyte Fuel Cells (SPEFCs)}

The SPEFC was the first FC system to find practical application when it was used as the non-propulsive power plant for the manned Gemini terrestrial-orbit missions in 1963-65. Since then, the SPEFC has been substantially refined, particularly with respect to membrane reliability and power density. The present design was developed by the General Electric Company for terrestrial applications and has the following advantageous features: relative simplicity, $\mathrm{CO}_{2}$ rejection, and moderately high cell voltages at acceptable power densities. Nevertheless, this system presently exhibits the following shortcomings, which render terrestrial non-military applications impractical: (a) high cost of the polymer membrane electrolyte $\left(\sim 400 / \mathrm{m}^{2}\right.$ or $\$ 200 / \mathrm{kW}$ ); (b) high Pt loadings for the cathode and a node (the combined loading is $8 \mathrm{mg} / \mathrm{cm}$ or $\approx \$ 450 / \mathrm{kW}$ for the catalyst alone); (c) satisfactory operation only on $\mathrm{H}_{2}$ with very low $\mathrm{CO}$ content (S I Ppm); (d) relatively low operating temperatures $\left(<100^{\circ} \mathrm{C}\right)$, which severely restrict the use of cell heat for fuel processing to produce $\mathrm{H}_{2}$.

The low tolerance of the $\mathrm{Pt}$ anode catalyst to $\mathrm{CO}$ with $\mathrm{H}_{2}$ fuel would be relaxed if the operating temperatures could be increased to about $150^{\circ} \mathrm{C}$ or if an effective, CO-tolerant alternative to $\mathrm{Pt}$ can be found. The vapor pressure of water with the presently used proton-conducting Nafion membranes is too high for operation at temperatures much above $100^{\circ} \mathrm{C}$, without de hydrating the membranes with the almost complete loss of conductivity. Pressurization of cells permits operation at somewhat higher temperatures but, to achieve temperatures of $\sim 150^{\circ} \mathrm{C}$, requires pressures that are impractically high for most applications. A more promising approach is identification of modified or new membrane materials.

If the indicated shortcomings can be overcome, the SPEFC system may be competitive with the PAFC for electric utility dispersed-power-plant applications and, provided catalysts other than noble metals can be found, superior for vehicular applications, including the personal transportation vehicle. The SPEFC system as a whole is probably about a decade behind the PAFC in the development for commercial use. Nevertheless, the potential transportation application justifies long-range research in support of this $F C$ system. The following is a listing of long-range research areas that have been identified.

\section{A. Polymer Membrane Electrolyte Research (Priority I)}

New and modified cation-exchange membranes (proton conductors) should be developed, offering the promise of lower cost and operation at temperatures $\geq 150^{\circ} \mathrm{C}$, with reasonable conductivities and affording reasonable kinetics for cathodic and anodic reactions with available electrocatalysts. This research should include the synthesis of new membrane materials and their electrochemical evaluations. A relatively large industrial effort is already in progress to find new, lower-cost membranes for the chlor-alkali industry and other applications. This work may become a source of promising new membrane structures for the SPEFC. Careful electrochemical evaluation will be necessary under conditions that are suitable for use in advanced SPEFC

For stability reasons, the most promising new polymeric materials are likely to involve fluorinated organic structures. Inorganic proton conductors should also be considered. Specific types of materials, which are possible candidates for electrolytes, include: (a) fluorinated organic proton-conducting polymers with alternative acid groups to the presently used sulfonic acids (e.g., phosphoric, phosphonic, phosphinic, silicic acids); (b) Nafion-type membranes (sulfonic acid groups), impregnated with very concentrated $(\sim 85 \%)$ phosphoric acid or other highly conducting acids to suppress the vapor pressure of water in the inverse micellar structure, 
while still retaining sufficient conductivity and stability at temperatures $2140^{\circ} \mathrm{C}$; (c) gelled ionexchange type proton-conducting polymeric materials; (d) gelled concentrated-acid electrolytes with adequate conductivities and stabilities at elevated temperatures (e.g., borophosphates, borofluorosulfonic acids); (e) multilayer polymeric proton-conducting membranes (e.g.., polymer membranes with different anolyte, bulk and catholyte layers, optimized for low electrode polarization while retaining high conductivity and low leakage of $\mathrm{H}_{2}$ and $\mathrm{O}_{2}$ between the anodes and cathodes; (f) proton-conducting inorganic solid membrane materials such as heteropolyacids (e.8.. polymolybdates and tungstates), including dispersions of these in teflon and other fluorinated polymer matrices.

The inorganic materials may be capable of operation at much higher temperatures than organic polymeric materials and offer the possibility of direct oxidation of methanol and other fuels. These are an extension of the SOFC concept but involve proton conductors rather than oxide-ion conductors.

\section{B. Electrocatalysts for SPEFCs (Priority I)}

\section{a. Cathode Electrocatalysts (Air Electrode)}

In contrast to oxygen cathodes in conventional liquid electrolytes, little information is a vailable concerning the factors controlling the polarization of Pt-catalyzed air cathodes. These studies are necessary as a first step in achieving more effective utilization of $P t$ and finding effective, lower-cost alternative catalysts to Pt (e.g., oxides, transition-metal complexes including macrocycles, and non-precious metals). Some catalysts may be stable in the polymeric electrolytes, while lacking adequate stability in concentrated phosphoric acid or KOH. This fact may make it possible to use some of the highly active macrocyclea which catalyze the 4-electron reduction of $\mathrm{O}_{2}$. Kinetic-mechanistic studies will be required with the se promising electrocatalysts in order to optimize them with respect to activity and long-term stability. Research on electrocatalysts for SPEFCs should include supported catalysts on various stable substrates.

\section{b. Anode Electrocatalysts}

The recommendations for the anode catalysts are similar to those for the cathode with $\mathrm{H}_{2}$ fuel. In addition, research on electrocatalysts for the direct oxidation of metha nol and other fuels should be initiated if new proposals are made on how to accomplish oxidation at reasonable potentials and current densities. Platinum is not sufficiently active at temperatures below $200^{\circ} \mathrm{C}$ and new ideas are needed before further research is initiated.

\section{Electrode Structures (Priority II)}

The electrode structures presently used in SPEFC $B$ do not appear to be near-optimal designs. Relatively little research has been reported on the se electrodes and the relation of transport processes to structures is not clear. It is questionable if substantial fractions of the catalysts are accessible to the chemical reactants and electrolyte, and are also in electronic contact in the present electrode structures. Specific research recommendations include: (a) characterization of the structures of existing SPEFC electrodes; (b) studies of $\mathrm{O}_{2}$ and $\mathrm{H}_{2}$ transport and electrolyte access to the catalysts in existing structures, including modelling;

(c) innovative development of more effective electrode structures.

\section{Innovations in Cell Design (Priority II)}

If improved membranes and electrocatalysts can be realized for the SPEFC, then it would be desirable to consider innovations in overall cell design. As an example, the monolithic structure proposed for the SOFC might be applicable also to the SPEFC. It is conceivable that a monolithic, all-polymer FC can be developed with polymer anodes, polymer cathodes and polymer electrolyte. The progress of this work is clearly contingent on new and innovative ideas.

\section{Molten Carbonate Fuel Cells (MCFC8)}

The MCFC, following a very intensive development effort during the last eight years, may now be about 7 to 9 years away from commercialization, depending on the particular application involved. An important attraction of the MCFC is the simpler overall plant design, made possible by its ability to accept $\mathrm{CO}$ and $\mathrm{CO}_{2}$ as well as $\mathrm{H}_{2}$; for this reason, a lower first cost of the overall MCFC plant is projected than for the PAFC, while still retaining high efficiency. The other inherent advantage of the MCFC is its favorable ratio of high-quality heat (above $550^{\circ} \mathrm{C}$ ) to total energy. This feature may open up a significant market for stand-alone applications, with the possibility of internal reforming of natural gas followed by early commercialization of MCFCs operated at atmospheric pressure and at a wide range of sites. There remain 
several technical difficulties that must be resolved before successful commercialization can occur. These relate to improved endurance and performance.

a. MCFC Endurance

Small amounts of electrolyte are lost from cells and stacks by corrosion and vaporization. The present stability of electrodes is not adequate for $40,000 \mathrm{~h}$ of operation under pressure; however, their stability under non-pressurized operation may be satisfactory for periods approaching this goal. Long-term creep under compressive stress and corrosion of non-electrode cell and stack parts are matters of continuing concern. Long range research is needed to understand the mechanisms of corrosion reactions. If the operating temperature could be lowered, cell endurance would be greatly improved (especially if it is limited by corrosion), even though overall performance would be somewhat less than at $650^{\circ} \mathrm{C}$.

\section{b. Performance}

Electrode performance, though adequate at present, can be further improved by optimization of electrolyte composition. Significant improvement in performance and lower firstcosts would be possible if direct $H C$ oxidation could be achieved. The effect of $S$ and other contaminants puts aignificant limitations on feed-gas quality. Improving the cell tolerance to contaminants would reduce the costs of cell clean-up and simplify the overall system. The quantitative effects of various structural and operating conditions on performance decay are poorly understood.

Although key issues are addressed, to some extent, in the present development programs, the needed fundamental research to improve understanding and solve the critical problems efficaciously is missing in many areas because of lack of funding. A stronger fundamental research program in MCFC development is required to define the ultimate performance possibilities and limitations of molten-salt fuel cells. This would also stimulate innovation in the important areas of improved cell materials and stack design.

\section{A. Conductive Ceramics (Priority I)}

Fundamental ceramic and solid-state science research are needed to support the development of a conductive ceramic that may be used either as an MCFC electrode substrate or electrode, or as a solid electrolyte usable at temperatures between 500 and $650^{\circ} \mathrm{C}$. Requirements include the development of alternative cathode materials but with emphasis on the interpretation of experimental results and the development of predictive theory pertaining to conducting ceramics as cathode materials.

\section{B. Electrode Kinetics and Mass Transport (Priority I)}

Elucidation of electrode kinetics is required, including studies of reaction mechanisms and transport in the electrolyte, as a function of electrolyte composition (not limited to alkali carbonates), temperature, and electrode material.

\section{Corrosion Mechanisms and Control (Priority I)}

Elucidation is required of corrosion mechanisms under representative three-phase (molten salt-solid-gas) contacts and polarization conditions, as well as modelling of corrosion rates.

\section{A Direct HC Electrode (Priority II)}

An important area for development is the design of a direct HC electrode and understanding of the kinetic issues involved in the operation of this device.

\section{E. S- and HCl-Tolerant Electrodes (Priority II)}

Development is needed of sulfur - and $\mathrm{HCl}$-tolerant electrodes, in conjunction with a sulfur - and chloride-rejecting electrolyte. The kinetic issues involved in understanding the operation of this electrode should be clarified.

\section{F. Fundamental Physicochemical and Engineering Studies (Priority II)}

(a) Fundamental physicochemical and engineering studies should include the determination and theoretical prediction of (i) gas and solid solubilities, (ii) gas and ionic transport properties, and (iii) capillary behavior of the molten electrolyte as a function of composition and temperature. (b) Of particular utility will be the development of novel performance and 
performance-decay models, in conjunction with experimental data to verify model validity. (c) The development of in situ diagnostic techniques is needed for applications to cells and cell stacks.

\section{G. Porous-Material Densification (Priority II)}

Experimental and theoretical analyses should be performed on the densification of porous materials that are in contact with gases and molten salts.

\section{Solid Oxide Fuel Cells (SOFCs)}

The solid oxide fuel cell (SOFC) has a moderately long history, with an early significant development effort emerging in industrial research laboratories in the mid-to-late 1950s. The development continued for 20-25 years at a low level. During the last five years, this effort has been expanded considerably. Currently, the major industrial development effort is located at Westinghouse. Development is at the demonstration stage for useful cell performance and endurance with a geometrically simple cell design. The present program calls for deployment at selected, technically sophisticated customers, of small laboratory demonstration test-units in early 1986. Other R\&D efforts are also in progress. A particularly noteworthy advanced development effort is focused on a monolithic cell design at the Argonne National Laboratory (ANL). This design is aimed at very high specific power and power density.

SOFCs have several characteristics that are distinct from those of other FCs. Chief among the se is operation at a temperature around $1000^{\circ} \mathrm{C}$. This feature allows electrochemical oxidation of $\mathrm{H}_{2}$ and $\mathrm{CO}$ without an added specific catalyst. Fuel versatility is a significant advantage in the use of SOFCs for some applications. The SOFC also offers the possibility of operating at higher power densities. The components of the SOFC are primarily ceramics. Although relatively cheap materials are used, the processing techniques needed to fabricate the final, composite article are very difficult to implement. Recent advances in processing techniques are viewed as major steps in advancing SOFC technology toward commercialization. The single most important need for research support falls in the area of ceramic science and involves finding improved materials and processing methods.

\section{A. Ceramic Science in Processing Technology (Priority I)}

Work on improved ceramics should focus on two size scales. These are: (a) macrostructure, where there is a need to develop processes suitable for use with new components and novel cell geometries, and (b) microstructures, where there is a need to understand atomicscale processes, which will allow creative engineering of cell components with desired pore structures or the use and/or control of sintering processes to prolong macrostructural stability. Other issues in this category include fundamental studies of multicomponent diffusion with various driving forces, segregation of impurities at grain boundaries, and solid/solid-surface interactions. Research on ceramic science is expected to have a significant and early impact on SOFC development. An important objective is advancing basic knowledge of the relation between ionic conductivity in solid oxides and their structures, with the goal of greatly increasing the number and range of substrates that may be used in SOFCs at temperatures even higher than those now employed.

\section{B. Fundamental Electrochemistry (Priority II)}

Although the complex overall cell reactions are established for the SOFC, the detailed electrochemical and chemical processes occurring at the atomic level at three-phase reaction sites (gas, electronically conducting solid, ionically conducting solid) are not well understood. At issue are such phenomena as adsorption/desorption, rate-controlling mechanisms, electrocatalysis, and the role of contaminants. The results of this research will assist in optimization of electrode microstructure and will also be applicable to near-term choices of components, such as $\mathrm{ZrO}_{2}$-based electrolytes.

\section{Materials Research (Priority I)}

In order to ensure technology improvements, it is necessary to perform studies of new materials for cell components. These studies should recognize that materials can only be used in compatible sets and that significant changes in one component may have ramifications for other parts of the cell and for the entire FC system. Research in this category should include studies of the electronic and ionic properties of oxygen-conducting electrolytes, and should include both contemporary and new electrolyte compositions. The effects of impurities, of the type that might arrive at the electrolyte by diffusion from the electrodes, should be considered. Similarly, studies are needed on the properties of new candidate materials for use as the anode, cathode, or interconnection. 


\section{Structural Modelling and Diagnostics (Priority II)}

For the purpose of obtaining novel and improved cell designs, we recommend the development of computer models for the SOFC stack. Important components of the model include kinetic processes at reaction sites, electrochemical mechanisms, and modelling of overall system performance. Models, when verified by critical measurements, provide insight and a useful design tool. Also required are detailed studies concerned with the design and optimization of electrode microstructures, from the point of view of heat transfer and kinetics. An integrated SOFC model is expected to be useful for the se studies.

\section{E. Thermochemistry (Priority II)}

The chemical behavior of cell components, under various cell environments, can often be understood in terms of thermodynamic analysis. However, some of the important required thermodynamic data for the complex phases are not known and need to be measured.

\section{F. Diagnostics (Priority II)}

Because the cell consists of layers of ceramic materials in intimate contact, it is inevitable that stresses will arise as the result of differential thermal expansion. Non-destructive techniques to measure these stresses in cells are needed, not only for quality control during production but also as research tools aiding in compatibility evaluations for new materials.

Recommendations listed under $A$ and $B$ should be initially focused on currently used materials for near-term technological advancement. Recommendation $C$ is an investment that should yield future benefits. Items $D, E$ and $F$ are generic studies that will be of general utility in both near-term and future cell designs.

\section{S-IV. Long-Range Interdisciplinary Research to Support FC Development}

In Sec. S-III, we have presented a prioritized research agenda for each of five selected FCs. Reference to this discussion shows recurring emphasis on auch topics as materials research and electrochemistry, although the particular problem areas within a given disciplinary field tend to be distinct for each FC type. Nevertheless, it is instructive to categorize the preference for studies in interdisciplinary research areas that results from examination of research needs for each FC type. The results of basic studies in the indicated interdisciplinary research areas should ultimately benefit all FC developments. We have identified nine primary interdisciplinary research areas. These are defined in the following enumeration: (1) fundamental electrochemistry, including interfacial structures, electrocatalysis, electrode kinetics, and electrode properties; (2) new materials and processes, including new catalysts, electrolytes and electrodes, as well as new procedures for the preparation of fuel-cell components; (3) corrosion, including studies of mechanisms and processes that limit cell life; (4) transport properties such as solid-, liquid-and gas-phase conductivities, diffusivities, viscosities, thermal conductivities, transport numbers, solid-state mobilities, and the development of new methods for determining these properties; (5) surface science defined as studies of surface p'lenomena in general and not included under fundamental electrochemistry, wetting and other ca illary phenomena, metal-ceramic bonding, surface-energy changes at electrodes, structure nd morphology of interfaces; (6) applications and development of new diagnostic techniques in order to gain improved understanding of fuel-cell behavior by performing incisive experimental measurements, including verification of fuel-cell models; (7) thermochemistry of materials such as studies of phase equilibria, solubilities, and solution behavior; (8) studies of electrode microstructure, including theoretical investigations of porous electrodes and utilization of the results of these investigations in the design and performance evaluation of fuel-cell electrodes; (9) novel cell concepts such as new approaches to the configuration of cell structures and their evaluations.

The immediate motivation for research support in the nine listed areas is based on their perceived importance in contributing, over the long-term, to the successful commercialization of improved cells. The relative distributions displayed in Fig. S-2 are AFCWG recommendations and serve to emphasize the evident preferred need for research on new materials and processes (2), studies of electrode microstructure (8), novel cell concepts (9), corrosion (3), and fundamental electrochemistry (1). Relatively high funding levels imply either (a) recognized long-term merit for the research or (b) need for substantial funding in order to obtain a proper assessment of long-term merit. There is a clear preference for basic investigations in electrochemistry, electrode microstructure, corrosion, and surface science for the low-temperature cells (PAFCs, AFCs, SPEFCs), whereas funding-level recommendations tend to be more uniformly distributed in all areas in support of the high-temperature cells (MCFCs and SOFC 8 ). 


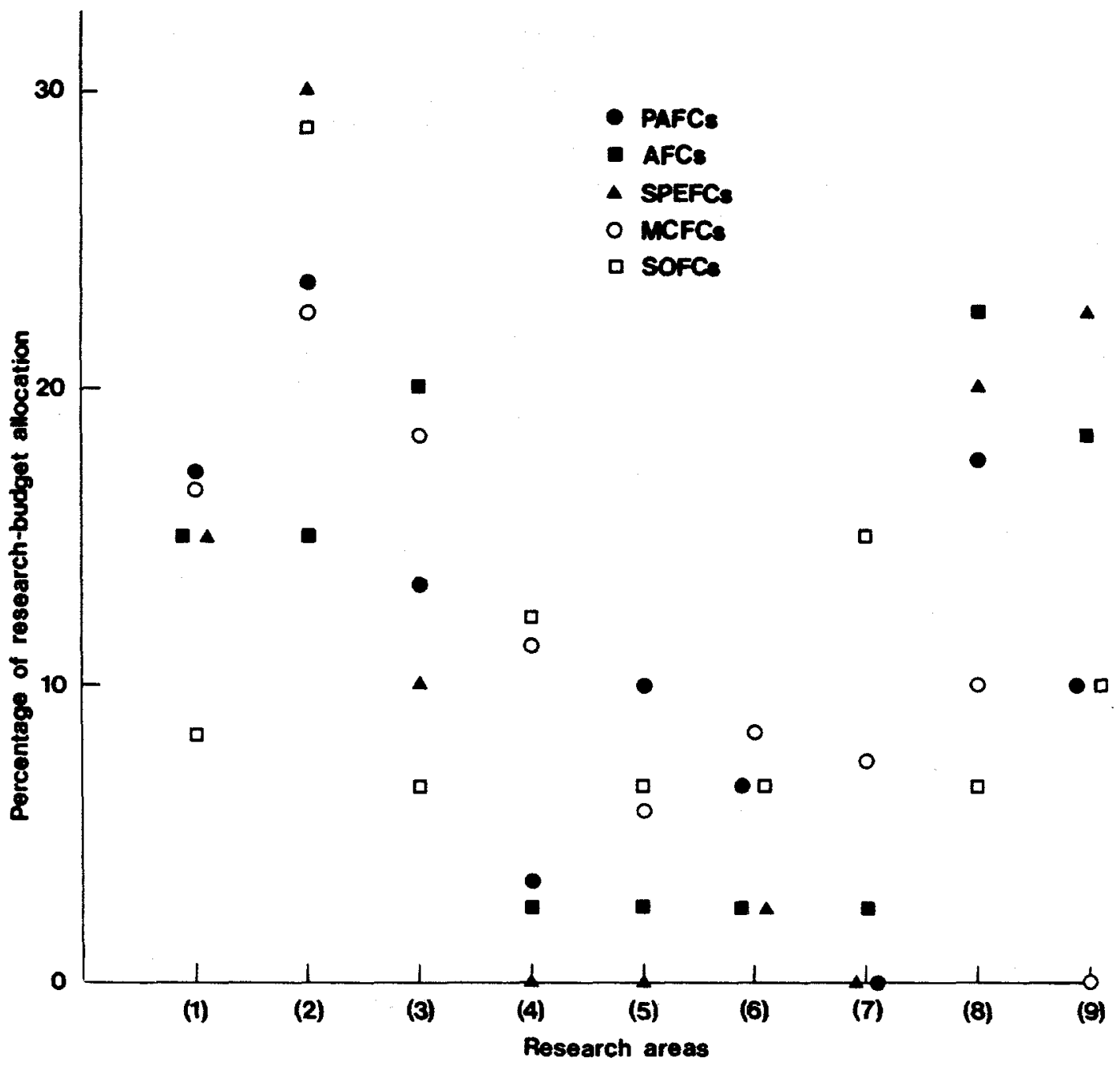

Fig. S-2. Recommended percentages of total research budgets, relating to perceived needs for specific $F C s$, are shown for allocations in identified interdisciplinery research areas. The preferred fund allocations are seen to be somewhat differently distributed for the identified $F C$ types (PAFCs, AFCs, $S P E F C s, M C F C s$, and SOFCs). The research areas are identified by numbers that correspond to the listing in the text; thus, fundamental electrochemistry is (1), new materials and processes (2), corrosion (3), transport properties (4), surface science (5), diagnostics (6), thermochemistry (7), electrode microstructure (8), and novel cell concepts (9). 
As in other fields of acience, funding for basic research will be effective only if it is stable, long-term, protected from pressures arising by unforeseen problems encountered in the developments of particular FC types, and redirected at appropriate intervals to remain pertinent to updated program objectives. While peer reviews should be carefully solicited and considered in making funding decisions for research, effective coordination with program objectives demands that strong management authority continue to be exercised.

Although there are a number of outstanding groups in industrial and government laboratories and in univer sities, at which first-rate research on fuel cells is in progress, it may be desirable not only to strengthen the competence of these existing groups but also to create several (two to four) additional research groups performing basic research pertaining to FCs. Joint funding for cooperative industry-university projects should receive preferential consideration for some but not for all of the contracts, provided the proposed work schedule clearly reflects a major commitment by the participants to advance FC science in an orderly manner, from an appropriate long-range perspective, and for the purpose of deriving ultimately improved designs and performance in FC operation.

It is una nimously recognized by AFCWG participants that the training of competent electrochemical engineers, electrochemists, material scientists, and related specialists working on FCs is an important obligation of the universities participating in these programs. This training function relating to $F C_{s}$ has been neglected in the U.S. Federal, as well as industrial funding, can contribute importantly to future FC development by supporting university-based research on FCs that involves the participation of competent people who are working toward graduate degrees.

\section{S-VI, Comments by AFCWG Members}

William E. Houghtby, International Fuel Cells, 195 Gover nors Highway, P.O. Box 739 .

South Windsor, CO 06074

\section{Comments to the Summary}

We (IFC) do not concur with the following statement appearing in the Summary under 4, Molten Carbonate Fuel Cells:

"This feature may open... at a wide range of sites."

This statement should be amended as follows:

"This feature may open up a significant market for stand-alone applications. It provides the opportunity to use stack 'waste' heat for reforming in a natural gas fueled power plant, which results in a significant improvement in efficiency for these applications. This could lead to the early commercialization of MCFCs operated at atmospheric pressure and at a wide range of sites." Supporting evidence for this revision may be found in GRI Report FCR-5196, "Evaluation of Natural Gas Molten Carbonate Power Plants" Final Report, February 1985.

IFC does not concur with the following statement appearing in the Summary under 4 , Molten Carbonate Fuel Cells:

\section{"C. Corrosion Mechanisms and Control (Priority 1):}

This statement should be amended as follows:

"C. Corrosion Mechanisms and Control (Priority 3)." Supporting evidence for this revision may be found in Chapter 5 of this document. Table 5.9-1, under Problem Status of the "Corrosion of the Cathode - Current (Collector)" and the "Corrosion of the Anode - Current Collector." 
IFC does not concur with the following statement appearing in the Summary under $4 a$, MCFC Endurance:

"The present stability of electrodes. . . may be satisfactory for periods approaching this goal."

This statement should be amended as follows:

"The present stability of electrodes is adequate for $40,000 \mathrm{~h}$ of operation for pressurized coal central station applications and for all non-pressurized applications. Significant cost savings may be realized with a more stable cathode. in stand-alone applications requiring pressurization. "Supporting evidence for this revision will be found in the April-June 1985 Quarterly Report for DOE Contract DE-AC-21-79ET 15440, "Development of Molten Carbonate Fuel Cell Powerplant Technology."

\section{Comments on Chapter 2}

Statements in Chapter 2 concerning future UTC technical approaches and offerings are the opinion of the author.

IFC (a UTC-Toshiba joint venture) has not announced the approaches or schedules to be used in a commercial offering.

\section{Comments on Chapter 5}

The suggested amendments to MCFC statements in the Summary should also be reflected in Chapter 5. 


\section{PHOSPHORIC ACID FUEL CELLS (PAFCs) ${ }^{\dagger}$}

\subsection{Introduction}

It is generally believed that the PAFC system represents a technology that is almost commercial and that no further $R \& D$ are required. This judgment is invalid. About $\$ 450 \mathrm{M}$ have been spent (in current dollars) since the $1960 \mathrm{~s}$ on AFC research, development, and demonstration. About $\$ 100 \mathrm{M}$ of this has come from electric and gas utilities, $\$ 100 \mathrm{M}$ from UTC, $\$ 150 \mathrm{M}$ from public bodies such as EPRI and GRI, and about $\$ 150 \mathrm{M}$ in recent years from DoE. Nevertheless, a great deal more research is needed to improve the product, to ensure that it will be competitive and, most importantly, introduce this technology into other commercial sectors in the future. This type of continuing $R \& D$ and product improvement has always represented the historical path by which applications of new technologies have entered the commercial world. In ge neral, new products fill an identified niche in the marketplace. Continued filling of that particular niche will then result in a sufficiently high production volume to reduce product cost, with the result that related but more competitive niches can be then tackled. A successful product will grow beyond this initial penetration area if it has advantages in displacing other technologies throughout a broader marketplace or if a need is created that did not exist previously.

In order to compete with and displace an existing technology, any new technology must be significantly more cost-effective than the state-of-the-art of the previous generation. Advantages must become apparent, consolidated, and expanded by $R \& D$. This process has traditionally been funded either by the cash flow generated during progressive market penetration or by capital raised as the result of promise for future cash-flow prospects of the new technology, or by both. On the other hand, if society sees a new use for the new technology (i.e., if the initial $R \& D$ investment has created a new need), penetration will be spontaneous and competition will arise from different compenies within the same industry rather than from different technologies. Finally, different technologies will develop competitively to serve the new need and, hence, the growth, maturity and replacement cycle will be repeated, with a new mix of technological species replacing obsolescent mixes in an evolutionary manner.

Good examples of technologies creating completely new needs in historical times have been steam engines (which created the industrial revolution and all that resulted from it), the internal combustion engine (which created personal automotive transportation and aviation) and, most recently, the integrated circuit. It is useful to attempt to look at the FC from this perspective. This discussion will serve to identify acid FC research needs and goals with longterm payoff (25-30 y or more). Future uses for FC applications may be determined as a function of performance and objectives. These, in turn, will determine research directions for achieving different goals, which will allow research funding-priority determinations for the acid FCs. When overlap occurs between projected market niches for acid and other FCs, it may be possible, in principle, to determine the better or more probable ultimate technology for each particular application. In this manner, a first approximation may be obtained for prioritizing long-term research directions for different types of FCs.

\section{2 The Marketplace for Present Acid FCs}

Current acid FCs use phosphoric acid (PA) as electrolyte with relatively clean, reformed fuels (light distillates, LPG, NG, etc.) or cleaned-up coal gas from a gasifier. The two applications envisaged for the initial commercialization effort are: (1) a dispersed power plant, in sizes of $\sim 7.5-11 \mathrm{MW} A C$, initially using reformed HCs but ultimately operating on clean coal gas (perhaps in larger units) and (2), an on-site cogeneration plant of about $0.2 \mathrm{MW}$ AC that is intended for the supply of electricity from reformed NG to commercial premises, apartments, or utility buildings, with FC waste energy used for water and space heating, absorption airconditioning $c y c l e B$, etc. These are the only major market segments that have so far been identified for cost-effective technology use of current PAFCs in mature volume production. In each sector, market penetration will be determined by the usual market-place considerations: final product cost compared with costs for competing technologies. This is an unsentimental statement that is nevertheless appropriate after considering the usual platitudes: the FC is very efficient, will conserve fos sil-fuel resources, will reduce acid rain and the greenhouge effect, will lead to a more natural energy situation in the sense of Rousseau by rejecting the Carnotcycle heat engine and introducing a more efficient isothermal energy-conversion device, etc.

† This chapter has been written by John Appleby, EPRI. The author is happy to express his appreciation to his colleagues on AFCWG for numerous helpful discussions and suggestions. 
Our conclusion is proper since the total product cost contains effective costs, as well as allowance for other advantages of the new technology. We note that the product is electricity for applications (1) and (2) and, when used in the induatrial cogeneration mode (1), electricity plus useful heat. The important assessment must therefore be based on a total cost comparison of the energy produced with that furnished by competitive technologies.

We must now identify competitive technologies and where the acid FC will find a predominant place. We will first consider the second question and attempt to identify those sectors of the energy economy for which the acid system may be suitable because it has inherent advantages. We may then compare these inherent characteristics with those of its potential competitors and thus arrive at some idea of the penetration of the PAFC into different sectors according to the organic growth predicted by marketplace considerations.

\section{3 The Acid FCs vs Competing Devices}

The characteristics of the acid FC are by now well known and may be summarized as follows: (a) very low chemical and acoustic pollution; (b) modular and hence available in small units $(200 \mathrm{~kW}$ to several $\mathrm{MW}$ ); (c) high efficiency with suitable fuels (i.e., efficiency is not limited by the Carnot cycle); (d) with correct design, the efficiency is independent of load; (e) cogeneration capability; (f) ease of siting; ( $g$ ) capability of hands-off operation leading to low labor-cost in mature units.

By contrast. PAFCs have the following disadvantages: (h) they require an inverter to produce utility quality $A C$ from DC power; (i) the only fuel that can be used directly is $\mathrm{H}_{2}$, which must contain less than about $1.5 \% \mathrm{CO}$ and 1 less than about 50 ppm of $\mathrm{S}$ as $\mathrm{H}_{2} \mathrm{~S}$ and $\mathrm{COS}$ (the sulfur compounds act as catalytic poisons); $\mathrm{H}_{2}$ must be manufactured from other suitable fuels before use, for example, by steam-reforming or gasification of coals, with suitable cleanup treatment; (j) the early units are costly, which is partially related to the high initial cost of the fuel-processing system (i) and, to a lesser extent, to (h). The characteristics (b), (c) and (d) are illustrated for FCs powered by clean fossil fuels in Figs. 2.3-1 and 2.3-2, in which we compare FC efficiencies as a function of size and load with competitive heat-engine systems. The heat-rates as a function of load for the FC system refer to a 33-MW plant (a combination of three 11-MW UTC electric utility units).

The PAFC modules require efficient fuel-processing systems to produce $\mathrm{H}_{2}$, which limits fuel choices to clean, light $\mathrm{HC}_{s}$ (NG and light distillates) and, where available, methanol. For these units, fuel cost will preclude use of the system for most baseload applications. However, at some later time, PAFCs may be widely considered for baseload use if low-S coal gas is used as fuel with larger integrated gasifier FC units. Uitimately and much later in time, PAFCs may find a place in the mobile transportation field. The degree of penetration in all markets will depend on competition.

For electric utilities, the most attractive features of PAFCs a re short required construction and installation times, modularity, efficiency, and ease of siting, probably in that order. Since FCs are modular, they can be on line and in the rate-base quickly after the decision to order has been made, thus avoiding the problem of long-term tie-up of capital that occurs with any large central station plant requiring many years for construction and licensing. An attractive additional feature of modularity is the fact that units can be ordered as the electricity market requires, thus avoiding uncertainties and often costly mistakes of long-range planning for anticipated demand. Demand must be evaluated over a $10-y$ period or longer for conventional nuclear or coal plants. In fact, one major feature of the modular $F C$ is the ability to defer investment in baseload plants into the future, especially if sudden surprises occur in load or in baseload plant costs and lead-times. A primary attraction for a utility depends on proportionally lower accounting costs, compared with conventional central power stations. As the result, we find a lower price per currently required and installed $\mathrm{kW}$ than for a $\mathrm{n}$ alternative large plant, which has lower cost per $\mathrm{kW}$ but for which the full power exceeds current demands. The importance of this advantage for a utility should not be underestimated.

The efficiency characteristics of PAFCs provide the utility with identified operating advantages. Thus, the high efficiency compared with the cheaper combustion turbines results in lower premium fuel costs. More important in the generating mix is the fact that, unlike combustion machines, PAFC efficiency is independent of load, which means that the baseload units (coal or nuclear in a typical utility) and the peaking units (combustion turbines) can always be operated at full load (maximum efficiency), with the PAFCs operating as constant-efficiency load-followers. In this way, the fuel economy of the whole utility system is maximized. Finally, successful licensing of PAFCs for use in downtown Manhattan means that the se can be sited anywhere, which results in highly advantageous credits for cogeneration, for the cost of additional power lines required with conventional equipment, etc. Flexibility in siting is a major attraction for the gas utilities. It is worth stressing that no other generating technology has this advantage. From this viewpoint, the PAFC system at the right price will have no competition, except from later-generation, more efficient, high-temperature FCs (MCFCs and SOFCs). However, the high-temperature FCs appear to be more uitable for baseload operation than for 


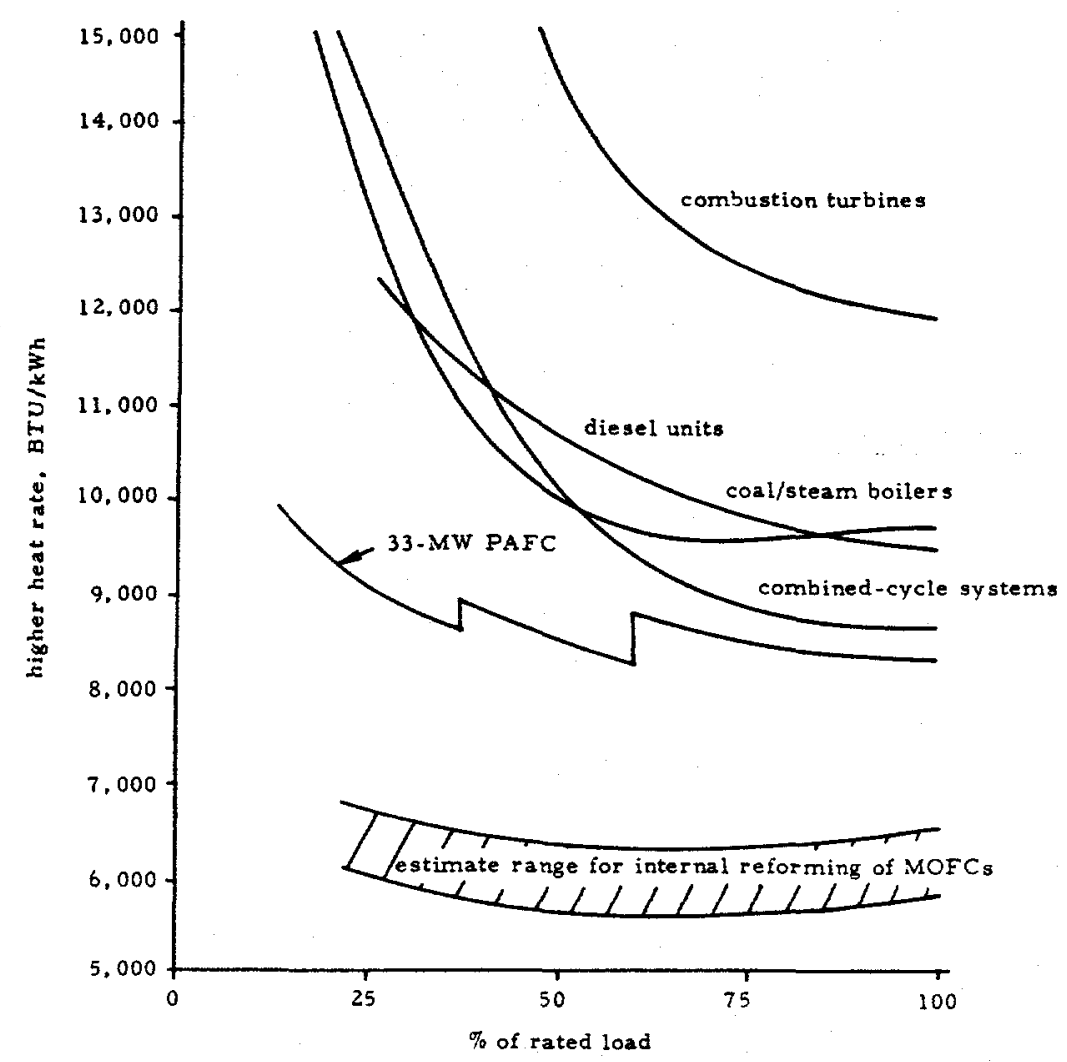

Fig. 2.3-1. Heat-rate (HHV) as a percentage of rated load for different generation technologies.

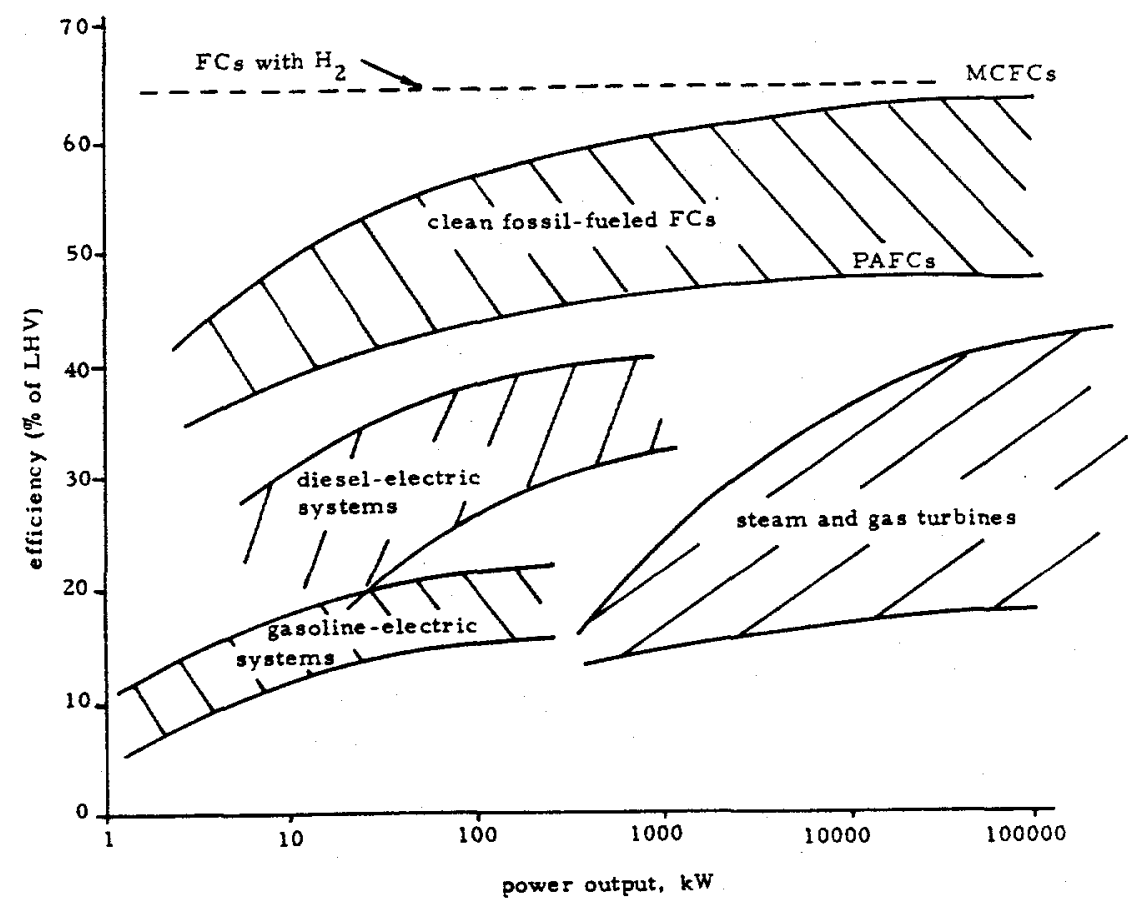

Fig. 2.3-2. LHV efficiency of different technologies as a function of scale. 
load-following because of their higher efficiencies and thermal characteristics, which may make them more difficult than PAFCs to operate at part load.

The only system which may be considered as a direct competitor of the FC for use by electric utilities is the combined-cycle unit, although we will show that, under some circumstances, the combustion turbine is also a possible competitor. Both of these systems also use premium fuel. The combined-cycle system has a high efficiency (average annual HHV heat-rates are about $8600 \mathrm{BTU} / \mathrm{kWh}$, which equals $40 \%$ efficiency, compared to about $41-43 \%$ for the commercial PAFC). Unlike the FC, the efficiency of the combined-cycle system will vary greatly with load. Its cost ${ }^{1}$ (about $\$ 450 / \mathrm{kW}$ in 1981 dollars) will be lower than that of the mature FC, for which the ultimate production cost is estimated to be 1 about $\$(1981) 600 / \mathrm{kW}$ or somewhat more than $\$(1985) 700 / \mathrm{kW}$, much of which is spent on the fuel-processing plant to produce $\mathrm{H}_{2}$-containing, high-BTU fuel. However, combined-cycle plants are large units ( $100+\mathrm{MW})$, are not easily sited in any location since they are combustion machines producing $\mathrm{NO}_{\mathbf{x}}$, and are not suitable for cogeneration. Their flexibility credits are therefore lower than those of FC plants, which helps to compensate for differences in capital cost. Provided that the FC operates within cost and efficiency specifications, there will be niches for both technologies in the marketplace. Improved, higher efficiency FCs, when they become available, will be preferable to combined-cycle plants.

\section{4 Economics of PAFCs}

When the PAFC is first introduced, its cost will be comparatively high since it will be in only limited production, far up on the cost-production learning curve. The best estimates of cost during the introductory period as an electric utility unit in the several $M W_{e}$ class are given in a recent EPRI study. ${ }^{2}$ Illustrative cost-production-rate curves are shown in Figs. 2.4-1 and 2. 4-2 for two different scenarios. The major difference between the two data sets is the effect of the (now expired) $10 \%$ cogeneration energy-tax credit to assist utilities using FCs. For the expected capital costs of the units, break-even will occur at a total installed cost of $\$ 1700 /$ $\mathrm{kW}$ and $\$ 1500 / \mathrm{kW}$, respectively, without and with the cogeneration credit, whereas break-even for electricity generation alone will occur at a total installed cost of slightly under $\$ 1000 / \mathrm{kW}$. The analysis is based on a fuel-cost model for NG of $\$ 4 / 10^{6}$ BTU for the 1982 base year, with price escalation of about $7 \%$ per annum. Actual fuel costs will be shown to be of minor relative importance sincs th.... fiect all competitive technologies. A high-heating-value (HHV) FC system heat rate of $83 \mathrm{i}$ BTU/kWh was assumed, and all costs are in 1982 dollars.

Figure 2.4-1 shows that the number of units that must be sold in order to reach an installed cost of $\$ 1500 / \mathrm{kW}$ with cogeneration but without the tax credit is $52(572-\mathrm{MW}$ ) over a period of $9 \mathrm{y}$. With the tax credit, the corresponding number from Fig. 2.4-2 is only 25 units or $275 \mathrm{MW}$ over a period of $7 \mathrm{y}$. On both plots, the slopes of the break-even costs vary with year because of the assumed escalation in fuel prices. All of these models involve uncertainties that are characteristic of the generation mix.

The first units off the production line should cost about $\$ 3000 / \mathrm{kW}$ installed and will only be economical for non-typical applications at special sites (military, remote areas, etc.). However, their development will serve market penetration for more standard commercial uses that will initially require subsidies (e.g., from the manufacturer, trade associations, federal, state, or local governments). As an example of such subsidies, EPRI intends to spend a substantial sum over the 5-y period between 1985 and 1989 to expedite commercialization.

The specified scenario is not very different from the launching of any other volume-production, high-cost, high-technology item, such as a new commercial transport aircraft. Production may continue at a loss for some years, until the product is sufficiently far down the learning curve to become economically self-sustaining. The scenarios in Figs. 2.4-1 and 2,4-2 show that, for $\mathrm{PAFC}_{3}$, a profit $w$ ill have to be made on the system in the cogeneration mode for $10 \mathrm{y}$ before a self-sustaining cost level is attained for stand-alone generation (about $\$ 970 / \mathrm{kW}$ installed, according to the economic model used). We should point out that this scenario is of heuristic value but does not represent the only way in which commercialization will occur.

If the lower cost, higher growth assumption (favorable economic climate, regulation, etc.) can be justified, the system may be economically self-sustaining for stand-alone use only $5 \mathrm{y}$ after reaching the breakeven point for cogeneration (i.e., in the early 1990s). This result is illustrated in Fig. 2.4-3, which is an overall progress chart on PAFC development.

For a very pessimistic business scenario, stand-alone breakeven will not occur until 15 y later than for cogeneration, i.e., after about the year 2010 . This scenario would probably result in the early commercial abandonment of electric utility PAFC technology in the US. The future of PAFCs must therefore not be considered to be assured. We believe that, if the acid technology fails for stand-alone electric utility generation, it will probably also fail for on-site use with limited volume production. Its fallure may also impact future application of advanced FCs.

An earlier study ${ }^{3}$ by Energy Management Associates (EMA) involved examination of FCs in the economically self-sustaining mode with similar mean fuel-cost and heat-rate assumptions to those used here, but considering PAFC $\mathrm{P}_{3}$ to be at the final point in the learning curve with an installed cost of about $\$(1981) 600 / \mathrm{kW}$, corresponding to $\$ 630 / \mathrm{kW}$ in Ref. 2 . Representative 


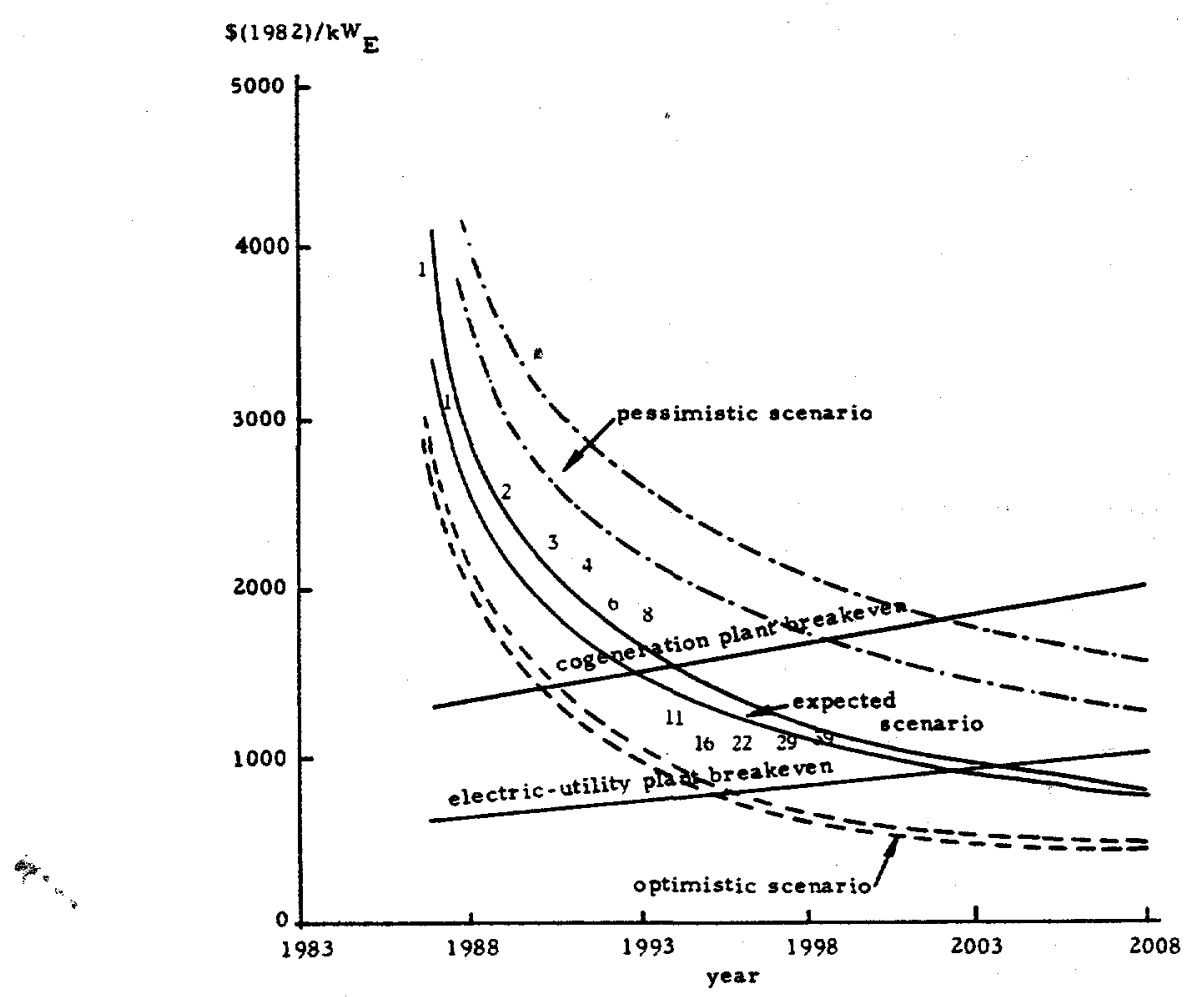

Fig. 2.4-1. Illustrative FC installed costs as functions of learning-curve position, without cogeneration credits. The numbers represent total $10+\mathrm{MW}$ PAFC units installed each year.

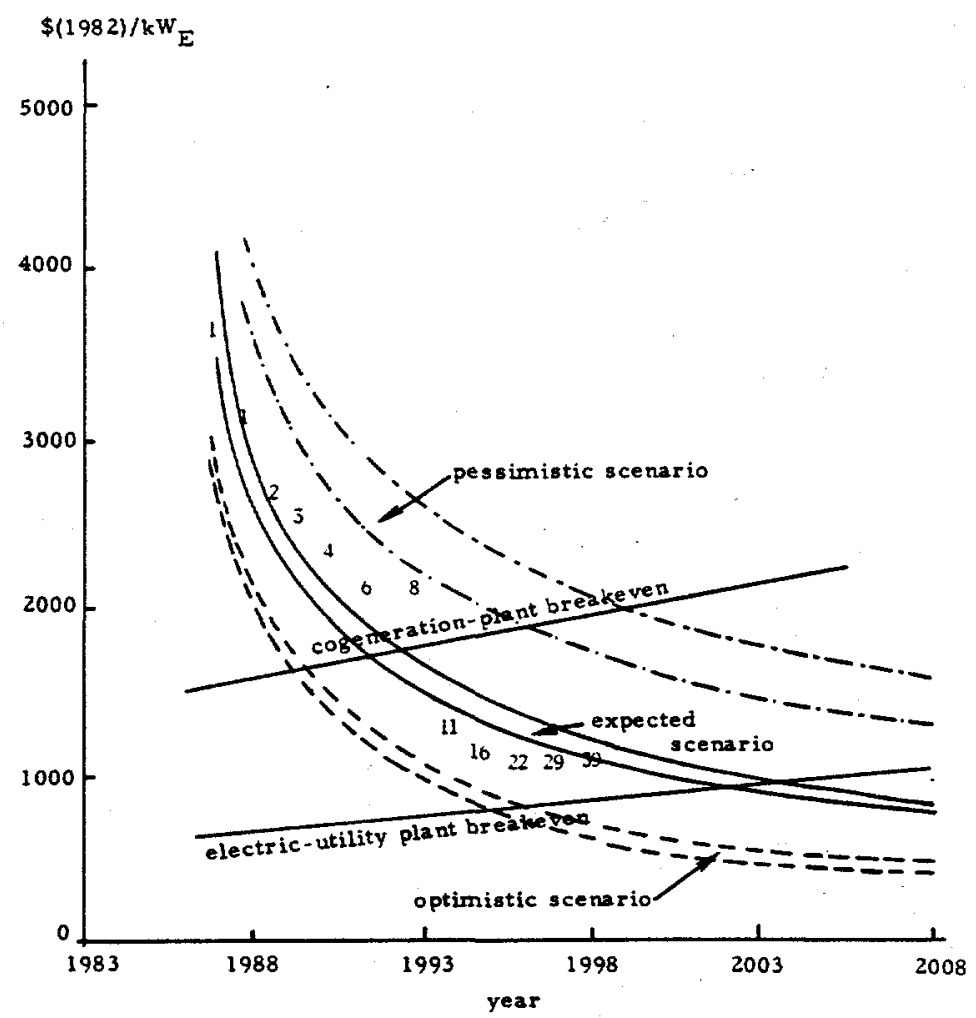

Fig. 2. 4-2. As in Fig. 2.4-1, but with 10\% cogeneration credit. 
utilities throughout the country examined their FC requirements within their expansion-planning studies for the time-frame 1990-2000, and the results were extrapolated nationwide. The combined results of Refs. 2 and 3 are shown in Table 2.4-1. It can be seen that Ref. 3 predicts a much higher demand than Ref, 2, which is to be expected considering the much higher installed costs presumed at the higher end of the learning curve in the latter study. In general, the re sults of Ref. 2 may be considered realistic to about the year 2000-2005 (see Figs. 2.4-1,2.4-2), after which production of the units will have been learned so that the Ref. 3 scenario will apply. The expected electric utility market between the years 2005 and 2015 will then be about $30,000 \mathrm{MW}$, giving a total capacity in 2015 equal to that in the center of the last column of Table 2. 4-1 or about 44,500 MW. The potential electric utility market outside the US, which will be shared with inter national competitor 8 (particularly in Japan if their present plans reach fruition), may be two to three times larger. Further details of the ultimate market as a function of FC cost and efficiency are given in Sec. 2.6.

Table 2. 4-1. Projected FC markets (Refs. 2 and 3).

\begin{tabular}{l|c|c|c|c|c|c}
\cline { 2 - 6 } & \multicolumn{5}{|c|}{ EPRI $^{2}$} & EMA $^{3}$ \\
\hline Scenario & 1988 & 1993 & 1998 & 2003 & 2008 & 2005 \\
\hline Optimistic & 60 & 500 & 3,400 & 14,000 & 30,000 & 104,000 \\
Expected & 50 & 300 & 1,600 & 6,000 & 14,000 & 44,500 \\
Pessimistic & 40 & 175 & 650 & 1,850 & 4,000 & 5,700 \\
\hline
\end{tabular}

If the highly optimistic scenario used in Figs. 2.4-1 and 2.4-2 (high utility growth rates, high tax credits, favorable regulation, optimistic FC costs and heat-rates) continues to hold through the early years of the next century, the capacity may be as high as 100,000 MW by 2018 . On the other hand, a poor business cycle and no credits may result in only a small total capacity (5700 MW in 2018), which will result in a yearly production rate that will not be sufficient to reduce costs quickly and this scenario will probably lead to the abandonment of PAFCs for commercial use. For this reason, a continuous program of product improvement will be needed during the early production period and beyond, so that costs can be brought down rapidly, for example, by the use of new materials that allow higher power densities and by the development of systems with higher efficiencies. Competitive systems (e.g., advanced gas turbines with combined cycles) will, of course, also be improved during this competitive introduction period.

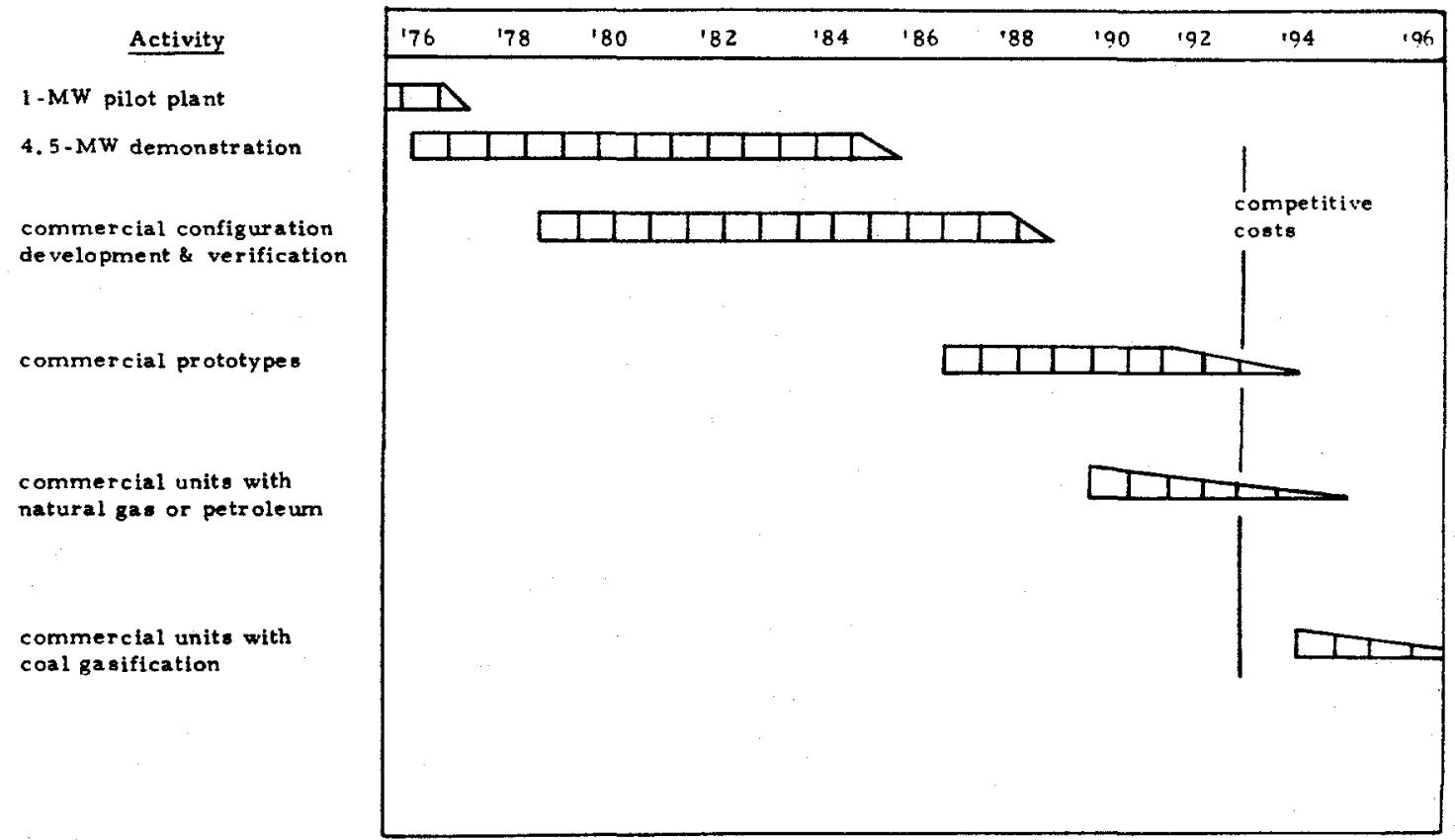

Fig. 2. 4-3. Mllestone chart for U.S. electric utility PAFC power-plant development. 


\subsection{Comparison of Acid and Alkaline FCs for Utility Use}

Some advantageous characteristics of FCs are well known to the general public from the space program, for example, their high efficiency and lack of environmental impact. Both of these require qualification since they may be misinterpreted. For the Gemini and Apollo capsules, the FCs were non-polluting and relatively efficient because they used pure $\mathrm{H}_{2}$ and $\mathrm{O}_{2}$ as fuel and oxidant, respectively. Well into the 21 st century, FCs will use carbonaceous fuels (NG, biomass material, coals) as primary energy sources. After about the year 2010, commercial, stored $\mathrm{H}_{2}$ may arrive. This hydrogen will probably have been produced from water by using nuclear or solar energy. It is premature to develop FCs only for use with non-fossil $\mathrm{H}_{2}$ now. Current commercialization efforts must involve carbonaceous fuels.

With our current limited knowledge of electrocatalysis, the only fuel which we can use efficiently in any of the presently known $\mathrm{FCs}$, at all temperatures, is $\mathrm{H}_{2}$. The rate of reaction of carbonaceous fuels is measurable (see Ref. 4 for a review of previous work, which was mostly carried out during the $1960 \mathrm{~s}$ at temperatures in the range between 100 and $200^{\circ} \mathrm{C}$ ) but is quite inadequate, even with high noble-metal catalyst loadings in PAFCs. In fact, direct HC oxidation rates do not come close to cathodic oxygen-reduction rates (which, in turn, are very low compared with $\mathrm{H}_{2}$-oxidation rates) under comparable overpotential conditions. In AFCs, the rates of oxidation of carbonaceous fuels are almost as low, although they are somewhat assisted by the affinity of the reaction products formed (in particular, carbonate ion) for the alkaline medium. Since energy - and cost-effective removal of carbonate ion from alkaline electrolyte is difficult, particularly if a practically attractive immobilized alkaline electrolyte $F C$ system is used, alkaline systems must be used with pure $\mathrm{H}_{2}$ as fuel and $\mathrm{CO}_{2}$-scrubbed air. The use of an acid $\mathrm{FC}$ eliminates this necessity. It has been generally accepted dogma since the late $1960 \mathrm{~s}$ that a ny useful electrolyte for carbonaceous fuels should be $\mathrm{CO}_{2}$-rejecting. Clearly, acid cells fall into this category, as do systems using aqueous carbonate buffers, 5 molten carbonates, and oxideion-conducting solid oxides as electrolytes.

The anode catalyst used in high-temperature cells is normally $\mathrm{Ni}$. Its intrinsic activity is lower than that of Pt at temperatures close to ambient but becomes acceptable in hightemperature environments. Most attempts to oxidize carbonaceous fuels in the high-temperature cells directly have failed because of thermal cracking of fuel and the accompanying irreversible deposition of carbon. This type of reaction can be prevented, both thermodynamically and kinetically, by the addition of steam to the fuel. 6 However, under these circumstances, complete or partial steam-reforming occurs in the cell, and the only product actually consumed in the charge-transfer process itself is $\mathrm{H}_{2}$.

Some authors (cf. Chapter 3) state that AFCs using pure $\mathrm{H}_{2}$ derived from reformed car bonaceous fuels may ultimately be more efficient and cheaper than acid systems, in particular, the PAFC. This claim may be the result of some confusion on the part of European ex-developers of alkaline systems, whose active work was mostly carried out during the 19608 and early 1970 s, and who have perhaps not utilized all of the available opportunities to compare hydrocarbon-fueled alkaline systems with those using acid electrolytes. Starting out with a carbonaceous fuel, any low-temperature cell system must use several fuel-processing steps to produce the hydrogen it will consume. First, fuel purification to avoid poisoning of the steam-reforming catalyst is required (clean-up to a fraction of a ppm of $S$, which normally requires hydrodesulfurization by using some of the $\mathrm{H}_{2}$ produced in the subsequent steam-reforming stage, followed by zinc-oxide polishing). This step is followed by steam-reforming and reaction in a water-gas shift-converter to reduce residual $C O$ to acceptable levels. All of these steps, except for the water-gas shift reaction, are endothermic processes and require a net heat input from the fuel used or from another source. If an HC such as methane is used, the most important part of this heat-input requirement is in the steam-reforming operation. To reform the HC efficiently, up to twice the calculated stoichiometric amount of steam is needed. The necessary heat requirement for this steam for reaction is therefore quite high. Heat for the endothermic reforming process is required at high temperature, typically about $750-800^{\circ} \mathrm{C}$. For maximum efficiency, the whole process must be integrated into the operating system to the highest degree possible.

With pure $\mathrm{H}_{2}$, an AFC operating at $0.9 \mathrm{~V}$ is more efficient than an acid cell operating at $0.7 \mathrm{~V}$. In a cell in which all of the fuel can be consumed, the energy conversion of the cell itself will be given by the cell voltage divided by the equivalent $\mathrm{HHV}$ of the $\mathrm{H}_{2}$ consumed. The latter is $1.48 \mathrm{eV}$ under standard conditions. Hence, a $0.9 \mathrm{~V}$ alkaline cell, consuming essentially all of its $\mathrm{H}_{2}$, with a feedback loop or other system to remove product water, would have an efficiency of $100 \times(0.9 / 1.48)=61 \%$. It should be noted that $0.9 \mathrm{~V}$ is not an impossible potential for a pure $\mathrm{H}_{2}$ /pure $\mathrm{O}_{2}$ alkaline cell operating at $100 \%$ reactant utilization. Indeed, the goal of a recent NASA program was to produce a pressurized cell giving $1 \mathrm{~A} / \mathrm{cm}^{2}$ at this potential. 7 Such a system would be capable of $250 \mathrm{~W} / \mathrm{kg}$ [in contrast, the PC-3A2 gystem used in the Apollo module delivered $13 \mathrm{~W} / \mathrm{kg}$ and cost $\$(1968) 250,000 / \mathrm{kW}]$. Bacon's original AFC ${ }^{+}$operated at $200^{\circ} \mathrm{C}$ and 45 bars and delivered $1 \mathrm{~A} / \mathrm{cm}^{2}$ at $0.85 \mathrm{~V}$, whereas the lightweight NASA cell operated at much lower temperatures and pressures $\left(\leq 120^{\circ} \mathrm{C}\right.$ and $\sim 7$ bars), but it required expensive hardware,

tSee Chapter 3 for details. 
including $20 \mathrm{mg} / \mathrm{cm}^{2}$ of $90 \%$ Au/10\% Pt cathodes. These catalysts were necessary for operation at $120^{\circ} \mathrm{C}$ to achieve the required performance but are far too costly and hence impractical except for special applications. More practical, inexpensive, C-supported cathode catalysts are limited to operation at about $80^{\circ} \mathrm{C}$ and are less active than high-loading, pure, noble-metal catalysts. In contrast, more recent work has shown that low-noble-metal-loading $\mathrm{C}-\mathrm{suppor}$ ted anodes have very high activity and are stable up to $120^{\circ} \mathrm{C}$ and beyond. 7 This difference in stability between a nodes and cathodes with respect to their electrochemical corrosion performance results from the electrochemical potential difference under which the anodes and cathodes operate.

At about $80^{\circ} \mathrm{C}$, an AFC with state-of-the-art C-supported catalysts, operating on scrubbed air at 1 bar and with pure $\mathrm{H}_{2}$. might deliver $0.80 \mathrm{~V}$ at $200 \mathrm{~mA} / \mathrm{cm}^{2}$ and yield a cell efficiency of $54 \%$. Since no waste heat will be available from the cell that could be used for compression work, the AFC will be limited to 1 bar operation (unlike the PAFC). The energy requirements for fuel processing and gas separation must also be taken into account. Methane entering the fuel processor emerges as a mixture of $\mathrm{H}_{2}, \mathrm{CO}_{2}$ (with some $\mathrm{CO}$ ) and excess steam, roughly in the ratio 1:4:1.5. If the cell could use $\mathrm{CH}_{4}$ directly or if the fuel-processing system could be totally integrated with a high-temperature cell so that the whole can be regarded as a $100 \%$ efficient black box, then a hypothetical system operating on $\mathrm{CH}_{4}$ at $100 \%$ utilization and $0.80 \mathrm{~V}$ would have an efficiency of $100 \times(0.80 / 1.143) \%(1.143 \mathrm{~V}=$ the equivalent high-heating value of $\mathrm{CH}_{4}$ in $\mathrm{eV}$ ). However, major energy inputs are required to convert $\mathrm{CH}_{4}$ to $\mathrm{H}_{2}$ for use in a low-temperature AFC, and these transformations are not $100 \%$ efficient.

The total energy inputs required to convert NG into $99.999 \%$ pure $\mathrm{H}_{2}$, using reforming followed by pressure-swing adsorption, ha ve been well documented. A typical ratio of $\left(\mathrm{CH}_{4}\right.$

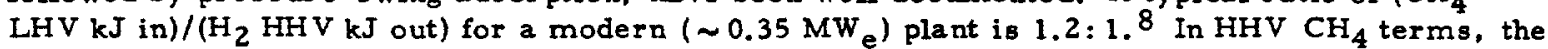
ratio corresponds to $1.33: 1$. In addition, about $11 \%(43 \mathrm{~kW})$ of the power-plant output is required for parasitic power to operate the pressure-swing absorption system. The HHV efficiency of a reformed $\mathrm{CH}_{4}$ AFC system operating at $0.80 \mathrm{~V}$ would therefore be $0.80 /(1.48 \times 1.33 \div 0.89)$ or $36 \%$. This value does not take account of inverter $108 s e s$ and any energy-storage requirements.

The efficiency will be improved significantly (by about $11 \%$ to $47 \%$ ) if methanol is used as fuel because of the improved efficiency of the lower-temperature methanol-reforming operation [(kJ in)/(kJ out) including parasitic power $\approx 1.17: 1, \mathrm{Rcf}$. 9]. However, the efficiency of a heatintegrated methanol PAFC system will improve by about $7 \%$ to about $50 \%$ using the same HaldorTopsde process. In any case, the production of methanol from methane is as energy-intensive as the preparation of $\mathrm{H}_{2}$ from the same source. Therefore, in terms of BTU content of methane feedstock, the primary energy efficiency of the overall process is still not very high.

We show in Sec. 2.9 that the WE PAFC system operating at $0.68 \mathrm{~V}$ per cell will have an overall system heat-rate $(B T U / \mathrm{kWh})$ of about 8100 , which is equivalent to $\approx 42 \%$ efficiency; the UTC system operating at $0.73 \mathrm{~V} / \mathrm{cell}$ is estimated to have an approximate heat-rate of $7700 \mathrm{BTU} /$ $\mathrm{kWh}$ or $\approx 44 \%$ efficiency. Therefore, in spite of the lower cell voltages obtained with PAFCs, their system heat-rates are greater than those of the best alkaline systems. Indeed, if a WE PAFC system could operate at the alkaline cell voltage, it would show an efficiency of $49 \%$, as compared with a value of $36 \%$ for the alkaline system. The major reason for the increased system efficiency of the se acid units is not only the avoidance of $\mathrm{H}_{2}$ clean-up operations, but particularly the use that can be made of the cell-operating temperature (close to $200^{\circ} \mathrm{C}$ ) in avoiding the burning of extra fuel to raise high-pressure steam for the reformer and to improve performance by providing compression work for the cathode-air supply. In addition, the PAFCs also provide useful cogeneration capability (an alkaline system would have no waste heat for this purpose).

Raising the excess steam is particularly important, since it reduces the effective heat required in reforming by more than $0.2 \mathrm{eV}$. The use of dirty $\mathrm{H}_{2}$ in the PAFC has a small thermodynamic penalty, since the exit $\mathrm{H}_{2}$ concentration limits the maximum theoretical cell voltage to that predicted by the Nernst equation for the anode exit-gas composition. The fall-off in current density as the $\mathrm{H}_{2}$ becomes progressively more dilute limits practical utilization to about $85 \%$. This tail gas can be used advantageously to supply the enthalpy of reaction in the reformer. Burning of $\mathrm{CH}_{4}$ is required for an alkaline system, even though some impure $\mathrm{H}_{2}$ from the tail gas of the purification system may be used in the reformer. PAFCs, with higher operating temperatures, zero gas-separation requirements, and utility in improved system integration, are therefore currently considered more desirable than cells using low-temperature alkaline stacks with somewhat higher potentials. The integrated high-efficiency PAFC system is covered by a number of UTC patents. 10

The question of the reformer-clean-up-AFC system vs the PAFC has been examined in a number of studies. 11 Plots of state-of-the-art cell performance for different technologies are shown in Fig. 2.5-1 under conditions of equal anode-gas utilization for non-dead-ended cells (the alkaline cell is assumed to be dead-ended). Utilization is taken as $85 \%$, and while all systems consume $\mathrm{H}_{2}$, the high temperature systems (MCFC and SOFC) are assumed to use internal methane reforming. The data show the high AFC performance on pure $\mathrm{H}_{2}$ and the apparently lower performance of IMCFC and particularly SOFC technology because of the thermodynamic penalty in high-temperature cells. In contrast, Fig. 2,5-2 shows HHV system efficiency (methane-AC).

tOther processes exist which require much less electrical work input. In these, a typical (HHV kJ in)/(HHV kJ H $\mathrm{H}_{2}$ out) value is $1.55: 1$, which yields similar $\mathrm{HHV}$ fuel/kW efficiencies. 


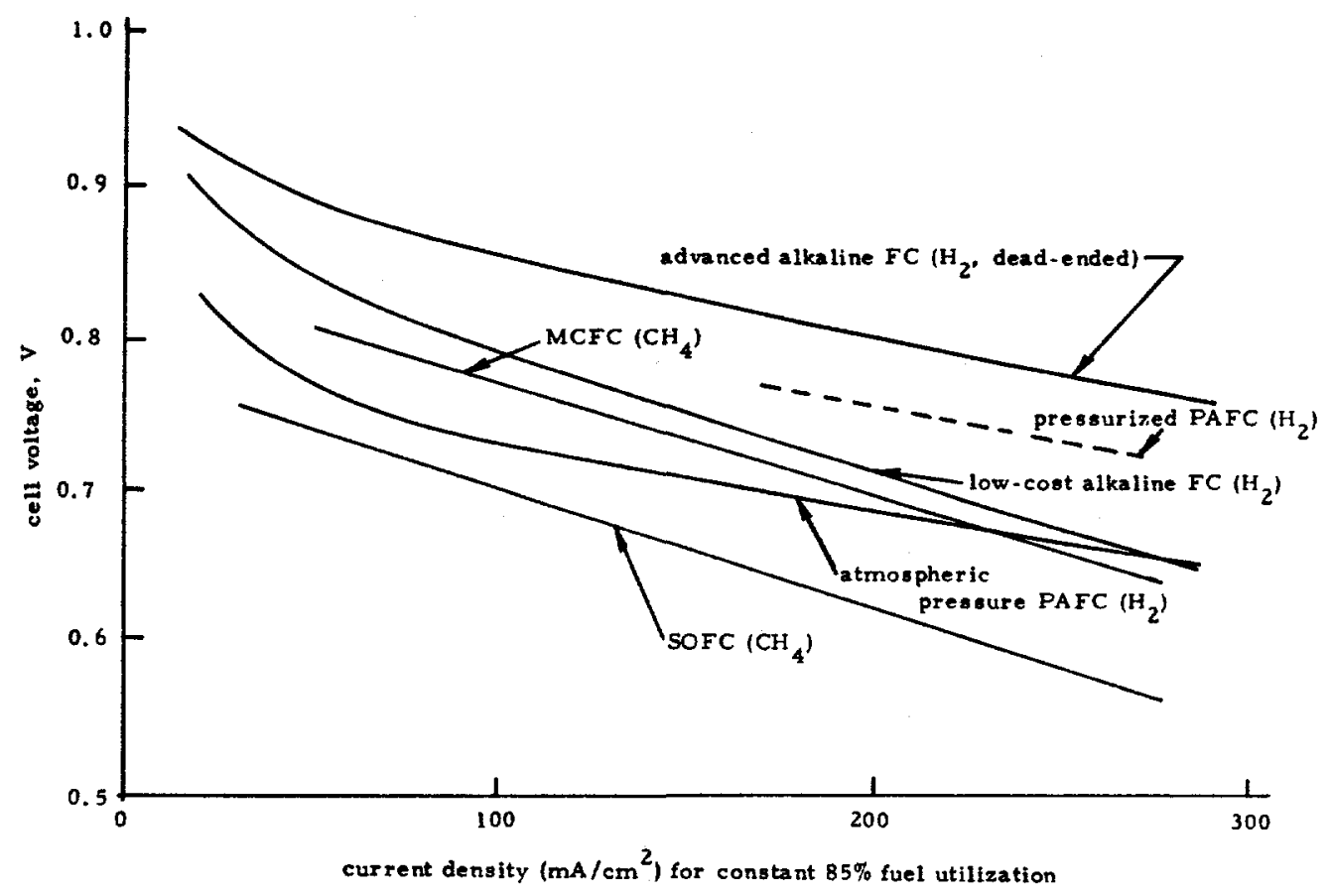

Fig. 2.5-1. Performances of different FCs at constant anode utilization (except for the alkaline cells). Low-temperature systems use $\mathrm{H}_{2}$ whereas high-temperature cells are internal reformers.

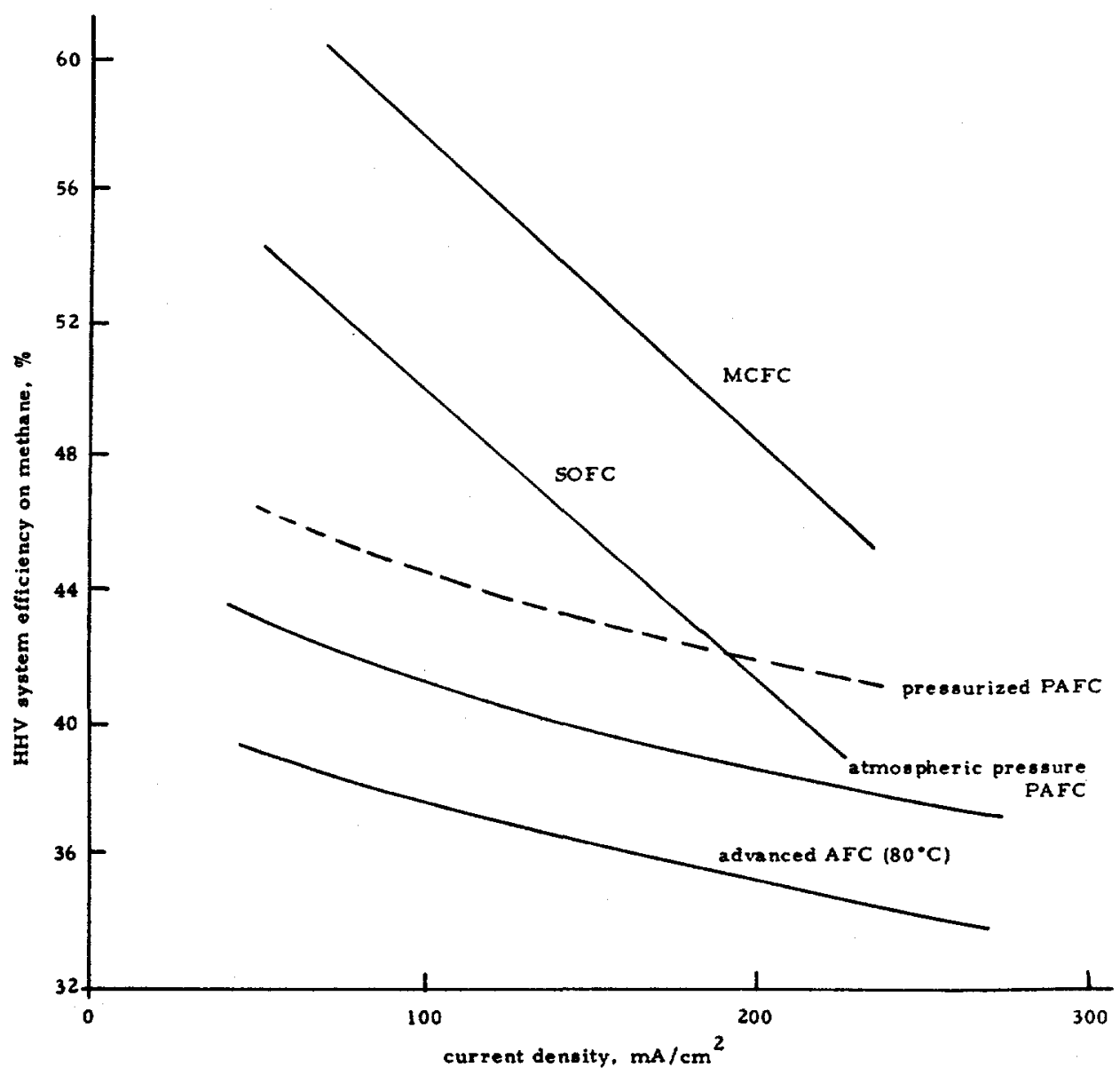

Fig. 2.5-2. Methane-fueled aystem efficiencies for the cells in Fig. 2,5-1. 
The advantage of the internal reforming systems, particularly the MCFC, is clear; as expected, the AFC has the lowest overall efficiency. According to Ref. 3, its efficiency $19700 \mathrm{BTU} / \mathrm{kWh}$ at $200 \mathrm{~mA} / \mathrm{cm}^{2}$ ) may not be economically acceptable for a U.S. electric utility.

A PAFC 24-cell stack has now run more than 25,000 h. 12 There has been no evidence that low-temperature $\left(80^{\circ} \mathrm{C}\right)$ alkaline stacks can be run longer than about $10,000 \mathrm{~h}(3 \mathrm{space}-$ shuttle orbiter stacks containing pure noble-metal electrodes have run for this period; $18,000 \mathrm{~h}$ have been registered on individual space-shuttle orbiter cells with the lifetime limited by dissolution of plastic frame components). ${ }^{\dagger}$ Cheap AFCs with carbon-based cathodes are limited to $10-15,000 \mathrm{~h}$ by $\mathrm{C}$ corrosion, and there is no evidence that such cells can be used at temperatures where waste heat for efficient reforming would be available. 7

The PAFCs operate at a relatively high current density $\left(267-325 \mathrm{~mA} / \mathrm{cm}^{2}\right.$ for WE cells). It is suggested in Chapter 3 that internal resistance losses in PAFCs should be much higher than in AFCs. At the PAFC operating temperature, the electrolyte exists essentially as pyrophosphoric acid, which is a stronger acid than orthophosphoric acid; its ionic conductivity increases rapidly with temperature to an anomalously high value at $200^{\circ} \mathrm{C}\left[>0.6(\Omega-\mathrm{cm})^{-1}, \operatorname{Ref}\right.$. 13]. In addition, the interelectrode gap in the immobilized-electrolyte PAFC is minimal (about $0.35 \mathrm{~mm}$ ), and internal resistance losses are only a few $\mathrm{mV}$. By contrast, the interelectrode gap in a circulating-electrolyte ATC is typically about $1 \mathrm{~mm}$ or more, which results in much greater resistance losses, despite the slightly higher specific conductivity of concentrated KOH compared with that of the PAFC electrolyte.

Acid cells that reject water in the vapor phase have relatively much simpler cell stacks, proven long lifetimes, and show few electrolyte-management problems. In general, AFCs require circulating electrolyte for electrolyte-volume control under load and heat rejection. In contrast, acid cells reject product water directly as steam and use immobilized electrolyte. Alkaline cells with non-circulating electrolyte have been made for specialized purposes, e.g., for the space-shuttle orbiter, but they require highly complex electrolyte-volume control and product-water rejection. In fact, the use of alkaline cells can presently only be justified if and when pure $\mathrm{H}_{2}$ will become available from non-fossil sources (e.g., from chlorine plants or in the context of the future hydrogen economy). The $\mathrm{H}_{2}$ will have to be suitably stored in the form of compressed gas or, possibly, as liquid $\mathrm{H}_{2}$, ammonia and other hydrides, or as an organic compound such as cyclohexane.

\section{6 The Incentive to Improve Present PAFC Technology}

Since the competitive systems to the PAFC are moving targets in terms of cost and efficiency, continued product improvement will be required if the PAFC is to remain viable within its initial market or to penetrate new markets. Two types of initial product improvements may be envisaged, both of which will affect the final cost of electricity. These are, respectively, an increase in plant efficiency (reducing fuel costs per $\mathrm{kWh}$ ) and a decrease in plant cost $(\$ / \mathrm{kW}$ ), which results in a reduction of annual charges. If all other factors remain the same, an improvement in efficiency will decrease capital cost proportionally, but the effects of both of these factors on the overall cost of electricity may be very small. This result can be illustrated by considering as an example a scenario in which the FC cost is taken to be $\$ 770 / \mathrm{kW}$, fuel cost = $\$ 4 / 10^{6} \mathrm{BIU}$ and $\mathrm{FC}$ heat rate $=8300 \mathrm{BTU} / \mathrm{kWh}$. For an average a nnual utilization of $50 \%$ for the power plant as a load-following unit, the fuel contribution to electricity cost is $3.3 \mathrm{c} / \mathrm{kWh}$, whereas FC amortization will contribute $\$ 3.5 \mathrm{c} / \mathrm{kWh}$ with annual carrying charges of $20 \%$ of capital cost. A $10 \%$ improvement in FC efficiency will result in a $10 \%$ decrease in both of these numbers, which by itself does not appear to yield a significantly reduced electricity cost. However, this analysis ignores the fact that the efficiency improvement may allow a utility to operate the FC economically during a greater number of hours per year, thus reducing the total capital contribution to the electricity cost. This result is illustrated by the screening curves in Fig. 2.6-1, where we compare baseload coal, intermediate duty FCs, combined cycles, and peaking combustion turbines, using 1981 capital costs and efficiencies given in Ref. 1 . The curve is illustrative only and does not allow for accounting subtleties or credits and for operational flexibility advantages of the FC. Delivered coal costs of $\$ 2 / 10^{6}$ BTU and NG costs of $\$ 4 / 10^{6}$ BTU have been used. The crossing points on the screening curve show that a $10 \%$ efficiency improvement (from $8300 \mathrm{BTU} / \mathrm{kWh}$ to $7500 \mathrm{BTU} / \mathrm{kWh}$ ) will allow the utility to run the FC economically with a baseload coal plant for 8200 instead of $5900 \mathrm{~h} / \mathrm{y}$. The corresponding mean levelized electricity cost will be reduced to 4.7 from $5.9 \mathrm{c} / \mathrm{kWh}$.

A striking result that is caused by low capital cost is lower cost of electricity production with the combustion turbine than with the FC to about $5000 \mathrm{~h} / \mathrm{y}$ (see Fig. 2.6-1). Similarly, the electricity cost for the combined cycle is less than that of any other technology, except for the combustion turbine to $2100 \mathrm{~h} / \mathrm{y}$. However, as the EMA report shows, ${ }^{3}$ many utilities will prefer to purchase FCs rather than turbine technologies because $F C_{s}$ are available in small sizes and there are potential credits, such as siting and pollution advantages. In contrast, the turbine

'Single PAFCs with representative technology have $\mathrm{r}$ un over $50,000 \mathrm{~h}$ at UTC, and cells with older technology have run up to about $100,000 \mathrm{~h}$. 
technologies suffer accounting debits, including pollution-control costs and difficulties in siting, which do not appear in the screening curve.

Discounting undesirable technologies, Fig. 2.6-1 illustrates the relative attraction of a $7500 \mathrm{BTU} / \mathrm{kWh}$ FC over an $8300 \mathrm{BTU} / \mathrm{kWh}$ unit for an average utility. The overall evaluation of the EMA report ${ }^{3}$ for base case FC technology ( $\$ 770 / \mathrm{kW}$ in 1981 dollars), with industry average credits, is shown ${ }^{3}$ in Fig. 2.6-2, which gives FC technology penetration into the total added capacity generating $\mathrm{mix}$ after the year 2000 as a function of FC heat-rate. It may be seen that attractiveness of the FC is marginal for heat-rates $\approx 9000 \mathrm{BTU} / \mathrm{kWh}$; even at the proposed introductory value of $8300 \mathrm{BTU} / \mathrm{kWh}$, penetration would amount to only about $6.6 \%$ of total added capacity. Figure 2,6-2 shows that the range of heat rates, for which expansion of the FC into the added capacity market would be greatest, will be between 8300 and $7500 \mathrm{BTU} / \mathrm{kWh}$. At $7500 \mathrm{BTU} / \mathrm{kWh}$, penetration would be $16.4 \%$; at $6500 \mathrm{BTU} / \mathrm{kWh}, 22.1 \%$; at $5000 \mathrm{BTU} / \mathrm{kWh}, 24.1 \%$. The relatively small further penetration of the most efficient units is made clear by ins pection of Fig. 2.6-1: after a minimal efficiency is reached, capital-cost considerations (i. e., the intercept in Fig. 2.6-1) become paramount. For $\$ 4 / 10^{6} \mathrm{BTU}$ fuel, the yearly fuel cost for a hypothetical baseload and $100 \%$ efficient FC would be $\$ 120 / \mathrm{kW}$, which must compete with the $\$ 393 / \mathrm{kW}-\mathrm{y}$ (fuel plus capital) of a baseload coal plant. This hypothetical, $100 \%$-efficient FC unit is therefore competitive for capital charges less than $\$ 273 / y$ or a total capital cost of less than $\$ 1365 / y$. If fuel costs were essentially zero (as with hydroelectric, solar, geothermal, and fusion energy), only plants costing less than $\$ 393 / \mathrm{kW}-\mathrm{y}$ would be competitive. For baseload, this estimate corresponds to $\$ 1965 / \mathrm{kW}$. For solar energy, which is available for only $2500 \mathrm{~h} / \mathrm{y}$ in favorable locations, breakeven occurs at $\$ 561 / \mathrm{kW}$. These estimates are not to be taken too seriously, but they illustrate methods used in discussions of economical generation options.

The $\$ 770 / \mathrm{kW}$ capital cost of the FC power plant in the EMA study ${ }^{3}$ and in Fig. 2.6-2 includes average credits of $\$ 166 / \mathrm{kW}$. Any further cost reductions, increased credits, or both will result in additional penetration. The effects of these credits and of FC capital cost on penetration of this new technology into the generating mix for the group of utilities studied by EMA is shown ${ }^{3}$ in Fig. 2.6-3 for a 10-y period of mature FC production (constant FC capital cost during the decade 2005-2015 and following the initial cost-reduction scenario in Ref. 2). With zero credits, breakeven capital cost (corresponding to marginal penetration) would be $\$(1981) 770 / \mathrm{kW}$, which is the intercept of the zero-credit curve with the $x$-axis. With the assumed $\$(1981) 600 / \mathrm{kW}$ mature learned-out capital cost and $\$ 166 / \mathrm{kW}$ average credits, the initial penetration of electric utility units is $10,000-\mathrm{MW}$ over the 10 -y period, corresponding to $6.6 \%$ penetration into the total added capacity mix shown in Fig. 2.6-2. Figure 2,6-3 also shows that penetration may double if FC capital cost is reduced to $\$ 400 / \mathrm{kW}$ or, alternatively, if credits can be assumed to double to about $\$ 300 / \mathrm{kW}(\mathrm{e} . \mathrm{g} .$. if pollution regulations become stricter and lead to special tax credits). This development would favor dispersed cogeneration technologies, which do not produce stratospheric $\mathrm{SO}_{2}$ and $\mathrm{NO}_{x}$ leading to acid rain. This proposed scenario is not inconceivable for the future.

It is interesting to note that there is an upper limit to $F C$ penetration, even if the $F C$ capital cost is zero. This limit results from the availability of premium fossil fuels for intermediate duty use. However, if the FC heat-rate were less than $8300 \mathrm{BTU} / \mathrm{kWh}$, the total added capacity would increase proportionately in this low capital cost (or high-credit) scenario.

Following study of the effects of heat-rates, capital costs and credits, EMA examined other factors which may influence penetration of the technology. ${ }^{3}$ Major sensitivity studies included the cost of capital (interest rates) and future fuel costs. The conclusion was that the effects of these factors on the percentage of FC penetration into the total added capacity would be minor, since they affect all competitive technologies to a greater or lesser degree. However, since they influence the total business climate, they can strongly affect the total MW of PAFC capacity installed. In particular, if fuel prices increase strongly, FC penetration will be higher because of the relatively higher efficiencies of this technology.

The EMA study was specifically aimed at an electric utility-market assessment of the PAFC. However, the general conclusions are also appropriate for other FC technologies, provided that they can operate in the same load-following mode and use the same fuel at the same heat-rate as this FC system. As we have seen, this may not necessarily be the case for systems based on the high-temperature cells, which will probably be most useful at the baseload end of the intermediate-duty spectrum.

A probable penetration scenario of the $8300 \mathrm{BTU} / \mathrm{kWh} F \mathrm{FC}$ into the generating mix of the electric utilities studied by $\mathrm{EMA}^{3}$ would therefore be $10,000-20,000 \mathrm{MW}$ over $10 \mathrm{y}$, depending on mature capital costs for the technology and/or credits. These figures may be multiplied by a factor of 3-4 for the whole of the continental U.S. and perhaps by a further factor of three to represent the whole of the developed world electric utility market. This mature market will therefore be very substantial (\$5-7 billion dollars per y). As is shown in Fig. 2.6-2, it will be greater by more than a factor of 2 if further research can decrease FC heat-rates to 7500 BTU/ $\mathrm{kWh}$ or less. In the distant future, identified possible improvements may enable PAFC systems to penetrate the baseload market when using, for example, high-or medium-BTU gas from coal as fuel. As already noted, this result will depend on the relative penetration of other modular FC systems, in particular, of the high-temperature technologies. 


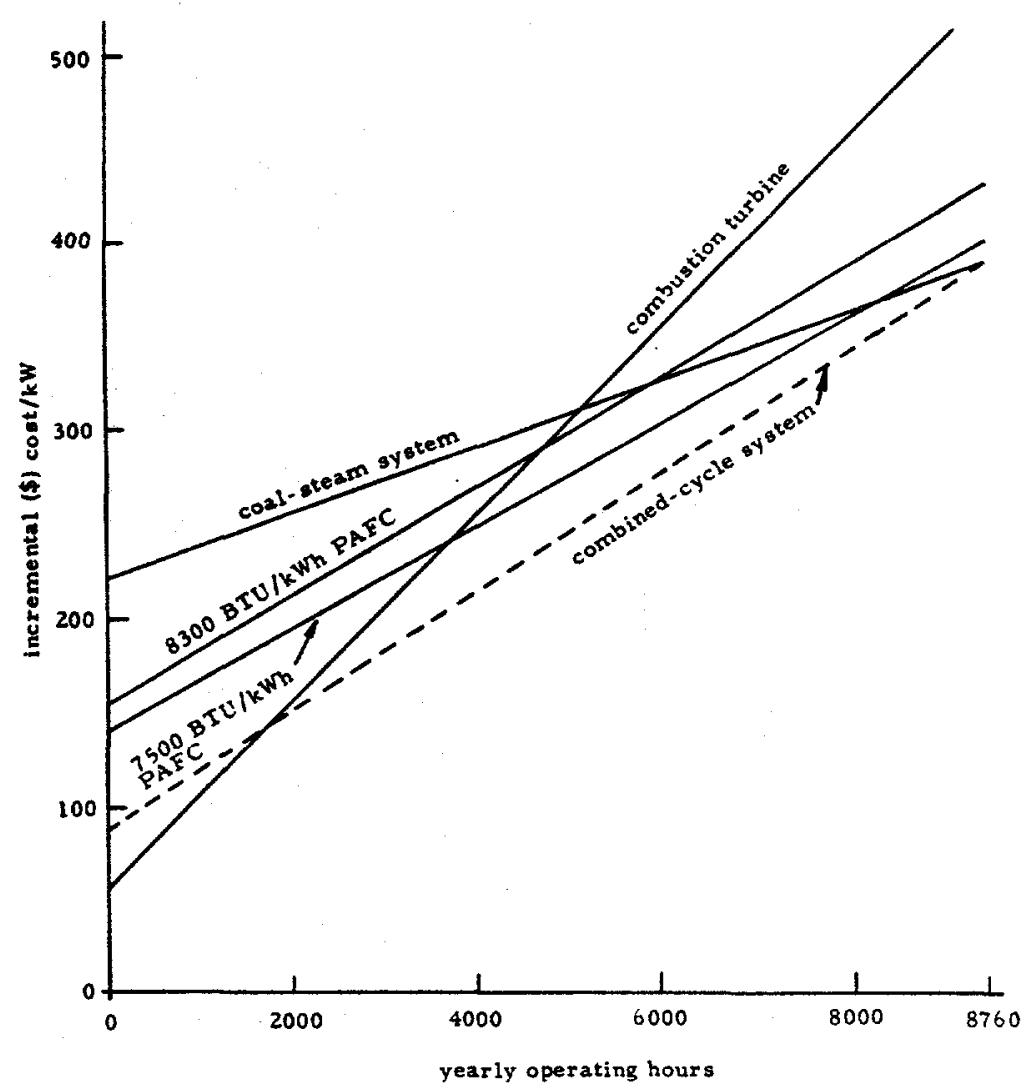

Fig. 2.6-1. Mlustrative screening curves are shown for different power plants. Assumption: capital costs as in Ref. 1 (20\% a nnual capital charges); coal cost, $\$ 2 / 10^{6}$ BTU; clean light HC cost, $\$ 4 / 10^{6}$ BTU.

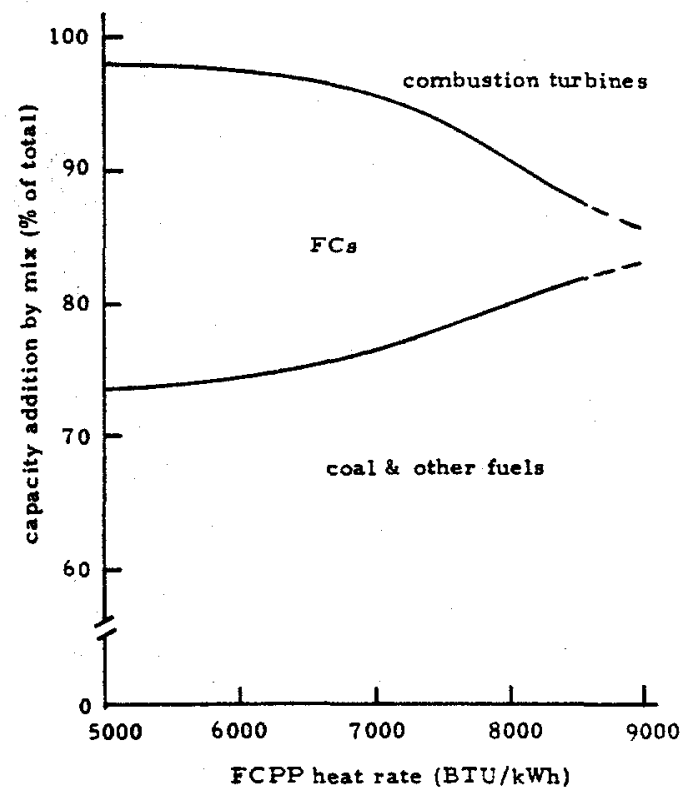

Fig. 2.6-2. Variation of capacity addition as a function of PAFC heat-rate. The assumed installed costs are $\$(1981) 770 / \mathrm{kW}$; coal cost $=$ $\$ 2 / 10^{6}$ BTU; FC and turbine fuels $=\$ 4 / 10^{6} \mathrm{BTU}$. 


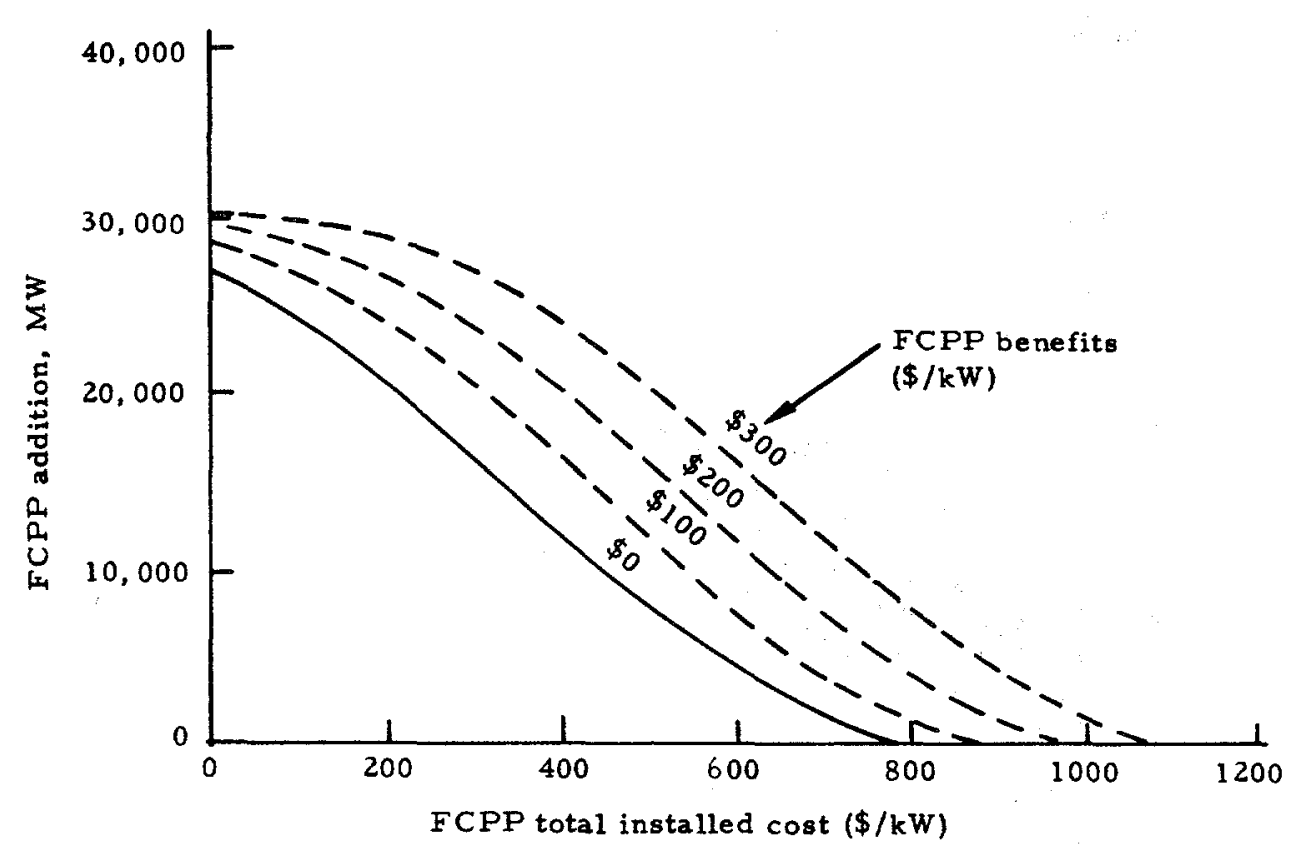

Fig. 2.6-3. Total added PAFC capacity $(8300 \mathrm{BTU} / \mathrm{kWh})$ as a function of installed costs and credits. The assumed fuel cost is $\$ 4 / 10^{6} \mathrm{BTU}$.

The EMA study did not consider the on-site market, where FCs would be used in either a residential and commercial or industrial cogeneration mode. There is an important difference between the electric utility and on-site markets. In the electric utility market, cogeneration (i.e., selling some steam) is simply a credit. On the other hand, the on-site market allows for the totally integrated energy requirements of the locality to be serviced. It is possible that the on-site market for acid (or high temperature) FCs may eventually become as large worldwide as that for electric utility PAFC systems. This result may be especially true in countries developing their resources in the early years of the 21 st century, for which the modular, on-site, highefficiency characteristics of the FC will have substantial advantages in the absence of large electrical utility networks. Its application for this use will particularly occur if mass production, combined with further research, can reduce its cost to more affordable levels.

Current projections for the on-site market in the U.S. appear to represent about $1000 \mathrm{MW} /$ $y, 14$ or about $25-30 \%$ of the total potential electric utility market. In Japan, 15 the electric utility market over the period 1991-2005 has been estimated at about 25,000 MW; the on-site market should contribute an additional $40 \%$ of this estimate. Clearly, the on-site market in developed economies will be a substantial fraction of the dedicated electric utility market. The potential industrial cogeneration market is at present unknown. It may be as much as $50,000 \mathrm{MW}$ in the U. S.

Beyond the electric utility intermediate load, on-site, and electric utility baseload markets (in that order), we can see the eventual use of FCs in the transportation sector if capital cost can be made low enough. An important factor will be availability of noble metal catalysts for use in acid FC systems. Research on catalysis to reduce our present dependence on noble metal supplies is therefore a matter of the greatest importance. Ideally, research on direct methanol cells using non-noble metal catalysts and acid (in particular, proton-conducting film) electrolytes would be desirable, both from the perspective of increasing basic understanding of electrocatalytic processes and for the initial introduction of advanced acid cells with simple, lightweight peripherals. This FC use has recently been identified as a target objective for the transportation sector by the EEC Commission. 16 Over the longer term, it is certain that the ultimate transportation fuel will not be methanol but rather stored, non-fossil $\mathrm{H}_{2}$, and the use of Pt-group metals in ECs must be eliminated if this technology is to be of major use in future transportation. This analysis argues for the ultimate ascendency of the alkaline system for transportation applications.

The important point about the need to eliminate Pt-group metals should be stressed. It is underlined by the fact that the lower of our two estimates for worldwide electric utility PAFC penetration on a yearly basis $(9000 \mathrm{MW} / \mathrm{y})$ would require the equivalent of the present U.S. annual Pt use $\left(\sim 1.4 \times 10^{6}\right.$ troy ounces/y). While the Pt producers assure us that this is possible, $9000 \mathrm{MW} / \mathrm{y}$ represent only the equivalent of 450,000 subcompact vehicles. Clearly, Pt requirements will prevent any major penetration of currently conceived acid cells into the worldwide transportation market. A major effort to replace Pt in acid systems, or the use of alkaline systems with stored non-fossil $\mathrm{H}_{2}$ (or, perhaps, initially fossil-derived $\mathrm{H}_{2}$ ) will be required in the future. Only in this manner can the outstanding efficiency of the FC for the transportation 
sector be used in the non-fossil-fuel economy which will emerge in the 21 st century. Non-fossil $\mathrm{H}_{2}$, produced at a very high capital cost compared with that for extracting and processing currently used fossil fuels, will be simply too valuable to burn in low-efficiency, Carnot-limited heat-engines. 17

\subsection{Historical Review of the Development of Acid FC Technology}

Since acid $\mathrm{FC}_{\mathrm{s}}$ reject $\mathrm{CO}_{2}$, they may, in principle, be used to oxidize carbonaceous fuels directly. The present acid FC, using PA as electrolyte, is the result of attempts to develop a direct HC FC in the period between 1955 and 1960. Most of this work took place at the General Electric Company Research and Development Center (GE) and is reviewed in Ref. 4. This work first demonstrated that the oxidation rates of typical $\mathrm{HCs}$ were very low, and that high noble-metal catalyst loadings were required to obtain rates of even a few mA $/ \mathrm{cm}^{2}$ at $80^{\circ} \mathrm{C}$ in sulfuric acid. Since $80^{\circ} \mathrm{C}$ is close to the operating-temperature limit of concentrated sulfuric acid, which is reduced at higher temperatures at the anode to $S$, PA was substituted as an electrolyte. PA is the only common non-volatile acid which is stable in the presence of both $\mathrm{H}_{2}$ and $\mathrm{O}_{2}$ at temperatures up to about $80^{\circ} \mathrm{C}$. Its rates for fuel oxidation and oxygen reduction, at equivalent catalyst loadings, were shown to be lower than those of sulfuric acid at the same temperature (below $80^{\circ} \mathrm{C}$ ), although they could, of course, be increased by raising the temperature. However, even at $150^{\circ} \mathrm{C}$, the rates of direct $H C$ oxidation were rather poor, and the concept was quickly abandoned as not cost-effective in view of the high loadings of pure noble metals which appeared to be necessary ( $P t$ costs alone were on the order of $\$ 15,000 / \mathrm{kW}$ in 1984 dollars). In addition, the materials technology available at that time for use in high-temperature acid electrolytes under highly oxidizing and reducing conditions was limited, and acid handling was complicated by the use of free-electrolyte cells. Only noble metals, Ta, and $\mathrm{Nb}$ appeared to be stable, and the $\mathrm{Ta}$ required gold-plating to give sufficient electrical conductivity for use as an electrode-support screen or as a bipolar plate.

One of the first developments in the direction of modern cell technology was the use of the matrix-immobilized electrolyte, in which the PA was held by capillary action. Some early attempts were made with glass and quartz fibers, which are eventually corroded by hot PA, but patents for more stable teflon-bonded $\mathrm{B}$ and $\mathrm{Zr}$ phosphates date from the mid-1960s. 18 The first cells using this technology were a vailable for purchase from Engelhard Industries in 1966.

In 1967, the American Gas Association started its support of the TARGET (Team to Advance Research on Gas Energy Transformation) program to supply small 12.5-kW, standalone (i.e., non-grid-connected) $\overline{F C}$ modules as total energy (cogeneration) packages for individual households. The intended fuel for these units was NG. The Pratt and Whitney Division of UTC was chosen as the prime contractor, after they had shown that PAFCs operating at $150^{\circ} \mathrm{C}$ or above would be able to use the $\mathrm{CO}_{2}$-containing $\mathrm{H}_{2}$ (containing small quantities of $\mathrm{CO}$ ) produced by steam-reforming of NG. The a nodes of the se cells were designed to operate close to thermodynamic reversibility on this fuel, and they would therefore not show either the potential or current-density penalties associated with attempts to oxidize $\mathrm{CH}_{4}$ directly. In addition, the operating temperature was high enough to allow waste heat from the cooling system to raise steam for reforming, so that system integration for maximum efficiency could be utilized, as is described in Sec. 2.5.

The principal problems with PAFCs were cost of the catalyst and of cell-construction materials. It was also unclear whether the chemical engineering system for fuel processing would prove practical in a $12.5-\mathrm{kW}$ size. However, the choice of PAFC technology was certain in 1967. In spite of the success of the Apollo FC program, UTC decided that improved alkaline cells of the Bacon type 19 would not be appropriate for the TARGET application. Such cells operated at high temperature and pressure, and their waste heat could certainly be used to raise steam at the appropriate pressure for the reforming of NG. They could operate, in a pressurized mode, at $0.8 \mathrm{~V}$ and $400 \mathrm{~mA} / \mathrm{cm}^{2}$ on pure $\mathrm{H}_{2}$ and $\mathrm{CO}_{2}-8 \mathrm{crubbed}$ air. In addition, they contained no precious metals, used Li-doped, sintered NiO for the cathode, and sintered Ni for the a node in the form of dual-porosity electrodes. In these, the electrolyte-gas interface was maintained by differential pressure between the reactant gases and the electrolyte. The technology is described in greater detail in Chapter 3. The cells had high structural Ni requirements and thus high containment cost per $\mathrm{kW}$, but the particular problems which made them unsuitable for the TARGET program were very poor durability at operating temperature and system complexity.

The poor durability resulted from cathode-component corrosion. The Apollo cells operated at $260^{\circ} \mathrm{C}$ in $75 \% \mathrm{KOH}$ at 7 bars and were barely suitable to complete reliably the 11 -day lunar landing mission. In contrast, Bacon's original technology yielded equivalent performance at lower temperature $\left(200^{\circ} \mathrm{C}\right)$, which was compensated for by the use of higher reagent pressures ( 45 bars) and had somewhat better lifetimes. However, the high pressure required for adequate performance would have made their use unsuitable for small, mass-produced units. Similarly, lower-temperature, longer-lasting alkaline cells with more active catalysts than sintered excarbonyl $\mathrm{Ni}$ could not be used because of poor efficiency and their inability to raise steam for reforming. Bacon-type cells required the complexity of circulating, pressurized electrolytes. All alkaline cells required reliable $\mathrm{CO}_{2}$ removal from both fuel and air, which was determined 
by the UTC engineering staff to be completely impractical for TARGET-sized units. The only choices were therefore $\mathrm{CO}_{2}$-rejecting systems, with acid cells as a first choice and molten carbonate cells as backup technology. Most of the studies on the acid cells were conducted at the Pratt \& Whitney Aircraft Power Systems Division in South Windsor, CT (later the same Division of UTC and now International Fuel Cells, Inc., a UTC-Toshiba joint venture). By 1969, the Power Systems Division had identified the matrix PAFC as the only technically acceptable acid FC choice. Most of the MCFC studies were conducted with IGT as subcontractor.

The challenges were to reduce the cost of the acid cell developed at GE to acceptable levels, while still maintaining adequate performance, and to make a sufficiently cheap and efficient fuel-processing system. An acceptable goal was considered to be a system operating at about $35 \%$ electrical efficiency, with a useful thermal output about equal to the electrical output. A capital cost of about $\$(1967) 150 / \mathrm{kW}$, equal to about $\$ 500 / \mathrm{kW}$ in $\$ 1985$ dollar s, with a unit life time of 40,000 hot $h$, was considered to be a reasonable economic goal, which would allow the gas utility delivering gas at (then) about $50 \mathrm{c} / 10^{6}$ BTU to compete with the electric utility supplying electricity at (then) $10 \mathrm{mils} / \mathrm{kWh}\left(\$ 3 / 10^{6} \mathrm{BTU}\right)$.

The first major materials breakthrough occurred in 1968-69, when graphite and selected carbons were shown to have sufficient stability as cell-construction materials to replace the gold-plated Ta boiler-plate hardware. Work conducted from about 1970 showed that it was possible to increase the effective surface area of $P t$ catalysts from the $20 \mathrm{~m}^{2} / \mathrm{g}$ for typical pure metal blacks to over $100 \mathrm{~m}^{2} / \mathrm{g}$ by preparing the $\mathrm{Pt}$ in a suitable way on a stable conductive support. Many carbons, particularly furnace blacks, had sufficiently high surface area and corrosion resistance for this application. An early favorite was Vulcan XC $-72(250 \mathrm{~m} 2 / \mathrm{gm})$, the most conducting furnace black produced by Cabot Corp. The most effective method of producing highly dispersed Pt on such supports was via Pt-sulfur complexes produced by reduction of, for example, chloroplatinic acid by sulfites, for which the key patents are given under Ref. 20 . In later work, dithionite was advantageously used as the reducing agent. 21 With improvements in teflon-bonded structure to allow better catalyst utilization, the latter apparently began to approach theoretical values. 22 Thus, $0.5 \mathrm{mg} / \mathrm{cm}^{2}$ of $\mathrm{Pt}$ in high-gurface-area form $(100 \mathrm{~m} / \mathrm{g})$ on a Vulcan XC-72 C-carrier was shown to be as active as $20 \mathrm{mg} / \mathrm{cm}^{2}$ of the pure teflon-bonded Pt-black used in older electrodes. 4 Typical cell performance in the early $1970 \mathrm{~s}$ was about $0.6 \mathrm{~V}$ at $150 \mathrm{~mA} / \mathrm{cm}^{2}$ in atmospheric pressure cells using low-loading, carbon-supported Pt electrodes of this type, corresponding then to Pt costs of about $\$ 50 / \mathrm{kW}$.

The emphasis of all systems work in the early stages of the TARGET program was on the use of atmospheric pressure PAFC technology. Engineering considerations pointed to the fact that only this technology would be appropriate in generating units of a few $\mathrm{kW}$, because of problems associated with air-compression efficiency and work recovery in the reject cathode air streams in very small units. The chemical engineering part of the plant (i. e.., fuel purification and processing to give a fuel gas mix acceptable to the FC anode at the chosen operating temperature) turned out to be more complex and costly than had been originally supposed. In order to achieve the heat-integration requirements, the number of heat exchangers required was high (typically 11-14 in each unit), and the use of atmospheric pressure gases required large gasvolume throughput and large piping diameters, which resulted in higher peripheral costs than were originally anticipated.

In spite of the se factors, a number of TARGET units, designated as PC-11, were built and tested at selected residential/commercial cogeneration sites throughout North America during the early to mid-70s. None of these units can be considered to have even approached the program commercial goals, especially with respect to required capital cost. However, the experimental installation and running of the test units can certainly be considered to be a technical success. In partic lar, they showed that small PAFC units were viable with $\mathrm{NG}$ in cogeneration applications. System reliability was not demonstrated to the degree required for stand-alone use. In addition, it became clear that back-up units to give the. required redundancy could never show the required cost-effectiveness. Therefore, electric utility grid connection was determined to be necessary. It should also be emphasized that the initial demonstration period over lapped the Yom Kippur war and the enormous increase in fuel and electricity prices which occurred at that time, as well as the start of major changes in interest rates and electricitycost growth rates from traditional levels. As a consequence, the economical capital cost of the on-site fuel cell remained a rapidly moving and rising target.

In order to reduce the capital cost, the number of individual components per $\mathrm{kW}$, especially in the cell stack and in the chemical engineering system, clearly needed to be reduced. An economy-of-scale reduction in the cost of the DC-AC inverter was also desirable. At the same time, better knowledge of materials problems involved in cell operation, particularly corrosion at the cathode and its associated components, allowed an increase in cell temperature to $190^{\circ} \mathrm{C}$. There was an associated improvement in cell potential (to $0.65 \mathrm{~V}$ ) at somewhat increased current density $\left(215 \mathrm{~mA} / \mathrm{cm}^{2}\right)$. In addition, the increased temperature allowed a relaxation of $C O$ levels in the anode inlet gas, since poisoning effects were then seen only above $1.5 \% \mathrm{CO}$. Together, these improvements allowed a simultaneous improvement in system efficiency and stack capital cost. As a consequence, the atmospheric-pressure, on-site generator was increased in size from 12.5 to $40 \mathrm{~kW}$, with a consequent redefinition of its sphere of applications (away from 
individual homes and towards commercial premises). This study was supported by the newlyestablished GRI. The $40-\mathrm{kW}$ unit is designated PC -18 by UTC.

In parallel, starting in 1972 , the first suggestions about a large electric utility generator, initially in a very large $(27 \mathrm{MW})$ size, were made by UTC to the Edison Electrical Institute and the newly-incorporated EPRI. This electric-utility unit was to be factory-assembled in trucktransportable modules and was to show sufficient economies of scale to be pressurized (thus increasing both cell potential and current density). Pressurization was to be largely carried out by using waste heat and/or gas from the unit, thus incurring no efficiency penalty in burning extra fuel to provide compression work.

The use of the pressurized system had two attractions for electrical utility use: an increase in individual cell potential and hence in overall system efficiency, and a reduction in cell cost per $\mathrm{kW}$ by the consequent increase in power density. In addition, larger cell components were used, thus reducing the number of components per $\mathrm{kW}$ and hence the ultimate manufacturing cost.

\subsection{PAFC Technology Demonstrations, 1976-1985}

It was clear that the development of the large $27-\mathrm{MW}$ unit (about substation size) would involve a considerable degree of risk and, in consequence, a decision was made to examine a scaled-down, pressurized demonstration system of $4.8 \mathrm{MW}$ for DC output from the fuel-cell modules or $4.5 \mathrm{MW}$ for $\mathrm{AC}$, after taking into account parasitic losses in the invertor and for auxiliary system power. System performance was fixed at $0.65 \mathrm{~V}, 250 \mathrm{~mA} / \mathrm{cm}^{2}$ at 3.4 bars and $190^{\circ} \mathrm{C}$. A $1-\mathrm{MW}$ demonstration unit, using the proposed technology (cell stacks with $0.30-\mathrm{m}^{2}$ components) was tested during 1976-77 at the UTC facility and supplied power to the utility grid. Starting in 1976, this and subsequent work on the UTC electric utility PA-power plant has been supported by the DoE or its predecessor, the Energy Research and Development Administration, jointly with EPRI. Work-sharing was conducted so that cell-stack technology improvement was DoE supported, whereas utility input (systems and grid integration) was provided by EPRI. In 1984, DoE spent $\$ 6 \mathrm{M}$ in support of this work, EPRI about $\$ 1.5 \mathrm{M}$.

After successful testing of the 1-MW unit, scale-up was conducted to the 4.5-MW demonstration size for installation at a Consolidated Edison site at 14 th Street and Franklin D. Roosevelt Drive in Manhattan. The original plan was that this unit should start to run before 1978, with testing to be completed by 1979 . Its subsequent history is by now well known. Installation and testing were slowed down by licensing regulations and by fire-department uncertainties concerning untested aspects of the new technology, particularly in the fuel storage and processing area and for $\mathrm{H}_{2}$ handling. This problem led to unforeseen pressure testing of parts of the system. including the one-of-a-kind advanced sheet-metal heat-exchangers, some of which were irreparably damaged by freezing of the water used for hydraulic testing during very cold weather. In consequence, installation of the stored power sections was not completed until the end of 1983 , by which time the 1976 -vintage technology cell stacks had exceeded their electrolyte storage inventory lifetimes. However, the plant did receive an operating license and thus became the only generating technology likely to be permitted in urban areas in the future. The total construction cost of the New York 4.5-MW demonstrator and of the check-out and testing of its chemical engineering system was about $\$ 70 \mathrm{M}$, of which DoE provided about $48 \%$, EPRI $25 \%$, UTC $20 \%$, and Consolidated Edison $7 \%$. The actual construction cost was approximately $\$ 35 \mathrm{M}$ or $\$ 7,800 / \mathrm{kW}$, with the stack cost alone contributing about $\$ 2000 / \mathrm{kW}$. While these figures are very high, it is reassuring to note that the second $4.5-\mathrm{MW}$ unit cost substantially less. This second 4.5-MW demonstrator was ordered from UTC by the Tokyo Electric Power Company (TEPCO) in 1980, was essentially identical to the New York unit, but contained cell stacks with new ribbed substrate technology described in Sec. 2.10. Since the cell stacks had more electrolyte inventory than the New York stacks, the shelf-life problems encountered in New York did not occur. Construction and licensing of the TEPCO unit, located at the Goi Power Plant on Tokyo Bay, proceeded close to schedule. It was modified in accord with requirements lear ned in New York, which involved improvements to the burner and insulation of the reformer and in the water-treatment and water-cooling system. The Goi unit was started up in April 1983 and, after some maintenance work in the summer of 1984, it was still (March 1985) operating satisfactorily. In view of the success at Goi, proposals to retrofit the New York unit with new stacks were abandoned as not cost-effective. A report is available on the New York unit, including the complete testing ot its $\mathrm{H}_{2}$-generating system. 23

After some maintenance, testing of the Goi unit is expected to continue until the end of 1985, after which decisions will be made on new installations. Up to mid-March 1985, the plant had run for a total of about $2000 \mathrm{~h}$ and had produced more than $5000 \mathrm{MWh}$. The longest continuous run was $500 \mathrm{~h}$. The plant demonstrated a period of satisfactory operation under full power on NG at the heat-rate and anticipated levels of chemical and acoustic emissions predicted by UTC. Heat-rates are shown in Fig. 2.8-1. The plant was maintained by the utility and produced utilityquality AC power with the correct start-up/shut-down and part-load to full-load characteristics. 23, 24 The Goi plant is a technical success and demonstrates that the PAFC will operate well for an electric utility. Concurrently, GRI and DoE have supported the manufacture and 


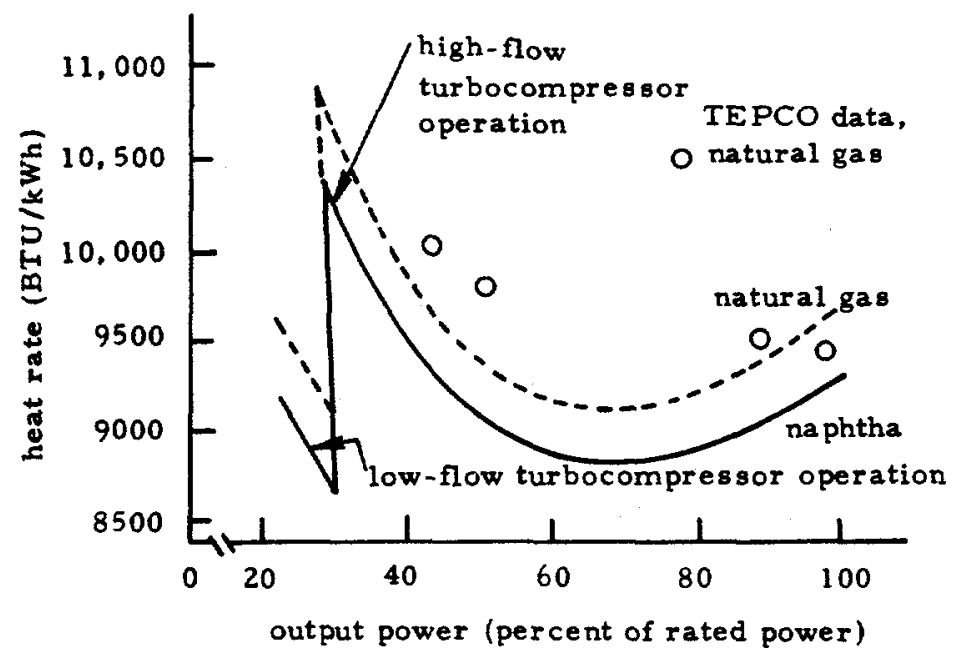

Fig. 2.8-1. Demonstrator heat rate, based on higher heat value of the fuel.

demonstration of approximately 49 UTC $40-\mathrm{kW}$ atmospheric pressure, on-site units using 36 host-gas utilities at various sites in the U.S., at three sites in Japan, and at one in Canada. In FY 83, GRI spent $\$ 24 \mathrm{M}$ on this project, DoE $\$ 8.25 \mathrm{M}$ and DoD $\$ 2 \mathrm{M}$. The DoE support was largely allocated to $R \& D$ on cell hardware. As of the end of February 1985, 41 of the se power plants had been delivered to 36 sites, one was operating in-house at UTC, and a total of $97,000 \mathrm{~h}$ of operation had been achieved $(50,000 \mathrm{~h}$ between October 1984 and February 1985). The longest total period of operation for a single unit was $7000 \mathrm{~h}$ at UTC, followed by $6470 \mathrm{~h}$ at Souther $\mathrm{n}$ California $\mathrm{Gas}$. Over 30 continuous periods of more than $1000 \mathrm{~h}$ of operation on different units have been recorded, and continuous runs over $2000 \mathrm{~h}$ have been recorded twice. A 24-cell onsite stack has exceeded $25,000 \mathrm{~h}$ of continuous running. 12

As expected, some difficulties were experienced with early units, but these were progressively eliminated or reduced as later units were produced. The major recurrent difficulty appears to be in the piping and manifolding to the pressurized water-cooling system in the cellstack cooling plates and will necessitate a manifold and tube redesign using teflon-coated stainless steel piping instead of teflon-coated copper (giving a slight heat-transfer penalty) in commercial developments of the technology. This redesign will reduce water-treatment subsystem purity requirements, improve reliability, and greatly reduce the need for frequent chemical cleaning of the cooling system. Cleaning has generally been required between 1500 and $2000 \mathrm{~h}$, with the longest continuous $r$ un to the end of October 1984 being $2165 \mathrm{~h}$ at Consumers Power, Jackson, MI.

The PAFC demonstration program will be completed by the end of 1985. Although a technological success, it is unlikely, as was the case for the PC-11, to meet the original commercial goals in the foreseeable future. The problem has been discussed in Sec. 2. 4: the system is so far up the learning curve that initial production costs are too high to allow spontaneous commercialization. The $40-\mathrm{kW}$ units have been manufactured at a cost of about $\$ 12,500 / \mathrm{kW}$. Considerations of economies of scale have forced a reorientation of the atmospheric-pressure on-site program towards a larger $200-\mathrm{kW}$ unit, which will hopefully be commercialized at an initial cost of about $\$ 2,500 / \mathrm{kW}$.

\subsection{PAFC Technology Plans}

\section{A. Work at UTC}

The 3.4-atm New York and Goi demonstration units operated at $0.65 \mathrm{~V} / \mathrm{cell}$ and $250 \mathrm{~mA} /$ $\mathrm{cm}^{2}$ but were only capable of heat-rates of 9300 or $9500 \mathrm{BTU} / \mathrm{kWh}$ (36 or $37 \% \mathrm{HHV}$ efficiency), depending on the fuel used (light distillate or NG). As is shown in Sec. 2.6, this heat-rate is marginal for practical utility use. Starting about 1980, UTC commenced the design of an advanced system 25 with the capability of achieving lower cost and higher efficiency. This design is commonly known as the FCG-1, for Fuel Cell Generator No. 1. It utilizes conceptually a reformer and fuel-processing system similar to that of the 4.5-MW units. However, the FCG-1 was designed to operate at a pressure of 8.2 rather than at 3.4 bars, so that its gas-volume throughput would be similar to that of the 4.5-MW demonstration units. Because of the use of higher gas pressure, the anode gas may be employed to feed a much larger total stack area at a higher unit cell potential. After performing a series of optimization studies, the cell temperature was raised by $15^{\circ} \mathrm{C}$ above that of the $4.5-\mathrm{MW}$ demonstrators to approximately $205^{\circ} \mathrm{C}$, 
thereby improving steam raising and cathode performance. The cell potential at the end of the specified useful cell life was to be $0.73 \mathrm{~V}$ at an average current density of $216 \mathrm{~mA} / \mathrm{cm}^{2}$. Aver age values for cell temperature and current density are stressed because the PAFC system operates in the cross-flow mode, so that reactant concentrations and hence current densities and temperatures vary across the cell face. These distributions are carefully optimized by using computer models. The FCG-1 was the first unit designed with a built-in electrolyte reservoir in the ribbed porous electrode substrates of the cell structure and sufficient electrolyte (about $0.8 \mathrm{~kg} / \mathrm{kW}$ ) to compensate for evaporation at a hot lifetime of at least $40,000 \mathrm{~h}$. The end-of-life heat-rate of the FCG-1 power plant (at $0.73 \mathrm{~V}$ average) was estimated to be about $8300 \mathrm{BTU} / \mathrm{kWh}$ (42\% HHV efficiency) at $35^{\circ} \mathrm{C}$, with a temperature coefficient of about $-4 \mathrm{BTU} / \mathrm{kWh}-{ }^{\circ} \mathrm{C}$ below this ambient temperature resulting from changes in compression work as a function of temperature. Since the average cell voltage decays somewhat with hot operating hour 8 because of slow catalyst recrystallization and other physicochemical changes, heat-rates early in life are lower at $\approx 7920 \mathrm{BTU} / \mathrm{kWh}$ at $35^{\circ} \mathrm{C}$ ambient. However, as is shown in Sec. 2.12, voltage degradation is a nearly linear function of the logarithm of hot operating time. After $10,000 \mathrm{~h}$, further decay should therefore be small (about $200 \mathrm{BTU} / \mathrm{kWh}$ in going from 10,000 to $40,000 \mathrm{~h}$ ). As a result, typical mid-life heat-rates were estimated to be $7990 \mathrm{BTU} / \mathrm{kWh}$ at $10,000 \mathrm{~h}$ on a $10^{\circ} \mathrm{C}$ day and $8080 \mathrm{BTU} / \mathrm{kWh}$ on $a 35^{\circ} \mathrm{C}$ day. These estimates 26 were calculated from UTC data. 27 The proposed plant was designed to yield a heat-rate nearly independent of load, as is required for optimal utility intermediate-load operating characteristics. It was designed to operate interchangeably on light distillate, NG and methanol, with a heat-rate almost independent of the fuel used. A dedicated methanol system could be supplied if this fuel becomes widely a vailable at low cost. Because of the advantageous low-temperature reforming characteristics of methanol, its processing would be much simpler and cheaper than the processing in the proposed FCG-1. For the same reason, the heat-rate would be improved above that for the NG or light-distillate units to $\approx 6970 \mathrm{BTU} / \mathrm{kWh}$, as extrapolated from system data given in Ref. 28. A schematic plan of the proposed FCG-1 is given in Fig. 2.9-1. The FCG-1 stacks were also designed to operate in a free-standing mode without a fuel-processing system and use of coal-derived gas or $\mathrm{H}_{2}$. Fuel clean-up is required 29 to only $\approx 50 \mathrm{ppm}$ of total $\mathrm{S}$ as $\mathrm{H}_{2} \mathrm{~S}$ and $\mathrm{COS}$ for efficient anode operation, but EPA regulations will necessitate sulfur reduction below this level. Characteristics of both the proposed FCG-1 and free-standing options are shown in Table 2.9-1.

\section{B. Work at Energy Research Corporation (ERC)}

ERC of Danbury, CT, now a subsidiary of Fluor Corporation, has been involved in the experimental development of the PAFC since 1969. Unlike UTC, ERC has always emphasized the use of air-cooling instead of pressurized water-cooling for acid FC stacks operating at temperatures above $150^{\circ} \mathrm{C}$. The reasons for this preference are associated with ERC's original goal of building small units $(0.5-3.0 \mathrm{~kW})$ for use by the U.S. Army. With methanol as fuel, steam-reforming may be accomplished at high space velocities, $250^{\circ} \mathrm{C}$, and atmospheric pressure on commercially a vailable catalysts. With methanol, the high-pressure steam produced in serpentine or manifolded cooling tubes inserted into the UTC graphite stack cooling plates (typically one for every five cells) becomes unnecessary. Instead, a simple air-cooling system is incorporated into the cell stack. With a heat-exchanger, this system produces a vaporized 60 volume\% methanol-water mixture (about $1.5: 1$ steam-carbon ratio). The depleted anode gas is then burned at 1 atmosphere in the reformer in the form of a tube-and-shell heat-exchanger. ERC subsequently considered the se imple air-cooled systems for use in utility-power generation. The initially favored system was known as DiGas $R$, in which the cell stack had a cross-flow configuration, and the process air stream was diverted into two types of channels in the stack: channels in individual cells, with relatively small cross-sectional area; and channels in the cooling plates (approximately one for every five cells), with greater cross-section. The channel areas and distributions were calculated according to required process air flows, oxygen utilization, current density, and heat-transfer requirements. 30 A perceived advantage of this system is that the cooling portion of the air supply does not come into contact with the electrolyte and therefore does not contribute to its evaporation. The straight-through cross-flow manifolding is very simple. The system is shown in Fig. 2.9-2.

ERC has always emphasized the use of inexpensive stack components. In work prior to 1972. which was conducted at relatively low cell temperatures of $\approx 150^{\circ} \mathrm{C}$, phenolic regins (Kynol) were shown to be sufficiently stable in fiber form to be used as a matrix material. Work at this time also emphasized the use of experimental bench-scale boiler-plate hardware or bipolar plates consisting of phenolic-bonded graphite powder materials. Later, as higher cell potential and current densities were recognized to be important in reducing system weight and volume, phenolics were found to be insufficiently stable, and attempts were made to use better resins. About 1975, polyphenylene sulfide, Hetron ${ }^{R}$ and H-Resin (Hercules) were examined, but these were either not cost-effective or were products which were abandoned because of manufacturing problems or because the potential markets were too small to justify continued production. Finally, major cell components consisting entirely of carbon were made for use at 


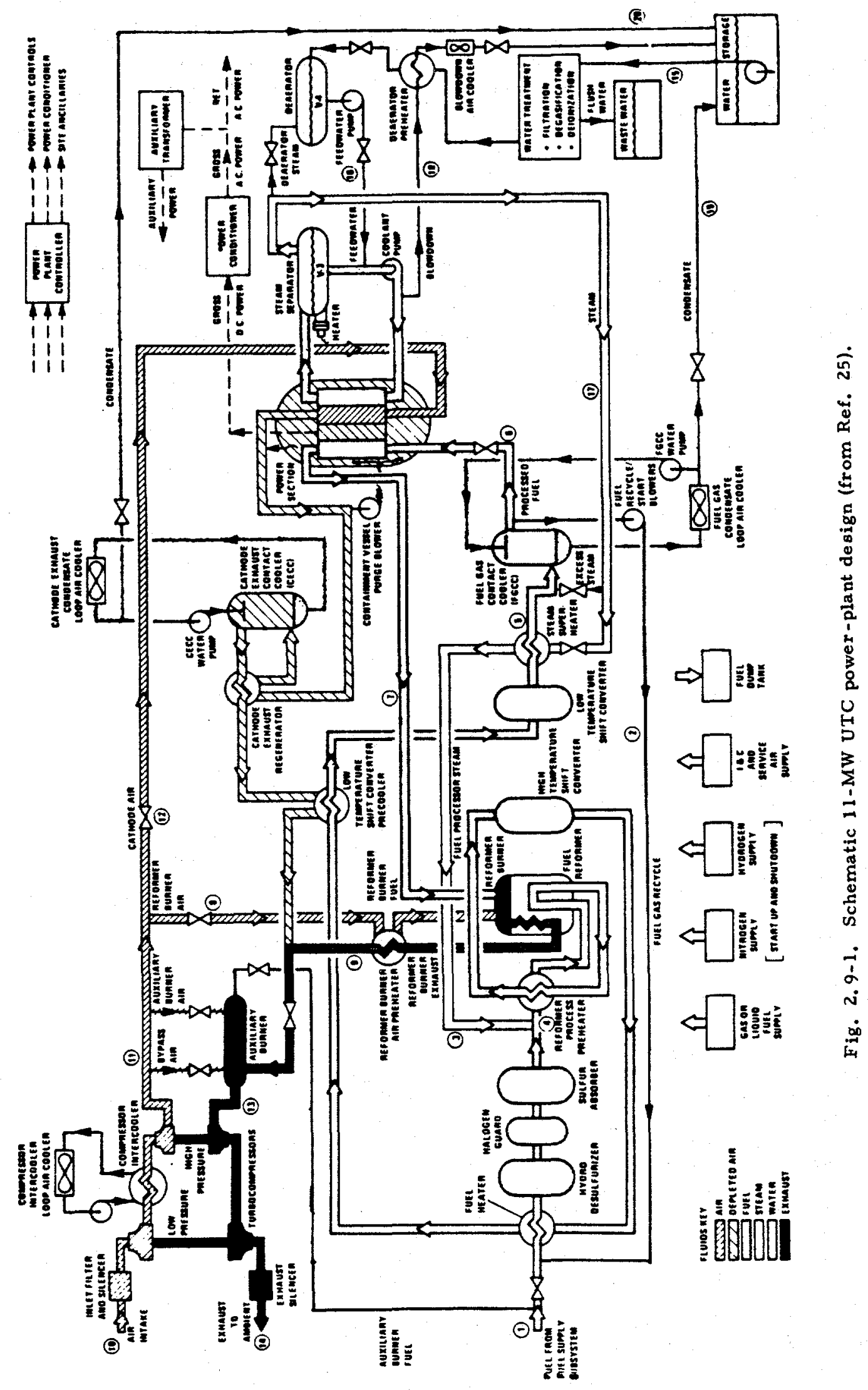


Table 2.9-1. The 1986-90 PAFC plant, which represents a first commercial target for the PAFC.

\begin{tabular}{|c|c|c|}
\hline Characteristics & $\begin{array}{l}\text { Dispersed } \\
\text { Applications }\end{array}$ & $\begin{array}{l}\text { Integrated with } \\
\text { Coal Gasifier }\end{array}$ \\
\hline fuel & $\begin{array}{l}\text { clean gaseous or } \\
\text { liquid fuel } \\
\text { NG } \\
\text { naphtha } \\
\text { methanol } \\
\text { coal gas }\end{array}$ & coal \\
\hline unit size & $\sim 10 \mathrm{MW}$ & $250 \mathrm{MW}$ \\
\hline $\begin{array}{l}\text { efficiency (fuel to } \mathrm{AC} \\
\text { power), } \%\end{array}$ & 41 & 35 \\
\hline $\begin{array}{l}\text { air emissions: } \\
\mathrm{SO}_{\mathbf{x}} \\
\mathrm{NO}_{\mathbf{x}} \\
\mathrm{TSP}\end{array}$ & $\begin{array}{l}3 \times 10^{-5} \mathrm{lb} / 10^{6} \mathrm{BTU} \\
2 \times 10^{-2} \mathrm{lb} / 10^{6} \mathrm{BTU} \\
\text { not detectable }\end{array}$ & $\begin{array}{l}4 \times 10^{-3} \mathrm{lb} / 10^{6} \mathrm{BTU} \\
2 \times 10^{-3} \mathrm{lb} / 10^{6} \mathrm{BTU} \\
\text { negligible }\end{array}$ \\
\hline $\begin{array}{l}\text { license \& construction } \\
\text { time, y }\end{array}$ & 3 & 5 \\
\hline $\begin{array}{l}\text { earliest time available } \\
\text { for orders }\end{array}$ & 1986 & 1992 \\
\hline
\end{tabular}

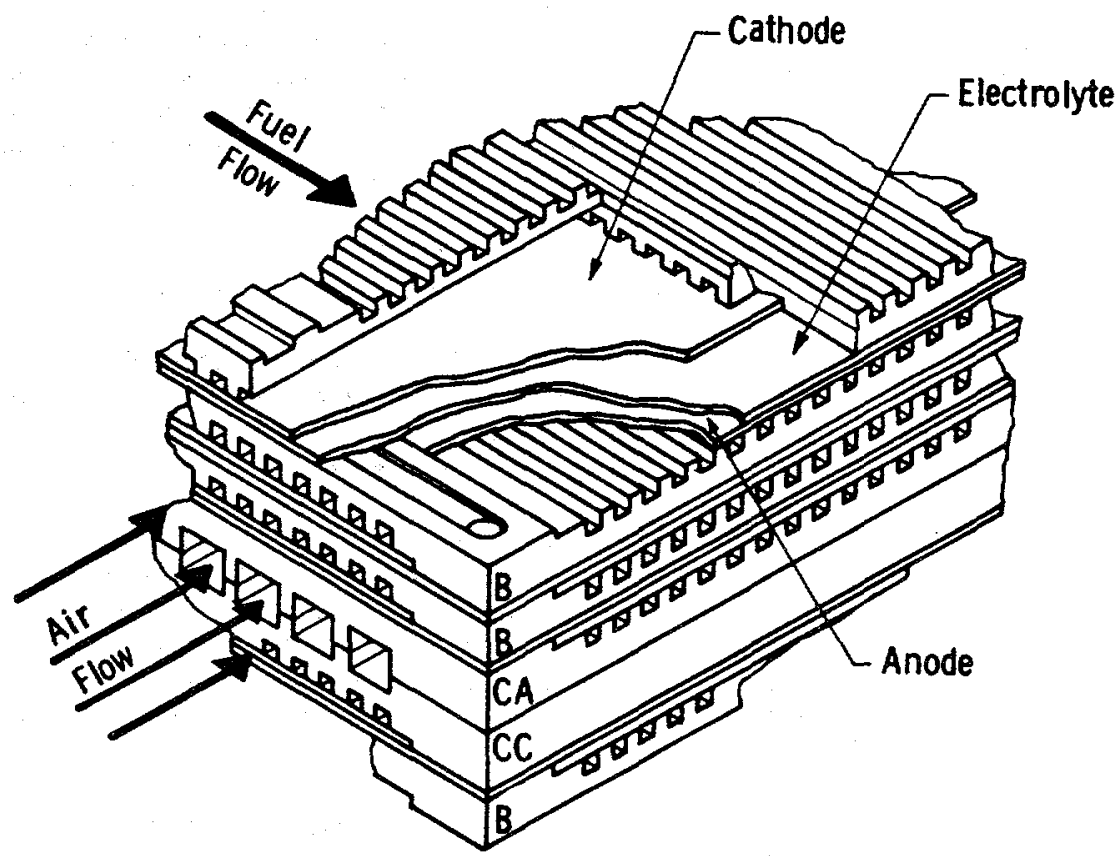

B - Bipolar plate with process air \& fuel channels

CA - Anode DIGAS cooling plate

CC - Cathode DIGAS cooling plate

Fig. 2, 9-2. The ERC-WE DiGas ${ }^{T M}$ system. 
temperatures in the $165-180^{\circ} \mathrm{C}$ range, with teflon-bonded silicon carbide as matrix material, following a UTC patent. 31

Bipolar plates were molded from a mixture of graphite (about 67 w\%) a nd phe nolic resin (33 wt\%) and were carefully heat-treated to carbonize the resin without introducing excessive porosity by rapid degassing. Typically, heat-treatment to $900^{\circ} \mathrm{C}$ was sufficient to give the required chemical, physical and mechanical properties. Early bipolar plates were molded flat and were machined to produce the required reactant gas-distribution grooves (or cooling grooves for the bipolar plate). Later, grooved plates were molded in a die (which was slightly oversized to compensate for shrinkage during baking) to produce the glassy graphitic, carbon-composite plate. The system was standardized at a plate size of $30 \mathrm{~cm} \times 42 \mathrm{~cm}$ with process and cooling air supplied along the longer side.

Electrodes used at ERC since about 1975 are similar to those used in the UTC 4.5-MW New York demonstrator: typically, $0.5 \mathrm{mg} / \mathrm{cm}^{2}$ of colloidal Pt in $10 \%$ loading on Vulcan XC-72 at the cathode, $0.25 \mathrm{mg} / \mathrm{cm}^{2}$ at the anode, combined with about $40 \mathrm{wt} \%$ colloidal teflon onto graphite-paper backing. The latter was pre-wetproofed with tellon to prevent excessive wetting by electrolyte and gave mechanical atrength to the electrode layers while providing good electronic contact to the bipolar plate. As has become commonplace in the industry, the required materials are supplied to specification by commercial vendor s (catalyst-on-carbon by JohnsonMatthey, graphite paper by Stackpole, teflon dispersion by Dupont). Unlike UTC, which used a spraying technique to place the electrode material mix onto the backing, ERC favored the preparation of films of catalyst and teflon by a calendering operation. The electrode preparation was completed by heating the catalytic layer and backing to $360^{\circ} \mathrm{C}$ in an inert atmosphere to sinter the teflon. This procedure provides optimal wetproofing and yields a good three-phase-boundary electrolyte-catalyst-gas interface for high reactivity, together with long electrode life.

\section{Work at Westinghouse (WE)}

In 1978-79, WE started work on PAFCs for utility use. After concluding a licensing agreement to use ERC technology, WE completed a study on the feasibility of the DiGas aircooled stack for this application. 32 Major conclusions were that auch a system appeared feasible but would require recirculation of most of the gas exhausted from the FC stack through an external heat exchanger for efficient heat recovery. Ratios of recirculating gas to make-up air of 15:1 to 60:1 were predicted, with fan-power and heat-exchanger-area considerations favoring the lower ratios. Despite large heat-exchanger area and piping-volume requirements, preliminary costs of components in the recirculatory coolant loop were estimated at only $\$(1979) 40 / \mathrm{kW}$. This low additional investment cost, together with the intrinsic reliability of an air-cooled system (in the event of tube rupture in water-cooled tubes, cells will electrically short out with catastrophic stack loss) made the concept look attractive. Heat-rates were calculated for two operational cases for systems operating at $3.4 \mathrm{~atm}$, at two different mean cell operating temperatures, with an average current density of $270 \mathrm{~mA} / \mathrm{cm}^{2}$ for both. The estimated values obtained were $9100 \mathrm{BTU} / \mathrm{kWh}$ at $190^{\circ} \mathrm{C}$ and $0.64 \mathrm{~V} / \mathrm{cell}$ and $8700 \mathrm{BTU} / \mathrm{kWh}$ at $205^{\circ} \mathrm{C}$ and $0.66 \mathrm{~V} /$ cell.

These preliminary investigations of the air-cooled PAFC system led WE to perform more detailed work to define parameters and costs. One of the first conclusions that became apparent was that a heat-rate of $9100 \mathrm{BTU} / \mathrm{kWh}$ was not commercially attractive. Therefore, attempts were made to adjust various aspects of the system to obtain a value closer to UTC's proposed $8300 \mathrm{BTU} / \mathrm{kWh}$. 77 This improvement could probably ha ve been effected by cathode-catalyst changes that would allow $0.70 \mathrm{~V} / \mathrm{cell}$ operation (up from $0.64 \mathrm{~V} / \mathrm{cell}$ ) at $190^{\circ} \mathrm{C}$, but the approach was not followed at WE. Instead, a number of air-cooled systems modifications were put into place, including abandonment of the $\mathrm{DiGas}^{\mathrm{R}}$ concept, for which separately manifolded processair and cooling-air streams were substituted. The new arrangement led to lower total compression-work and pumping-energy requirements. A schematic view of the proposed configuration is shown in Fig. 2.9-3. According to the original calculations, this system design would yield $8300 \mathrm{BTU} / \mathrm{kWh}$ at unit cell voltage of $0.68 \mathrm{~V}$. The system includes a stage for condensation of product water via a turbine/condenser in the turbocompressor, which essentially amounts to an energy-saving bottoming cycle. UTC's unit does not contain a cycle of this type but requires the burning of a small amount of fuel up-stream of the turbine to balance the turbine and compressor work at part load.

Separation of WE's process air and air-cooling streams had important consequences, not only for cell manifolding but also for design of the cell stack and DC power section of their PAFC unit. Some of these have been described recently. 33 The WE approach has involved a total redesign of the gas-distribution grooves on the bipolar plate to yield an arrangement which has become known as the $Z$ plate.

In the $Z$-plate design, the inlet manifolds for the two reactants are side by side on the narrower side of the bipolar structure, and the appropriate gases for adjacent cells enter on opposite sides of the bipolar plate. The channel arrangement is illustrated in Fig. 2.9-4. Basically, the gas channel nearest the centerline of the plate makes an immediate right-angle turn to the far side of the plate, followed by a further turn along the length of the plate. Subsequent 


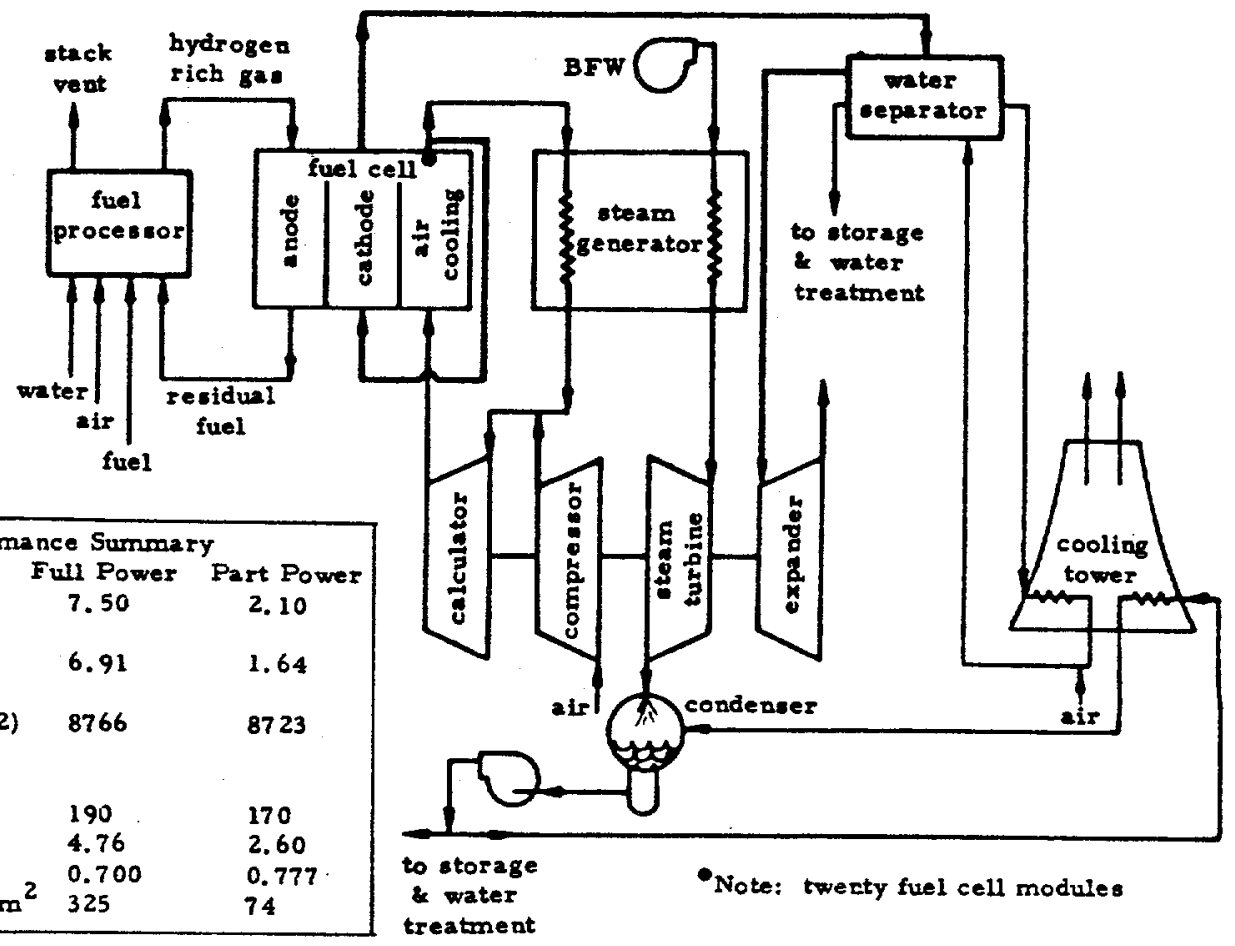

Fig. 2.9-3. The WE PAFC plant (1985, $8100 \mathrm{BTU} / \mathrm{kWh}$ at $0.69 \mathrm{~V} / \mathrm{cell})$; a condenser-bottoming cycle is located in the turbine.

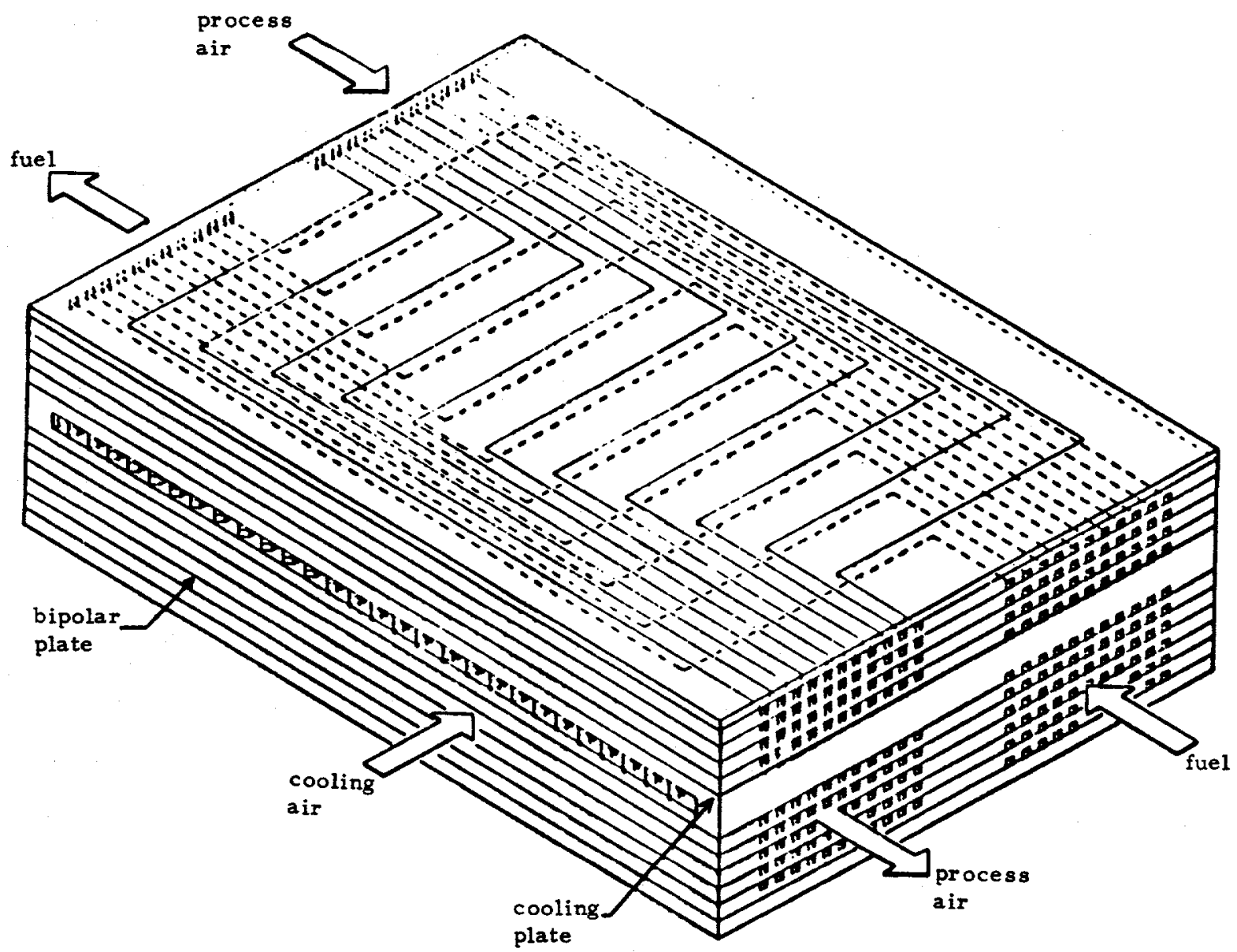

Fig. 2.9-4. The WE air-cooled stack concept. 
channels make the initial turn progressively farther down the plate, the length of the right-angle flow portion being constant for each channel. Finally, the gas channel nearest the plate edge runs the whole length of the plate before the first turn. Looked at horizontally, the fuel-flow and oxidant channels in each cell are mirror images, so that the exit manifolds for one gas are opposite the inlet manifolds of the other. The net result is a clever combination of cross-flow and counter -flow channels with good local gas partial pressure, temperature, and current density contour $B$ across the surface of the plate. It is expected that this design will be further refined.

The longer side of the plate is reserved for the cooling air, which flows through cooling channels located approximately 5 cells apart. The cooling plate has the flow-channels of the anode or cathode of an active cell on one side and cooling air channels on the other. The channels are of a straight-through tree type, with a large cross-section on the inlet side and then branching into many smaller channels as the coolant crosses the plate. This design provides most efficient cooling across the plate.

The new system manifolding required a change in the arrangement of the FC stacks within the pressure vessel. With pressure-vessel dimensions dictated by practical considerations of size, wall thickness and a vallability, the most cost-effective diameter for 3.5 bar operation was found to be just over $1 \mathrm{~m}$ and will accommodate four $30 \mathrm{~cm} \times 43 \mathrm{~cm}$ cell stacks arranged in a cruciform manner, with their longer sides forming a square in an internal duct. This duct serves to evacuate hot air downwards, after it has passed through the cells from segmental ducts between the outside of each stack and the pressure-vessel walls (Fig. 2.9-5).

The arrangement (see Fig. 2,9-5) thus consists of an elegant package of small, easily manufactured components. The cell-stack arrangement has a cooling system that is relatively fail-safe. It does, however, have a number of disadvantages compared with the UTC system, which may offset these advantages. One has been referred to and relates to the piping diameters and heat-exchanger area required for the recirculating air-cooling system. Another is discussed in Sec. 2,13 and relates to system-efficiency loss in cogeneration or when integrated with a coal gasifier. A major factor, which has had a large effect on cell-performance requirements, is the relatively lower power footprint of the WE system within its pressure vessel compared with UTC's FCG-1 design and the originally specified mean current densities. The UTC design, with $0.98-\mathrm{m}^{2}$ cells, was intended to give $1.8 \mathrm{~kW} /$ cell, whereas WE originally estimated a total of about $0.8 \mathrm{~kW}$ for a combination of the four small cells, corresponding to each element of the four-stack combination. Since this lower footprint would lead to high pressure-vessel costs, the design current density was first raised to $325 \mathrm{~mA} / \mathrm{cm}^{2}$, thereby increasing the power per unit four-cell combination to $1.0 \mathrm{~kW} / \mathrm{m}^{2}$. This value has now (May 1985) again been reduced to $0.8 \mathrm{~kW} / \mathrm{m}^{2}$, corresponding to $267 \mathrm{~mA} / \mathrm{cm}^{2}$, to yield a higher cell potential.

WE has made important progress, both in design and development, since 1980 with internal support and funding from DoE-NASA (about $\$ 12 \mathrm{M}$ in 1984 for stack-component improvement) and EPRI (about $\$ 2 \mathrm{M} /$ year for systems). WE's present plans are to build 7.5-MW electric utility units. Each stack will contain about 400 cells, so that the individual power sections (i.e.. the four-stack systems in each pressure vessel) will produce between 375 and $400 \mathrm{~kW}$, depending on the exact active cell area chosen for the final product. This unit is expected to be installed by about 1988 and should be followed by further utility orders. As will be discussed in Sec. 2.13, the design of the 7.5-MW plants is not yet frozen, and continuing product improvement may be expected to result in improved heat-rates in the future. A part of this work includes system improvements, particularly in the advanced reformers. New developments in this area are being partially financed by EPRI and involve two approaches: a high-risk advanced system with a large, unitized, fluidized-bed reformer, which is under development at Mitsubishi Heavy Industries (MHI), and a modular reforming system of Haldor-Topsbe (Denmark) design. WE will deliver a $375-400 \mathrm{~kW}$ stack to MHI for testing at Kyushu Electric Power Company in Japan in 1986. Testing on a $10-\mathrm{kW}$ scale is now in progress. The next stage is the detailed design and development of the $100-\mathrm{kW}$ stack (one quarter of the $400-\mathrm{kW}$ unit).

\section{Work at Sanyo}

Sanyo in Japan has also become a licensee of ERC's air-cooled PAFC technology. Their objective appears to be similar to that of $\mathrm{WE}$, namely, the development and manufacture of an electric utility unit, with emphasis on the use of NG. Since an agreement was signed with ERC in 1980. Sanyo has made significant progress in defining stack and systems requirements for this application. The development is internally financed. Although little is known about the latest work, it appears that Sanyo is actively pursuing the design of a stack with dimensions and reactant-gas manifolding similar to those of WE. Separated process- and cooling-air flows are used. All stack components will be graphitic for added corrosion resistance. Sanyo has signed an agreement with TEPCO to build a $600-\mathrm{kW}$ demonstration unit for operation in June 1986.

\section{E. Work at Engelhard Industries (EI)}

EI has been working on some form of the PAFC for the past $20 \mathrm{y}$. Its research on the FC utilizes EI expertise in catalysis. Thus far, EI has not been involved in the larger units for 


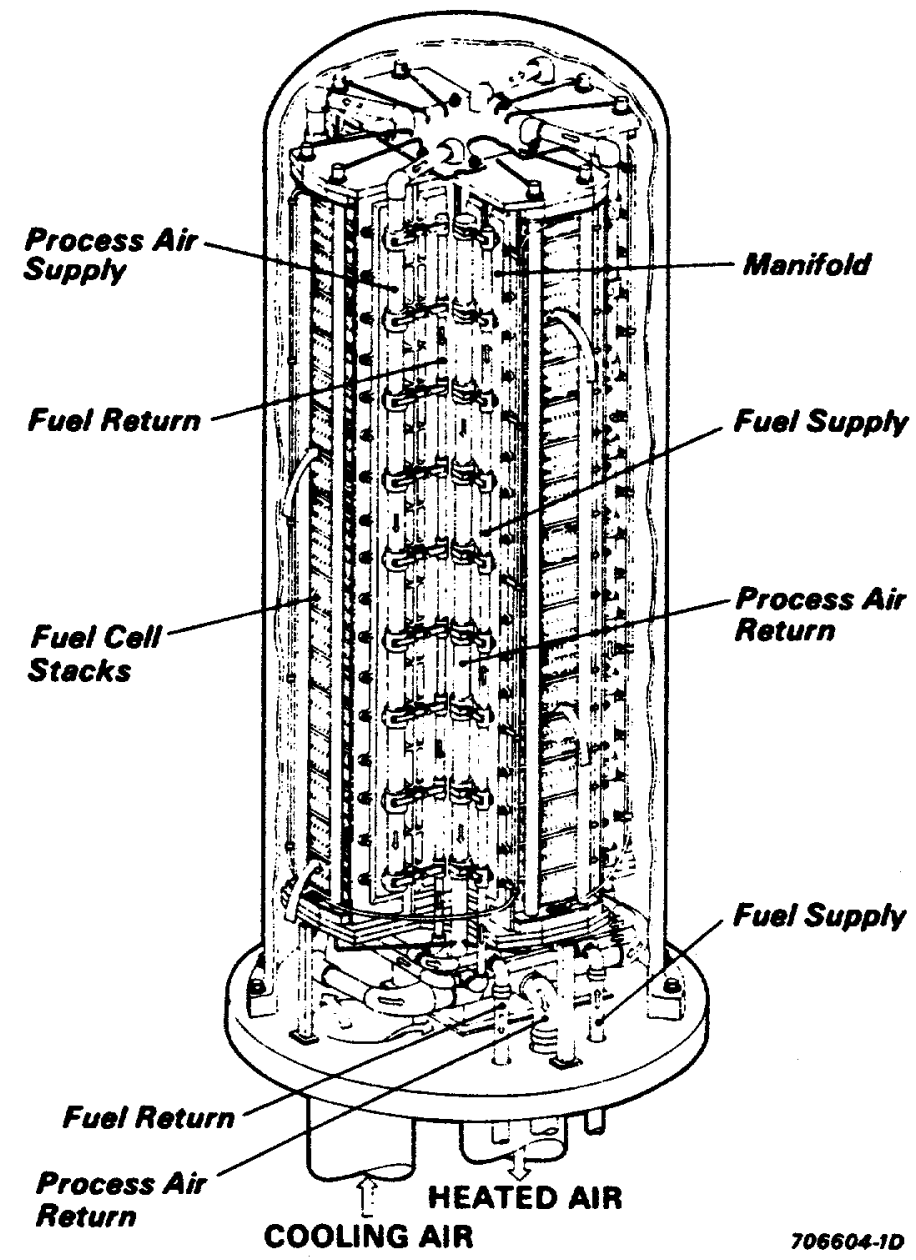

Fig. 2.9-5. The $375-400 \mathrm{~kW}$ WE $1-\mathrm{m}$ diameter cell stack (four rectangular air-cooled stacks in cruciform configuration). 
the electric utility market. Instead, emphasis has been on mobile applications (e.g., an experimental fork-lift truck) and on remote on-site markets, with reformed methanol as fuel. Their PAFC system concept is supported by DoE to the extent of about $\$ 2 \mathrm{M} / \mathrm{y}$ as a back-up technology for on-site use. Cell-size and operating conditions are similar to those of the UTC 40-kW atmospheric pressure, on-site cell. While the cell is liquid-cooled, a notable design characteristic of the system is the use of a dielectric liquid coolant in place of pressurized water. In addition, the structure of the bipolar plate is unique. It is ribbed on both sides in a cross-flow arrangement that is similar to that of the 4.5-MW New York demonstrator and also to ERC's system. It consists of two separate pieces bonded together by dense carbonized resin (the socalled ABA structure). This item is of practical interest in terms of cost and manufacturing ease and is described in Sec. 2.13.

\section{F. Work Supported by the Japanese Government}

A national effort to develop a commercial PAFC started in Japan in 1980 . The original plan was to produce an indigenous version of the 4.8-MW demonstrator, with a parallel effort to produce a ribbed substrate stack technology of the UTC type, as used in the TEPCO demonstrator and in the $49-\mathrm{kW}$ on-site units. The program is financed by the National Energy Development Organization (NEDO) under the Japanese Government's Moonlight Program for national energy independence. Probably less than half of the costs are being provided by NEDO. NEDO provided the original specifications for the cell technology to be developed in the initial learning exercise that was to have two I-MW demonstrators on line by the middle of 1986 . During this learning process, government-funded developers are designing their own, definitive commercial technology, which we anticipate to be different in detail and scale from the original NEDO guidelines. At the present time, plans beyond the two 1-MW units, which will be installed in the networks of the Chubu Electric Power Company (CHEPCO, Nagoya) and the Kansai Electric Power Company (KEPCO, Osaka), are not known.

Starting in 1981, NEDO has asked two groups of developers to conduct R\& D on two systems that are apparently not intended to be directly competitive. Nevertheless, there exists strong competition between individual Japanese developers involved. The ground rules laid down by NEDO were to develop square cell-stack technology with an active area of $0.36 \mathrm{~m}^{2}$, containing not more than $6.5 \mathrm{~g}$ of Pt per $\mathrm{kW}$ (WE cells currently use about $4.1 \mathrm{~g} / \mathrm{kW}$; since the end of 1984, UTC cells have used between 4.8 and $7.3 \mathrm{~g} / \mathrm{kW}$, depending on cell specification). These ranges represent $\$ 32-52 / \mathrm{kW}$ at the present Pt price of $\$ 295 /$ troy-ounce, which is depressed compared with the $\$ 475 /$ troy-ounce price fixed by $P t$ producers. Even at the producer price, which is probably a realistic longer-term value, high loadings correspond to a catalyst cost of only $\$ 84 / \mathrm{kW}$, which is small compared to that of the balance-of-plant cost.

NEDO has fixed $0.7 \mathrm{~V}$ as the target cell-potential, at current densities in the 200$220 \mathrm{~mA} / \mathrm{cm}^{2}$ range, depending on the temperature and pressure characteristics of the technology $\left(190^{\circ} \mathrm{C}\right.$ and 4 bars or $205^{\circ} \mathrm{C}$ and 7-8 bars). Comparison with U.S. experience shows that these goals should be relatively easily attainable with state-of-technology components.

NEDO selected TOshiba and Hitachi to develop higher temperature and pressure technology and Fuji Electric and Mitsubishi Electric (MEICO) to develop a lower pressure and temperature system. These two variants were selected because of the greater suitability of the $190^{\circ} \mathrm{C}, 4 \mathrm{~atm}$ technology for dispersed systems in the 1-5 MW class, whereas the 205 ${ }^{\circ} \mathrm{C}, 7-8$ atm system was considered to be optimal for $10+\mathrm{MW}-$ scale units. The lower temperature-pressure system clearly follows the conditions and stack active areas of the TEPCO unit but somewhat derated in current density and up-rated in cell potential, whereas the higher-pressure and highertemperature unit corresponds closely to somewhat derated (in cell potential) FCG-1 operating conditions, although its stack area is only $37 \%$ of that of UIC's proposed commercial unit. Both design teams have made good progress and have tested $30-k W$ pressurized prototypes, which were designed for component demonstration and not optimal heat integration for highest efficiency. A milestone chart for the development of the technology is given in Table 2.9-2.

Japanese national funding was $200 \mathrm{M}$ in $1981, \% 600 \mathrm{M}$ in 1982 and reached $2.5 \mathrm{~B}$ in $1983(\$ 10 \mathrm{M})$. Funding has been divided in the ratio of about 53:47 between the two groups, with each partner in the two groups receiving equal NEDO funding. NEDO is insisting on cost sharing. According to a relatively complex formula, a partner developing a NEDO-supported part of the technology (for example, the cell-repeat components) must fund a simultaneous program to develop the balance-of-plant. For example, Hitachi and Toshiba are spending more than 11 each, on an annual basis, on FC systems and stack components, respectively, to complement NEDO funding on cell stacks at Toshiba and power plant chemical and electrical engineering at Hitachi. Similarly, MELCO is being supported by NEDO for stack-component development and by Fuji Electric for the balance-of-plant. Fuji has been a pioneer in the development of the PAFC in Japan. A 30-kW Fuji process-air-cooled NG PAFC was developed before the start of the NEDO program and installed at a KEPCO generating unit in July 1982, with now successfully completed testing. These arrangements have stimulated extensive competition and provide NEDO and other Japanese customers with a possible choice of many different technological combinations. 
Table 2.9-2. The Japanese national PAFC milestone chart.

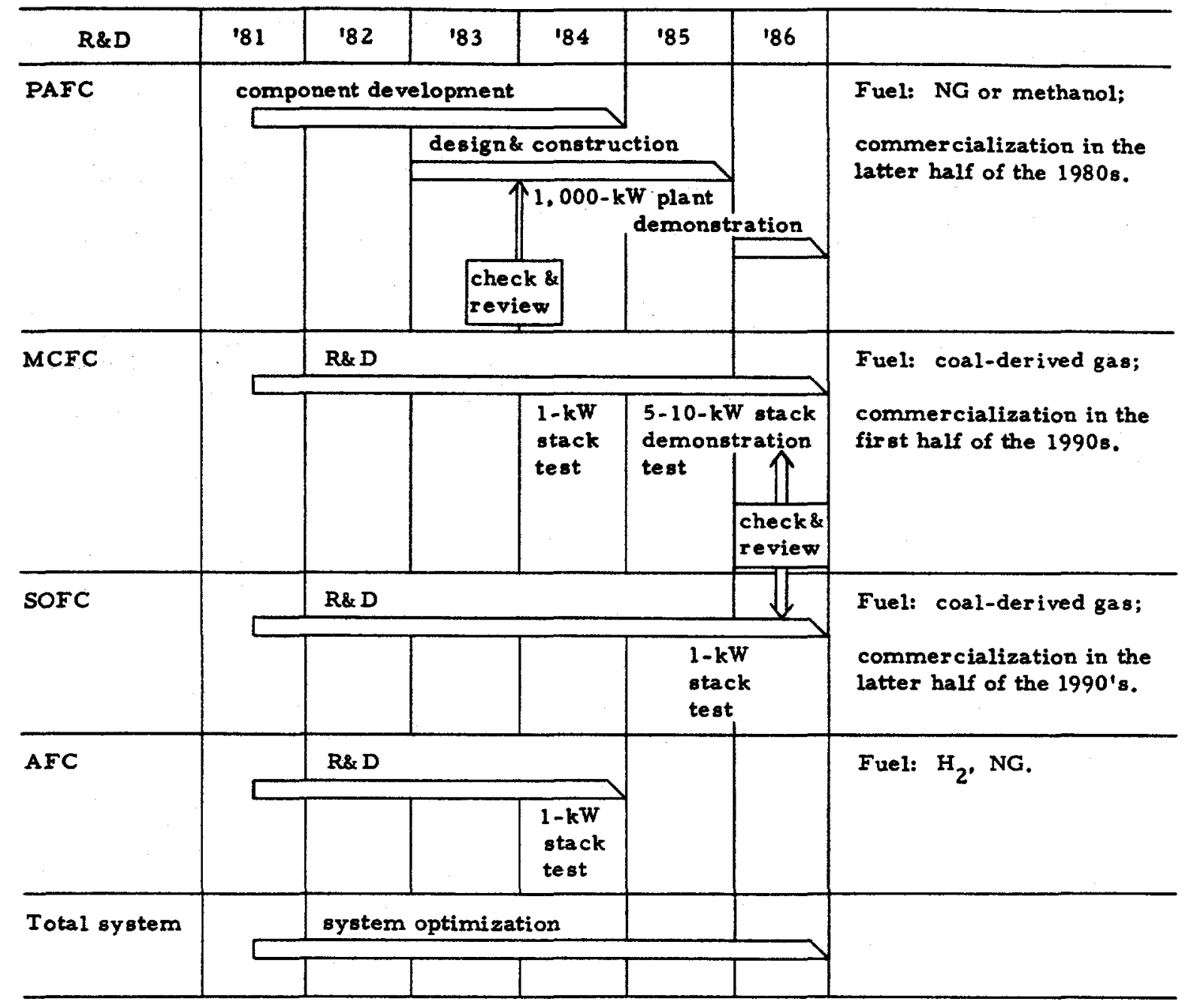

The work in Japan has led to a great deal of system innovation. For example, a catalytic combustion reformer has been developed at Toshiba with a burner that uses the depleted cathode air as oxidant. This system not only reduces the reformer-burner temperature but also reduces the compression-work requirements of the whole plant and hence increases total system efficiency. The NEDO-supported effort at Toshiba is completely independent of any developments at the UTC-Toshiba joint venture (International Fuel Cells, Inc.), which will be responsible for manufacturing and selling the FCG-1 technology or that of its successors. Similarly, cell-stack technology developed at UTC under partial DoE funding will be retained for use in the FCG-1, and future Toshiba commercial units will apparently use Japanese-developed stack technology.

\section{10 Probable FC Component Costs}

We have emphasized in Sec. 2. 4 that cost reduction will be of prime importance during the introduction of the PAFC into commercial use. An important method for lowering cost is reduction of the total number of parts and total weight per $\mathrm{kW}$. For the chemical engineering items in the system, this can be achieved by an increase in the scale of each individual item, as in the scale-up of the $12.5-\mathrm{kW}$ TARGET PC-11 unit, first to the 40-kW PC-18 demonstration on-site unit and then to the proposed $200-\mathrm{kW}$ atmospheric pressure on-site unit; the reformer, heat-exchanger 8 , etc. are thereby reduced in cost. per $\mathrm{kW}$. Increasing the system pressure in the larger electric utility systems also reduces cost by reducing the total weight and volume of piping, making better use of pressure vessels, and incidentally reducing pumping-power requirements that lead to slight increases in efficiency. For the cell stack, component cost can be reduced by the use of cheaper materials. However, a major factor involves a reduction in the number of cell components per $\mathrm{kW}$. For the 4.5-MW demonstrators, the active area of the cell components was $0.34 \mathrm{~m}^{2}$. For the FCG-1 system, UTC proposes to use cells with an active area of $0.98 \mathrm{~m}^{2}$, thus reducing the total number of components that must be manufactured, handled and stacked by $15 \%$ overall compared with the $4.5-\mathrm{MW}$ demonstrator 8 , despite the more than doubled system output. This change means that the total number of cell-stack items per 
$\mathrm{kW}$ will decline by a factor of three, which should lead to substantial overall cost savings. Finally, cheaper starting materials and methods of manufacture are being explored under DoE/ NASA contracts so that the early rapid descent down the learning curve will occur expeditiously. A question which must be addressed is just how realistic the costs are which will be required for spontaneous market penetration of the PAFC. The developers' business plans and strategy are confidential but it has nevertheless been possible to use outside architectural engineering companies to provide internal audits of the technology, both of the chemical engineering to verify workability of the system ${ }^{34}$ and of the manufacturing cost. 35 The conclusions were that both were realistic. We must therefore assume that the efficiency and cost goals to achieve projected market penetration will be met. This conclusion can also be verified by examining the most uncertain costs of the truly new-technology parts of the PAFC system. These are the stack components in mass production, which are also key elements in ensuring technical and commercial success. The components are made of graphite and are presently manufactured by the Acheson process. With linear dimensions of $1 \mathrm{~m} \times 1 \mathrm{~m}$, they are at the upper size limit that can be made in available Acheson furnaces and beyond the size limits of presently available graphite-induction furnace technology, which represents an alternative future method of production that may lead to further cost reduction. In the 4.5-MW New York demonstrator, the $0.34-\mathrm{m}^{2}$ separator plates lying between the anodes and cathodes of adjacent cells were ribbed on both sides to provide gas channels arranged perpendicular to each other. These ribs contacted electrode supports made from thin $(=1 \mathrm{~mm})$, commercially-available graphite felts or papers and carrying the catalyzed electrode surfaces on their inner sides, which were themselves in contact with the immobilized PA electrolyte. The electrolyte was held by capillary action in a fine silicon carbide powder matrix bonded with a small amount of teflon. This structure is shown in Fig. 2,10-1. All of the components had been shown in laboratory tests to be stable against corrosion under both anode and cathode conditions.

The bipolar separator plate in the earlier (1976), l-MW demonstration stack was molded from graphite powder and polyphenylene sulfide resin (cf. Sec. 2.9). The corrosion resistance of this plate was shown to be only marginally acceptable in the finished demonstrator. Therefore, the plates in the 4.5-MW New York demonstrator and also in subsequent UTC stacks were prepared by molding from graphite powder and inexpensive resins, followed by baking and graphitization at about $2700^{\circ} \mathrm{C}$. The surfaces of the molded ribs were then finished by sanding. The electrolyte was partially stored in the matrix, the thickness of which was necessarily as small as possible to minimize internal cell resistance; the electrolyte was partially stored also in the anode-support paper, which on this side consisted of a double-backing layer which had been selectively wet-proofed with teflon via a patented technique 36 that allowed some storage of electrolyte in partially wetted areas, without markedly interfering with hydrogen diffusion. In contrast, the cathode substrate (a single layer) was wet-proofed to the greatest extent possible.

The electrolyte inventory was low (about $0.2 \mathrm{~kg} / \mathrm{kW}$ ), but no allowance had been made for electrolyte logs by evaporation (as $\mathrm{P}_{2} \mathrm{O}_{5}$ ) over some thousands of hours of operation. The residual porosity in the bipolar plate tended to absorb electrolyte in time, as had been observed in

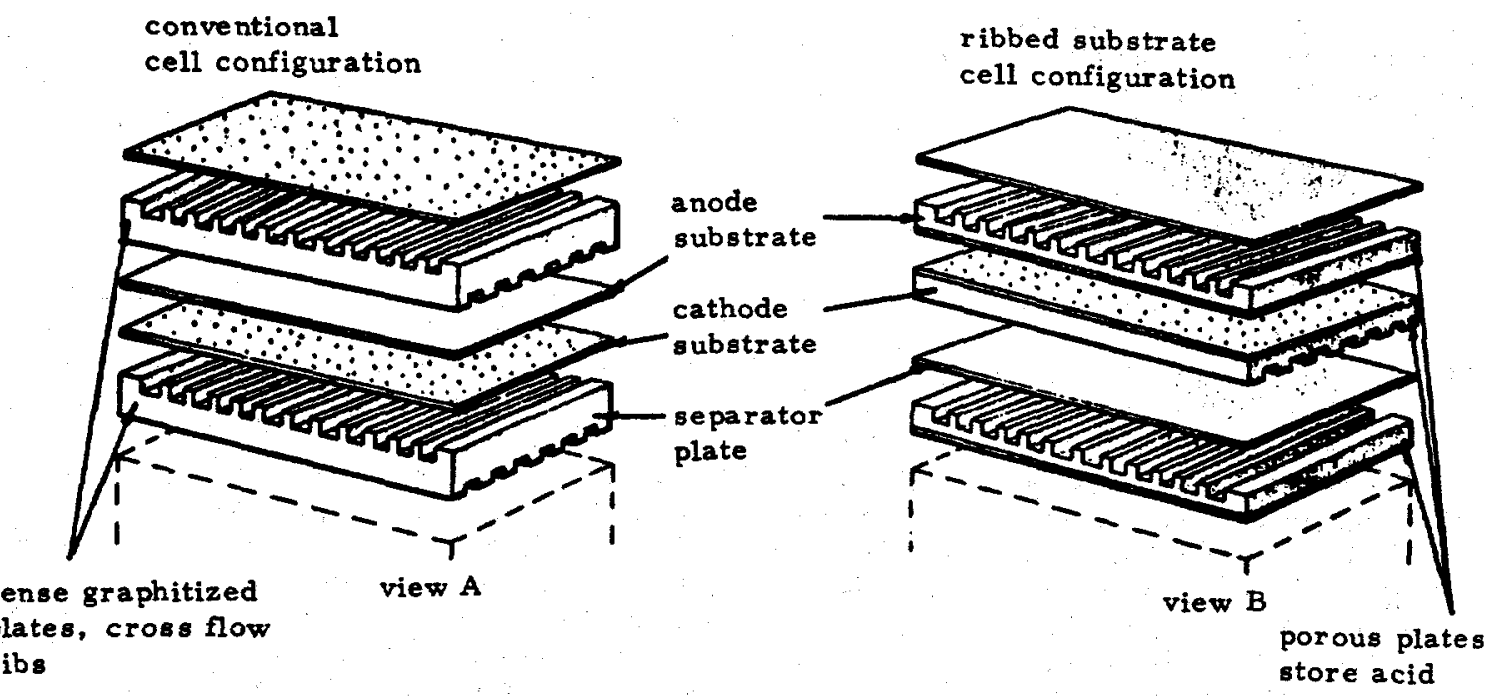

Fig. 2.10-1. Comparison of UTC's conventional stack (4.5-MW, 0.34-m ${ }^{2}$ Manhattan System) and later stacks (ribbed substrate configuration). 
the New York stacks during their long period of storage at $38^{\circ} \mathrm{C}$; this high temperature was necessary to prevent freezing of the concentrated electrolyte in the electrodes, which would destroy their microstructure. As a result, the electrolyte inventory of many of the New York stacks was depleted after about 6 y of storage, thus leading to unacceptable gas crossover when they were finally installed in the power plant. Attempts to refill the stacks in situ failed, and dismantling and rebuilding of the obsolete stacks would have been wasted effort in view of the success of the TEPCO plant.

The 40-kW on-site units and the TEPco Goi system used a new ribbed substrate stack. This sytem placed the gas-distribution channels in the porous electrode substrate itself, rather than in the flat bipolar plate, which was about $1 \mathrm{~mm}$ thick. The ribbed sides of the substrates contacted the surface of this flat bipolar plate. The catalytic electrode mix was applied to the opposite sides. The concept is shown in Fig. 2, 10-1. The initially perceived advantage for this technology was reduced cost. The ribbed substrate configuration promised the possibility of molded bipolar plates requiring a minimum of surface finishing, together with ribbed substrates of relatively low porosity that would be easy to machine. Eventually, if problems of substrate flatness after bake-out and graphitization heat-treatment can be overcome, the green substrate could, in principle, be made in a molding operation using cheap starting materials such as chopped carbon or graphite fibers, perhaps followed by acrylic or other organic fibers that are mixed with resins or pitch. This a pproach promises low ultimate costs.

A further advantage was the possibility of storage of excess electrolyte in the ribbed substrate itself, without interfering with gas diffusion from the ribbed channels to the electrodes. This procedure allowed expansion space for acid-volume change (dilution or concentration as a function of load). The lower pore size of the silicon carbide matrix compared with that of the substrate ensured that the matrix would always be correctly wetted by electrolyte, so that gas crossover would not occur even under moderate conditions of differential pressure. A $40 \%$ fill of the ribbed substrate could accommodate $0.8 \mathrm{~kg} / \mathrm{kW}$ of electrolyte, which (see Sec. 2. 9) should have been sufficient to compensate for electrolyte evaporation over $40,000 \mathrm{~h}$ of operation with gas throughput at 8.2 -bar pressure and $205^{\circ} \mathrm{C}$, as proposed for the electric utility FCG-1.25 In the atmosphere pressure on-site system, the lower operating temperature $\left(190^{\circ} \mathrm{C}\right)$ compensates, at least partially, for the effects of the higher gas-volume throughput on evaporation rate. A full report on the development of the integral stack with ribbed substrate has been published. 37

About $\$ 30 / \mathrm{m}^{2}$ have been estimated by one graphite producer as a likely ultimate cost for each of the three components (two ribbed substrates and one bipolar plate) produced in quantities of $\sim 100,000 \mathrm{~m}^{2} / \mathrm{y}$. This estimate represents an annual production of about $50 \mathrm{MW}$ of $\mathrm{FC}$ parts at proposed UTC current densities 23,25 and corresponds to a situation well up the learning curves in Figs. 2.4-1 and 2.4-2. The resulting stack-parts cost is $\$ 62 / \mathrm{kW}$ for a component weight of about $3.4 \mathrm{~kg} / \mathrm{kW}$ or a cost of $\$ 18.2 / \mathrm{kg}(\$ 8.30 / \mathrm{b})$. To this component cost must be added the cost of electrodes and catalyst, matrix, teflon used in substrate preparation, and assembly. Using the Pt-catalyst costs given in Sec. 2.9, a total cell-stack cost of $\$ 200 / \mathrm{kW}$ out of a total system cost of $\$(1985) 700 / \mathrm{kW}$ appears to be reasonable. Although the Pt may be recycled, no credits are assumed since an initial investment must be made for each $\mathrm{kW}$ of new capacity. However, a credit may be taken in stack replacement.

The estimated costs of the graphite parts can be checked by using published estimates 38 from Airco Carbon Division of Airco, Inc., for machined graphite parts given for another EPRI electrochemical application (electrodes and separator plates for the zinc-chloride battery), which will be required in similar annual production quantities. Extruded, molded and porous grades of graphite are required for this application; these are similar to those used for the FC. After a detailed study of both a greenfield and an incremental plant, the costs of the finished products ready for assembly were estimated to be in the range $\$(1981) 14.10-14.80 / \mathrm{kg}$ for a production of $20 \times 10^{6} \mathrm{lb} / \mathrm{y}$. This production level is more than an order of magnitude greater than that anticipated by the first manufacturer, and the costs therefore do not appear to be out of line. We conclude that the cost of FC stack graphite components, in mature volume production, will probably be under $\$ 60 / \mathrm{kW}$. A major problem is component costs from limited production runs in early units. A method of handling this problem and other methods for cutting costs, by taking advantage of component simplification and performance improvements, is discussed in Sec. 2.13.

\section{11 Carbon Corrosion and Cathode-Catalyst Stability in the PAFC}

The cathode-catalyst potential controls the heat-rate of the PAFC system. This potential affects the corrosion environment of the cathode catalyst and support, its substrate, and that of the cathode side of the bipolar plate. $A_{B}$ is indicated in Sec. 2.7, the use of carbon-graphite components is a key element in the production of a cost-effective PAFC system. In Sec. 2.10, the use of graphite rather than of carbon parts has been assumed, and we pointed out in Sec. 2.9 that ERC successfully used much cheaper carbon parts in atmospheric pressure cells. We will now discuss the necessity for using graphitized or at least high-temperature, heat-treated components in the electric utility FC. 
Operations of the PAFC under pressure conditions and at atmospheric pressure provide greatly different materials environments for the cell components. At $190^{\circ} \mathrm{C}$ and 1 atm, the acid concentration in typical cells corresponds to about 98-100 wt\% of acid expressed as $\mathrm{H}_{3} \mathrm{PO}_{4}$. At $205^{\circ} \mathrm{C}$ and $8.2 \mathrm{~atm}$, the acid concentration corresponds to about $93 \mathrm{wt} \% \mathrm{H}_{3} \mathrm{PO}_{4}$. The volatilities of the acids are not only different, but they also contain different proportions of different condensed PA, which behave as different chemical species insofar as carbon corrosion is concerned. This fact is well illustrated by the work reported in Ref. 39 (cf. Fig. 2.11-1) for the rate of corrosion of a carbon-catalyst support (Shawinigan acetylene black) as a function of PA concentration or water-vapor pressure at $150^{\circ} \mathrm{C}$ and a potential of $1.0 \mathrm{~V}$ on the hydrogen scale. The curve shows a break at a concentration between 93 and $97 \mathrm{wt} \%$, which represents a change in kinetics. In this concentration range, PA changes from the ortho- to the pyro-form. In the range 85 to $93 \mathrm{wt} \%$, carbon corrosion is rapid, and the reaction order for water (the corrosion reactant) appears to be approximated unity. Later data on glassy carbon, conducted under pressurized conditions, 40 show an even longer linear region (to $100 \%$ acid) or a pressure of 5.4 bars (Fig. 2.11-2). However, it should be noted that the break to unit reaction order occurs at the same vapor pressure (100 torr) in both figures. Thus, above 100 torr of water-vapor pressure, the rate-determining step in the corrosion process is probably $\mathrm{C}+\mathrm{H}_{2} \mathrm{O} \rightarrow$ products.

In the more concentrated acid range, where condensed acids are present, the corrosion rates are lower, and the water-reaction order is close to zero. Arrhenius plots for the corrosion of carbon black as a function of acid concentration and temperature indicate that the corrosion rate at $205^{\circ} \mathrm{C}$ in $93 \mathrm{wt} \% \mathrm{PA}$ is perhaps a factor of 3 to 4 higher than at $190^{\circ} \mathrm{C}$ in 98 wt\% acid at the same electrode potential. However, cells operating under pressure do not only see this effect of acid concentration and/or water-vapor pressure but also the effect of increased cathode potential when pressurized operation is used to improve system efficiency.

Figure 2.11-3 shows a series of plots obtained at WE on the corrosion of bipolar plate material as a function of heat-treatment temperature and potential. These show not only the much greater corrosion resistance of graphitized parts compared with those consisting of low-temperature vitreous carbon but also that an increase in cell potential by about $50 \mathrm{mV}$ (corresponding to increased pressure) increases the electrochemical corrosion rate by about a factor of three, in addition to the 3-4 fold increase involved in the change in temperature and acid concentration, giving overall corrosion rates that are more than a factor of 10 higher. When tests conducted in pressurized cells at WE are compared with tests of equivalent ERC components at atmospheric pressure, this last conclusion is confirmed. While the results contained in Refs. 39 and 41, together with the data in Fig. 2.11-3, show a wide range of corrosion rates for different types of carbon, we are probably close to the operating limits of many of the commonly a vailable heat-treated carbon or graphite materials at the PAFC cathode. To achieve a 40,00060,000 hot-hour lifetime requires pushing the state-of-the-art. Corrosion definitely defines the limits of the potential to which the cathode can be exposed at part load. On standby, the cathode gas must be diluted by nitrogen to prevent the potential from rising into the danger zone.

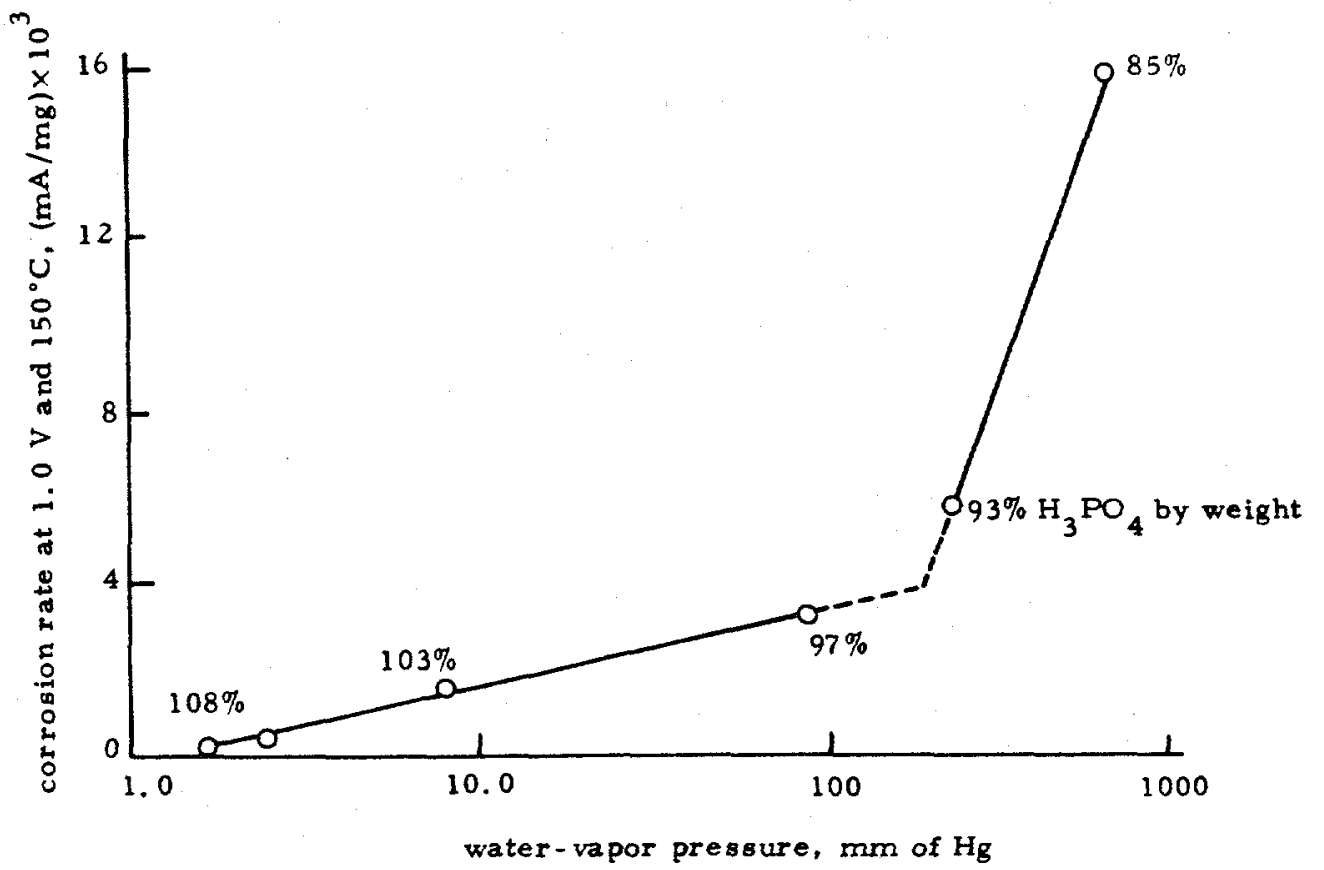

Fig. 2.11-1. The effect of water-vapor pressure on the corrosion rate of Shawinigan acetylene black in concentrated phosphoric acid. 


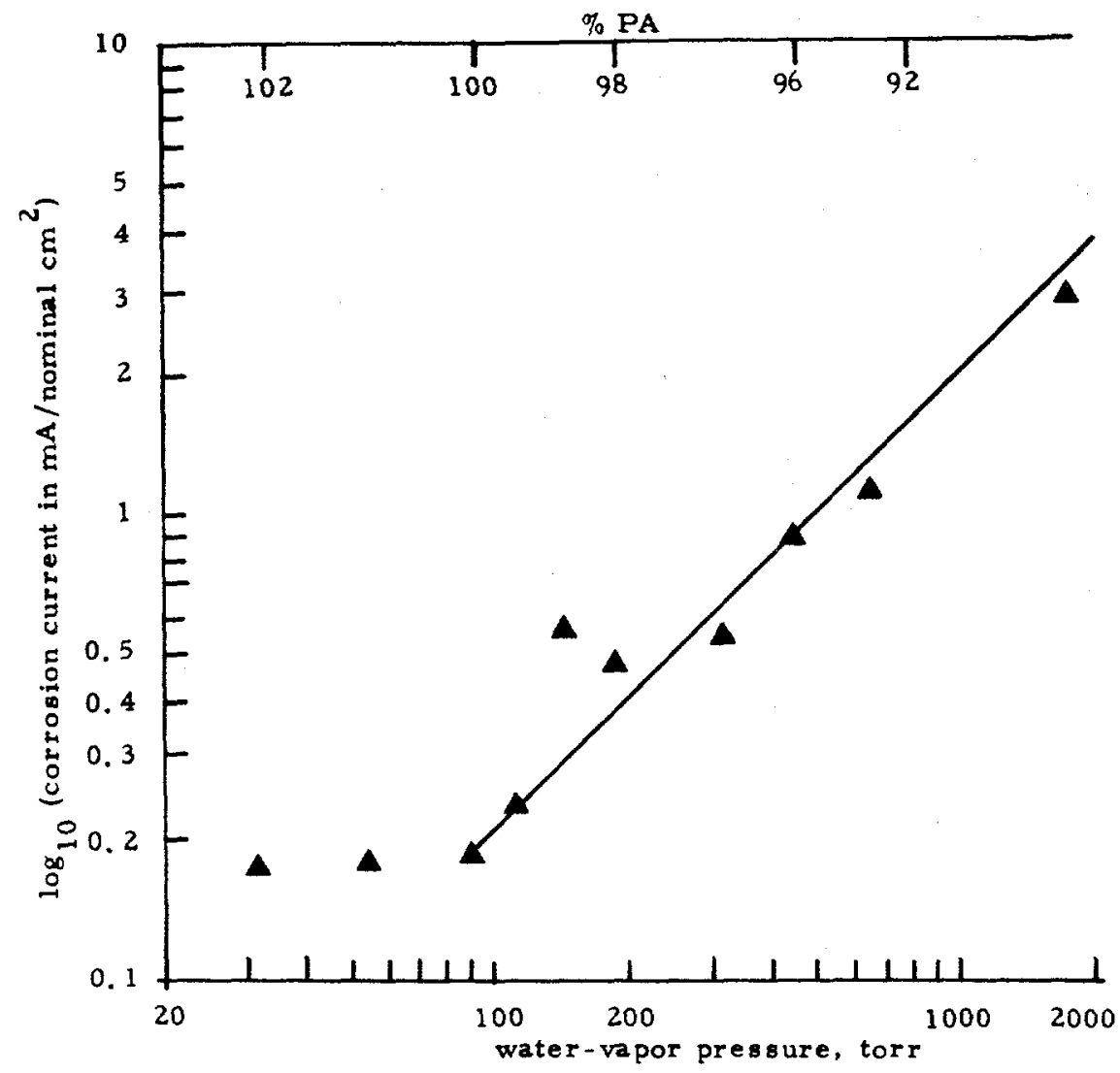

Fig. 2.11-2. The relation is shown between corrosion current on glassy carbon and water-vapor pressure above phosphoric acid (1 V/HE, $180^{\circ} \mathrm{C}$, total pressure $=5.4 \mathrm{bar}$ ).

The use of carbon in the PAFC and in other electrochemical systems has recently been extensively reviewed. 42 Questions arising from the use of carbon-graphite in the PAFC at the cell anode, cathode and bipolar plate are summarized in Ref. 43, which contains a review of the history of the use of carbon in the cell and of the UTC patents covering its use for the support, substrate and bipolar plate applications. Figure 2.11-4 shows a large collection of carboncorrosion Tafel plots obtained under steady-state conditions in PA with approximately 100 wt $\%$ $\mathrm{H}_{3} \mathrm{PO}_{4}$ concentration at $200^{\circ} \mathrm{C}$. The plots were obtained under the conditions used in Refs. 39 and 41. They involved, in good approximation, precorrosion of the electrode to the steady state at $1.0 \mathrm{~V}$ vs the reversible hydrogen electrode in the same medium. On the other hand, the results shown in Fig. 2.11-3 refer to specimens precorroded at $0.9 \mathrm{~V}$, which results in higher absolute corrosion current densities when the specimens are farther away from their long-term corrosion condition. The comparison stresses the fact that the corrosion rates always fall off as a function of time since the highest energy material is consumed first, thus leaving structures that are progressively more stable. For Vulcan XC-72 catalyst supports (a turbostractic oilfurnace black), corrosion has been shown to take place on the inside of the carbon particles. thus leaving more stable skins which have grown at higher temperatures than those of initial nucleation and hence show more order and greater stability.

Plot D in Fig. 2.11-4 holds for a non-treated bipolar plate graphite-resin composite material, which shows the expected and totally unacceptable performance. The plot range for Vulcan XC-72 electrode supports is also known from experience to be unacceptable under pressure conditions, although not at lower temperatures and atmospheric pressure. The most corrosion-resistant supports currently available are acetylene blacks, which are actually better if they are precorroded by activation, i.e., steam- or $\mathrm{CO}_{2}$-treatment at about $1200^{\circ} \mathrm{C}$. This pre corrosion increases the surface area from about 70 to $250 \mathrm{~m} 2 / \mathrm{g}$, thereby making acetylene blacks equivalent to Vulcan $\mathrm{XC}-72$ in BET area. Furnace blacks heat-treated to graphitication show high corrosion resigtance but low BET areas (Vulcan XC-72 $2700^{\circ} \mathrm{C}$ is $80 \mathrm{~m} / \mathrm{g}$ ), and are not necessarily optimal for the long-term stabilization of high-surface Pt, for which $200 \mathrm{~m} 2 / \mathrm{g}$ or more is desirable. Some promising recent data 44 on Cabot Black Pearls $2000\left(a 2000 \mathrm{~m}^{2} / \mathrm{g}\right.$ furnaceblack after graphitizing at $2700^{\circ} \mathrm{C}$ ), with a surface area of $214 \mathrm{~m}^{2} / \mathrm{g}$, are shown in Fig. $2.11-5$.

Figure 2.11-4 is useful in obtaining an indication of the order of magnitude of the corrosion rate per $\mathrm{cm}^{2}$ of geometric area for each individual cell component under cathode operating 


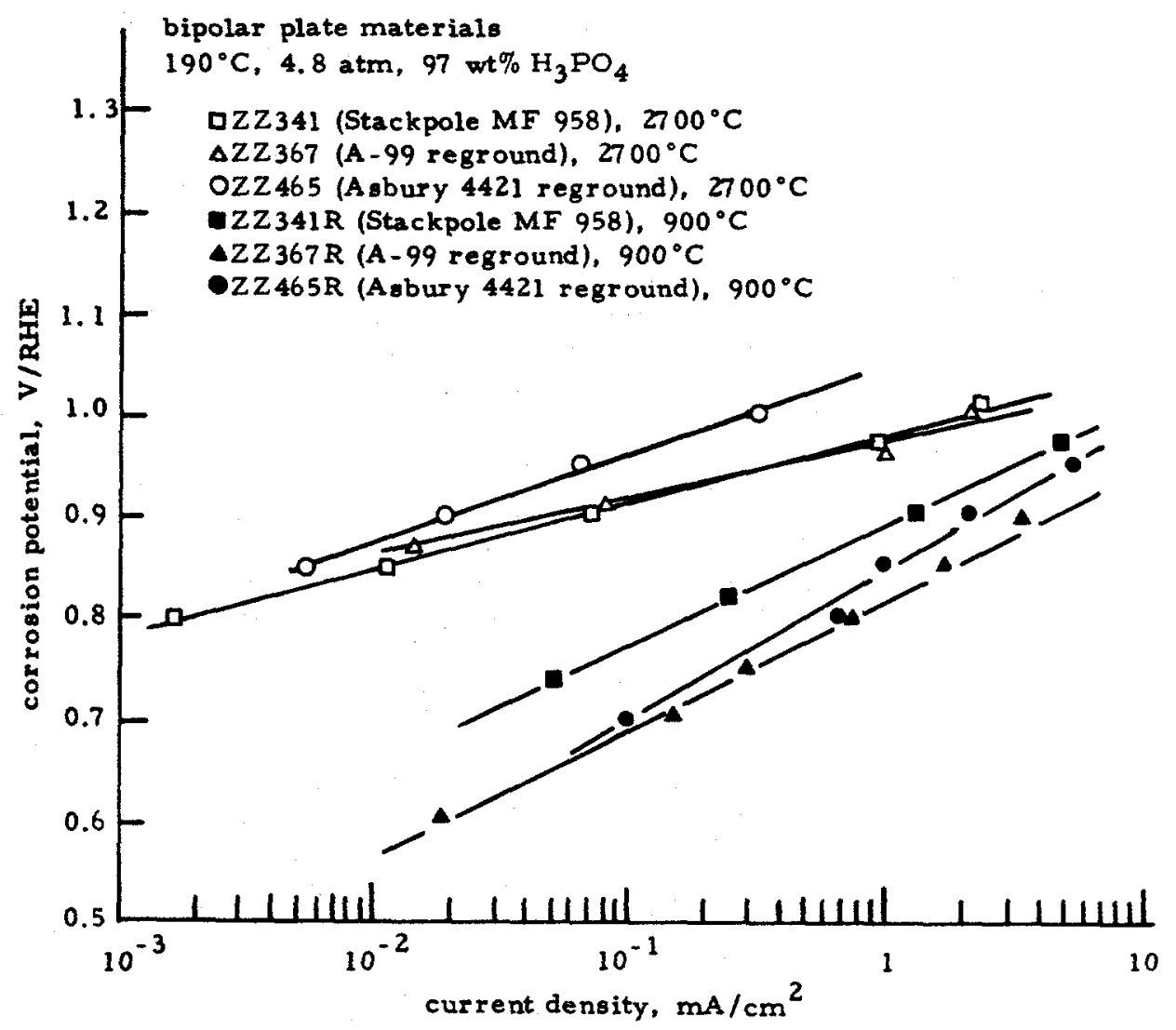

Fig. 2,11-3. The corrosion current is plotted vs the corrosion potential for various heat-treated bipolar plate materials (held at $0.9 \mathrm{~V} / \mathrm{HE}$ in PA at the indicated conditions).

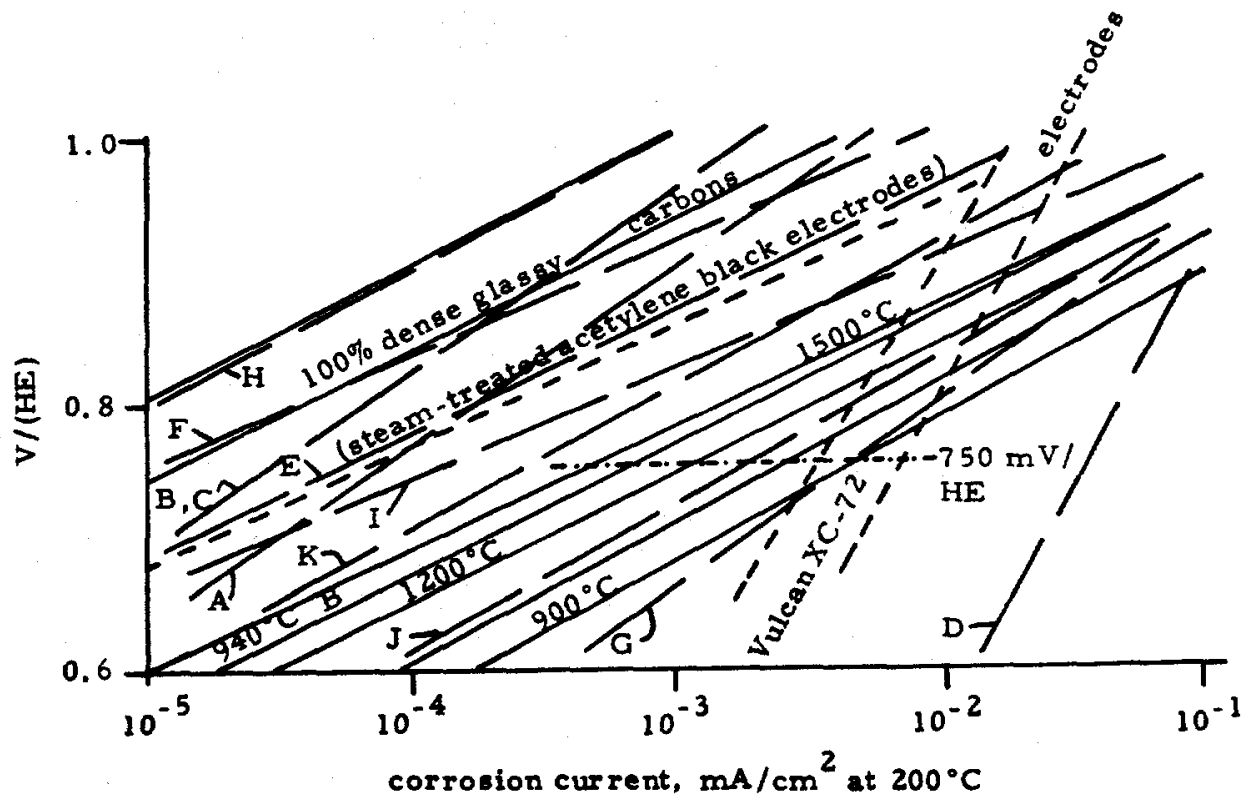

Fig. 2.11-4. Composite Tafel plots are shown for corrosion of various carbon materials in $200^{\circ} \mathrm{C}$ PA. Plots are generally taken after 10,000 min. Dashed lines: corrosion behavior of $5 \mathrm{mg} / \mathrm{cm}^{2}$ Vulcan XC72 supports and steam-treated acetylene black supports (right and left, respectively). Lines show heattreatment $T$ in ${ }^{\circ} \mathrm{C}$. Plots are shown for typical 67 wt\% graphite powder $/ 33$ wt $\%$ phenolformaldehyde resins, heat-treated at the indicated temperatures. D: non-heat-treated material. G-J; range of $900^{\circ} \mathrm{C}$ heat-treatments. $940^{\circ} \mathrm{C}$, B: boron-doped materials, heat-treated at $940^{\circ} \mathrm{C}$, $\mathrm{K}: 5 \%$ borondoped resin treated at $900^{\circ} \mathrm{C}$. I; $1200^{\circ} \mathrm{C}$ heat-treated resin. A-C: commercially available graphite felts or papers. Range between $F$ and $H$ : dense glassy carbons and graphitized materials. 


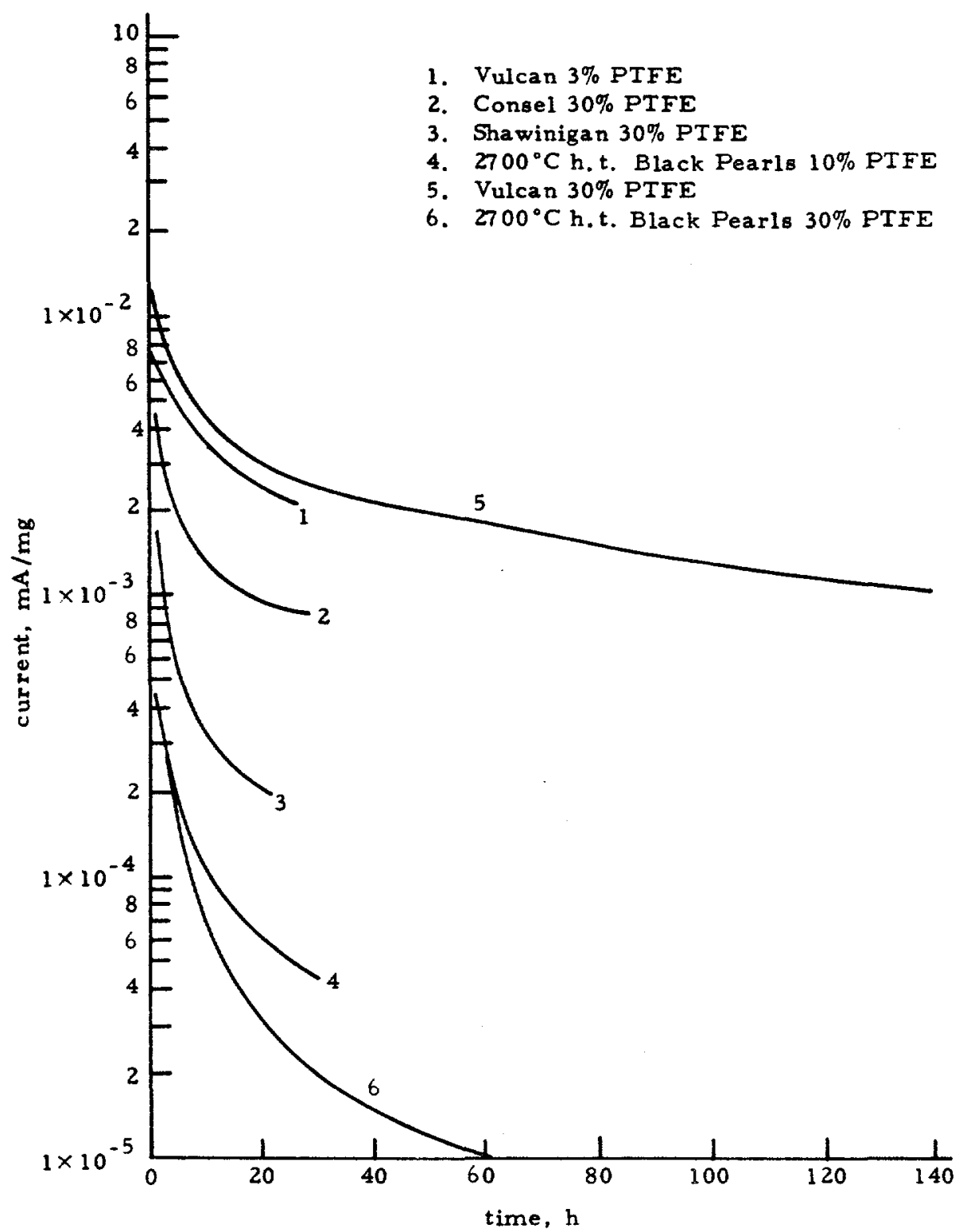

Fig. 2.11-5. The corrosion characteristics are shown for various carbon-support materials at $0.8 \mathrm{~V}$.

conditions. Since corrosion is an electrochemical process and occurs at a much higher rate under cathode conditions, there is no concern about the PAFC anode. Corrosion rates on the order of $10^{-4} \mathrm{~mA} / \mathrm{cm}^{2}$ or less, corresponding to the total corrosion of the acetylene black electrode support, are desirable. For the electrode support, $10^{-4} \mathrm{~mA} / \mathrm{cm}^{2}$ over $40,000 \mathrm{~h} \mathrm{repre-}$ sents the $108 \mathrm{~s}$ of $10 \%$ of material at $5 \mathrm{mg} / \mathrm{cm}^{2}$ loading. Hence, pressurized cells require the use of fully or at least partially graphitized bipolar plates and substrates, with heat-treatment temperature of at least $1800^{\circ} \mathrm{C}$ and preferably $2700^{\circ} \mathrm{C}$ or, alternatively, high-temperature glassy carbons. The use of boron as a graphitization catalyst for the starting materials used in forming the bipolar plate has been shown to be desirable. 39,41 As is shown in Fig. 2. 11-4, boron confers equivalent corrosion resistance at lower heat-treatment temperatures and has no adverse effects on cell operation.

A nother important point should be emphasized. Some manufacturers have observed accelerated corrosion on some cathode components or on components connected to the cathode, especially under pressure conditions. Frequently, this corrosion has been localized and has affected parts of the electrode support and the bipolar plate. A possible explanation for the initiation of this effect has been suggested by Jalan, 45 and it is illustrated in diagrammatic form in Fig. 2,11-6. The essence of the explanation is as follows. If an electrode structure has nonuniform electrolyte wetting, PA films, which show a high electrolytic resistance, may form in certain parts of the electrode. Any Pt catalyst that is in contact with these films will have a 


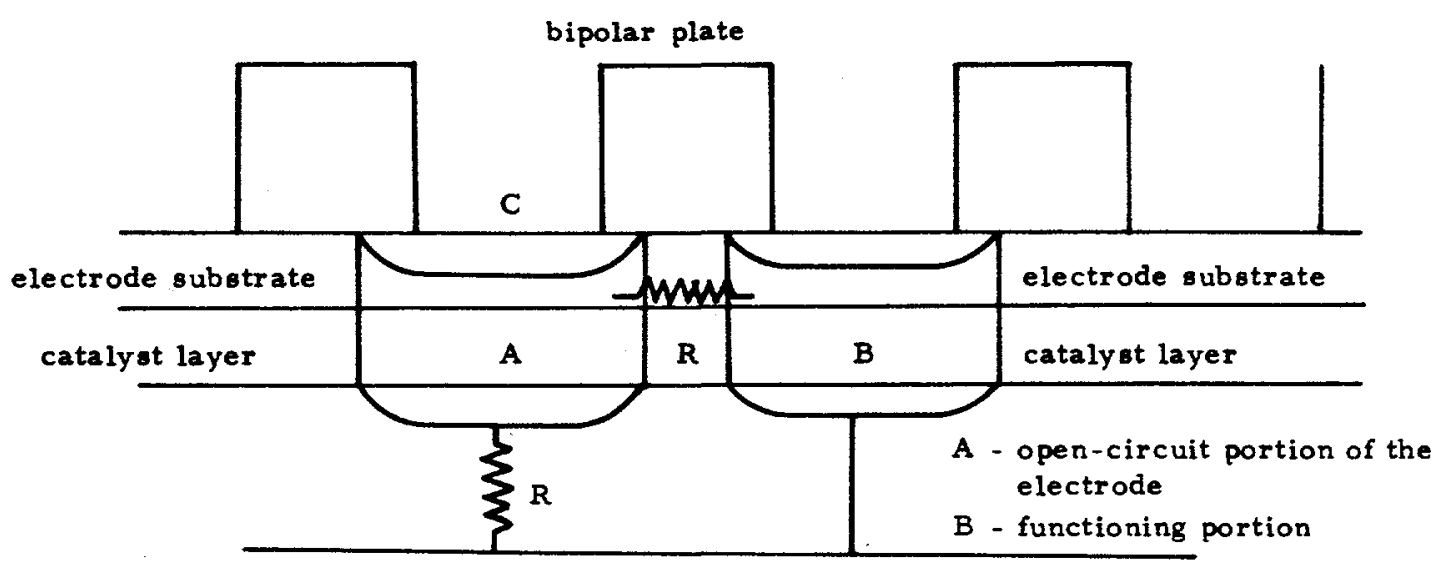

common electrolyte pool

Fig. 2.11-6. A model is shown for corrosive degradation of cathode components in the PAFC (cf. Ref. 45). If the resistances $R$, resulting from poor electrolyte filming, are high $(=500 \Omega)$, A will be effectively at open circuit. This process will cause corrosion in the electrolyte film at $C$ (the open-circuit corrosion resistance is $10^{5}-10^{6} \Omega$ and, therefore, the reaction will be unaffected by $R$ ).

local electrode potential approaching open circuit values. Any such parts of the electrode, as well as any other components in the presence of a PA film in contact with this open-circuit Pt, will be subject to accelerated (by about two orders of magnitude) corrosion. The solution to this problem is to have electrodes of reproducible wettability (or hydrophobicity) and to make certain that the electrolyte resistance between the Pt catalyst layers and the bipolar plate is as high as possible. This result may be achieved by increasing the hydrophobicity of the substrate and of the bipolar plate in order to maximize the electrolytic film resistance across these components. Hydrophobicity should not be taken too far; otherwise, electronic contact between the different components may become compromised. Once corrosive attack on the bipolar plate structure becomes significant, electrolyte contact between the anode of one cell and the cathode of an adjacent cell may occur, which will result in rapid failure of the bipolar plate via the shunt current effect, since the plate will see an effective local electrochemical potential that is additive throughout the stack via the ionic short-circuit between individual cells or groups of cells.

\subsection{The PAFC Cathode Catalyst}

The cathode catalyst must have long-term stability. Platinum in high-surface-area form is currently the most favored-catalyst material. Use of the maximum number of Pt catalytic $s$ ites is important for system cost-effectiveness. The smallest possible stable Pt particles are used to give the largest possible number of $\mathrm{Pt}$ atoms on particle surfaces. In addition, these particles must be dispersed on the support in such a way that they are equally accessible to the electrolyte and to gaseous oxygen. They must be used equally effectively for oxygen reduction, which requires a three-phase contact between the electrolyte film, gaseous reactant, and electronically conducting catalyst and support. This process requires both the most even possible distribution of catalyst particles over the support surface and an optimized electrode structure in which all catalyst-containing parts have equal accessibility to electrolyte and gaseous phases. A discussion of the methods used in obtaining optimized electrode structures is given in Sec. 2. 15 .

Yeager et al ${ }^{46}$ have measured the solubility of bulk $\mathrm{Pt}$ (or rather of $\mathrm{Pt}^{2+}$ ion) under utility PAFC conditions. They showed that the relation between potential and $\log$ solubility had a slope of $\mathrm{RT} / 2 \mathrm{~F}$, which had previously been also observed for $\mathrm{Pd}$. 47 It may be attributed to the Pt/Pt $2+$ Nernst equation. 46,47 The observed solubilities were not negligible. Very small Pt crystallite $s$ are free in the electrolyte in colloidal form and are expected to have appreciably higher solubilities than the measured values because of the effect of the Kelvin equation. In fact, particles of the size commonly used in FCs would be expected to have such a high solubility that the whole of the cathode catalyst should rapidly saturate the electrolyte and reprecipitate at the anode. This proces does not happen, for reasons which can only be regarded as speculative at the present time. There must be strong catalyst-support interactions, which have a strong stabilizing effect on all of the atoms present in small $(2-5 \mathrm{~nm})$ Pt agglomerates. The se interactions may have an effect on the rates of electrocatalytic processes per unit area of catalyst. However, these strong support interactions allow the use of very small crystallites with the maximum number of surface sites. These crystallites tend to grow with time, either by twodimensional surface Brownian motion (which would be potential-independent and hence would 
occur only at high overpotentials) or by Ostwald ripening via a potential-dependent dissolutionprecipitation mechanism. The latter would be more marked at low current densities or, if parts of the cathode are effectively at open circuit, would occur as outlined in Sec. 2.11. Both carbon dissolution (which may result in $\mathrm{Pt}$ loss) and Pt dissolution must be prevented in the PAFC by not allowing the cathode potential to exceed about $0.80-0.85 \mathrm{~V} \mathrm{vs} \mathrm{H}_{2}$. for example, by the use of nitrogen blanketing at open circuit.

A maximum number of surface sites is obtained with the smallest possible Pt agglomerate size. For example, spherical Pt particles containing about 15 atoms $(0.8 \mathrm{~nm}$ diameter) would have more than $90 \%$ of the atoms on the surface and a BET area of about $200 \mathrm{~m} / \mathrm{g}$. This value probably represents the upper limit for the smallest crystallite nuclei. By the time particles reach a diameter of about $1.8 \mathrm{~nm}$ and contain about 150 atoms, about $2 / 3$ of these are on the surface, and the BET area has decreased to $130 \mathrm{~m}^{2} / \mathrm{g}$. These are the initial sizes of typical Pt-catalyst particles made by the colloidal sulfur-chemistry method. 20,21 This method probably produces the smallest dispersion of $\mathrm{Pt}$ on carbon in a reliable manner and is generally favored by developers and catalyst manufacturers. In impregnation techniques, the Pt particles are directly produced on the carbon support, e.g., by $\mathrm{H}_{2}$ reduction of adsorbed chloroplatinic acid. In the colloidal method, Pt is fir st dispersed and then adsorbed onto the carbon support. This process occurs spontaneously because of static electrical charges on the Pt crystallites and on the support. The use of ultrasonic stirring yields an extremely uniform dispersion. Iransmission electron micrographs of typical dispersions are shown in Figs. 2.12-1 and 2.12-2 for Vulcan XC-72 and graphitized Vulcan XC-72 supports, respectively.

Crystallites with about $5 \mathrm{~nm}$ diameter contain more than 20 times as many atoms as the initial colloidal material, and their specific surface areas have fallen to $50 \mathrm{~m} / \mathrm{g}$, with about $25 \%$ of the total atoms on the particle surface. Particles in this size range are formed by heattreatment of colloidal particles adsorbed on carbon supports in a reducing atmosphere at $900^{\circ} \mathrm{C} .48$ Similarly, prolonged exposure of colloidal Pt on a carbon support to hot PA results in grain growth, yields similarly-sized particles, and will eventually result in particles in the $8-10 \mathrm{~nm}$ diameter range after about $100,000 \mathrm{~h}$ of exposure to PAFC utility cathode conditions. Plots illustrating the change in Pt-crystallite surface area on different carbons (Vulcan XC-72, Shawinigan acetylene black, and steam-treated acetylene black) as functions of time in PA that is close to utility cathode PAFC conditions, are shown in Figs, 2.12-3 and 2.12-4. 39

These plots are significant for two reasons. First, the initial surface areas of the Pt crystallites are affected by the type of carbon support used, and these initial areas, in turn, influence the changes that occur during the lifetime of the electrode under cathode conditions. Second, the relationship between the logarithm of the average Pt BET surface area, which is inversely proportional to the crystallite size, is a linear function of the logarithm of time. This fact has profound consequences for long-term FC operation since, in good approximation, Pt activity on a per $\mathrm{cm}^{2}$ of active area basis is independent of crystallite size in the $5+\mathrm{nm}$ diameter range. Hence, at constant cell current, log (local current density) should increase linearly with $\log$ (time). Since $\log i \propto V$ from the Tafel relation, the overall system efficiency will decay as a function of the logarithm of hot time, all other factors being equal.

This result is important since it implies that the cell-voltage decay between 100 and $1000 \mathrm{~h}$, which is easily measurable in a rapid and routine laboratory test of a new catalystsupport combination, should be similar to the expected decay between 1,000 and $10,000 \mathrm{~h}$ or be tween 10,000 and $100,000 \mathrm{~h}$, provided other factors such as electrode structure, electrolyte loading, and internal cell resistance remain the same. The relation may therefore be used for the predictions of ultimate cell heat-rates at the end of life, as has already been alluded to under the UTC FCG-1 work described in Sec. 2.9.

Unfortunately, the processes involved in the growth of Pt crystallites at the oxygen cathode in hot PA are somewhat more complex than the indicated simple picture. Thus, the linearity of the $\log$ (crystallite size or BET area) vs $\log$ (time) relation does not appear to be absolute, and there is evidence of some leveling off to reach an asymptotically constant mean crystallite diameter at long times. Furthermore, the supposition of constant electrocatalytic activity (i.e.. the turnover number at constant potential on a standard scale and at constant temperature and reactant concentration), which is independent of Pt crystallite diameter, surface treatment, and other variables, may not be accurate. Plots of apparent catalyst activity for 1 atm of oxygen at $900 \mathrm{mV}$ vs $\mathrm{H}_{2}$ in $99 \mathrm{wt} \% \mathrm{H}_{3} \mathrm{PO}_{4}$ at $177^{\circ} \mathrm{C}$ as a function of catalyst crystallite size (taken from data in Ref. 49) are given in Fig. 2.12-5. While some of the effects noted may be caused by problems of electrode structure (i, e., overall utilization of the total a vailable Pt catalyst in the electrode), the effect may be real, although the data show a great deal of scatter.

The three lines in Fig. 2,12-5 are fits assuming that the specific activity is independent of Pt specific surface area in this crystallite size range. They refer, respectively, to constant activities of 15,25 and $35 \mathrm{~mA} / \mathrm{mg}$. It is seen that the extreme values contain the majority of the experimental data points. The solid line is a best fit to the available data and indicates about a factor of two improvement in activity in going from about 15 to $45 \mathrm{~m} 2 / \mathrm{g}$, with little change thereafter if the large scatter of points can be believed to be independent of electrode structure and catalyst-utilization effects. The absolute activity, ignoring the specific surface area in this range, varies by a factor of two between different samples at $900 \mathrm{mV}$. This value of 


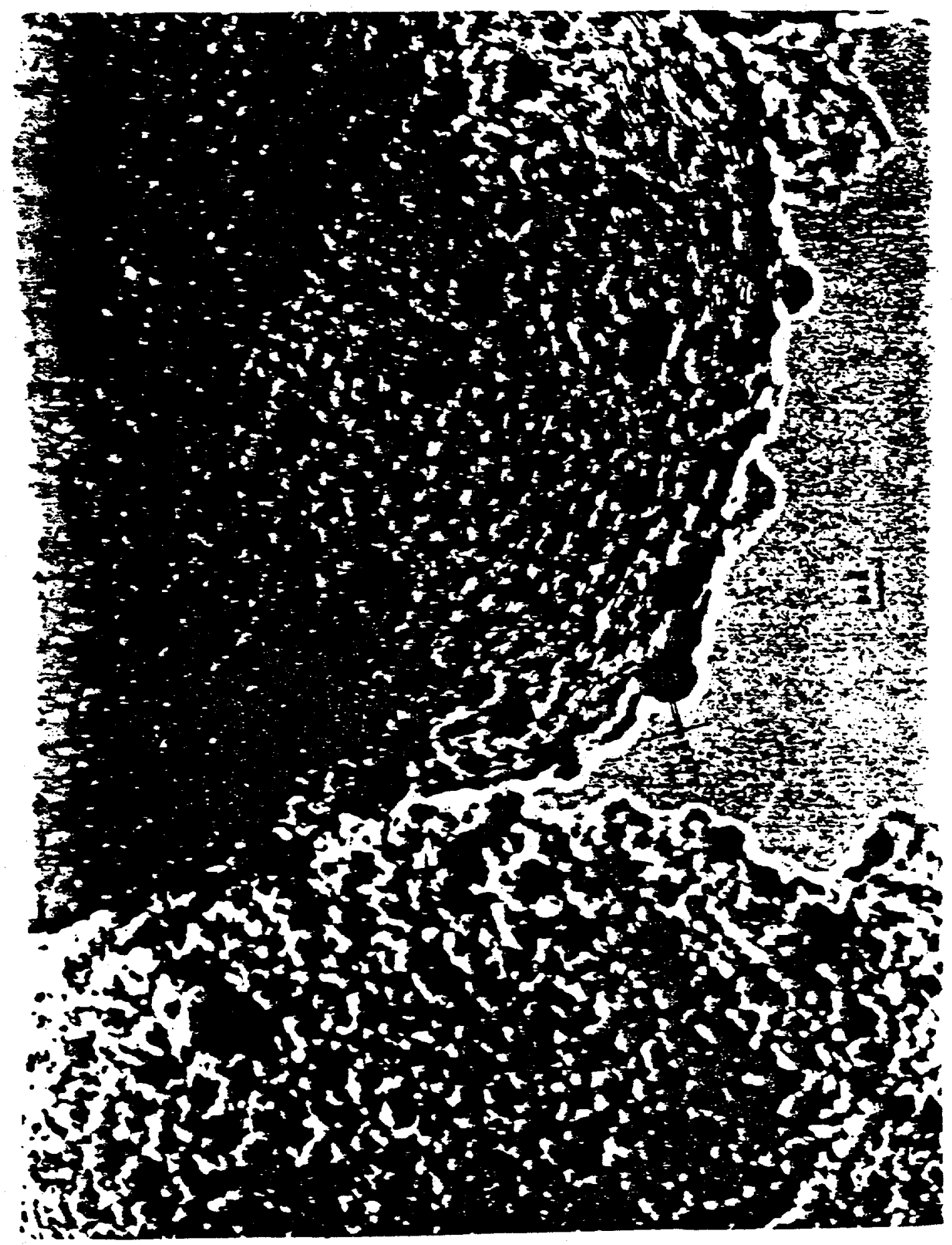

Fig. 2.12-1. TEM view of Pt microcrystallites on untreated Vulcan XC-72 (Cabot); the amorphous carbon structure should be noted. 


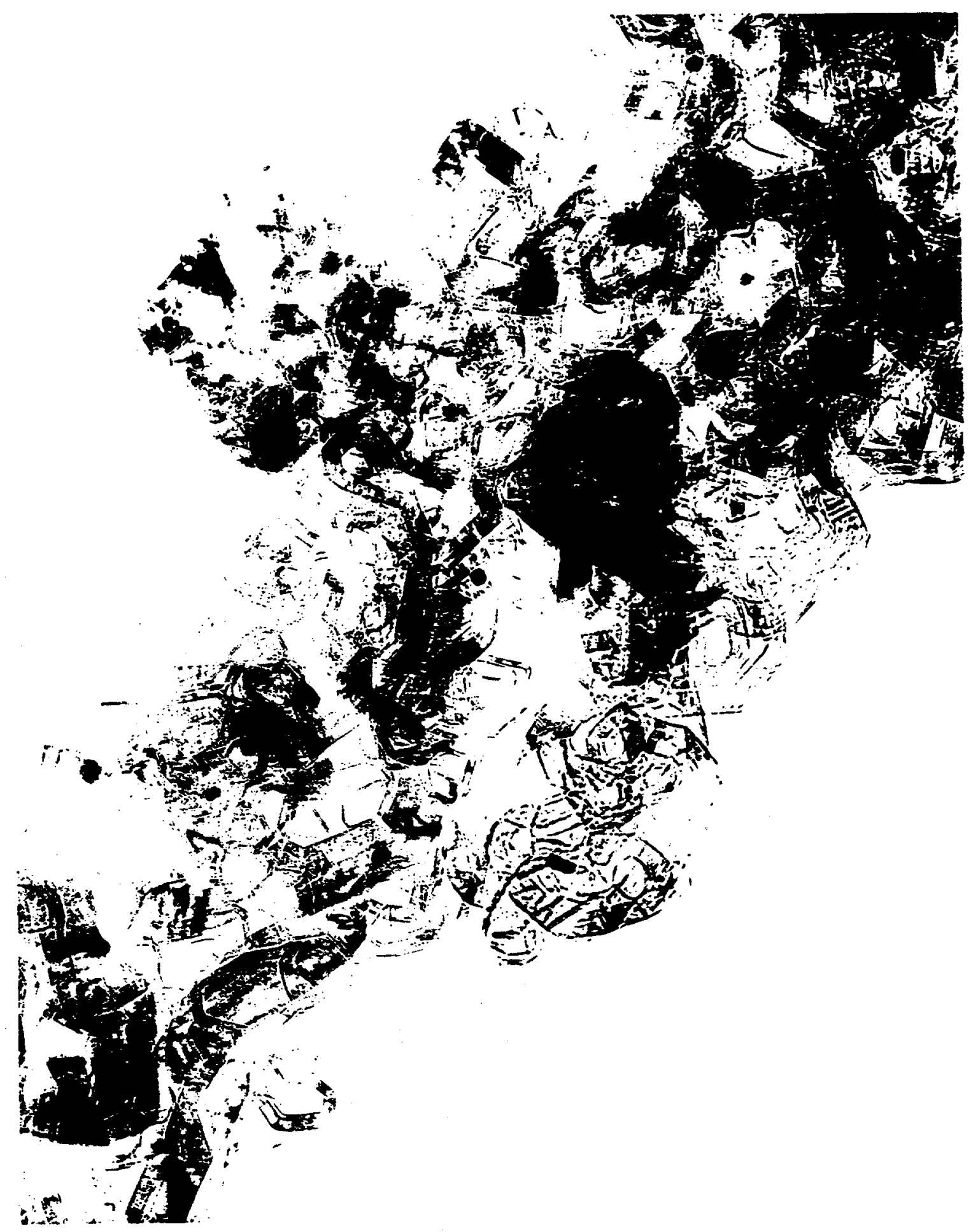

Fig. 2. 12-2. EM view of Pt microcrystallites on graphitized Vulcan XC-72. 


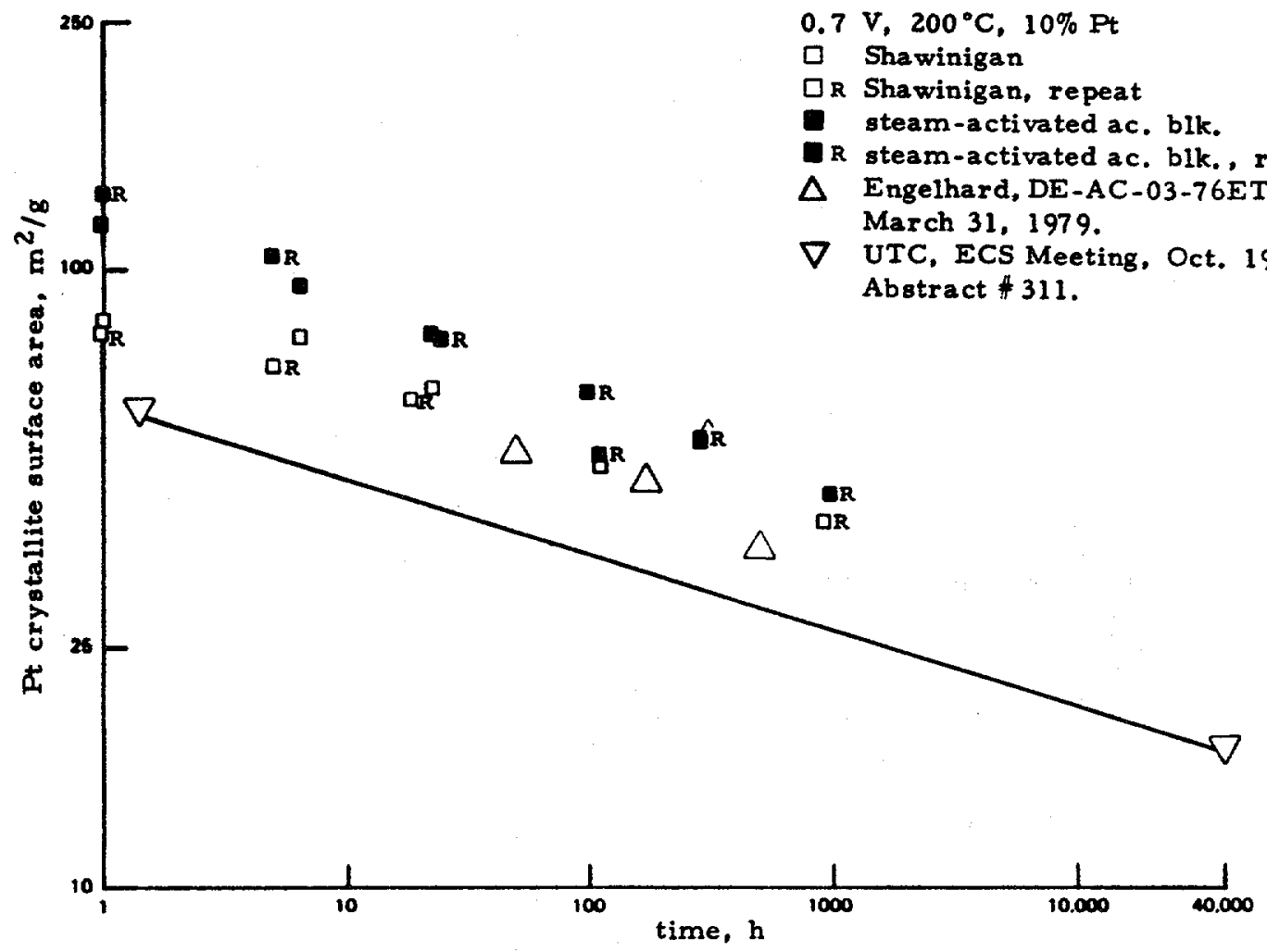

Fig. 2.12-3. Platinum crystallite surface area losses are shown as a function of time (log-log scale) for Vulcan XC-72, Shawinigan acetylene black, and steam-activated Shawinigan at $0.7 \mathrm{~V} / \mathrm{HE}$ in $200^{\circ} \mathrm{C}$ $\mathrm{H}_{3} \mathrm{PO}_{4}$.

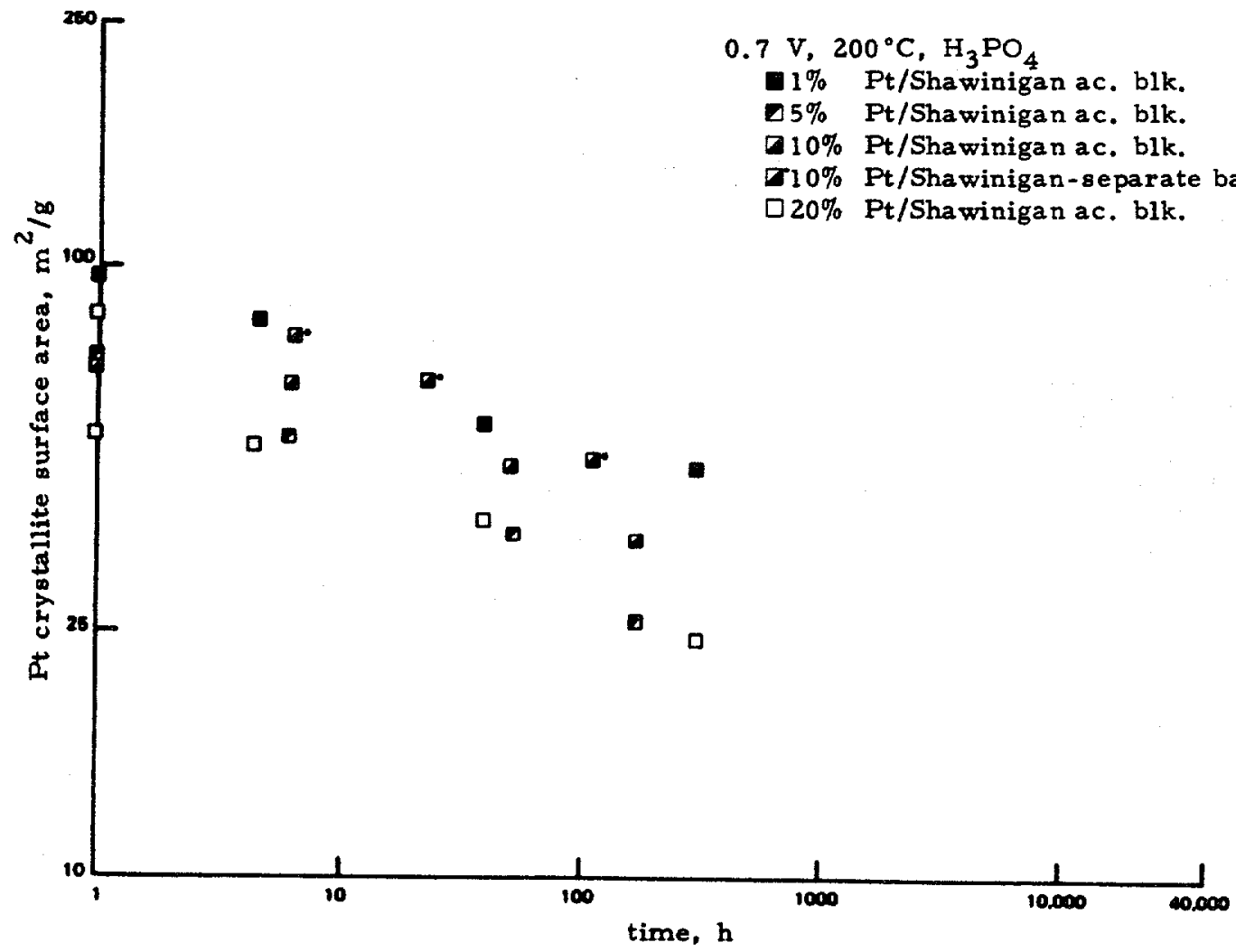

Fig. 2.12-4. As in Fig. 2,12-3 but for different loadings of Pt on Shawinigan acetylene black. 


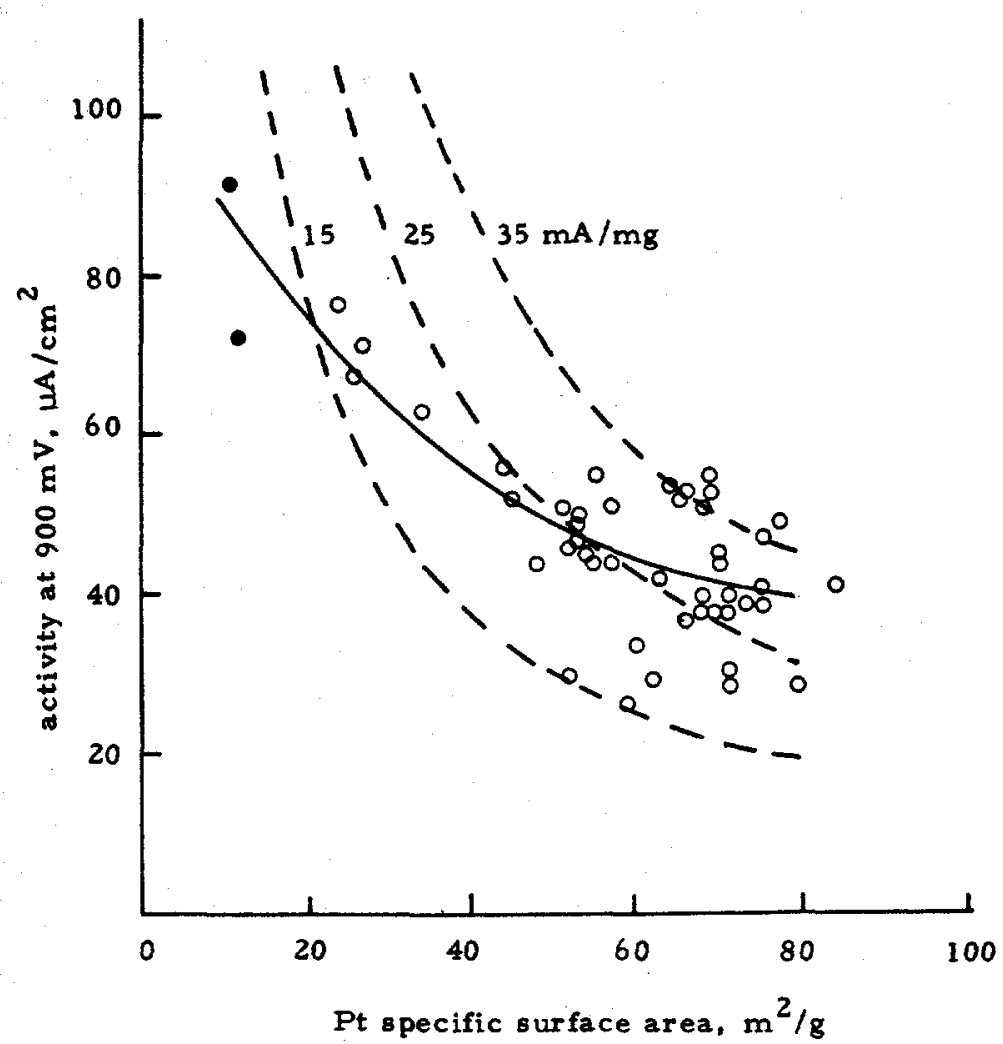

Fig. 2.12-5. The specific activity $\left(\mathrm{A} / \mathrm{cm}^{2}\right)$ is shown as a function of BET crystallite area $\left(177^{\circ} \mathrm{C} ; 99 \% \mathrm{H}_{3} \mathrm{PO}_{4}\right)$; solid points: pure $\mathrm{Pt}$ black; open points: Pt on Vulcan XC-72. The dashed lines refer to constant activities per unit mass $(15,25$ and $35 \mathrm{~mA} / \mathrm{mg} \mathrm{Pt}$ ). These results are taken from Ref. 49.

electrode activity is traditionally measured in a convenient laboratory test in order to compare different catalysts or electrode structures, since the low currents at this potential will be largely independent of diffusion problems within the electrode. The value $35 \mathrm{~mA} / \mathrm{mg}$ is generally considered to apply to a very good electrode.

Figure 2. 12-6 shows the Tafel slopes of these same electrodes. The net Tafel slope trend is from rather high values for the materials of highest turnover number to lower values for the less intrinsically active, high-surface-area materials. Since the FC cathode operates at approximately $750 \mathrm{mV}$, the effect of Tafel slope on the activity of a given catalyst at this potential may be important. In general, the less active high-surface-area materials have Tafel slopes of about $90 \mathrm{mV} /$ decade, whereas the more active low-surface-area materials show slopes a proaching $115 \mathrm{mV} /$ decade. As a result, an electrode with a fairly high surface area $\left(70 \mathrm{~m}^{2} / \mathrm{g}\right)$ and an activity of $20 \mathrm{~mA} / \mathrm{mg}$ at $900 \mathrm{mV}$ would show $928 \mathrm{~mA} / \mathrm{mg}$ at $750 \mathrm{mV}$. Furthermore, an

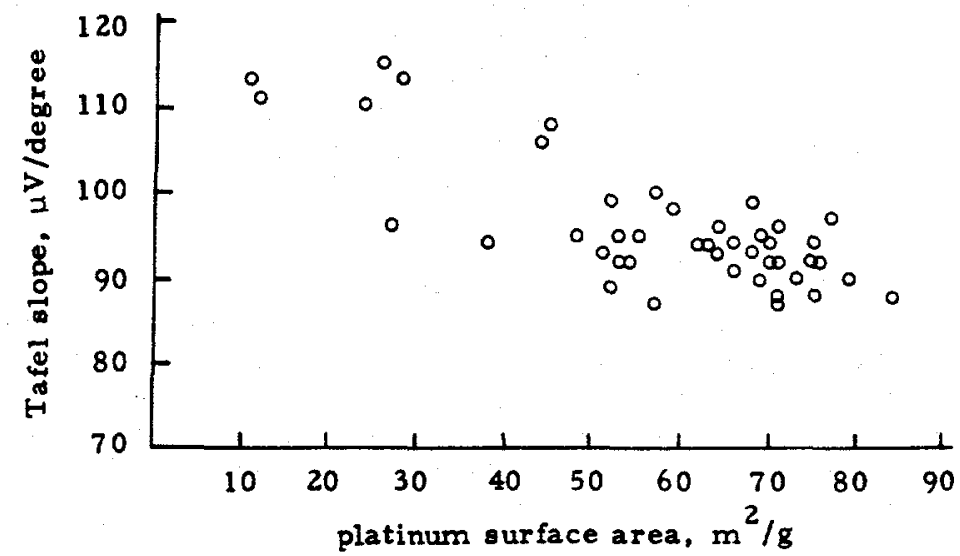

Fig. 2.12-6. The $\mathrm{O}_{2}$ reduction Tafel slope is shown as a function of BET. 
electrode with the same catalyst loading but with a specific area of $35 \mathrm{~m}^{2} / \mathrm{g}$ may have twice this specific activity. It would therefore show the same $20 \mathrm{~mA} / \mathrm{mg}$ absolute activity at $900 \mathrm{mV}$ as that of the high-aurface-area electrode but only $400 \mathrm{~mA} / \mathrm{mg}$ at $750 \mathrm{mV}$ because of its higher Tafel slope. Hence, at the real FC operating point, the activity decreases roughly linearly with crystallite size for electrodes with BET areas less than about $70 \mathrm{~m}^{2} / \mathrm{g}$. For BET areas greater than $70 \mathrm{~m} / \mathrm{g}$, the activity falls with time because of the $108 \mathrm{~B}$ of BET area (increasing current density) at an approximately constant Tafel slope that is not shown in Fig. 2.12-6. If this is a general result, the performance of all Pt electrodes would be expected to decay with time, first and at short times because of the effect of decreasing crystallite size and, then, because of the Tafel slope alone, rather than because of a change in specific activity measured at low current density. Figure 2.12-6 also shows that the Tafel slope decreases roughly linearly with crystallite size. Since the over potential is proportional to the Tafel slope, and ance crystallite BET area falls linearly (Figs. 2.12-3 and 2.12-4) with $\log$ (time), the over potential will increase linearly with $\log$ (time) because of combined current-density, crystallite-size and Tafel slope effects.

Experimental data support the model and form the basis of UTC heat-rate-decay calculations as a function of time, as given in Sec. 2.9 and, in greater detail, in Sec. 2.14. The fall in absolute activity of the particles as a function of crystallite size is certainly real and is not caused by the electrode structure, which may, however, be instrumental in reducing Pt utiliza tion. The main evidence for this last statement is the Tafel slope relation: the low slope for small crystallite particles would be expected to be greater than that for larger particles if electrode structural effects are involved in reducing small-particle activity. When electrodes do not show a linear voltage decay as a function of $\log$ (time), for example, if the decay is proportional to time as has sometimes been claimed, something is probably amiss with the PAFC (e.g., corrosion effects may be apparent, or an IR rise is occurring as the result of electrolyte loss). If corrosion of the cathode occurs because of use of an inappropriate support, the physical cathode degradation may indeed be proportional to time.

An interesting suggestion to improve catalyst activity at long lifetimes involves manipulation of the Tafel slope, if this could be done in some practical manner. The higher activities of the low-surface-area catalysts at $900 \mathrm{mV}$ could be maintained if means could be found to decrease the Tafel slope on low-surface-area material from 120 to $90 \mathrm{mV} /$ decade. A discussion of the physical significance of these values of the Tafel slope, which correspond to transfer coefficients of 1.04 to 1.28, is given in Sec. 2.15. The indicated approach was examined at sereral developers during the $1970 \mathrm{~s}$. One of the best demonstrations of the effect is shown in Fig. 2, 12,-7, 48 which illustrates the difference in activity between a $140 \mathrm{~m}^{2} / \mathrm{g}$ colloidal $\mathrm{Pt}$ on $\mathrm{C}$ (Vulcan XC-72) electrode in its as-received condition and after heat-treatment in an inert atmosphere at $900^{\circ} \mathrm{C}$. Transmission-electron micrographs of specimens are included. After heattreatment, the BET area of the specimen fell to $55 \mathrm{~m} / \mathrm{g}$, but its net activity at $900 \mathrm{mV}$ increased by about $30 \%$. This result is not unexpected in view of the data of Fig. 2.12-5. Most surprising, however, is the fact that the Tafel slope improves somewhat, going from about $105 \mathrm{mV} / \mathrm{decade}$ for the untreated material to $100 \mathrm{mV} /$ decade for the untreated sample.

The overall result is an activity that is higher by about a factor of two at 750 than at $900 \mathrm{mV}$. Similar data, although with slightly different Tafel slopes, are shown from work by Ross in Fig. 2.12-8. 51 Jalan 48,50 attributes this increased activity to the formation of a $\mathrm{Pt}$-C alloy with electrocatalytic properties different from those of pure colloidal Pt. The alloy may be formed either by direct reaction of the $\mathrm{Pt}$ particles with $\mathrm{C}$ or by the reaction $2 \mathrm{CO} \rightarrow \mathrm{C}+\mathrm{CO}_{2}$ if a $\mathrm{CO}$ atmosphere is used. 48 . The formation of this alloy has a significant effect on the rate of electrode degradation as a function of time, as is shown in Fig. 2.12-9.50

Examination of other Pt alloys started in the mid 19708 at UTC, with emphasis on the use of base transition metals as alloying agents. For proprietary reasons, no work was published during this period; UTC's first patent applications were allowed in 1980. 52 This patent concerned the use of a $\mathrm{Pt}-\mathrm{V}$ alloy, with a composition of about $\mathrm{Pt}_{3} \mathrm{~V}$, that was prepared by hightemperature reaction of a vanadium oxide with colloidal $\mathrm{Pt}$ on $\mathrm{C}$, the latter acting as the reducing agent as well as the catalyst support. About a factor of two improvement in performance over the colloidal Pt precursors was reported, in spite of the fact that the alloy, after necessary heat-treatment, had a specific BET area of only about $50 \mathrm{~m} 2 / \mathrm{g}$, down from the original value of about $130 \mathrm{~m}^{2} / \mathrm{g}$.

Similar results on Pt base-metal alloys or intermetallic compounds were reported by Ross in 1980. 51 Some of his results on four different alloy systems are contrasted with pure colloidal Pt (Protech) in Fig. 2. 12-10. This experimental work was based on theoretical concepts derived from the Brönsted theory of heterogeneous catalysis, 53 which is based on the later concepts of Horiuti and Polanyi ${ }^{54}$ as applied to both chemical reactions on different substrates and to comparisons of electrochemical processes on different electrode materials at constant potential. This work has been discussed in detail in the electrochemical literature.55, 56 The essence of the theory is that the rates of the same rate-determining step, at the same reactant concentrations, potentials, and temperature, vary as exp-( $\left.8 \Delta G_{a d s} / R T\right)$, where $\Delta G_{a d s}$ is the free energy of adsorption of each reactant or product involved in the rate-determining step, and $\beta$ is a constant known as the Brönsted slope, which is normally close to $1 / 2$. The theory used to 


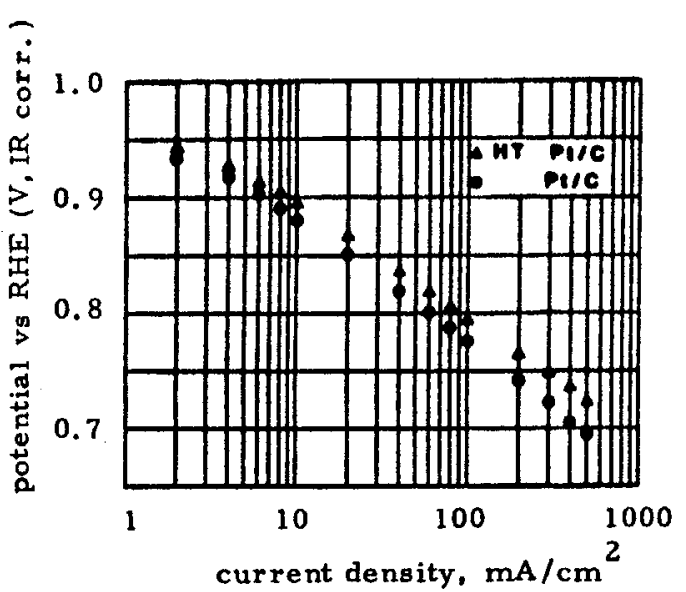

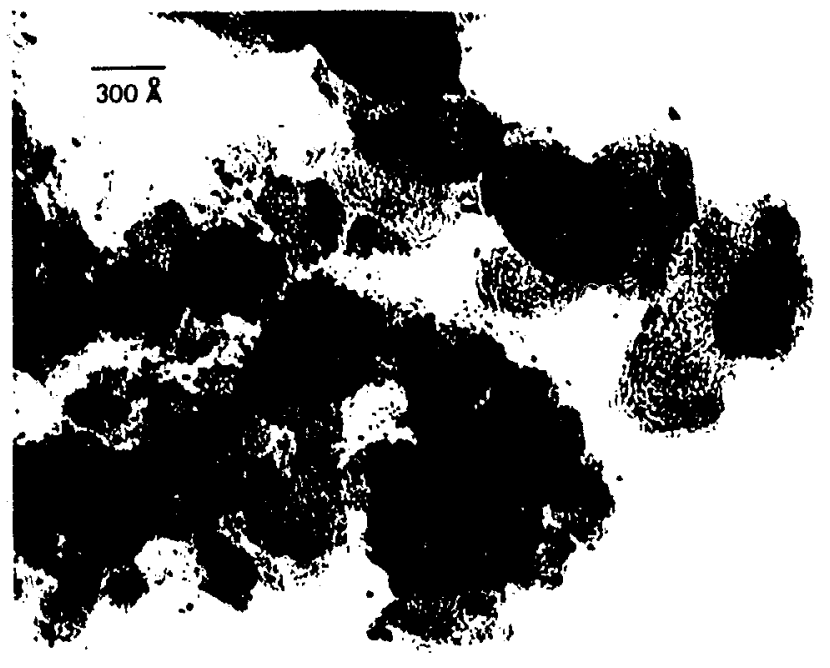

(a) no heat treatment

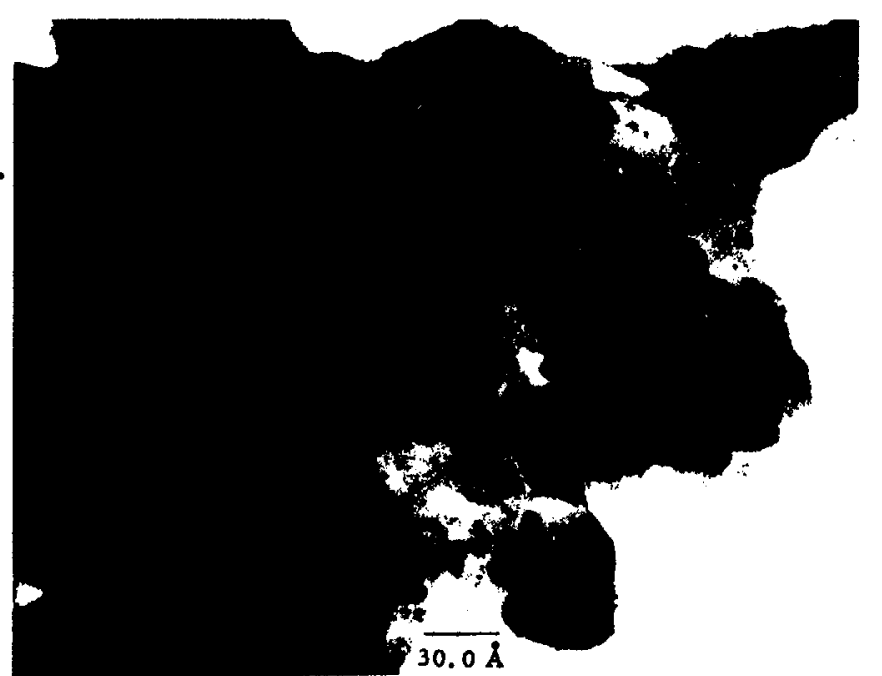

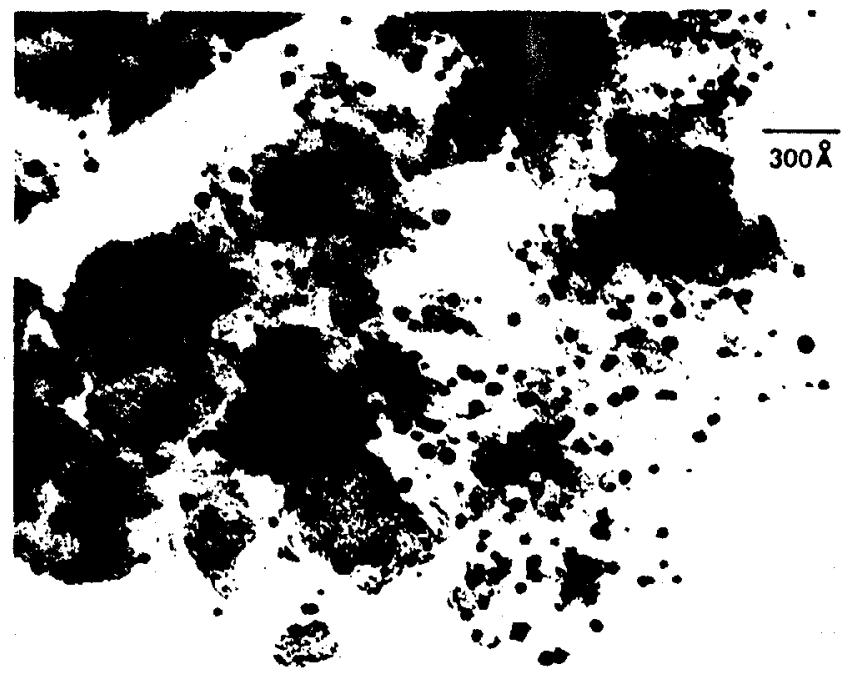

(b) heat treated to $900^{\circ} \mathrm{C}$

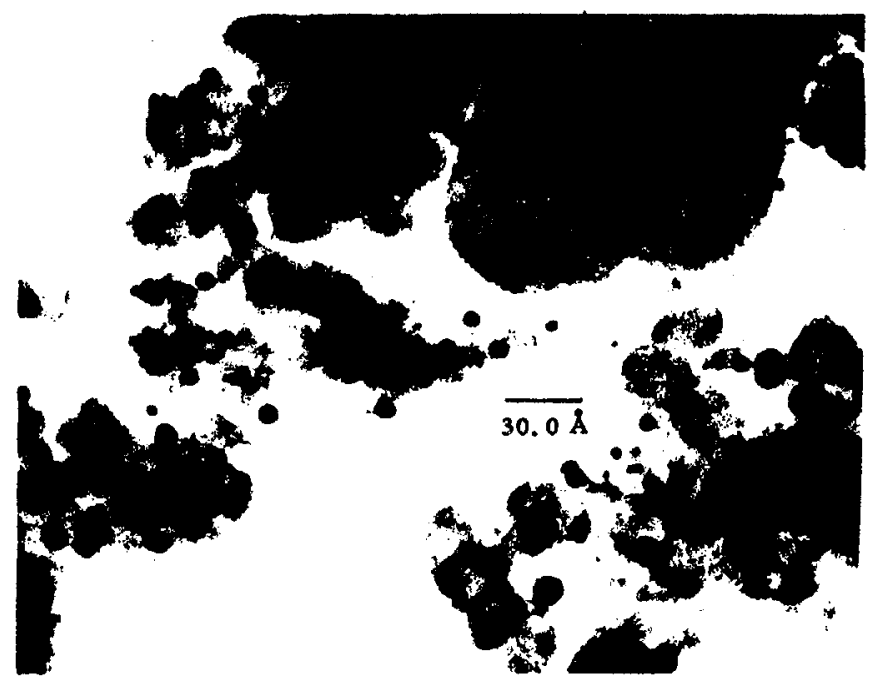

Fig. 2, 12-7. Comparison of as-prepared colloidal Pt on Vulcan XC-72 and heat-treated material (to $900{ }^{\circ} \mathrm{C}$ ). Changes in the TEM photographe should be noted; reproduced from Ref. 48. The bottom pictures have 10 times greater magnification. 


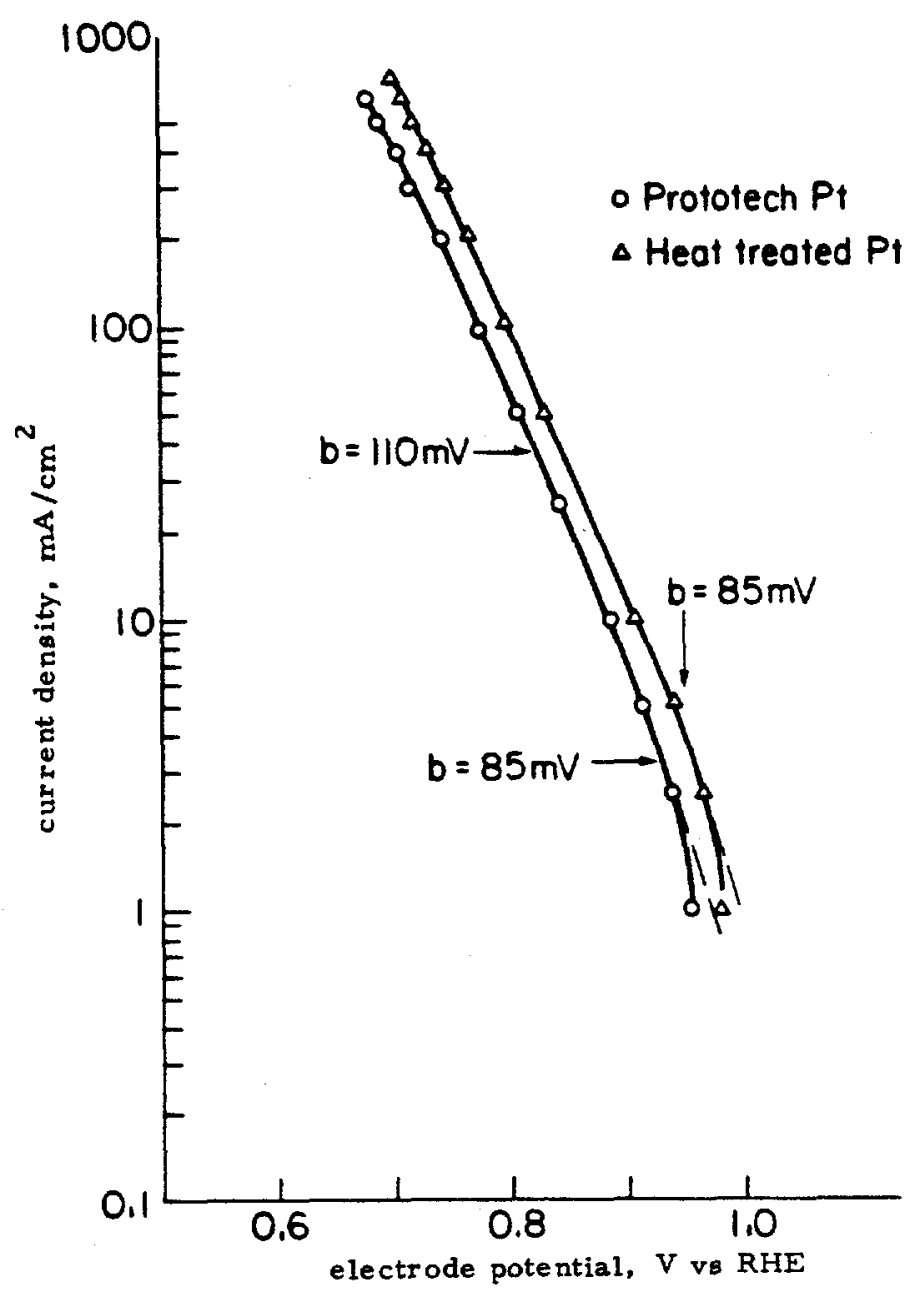

Fig. 2.12-8. The effect of heat-treatment on activity of Pt microcrystallites is shown (from Ref. 51); $440 \mathrm{~K}, 97 \mathrm{wt} \% \mathrm{H}_{3} \mathrm{PO}_{4}, 0.33 \mathrm{mg} \mathrm{Pt} / \mathrm{cm}^{2}$.

derive this expression is given in Refs. 55 and 56. In general, it will only apply if adsorbate coverates are low. At high coverage, the corresponding multiplier becomes the reciprocal, exp + $\left[(1-B) \Delta G_{a d s} / R T\right]$. Coverages are low only if $\Delta G_{a d s}$ is positive and high if $\Delta G_{a d s}$ is negative. In the intermediate region, around $\Delta \mathrm{G}_{\mathrm{ads}}=0$, the reaction rate is approximately independent of $\Delta G_{\text {ads. }}$. The resulting $\log$ (rate) vs $\Delta G_{\text {ads }}$ plot is known as a Volcano plot, 57 since it is a $r$ ising and falling function of $\Delta G_{a d s}$ centered around $\Delta G_{a d s}=0$. The theory of the concept, as applied to electrochemical reactions (the $\mathrm{H}_{2}$-electrode process) was first given by $\mathrm{Parsons} 58$ and Gerischer, 59 and experimental evidence for the more complex case of three complementary and/ or competing steps in the hydrogen electrode reaction was first given by Bockris and Conway. 60 A relatively complete analysis of this process is given in Ref. 56.

Only a reactant or a product adsorbate will be expected to enter in the rate-determining step. If both a reactant and a product are involved, the reaction is expected to be rapid and not rate-determining. 56 The preceding expressions may be of some value in explaining the activity difference between bulk and small $P_{t}$ particles, as is shown in Fig. 2.12-5.49 The oxygen electrode process in acid solution is almost certainly a one-electron transfer to molecular dioxygen and involves a proton to give an adsorbed product, $55,61 \mathrm{viz} . \mathrm{O}_{2}+\mathrm{H}^{+}+\mathrm{e}^{-} \rightarrow \mathrm{O}_{2} \mathrm{H}_{\text {ads }}$. As long as the free energy of adsorption of $\mathrm{O}_{2} \mathrm{H}_{\mathrm{ads}}$ or, at least, the difference in free energy of adsorption between this radical and that of $\mathrm{H}_{2} \mathrm{O}$ on the metal surface is positive, the free energy will become more positive according to the Kelvin equation applied to the surface free energy as the particle size decreases, 62 Using this argument, the free energy of evaporation of the molecules in the small particle, or any other free-energy function including free energy of adsorption, will be increased by $2 \mathrm{eV} / \mathrm{rRT}$, where $\varepsilon$ is the interfacial energy, $V$ the molar volume of the metal, and $r$ the radius of the spherical particles. This process explains a two-fold fall-off in rate at $900 \mathrm{mV} / \mathrm{HE}$ in going from bulk material to $5 \mathrm{~nm}$ particles, but it will not explain the more finely detailed changes that are observed, for example, in the Tafel slope. This question is addressed in Sec. 2.15. 


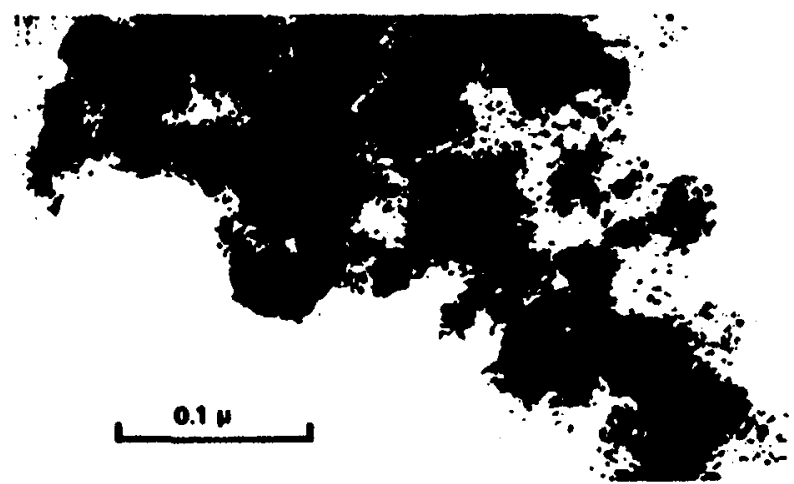

A. Transmisgion electron micrograph of $10 \mathrm{w} / 0 \mathrm{Pt}$ supported on Vulcan XC-72

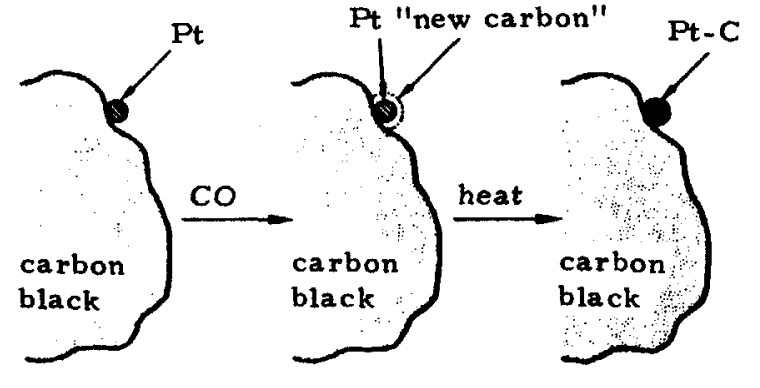

catalyst $A$

catalyst B

catalyst C

B. Schematic presentation of carbiding processes of Pt supported on carbon black.

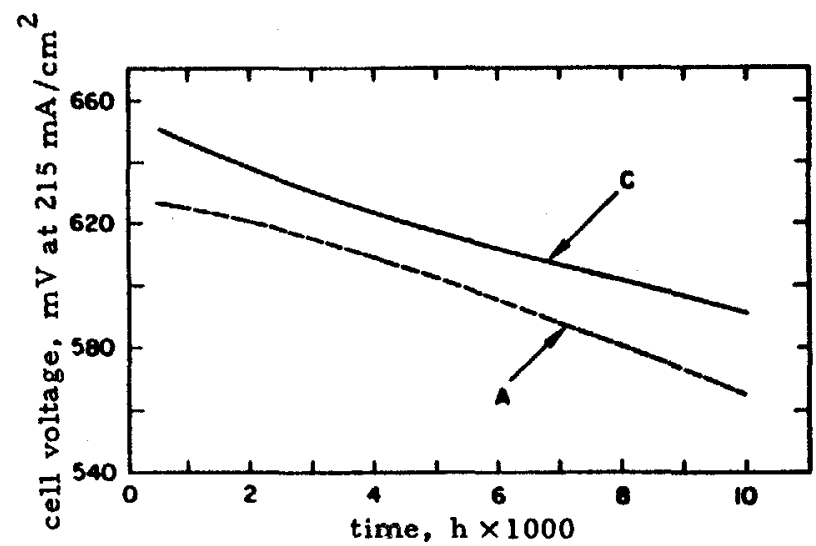

D. Cell-terminal voltage at $190^{\circ} \mathrm{C}$ in $100 \%$ $\mathrm{H}_{3} \mathrm{PO}_{4}$ vs operating time for $\mathrm{Pt}$ and Pt - C aupported on carbon black.

Fig. 2.12-9. The effect of heat-treatment on $\mathrm{Pt},{ }^{50}$ showing improvement of time-dependence of activity for heat-treated $\mathrm{Pt}$, perhaps indicating $\mathrm{Pt}-\mathrm{C}$ alloy formation. 


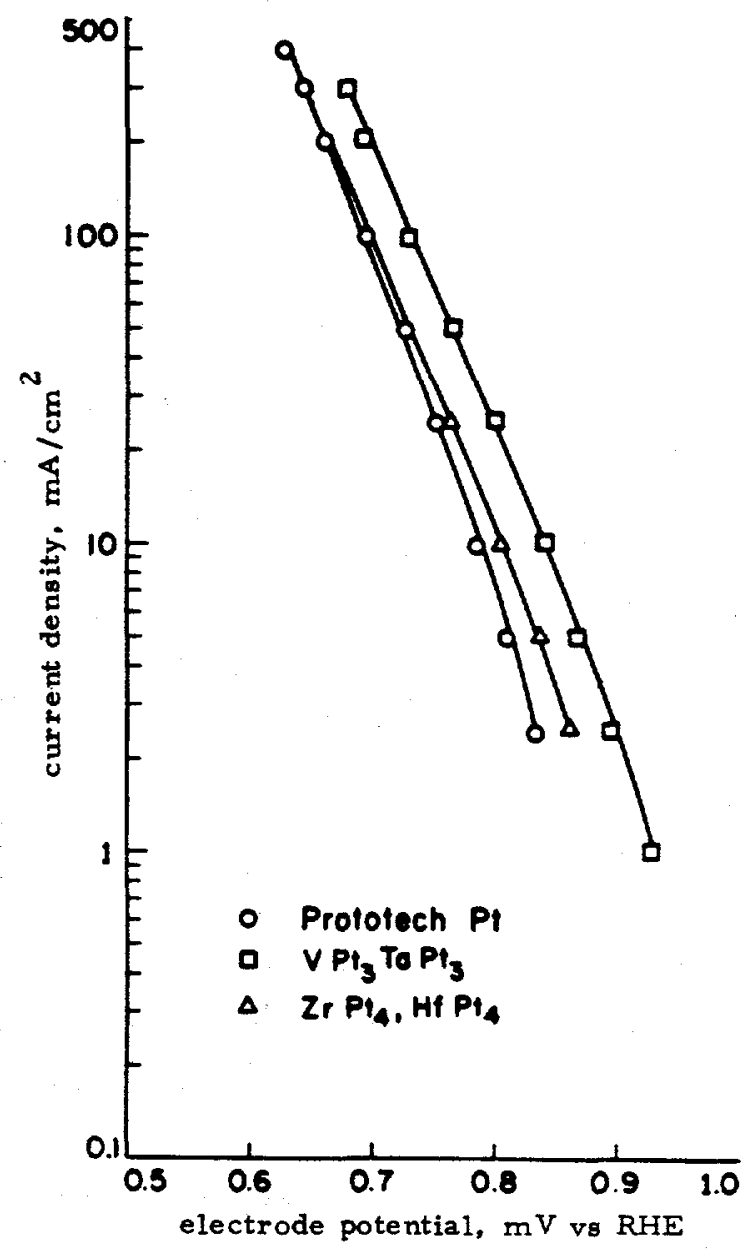

Fig. 2.12-10. The enhanced activity of $\mathrm{Pt}$ alloys for oxygen reduction is shown (from Ref. 51 ); $388 \mathrm{~K}, 88 \mathrm{wt} \% \mathrm{H}_{3} \mathrm{PO}_{4}, 0.18 \mathrm{mg} \mathrm{Pt} / \mathrm{cm}^{2}$.

It is generally accepted that changes in $\Delta \mathrm{H}_{\mathrm{ads}}\left(\Delta \mathrm{H}_{\mathrm{ads}}=\right.$ the heat of adsorption $)$ and hence in $\Delta G_{\text {ads }}$ are directly affected by changes in the bond strength of the adsorbate. However, as is shown in Sec. 2,15, simultaneous changes in adsorbate entropy as a function of the substrate should not be discounted. On the assumption that the bond strength of the adsorbate is affected by that of the substrate via the Ely-Pauling equation, 63 a tighter substrate bond means a stronger substrate-adsorbate bond. If the catalytic system is on the rising side of the Volcano curve [i.e., if $\log$ (rate) increases with increasingly negative free energy of adsorption], then increasing the substrate bond strength will be expected to increase the rate. It is generally accepted that $\mathrm{Pt}$ is very close to the top of the Volcano expression for the process $\mathrm{O}_{2}+\mathrm{H}^{+}+\mathrm{e}^{-} \rightarrow \mathrm{O}_{2} \mathrm{H}_{\mathrm{ads}}$ in the rate-determining-step for acid solutions (Fig. 2.12-11) for oxygen-reduction results in concentrated PA, as given in Ref. 64. The reaction-rate scale in Fig. 2.12-11 involves many decades, and Pt may not be precisely at the top, so that some fine-tuning can perhaps increase rates by a fraction of a decade. Indeed, there is evidence that some Pt noble-metal alloys, for example, those containing small amounts of $R a, 65$ may be more active than pure Pt.

Ross 51 used the preceding argument to examine base-metal alloys, particularly those of the Engel-Brewer type. ${ }^{66} \mathrm{Pt}_{3} \mathrm{~V}$ and $\mathrm{Pt}_{4} \mathrm{Z}_{\mathrm{r}}$ are good examples of the se compounds. Normally, base metals (except for $\mathrm{Nb}$ and $\mathrm{Ta}$, cf. thermodynamic data given by Pourbaix in Ref.67) alloyed with $P t$ would be expected to dissolve at the oxygen-electrode potential at relatively high temperatures in acid solution. Even $\mathrm{Ag}_{\mathrm{g}}$ is very soluble and $\mathrm{Pd}$ has a measurable solubility, which prevents its use as an effective cathode material, 47 even though its activity is high (Fig. 2. 12-11). Ross' argument was based on the fact that the additional stability conferred by bond formation in the class of Engel-Brewer valence-band intermetallic compounds should prevent dissolution of the base-metal-alloying element. Since the bond strength may be as much as $-250 \mathrm{~kJ} / \mathrm{mole}$, this assumption is reasonable. However, the higher bonding energy of the intermetallic material compared'with pure Pt would be expected to increase the bonding energy of the rate-determiningstep adsorbate at the surface $P t$ sites and thus render the relatively more widely spaced and 


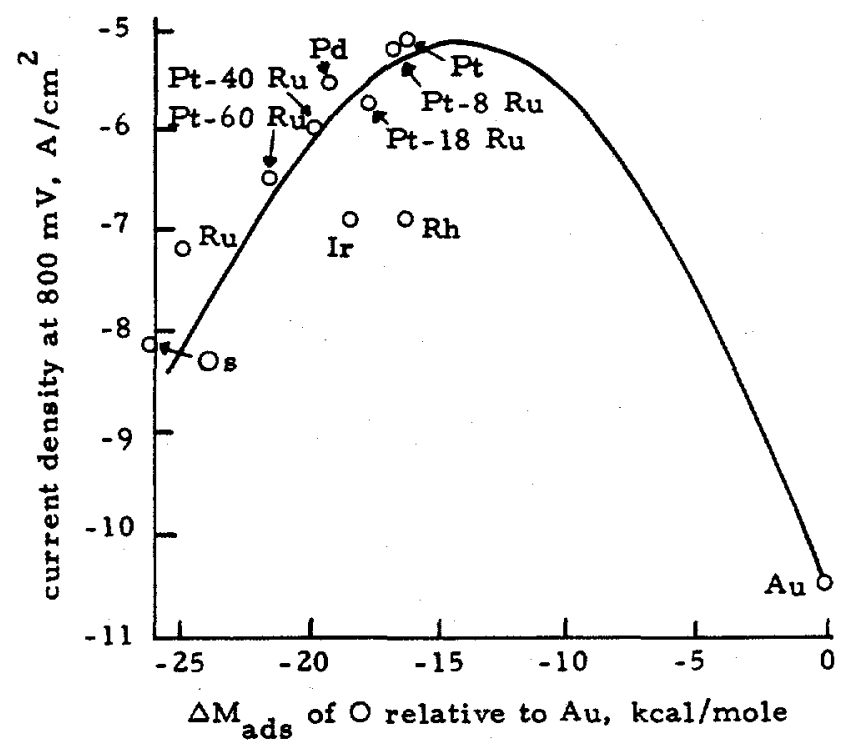

Fig. 2.12-11. The Volcano plot is shown for oxygen reduction in PA (Ref. 64). The concentrations of Pt-Ru alloys are expressed in atom \%.

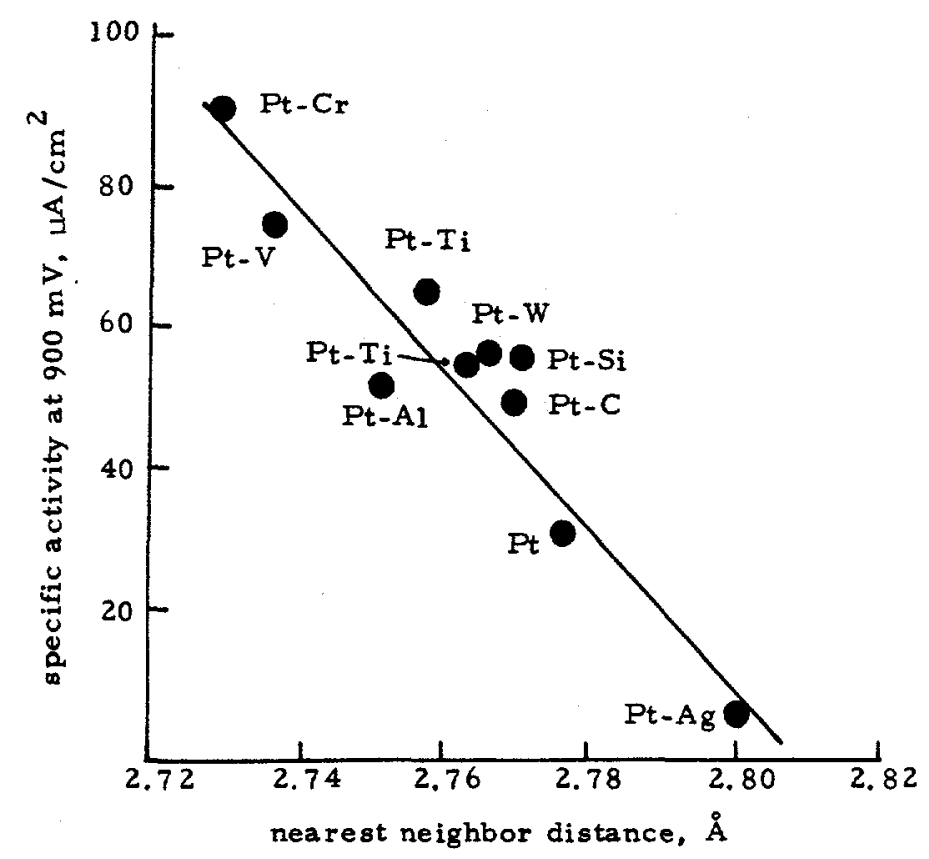

Fig. 2.12-12. The correlation is shown between Pt-alloy activity for oxygen reduction in PA with nearest neighbor distance (i.e., bond-strength of reaction intermediates; $c f$. Ref. 69). 
more weakly bonded surface Pt atoms present in colloidal particles more like bulk Pt. This weaker bonding and correspondingly wider spacing are implied by the effect described by the classical Kelvin equation. The alloys examined by Ross appeared to show improved activity by about a factor of 2, or the equivalent of $30 \mathrm{mV}$, at constant current density and apparently good stability.

It is not known whether similar arguments involving the effect of adsorbate bonding and the Engel-Brewer compounds were used by researchers at UTC who developed alloy materials of the type described in their Pt-V patent. 52 The 8 cope of their development work was later described by Jalan, 68 who indicated that a wide range of binary Pt-based alloys was considered, and that work also involved ternary alloys of Pt-Al-Si and Pt-Sr-Ti. The fact that alloys containing metals as reactive as $\mathrm{Al}$ and $\mathrm{Sr}$ showed reasonable stability is surprising and has led to arguments that the higher activity of the catalyst results from formation of a surface skin of a Raneytype Pt. Although this hypothesis has not been substantiated, there is no evidence that any of the alloying metal is present in the surface Pt layer, which may simply have its surface properties modified by those of the bulk-alloy material. The most effective material examined was the Pt-V alloy, which was about three times as active (in $\mathrm{mA} / \mathrm{mg}$ ) as its pure Pt precursors, which had substantially higher BET surface areas.

In 1983. Jalan tried to correlate alloy catalyst activity as an indirect function of adsorbate bond-strength. 69 The function used was the bulk interatomic distance in the alloy, which is related to the bond tightness and hence to the strength of the $\mathrm{O}_{2} \mathrm{H}_{2}$ ads adsorbate bond involved in the rate-determining step for molecular dioxygen reduction. Jalan's correlation, which can be regarded as a fine-tuning of the basic Volcano plot shown in Fig. 2.12-11, is shown in Fig. 2.12 12. Figure 2.12-13 show a composite Volcano plot as a function of nearest neighbor distance and incor porates the data of Figs, 2.12-11 and 2.12-12.

It is reasonable to presume that some of the $\mathrm{Pt}-\mathrm{V}$ and other base-metal alloys described in Refs. 51 and 52 were used in cell and stack demonstrations quoted in published reports and describing DoE-supported work at UTC in the period following 1980, although they were not used in the Manhattan 4.5-MW cells but were employed in the TEPCO 4.5-MW system. Some measurements of the properties of the $\mathrm{Pt}_{3} \mathrm{~V}$ material as an electrocatalyst were carried out by other workers, following publication of the work described in Refs. 51 and 52 . In 1980 , Pt-V catalysts were prepared under EPRI sponsor ship on a proprietary UOP C catalyst (Kocite ${ }^{R}$, which is prepared by cracking aromatic hydrocarbons on high-surface-area alumina). These catalysts were tested for durability under pressure at ERC and showed high initial performance (Fig. 2.12-14) but decayed fairly rapidly as a function of time. The question remains whether the decay was intrinsic to the catalyst itself or was associated with the Kocite ${ }^{R}$ support.

A series of analytical measurements on the constituents of ERC cells with the se catalysts was conducted at LANL using the Rutherford back-scattering method. 70 It was shown that $V$ had

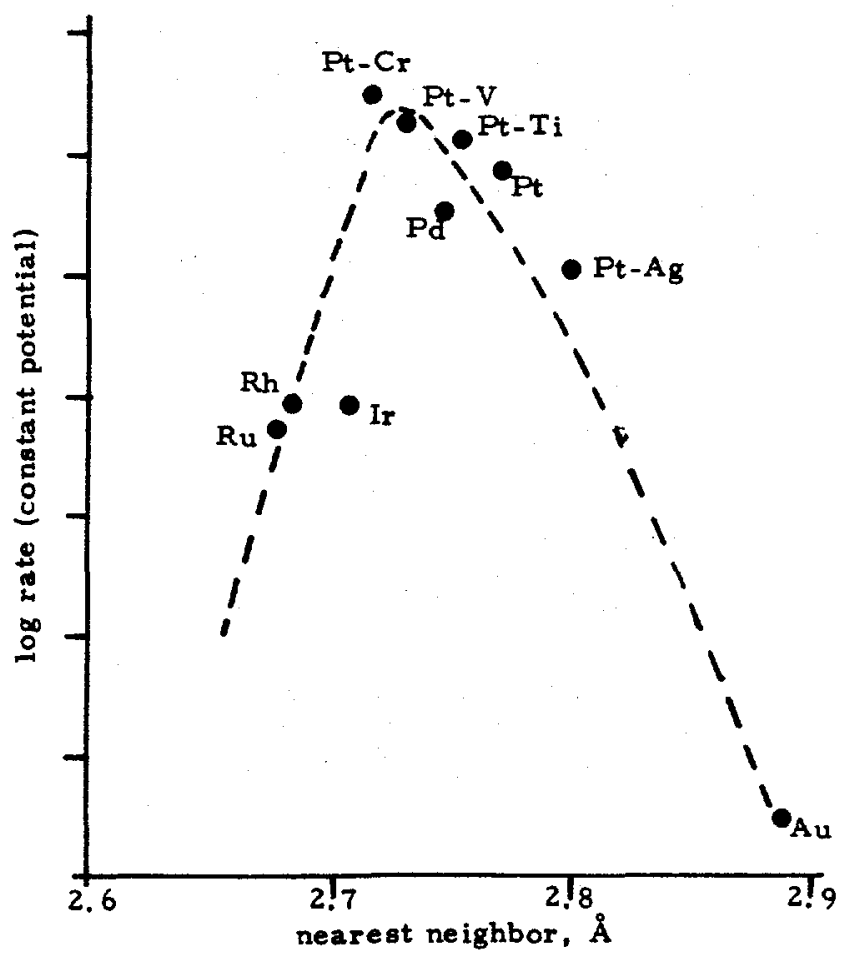

Fig. 2.12-13. The composite Volcano plot is shown which incorporates Figs. 2.12-11 and 2.12-12.55,69 
largely dissolved from the catalyst and diffused towards the anode, where it had apparently precipitated as an insoluble compound (a vanadium bronze?). On the other hand, a series of studies carried out by Stonehart 41 shows that the long-term 1088 in surface area of a $\mathrm{Pt}-\mathrm{V}$ alloy under utility PAFC cathode conditions is less than that for pure Pt (Fig. 2.12-15). Clearly, the V in Stonehart's tests had some effect in modifying the Pt crystallites to prevent sintering. Stonehart used drowned electrode structures, which may explain the difference between the Stonehart and ERC results. According to Sec, 2.11,45 it is possible that a high ionic IR drop in parts of the teflon-bonded electrode structure may result in some areas of the electrode being effectively at open circuit (Fig. 2.11-6), so that much higher dissolution rates are observed compared with those seen with drowned catalysts.

The next significant step involved two patents of Landsman and Luczak, ${ }^{71}$ assigned to UTC, with claims that Pt-Cr catalysts were more stable than Pt-V materials. One of these $(4,316,944)$ stated "in an accelerated catalyst corrosion test,... Pt-V and Pt-Cr alloy catalysts were immersed in $99 \%$ phosphoric acid saturated with air at $350^{\circ} \mathrm{F}$... after 48 hour s, $65.5 \%$ by weight of the vanadium had been dissolved from the V-alloy catalyst, whereas only $37.5 \%$ of the chromium had been dissolved from the $\mathrm{Cr}$-alloy catalyst." This work acknowledges UTC's acceptance of the long-term instability of at least some base-metal Pt-alloy catalysts. Since then, Jalan has examined a range of ternary alloy catalysts for DoE (NASA-Lewis, Cleveland). Some of these, particularly a composition code-named GS82-5-34, have shown good stability and a higher activity than pure $\mathrm{Pt}$, with over $6000 \mathrm{~h}$ of operation. Comparisons of $\mathrm{Pt}-\mathrm{Cr}_{\mathrm{r}}$, heattreated $P t$ and a ternary catalyst are shown in Fig. 2.12-16 as a function of time. While the Pt-Cr catalyst shows a very high initial activity, the activity rate of decay is very marked compared with that for both the ternary catalyst and pure Pt.

A recent UTC catalyst patent (dated May 8, 1984) refers to the advantages of a Pt-V-Co or $\mathrm{Pt}-\mathrm{Cr}$-Co ternary catalyst. 72 Their comparative performance under utility PAFC conditions is shown in Fig. 2.12-17. No data have been published on the lifetimes of these materials.

It seems clear that further work on the development of noble-metal alloy catalysts will be profitable if it leads to improved intrinsic activity or changes the Tafel slopes to lower values. The field of non-noble metal catalysts for use in acid solution has scarcely been touched. Some of the se possible improvements will be discussed in Sec. 2. 15.

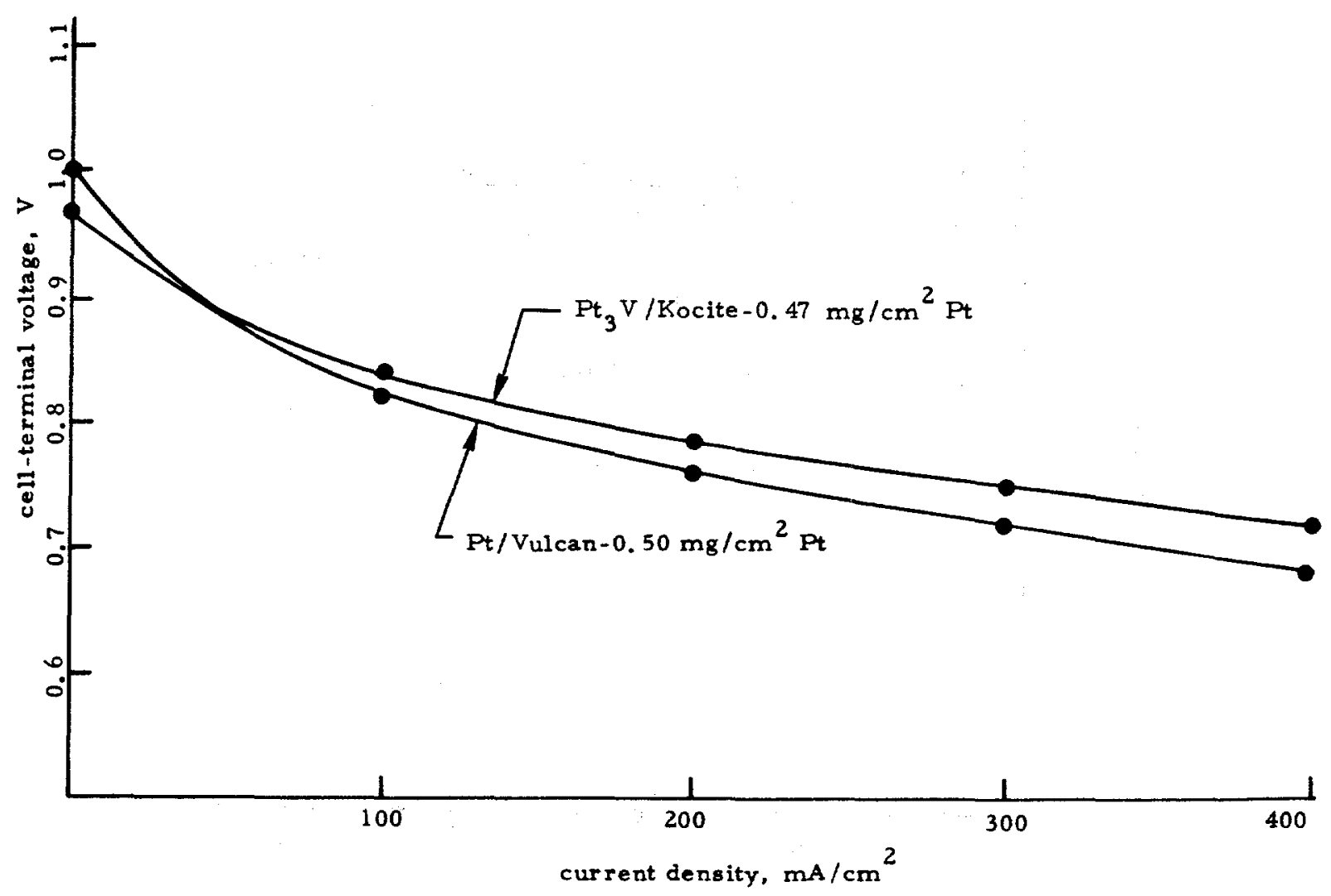

Fig. 2.12-14. $\mathrm{O}_{2}$ reduction in the $P A F C$ is shown with intermetallic $\mathrm{Pt}_{3} \mathrm{~V}$ (on proprietary Kocite ${ }^{R}$ carbon support), compared with colloidal Pt on Vulcan XC-72. 


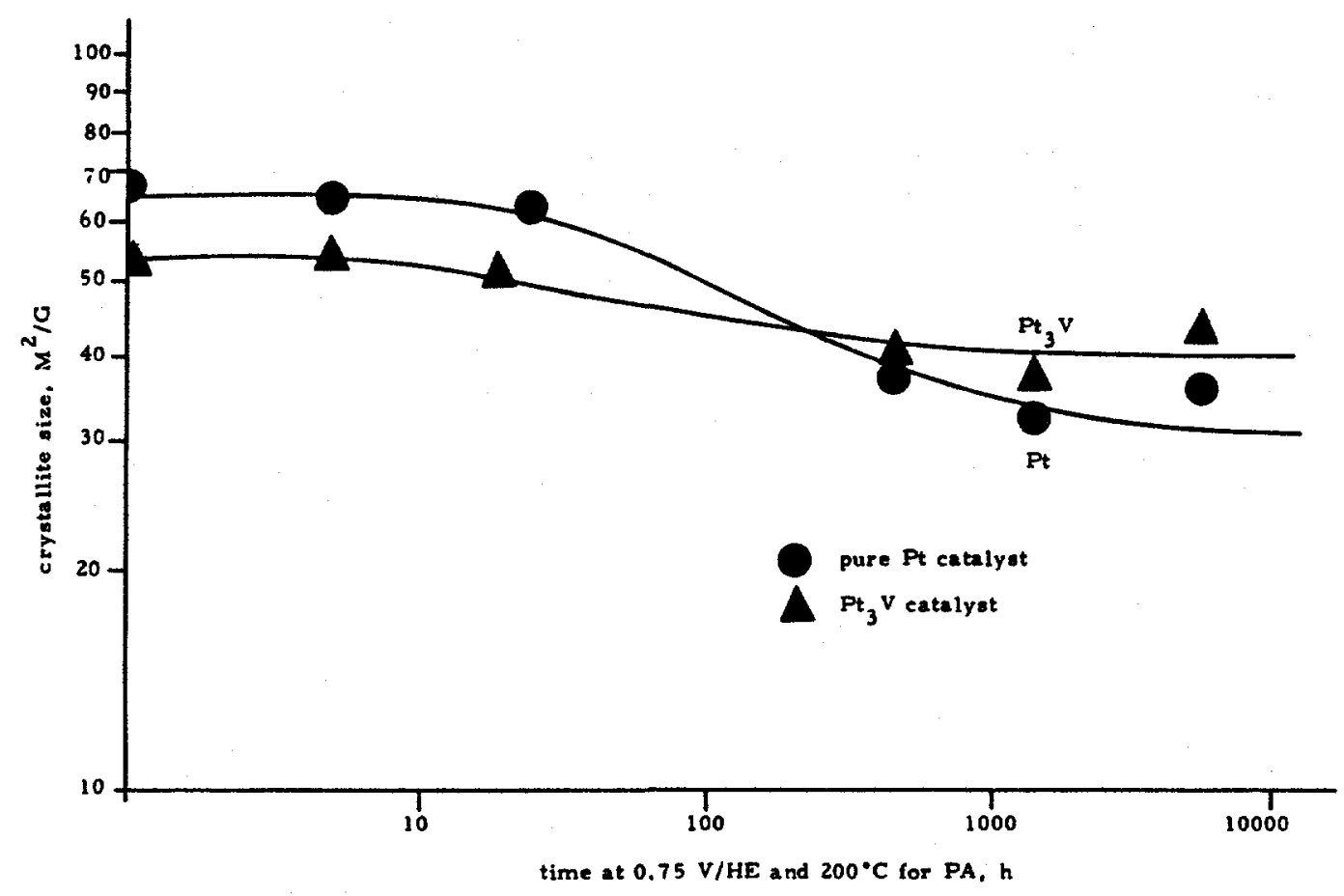

Fig. 2.12-15. The change in crystallite size is shown for $\mathrm{Pt}_{3} \mathrm{~V}$ on Vulcan XC-72 and also for Pt on Vulcan XC-7

(log-log scale) under PAFC conditions

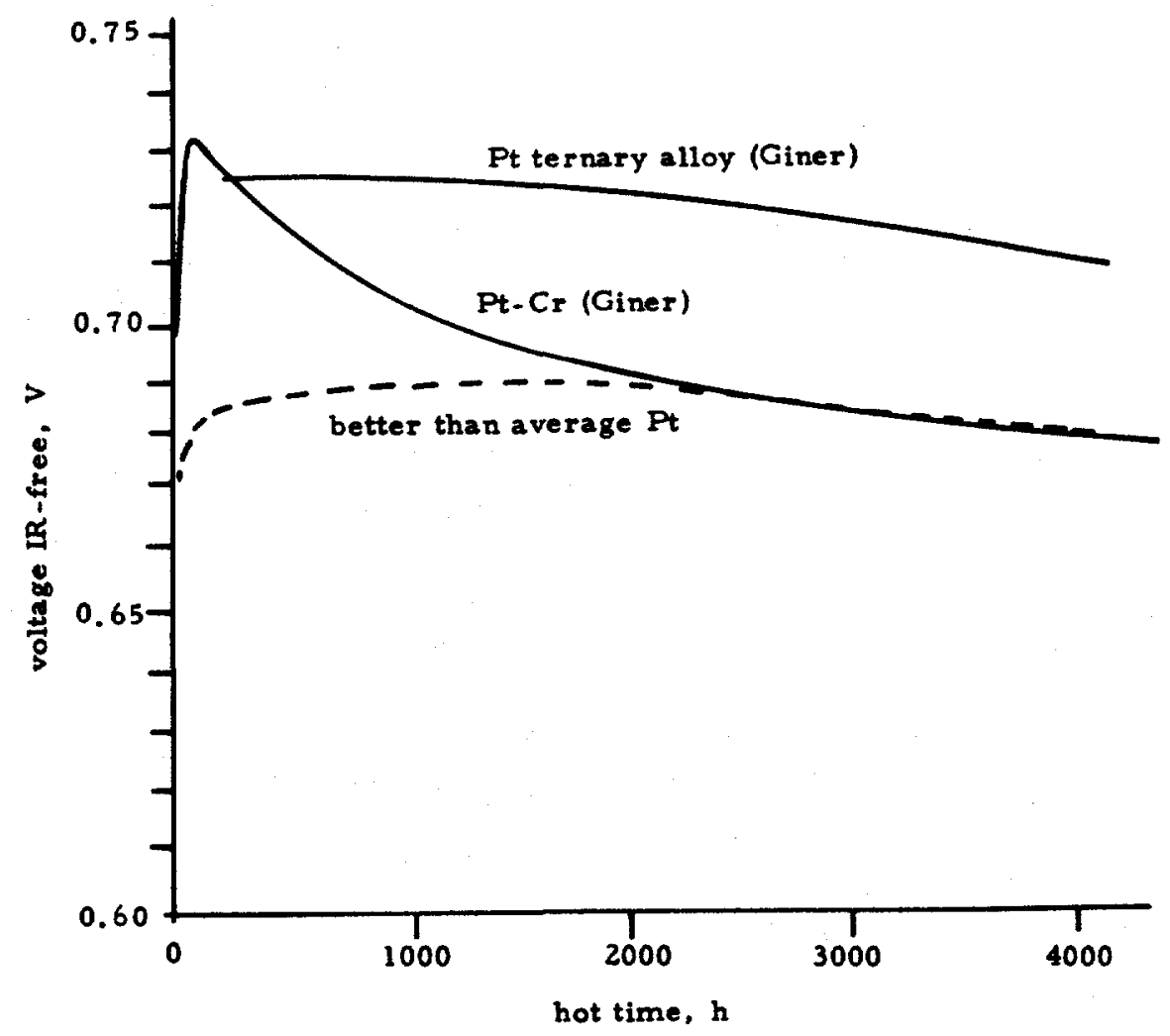

Fig. 2.12-16. The oxygen activity is shown as a function of time of heat-treated Pt, Pt-Cr, and of a ternary alloy (Giner, Inc.), all equivalent to $0.5 \mathrm{mg}$ / $\mathrm{cm}^{2}$ of $\mathrm{Pt}$ on Vulcan XC-72; $\mathrm{T}=190^{\circ} \mathrm{C}, 80 / 50$ utilization. 


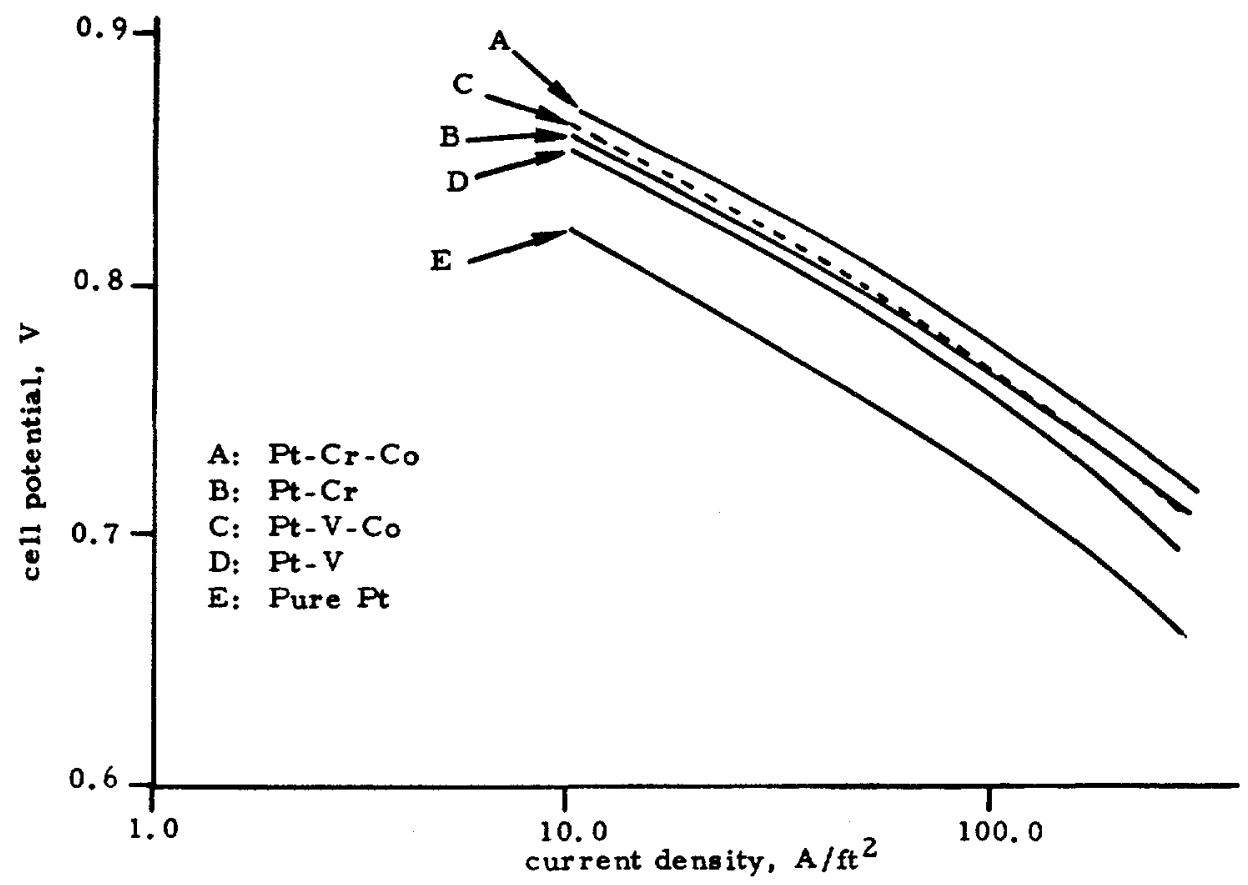

Fig. 2. 12-17. UTC comparisons of pure Pt, Pt binary and Pt-ternary alloys for oxygen reduction under PAFC conditions (Ref. 72); $\mathrm{T}=191^{\circ} \mathrm{C}, \mathrm{PA}, \mathrm{H}_{2}$-air.

\section{13 Potential Improvements in PAFC Performance and Costs}

We have stressed the importance of cost and heat-rate on the potential market for the PAFC. In particular, the EMA study ${ }^{3}$ showed that a heat-rate of $8300 \mathrm{BTU} / \mathrm{kWh}$ is a marginal minimum value for introduction into the electric utility market. As shown in Sec. 2.9, 8300 BIU/ $\mathrm{kWh}$ was the proposed introductory heat-rate value of the UTC $11-\mathrm{MW}$ unit, as defined in 1980 specifications, as well as that of the proposed 7.5-MW WE unit. Improvements in the se values would facilitate electric utility market penetration, as would decreases in initial cost. However, the situation may be different for cogeneration applications, since progressively less useful steam is produced as the heat-rate falls. It is shown in Ref. 2 (compare Sec. 2. 4) that, for $8300 \mathrm{BTU} / \mathrm{kWh}$, a generic FC plant with cogeneration may have a market-place break-even cost of $\$(1982) 1500 / \mathrm{kW}$ (electric), whereas, for a stand-alone electric utility system, the corresponding figure might be slightly under $\$(1982) 1000 / \mathrm{kW}$. The latter is much more sensitive to overall electrical efficiency than the former, since a more expensive dispersed cogeneration system will be sited so that all of the available waste heat can be sold. In addition, a cogeneration plant will be used more intensively than a load-following electric utility plant. Hence, its capital cost can be economically levelized over more operating hours, i.e.. it may therefore cost more and still be economical. This fact is behind the gas utility on-site program, for which an introductory cost of $\$ 2500 / \mathrm{kW}$ (electric) for a $200-\mathrm{kW}$ on-site cogeneration unit with a $9300 \mathrm{BTU} / \mathrm{kWh}$ (electric) heat-rate is not considered excessive.

If a developer is high up the learning curves in Figs. 2. 4-1 and 2. 4-2, it may be better to aim for a lower-cost, lower-efficiency system for cogeneration purposes, until the cogeneration break-even point is reached. This stage will then be followed by further cost reduction and improvements in heat-rate until a definitive, stand-alone electric utility system is reached.

The UTC plant was intended to operate at $0.73 \mathrm{~V}$ (end-of-life), whereas the WE plant goal (at an unspecified point in life) was a $0.68 \mathrm{~V}$ unit cell potential. Both systems were planned for operation at the same overall BTU/kWh (electric) heat-rate, which is obtained by better integration of system components in the WE unit. As noted in Sec. 2. 9, this goal is reached by using a steam-condensing bottoming cycle in the turbo-compressor in the WE unit to eliminate the need for fuel burning in the turbine. The result is virtually no available waste heat in the cogeneration mode. If the WE system is redesigned for use in cogeneration, its heat rate will rise to over 8700-8900 BTU/kWh. 73 According to a recent study, 74 if pressurized WE FC stacks are examined in a generation plant in which they are integrated with a coal gasifier, their low steam production and lower unit cell voltage compared with that of a competing UTC unit will result in an overall coal-AC heat-rate of $11,500 \mathrm{BTU} / \mathrm{kWh}$, compared with $9600 \mathrm{BTU} / \mathrm{kWh}$ if presaurized UTC cell stacks are used. 75 These figures assumed BTU credit for tars and coal fines produced in the gasification process. Without this credit, the corresponding figures were $12800 \mathrm{BTU} / \mathrm{kWh}$ 
and $11100 \mathrm{BTU} / \mathrm{kWh}$, respectively. Because WE cells require a larger balance-of-plant per $\mathrm{kW}$, their investment cost is necessarily higher.

Even with the UTC unit, there are heat-rate limits with NG or light distillate because a large excess of steam is required in the reforming operation. If the cell potential could be raised, the system efficiency would increase. Eventually, a cell potential $(\sim 0.8 \mathrm{~V})$ will be reached at which insufficient waste heat will become a vailable to operate the reformer. The system also requires desulfurized fuels for effective operation of the present reformer. The necessity of using vapor-phase hydrodesulfurization for fuel clean-up limits fuels to those with an end-point of about $215^{\circ} \mathrm{C}$. As the result, JP-4 or No. 2 fuel oil cannot be used, since they tend to crack when fully vaporized. Fuel requirements set economic limits on the present system. Even with clean and light fuels, the system heat-rate will be limited to the range 7200 $7500 \mathrm{BTU} / \mathrm{kWh}$, depending on efficiency of system components.

As was pointed out in Sec. 2. 4, efficiency improvements beyond those currently envisaged will be needed after introduction of the PAFC if it is to remain competitive and find broader markets than its initial niche. The other major problem is the necessity for rapid cost reduction during the introduction period. Cost and efficiency are closely related, as is shown by the fact that, for otherwise equal cell parameters such as catalyst, temperature and pressure, a more complex and costly chemical engineering system will give better heat integration and higher efficiency. A cell stack operating at lower current density and hence higher cost per $\mathrm{kW}$ will operate at higher voltage and will therefore be more efficient.

The system efficiency can be improved in a number of different ways, involving any combination of systems improvements and including also catalysts at the cell cathode and different cell operating conditions. By far the cheapest way to improve efficiency is improved catalysis, even if a higher cathode $\mathrm{Pt}$ loading is needed and can be used effectively in existing electrode structures at a cost of $\sim \$ 40 / \mathrm{kW}$. Lowering of stack current density and, particularly, redesigning of the chemical engineering system to obtain a better-integrated unit, will be much more costly.

In the proposed UTC system, 25, 27 a number of gains in overall efficiency can be effected by making small improvements to the system as a whole. One obvious example is in the DC-AC inverter, for which the target efficiency has been raised from about 97.5 to $98.5 \%$. Small efficiency improvements can be made in the turbocompressor. Perhaps the most important but difficult and costly improvement that can be made without degrading overall system performance in a cogeneration mode involves use of the spent cathode air in the reformer burner. This procedure was first examined (cf. Sec. 2. 9) by Toshiba in Japan. Use of the fact that the cathode exhaust is already at high pressure and at the cell temperature saves some heat and compression work and allows utilization of a smaller turbocompressor. Since the cathode exhaust air is depleted in $\mathrm{O}_{2}$, the resulting flame temperature is reduced from 1700 to about $1250^{\circ} \mathrm{C}$, which allows replacement of zirconia insulation by alumina and presumably yields a significant improvement in system reliability. Toshiba plans to use a catalytic burner in their reformer, which may further reduce the flame temperature, perhaps at the rigk of relatively short combustion-catalyst life. UTC appears to prefer preheated gases and a conventional burner. The indicated improvements should allow UTC to reach a system heat-rate of about $7700 \mathrm{BTU} / \mathrm{kWh}$ (down from $8300 \mathrm{BTU} / \mathrm{kWh}$ ), based on a $0.73 \mathrm{~V}$ cell. WE may also introduce system changes to obtain a heat-rate of about $8100 \mathrm{BTU} / \mathrm{kWh}$ at $0.68 \mathrm{~V}$ (cf. Fig. 2.9-3).

Further heat-rate improvements with methane or light distillate fuel will probably require increased cell voltage, which means improved catalysis and/or increased system pressure and temperature. A plot of the effect of cell temperature on system heat-rate is given in Fig. 2. 13-1 and assumes a system heat-rate of $7730 \mathrm{BTU} / \mathrm{kWh}$ at $0.73 \mathrm{~V}$, an a verage cell temperature of $205^{\circ} \mathrm{C}$ and a pressure of $8.2 \mathrm{~atm}$. Heat-rates calculated at different temperatures and pressures are based on the assumption that the operating pressure can be increased proportionately with an increase in water vapor pressure as the cell temperature increases. Figure 2.13-1 is based on a dependence of cell performance on temperature and pressure of the type shown in Figs. 2.13-2 and 2.13-3. Figure 2.13-1 shows that the unit cell voltage increases as $1 / \mathrm{T}(\mathrm{K})$, and a $7000 \mathrm{BTU} / \mathrm{kWh}$ heat rate should be attainable for a cell potential of $0.8 \mathrm{~V}$ at about $225^{\circ} \mathrm{C}$, with $7500 \mathrm{BTU} / \mathrm{kWh}$ at $0.76 \mathrm{~V}$ and about $215^{\circ} \mathrm{C}$. The latter value may be reasonably expected to be attainable in the future with some materials improvements, whereas the former value is more doubtful.

In the preceding discussion, we have not assumed any progress in catalysis, which requires innovation. There are other ways to improve system heat-rate, which do not require increased cell temperature and will therefore be less likely to augment corrosion of cathode components. One of these is to $r$ un the cell at reduced current density, which may improve sys tem heat-rate by decreasing cell overvoltage. Unfortunately, this approach requires an increase in system capital cost because a large cell-surface area is needed.

The simplest method for improving heat-rate, which requires no systems changes and will have no effect on the corrosion environment of the cathode, is to reduce the internal cell resistance. This procedure results in an increase in cell potential of $15 \mathrm{mV}$, which is sufficient to take the system from 7730 to $7570 \mathrm{BTU} / \mathrm{kWh}$. Table 2.13-1 lists options for improving PAFC 


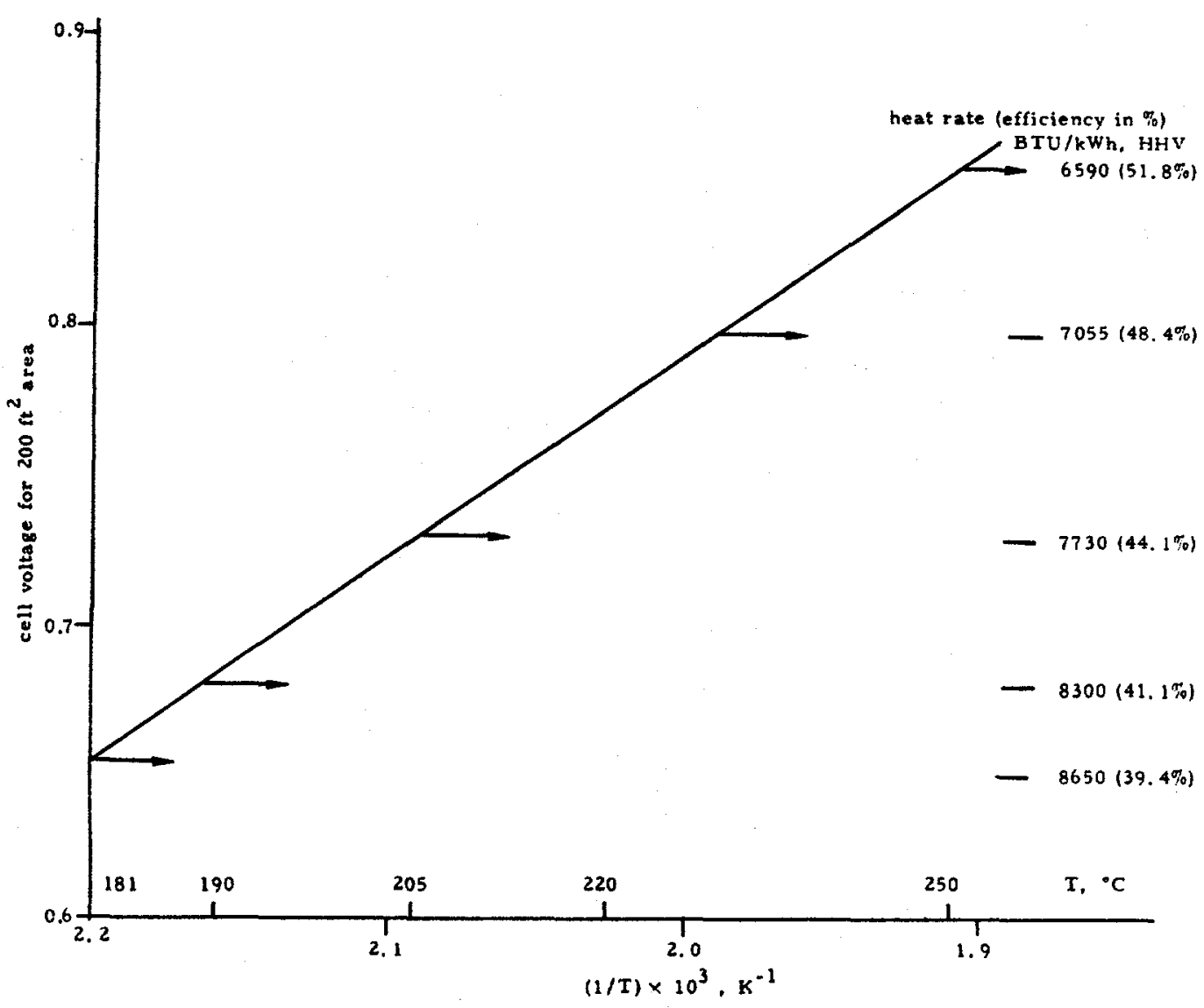

Fig. 2.13-1. The extrapolated UTC system heat-rate is shown as a function of cell temperature $\left(7730 \mathrm{BTU} / \mathrm{kWh}\right.$ are assumed at $\left.205^{\circ} \mathrm{C}, 0.73 \mathrm{~V}\right)$.

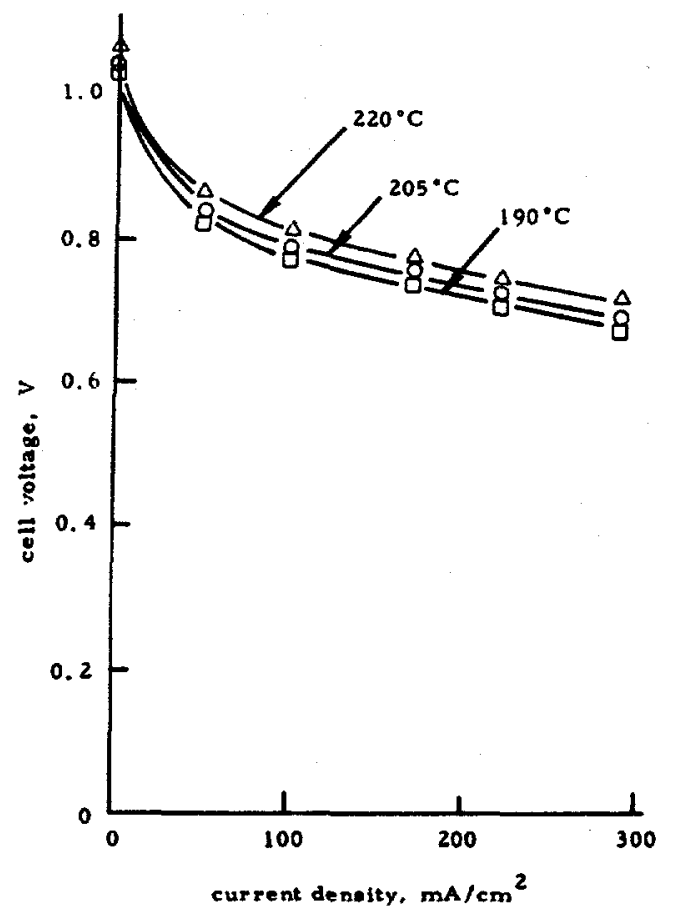

Fig. 2.13-2. The performance at 5.0 atm is shown as a function of temperature (from Hitachi).

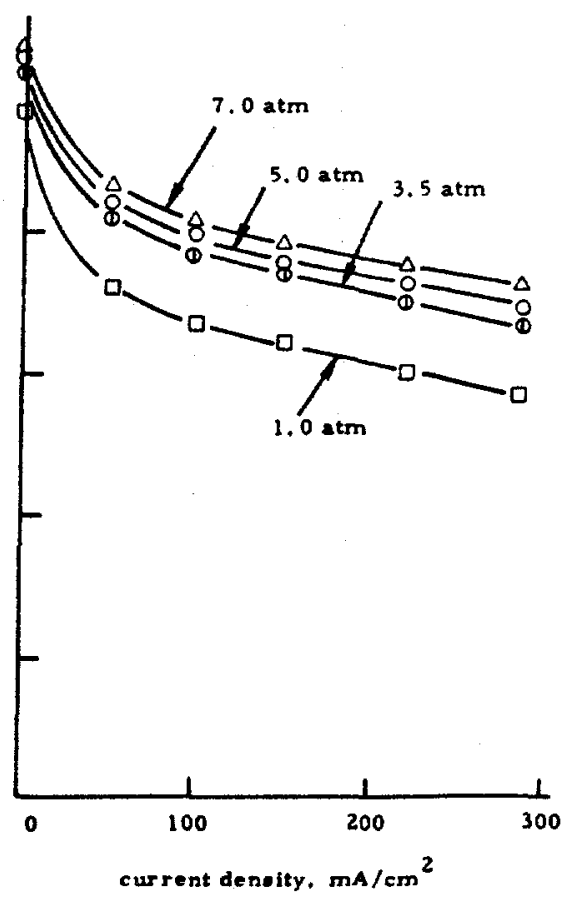

Fig. 2.13-3. The performance at $205^{\circ} \mathrm{C}$ is shown as a function of preseure (from Hitachi). 
Table 2.13-1. Options for PAFC heat-rate improvements.

\begin{tabular}{|c|c|c|c|c|}
\hline Action & BTU/kWh Gain & Problems & Advantages & Change of Succes $8, \%$ \\
\hline $\begin{array}{l}\text { lower IR drop } \\
\left(15 \mathrm{mV} @ 216 \mathrm{~mA} / \mathrm{cm}^{2}\right) \\
\left(23 \mathrm{mV} @ 324 \mathrm{~mA} / \mathrm{cm}^{2}\right)\end{array}$ & $\begin{array}{l}150 \\
225\end{array}$ & tolerances & $\begin{array}{l}\text { no corrosion } \\
\text { changes }\end{array}$ & $\begin{array}{l}70 \\
70\end{array}$ \\
\hline $\begin{array}{l}\text { lower current density } \\
\left(162 \mathrm{~mA} / \mathrm{cm}^{2}\right)\end{array}$ & 300 & $\begin{array}{l}\text { corrosion } \\
\text { capital cost }\end{array}$ & easy & 60 \\
\hline system improvements & 300 & $\begin{array}{l}\text { development } \\
\text { cost }\end{array}$ & $\begin{array}{l}\text { does not } \\
\text { affect cells }\end{array}$ & 60 \\
\hline catalysis $(50 \mathrm{mV})$ & 500 & technical & $\begin{array}{l}\text { cheap } \\
\text { (if possible) }\end{array}$ & 40 \\
\hline
\end{tabular}

heat-rate and shows advantages and disadvantages and also probabilities of success. Long-term research should clearly emphasize catalysis and improved cathode materials.

Section 2.4 shows that, in the near term, cost will be the driving factor in market penetration, since the heat-rates of the first units will be adequate for commercial use. The system cost should decline rapidly after the first few units have been built, but the learning curve for this subsystem will be different from that for the FC units.

The proposed UTC $11-\mathrm{MW}$ unit 25,27 will contain over 8000 cells, as suming specification current density and cell potential. The learning curve for cell components may well be different from that for the system as a whole, since a semi-automated production line will have to be installed when the first unit is ordered. It is hard to predict the precise costs of individual components during the early period of cell commercialization, since ratios of cell-stack cost to balance-of-plant will vary as plants come off the production line. This fact makes it difficult to estimate the effects of changing parameters such as stack-power density on total FC plant cost. The present stack components are not final, even though their materials and dimensions may be frozen (e.g., graphitization, oven size, pressure-vessel size, and wall thickness) by the corrosion environment and by economic and systems considerations. However, the nature and design of the repeating cell components may well change, with potentially great cost advantages. Some of the possible variations of repeating cell parts are shown in Fig, 2.13-4, which should be compared with the first two generations of UTC structures shown in Fig. 2. 10-1. Concept $A$ is known as the ABA structure of Engelhard and shows a bipolar plate that is similar in geometry to the conventional bipolar plate used in the New York 4.5-MW demonstrator (see Fig. 2. 10-1), with cross-flow structure and integral gas channels. The major difference between the conventional ribbed bipolar plate structure and the Engelhard structure is the fact that the latter is built up from two separate, flat, porous components, which are commercially available and relatively cheap. Originally, the material used was porous Pfizer vitreous carbon foam, but this has been superseded by another commercial product. These materials are likely to cost $\$ 30 / \mathrm{m}^{2}$ in quantity. As for the UTC ribbed substrate, the gas-flow distribution ribs can be machined relatively easily into the porous material, which, if it is vitreous carbon or graphite, will show adequate corrosion resistance. To form the complete bipolar plate, the two halves must be cemented together by an impervious, gas-tight layer with adequate corrosion resistance. This layer is a heat-treated carbonized or graphitized resin. The bipolar plate is completed by application of two electrodes, with the catalyst layer introduced into graphite felts; papers or cloths are used in the normal manner to give two complete half-cells, which can be assembled into a stack by using the standard silicon carbide matrix that holds electrolyte between the anode of one bipolar plate and the cathode of another.

The following speculations relate to electrolyte management. Since the bipolar plate consists of two porous halves, it may be constructed so that it contains an electrolyte reservoir. This goal is usually considered to be desirable, even if it means occasional addition of electrolyte to the cell-stack, since a buffer reservoir is needed to take up acid volume as a function of dilution changes with load. If a reservoir is required on both the anode and cathode sides, then each will require little or no wet-proofing but has a ppropriate porosity to retain electrolyte without withdrawing it from the matrix. In this case, the carbonized resin layer on the cathode side will be in contact with the electrolyte at the oxygen-electrode potential and liable to corrosion. The whole plate will therefore need graphitization or, at least, high-temperature heattreatment and will, therefore, require one further, relatively costly manufacturing step. This complication can be avoided if the reservoir side is only on the anode, which is operationally desirable. The cathode side can then be thoroughly wet-proofed by teflon so that it retains no electrolyte at all. In this way, the carbonized resin can be protected against corrosion; in fact, 


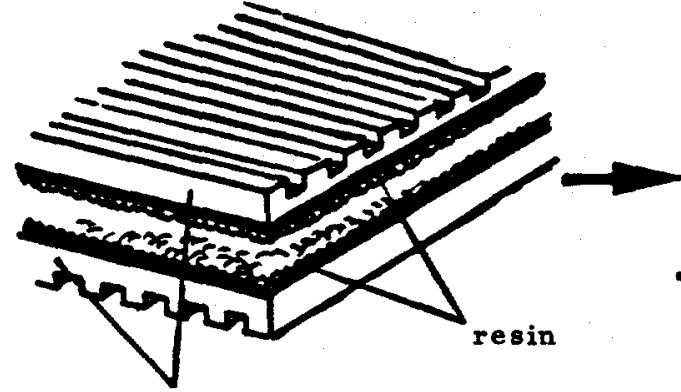

machined Pfizer vitreous carbon foam parts

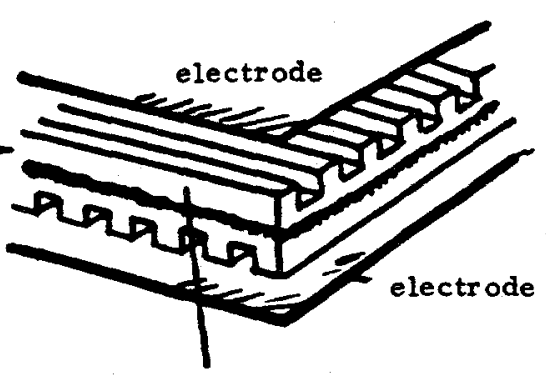

completed bipolar plate (after heat-treatment)

B: Toshiba Hybrid Stack

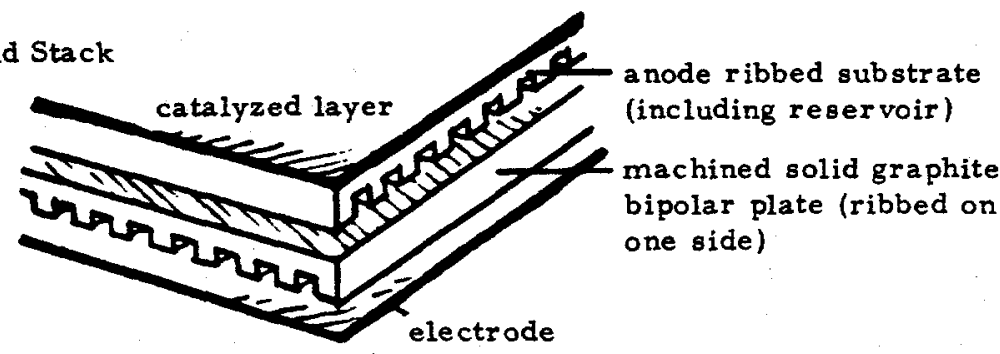

C: Alternative Configuration

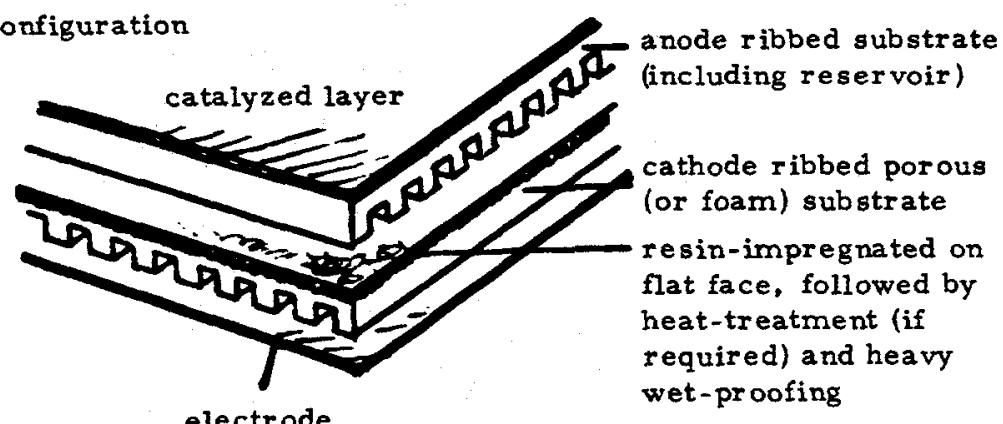

D: Kureha KES-1 Integrated Electrode Substrate

ribbed electrode
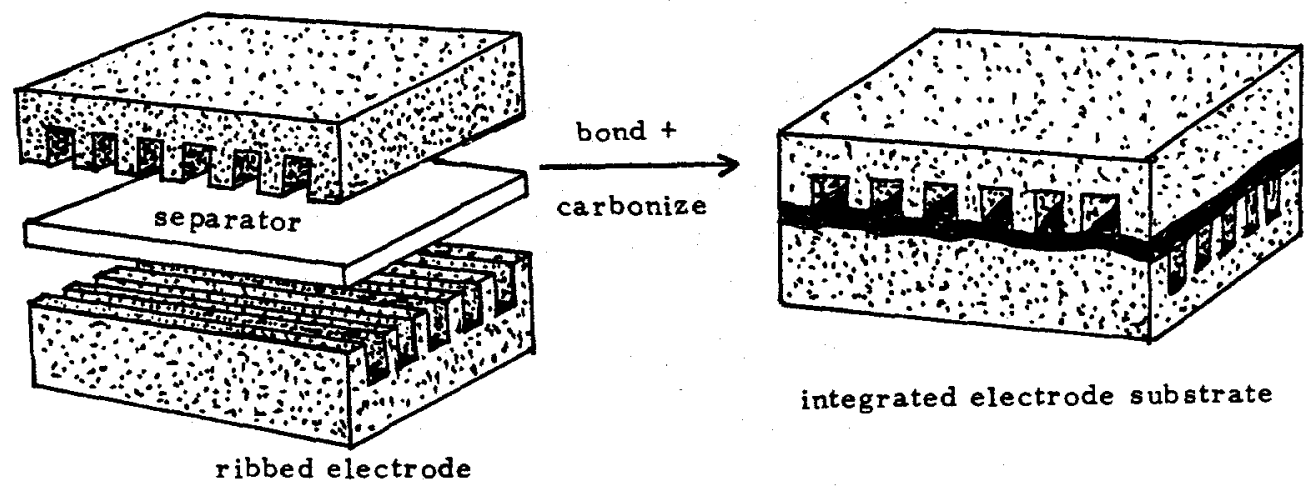

integrated electrode substrate

NOTE: All electrodes are catalyzed carbon paper or graphite cloth layers.

Fig. 2.13-4. Stack component alternatives are shown. 
if the correct resin is chosen, it need not even be carbonized. Electronic contact will be no problem since the internal structure will remain conducting.

Concept $B$ is the Toshiba 76 hybrid stack and combines features of both the conventional and ribbed substrate stacks of Fig. 2.10-1. The bipolar plate is ribbed on the cathode side and flat on the anode side of the adjacent cell. The cathode ribs are in contact with a conventional cathode on a graphite paper or cloth and no reservoir exists on that side. The anode, on the other hand, has a non-wetproofed or partially-wetproofed ribbed substrate to provide an electrolyte reservoir. While Toshiba's concept may have been developed in order to find a structure which would not infringe on UTC's ribbed substrate stack patent, 77 experience has shown that a reservoir in a ribbed substrate at the cathode side may not be desirable. When it is filled to a reasonable extent, electrolyte can be pumped during operation by electroendosmosis through the cell to the anode receiver, which then floods and causes high anode polarization. This problem is discussed in greater detail in Sec. 2.14.

Toshiba's structure is attractive because the anode substrate can act as an effective reservoir (hydrogen-diffusion polarization is not appreciable for a reasonable acid fill). Furthermore, it is in contact with a barely corrosive environment and hence does not need to be graphitized and is only carbonized at $900^{\circ} \mathrm{C}$. The bipolar plate should be easy to mold, bake out and graphitize but, since it is ribbed on one side only, it may suffer from flatness problems. Like the UTC ribbed substrate stack, it contains only three components, whereas the Engelhard ABA stack has four. This fact should reduce the ultimate cost.

One of UTC's ribbed-substrate stack components, the large flat graphite bipolar plate, is costly and difficult to manufacture. Its dimensional tolerances must be excellent, and it must be uniformly graphitized, which is difficult in $1-\mathrm{m}^{2}$ sizes. It is also fragile during handling. As a result, the reject rate for this item is not likely to be negligible. Configuration $C$ in Fig. 2.13-4 is a speculative suggestion, which combines the best features of the ABA system and of the Toshiba hybrid stack in a dimensionally tolerant structure, although surface finishing of the combined bipolar structure may be needed. The separate bipolar plate is replaced by a carbonized resin (or pure stable resin) layer between two ribbed substrates, one of which is a corrosion-resistant foam-type material on the cathode side with the flat face inwards. As noted, this component may be fully wetproofed. The other component is a UTC-type ribbed substrate carrying the anode layer, which need not be graphitized and is partially wetproofed to act as an electrolyte reservoir. This three-component system requires a minimum number of high-temperature manufacturing steps and should lead to low production investment and cost.

It is difficult to predict the total per $\mathrm{kW}$ cost of an FC stack as a function of cumulative production, since component technology and power density may change with time. However, ultimately, the installed cost of the stack should be about $20 \%$ of the installed initial plant cost. 35 For the mature system, manufactured cost and the selling price as paid by the utility will be very different, since the manufacturer will be making a profit to cover his development costs for early-model plants. For the early model plants high up on the learning curve, manufactured cost and selling price may be much closer, and the stack cost may be as high as $40 \%$ of manufactured cost or $30 \%$ of selling price. Therefore, it may be profitable for the developer to increase power density in initial stacks in order to reduce the proportional stack cost in early plants. This procedure will reduce the overall system selling price and thus help the early plants to reach the marketplace. Such a selling strategy would be perfectly reasonable, provided the system heat-rate is acceptable. Figures 2,4-1,2,4-2 and 2.6-2 show that the best strategy may be to aim for the lowe st cost unit with a heat rate $<8500 \mathrm{BTU} / \mathrm{kWh}$ in order to descend on the learning curve as rapidly as possible and quickly reach spontaneous market penetration cor responding to the right-hand side of Fig. 2.6-2. When this end of the market is satisfied, the strategy should change and emphasis should be on higher heat-rates to fill in the center and left side of Fig. 2.6-2, i.e., to broaden the market, which will be especially true if fuel costs rise and the economic effects of improved heat-rate become more important.

A reexamination of Fig. 2.6-1 shows that a 10\% improvement in heat-rate $(8300$ to 7500 BTU $/ \mathrm{kWh}$ ) is equivalent in total cost of electricity to a decrease in capital cost of $11 \%$ when fuel prices are $\$ 4 / 10^{6}$ BTU. At $\$ 6 / 10^{6}$ BTU, the corresponding equivalent decrease in capital cost is $16 \%$. Doubling the stack-power density involves a heat-rate penalty, but it would save about $10 \%$ of the capital cost in a mature plant. For situations which are capital-cost intensive (i.e. , cheap fuel), this trade-off may be useful. In fuel-cost-intensive situations, it would not be. The marketing strategy of the FC developer $B$ will have to take these factors into account, which will be much more important farther up the learning curve, where the $F C$ is effectively being sold for special uses when the capital cost to the utility is of primary importance. The developed might therefore build early systems using high power densities in order to reduce stack cost in an initial marketing strategy.

The Kureha Chemical Industry Co., Tokyo, Japan, has just announced (May 1985) the KES- 1 bipolar plate structure, for which patents are pending. It is an ABA system in which the porous ribbed plates are inverted so that the flat surfaces supporting the electrodes face the electrolyte, as in the UTC ribbed substrate stack (Fig. 2,10-1, Ref. 37). The Kureha system is shown in Fig. 2.13-4 as Configuration D. 
Another practical advantage of a high power-density system is that it will $r$ un at a slightly lower cathode potential. As we have seen in Sec. 2.11, this decrease reduces the rate of corrosion of the catalyst support and will therefore probably increase stack endurance. Guaranteeing endurance will be of prime importance with early stacks so that user acceptance and enthusiasm can be generated for the new technology, while confidence is gained and labor costs are reduced through hands-off operation. Finally, the developer has another trade-off option: that of increasing the catalyst loading as a function of current density. If electrodes of correct structure are used, the stack-power density can thereby be doubled at constant heat-rate for a total increase in catalyst cost of about $\$ 50 / \mathrm{kW}$ (based on producer price) but at a great saving in stackcomponent cost.

To study the effect of current density on unit cell potential at constant catalyst loading, a plot derived from recent UTC data on expectations for the 11-MW unit is shown in Fig. 2.13-5 (these and other data are available in Ref.78). The solid line shows the goal for a proposed commercial $0.98-\mathrm{m}^{2}$ cell-stack performance at $1000 \mathrm{~h}$. The data points shown for the particular stack (the first $0.98-\mathrm{m}^{2}$ short stack) are slightly below the goal; however (cf. Sec. 2, 14), more recently built stacks have performed at, or even higher than, this level. At $1000 \mathrm{~h}$, the target unit-cell potential is $0.76 \mathrm{~V}$ at $200 \mathrm{~A} / \mathrm{ft}^{2}\left(=216 \mathrm{~mA} / \mathrm{cm}^{2}\right)$; this current density is the present DoE/NASA/UTC system design point. Using this value as basis, we may assess the effect of current density and hence of cell-stack capital cost on system heat-rate. Assuming that the cellstack cost is $20 \%$ of installed system cost for a mature unit and $30 \%$ of installed cost for a unit well up the learning curve, the heat-rate as a function of total installed system cost is plotted for both cases in Fig. 2.13-6. The balance-of-plant cost for the fuel processor but not including the inverter, on a per $\mathrm{kW}$ basis, has also been corrected for system heat-rate, since the upstream equipment cost is inversely proportional to cell efficiency for a given output. The system heatrate is assumed to be proportional to the cell potential, and values have been changed proportionately from the estimate at $1000 \mathrm{~h}$ (corresponding to the cell potentials in Fig. 2. 13-5) to end-oflife values, assuming that other system improvements result in a $7730 \mathrm{BTU} / \mathrm{kWh}$ heat-rate at $216 \mathrm{~mA} / \mathrm{cm}^{2}$.

The illustrative capital-cost curves are based on $\$ 2,000 / \mathrm{kW}$ for the system fairly high up the learning curve in Figs. 2. 4-1 and 2.4-2 and $\$ 1,000 / \mathrm{kW}$ for a system that can be considered to be reasonably mature. The illustration in Fig. 2-13-6 demonstrates the wide range of heatrates that may become available by changing the current density from 400 to $50 \mathrm{~A} / \mathrm{ft}^{2}$, corresponding to values varying from $8640 \mathrm{BTU} / \mathrm{kWh}(39.5 \% \mathrm{HHV}$ efficiency) to $6830 \mathrm{BTU} / \mathrm{kWh}(50 \%$ HHV efficiency), assuming that cathode corrosion will not be a limiting problem. The large effect of heat-rate on capital cost for systems up the learning curve is also shown, together with the much smaller effect for mature systems.

Referring to the screening curve in Fig. 2.6-1, a $10 \%$ improvement in heat-rate for a mature unit is worth about a $10 \%$ increase in capital cost and vice versa. For more costly systems, the proportion of capital cost has a larger effect on the ultimate cost of electricity. A

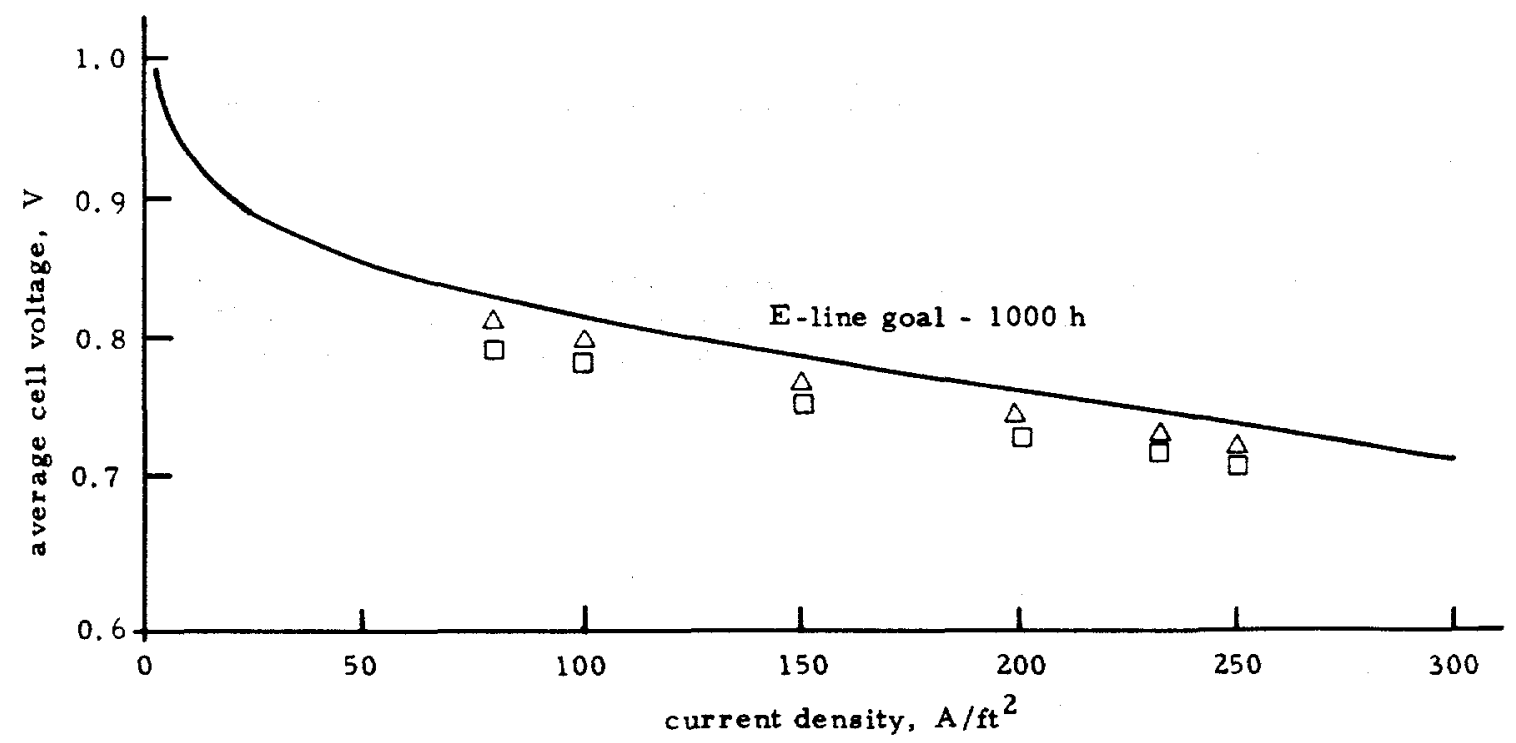

Fig. 2.13-5. The UTC cell performance (1982 specification) as a function of stack-current density; $500 \mathrm{~h}$ of test time, $\triangle ; 1600 \mathrm{~h}$ of test time, $\square ; 29$ cells were used, $10.8 \mathrm{ft}^{2}, 8 \mathrm{cells} /$ cooler, $120 \mathrm{psia}, 405^{\circ} \mathrm{F}$ average temperature. 


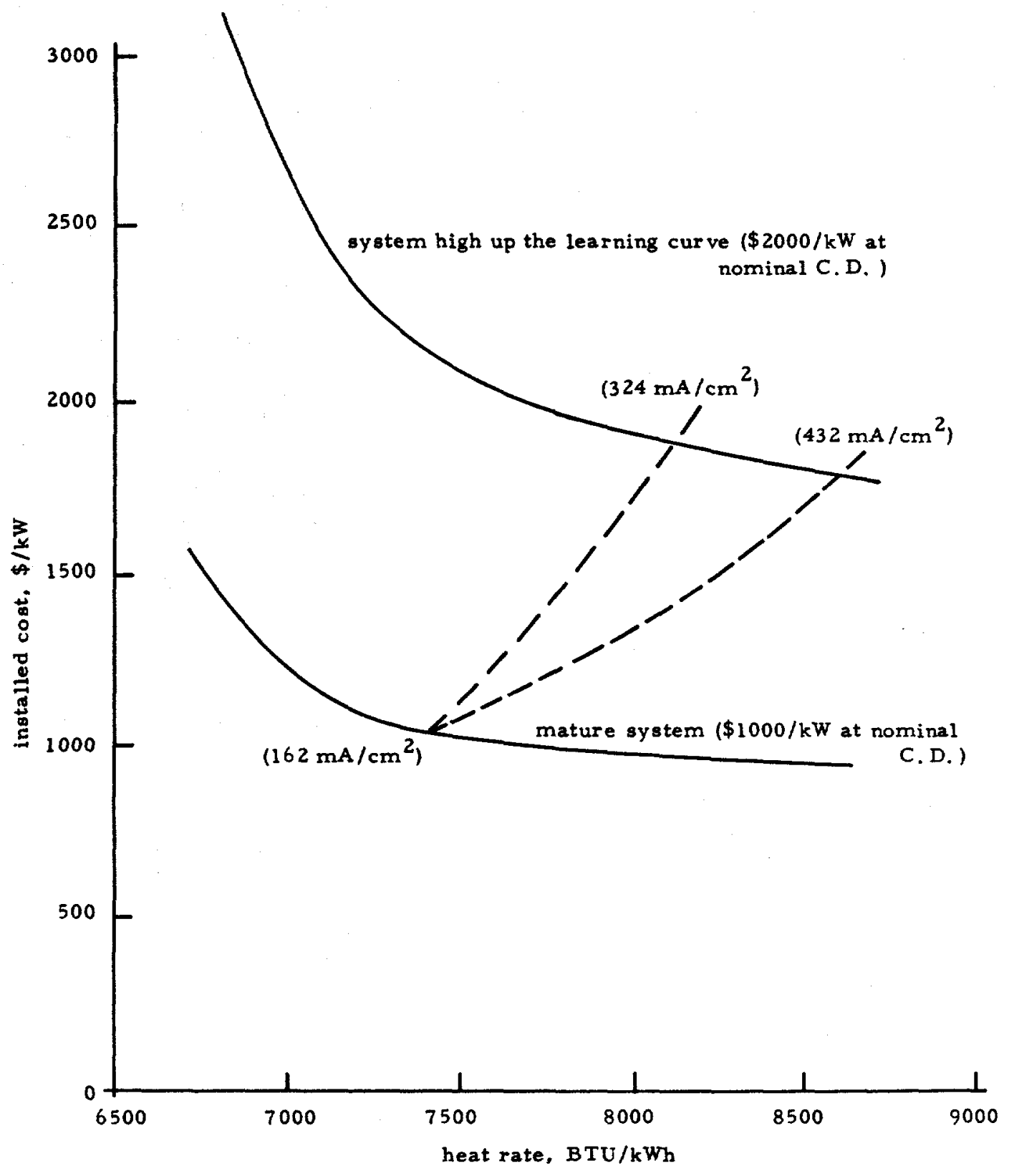

Fig. 2.13-6. Developer's commercialization planning: the capital cost is shown as a function of heat-rate for systems high up the lear ning curve and is compared to mature systems. 
developer might therefore introduce his first units at a heat-rate of $8640 \mathrm{BTU} / \mathrm{kWh}$ at the lowe st possible introductory capital cost (illustrated $28 \$ 1800 / \mathrm{kW}$ in Fig. $2.13-6$ ), since this is the most attractive economic value. As the developer comes down the learning curve, the effect of heat-rate will become progressively more important in terms of market economics (Fig. 2.6-2), and the capital-cost vs heat-rate locus might be one of the dotted lines on Fig. 2.13-6, which shows the system moving to an ultimate heat-rate of about $7400 \mathrm{BTU} / \mathrm{kWh}$ at a current density of $162 \mathrm{~mA} / \mathrm{cm}^{2}\left(150 \mathrm{~A} / \mathrm{ft}^{2}\right)$, assuming that no improvements in catalysis have occurred. One of the line 8 is based on the assumption that early units will be $r$ un at $432 \mathrm{~mA} / \mathrm{cm}^{2}$ and about 8600 BTU/ $\mathrm{kWh}$, the other refers to $324 \mathrm{~mA} / \mathrm{cm}^{2}$ and about $8100 \mathrm{BTU} / \mathrm{kWh}$. In practice, the latter may be preferred since, in an 11-MW design of FCG-1 type, 25, 27 it will lead to a reduction of the number of stacks from 18 to 12, which will interface better with the DC/AC inverter than a 9-stack system, in the opinion of the author and not necessarily of the developers.

We stress again that successive materials improvements must be put in place to allow the cell to operate to an end-of-life voltage of $0.76-0.77 \mathrm{~V}$ and achieve the ultimate $7400 \mathrm{BTU} /$ kWh heat-rate. Finally, we envisage further materials improvements that will allow the use of higher temperatures and pressures, thus further improving capital costs, especially if improved catalysts are found that allow higher efficiencies. The result will be a broader field of applications, including transportation use of methanol as fuel provided fossil fuels remain available at reasonable cost.

The curve shown in Fig. 2.13-6, even though it was obtained on a different basis, is consistent with that of Figs. 2.4-1 and 2.4-2. High up the learning curve, the FC will first be aimed at the specialty cogeneration market, where it will be used at high annual utilization (capacity factor) so that its high capital cost is amortized over a maximum number of hours per year and over the cost of both the electricity and heat produced. For this market, the highe st electrical efficiency is not necessary. As the FC system matures and reaches about $\$ 1500 / \mathrm{kW}$, it will break into the broad cogeneration market predicted in Ref. 2 at a heat-rate of 8300 BTU $/$ $\mathrm{kWh}$. Finally, it will reach the stand-alone electric utility market for a unit costing less than $\$ 1000 / \mathrm{kW}$ that is required in the load-following mode with about a $10-25 \%$ annual capacity factor, as shown by EMA in a successor report, ${ }^{79}$ which updates the previous study (Ref, 3 ), although the essential conclusions remain the same. Utilization of the system over a relatively small number of hours per year is not consistent with cogeneration and, therefore, operation at the highest possible electrical efficiency will become desirable. A heat rate of $7400 \mathrm{BTU} / \mathrm{kWh}$, as shown in Fig. 2.13-6, will be a good target; under these conditions, no steam will be available for cogeneration; Fig. 2.6-2 shows that the market will be large for a dedicated electric utility cell with these specifications.

\section{14 The Current State of PAFC Technology; Performance Life}

The preceding discussion of projected PAFC performance is based on the performance of laboratory cells or on mathematical models derived from laboratory-cell data. We now review the state of the art, with emphasis on current full-scale cell performance characteristics as a function of lifetime. These data are required for potential customers to provide confidence in developer specifications, especially those involving cell voltage (i.e., system efficiency) as a function of the lifetime of the FC stack. Lifetimes of the active elements (cell components) in the FC generator are clearly a major factor in determining the overall economic viability of the FC system. As an example, we consider the case of an installed replacement cell-stack cost equal to $40 \%$ of the total system cost, with a ten-year balance-of-system lifetime. These assumptions are reasonable if we consider stack-manufacturing costs to be $20 \%$ of installed system cost. If a 10 -y system life is assumed to have $20 \%$ annual carrying charges, then stack replacement every 5 y would carry total proportional charges of $33 \%$ of the total system charges, which may be affordable, whereas replacement every 2.5 y would raise this cost considerably, particularly when labor costs and contingencies, such as spare stacks and installation funds, are included. Reliable cell-stack life, including cooling systems and associated peripherals, will therefore be of major importance.

Here, we consider only the electrochemical aspects of stack life as a function of time, since statistical analyses of imponderable performance-loss modes (for example, catastrophic cooling-system failures) are at present not known because the number of stacks tested for a sufficiently large number of hours is too small. It should, however, be noted that the number of total failures of stacks of reasonably mature technology has so far been zero. Thus, whereas the scientific limitations which determine the life of electrochemical cells are reasonably well known, lives for the various subsystems are much less well defined, and the se will consequently have to be overengineered in early units to provide required reliability. All subsystems, including the water-feed system for pressurized water-cooled stacks, will require special attention, particularly the use of multiple, redundant, computer-controlled sensors and controls, which may be progressively eliminated as experience is gained with the system as a whole. To make the dispersed FC a truly economical electrical generator, a stand-alone system is needed that requires only a nnual maintenance. Achievement of this design goal will take considerable development time. 
Compared with the peripheral auxiliaries, the electrochemical system is more forgiving because it contains a very large number of elements in parallel arrays. Unfortunately, systems considerations require that a large number (about 400-450) of cells must be in series. A loss in performance of one of these cells will be tolerable, provided that this cell allows electricity to pass after failure. Unfortunately, cells are designed to eliminate the possibility of electronic short circuit so that the failure of any cell as an ionic conductor will result in failure of the entire cell stack. Furthermore, since it is almost certain that it will not be worthwhile to rebuild failed stacks (instead, these will only be used for the recovery of scrap catalyst), the loss of a single cell may prove to be an economic disaster. To a void cell failure, careful attention must be paid to electrolyte distribution within the stack in order to prevent cells becoming star ved of electrolyte, for example by osmotic pumping effects of the type observed in early experimental MCFC stacks. $80,+$

Since it seems almost impossible to design cells which will have a sufficiently large electrolyte reser voir to contain all of the electrolyte necessary to compensate for evaporation and other losses over the entire stack lifetime, an electrolyte-addition system must be designed to distribute (for example, by capillary action) electrolyte uniformly. Means are needed to ensure occurrence of anode and cathode cell reactions. This goal implies hydrogen oxidation and oxygen reduction, rather than cathode-support corrosion and hydrogen-evolution processes in cells lacking gaseous reactants or appropriate catalysts. The production line for cell components must ensure proper quality control for the catalyst layers, including uniformity in cell construction. The matrix layers must also not allow development of electronic shorting. Reacting gases must be properly supplied to the cell stack, and blockage of gas channels must, therefore, be a low-probability event.

Uniformity of the cathode-catalyst layer must be ensured in order to prevent problems of the type described in Sec. 2.11, which cause some areas of the cathode-current collectors to be effectively at open circuit in some structures. 45 Edge-seal problems may become important in the designs of practical stacks. The use of materials that cause poisoning of electrocatalytic processes at the anode and cathode should be avoided. At the cathode, ferric chloride complexes have been found as a poison. 46 Therefore, the use of materials containing traces of iron or substances that may leach chloride ion (e.g. . Viton) should be a voided.

Some idea of the degree of confidence of developers in meeting performance goals may be obtained from recent data of UTC, WE and other developers. Figure 2.14-1 shows a plot of the performance of the DoE/NASA/UTC $0.34-\mathrm{m}^{2}, 20$-cell stack No. 39488-1 (about 1982). The conditions of operation are those of the FCG-1 $\left(205^{\circ} \mathrm{C}, 8.2 \mathrm{~atm}\right) .25,27$ The solid line is the UTC E-line. This E-line performance is established as a guideline based on overpotential measurements of electrodes under utility conditions, at the correct local gas compositions and cell temperatures, as a function of time and corrected for the measured internal resistance of a typical cell structure. The E-line has a potential at a current density of $232 \mathrm{~A} / \mathrm{ft}^{2}\left(250 \mathrm{~mA} / \mathrm{cm}^{2}\right)$ at $1000 \mathrm{~h}$, corresponding to the value used on the plot in Fig. 2.13-5. As indicated in Sec. 2.14, the E-line will descend to a design standard potential of $0.73 \mathrm{~V}$ at $40,000 \mathrm{~h}$. As discussed in Sec. 2.12, the decay of voltage is proportional to the logarithm of time. The results shown were obtained on a heat-treated Pt alloy cathode catalyst with corrosion-resistant support (Code GSB15). The results fall consistently below the E-line by about $10 \mathrm{mV}$, which may be attributed to somewhat high contact resistances. The performance as a whole is acceptable, with little dispersion between cells. The deviation of measured performance from expected values corresponds to only a $1.4 \%$ increase in overall system heat-rate $\left(i_{.} e ., \sim 8400 \mathrm{BTU} / \mathrm{kWh}\right.$ at the end of life rather than the expected value of $8300 \mathrm{BTU} / \mathrm{kWh}$ ). This stack was $\mathrm{r}$ un for $7000 \mathrm{~h}$.

Figure 2.14-2 shows the corresponding plot for the next NASA/DoE electric utility stack (39569), using the same catalysts under exactly the same temperature and pressure conditions as Fig. 2. 14-1. Some important differences should be noted between the two plots. Most importantly, the E-line has been raised from $0.74 \mathrm{~V}$ at $1000 \mathrm{~h}$ to $0.76 \mathrm{~V}$; this difference corresponds to a current-density reduction from $250 \mathrm{~mA} / \mathrm{cm}^{2}$ to $216 \mathrm{~mA} / \mathrm{cm}^{2}\left(200 \mathrm{~A} / \mathrm{ft}^{2}\right)(\mathrm{see}$ Fig. 2.13-5). This result indicates data revision by UTC, so that a terminal voltage of $0.73 \mathrm{~V}$ can be achieved after $40,000 \mathrm{~h}$. Furthermore, the cells in Fig. 2. 14-2 registered only an average potential of $0.725 \mathrm{~V}$ at $1000 \mathrm{~h}$ and $216 \mathrm{~mA} / \mathrm{cm}^{2}$, which would be equivalent to $\sim 0.705 \mathrm{~V}$ at $250 \mathrm{~mA} / \mathrm{cm}^{2}$. This value is $35 \mathrm{mV}$ below the earlier datum shown in Fig. 2,14-1. The initial rate of decay of the cells in Fig. 2. 14-2 is also high: about $8 \mathrm{mV} / 1000 \mathrm{~h}$ between 1000 and $2000 \mathrm{~h}$ of operation, compared with about $3 \mathrm{mV} / 1000 \mathrm{~h}$ in Fig. 2.14-1. Actually, the tangential linear decay rates are inaccurate but nevertheless used by developers in making comparisons.

There are clearly some differences between the components used in Figs. 2. 14-1 and 2.14-2. The relatively high decay rate in Fig. 2.14-2 suggests some catalyst differences. It is instructive to compare Fig. 2.14-2 with Fig. 2.12-14, which shows the decay behavior of a PtCr alloy catalyst. Inspection shows that the decays are similar; it is interesting to note that the decays are close to those predicted for the New York 4.5-MW demonstrator stacks, which used untreated Vulcan XC-72 cathode supports. Since the cells in Fig. 2,14-2 contain the same alloy

\footnotetext{
These statements and the following are the author's opinion.
} 


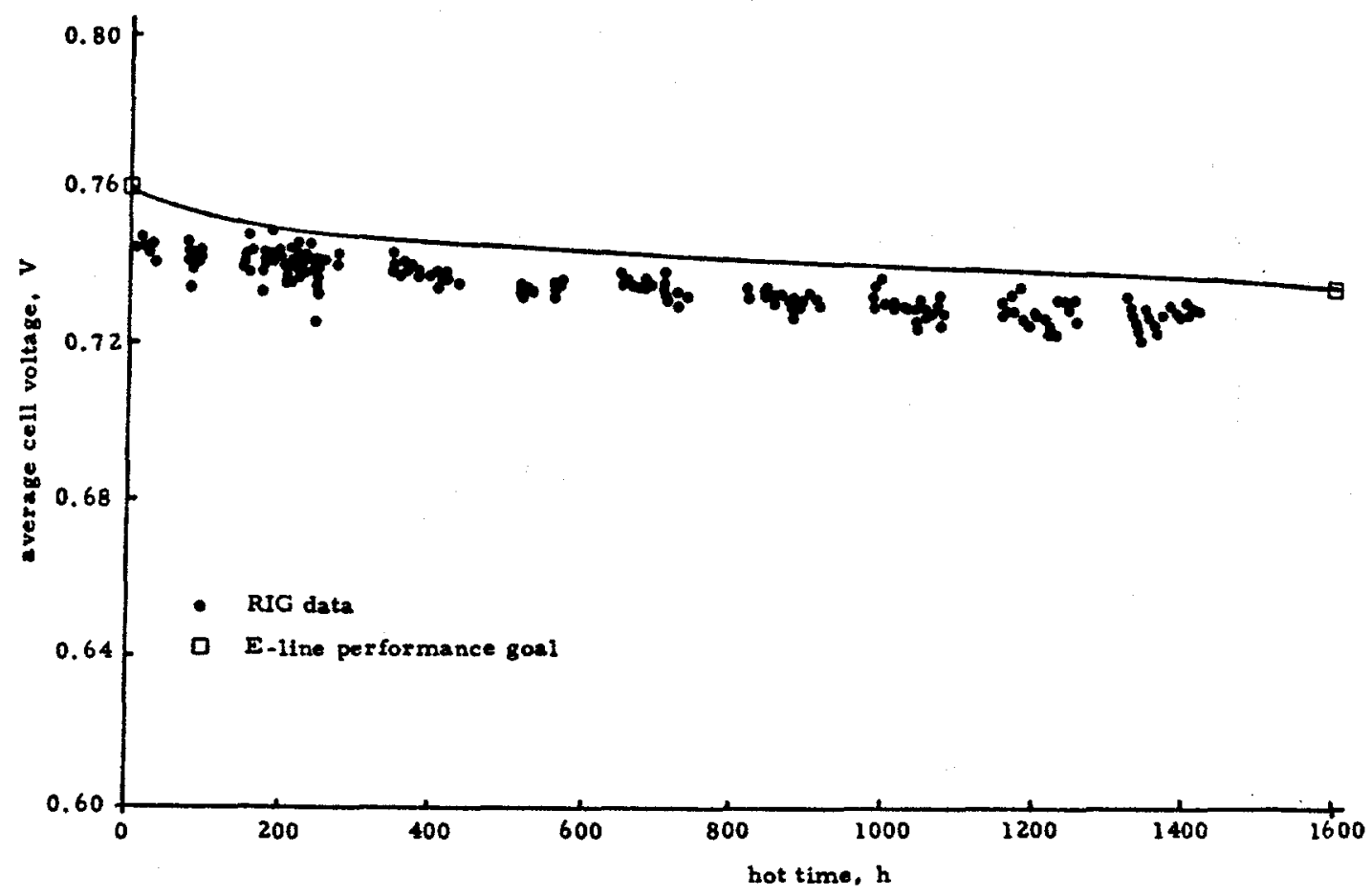

Fig. 2. 14-1. Performance history of a 1982 DoE/NASA/UTC $0.34-\mathrm{m}^{2}$ stack; $\mathrm{p}=120 \mathrm{psia}, \mathrm{T}=466^{\circ} \mathrm{F}, 232 \mathrm{~mA} / \mathrm{ft}^{2}$.

as those in Fig. 2.14-1, they should possess an initial activity about 15-30 mV higher than pure Pt, depending on whether or not the Pt has been heat-treated. It thus appears that the cell stack of Fig. 2.14-2 has been constructed in such a way that about $50 \mathrm{mV}$ was lost in real activity compared with that of Fig. 2. 14-2.

Both stacks were constructed with ribbed porous substrates that contained sufficient reservoir capacity to store electrolyte for a hot lifetime of $40,000 \mathrm{~h}$ or more. A possible explanation for the lower overall performance may have been diffusion losses, particularly at the anode. 78 The problem involved was seen in an early stack (39486-1) and resulted from electroosmotic pumping under load, so that the electrolyte stored in the cathode reservoir is transferred to the anode, which results in drying out of the former and flooding of the latter. If this occurs, the cure appears to be to abandon the reservoir on the cathode side completely (see Fig. 2. 13-4). Stacks may then require provision for electrolyte replenishment, particularly for gas-utility on-site systems at atmospheric pressure; for these, the high gas-volume throughput will probably cause evaporation losses requiring annual replenishment.

However, hydrogen gains with the 39569 stack were apparently not high, indicating that little electrolyte redistribution actually occurred. Aside from this explanation, the only reason for the relatively low stack performance can be attributed to an inferior batch of electrodes and/ or catalyst. In spite of its low performance, the experiment with this electric utility stack was nevertheless highly successful. The stack was a run for $16,000 \mathrm{~h}$ with 38 thermal cycles. At the end of the test, the stack performance fell $74 \mathrm{kmV}$ below the new E-line value (Fig. 2.14-3, which is reproduced from Ref, 78); this performance decay was properly predicted from singlecell tests and the theoretical logarithmic relation.

UTC next built and tested stacks containing the $0.98-\mathrm{m}^{2}$ components. Some early results on the fir st stack are shown in Fig. 2. 14-4. Performance at $1500 \mathrm{~h}$ is a few $\mathrm{mV}$ better than for the Fig. 2. 14-3 stack (0.725 V), and the decay rate is also somewhat improved. The performance of a later $0.98-\mathrm{m}^{2}$ stack (Figs. 2, 14-4 and 2.14-5) is even more impressive: the voltage is several $\mathrm{mV}$ higher and corresponds closely to $\mathrm{E}$-line values, while decay is much lower than in Fig. 2.14-3 (about 3-4 mV/1000 h).

Performance tests in large stacks require scale-up not only of components but also of preparation and manufacturing procedures. They are not representative of the latest technology. 


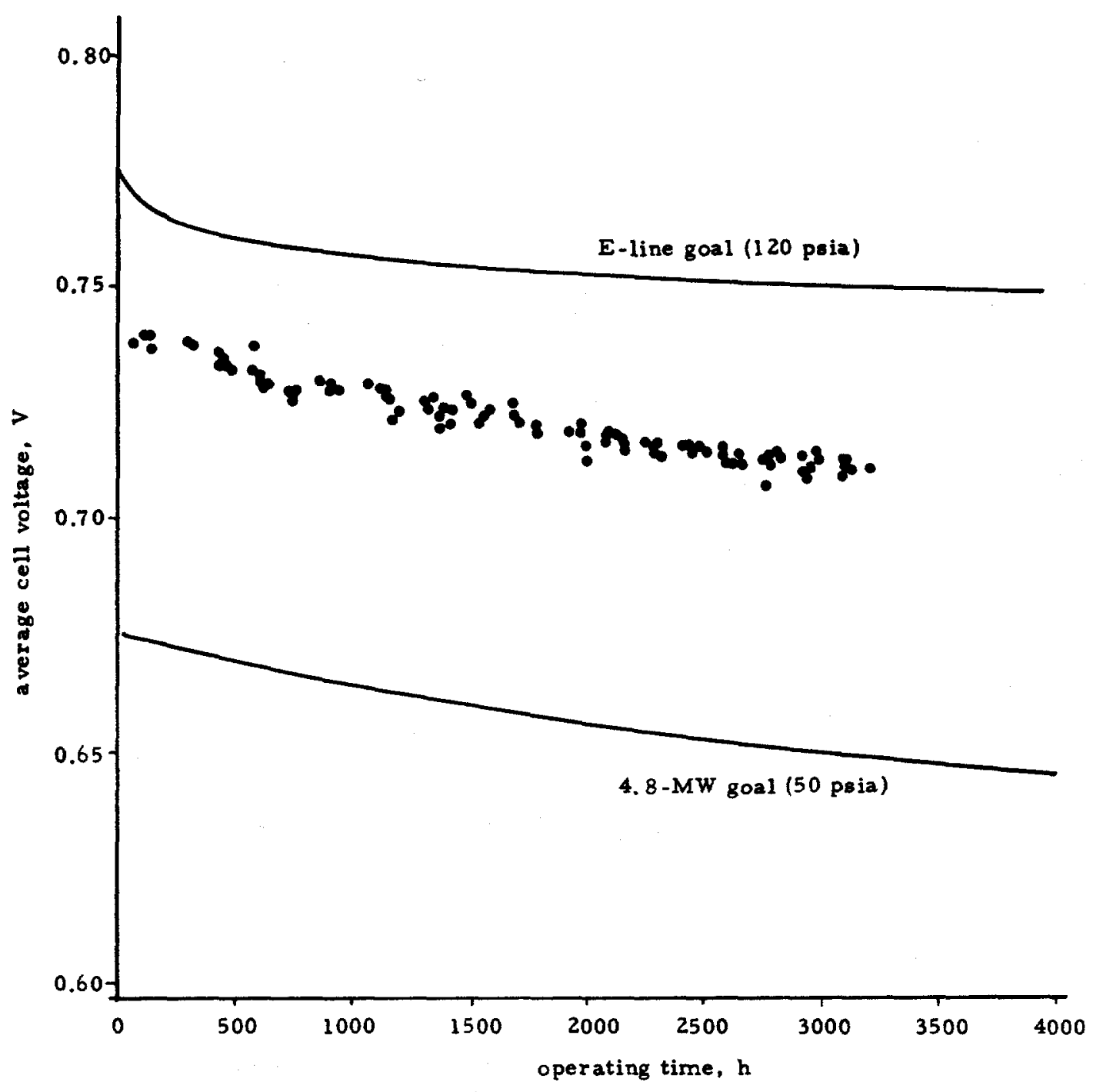

Fig. 2. 14-2. Performance history for a 1982-83 DoE/NASA/UTC $0.34-\mathrm{m}^{2}$ electric utility stack (to $3000 \mathrm{~h}$ ); 30 cells, $3.7 \mathrm{ft}^{2}, 8$ cells/ cooler, $120 \mathrm{psia}, 405^{\circ} \mathrm{F}$ a verage temperature, $200 \mathrm{~A} / \mathrm{ft}^{2}$. $85 / 70$ reactant utilization.

Some data are shown in Figs. 2. 14-6 and 2.14-7. Figure 2.14-6 indicates the difference between cathode-catalyst-layer preparations of the DoE/NASA/UTC traditional wet-mix electrodes using deflocculated colloidal teflon 81 and a new technique with a dry-mix teflon, which allows loadings to be increased from 0.5 to $0.9 \mathrm{mg} / \mathrm{cm}^{2}$, with at least the theoretical $23-\mathrm{mV}$ increase in performance and improved stability at an additional $P t$ cost of about $\$ 40 / \mathrm{kW}$. The dry-mix technique results in electrodes of better structure and higher performance; at $4500 \mathrm{~h}$, the results are about $15 \mathrm{mV}$ above the E-line. The results in Figs. 2.14-6 and 2.14-7 were obtained with a proprietary catalyst (GSB-18), which is presumably a Pt alloy on a heat-treated, corrosionresistant support. Later results have been obtained on a catalyst code-named GSB-26. With standard wet-mix electrodes, this catalyst yields higher initial performance than GSB-18, as is shown in Fig. 2.14-8. Other cells are currently operating up to $18 \mathrm{mV}$ above the E-line (cf. the example of Fig. 2.14-9).

Perhaps the most remarkable result is shown in Fig. 2. 14-10, which refers to a cell of advanced structure at a cathode-catalyst loading equivalent to $0.9 \mathrm{mg} / \mathrm{cm}^{2}$; it operates at a current density of $432 \mathrm{~mA} / \mathrm{cm}^{2}$ (almost $50 \mathrm{mV}$ over the calculated E-line for this current density), with the voltage close to the previously obtained voltage for $216 \mathrm{~mA} / \mathrm{cm}^{2}$. Thus, the strategy out lined in Sec. 2. 13 for stack-cost reduction may be pursued by UTC. A similar advanced-structure stack is intended as a prototype for the $200-\mathrm{kW}$ on-site atmospheric pressure unit and is currently operating about $25 \mathrm{mV}$ above the $\mathrm{E}$-line for that system (Fig. 2.14-11), at a potential corresponding to UTC's development goal. A notable characteristic of this stack is an increase in cell potentials over the first few hundred hours of operation, as the highly wet-proofed electrodes develop the correct fill of electrolyte. This behavior is normal. 


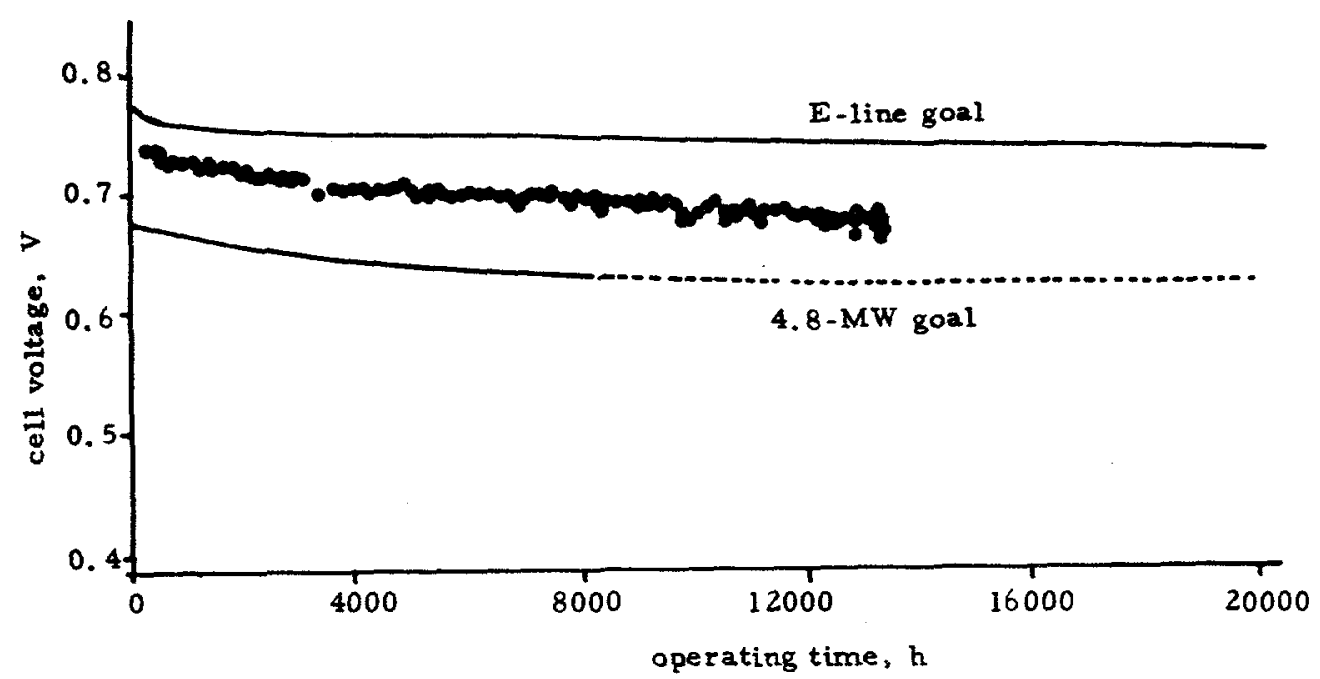

Fig. 2-14-3. As in Fig. 2.14-2, to $13,500 \mathrm{~h}$ (the test was terminated in midJanuary, 1985 , at $16,000 \mathrm{~h}$ ); 30 cells, $3.7 \mathrm{ft}^{2}, 8$ cells $/$ cooler, 120 psia, $405^{\circ} \mathrm{F}$ average temperature, $200 \mathrm{~A} / \mathrm{ft}^{2}, 85 / 70$ reactant utilization.

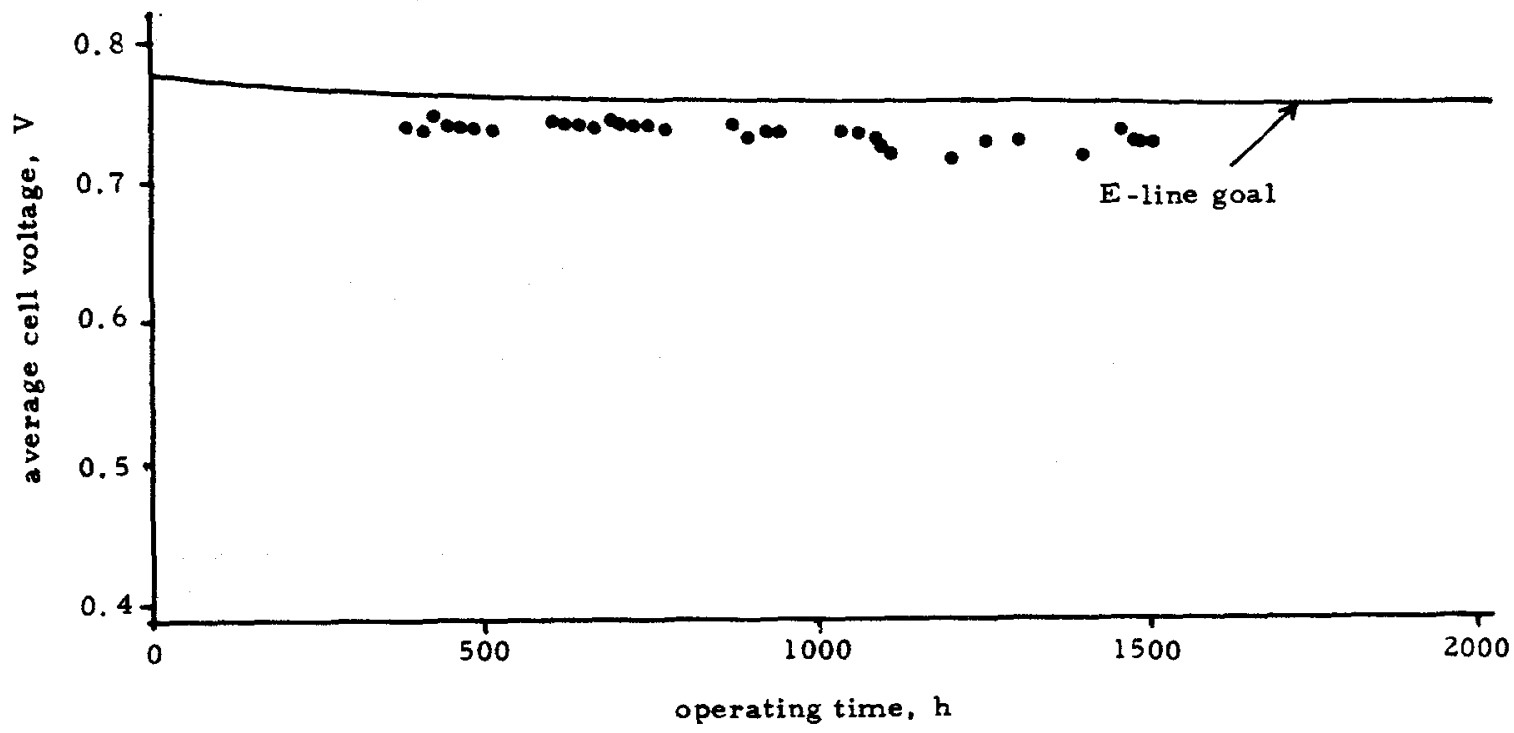

Fig. 2. 14-4. The first DoE/NASA/UTC $0.98-\mathrm{m}^{2}$ short-stack test; 20 cells, $10.5 \mathrm{ft}^{2}, 8 \mathrm{cells} /$ cooler, $120 \mathrm{psi}, 405^{\circ} \mathrm{F}$ average temperature, $200 \mathrm{~A} / \mathrm{ft}^{2}, 85 / 70$ reactant utilization. 
A

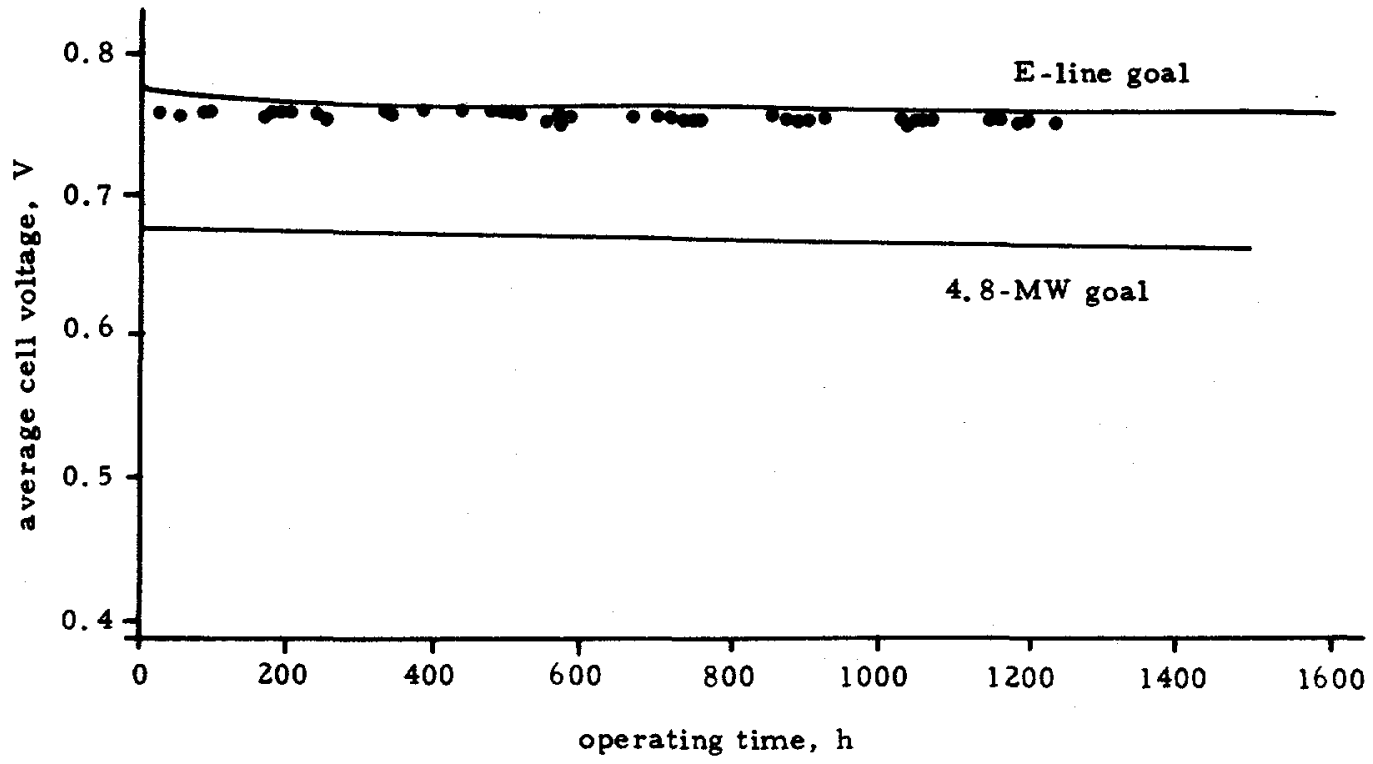

B

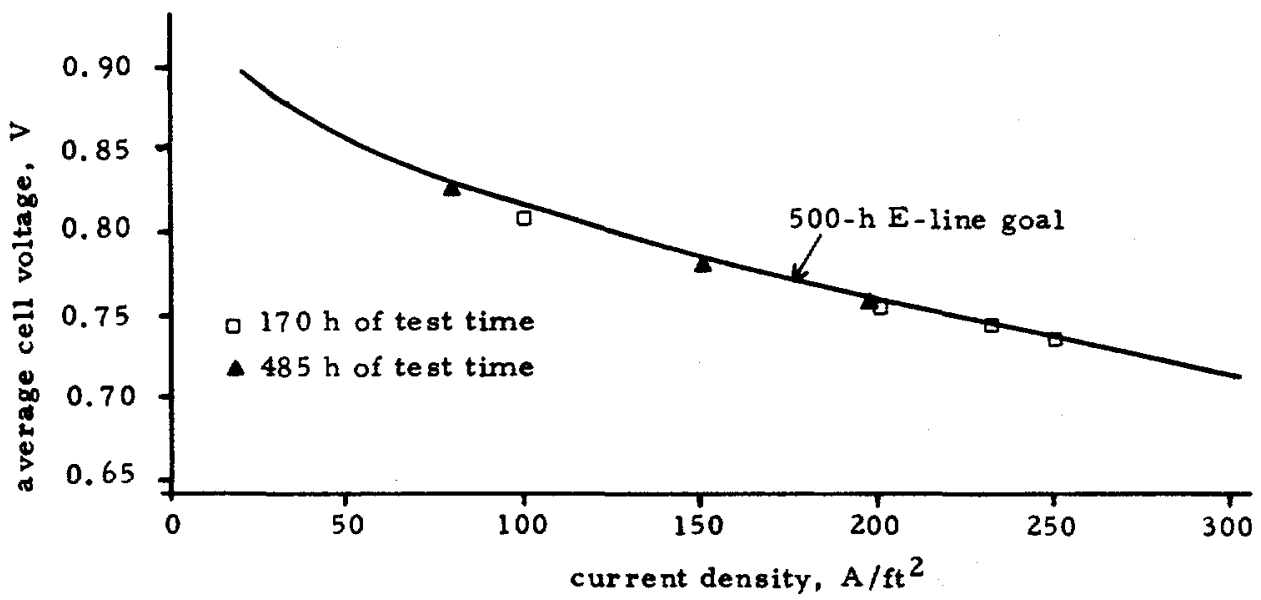

Fig. 2.14-5. A - Further DoE/NASA/UTC 0.98-m² short-stack tests, which show improvements compared to Fig. $2.14-4 ; 28$ cells, $10.3 \mathrm{ft}^{2}, 7$ cells/ cooler, $120 \mathrm{psia}, 405^{\circ} \mathrm{F}$ average temperature, $200 \mathrm{~A} / \mathrm{ft}^{2}, 85 / 70$ reactant utilization. B - Performance as a function of current density for the second $0.98-\mathrm{m}^{2}$ DoE/NASA/UTC short stack; 28 cells, $10.3 \mathrm{ft}^{2}, 7$ cells/ cooler, $120 \mathrm{pgia}, 405^{\circ} \mathrm{F}$ average temperature, $85 / 70$ reactant utilization. 

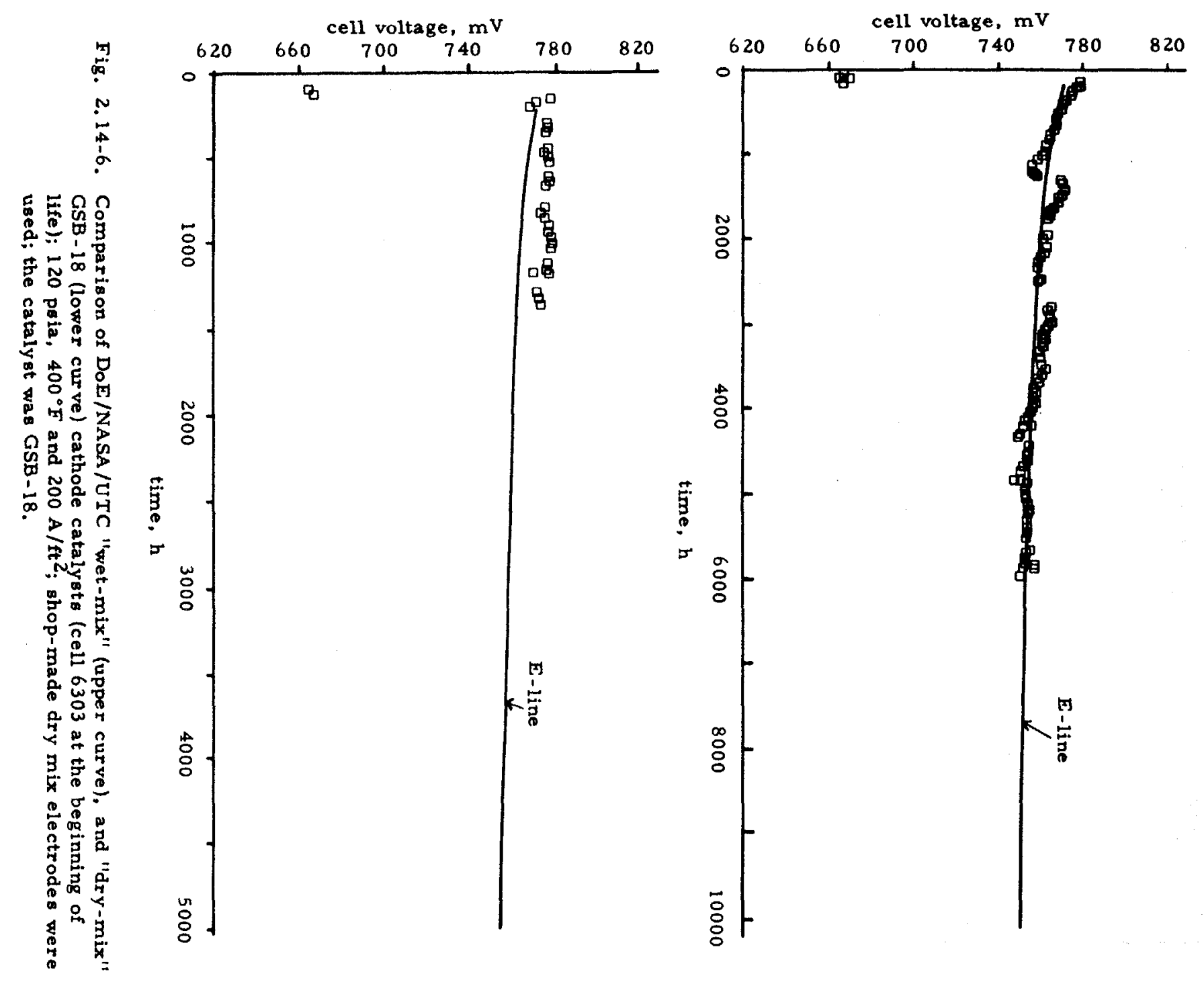


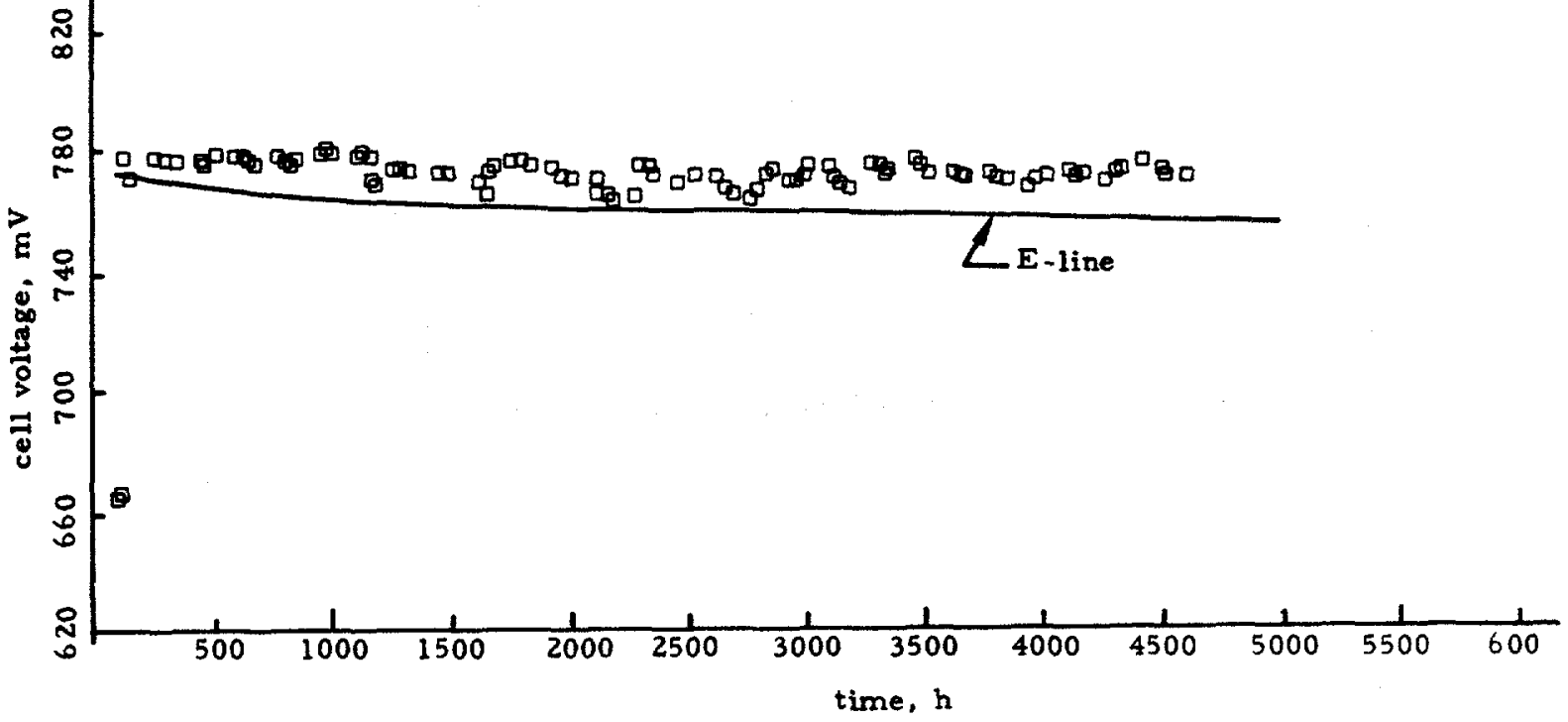

Fig. 2.14-7. Voltage vs time for the DoE/NASA/UTC "dry-mix" cell (6303) after $4500 \mathrm{~h} ; 120 \mathrm{psia}, 400^{\circ} \mathrm{F}$, and $200 \mathrm{~A} / \mathrm{ft}^{2}$. This cell had a $0.9 \mathrm{mg} / \mathrm{cm}^{2}$ platinum-equivalent cathode catalyst.

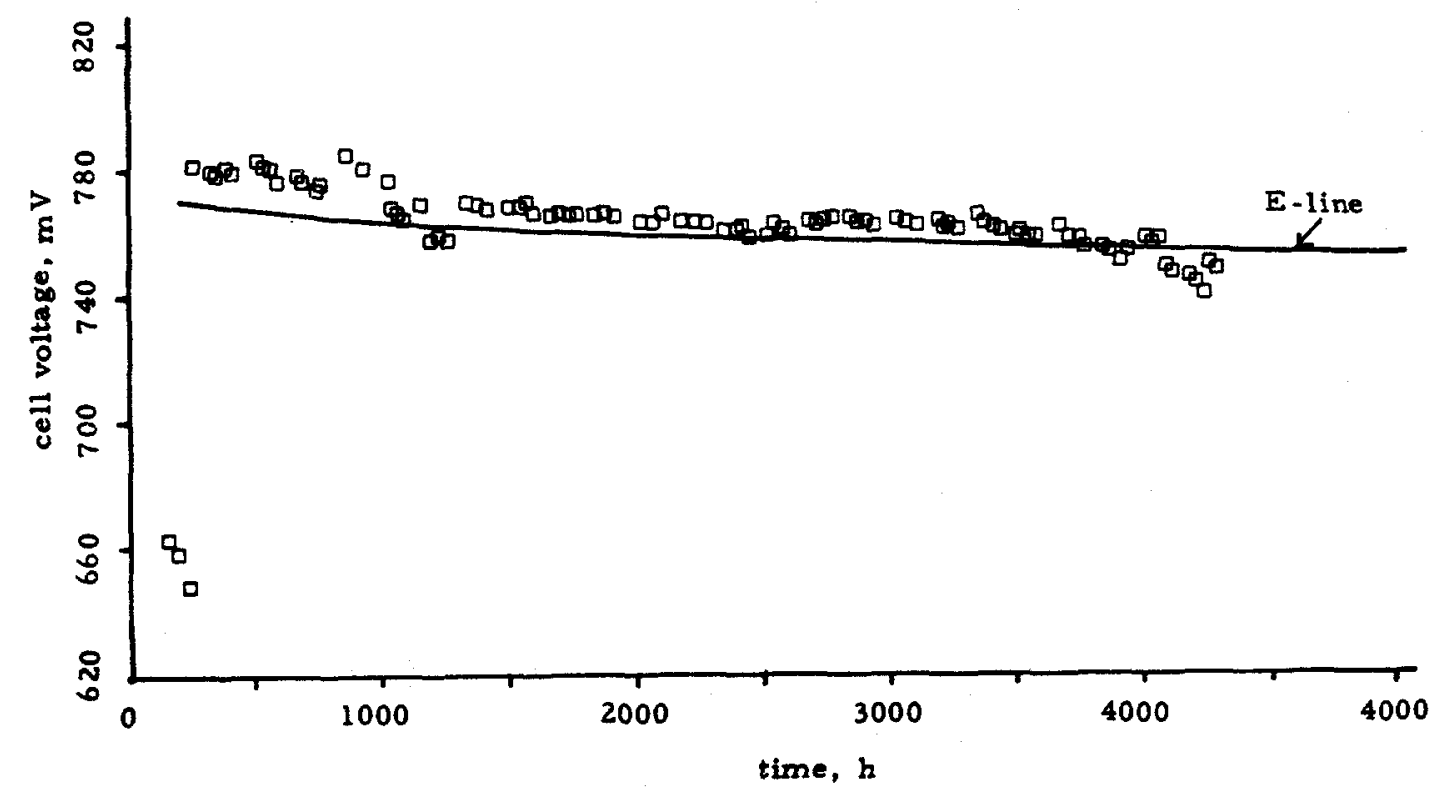

Fig. 2, 14-8. Voltage vs time for the DoE/NASA/UTC "wet-mix" GSB-26 catalyat cell at $4000 \mathrm{~h} ; 120 \mathrm{psia}, 400^{\circ} \mathrm{F}, 200 \mathrm{~A} / \mathrm{ft}^{2}$; shop-made dry mix electrodes were used. 

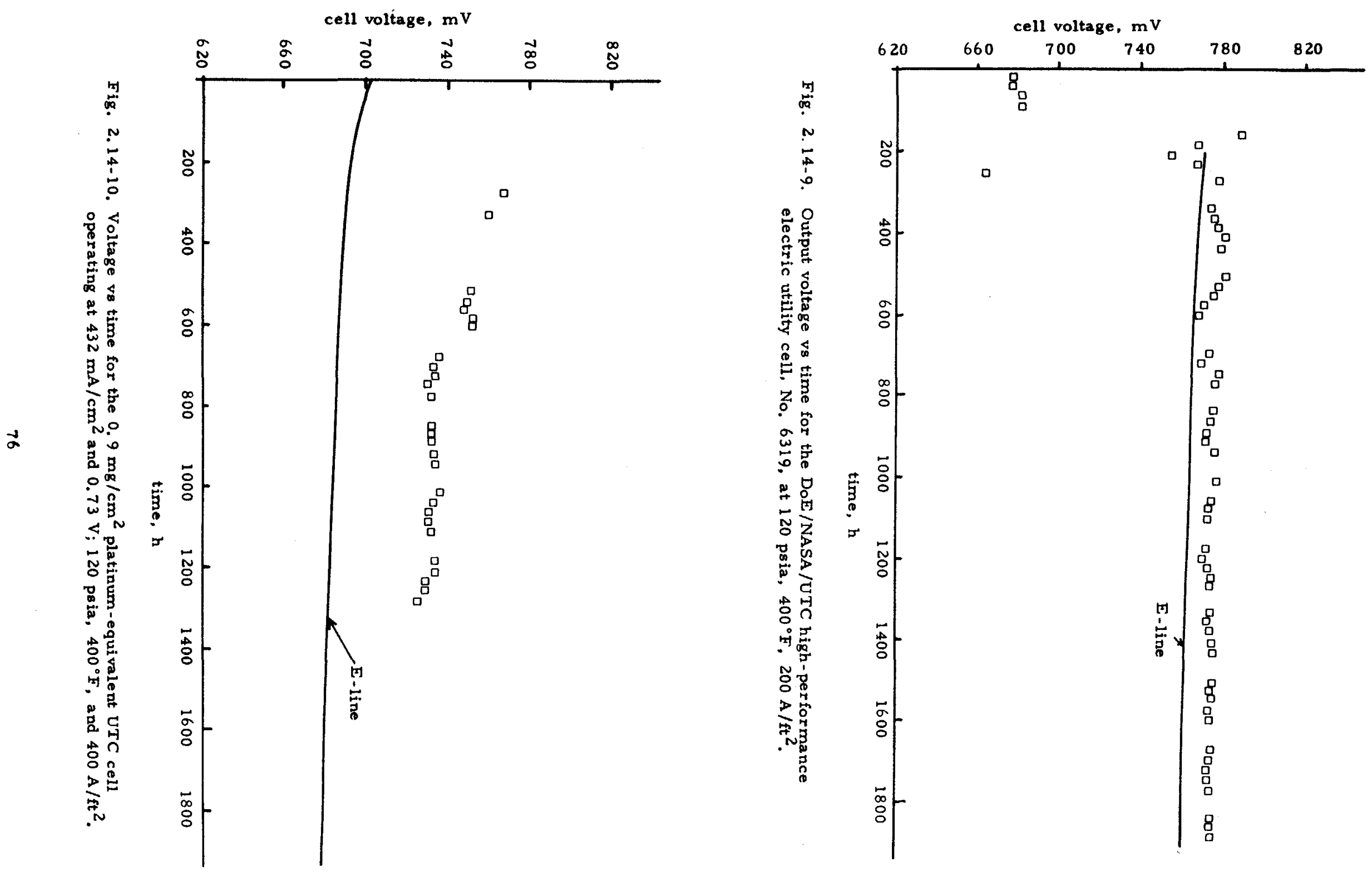


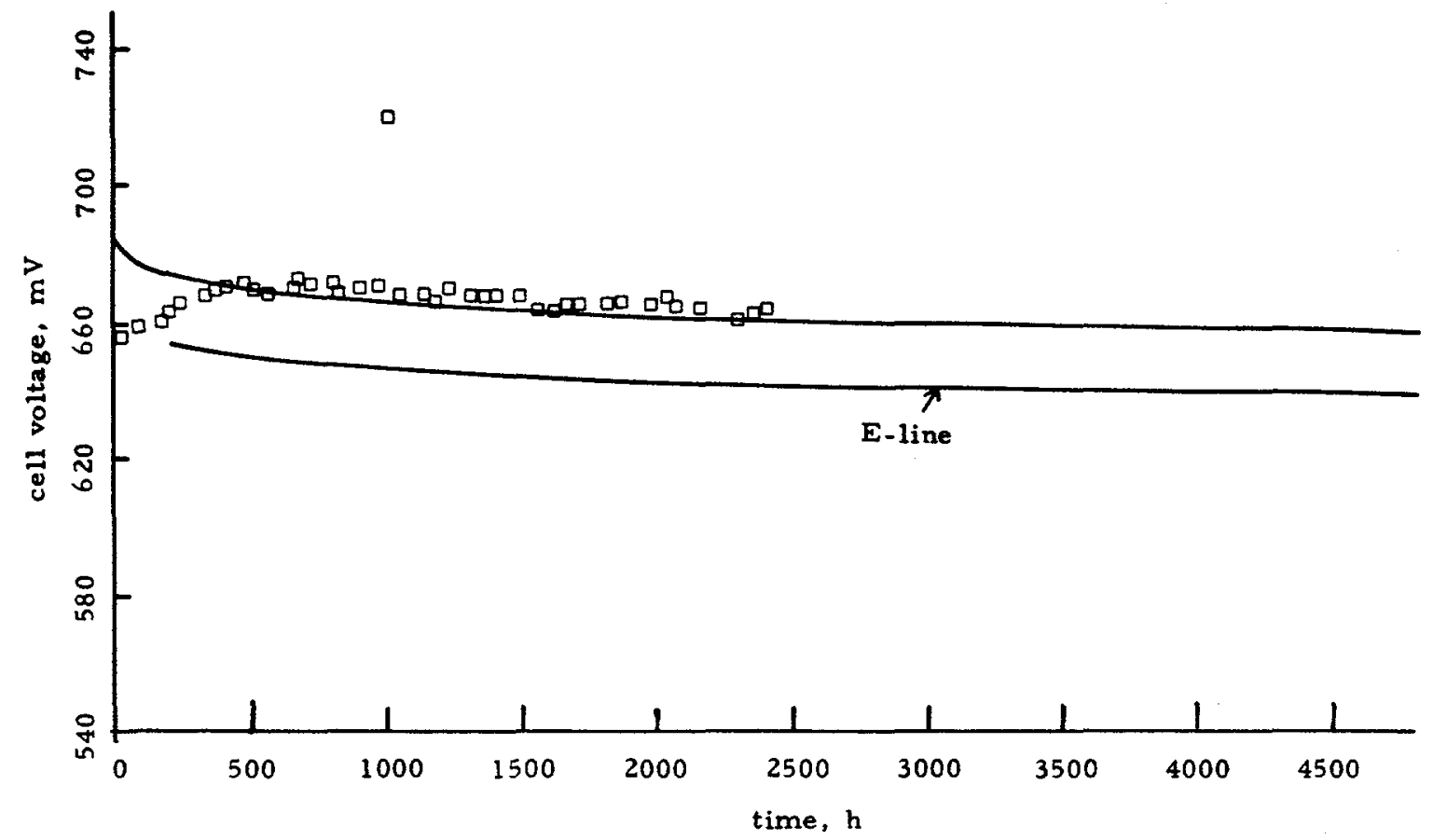

Fig. 2. 14-11, Performance of an on-site cell using an alternative ribbed substrate configuration; $0.66 \mathrm{~V}$, atmospheric pressure, and $190^{\circ} \mathrm{C}$.

Engelhard's recent data also indicate a high level of achievement, as is shown in Fig. 2.14-12 for atmospheric pressure cells at $191^{\circ} \mathrm{C}$. The results indicate $0.7 \mathrm{~V}$ at $200 \mathrm{~A} / \mathrm{ft}^{2}$, which corresponds to about $0.75 \mathrm{~V}$ for UTC's electric utility cell gas mixtures, operating pressures and temperatures. The results are probably about the same as those in the UTC atmospheric pressure stack in Fig. 2.14-11, since the latter were obtained with reformed NG at high utilization rather than with pure $\mathrm{H}_{2}$.

Recent Japanese results fall substantially above NEDO's target potential goal of $0.7 \mathrm{~V}$ at $220 \mathrm{~mA} / \mathrm{cm}^{2}$ and show the very rapid progress that has been made in the last two years of stackbuilding in the Japanese national program. As in the work at UTC, components in laboratory cells are superior to those in operating stacks (cf. performance 82 at Mitsubishi Electric shown in Figs. 2.14-13 and 2.14-14). When corrected for pressure, these data are close to UTC's results in Fig. 2.14-10. Pt loadings in the Japanese stacks are somewhat higher than UTC's standard value and correspond to $6.5 \mathrm{~g} / \mathrm{kW}$ or a total loading of $1 \mathrm{mg} / \mathrm{cm}^{2}$. Performance improvements in MELCO stacks since 1981 are shown in Fig. 2.14-15.82 Results have been improving by about $30 \mathrm{mV} / \mathrm{y}$; it is possible that final results will allow operation at about $0.75 \mathrm{~V}$, which corresponds to a system heat-rate close to $7500 \mathrm{BTU} / \mathrm{kWh}$ or a $45.5 \% \mathrm{HHV}$ efficiency.

We conclude that electric and gas-utility FCs will perform as expected and that ongoing improvements will allow more rapid stack-cost reductions than had been anticipated. The excellent results allow technological redundancy, which corresponds to an operating contingency in commercial stacks or, alternatively, can be used to improve overall system heat-rate if this becomes desirable with fuel costs rising at a greater rate than anticipated. 

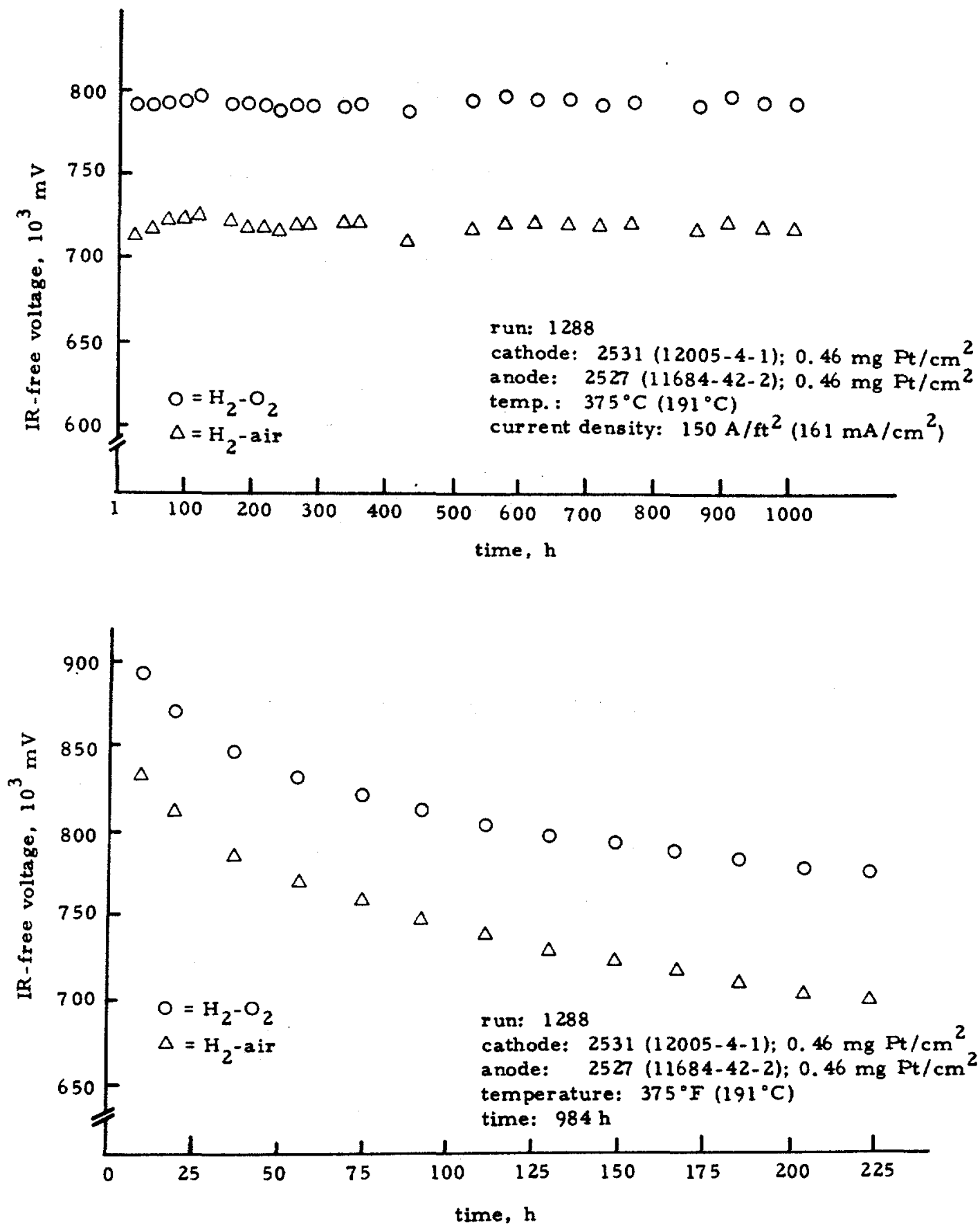

Fig. 2.14-12. Steady load performance of a single Engelhard cell utilizing the E-3 cathode catalyst at atmospheric pressure. 


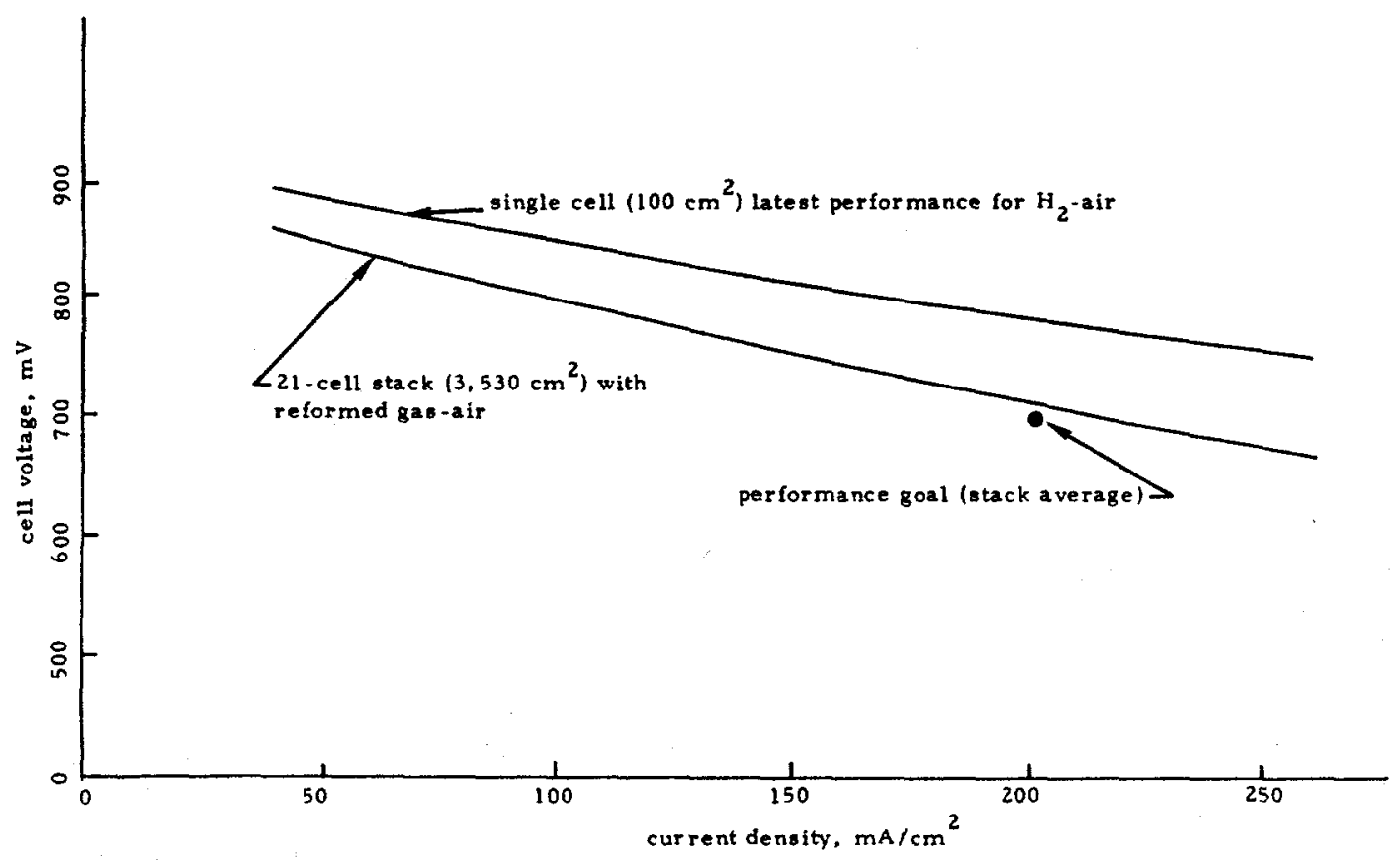

Fig. 2. 14-13. The MELCO 21 -cell stack performance is shown under utility conditions and compared with single-cell (1983) $\mathrm{H}_{2}$-air performance (from Ref. 82); $\mathrm{P}=4.0 \mathrm{~kg} / \mathrm{cm}^{2} \mathrm{~g}, \mathrm{~T}=190^{\circ} \mathrm{C}, \mathrm{U}_{\mathrm{H}_{2}}=75 \%, \mathrm{U}_{\mathrm{air}}=50 \%$.

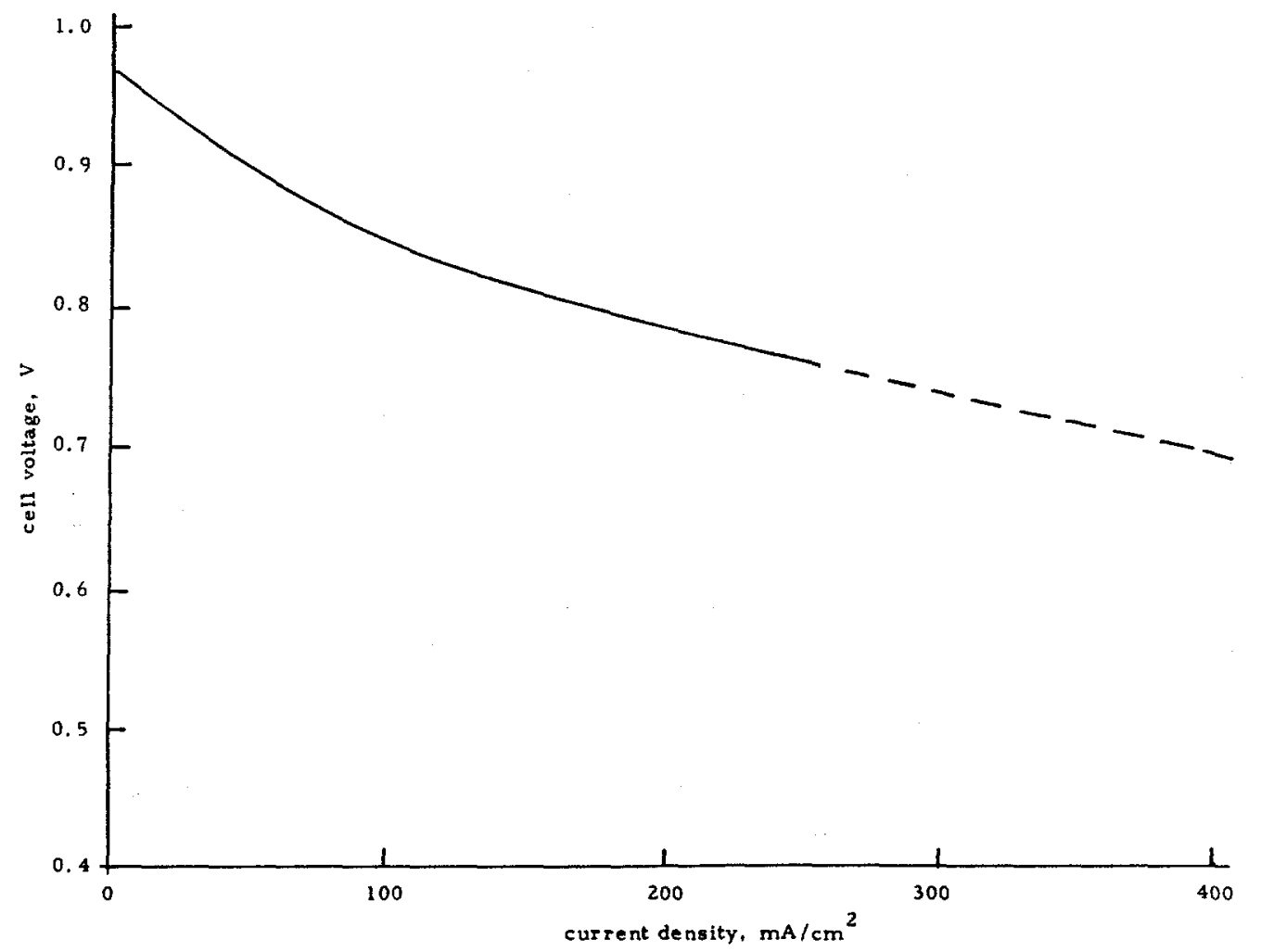

Fig. 2. 14-14. MELCO H2-air cell performance, Mitsubishi Electric (1983) at $190^{\circ} \mathrm{C}$, 4 atrn. 


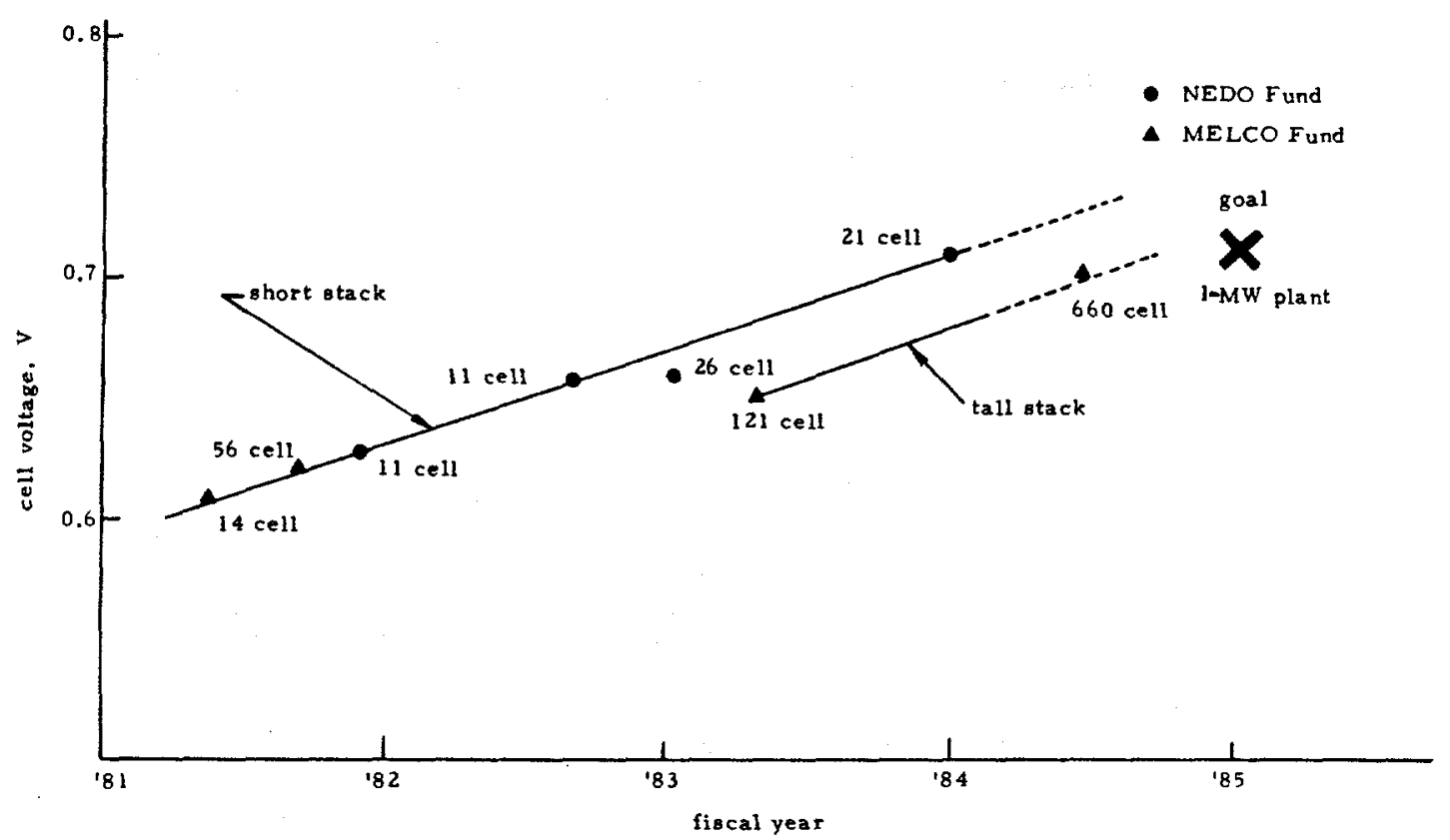

Fig. 2.14-15. The MELCO cell performance improvement since 1981 (see Ref. 82); all data refer to $190^{\circ} \mathrm{C}, 4 \mathrm{~kg} / \mathrm{cm}^{2} \mathrm{~g}, \mathrm{RG} / \mathrm{air}, 200 \mathrm{~mA} / \mathrm{cm}^{2}$.

\subsection{Conclusions and Recommendations for Future Research}

\section{15A Systems}

It is apparent that a major advantage of PAFCs derives from the possibility of using conventional carbonaceous fuels. Since state-of-the-art technology steam-reforming is necessary to make the $\mathrm{H}_{2}$ that $\mathrm{FC}$ s consume efficiently, low-sulfur fuels must be used in the reformer. The only a vailable technique for producing sufficiently desulfurized fuels efficiently is vapor phase hydrodesulfurization. Therefore, low end-point $\left(215^{\circ} \mathrm{C}\right)$ fuels are needed to prevent cracking. Advanced high temperature (adiabatic) reformer concepts have been examined that would allow handling of heavier S-containing fuels (e.g., No. 2 fuel oil $\left.{ }^{83}\right)$, but some heat-rate penalties occur. In general, the heavier the fuel, the more excess steam is required in reforming, which limits system heat-rate. Very heavy fuels (crude, residual fuel, bunker fuel, etc.) can only be handled in a gasifier that is similar to those used for coal gasification. The result is about a $20 \%$ heat rate penalty because the fuel-treatment system uses partial oxidation. A great deal of further work is required in this area, especially on innovative desulfurization methods and new fueltreatment catalysts. This work has evident and useful applications outside of the FC field. Systems developments (i.e., innovative chemical engineering subsystems, a method for separating $\mathrm{H}_{2}$ and steam without condensation in order to lower system steam requirements) should be left to $F C$ developers and users.

\section{15B Electrolytes}

Requirements of an acid electrolyte for an electric or gas utility FC system using reformed $\mathrm{HC}$ are shown in Table 2.15-1. As we have seen in Sec. 2.5, a high cell temperature is not only important for good kinetics in acid systems (yielding $C O$ and limited $S$ tolerance at the a node, together with good cathode kinetics) but is also required so that the a vailable heat in the cell can be used to supply the large excess of steam needed for fuel reforming to produce $\mathrm{H}_{2}$; $\mathrm{H}_{2}$ is the only easily manufactured fuel with sufficiently rapid oxidation kinetics for practical use in the cell. The available heat is the difference between the actual cell potential (with $\mathrm{eV}$ units converted to $\mathrm{kJ}$ ) and the heat of reaction for $\mathrm{H}_{2}$ oxidation and corresponds to $T \Delta \mathrm{S}_{\text {irrev, where }}$ $\Delta S_{i r r e v}$ is the irreversible entropy of reaction. The excess-gteam requirement sets an upper 
Table 2.15-1. Requirements for a utility FC electrolyte with HC fuel.

\author{
high temperature $\left(\sim 200+^{\circ} \mathrm{C}\right)$ for the reforming system \\ good kinetics at the anode and, particularly, at the cathode \\ good conductivity \\ very low to zero volatility (high boiling point) \\ very high chemical stability \\ very high cathode stability (Pt initially, later $\mathrm{X}$ ) \\ low $\mathrm{C}$ oxidation, which may be inferred from rest potentials \\ low impurity or poison level for electrocatalytic processes \\ for use with present electrodes that vary from lyophobic to teflon
}

The only known material is $\left(\mathrm{H}_{2} \mathrm{O}\right)_{x} \mathrm{P}_{2} \mathrm{O}_{5}$, but the system has slow kinetics.

limit to the cell potential and system heat-rate. Presently available cells operate far from this limit, which is probably slightly higher than $0.8 \mathrm{~V}$. Thus, potential exists for further systemefficiency increases.

Requirements for a suitable electrolyte include stability, non-volatility (unless some highly complex electrolyte recycle is devised), suitability for use with high-surface-area, threephase-boundary electrodes, good ionic conductivity, and physical and chemical stability with respect to cell components. The material must also be capable of sustaining the highest possible reaction rates at both the anode and cathode. It should not contain impurities which poison electrode processes. PA in high concentration at about $200^{\circ} \mathrm{C}$ is the only common material with nearly the desired properties. However, with PA, electrode kinetics are not as desired. This fact is illustrated in Fig. 2.15-1 by oxygen-reduction data at 1 atm for low-loading FC cathodes. Figure 2.15-1 shows data for concentrated PA at $165^{\circ} \mathrm{C}$ and also for two fluorinated sulfonic acids [TFMSA or trifluoromethane sulfonic acid, $\mathrm{CF}_{3} \mathrm{SO}_{3} \mathrm{H}$, and TFEDSA or tetrafluoroethane 1,2 disulfonic acid, $\left.\left(\mathrm{CF}_{2} \mathrm{SO}_{3} \mathrm{H}\right)_{2}\right]$, at $70^{\circ}$ and $110^{\circ} \mathrm{C}$, respectively. The sulfonic acids are seen to have greater activity for $\mathrm{O}_{2}$-reduction than $\mathrm{PA}$, even though the test temperatures were much lower. It is known that PA becomes more active as it is diluted, but it is always less active than the sulfonic acids, typically by about $40-50 \mathrm{mV}$ under the same conditions in porous electrodes. 84 However, on smooth Pt electrodes at the same pH, PA is more than $100 \mathrm{mV}$ less active than TFMSA, 85 which may indicate that porous electrodes used for PAFCs are not optimized for materials such as TFMSA. While the deleterious effects of even small amounts of PA on performance 85,86 may be attributed to adsorption of the neutral molecule, which has an inhibiting effect on $\mathrm{O}_{2}$ reduction, 86,87 the major difference between $\mathrm{O}_{2}$ kinetic $\mathrm{s}$ in concentrated solutions of fluorinated sulfonic acids and $P A$ results from differences in $\mathrm{O}_{2}$ solubility. 86,88

Fluorinated sulfonic acids, particularly TFMSA, were examined as possible FC electrolytes in 1971 at $E R C$, but the first results were published later. 89 Since early work showed that TFMSA was too volatile for use under utility FC conditions and also wetted teflon-bonded electrodes at high concentrations, the use of higher polymers such as TFEDSA was emphasized 90 because wetting was found to be associated with the presence of the CF 3 group. Since TFEDSA is somewhat volatile at temperatures above $150^{\circ} \mathrm{C}, 84$ higher molecular weight materials such as 1, 2, 3, 4 perfluorobutane tetrasulfonic acid (HFBTSA, see Fig. 2.5-10) are being synthesized. Without impurities, all of the fluorinated sulfonic acid family share high $\mathrm{O}_{2}$ electrode activity, which also occurs with solid polymers of the Nafion ${ }^{R}$ family of compounds (cf. Chapter 4). They are also stable under utility FC conditions. Very few potential members of the family have been synthesized. Their possible application as FC electrolytes has recently been reviewed. 91 There is evidence that they have much better CO tolerance than PA; 92 their high cathode open-circuit potentials indicate that they are not particularly likely to cause carbon corrosion.

Unfortunately, the sulfonic acids possess a major and originally unanticipated drawback, which was not immediately apparent in early work. 90 For $\mathrm{T} \geqslant 110^{\circ} \mathrm{C}$, they lose water and exist as hydrates. While these compounds are fully ionized, they contain protons exclusively as $\mathrm{H}_{3} \mathrm{O}^{+}$ ions, and the se ions are relatively immobile. No Grotthus-chain mechanism is available for enhanced proton conductivity via a hopping mechanism. Therefore, at constant water-vapor pressure, their conductivities fall with increasing temperature and eventually become less than $0.1(\Omega-\mathrm{cm})^{-1}$ (cf. Fig. 2.15-2, which is taken from Refs. 13 and 84). The monohydrates behave like low-melting potassium salts, since $\mathrm{K}^{+}$and $\mathrm{H}_{3} \mathrm{O}^{+}$have about the same ionic radius. By contrast, PA has increasing conductivity at constant water-vapor pressure as the concentration and temperature are raised, reaching the high value of $0.6(\Omega-\mathrm{cm})^{-1}$ at $170^{\circ} \mathrm{C}(\mathrm{Fig} .2 .15-2)$. These changes result from the presence of protons as $\mathrm{H}_{4} \mathrm{PO}_{4}^{+}$ions $\{$actually, the condensed 


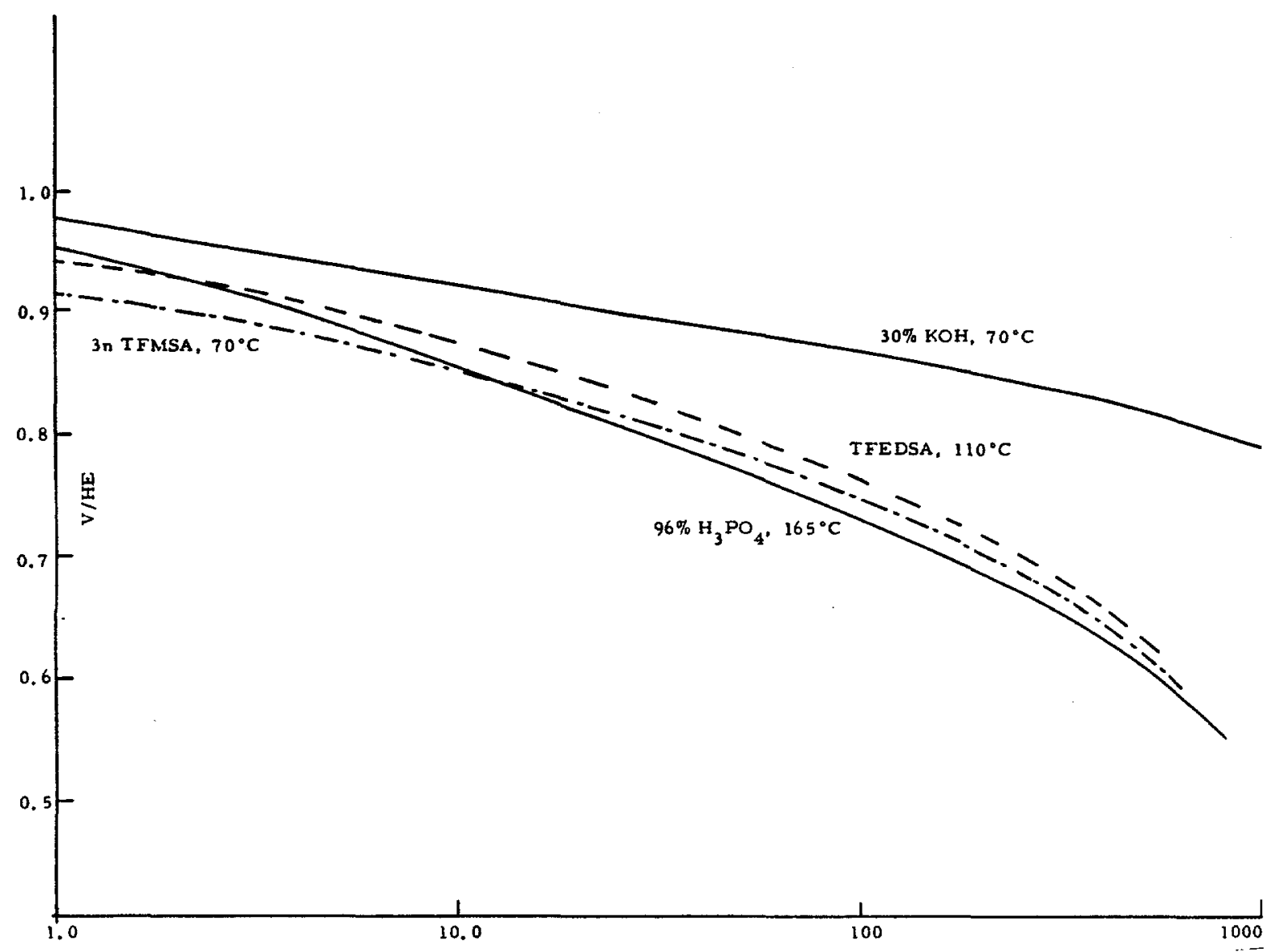

Fig. 2.15-1. Comparisons of low-loading Pt FC cathodes in different media; IRfree, oxygen $(1 \mathrm{~atm}), 0.25 \mathrm{mg} / \mathrm{cm}^{2}$ of $\mathrm{Pt}$.

8 pecies $\left[\mathrm{H}_{3} \mathrm{O}\left(\mathrm{H}_{2} \mathrm{O}\right)_{x}\left(\mathrm{P}_{2} \mathrm{O}_{5}\right)_{y}\right]^{+}$is present $\}$, which are accompanied by neutral molecules. A Grotthus proton-transport chain can therefore be set up which leads to high conductivity. Since the PA is stronger as it becomes more condensed, its conductivity rises with temperature. However, condensation has the adverse effect of reducing $\mathrm{O}_{2}$ solubility and, therefore, little improvement occurs in the kinetics with rising temperature.

The overall effect is that FCs operating on polymer sulfonic acids have about the same performance under utility conditions as PAFCs, since their improved cathode kinetics are counteracted by poor conductivity of acid hydrates. Attempts are being made to improve the conductivity by the use of additives which introduce a Grotthus -type proton-conduction chain. Additions should be made of phosphates, borates and silicates to test the concept. Thus far, 88 PA has been added to sulfonic acids in equimolar mixtures. About a $50-\mathrm{mV}$ activity improvement compared with PA was seen, while the inter nal resistance was approximately doubled. This behavior leads to only a few $\mathrm{mV}$ increase in overall performance in a practical FC.

Alternative fluorinated phosphonic and phosphinic acids may hold greater promise, since they may form Grotthus chains at high $\mathrm{O}_{2}$ solubility and hence exhibit excellent cathode kinetics. Work is required on the synthesis of $\mathrm{C}_{x} \mathrm{~F}_{y} \mathrm{PO}_{3} \mathrm{H}_{2}$ and $\left(\mathrm{C}_{x} \mathrm{~F}_{y}\right)_{2} \mathrm{PO}_{2} \mathrm{H}$, as well as on their $\mathrm{SO}_{3} \mathrm{H}$ equivalents. These compounds must be polymeric to give low volatility, and they must not contain a significant number of terminal $\mathrm{CF}_{3}$ groups to avoid teflon wetting in the present electrodes. The absence of the $\mathrm{CF}_{3}$ group may not yield a non-wetting material since $\mathrm{FPO}_{3} \mathrm{H}$ wets teflon. 88 The only published work on phos phonic acids is the synthesis of $\mathrm{CF}_{2}\left(\mathrm{PO}_{3} \mathrm{H}_{2}\right.$ in 1980.93 The compound was tested for toxicity with negative results. Some limited FC testing was conducted, ${ }^{2}$ and the compound appeared to exhibit poor conductivity at $\mathrm{T}>150^{\circ} \mathrm{C}$. Hence, its behavior appears to be similar to that of the fluorinated sulfonic acids, except that the onset of hydrate formation occurs at much higher temperatures, which would be advantageous. 


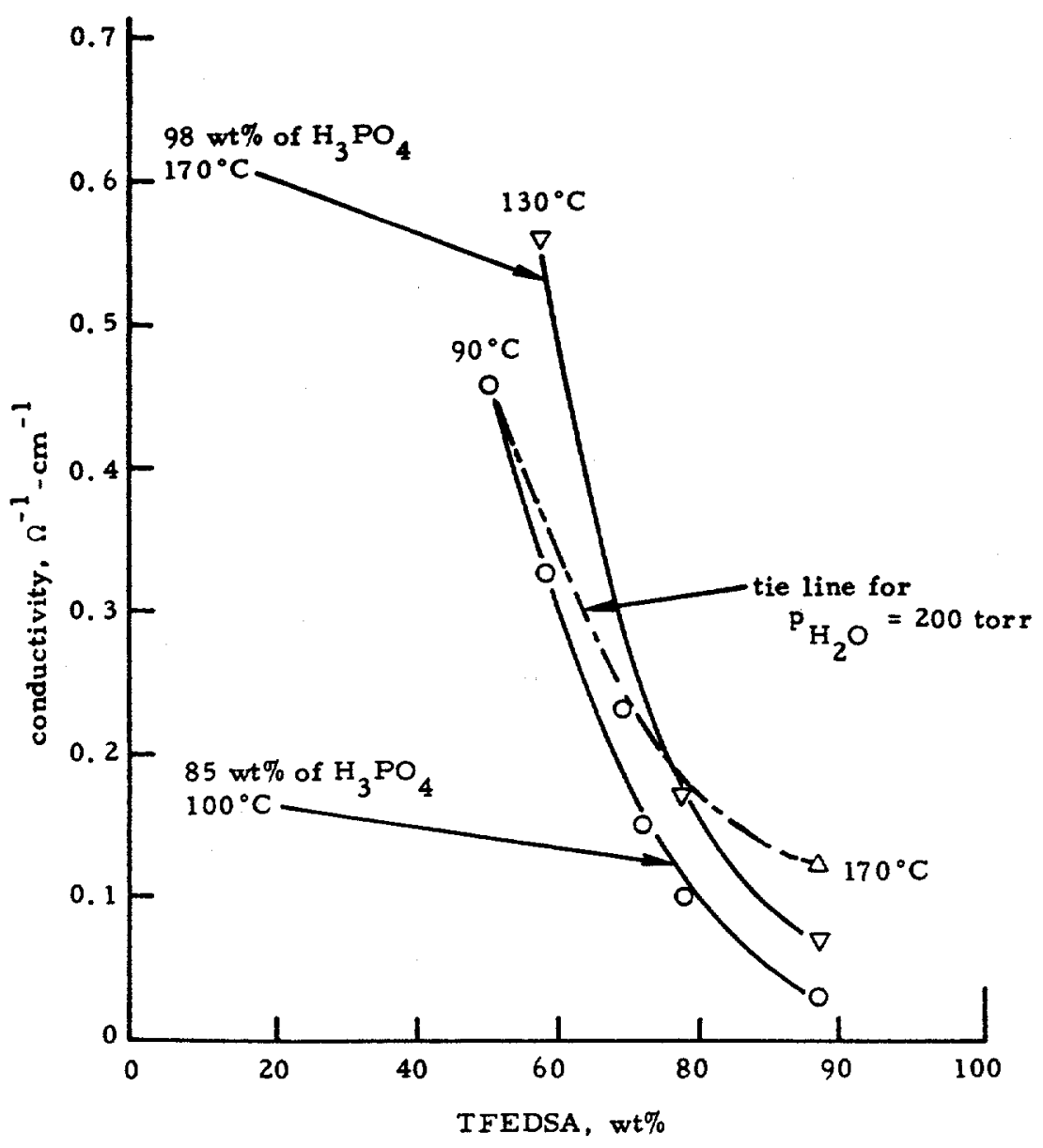

Fig. 2. 15-2. The conductivities of TFEDSA and PA are shown as functions of concentration.

Other compounds should be studied, such as fluorinated organo-phosphinic acids. Attempts are currently being made to synthesize lower members of the series under a GRI program. Entirely new classes of electrolytes are possible. If the presence of fluorine is the key to enhanced $\mathrm{O}_{2}$ solubility and improved kinetics, new polymer systems with non-C skeletons may be envisaged, perhaps based on B-O-B or Si-O-Si systems. It should be recalled that PA is based on the P-O-P system at utility operating temperatures. The new polymers could be fluorinated if the appropriate $F-X(X=B$, etc.) bonds are stable or, alternatively, they could contain -C-F links attached to the polymer backbone. Fluorocarbon boric and silicic acids should be strong and have properties appropriate for FC electrolytes. Blends of these materials may be useful, even such simple blends as a fluorinated polyether in a polymer fluorosulfonic acid, where the stable fluorinated polyether not only enhances $\mathrm{O}_{2}$ solubility but also provides a mechanism for proton hopping from $\mathrm{H}_{3} \mathrm{O}^{+}$lons to - $\mathrm{O}$ - groups, thus increasing conductivity. A similar soluble fluorocompound added to PA could augment its $\mathrm{O}_{2}$ solubility and leads to improved kinetics. The development of new families of inorganic-organic fluorinated polymers may also have significant applications outside of the FC field.

Another approach is separation of the catalytic and transport functions of the electrolyte. Because of the problem of conductivity at high temperature, perfluorocarbon sulfonic acid polymers can only be used in extremely thin films in practical cells. There is a limit to the thicknes 8 of the electrolyte film that can be placed in cells with liquid electrolyte. This limit is probably about $0.2 \mathrm{~mm}$. At a current density of $250 \mathrm{~mA} / \mathrm{cm}^{2}$, the IR drop may be tolerable in such a layer if we allow for the presence of matrix material and tortuosity factors. Howe ver, a better solution would be to place a layer of a catalytic electrolyte, which is insoluble in PA, within the cathode structure itself so that it alone is in contact with the catalyst, while we rely on the PA in the matrix for conduction between electrodes. 94 The catalytic polymer electrolyte within the electrode will be present in the form of $1-10 \mathrm{um}$ thick films, which will have negligible IR drops. This concept bridges the gap between the acid cell proper and modifications to the SPE cell, in which attempts are being made to improve polymer conductivity at high temper ature by incorporating a Grothus proton conductor into the electrolyte polymer film. 


\section{15C Electrodes}

The electrode must contain the proton-conducting catalytic electrolyte, the electronically conducting catalyst support, and the catalyst itself, all disposed in such a way that the ionic and electronic pathways (in opposite directions) are as short and non-tortuous as pos sible, so that the catalyst has maximum exposure to electrolyte and $\mathrm{O}_{2}$. The catalyst can only operate if it is simultaneously in contact with the electronically conducting support, the electrolyte and $\mathrm{O}_{2}$. In present electrodes, a mixture of catalyst on carbon black (the conducting support) is used. The carbon black is mixed with teflon and then sintered at about $360^{\circ} \mathrm{C}$. It must have suitable corroHetace, exally at the cathode, and typically has $70-200 \mathrm{~m} / \mathrm{g}$ BET area. The catalyst consists of $70-100 \mathrm{~m} / \mathrm{g}$ of $\mathrm{Pt}$ or of a Pt alloy at a loading of about 10 wt $\%$ on $\mathrm{C}$, so that the ratio of $\mathrm{Pt}$ surface to support area is about $5 \%$.

Tranumission electron micrographs of catalysts on their supports are shown in Figs. 2.12-1, 2.12-2 and 2.12-7. Pt loadings are $0.2-0.25 \mathrm{mg} / \mathrm{cm}^{2}$ for the anode and $0.3-$ $1.0 \mathrm{mg} / \mathrm{cm}^{2}$ (normally $0.5 \mathrm{mg} / \mathrm{cm}^{2}$ ) for the cathode. The electrode mix (catalyst with about 40 w $\%$ of colloidal teflon and about $0.3 \mu \mathrm{m}$ diameter particle size) can be laid down on the $80 \%$ porous graphite cloth or paper substrate by a variety of methods. A dry or wet process may be used (dry teflon or teflon in aqueous suspensions). Some of these are discussed in Ref. 81 . The film may be prepared by calendering, electrostatic spraying, silk-screen printing or other inking processes. Finally, the whole structure is sintered.

The electrode structure and the teflon-sintering process are shown schematically in Fig. 2.15-3. Sintering appears to be very important in obtaining longevity and high performance in acid cells although, in the past, unsintered electrodes have been used in alkaline electrolytes. During sintering, the teflon partially depolymerizes and wets the carbon 95 in a random manner, so that some parts of the structure are more highly wet-proofed than other 8 . Some of the catalyst is covered by teflon or is present in highly wet-proofed pores, which are not filled with electrolyte and cannot operate electrochemically. Other catalyst may be present in wettable pores, which are drowned with electrolyte, where the diffusion pathway for the gaseous reactant is too long for effective reaction rates. Only catalyst covered by a thin film of electrolyte, which allows a short diffusion pathway for the reactant gas through empty pores, is truly effective. Even then, the thin meniscus should not be too long. Otherwise, the ionic pathway will be too resistive and will allow that part of the electrode to approach open-circuit conditions, which may have important corrosion consequences (see Fig. 2.11-6 and the discussion in Sec. 2. 11).

The art in electrode-making is to make certain that as much of the catalyst as possible is working at any given time. Some parts of the electrode structure may become more strongly wetted with time. Low teflon loading s may lead to drowning of the catalyst, whereas high teflon loadings may reduce wetting below operational limits. Ideally, the structure should be about $50 \%$ wetted; reference to Fig. 2.15-3 shows flooded pores (the flooded agglomerate) and open pores, with the former conducting the current and the latter allowing entry of gas and exit of product water at the cathode. Most of the reaction takes place on the wetted surface of the empty pores, i.e., on the outside of the flooded agglomerate radius, which is typically about $1 \mathrm{um}$. The Giner-Hunter flooded agglomerate model 96 of the porous electrode as an electrochemical reactor has been used with reasonable success in describing reaction rates, but it contains a number of adjustable parameters. More recent attempts have been made to describe the diffusion processes using the Stefan-Maxwell equations for multicomponent diffusion, 97 but the results remain ambiguous. 98

While progress has been made in understanding electrode microstructure and flooded agglomerate radius as a function of weight fraction of teflon, sintering time and temperature, 99 electrode making is still an art, and it is never certain how effectively the catalyst is being used in typical electrodes. A guess is that $30 \%$ is in use at any given time, with the particular $30 \%$ that is involved changing as the electrode ages. Doubling or tripling of catalyst utilization in the electrode would clearly be desirable if electrode life were not affected, since $30-40 \mathrm{mV}$ higher performance or a heat-rate reduction of about $400 \mathrm{BTU} / \mathrm{kWh}$ would result. Improved catalyst utilization may perhaps be effected by redistributing the catalyst in a redesigned electrode that has most of the catalyst present on the flooded agglomerate surface. An attempt to accomplish this goal was made by Motoo, Watanabe and Furuya, 100 who made electrodes in which gas pores are uncatalyzed and have a higher teflon content than flooded catalyzed, agglomerate pores. The gas-pore and flooded pore materials were prepared separately, ground in liquid nitrogen, mixed, and sintered. A strong relation was found between teflon content and current density (Fig. 2.15-4, which has been reproduced from Ref. 101). Some performance curves 100 are shown in Fig. 2.15-5, but the very high performance claim, about 10 times that seen on state-of-the-art electrodes at the same potential, has not been reproduced. Instead, recent independent tests have shown that the electrodes are more than twice as active as state-of-the-art electrode structures made by the FC developers. Doubts remain about the longevity of the electrodes. More work is needed to understand model electrode structures. It seems certain that catalyst utilization can be improved. This development will be particularly important if polymers are made with separation of the electrolyte into catalytic and transport components. 

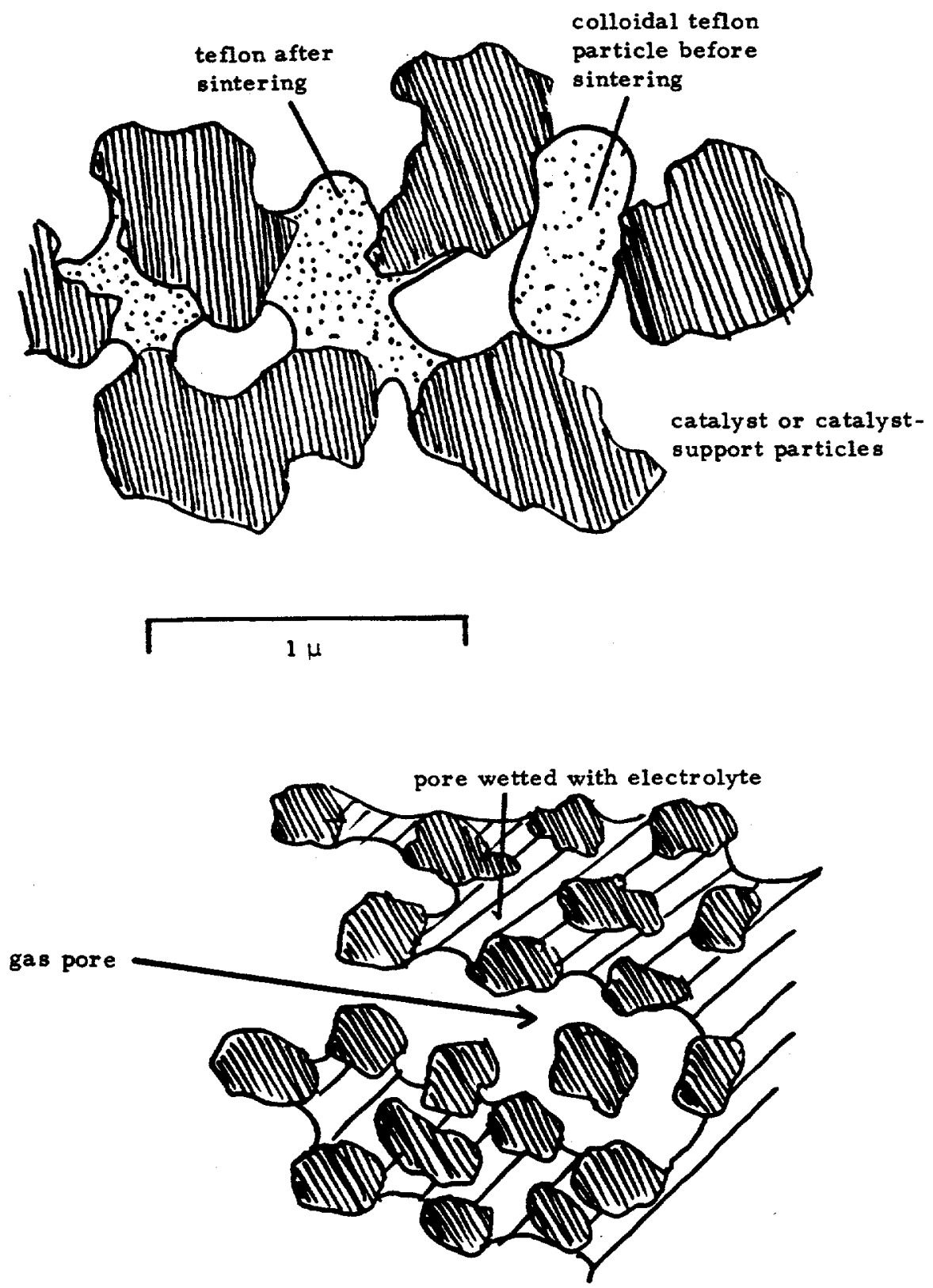

Fig. 2.15-3. Schematic models of teflon-bonded electrodes. 


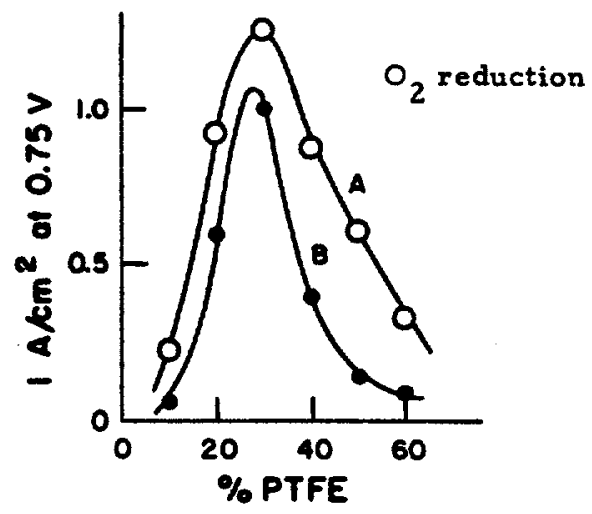

Fig. 2. 15-4. The Watanabe-Motoo relation is shown for electrode activity as a function of teflon content; $A=$ colloidal method, $B$ = impregnating method.

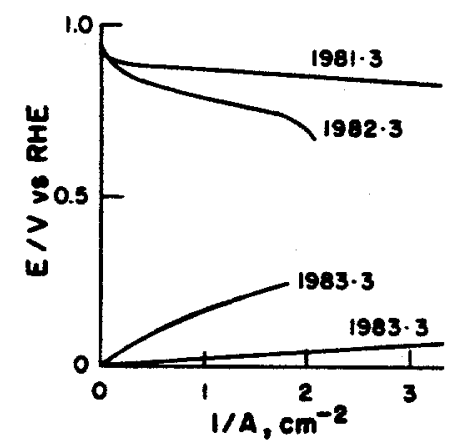

A. History of cathode and anode performance for Pt $\left(0.56 \mathrm{mg} / \mathrm{cm}^{2}\right)$ supported on carbon black (Vulcan XC-72) at $60^{\circ} \mathrm{C}$ in $25 \% \mathrm{KOH}$.
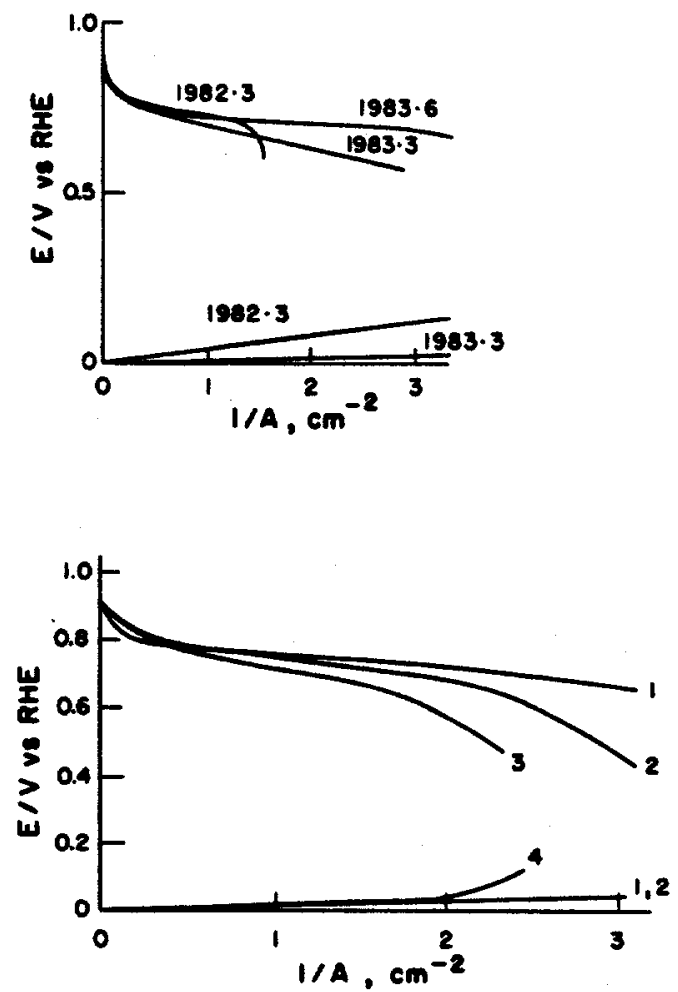

B. History of cathode and anode performance of Pt $\left(0.56 \mathrm{mg} / \mathrm{cm}^{2}\right)$ supported on Vulcan XC72 at $60^{\circ} \mathrm{C}$ in $20 \% \mathrm{H}_{2} \mathrm{SO}_{4}$.

C. Cathode and anode performance of $\mathrm{Pt}(0.56$ $\mathrm{mg} / \mathrm{cm}^{2}$ ) supported on Denka Black at $190^{\circ} \mathrm{C}$ in $\mathrm{H}_{2} \mathrm{PO}_{4}$. Curve 4: $\mathrm{Pt} 0.28 \mathrm{mg} / \mathrm{cm}^{3}$, cold pressed at $25 \mathrm{~kg} / \mathrm{cm}^{3}$; curve 2: Pt $0.56 \mathrm{mg} /$ $\mathrm{cm}^{2}$, cold pressed at $50 \mathrm{~kg} / \mathrm{cm}^{2}$; curve $3: \mathrm{Pt}$ $0.56 \mathrm{mg} / \mathrm{cm}^{2}$, cold pressed at $36 \mathrm{~kg} / \mathrm{cm}^{2}$; curve 1: Pt $0.56 \mathrm{mg} / \mathrm{cm}^{2}$, cold pressed at $200 \mathrm{~kg} / \mathrm{cm}^{2}$.

Fig. 2.15-5. The Watanabe-Motoo electrode activities are shown in different media. 
It is conceivable that new polymers will be developed which incorporate non-wetting and active areas on the scale required in FC electrodes. New supports consisting of stable electronconducting polymers containing redox groups may eventually replace the currently used $\mathrm{Pt}$ as catalyst. Finally, advances including a combination of improvements may occur. Since electrodes are of microbiological dimensions, it may be possible to invent replica structures that are perhaps based on genetic engineering templates. Biological cells are, in principle, FCs 102 making use of a proton gradient to create useful work. They must therefore have a shortcircuited electron pathway through the cell membrane and are, in a sense, the opposite of our conception of the acid FC, which is short-circuited via the proton gradient (i.e., the electrolyte) and performs useful work via the electron gradient (the potential difference between the electrodes).

\subsection{Electrocatalysts}

Future improvements of electrocatalysts or the use of higher cell temperatures and pressures will also require improved corrosion-resistant supports. Innovative construction of the cell stack (Sec. 2.14) and incorporation of catalytic polymer electrolyte in the electrodes may ensure that the contact between the highly graphitized and wet-proofed electrode substrate and the bipolar plate will not contact PA or other acid electrolytes used in the future. Therefore, corrosion should not be a special problem for the se components.

Since silicon carbide used as matrix showed a negligibly small corrosion rate under FC conditions, an EPRI project was started in 1980 to explore the possibility of using conducting carbides as supports. 103 High surface area titanium carbide was the favored material. 104 Pt deposited on this material showed a considerably higher activity than Pt on carbon (see

Fig. 2.15-6, which includes a transmission electron-micrograph of the approximately $70 \mathrm{~m} / \mathrm{g}$ material that is volumetrically equivalent to about $200 \mathrm{~m}^{2} / \mathrm{g}$ carbon). This higher activity stresses the effect of support interaction in catalysis by $\mathrm{Pt}$ microcrystallites and also increa ses crystallite stability (see Sec. 2.12).

It has been shown that some samples of titanium carbide prepared at low temperature, for example, by a low-gas-temperature plasma-process, are soluble in PA, but the method of preparing corrosion-resistant samples may be difficult to scale up. More work is required in this area, especially in attempting to stabilize other carbides (e.g., $\mathrm{TaC}$ ) and to dope silicon carbide and make it electronically conductive, for example with Ta $5+$. Such materials may not only be highly corrosion-resistant but may also show favorable support-interaction effects that increase the activity of present Pt-based catalysts. 105 Innovative work with new materials (e.g. conducting fluorinated polymers) is required.

Figure 2.15-1 show s what might be achieved in electrocatalysis at the cathode. The Tafel slope for oxygen reduction on Pt-black electrodes in the acid electrolytes lies between $110 \mathrm{mV} /$ decade for TFMSA at $70^{\circ} \mathrm{C}$ and $130 \mathrm{mV} /$ decade for $\mathrm{PA}$ at $165^{\circ} \mathrm{C}$. The se values correspond, respectively, to transfer coefficients between 0.67 for PA and 0.71 for TFMSA. Neither of these values has any significance according to the classical theory of the transfer coefficient under
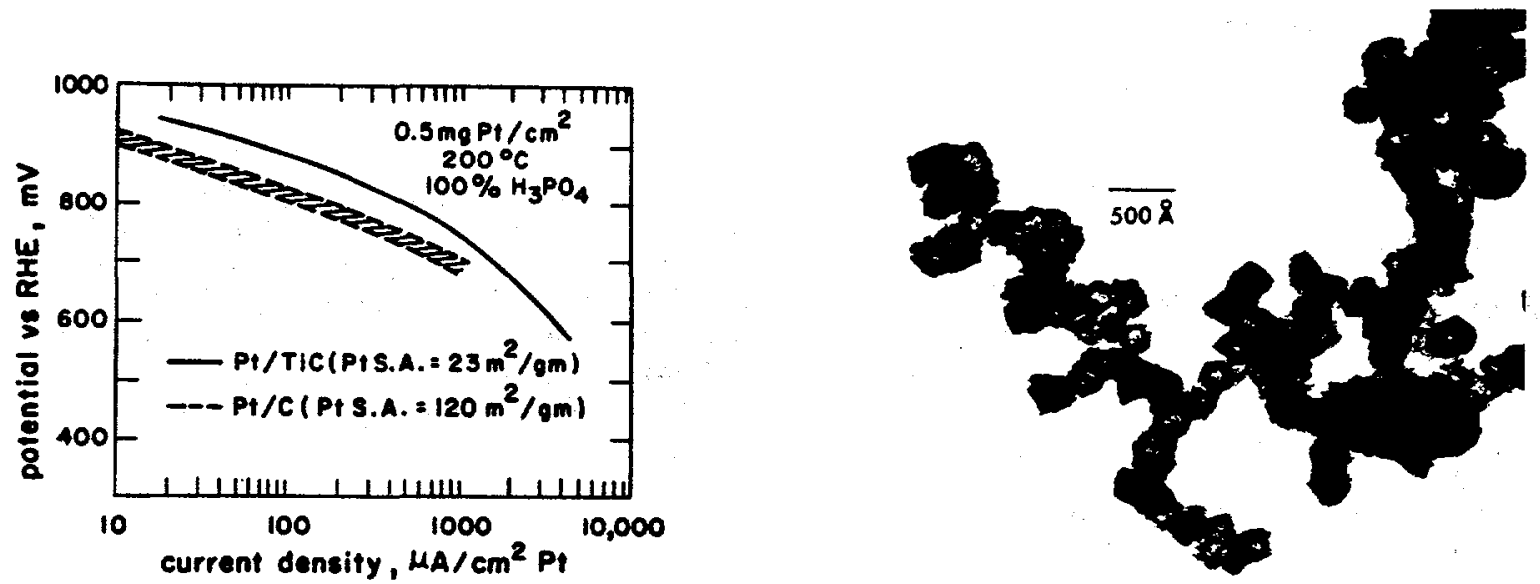

Fig. 2. 15-6. Comparison of the activity of Pt for oxygen reduction on high-surfacearea titanium carbide and on Vulcan $X C-72 R$ carbon black (cf. the TEM photographs of the TiC support). 
either Langmuir or other adsorption conditions of reaction intermediates. 56 On the other hand, the results obtained in $30 \% \mathrm{KOH}$ at $70^{\circ} \mathrm{C}$ not only show more activity than those in acid solution at reasonable current densities, but the Tafel slope is also smaller at about $50 \mathrm{mV} /$ decade. This value is consistent with the classical theory for an electron-transfer rate-determining step that follows primary, pseudoequilibrium electron transfer. The mechanistic analysis is supported by evidence on reaction order. 106 Thus, the oxygen electrochemical reduction reaction is not only less polarized (i.e., has a lower overpotential because of its low Tafel slope) in $\mathrm{KOH}$ solution than in PA, but it is also reasonably described by classical electrochemical theory.

The reaction in PA is not at all under standable, since the evidence points to a Tafel slope that is independent of temperature rather than proportional to the absolute temperature, as is required by the classical theory. This fact was first noted 107 on Pt, which was said to be active at the time but in view of later work, 108 "clean" might be a more accurate designation, since the results are virtually identical to those in Ref. 107 for a reasonable range of the roughness factor. The same behavior was later observed for $\mathrm{O}_{2}$ reduction on other noble metals 109 and a recent extensive review by Conway 110 shows that this is by no means an uncommon phenomenon.

Unfortunately, no satisfactory theory exists to explain the observations, although it has been suggested that they may be associated with an entropy of activation resulting from the change in free volume of condensed liquids as a function of temperature. 111

We have presented speculations on what might become possible if we had more knowledge of the mechanisms that are responsible for a change in the theoretical Tafel slope as a function of temperature and crystallite size for $\mathrm{Pt}$ microcrystallites in concentrated PA. 49 If means could be found to change the course of the rate-determining step in acid solution, it is possible (cf. Fig. 2.15-1) that a $150 \mathrm{mV}$ improvement might occur in $\mathrm{O}_{2}$ electrode performance. Since the Tafel slope does not appear to follow predictions of the classical mechanistic theories, especially on microcrystallites, it may be possible to reduce it further and thus increase $\mathrm{Pt}$ activity if the physical basis behind the measured values could be determined.

The oxygen-reduction reaction is notably ir reversible in acid solutions, even more so than in alkaline media. We understand neither the significance of the Tafel slope in concentrated PA nor correlations between activity for $\mathrm{O}_{2}$ reduction in this medium and the usual properties that govern the rates of electrocatalytic 55,56 processes.

It is well known that the $\mathrm{O}_{2} / \mathrm{O}_{2} \mathrm{H}^{-}$couple is reasonably reversible in alkaline solution. 112 It is difficult to find an electrode surface that is stable in alkaline solution but will not reduce $\mathrm{O}_{2}$ close to the reversible potential of the couple $(\sim 0.68 \mathrm{~V} / \mathrm{HE})$. Any surface which can decompose the peroxide ion or which can provide an alternative pathway for oxygen reduction in which peroxide is not a free reaction intermediate (i.e., in which the dioxygen bond is broken early in the reaction sequence) will give a higher $O_{2}$ electrode potential than this value. As a result, a wide range of materials shows high activity in alkaline solution. The activities tend to fall fairly close togethe $r$, and there is little correlation between electrocatalytic rate and other properties, such as those predicted from the Brdnsted and Horiuti-Polanyi relations. 53,54 This result suggests a reexamination of the possibilities of $\mathrm{CO}_{2}$-rejecting alkaline electrolytes. 5 However, the rate of the $\mathrm{O}_{2}$-reduction reaction on the more restricted set of materials that are stable in concentrated PA appears to follow the classical Volcano relations, $55-60,64$ as is shown in Figs. 2.12-11 and 2.12-13.

The classical Volcano relation, as developed in Refs. 58 and 59, assumed that a ratedetermining step was involved with a reversibly adsorbed reaction intermediate which is formed by a subsequent or preceding reversible step. For these cases, the activation energy is expected to fall along the rising side of the Volcano curve. Activation-energy data55,61, 113 a re shown in Figs. 2.15-8 for the same potential conditions for $\mathrm{O}_{2}$ reduction in $\mathrm{PA}$ and for what is apparently the same one-electron rate-determining step on a series of noble metals. These are given as a function of heat and free energy of adsorption, the former derived by Pauling's method 63 and the latter from actual electrochemical measurements. It can be seen that the activation energies of the $\mathrm{O}_{2}$-electrode process on noble metals decline with increasing adsorption of a reaction intermediate, as is predicted by the Brdnsted theory. 53 The series of materials lie on the falling side of the Volcano curve. According to the classical theory, the series of activation energies should be reversed. Figure 2.15-8 provides the clue to the problem: the a pparent entropy of activation falls as the activation energy decreases, which gives rise to a compensation effect that is probably the result of temperature-independent blockage of active sites. 56 If there were some way to mitigate this problem, much lower $\mathrm{O}_{2}$ over potentials could be envisaged, which would be very desirable. Other methods for reducing the $\mathrm{O}_{2}$-electrode potential also require consideration. These include the use of intermediary redox couples which react chemically with dioxygen.

A nother problem in electrocatalysis is that of the direct FC. Even if direct oxidation of HCs at reasonable rates is a long way off, it merits research, since it has far-reaching implications for utility acid FCs, as well as for implanted FCs in the body using glucose or other physiological materials as fuels to provide a permanent power source for an artificial heart. This last goal was the focus of an NIH program in the late 1960s. As a first step, a direct methanol FC, with or without internal reforming and operating at about $250^{\circ} \mathrm{C}$, could be considered, as was proposed in Ref. 16. If this system does not require a Pt-based catalyst, it would 


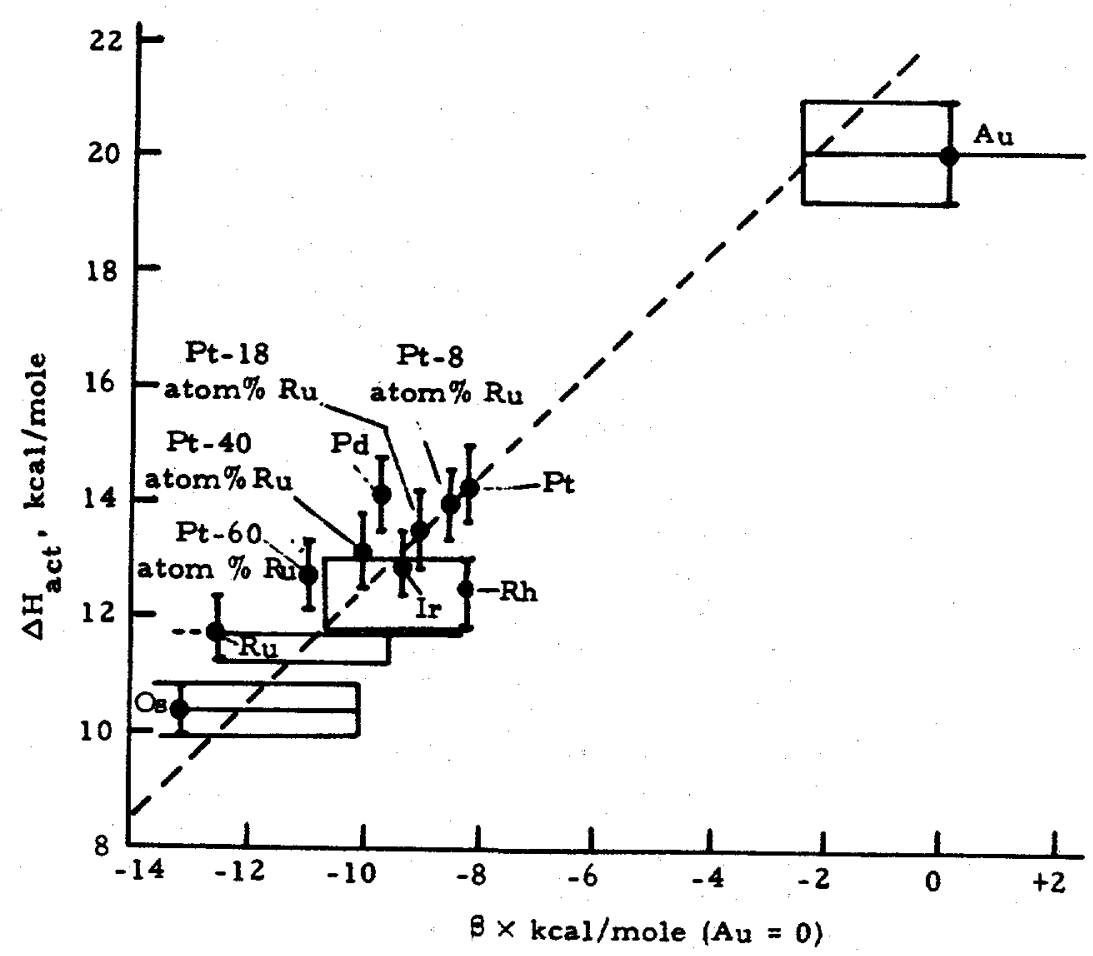

Fig. 2, 15-7. Relations between activation energy for oxygen reduction on noble metals are shown in PA, together with heats and free energies of adsorption of reaction intermediates. 55,113

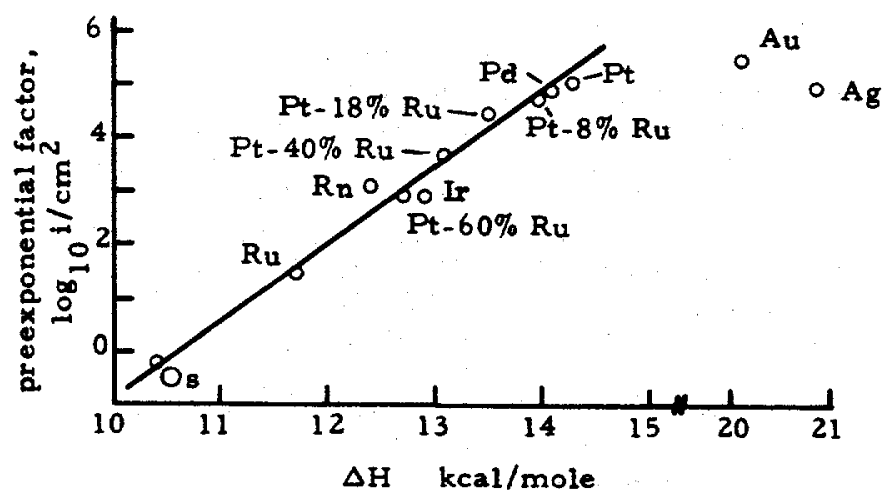

Fig. 2.15-8. The compensation effect between the Arrhenius preexponential factor and the activation energy is shown for oxygen reduction on noble metal in PA. The data refer to $\mathrm{O}_{2}$ reduction in $85 \%$ orthophos phoric acid at $\eta=-460 \mathrm{mP}$.

be an ideal power source for a personal transportation vehicle (perhaps in the first part of the 21 st century), provided that fossil-based fuels are still available.

A large research effort will be required to find an effective substitute for $P t$, especially at the acid FC cathode. In alkaline solution, excellent activity is obtained with macrocyclics of the type shown in Fig. 2.15-9 that are suported on carbon and heat-treated beyond their decomposition temperatures. The end-products appear to be oxides.114 These materials would not be expected to be stable in acid electrolytes at high temperature; however, as is shown in Fig. 2.1510, not only was the iron compound stable for the duration of the test, but it was also very active. Confirmatory results have been obtained under a UTC program. 115 If suitably-structured electrodes could be made, macrocyclics might even prove to be more active than $\mathrm{Pt}$ at high current density. These results are unexpected and suggest many possibilities for future electrocatalysis.

The identification of research areas in this Sec. 2.15 has led to the specific recommendations listed in the Summary. We regard all of the listed recommendations to be of equal importance in obtaining improved PAFCs. 


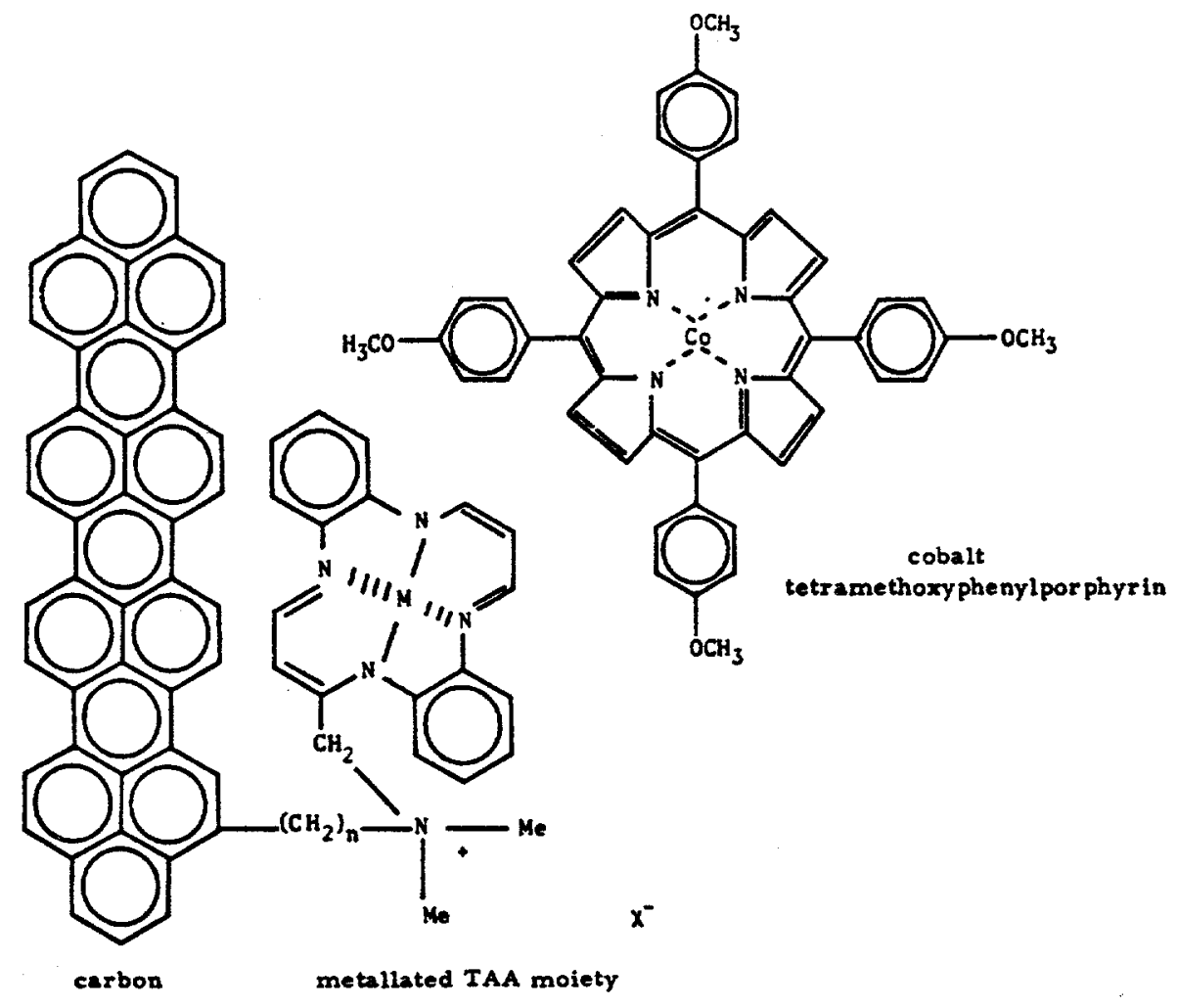

Fig. 2. 15-9. Structures of typical macrocyclic catalysts before pyrolysis on carbon supports.

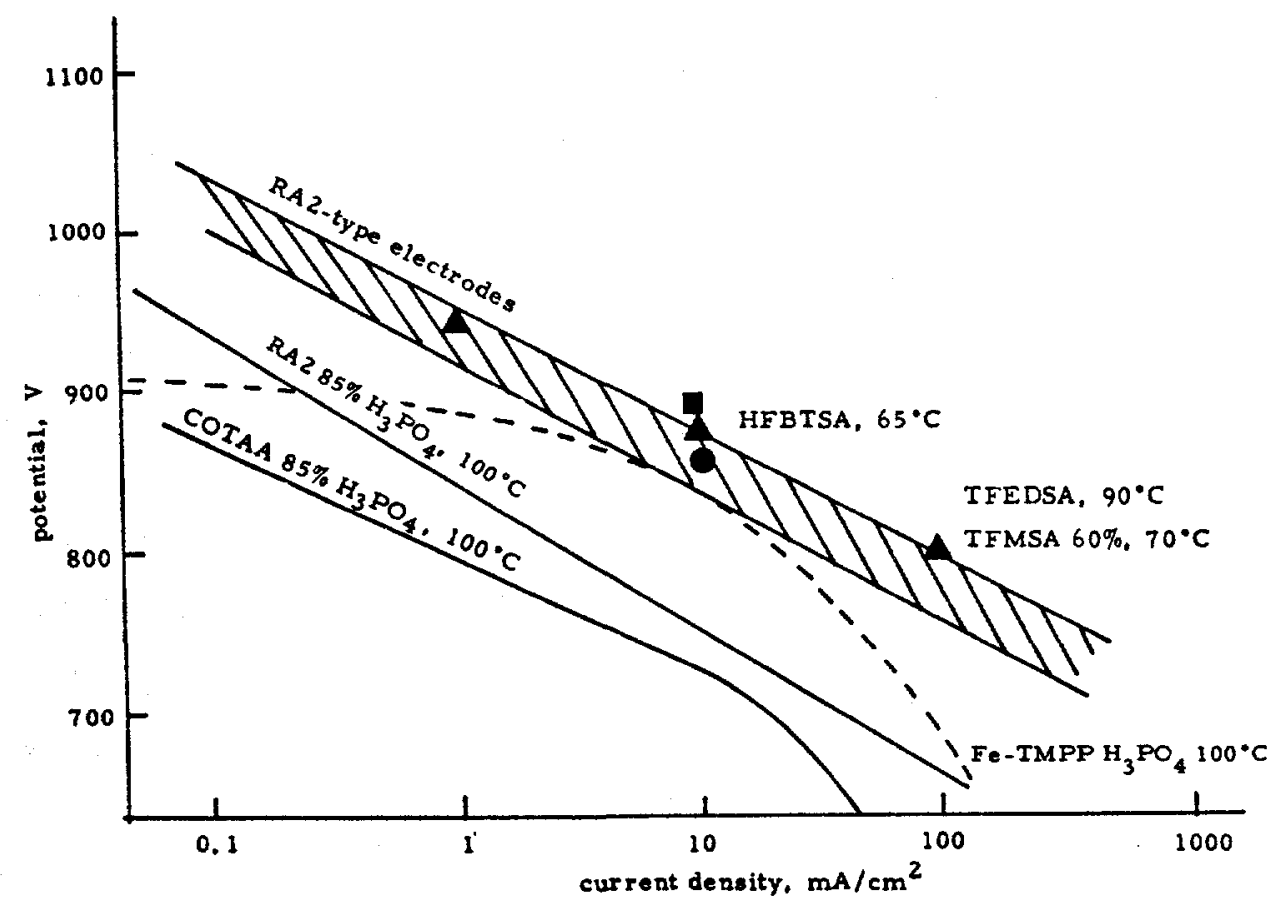

Fig. 2. 15-10. Oxygen-electrode activity of a pyrolyzed iron macrocyclic in PA. RA2: Commercial $0.3 \mathrm{mg} / \mathrm{cm}^{2}$ Pt electrode (Prototech, Inc.). The figure includes data for RA2 electrodes in fluorinated sulfonic acids (HFBTSA, hexafluoro n-butane tetrasulfonic acid). 


\section{REFERENCES FOR CHAPTER 2}

1. "Technical Assessment Guide," EPRI P-2410-SR, EPRI, Palo Alto, CA (1982).

2. E. A. Gillis, Testimony to California Public Utilities Commission, March 14, 1985.

3. C. K. Pang, S. T. Lee, K. Lee, D. T. Imamura, "Application of Fuel Cells on Utility Systems, Vol. 1: Study Results," EM-3205, Vol. 1, EPRI (1983).

4. H. A. Liebhafsky and E. J. Cairns, Fuel Cells and Fuel Batteries, Wiley, New York (1968), Pp. 458-523.

5. E. J. Cairns and D. I. Macdonald, Electrochem. Tech. 2, 65 (1964); E. J. Cairns and D. C. Bartosik, J. Electrochem. Soc. 111, 1205 (1964); E. J. Cairns and A. M. Breitenstein, J. Electrochem. Soc. 114, 349 (1967).

6. Ref. 4, pp. 654-668; I. J. Bregoli et al, "Development of Improved Molten Carbonate Fuel Cell Technology," Final Report, RP1085-4, EPRI (1983).

7. R. E. Martin, "Advanced Technology Lightweight Fuel Cell Program," NASA CR-159653, FCR-1017, NASA Lewis Research Center, Cleveland, OH (1979).

8. J. Pascual and B. Evans, Proc. KTI Symposium V, Napa, CA (May 9-12, 1982 ).

9. Commercial Brochure, "Methanol/Hydrogen Topsie Package Plants," Haldor-Topsфe A/S, Lyngby, Denmark.

10. D. P. Bloomfield and M. B. Landau, U.S. P. 3, 973,993 (8/10/76); D. P. Bloomfield and R. Cohen, U.S.P. 3,972,731 (8/3/76); M. B. Landau, U.S. P. 3,976,506 (8/24/76);

D. P. Bloomfield, U.S. P. 3,982,962 (9/28/76); M. C. Menard, U.S. P. 4,001,04I (1/4/77); D. P. Bloomfield, U.S. P. 4, 004, $947(1 / 25 / 77)$.

11. J. M. King, "Advanced Technology Fuel Cell Program," EPRI EM-335, 1976; E. R. Elzinga et al, "Application of the Alsthom/Exxon Alkaline Fuel Cell System to Utility Power Generation," EPRI EM-384 (1977).

12. Anon., UTC Power Systems Division, On-Site Fuel Cell Power Plant Technology and Development Program, 1982-83 Annual Report, GRI 82099, Gas Research Institute. Chicago, IL (1983).

13. P. N. Ross, Ext. Abs. 1983 National Fuel Cell Seminar, p. 141, Nov. 13-16, 1983, Orlando, FL; EPRI, Palo Alto, CA (1983).

14. D. T. Hooie and E. H. Camara, Ext. Abs. 1985 Fuel Cell Seminar, Tucson, AZ, p. 182, EPRI, Palo Alto, CA, May 19-25, 1985.

15. Y. Narimatsu, N. Niguchi, M. Simuzu and M. Nagashima, p. 6, Ref. 12.

16. P. Zegers, European Economic Community Fuel Cell Meeting, Brussels, Belgium, Jan, 10, 1985.

17. A. J. Appleby, Energy Policy 4, 87 (1976); "Energy Use and Society in the 21 st Century," in Alternative Energy Sources, A Global Compendium, p. 4603, T. N. Veziroglu, ed., Hemisphere, Wa shington, D.C. (1978).

18. C. Berger and A. D. Kelmers, U.S. P. 3,497,389 (F. P. 1,473,478); Patent filed 10/20/1964, issued 2/24/1970; C. Berger and M. P. Strier, Am. Chem. Soc. Div. Fuel Chem. Preprints, 9, No. 3, 143 (September 1965); R. P. Hamlen and E. J. Szymalak, Electrochem. Technology $\underline{4}, 172$ (1966).

19. F. T. Bacon in Trends in Electrochemistry, J. O'M. Bockris, D. J. Rand and B. J. Welch, eds., Plenum, New York (1977); J. Electrochem. Soc. 126, 7C (1979).

20. H. G. Petrow and R. J. Allen, U.S. P. 3, 992, 331;3,992, $512(11 / 16 / 1976) ; 4,044,193$ $(8 / 23 / 1977) ; 4,059,541(11 / 22 / 1977) ; 4,082,695$ (4/4/1978).

21. V. M. Jalan and C. L. Bushnell, U.S. P. 4,136, 056, 4, 137, $373(1 / 23 / 79)$.

22. H. R. Kunz and G. A. Gruver, J. Electrochem. Soc. 122, 1279 (1975); W. M. Vogel and J. Lundquist, ibid. 117,1517 (1970).

23. Anon., "4.5 MW Fuel Cell Power Plan Development Program, "EM-3856-ID, EPRI, Palo Alto, CA (1984).

24. M. Kobiashi, Presentation at the 1985 National Fuel Cell Seminar, Tucson, AZ, May 1922, 1985.

25. L. M. Handley, "Description of a Generic 11-MW Fuel Cell Power Plant for Utility Applications," EM-3161, EPRI, Palo A1to, CA (1983).

26. A. J. Appleby and E. A. Gillis, "Fuel Cell Developments in the United States," in International Energy Agency. New Energy Conservation Technologies and Their Commercialization, p. 2618, Springer, New York (1981).

27. L. M. Handley and R. Cohen, "Specification for Dispersed Fuel Cell Generator," EM2123, EPRI, Palo Alto, CA (1981).

28. T. G. Bengamin, "Handbook of Fuel Cell Performance," p. 104, Institute of Gas Technology, Chicago, IL (1980).

29. P. N. Ross, Ext. Abs. 1985 National Fuel Cell Seminar, Tucson, AZ, May 19-22, 1985, p. P2-12A, EPRI, Palo Alto, CA. 
30. B.S. Baker et al, "Development of Phosphoric Acid Fuel Cell Technology, "Final Report, DoE Contr. No. EC-77-C-03-1404, September 1978.

31. R. D. Breault, U.S. P. 4,017,664 (4/12/1977).

32. E. V. Somers, R, E. Grimble and E. J. Vidt, "Evaluation of Digas TM Cooling for Utility Fuel Cell Power Plants," EPRI EM-1365, EPRI, Palo Alto, CA (1980).

33. D. Newby and J. M. Feret, "Phosphoric Acid Fuel Cell 7.5 MWe Electric Power Plant Conceptual Design Report," WAESD TR-83-1002, Westinghouse Electric Corporation, Pittsburgh, PA (May 1983).

34. S. J. Markbreiter and I. Weiss, "Technical Assessment of UTC FCG-1 Design." EPRI RP-1777 - 2, EPRI, Palo Alto, CA (1983).

35. P. Mighdoll, M. A. Cobb, K. Meacham and C. S. Skinner, "Fuel Cell Power Plant Manufacturing Cost Analysis, "EPRI, Palo Alto, CA (1984).

36. J. C. Trocciola, U.S. P. 3,905,832 (9/16/75); D. A. Landsman and E. J. Thiery, U.S.P. 3, 956,014(5/11/1976).

37. L. M. Handley, "Integrated Cell Scale-Up and Performance Verification, "EPRI EM-1134; R. D. Breault, "Improved FCG-1 Technology," EPRI EM-1566, EPRI, Palo Alto, CA $(1979,1980)$.

38. Anon., "Zinc-Chlorine Battery Graphite Costs," Interim Report, RP1198-9, EPRI, Palo Alto, CA (1981).

39. P. Stonehart and J. P. MacDonald, "Stability of Acid Fuel Cell Cathode Materials," EPRI EM-1664, EPRI, Palo Alto, CA (1981).

40. P. N. Ross, Ext. Abstr. Electrochem. Soc. Fall Meeting, Las Vegas, NV, 1985.

41. P. Stonehart and J. P. MacDonald, "Stability of Acid Fuel Cell Cathode Materials," Final Report, RP1200-2, EPRI, Palo Alto, CA (1984).

42. S. Sarangapani, J. R. Akridge, and B. Schumm, eds. , The Electrochemistry of Carbon, The Electrochemical Society, Princeton, NJ (1984).

43. A. J. Appleby in Ref. 38, pp. 251-272.

44. J. M. Feret, Quarterly Technical Report, NASA DEN3-290, December 1984-February 1985.

45. V. M. Jalan, private communication, Aprîl 1985.

46. E. B. Yeager, P. Bindra, S. Clouser, and S. Sarangapani, "O Reduction on Platinum Catalysts in $\mathrm{H}_{3} \mathrm{PO}_{4}$," EPRI EM-1814, EPRI, Palo Alto, CA (1981).

47. A. J. Appleby, J. Electrochem. Soc. 117, 1373 (1970).

48. V. Jalan, E. J. Taylor, M. Desai, and B. Morriseau, Ext. Abs. Electrochem. Soc. Fall Meeting, p. 808 , New Orleans, LA (1984).

49. L. J. Bregoli, Electrochem. Acta 23, 489 (1978).

50. V. Jalan, Ext. Abs. Electrochem. Soc. Spring Meeting, p. 584, Montreal, QUE (1982).

51. P. N. Ross, "Oxygen Reduction on Supported Pt Alloys and Intermetallic Compounds in Phos phoric Acid," EPRI-EM-1553, EPRI, Palo Alto, CA (1980).

52. V. M. Jalan and D. A. Landsman, U.S. P. 4, 186,110 (1/29/1980); V. M. Jalan, D. A. Landsman and J. M. Lee, U.S.P. 4,192,907 (3/11/1980); V. J. Jalan, U.S.P. $4,202,934(5 / 13 / 1980)$.

53. J. N. Bronsted, Chem. Rev. 5, 231 (1928).

54. J. Horiuti and M. Polanyi, Acta Physicochim. USSR 2, 505 (1935).

55. A. J. Appleby in Modern Aspects of Electrochemistry, Vol. 7, B. E. Conway, J. O'M. Bockris, E. Yeager, S. U. M. Khan, and R. E. White, eds., p. 369, Plenum, NY (1974).

56. A. J. Appleby in Comprehensive Treatise of Electrochemistry, Vol. 7, B. E. Conway, J. O'M. Bockris, E. Yeager, S. U. M. Khan, and R. E. White, eds., p. 173, Plenum, NY (1983).

57. A. A. Balandin, The Problems of Chemical Kinetics, Catalysis and Reactivity, p. 462 , Academy of Sciences, Moscow (1955).

58. R. Parsons, Trans. Farad. Soc. 54, 1053 (1958).

59. H. Gerischer, Bull. Chim. Soc. Belge 67, 506 (1958).

60. B. E. Conway and J. O'M. Bockris, J. Chem. Phys. 26, 532 (1956).

61. A. Damjanovic and V. Brusic, Electrochim. Acta 12, 615 (1967); A. J. Appleby, J. Electrochem. Soc. 117, 328 (1970).

62. E. A. Moelwyn-Hughes, Physical Chemistry, p. 934, Pergamon, NY (1965).

63. L. Pauling. The Nature of the Chemical Bond, Cornell Univ. Press, Ithaca, NY (1948); D. D. Eley, J. Phys. Coll. Chem. 55, 1017 (1961).

64. A. J. Appleby, Catalysis Reviews 4 (2), 221 (1970).

65. A. J. Appleby, J. Electroanal. Chem. 27, 347 (1970); J. Electrochem. Soc. 117, 1159 (1970).

66. L. Brewer in Phase Stability in Metals and Alloy g, p. 39. P. Rudman, J. Stringer and R. Jaffee, eds., McGraw-Hill, NY (1967).

67. M. Pourbaix, Atlas d'Equilibres Electrochimiques. Gauthier-Villars, Paris (1963).

68. V. Jala n, Ext. Abs. Electrochem. Soc. Spring Meeting, p. 581, Montreal, QUE (1982).

69. V. Jalan and E. J. Taylor, J. Electrochem. Soc. 130, 2299 (1983).

70. C. J. Maggiore, P. J. Hyde, and S. Srinivasan, Ext. Abs. Electrochem. Soc. Spring Meeting, P. 605, Montreal, QUE (1982). 
71. D. A. Landsman and F. J. Luczak, U.S. P. 4, 316, $944(2 / 23 / 1982) ;$ U.S. P. 4, 373,014 (2/8/1983).

72. F. J. Luczak and D. A. Landsman, U.S.P. 4,447, $586(8 / 5 / 1984)$.

73. J.Buggy, Westinghouse presentation to EPRI, May 16, 1984.

74. J. Brown, B. Nazar, and V. Varma, "Site-Specific Assessment of a 50 MW Coal Gasification-Air-Cooled Fuel Cell Power Plant," RP1041-11, EPRI, Palo Alto, CA (1983).

75. J. Brown, A. Murphy, P. Pietrogrande, and J. Tien, "Site-Specific Assessment of a 150-MW Coal Gasification Fuel Cell Power Plant, EPRI-EM-3162, EPRI, Palo Alto, CA (1983).

76. M. Ueno, Presentation at a "Seminar on New Japanese Technology," Cleveland, $O H$, October 25, 1982.

77. L. G. Christner and D. C. Nagle, U.S. P. 4,115,627 (9/19/1978).

78. W. H. Johnson, T. G. Schiller et al, 'Electric Utility Acid Fuel Cell Stack Technology Advancement, Final Report," DOE/NASA/01910-1, NASA CR-1748094; NASA-Lewis Regearch Center, Cleveland, OH (1984).

79. S. T. Lee, "A pplication of Fuel Cells on Utility Systems, Final Summary Report," RP1677-6, EPRI, Palo Alto, CA (1984).

80. J. P. King, A. P. Meyer, C. A. Reiser, and C. R. Schroll, 'Molten Carbonate Fuel Cell System Verification and Scale-Up," RP1273-1, EPRI, Palo Alto, CA (1984).

81. R. D. Breault, R. P. Harding, and F. S. Kemp, U.S. P. 4, 043, $933(8 / 23 / 1977)$; G. M. Kohlmayr and P. Stonehart, U.S.P. 4, 163,811(8/7/1979); R. D Breault and G. J. Goller, U.S. P. 4, 233,181 (11/11/1982); R. D. Breault, A. J. Decasperis and R. J. Rothlein, U.S.P. 4, 269,642 (5/26/1981).

82. Presentation by MELCO to Netherland's Fuel Cell Group, Amsterdam, October 1984.

83. J. Houseman, G. Voecks, and R. Shah, "Autothermal Reforming of No. 2 Fuel Oil," EPRI-EM-1126, EPRI, Palo Alto, CA (1979).

84. P. N. Ross, J. Electrochem. Soc. 130, 882 (1983).

85. P. C. Andricacos and P. N. Ros 8, Ext. Abs. Electrochem. Soc. Spring Meeting, p, 1187 , San Francisco, CA (1983).

86. O. Petrii, S. Vasina, and L. Lukyanycheva, Sov. Electrochem. 17, 1144 (1982).

87. J. O'M. Bockris, M. A. Habib, and P. Zelenay, ongoing work under EPRI 1200-9, to be published.

88. E. Yeager, ongoing work under EPRI 1200-7, to be published.

89. A. A. Adams and H. Barger, J. Electrochem. Soc. 121, 987 (1974); A. A. Adams, R. Foley, and H. Barger, ibid. 124, 1228 (1977); A. J. A ppleby and B. S. Baker, ibid. 125, 404 (1978).

90. B. S. Baker, Final Technical Report, U.S. Army Mobility Equipment Research and Development Command, Contract No. DAAK02-73-C-0084, February 1975.

91. A. J. Appleby in Progress in Batteries and Solar Cells, 5, p. 246, H. Shimotake et al, eds., JEC Press, Inc., Cleveland, OH (1984).

92. P. N. Ross, Lawrence Berkeley Laboratory, Berkeley, CA, private communication.

93. D. Crouse, ECO, Inc., Cambridge, MA, work performed under EPRI RP1676-1 (1980).

94. A. J. Appleby, EPRI, patent applied for.

95. W. M. Vogel and K. Klinedinst, J. Adhesion 9(2), 123 (1978).

96. J. Giner and C. Hunter. J. Electrochem. Soc. 117.1124 (1969).

97. R. P. Iczkowski and M. B. Cutlip, ibid. 127,1433 (1980).

98. P. N. Ros8, ibid. 127,2655 (1980).

99. K. Klinedinst, W. Vogel, and P.Stonehart, J. Mater. Sci. 11, 794 (1976).

100. S. Motoo, M. Watanabe, and N. Furuya, J. Electroanal. Chem. 160, 351 (1984).

101. M. Watanabe, M. Tomkawa, and S. Motoo, Ext. Abs. Electrochem. Soc. Spring Meeting, p. 576, Cincinnati, OH (1984).

102. J. O'M. Bockris, Nature 224, 775 (1969); cf., L. J. Mandel, ibid. 225, 450 (1970).

103. V. M. Jalan, onging work under EPRI RP1200-8, to be published.

104. V. M. Jalan, E. J. Taylor, D. Frost, and B. Morriseau, Ext. Abs. 1983 National Fuel Cell Seminar, p. 127. Nov, 13-16, 1983 Orlando, FL, EPRI, Palo Alto, CA (1983).

105. P. N. Ross and A. J. Appleby, presentation at ACS Meeting on "New Surface Science in Catalysis," Philadelphia, PA, August 26-31, 1984; to be published in the proceedings of the meeting.

106. A. J. Appleby and M. Appel, C. R. Acad. Sci. Paris Ser. C. 280, 551 (1975).

107. A. J. Appleby, J. Electrochem. Soc. 117, 641 (1970).

108. E. Yeager, D. Scherson, and B. Simic-Glavaski, Ext. Abs. Electrochem. Soc. Spring Meeting, p. 1043, San Francisco, CA (1983).

109. A. J. Appleby, J. Electroanal. Chem. 27, 324, 335 (1970); J. Electrochem. Soc. 117. $1158(1970)$.

110. B. E. Conway, to be published.

111. A. J. Appleby, presentation at Kendell Award Symposium, ACS Meeting, St. Louis, MO, April 1984.

112. V. S. Bagotskii and I. Yablokova, Zh. Fiz. Khim. 27, 1663 (1953). 
113. A. J. Appleby, Surface Science 27, 225 (1971).

114. D. A. Scherson, R. W. Grimes, R. Holze, A. Tanaka, C. Fierro, E. Yeager, and R. Lattimer, Ext. Abst. Electrochem. Soc. Fall Meeting, p. 810, New Orleans, LA (1984).

115. J. A. S. Bett, H. R. Kunz, and S. W. Smith, "Investigation of Alloy Catalysts and Redox Catalysts for Phosphoric Acid Electrochemical Systems," FCR-6440, prepared for the Los Alamos National Laboratory, Los Alamos, NM (1984). 


\section{ALKALINE FUEL CELLS (AFCs) ${ }^{\dagger}$}

\subsection{Introduction}

The first successful, semi-practical fuel cell was the $\mathrm{H}_{2}-\mathrm{O}_{2}$ alkaline cell, engineered by Bacon. His work started in 1932, and culminated in a $5-\mathrm{kW}$ system in 1955 . Bacon's FCs used $30 \% \mathrm{KOH}$ as electrolyte and operated at $200^{\circ} \mathrm{C}$ and a pressure between 45 and 50 bars. 1 The anode consisted of a sintered nickel electrode of dual porosity, and the cathode was lithiated nickel oxide. The original cell was a sandwich arrangement of two circular disk electrodes, face-to-face, with a thin circulating electrolyte layer between them. The three-phase boundary (compare Chapter 2) in the electrode was maintained by differential gas pressure, since wetproofing agents such as teflon are not stable at high temperatures in alkaline solution, and indeed did not exist until well after Bacon's work started. 1 Basically, Bacon used an engineer's approach to high cell performance: for the highest reaction rates, high pressures and temperatures were used in an electrolyte (KOH) in which easily a vailable materials (nickel) are stable.

In this chapter, the development of AFCs will be described briefly. A reexamination of $A F C_{B}$ is appropriate because active development of FCs in the U.S. and Japan has emphasized the higher temperature $\left(190-210^{\circ} \mathrm{C}\right)$ phosphoric acid fuel cell (PAFC) described in Chapter 2. The concentrated PA as electrolyte involves acceptance of low conductivity at low cell operating temperatures $\left(\sim 100-120^{\circ} \mathrm{C}\right)$, although, at normal operating temperature, the PA conductivity is acceptable and equals about one half of that of concentrated KOH. Furthermore, freezing of concentrated PA occurs between 40 and $50^{\circ} \mathrm{C}$, so that the cell has no self-starting capability in cold weather. Since freezing may cause electrode degradation, it is difficult to envisage the presently conceived PAFC as a small power unit for personal transportation vehicles. Another practical problem involved in the PAFC is the limited range of electrode catalysts which are usable at high anodic potentials (i.e., at the oxygen cathode) in acidic solutions. This has thus far been limited to the noble metals and their alloys. Since the loadings used are very low, their cost $/ \mathrm{kW}$ is presently not an important factor (about $\$ 70 / \mathrm{kW}$ at present $\mathrm{Pt}$-producer prices). However, if the PAFC finds a broad-based future market, noble metal availability may become a limiting problem (cf. Sec. 2.6).

As is discussed in Sec. 2.15, it has been suggested that adsorption of PA on the electrocatalyst reduces the rates of oxygen-electrode kinetics. 2 The use of superacids such as fluorinated sulfonic, phosphoric, or phosphinic acids (Chapter 2) may be a solution to this difficulty, but these have relatively high costs and, at high temperatures, they have limited ionic conductivity and possibly marginal stability.

AFCs have the following advantages: (1) Their cell life may ultimately be longer ${ }^{\delta}$ than that of acid FCs because of greater compatibility of alkaline electrolytes with practical cell materials. A 10,000-h life has already been achieved by Alsthom with low-cost half-cell components, while NASA and UTC have achieved over $15,000 \mathrm{~h}$ with space-shuttle AFC stacks.

(2) Thermodynamic considerations $s^{3}$ hw $^{3}$ that the range of possible catalysts is greater. (3) ATC can operate at significantly higher efficiencies (up to $60 \%$, based on the HHV) on pure $\mathrm{H}_{2}$ than present acid cells $\left(50 \%\right.$, on pure $\left.\mathrm{H}_{2}\right)$. (4) The cell-component cost per $\mathrm{m}^{2}$ of AFCs are substantially lower than for PAFCs.

Alkaline cells are particularly suitable for direct use with pristine $\mathrm{H}_{2}$, since no special equipment for $\mathrm{CO}_{2}$ removal is needed. Pure $\mathrm{H}_{2}$ may be obtained from hydroelectric resources or nuclear electricity. 4 Pure $\mathrm{H}_{2}$ may also be obtained from the steam-iron process (using coal or biomass to reduce the ferric oxide) and from other coal-based technologies. The cost of $\mathrm{H}_{2}$ from this source is unlikely to be significantly greater than that of synthetic NG. 4 Hydrogen

t This chapter was written by J. O'M. Bockris and A. J. Appleby. The authors are indebted to several of their AFCWG colleagues for helpful suggestions, especially H. Wroblowa.

$F_{R}$. Watson of the Imperial College (Iondon) Electrochemistry Group carried out the experimental work on the electrochemical engineering of the $5-\mathrm{kW}$ cell.

Indeed, lifetimes of $15,000 \mathrm{~h}$ have been obtained by UTC for AFCs. The mechanism of failure at this time is attack on the frames and may be subject to correction in future AFCs. Indeed, application of a "rejuvenation process" every $15,000 \mathrm{~h}$ has enabled $40,000 \mathrm{~h}$ "Iifetimes" to be achieved. 
from nuclear and hydroelectric sources may be available in some countries (in particular, Canada) at a cost of less than \$10/MMBTUं and the CANDU Th-cycle reactor, coupled in 1,000-MW units to water-electrolysis plants, could yield inexpensive $\mathrm{H}_{2}$ by using electricity costing $1.5 \mathrm{c} / \mathrm{kWh}$. With an electrolysis cell potential of $1.6 \mathrm{~V}$, the cost of $\mathrm{H}_{2}$ produced would be about $\$ 9 / \mathrm{MMBTU}$, assuming a plant cost of about $\$ 400 / \mathrm{kW}$.

The Hot-Elly process was developed by Jülich ${ }^{5}$ and uses solid-oxide cells similar to those described for SOFCs in Chapter 6. As electrolyzer s, these cells have a large thermody. namic advantage over low-temperature systems, whereas the converse is true when they are used as FCs. Typically, they possess an open-circuit potential at $85 \%$ steam utilization of about $1.0 \mathrm{~V}$ at $1000^{\circ} \mathrm{C}$ and operate at $400 \mathrm{~mA} / \mathrm{cm}^{2}, 1.3 \mathrm{~V}$ (thermoneutral potential for $\mathrm{H}_{2} \mathrm{O}$ electrolysis).

Coupled with hydroelectric electricity at $1.0 \mathrm{c} / \mathrm{kWh}, \mathrm{H}_{2}$ costs using this process would be c. $\$ 6 / \mathrm{MMBTU}$, but only if cell costs for this solid oxide path are not substantially greater than those for low temperature cells. In summary, substantial amounts of low-cost electricity that may be used for the production of $\mathrm{H}_{2}$ with advanced electrolyzers exist in various parts of the world. 6 Greenland has particularly large, but undeveloped, resources and, if the $\mathrm{H}_{2}$ generated could be transported economically to the U.S. (in the form of $\mathrm{NH}_{3}$ ), then about $20 \%$ of the automotive fuel needs could be supplied (see Sec. 3.10).

The Pratt and Whitney Division of UTC proposed electric utility use of FCs in the early $1970 \mathrm{~s}$ at the conclusion of the gas - utility TARGET program (Sec. 2.7). TARGET was a total energy concept using NG for heating and the electricity supply to residential and commercial buildings. For this program, heat is an essential product of FC operation, since waste heat from the FC is required in both steam-reforming of methane to hydrogen and space-heating onsite. UTC pointed out to the utilities that the PAFC technology could be applied with little additional development cost for the stand-alone production of electricity. They said that, if AFCs were used, the system-development cost, including gas clean-up, from $\mathrm{CO}_{2}$, would be greater, despite the acknowledged higher performance of $A F C_{s}$ compared with acid cells on pure $\mathrm{H}_{2}$.

\section{2 AFC Development}

An excellent review of early AFC electrodes and of cell electrochemistry prior to 1968 is given in Ref.7. A valuable account of early engineering and applications was published in 1973 by NASA. 8

The performance of the Bacon cell of $1955-60$ is still considered to be excellent (Fig. 3, 2-1). This fact is not surprising in view of the high operating pressure $(\sim 42$ bars) used. Starting about 1956, workers at UTC began a large FC program, based on the cell concepts of Bacon and co-workers. For weight and safety reasons, they modified the Bacon cell to operate at a higher temperature $\left(260^{\circ} \mathrm{C}\right)$ but at lower pressure. This work led to the cell stacks used on the Apollo lunar missions during the period 1968-75. Because of the use of reduced operating pressure, the performance was inferior to that shown in Fig. 3.2-1.

About the same time, Union Carbide (UC) developed a different type of AFC, using thin active carbon electrodes. This cell is shown schematically in Fig. 3.2-2. The porous metal backing plate supplying the gas was a nickel screen with carbonyl nickel powder sintered to its surface. It was impregnated with teflon, which is stable at the 10 $50-80^{\circ} \mathrm{C}$ operating temperature and makes the electrode water repellent. Adjacent to the screen was the carbon catalyst with a teflon binder. These cells attained $0.8 \mathrm{~V}$ at $150 \mathrm{~mA} / \mathrm{cm}^{2}$ using $\mathrm{CO}_{2}-8 \mathrm{crubbed}$ air.

Allis-Chalmers developed an FC tractor in 1959, and this was followed by the first FCpowered road vehicle, the GM Electrovan of 1964, which used a $32-\mathrm{kW}$ UC AFC system. 8 This was a demonstration unit and not a practical vehicle, since it was fueled by liquid $\mathrm{H}_{2}$ and liquid $\mathrm{O}_{2}$. During the late $1960 \mathrm{~s}$, Kordesch 8 , 9 built a six-kW $\mathrm{e}$ F-battery vehicle power-source using UC monopolar back-to-back bicells operating on compressed $\mathrm{H}_{2}$ and scrubbed air. The system was a hybrid that used lead-acid batteries for start-up and acceleration. The vehicle was based on an Austin A40 sedan and had a cruising speed of $40 \mathrm{mph}$ with a range of $200 \mathrm{miles}$.

The principal early rival of Bacon was Justilo in Germany. Justi's cells had dual porosity for capillary interface control, and the support layer of sintered carbonyl nickel was homoporous with each pore active at a given pressure. The catalyat layers in the electrodes were made from Raney $\mathrm{Ni}$ and $\mathrm{Ag}$, and, therefore, had very high surface area compared with the sintered carbonyl nickel used by Bacon. Justi referred to his electrodes as DSK (in English, double skeleton catalyst) electrodes, because of their dual-layer structure. A detailed account of their preparation is given in Ref.7. Justi's cell design was developed by Varta, Siemens, and Brown Boveri, and strongly influenced other AFC designs (for example, that of the CGE and IFP). All of the cells used circulating $\mathrm{KOH}$ as the electrolyte for heat removal and water elimination by evaporation.

${ }^{\dagger}$ Some Canadian sources (1985) sell hydroelectric electricity at $0.42 \mathrm{c} / \mathrm{kWh}$ in massive purchases. Taking a high-temperature electrolyzer potential to be c. $1.3 \mathrm{~V}$, the corresponding cost of electrolytic $\mathrm{H}_{2}$ would be about $\$ 4.50$ and in the cost range of natural gas at this time. 


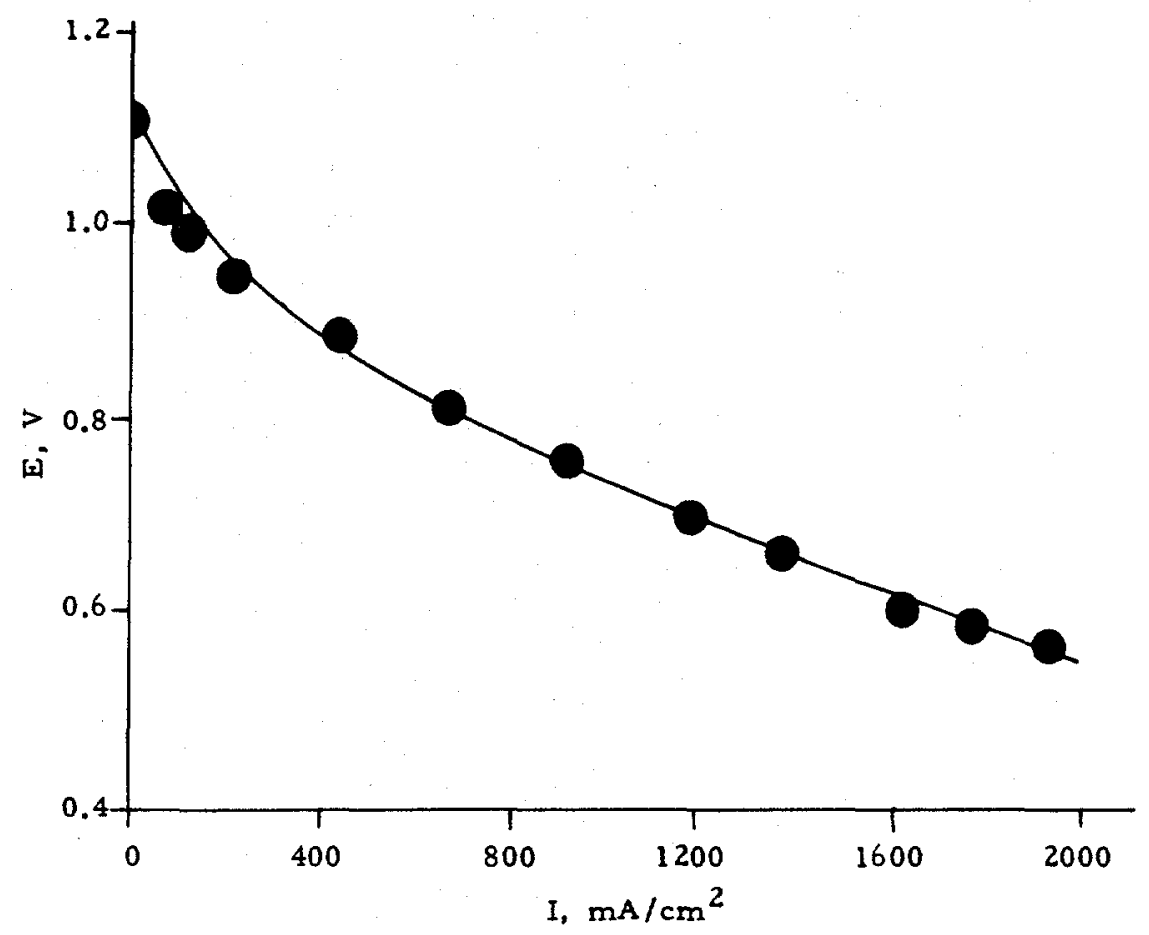

Fig. 3.2-1. Performance of a Bacon cell (1955-60) at $200^{\circ} \mathrm{C}$, 45 bars, with $\mathrm{H}_{2} / \mathrm{O}_{2}$.

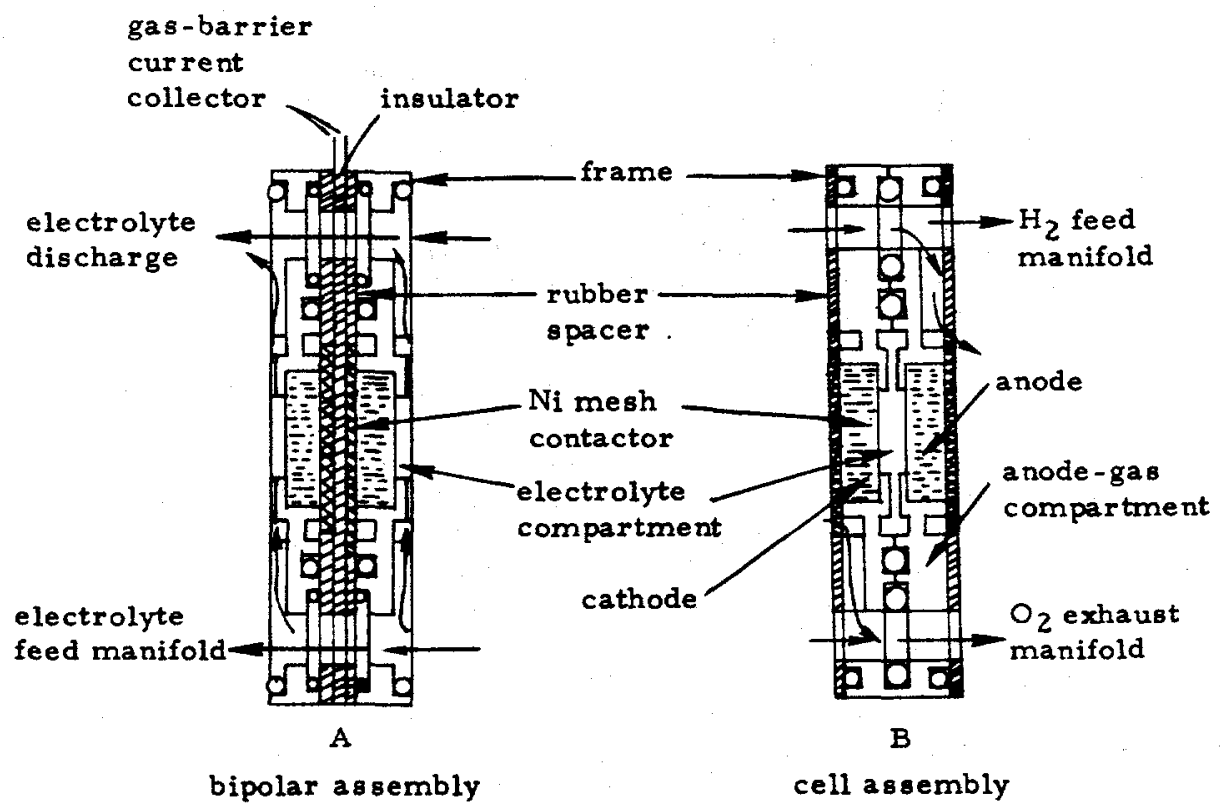

Fig. 3. 2-2. A basic cell assembly of the Union Carbide $\mathrm{H}_{2}-\mathrm{O}_{2} \mathrm{FC}$. 
The third U.S. corporation to enter FC development in the 19608 was Allis-Chalmers. Workers there used $\mathrm{Ni}$ a nodes and $\mathrm{Ag}$ cathodes, following Justi's work. They employed a sys tem with immobilized electrolyte, an interesting feature of which was the device used for water removal. This consisted of a second sintered Ni membrane in each cell with higher $\mathrm{KOH}$ concentration than in the active electrolyte. In the space version, the cell was arranged to eliminate water by evaporation to vacuum on its reverse side. This design is illustrated schematically in Fig. 4.8-3. The major advantage of this design was bipolar construction with a narrow gap between the electrodes, which led to low IR losses.

$A$ number of terrestrial versions of the Allis-Chalmers cell design were tested, one being a $5-\mathrm{kW}$ unit which ran intermittently for $4,500 \mathrm{~h} .8$ Instead of using active water loss by evaporation from the back of the anode-reservoir plate, a concentrated flowing electrolyte solution was employed for water removal. All designs were, however, bipolar with low cell IR drop. In 1959, a $20-\mathrm{kW}$ unit of this type was mounted in a tractor, 8 which was shown to function adequately. Examination of this tractor led A. N. Frumkin to initiate the Soviet FC program around $19610^{+}$

Organic materials, especially saturated $\mathrm{HC}_{s}$, were used as a fuel in research by a number of oil companies, particularly Exxon (then Esso) and Shell, during the 1960s. As is indicated in Sec. 2.7, these efforts failed because of the very low equilibrium reaction rate or exchange current ( $i_{0}$ ) obtained for HC oxidation, even on Pt. 7,11 In 1969, workers at Esso concluded that further electrocatalytic research would probably result in workable hydrocarbonbased FCs but would require very costly high-loading noble metal catalysts.

Ammonia and hydrazine were also examined as direct fuels, and the use of low-temperature molten salts (e.g., 50-85\% $\mathrm{KOH}$ ) as electrolytes was attempted. These cells were less successful than those using $\mathrm{H}_{2}$; cells using ammonia showed low reaction rates, whereas hydrazine, though active, was expensive and carcinogenic. Another possible fuel was methanol. Despite relatively favorable kinetics, non- $\mathrm{CO}_{2}$ rejecting electrolytes such as $\mathrm{KOH}$ could not directly consume methanol because of carbonation. The only usable $\mathrm{CO}_{2}$-rejecting media at moderate temperatures appeared to be acids or buffers such as cesium carbonate-bicarbonate. 12

\section{3. $3 \quad \mathrm{CO}_{2}$ Removal}

AFCs suffer from the difficulty that a fuel mixture containing $\mathrm{CO}_{2}$ from steam reforming or partial oxidation of coal must have the $\mathrm{CO}_{2}$ eliminated after the water-gas shift of any $\mathrm{CO}$ present, before the $\mathrm{H}_{2}$ is consumed in the cell. Furthermore, there are about $384 \mathrm{ppm}^{\text {of }} \mathrm{CO}_{2}$ in the ambient air used at the cathode.

Three negative performance effects arise from the presence of carbonate in the alkaline electrolyte. First, $\mathrm{CO}_{2}$ removes $\mathrm{OH}^{-}$by the process $\mathrm{CO}_{2}+2 \mathrm{OH}^{-} \rightarrow \mathrm{CO}_{3}^{-}+\mathrm{H}_{2} \mathrm{O}$, thus reducing the $\mathrm{OH}^{-}$ion concentration and interfering with the electrode kinetics, especially in porous electrodes. Furthermore, the presence of carbonate causes electrolyte viscosity to increase. Since the diffusion rate varies inversely with viscosity, a decline occurs in the limiting current. In addition, the electrolyte surface tension changes and, thus, the non-wetting properties of the porous electrode eventually cause electrolyte precipitation in the micropores of the electrode, which may thereby become partially inactive, as will be discussed in connection with the decay of $\mathrm{C}$ electrodes.

It is important to remove $\mathrm{CO}_{2}$ to about $10 \mathrm{ppm}$ before entry of reactant gases into the $A F C$. This reduction can be carried out by passing the $\mathrm{CO}_{2}$-containing gas through a preliminary scrubber containing $\mathrm{KOH}$ or $\mathrm{NaOH} .13$ The spent alkali can be removed and transferred to an electrodialysis cell, where fresh alkali may be regenerated. Alternatively, the electrolyte can be circulated over a $\mathrm{CO}_{2}$ transfer membrane, which is in contact with an acid solution. A membrane of ion-exchange type can also be used, the $\mathrm{CO}_{2}$ being removed by electrodialysis. $14 \mathrm{Al}-$ though these methods are usable, $\mathrm{CO}_{2}$ removal is so important in AFC operation that this problem will be discussed quantitatively in Sec. 3.8.

\section{4 Electrocatalysis}

The performance of a successful FC is described by the Tafel slopes (i.e., the slope of overpotential as a function of the logarithm of the current density) of the individual anodic and cathodic reactions, together with their exchange current $\left(i_{0}\right)$ values. The oxygen-reduction reaction is the primary target for overpotential reduction in the AFC, as it is in acid solution. However, as is shown in Fig. 2.15-1, the oxygen-reduction Tafel slope in the AFC is typically much lower than that in the PAFC, leading to much lower oxygen over potentials. Hence, at high

†Fumkin, who effectively decided the path of Soviet electrochemical research, was skeptical of FCs because he did not understand the effect of the 3-phase boundary, which greatly increases the limiting current in porous structures involving electrochemical reactions of gases. This skepticism delayed the Soviet program, which was later taken over by Didorenko at the Institute for Battery Research, where significant progress was achieved. 
current density in the AFC, $\sim 20 \%$ of the polarization may be due to $\mathrm{H}_{2}$ oxidation. Electrocatalysis of the AFC anodic reaction therefore remains a significant field for further study.

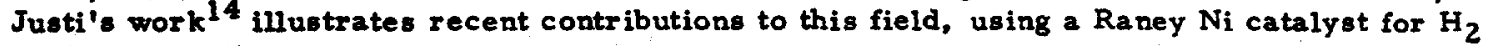
oxidation in alkaline solutions.

The alloys used by $J u s t i$ contained $T_{i}$ for control of sintering, as well as $A 1$ and $N_{i}$. A typical mixture consisted of $50 \% \mathrm{Al}, 48 \% \mathrm{Ni}$, and $2 \% \mathrm{Ti}$, with a particle size of $\sim 50 \mathrm{um}$. After leaching, the mixture spontaneously oxidized so rapidly in air that the catalyst became useless. Thus, air oxidation of $\mathrm{Ni}$ had to be slowed down. This goal was achieved by formation of $\mathrm{O}$ and $\mathrm{OH}$ films on the $\mathrm{Ni}$ surface, which were then reduced by $\mathrm{H}_{2}$. Justi and co-workers described the $\mathrm{H}_{2}$-oxidation process according to Fig. 3.4-1. Two different Tafel slopes were observed, which they ascribed to polarization caused by rate-determining surface diffusion and by electron transfer accompanied by proton discharge, respectively. They ${ }^{15}$ measured the amounts of $\mathrm{Ni}(\mathrm{OH})_{2}$ formed by partial oxidation of the Raney $\mathrm{Ni}$ at $25 \mathrm{mbar} \mathrm{O}_{2}$ partial pressure, and they then related the $\mathrm{Ni}(\mathrm{OH})_{2}$ content to the diffusion over potential ( $\eta_{\text {diff }}$ ), and to the charge-tranafer over potential $\left(\eta_{\mathrm{ct}}\right)$. $\mathrm{Ni}(\mathrm{OH})_{2}$ contents up to $5 \mathrm{wt} \%$ increased the current density three-to fourfold; however, at larger $\mathrm{Ni}(\mathrm{OH})_{2}$ contents, the current became constant. Figure 3.4-2 shows how the values of ratios $\eta_{\text {diff }} / \eta_{\text {ct }}$ varied with wt $\%$ of $\mathrm{Ni}(\mathrm{OH})_{2}$. As the $\mathrm{Ni}(\mathrm{OH})_{2}$ w $\%$ increases, the diffusional overpotential increases and compensates for a decrease in $\eta_{c t}$. The best results were obtained by using $4.5 \% \mathrm{Ni}(\mathrm{OH})_{2}$ (see Fig. 3. 4-3).

Electrocatalysis of the $\mathrm{O}_{2}$-reduction reaction appears to be the key barrier to the prospects of the AFC as an energy-conversion device. The rate-determining steps and mechanisms for reaction in AFCs are not as well known as for PAFCs, though some data are available for noble metal electrodes. In alkaline solutions, $\mathrm{O}_{2}$ evolution on a perovskite surface has been proposed to occur 16,17 as follows:

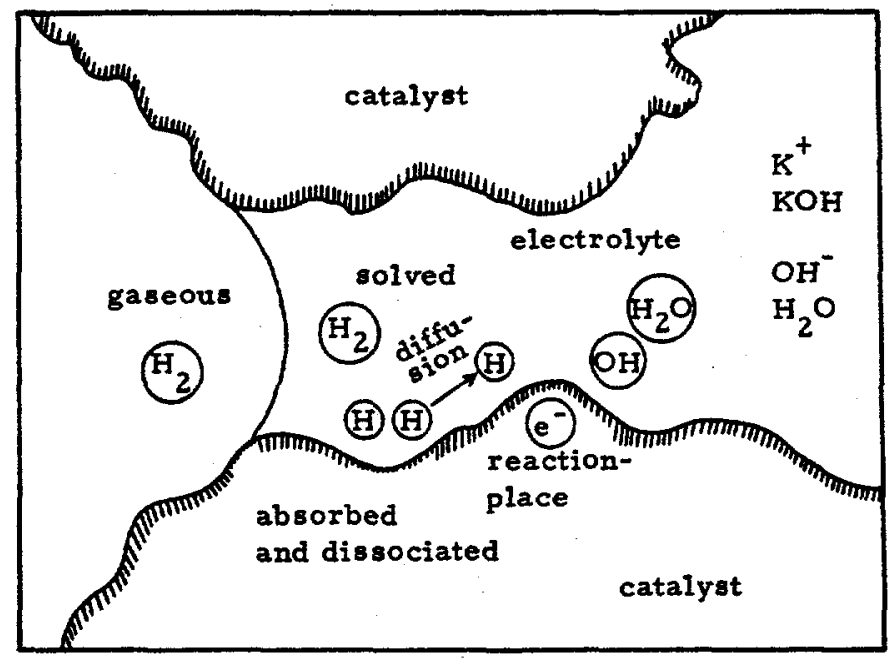

Fig. 3.4-1. A simple model is shown of the reaction steps occurring during the anodic oxidation of hydrogen. 11

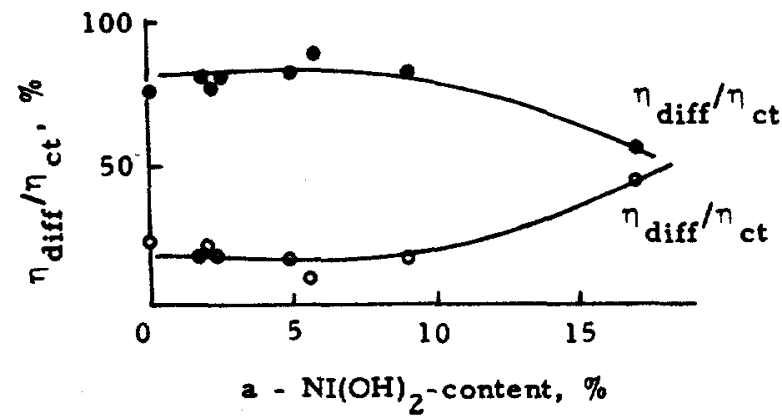

Fig. 3.4-2. Separation of the total polarization of $100 \mathrm{mV}$ into diffusion and charge-transfer polarization as a function of the $\mathrm{Ni}(\mathrm{OH})_{2}$-content of the catalysts. 14 


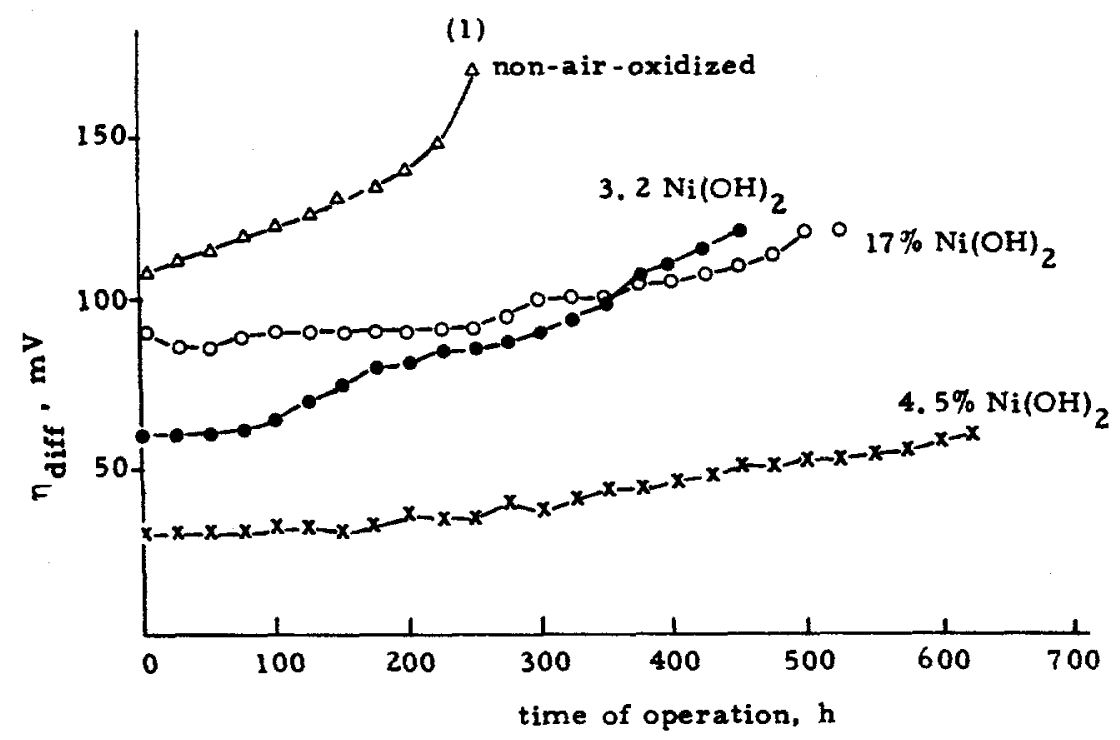

Fig. 3.4-3. Galvanostatic long-term performance tests with supported Raney-NiTi $i_{2}$ electrodes and with non-air-oxidized (1) and air-oxidized catalysts at $80^{\circ} \mathrm{C}$; the catalyst loading was $30 \mathrm{mg} / \mathrm{cm}^{2}$, and the current density ${ }^{14}$ was $4 \mathrm{~A} / \mathrm{g}$. The percentages are wt\%.

$$
\begin{aligned}
& \mathrm{M}^{\mathrm{z}}+\mathrm{OH}^{-} \rightleftharpoons \mathrm{M}^{\mathrm{z}}+\mathrm{OH}+\mathrm{e}^{-}, \\
& \mathrm{M}^{\mathrm{z}}+\mathrm{OH}+\mathrm{OH}^{-\left(\mathrm{x} \cdot \mathrm{d} . \mathrm{s}^{\prime}\right)} \mathrm{M}^{\mathrm{z}}+\mathrm{H}_{2} \mathrm{O}_{2}+\mathrm{e}^{-}, \\
&\left(\mathrm{H}_{2} \mathrm{O}_{2}\right)_{\text {phys.ads. }}+\mathrm{OH}^{-}=\left(\mathrm{HO}_{2}^{-}\right)_{\text {phys.ads. }}+\mathrm{H}_{2} \mathrm{O}, \\
&\left(\mathrm{H}_{2} \mathrm{O}_{2}\right)_{\text {phys.ads. }}+\left(\mathrm{HO}_{2}^{-}\right)_{\text {phys.ads. }}=\mathrm{H}_{2} \mathrm{O}+\mathrm{OH}^{-}+\mathrm{O}_{2},
\end{aligned}
$$

where $M$ is the $B$ ion in $\mathrm{ABO}_{3}$ (usually a first-row transition metal), and the ion $A$ is usually La. According to the principle of microscopic reversibility, near the reversible potential the same reaction path and rate-determining step apply for evolution and dissolution. Hence, if there is no change in the mechanism between the reversible potential and at the cathodic potential of interest, the anodic evolution processes may be used to define the cathodic reduction-reaction steps. This type of information must, however, be used with caution since a change of substrate surface may occur by oxidation between the cathodic and anodic regions, e.g., on Pt in acid solution.

It is important to ascertain if $\mathrm{HO}_{2}^{-}$is a reaction intermediate in $\mathrm{O}_{2}$ reduction, i.e., whether one or both of the following mechanisms occur:

$$
\begin{aligned}
& I_{1} \text { (direct reduction) }
\end{aligned}
$$

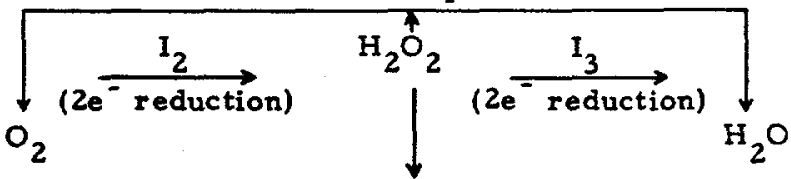

$$
\begin{aligned}
& \text { diffuses away from the electrode surface }
\end{aligned}
$$

Formation of peroxide as an intermediate may be energetically favored, since all multiple electron-transfer processes, either alone or coupled with proton transfer, proceed stepwise. Many reaction intermediates and pathways are therefore possible in the 4-electron, 2-proton process in the AFC, including those with free peroxide as an intermediate. $\mathrm{HO}_{2}^{-}$tends to be 
stabilized in alkaline solution, because of its solvation energy. A rotating ring-disk electrode 18 has often been used to study these processes. If $\mathrm{H}_{2} \mathrm{O}_{2}$ is formed as an intermediate on the disk at a varying series of potentials, an amount of $\mathrm{H}_{2} \mathrm{O}_{2}$ that is known from hydrodynamic theory to be a function of $w^{-1 / 2}$. 19 where $w$ is the rotation rate, will be transmitted from the disk to the concentric external ring electrode. The quantity of peroxide produced is determined from its oxidation current on the ring.

For a simple process, Damjanovic, Genshaw and Bockris ${ }^{18}$ showed that

$$
I_{\text {disk }} / I_{\text {ring }}=(x+1) / N+(x+2) k^{1} / N \omega{ }^{+1 / 2}
$$

where $x$ is the fraction of the reaction which proceeds via $\mathrm{H}_{2} \mathrm{O}_{2}, \mathrm{i}$.e., $\mathrm{I}_{1}=\mathrm{xI}_{2} ; \mathrm{k}^{l}$ is the rate constant for $\mathrm{H}_{2} \mathrm{O}$ reduction on the disk; $\mathrm{N}$ is the hydrodynamic collection efficiency for the particular electrode geometry. If $\mathrm{H}_{2} \mathrm{O}$ is not formed, then there is no ring current. If only $\mathrm{H}_{2} \mathrm{O}_{2}$ is formed and it does not react further, then $I_{\text {disk }} / I_{\text {ring }}=1 / N$ and the ratio of the disk to the ring current is a straight line parallel to the $w^{-1 / 2}$ axis. Alternatively, the pathway may only involve $\mathrm{H}_{2} \mathrm{O}_{2}$, the latter being reduced at the disk. In this case, $x=0, k^{l}$ is finite, and

$$
I_{\text {disk }} / I_{r i n g}=(1 / N)+2 k^{1} / N w^{+1 / 2} \text {. }
$$

The slope will thus depend on the potential, since $\mathbf{k}^{1}$, the electrochemical rate constant, is potential-dependent.

Oxygen may also be reduced to $\mathrm{H}_{2} \mathrm{O}$ and $\mathrm{H}_{2} \mathrm{O}_{2}$ in parallel reactions. If $\mathrm{H}_{2} \mathrm{O}_{2}$ is not further reduced, $k^{l}=0$ and

$$
I_{\text {disk }} / I_{\text {ring }}=(x+1) / N
$$

There is now no dependence of $I_{d i s k} / I_{r i n g}$ on $\omega$, but a dependence remains on potential, since $x$ may be potential-dependent. If all the indicated processes occur, $I_{d i s k} / I_{r i n g}$ has a finite slope and is independent of potential. An early example of this analysis is provided by the work of Damjanovic. 18 In alkaline solution on noble metals, both the direct reduction to $\mathrm{H}_{2} \mathrm{O}$ and the reduction of $\mathrm{H}_{2} \mathrm{O}$ to $\mathrm{H}_{2} \mathrm{O}_{2}$ occur. The peroxide ion is then further reduced at the disk (Fig. 3.4-4). These ideas 18-20 have been modified, corrected, and improved by Wroblowa, Appleby and other 8 , taking into account adsorption processes and more complex sequencesof steps. $21-23$

$\mathrm{Berl}{ }^{24}$ showed that, in alkaline solutions, oxygen reduction on carbon produces $\mathrm{H}_{2} \mathrm{O}$, and the $\mathrm{O}_{2} / \mathrm{H}_{2} \mathrm{O}_{2}$ couple then behaves reversibly. Similar results occur on Ag and Au. Krasilchikov 25 was the first to suggest that the primary transfer reaction $\mathrm{O}_{2}+\mathrm{e}^{-} \rightarrow \mathrm{O}_{2}^{-}$may be ratedetermining and is followed by $\mathrm{O}_{2}^{-}+\mathrm{H}_{2} \mathrm{O} \rightarrow \mathrm{HO}_{2}(\mathrm{ads})+\mathrm{OH}^{-}, \mathrm{HO}_{2}(\mathrm{ads})+\mathrm{e}^{-} \rightarrow \mathrm{HO}_{2}^{-}$. If all these steps are reasonably rapid, the potential of the electrode will be controlled by the activities of $\mathrm{O}_{2}, \mathrm{H}_{2} \mathrm{O}_{2}$ and $\mathrm{OH}^{-}$. Other reactions may regenerate some of the oxygen via the process

$$
2 \mathrm{H}_{2} \mathrm{O}_{2}=2 \mathrm{H}_{2} \mathrm{O}+\mathrm{O}_{2}
$$

Therefore, the behavior of the electrode depends on the ratio of the active surface to the free volume of the electrolyte. If this ratio is large, as in a porous electrode, reaction control by diffusion away of the $\mathrm{HO}_{2}^{-}$is unlikely. These cases require complex kinetic analysis when oxygen recycling occur 8 ; they have been examined in some detail in Ref. 23.

The process $\mathrm{O}_{2}+\mathrm{e}^{-} \rightarrow \mathrm{O}_{2}^{-}$may be a rate-determining step in alkaline solution because of the frequently observed near-independence of $i_{0}$ on the substrate. A volcano relation (cf. Sec. 2.12 and Fig. 2.12-11 and Fig. 3.4-5) for oxygen reduction in acid electrolytes will, therefore, not be expected on noble metals in alkaline solution. Electrocatalysis is, however, ouggested by mechanistic determinations 26 on $\mathrm{Pt}$ which involve $\mathrm{HO}_{2}^{-}$and the rate-determining step

$$
\mathrm{O}_{2}+\mathrm{H}_{2} \mathrm{O}+\mathrm{e}^{-} \rightarrow \mathrm{HO}_{2}(\mathrm{ads})+\mathrm{OH}^{-}
$$

under Temkin isotherm adsorption conditions. In both cases, there should be an increase of reaction rate with increase of adsorption strength of $\mathrm{HO}_{2}^{-}$on the substrate. On the other hand, there are some indications 27,28 for the subsequent rate-determining step

$$
\mathrm{O}_{2} \mathrm{H}(\mathrm{ads})+\mathrm{OH}(\mathrm{ads})+\mathrm{O}(\mathrm{ads})
$$

for which the same dependence on the substrate as before would qualitatively apply.

In summary, electrocatalysis for $O_{2}$ reduction in alkaline solution is less clearly defined than the volcano curve (see Fig. 3.4-5) obtained in acid solution. There are not enough data to ascertain if the volcano relations exist in alkaline solutions. A possible volcano relation seems to be inferred from oxygen evolution work on perovskites (Fig. 3.4-6). Such a dependence of 


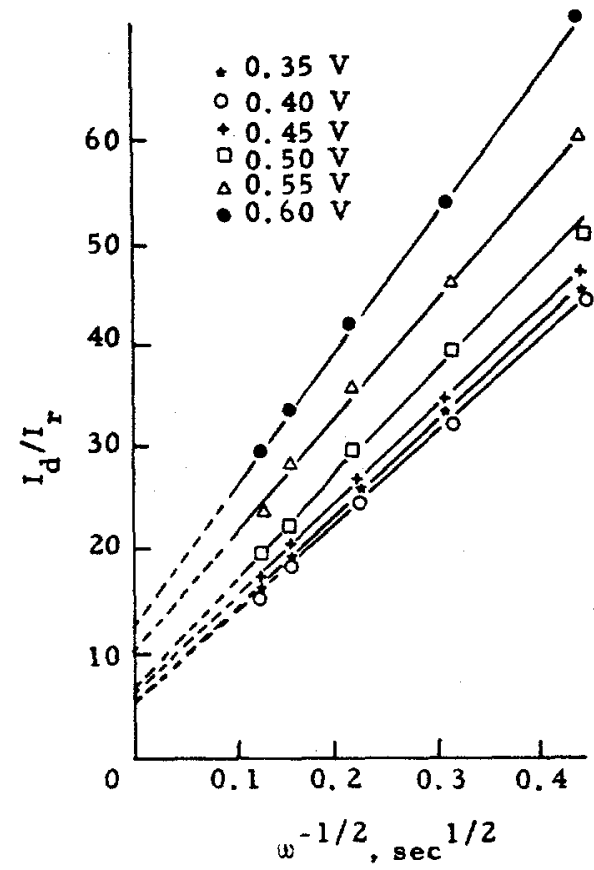

Fig. 3.4-4. Plots of the ratio of the currents at disk and ring electrodes $\left(I_{d} / I_{r}\right)$ in $1 \mathrm{~N}$ KOH vs $w^{-1 / 2}$ at various potentials of the disk electrode. 20

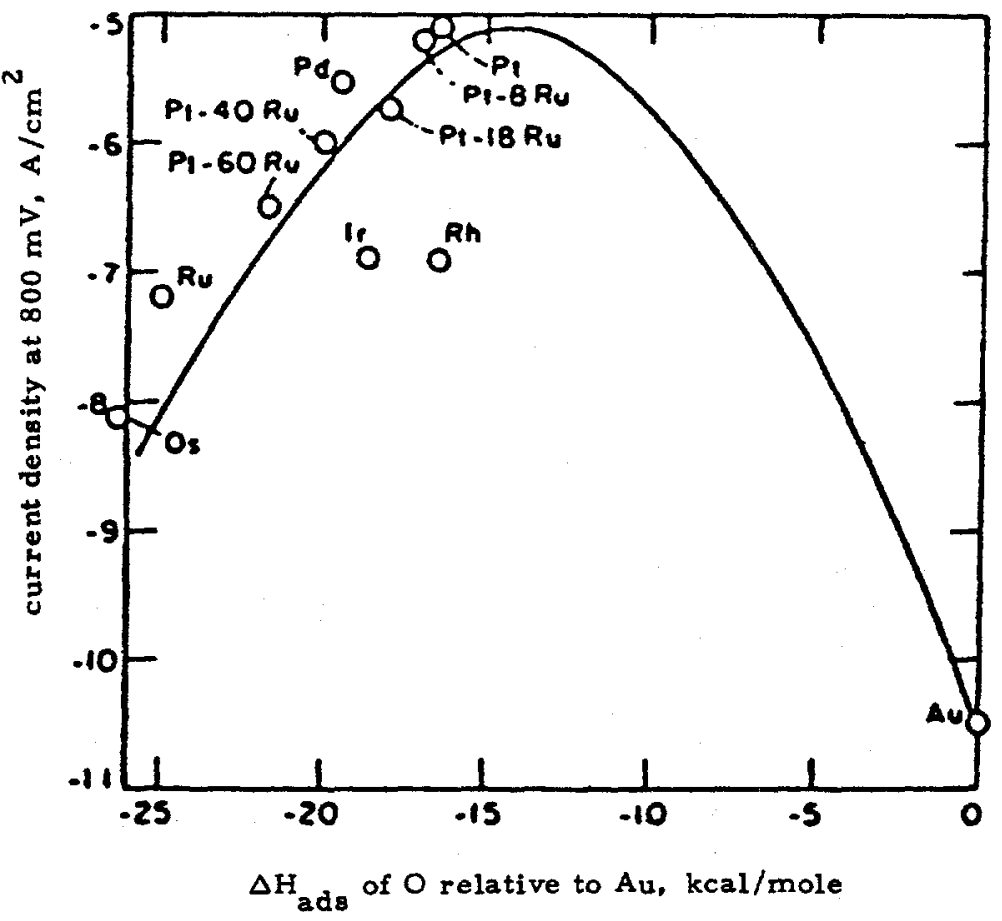

Fig. 3.4-5. O reduction in $85 \%$ orthophosphoric acid. A plot of $i$ at $\eta=-460 \mathrm{mV}$ at $25^{\circ} \mathrm{C}$ is shown vs the calculated $\mathrm{M}-\mathrm{O}$ adsorbate bond atrength using Pauling's methd. (M-O bond strengths are given relative to that of $\mathrm{Au}-\mathrm{O}$. $)^{28}$ The Pt-Ru alloys are expressed in atom $-\%$. 


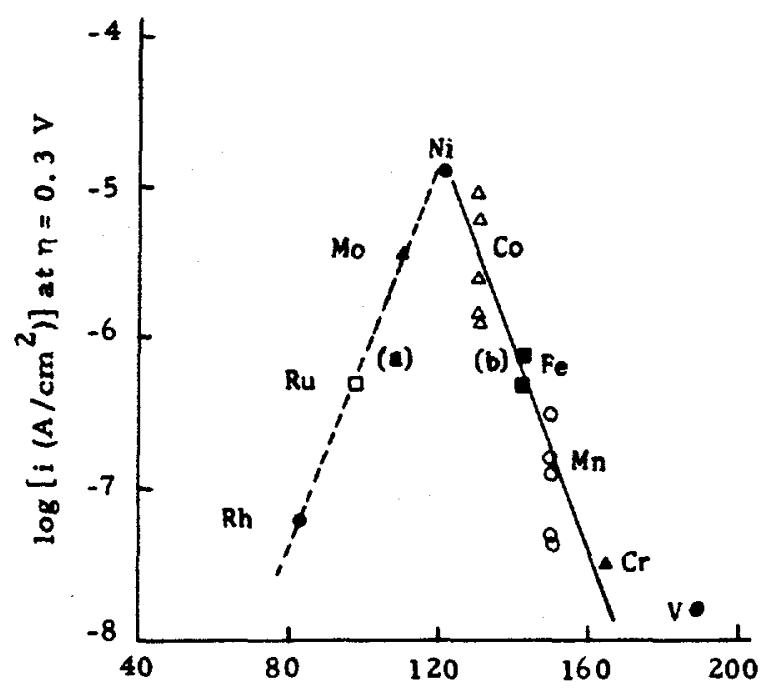

$\mathrm{M}-\mathrm{OH}$ bond strength, $\mathrm{kcal} / \mathrm{mol}$

Fig. 3.4-6. Hypothetical volca no plot for oxygen evolution on perovskites. Transition metal $B$ ions in perovskites are indicated by different symbols; 16 (a), hypothetical relation; (b), real relation.

current density on $\Delta G_{a d s}$ would be important, according to the mechanisms of Damjanovic et al 18 and Appleby. 27,28 On a catalyst showing little variation of $i_{0}$ with substrate, $\mathrm{O}_{2}+e^{-} \rightarrow \mathrm{O}_{2}^{-}$is probably the rate-determining step, and the alkaline solution itself may be regarded as the catalyst because it stabilizes and solvates $\mathrm{O}_{2}^{-}$.

Trangition-metal macrocyclic catalysts have been examined extensively, particularly by Anson et al. 29 Examples of these compounds (porphyrins, phthalocyanines, etc.) are shown in Table 3.4-1. Normally, they are supported on high surface-area carbon to ensure maximum distribution and high conductivity.

The theory of catalysis by porphyrins is ill defined. It is generally supposed that the central ion undergoes the redox process

$$
\mathrm{Mn}^{3+}+\mathrm{e}^{-} \rightarrow \mathrm{Mn}^{2+}
$$

followed by

$$
\mathrm{Mn}^{2+}+\mathrm{O}_{2} \rightarrow \mathrm{Mn}^{3+}+\mathrm{O}_{2}^{-}
$$

According to Appleby et $a 1,{ }^{30}$ if the standard redox potential ( $\mathrm{E}^{\circ}$ ) of the central ligand is more positive than that for the reaction $\mathrm{O}_{2}+2 \mathrm{H}_{2} \mathrm{O}+4 \mathrm{e}^{-} \rightarrow 4 \mathrm{OH}^{-}$(i. e., $\mathrm{E}^{\circ}=+0.4$ at $\mathrm{pH}=14$ ), then secondary reduction of $\mathrm{O}_{2}$ will occur. The mechanistic advantage would be that the $i_{0}$ for the redox reaction would be expected to be rather high, so that the subsequent reaction with $\mathrm{O}_{2}$ would yield higher io compared to those corresponding to a direct 4-e transfer to oxygen. In this way, the effect of adsor ption forces and of other factors that are responsible for electrocatalysis would be a voided. The function of the surrounding porphyrin or phthalocyanine groups is presently unclear, although the precise configuration is considered to be important by Anson and Collman. 31 Adjustment of the organic framework around the metal ion may conceivably give rise to a fine-tuning of the redox potential.

A method of circumventing the fundamental problems of such mechanistic explanations has been reported by $Z_{a g a l}$ et al. 32 If CO-containing macrocyclics supported on high surface area carbons are heated to $800^{\circ} \mathrm{C}$, the resultant layer clearly no longer contains the original porphyrin structure. However, it appears to provide better catalysis than that given with the non-pyrolyzed porphyrins. ' Recent work has shown that pyrolyzed macrocyclic electrodes are

'On the other hand, if the metal oxide alone is deposited on $C$ without preliminary formation of porphyrin before pyrolysis, little electrocatalysis is seen. Thus, the formation of the metal center on the $C$ surface is in some way influenced by the $\mathrm{MN}_{4}$ structure of the porphyrin. However, both the effect of the structure of the porphyrin and its influence on electrocatalysis are unclear since, at $800^{\circ} \mathrm{C}$, organic material has probably been largely decomposed. 
Table 3.4-1. Some ligands used in the preparation of chelate electrocatalysts.

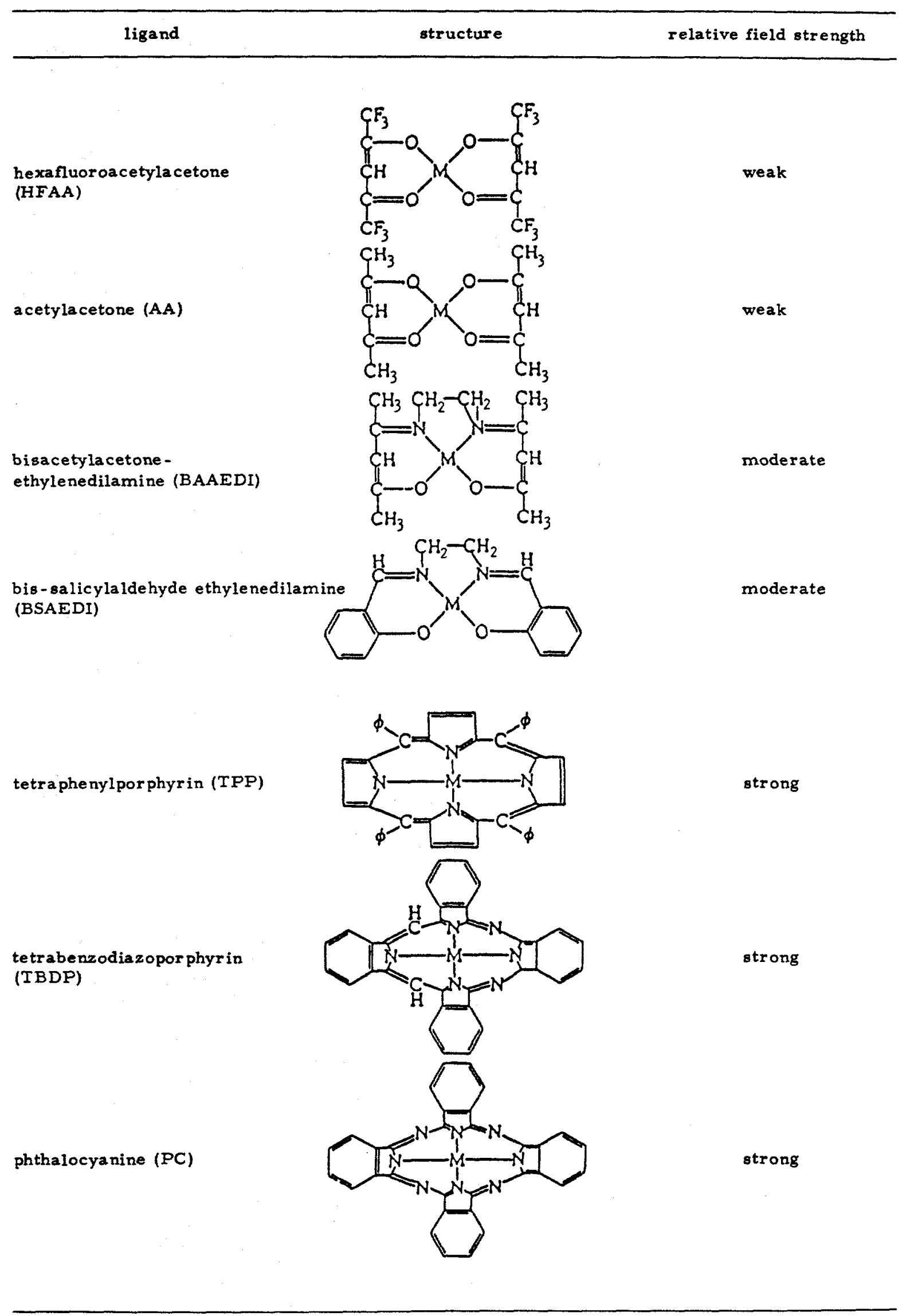


superior under AFC conditions to high surface area Pt carbon, both from the viewpoint of performance and lifetime, 35 and are likely to be prime candidates for AFCs in future transportation applications using stored hydrogen as fuel.

It is likely that electrocatalysis on the pyrolyzed substrates occurs via the step $\mathrm{O}_{2}+\mathrm{H}_{2} \mathrm{O}$ $+\mathrm{e}^{-} \rightarrow \mathrm{HO}_{2}$ (ads) $+\mathrm{OH}^{-}$or via some subsequent electron-transfer step, which would be expected to have a lower Tafel slope. It will therefore result from the binding of a dioxygen molecule or dioxygen radical ion to the central ion.

Work is required in which 6-10 transition elements are examined for their electrocatalytic properties, keeping the surrounding porphyrin structure constant. Plots should then be made relating the resulting $i_{0}$ to the bond strength of oxygen to the transition metal (Fig. 3.4-6). Similarly, results on activation-energy changes as a function of changes in bond strength are necessary. The theory of electrocatalysis, with the effect of bond strengths of reaction rates in the form of volcano plots and the classical theory of the Tafel slope in electrocatalytic processes, has been outlined in Refs. 28 and 33, but it requires much more work for confirmation under AFC conditions. In any case, as discussed in Chapter 2, Fig. 2.12-6, the classical theory of Tafel slopes, which assumes that slopes are proportional to absolute temperature and independent of the form or crystallite size of the substrate, leaves much to be desired. This area will provide some very fruitful future research.

\section{4-1. Chemibonding of Molecular $\mathrm{O}_{2}$ to the Electrode Surface}

When the question of oxygen electrocatalysis in the AFC has been considered, particularly in steps such as

$$
\mathrm{O}_{2}+\mathrm{H}_{2} \mathrm{O}+\mathrm{e}^{-} \rightarrow \mathrm{HO}_{2}(\mathrm{ads})+\mathrm{OH}^{-}
$$

or in subsequent electron-transfer rate-determining steps, it has mostly been assumed that $\mathrm{O}_{2}$ does not have free orbitals that are a vailable for bonding to transition metals. This assumption makes the electrocatalytic effects implied by this mechanism clear, since only the bonding of the adsorbed radical will affect reaction velocity and lead to increasing rate with increasing $\mathrm{M}-\mathrm{O}_{\mathrm{ads}}$, at least under low-coverage conditions. However, if $\mathrm{O}_{2}$ is also bonded to the electrodes by chemi-bonds, the inference is less clear. This consideration is important for the future direction of electrocatalytic research. In particular. Tseung's ideas 34 depend on the effect of the heat of adsorption of molecular oxygen on reaction rate.

A future test of the $\mathrm{O}_{2}$ bonding hypothesis on electrocatalysis is important. 33 If $\mathrm{O}_{2}$ is physisorbed on the electrode surfaces, the slope of the $\log i_{0}$ vs $\Delta H_{a d s}$ plot (or better, that of the $E_{\text {act }} v s \Delta H_{2 d s}$ plot, if these data can be obtained) should be 0.5 ; on the other hand, if it is chemisorbed, the slope should be very different. Indeed, it could even be negative, depending on the adsorbate coverages, 33 In alkaline solution, only a few results are available, and these tend to favor the zero molecular $\mathrm{O}_{2}$ chemisor ption case. On the other hand, Rao, Damjanovic and Bockris ${ }^{36}$ observed a parallelism of $O(a d s)\left[O_{2}(a d s)\right.$ ? ] with the magnetism of the substrate in experiments on the adsorption of oxygen on noble metals (cf. Table 3.4-2 and Fig. 3.4-7). The scarce available results suggest that $\mathrm{HO}_{2}(\mathrm{ads})$ is adsorbed much more strongly than molecular oxygen on most electrocatalytic surfaces.

\section{4-2 Optimal Oxygen Reduction Catalysts for AFCs}

Research on oxygen reduction catalysts for $A F C s$ is quite incomplete, 37 as is shown by the data of Table 3.4-3. Although Pt and Ru appear to be the most effective, Ag may be the least costly, even though $0.1 \mathrm{~kg} / \mathrm{m}^{2}$ has been normally used vs $0.002-0.01 \mathrm{~kg} / \mathrm{m}^{2}$ for Pt. Mercury appears to be a suitable additive to reduce the rate of dissolution and recrystallization of Ag. Addition of $11 \% \mathrm{Hg}$ seems to be optimal. 38

This brief account of oxygen electrocatalysts for alkaline solutions is incomplete. The most likely future electrocatalysts, apart from pyrolyzed transition metal macrocyclics on carbon, are likely to be mixed inorganic oxides such as perovskites. As an example, $\mathrm{LaNiO}_{3}$ is about 1,000 times better than $\mathrm{Pt}$, in the region of current densities of $100 \mathrm{~mA} / \mathrm{cm}^{2}$, for oxygen evolution in alkaline solution. 16,17

\subsection{Cathode Catalyst Supports}

Carbon supports in $A F C_{s}$ play an important part in the efficacy of the catalyst material because $C$ is itself a viable electrocatalyst in strongly alkaline solution. $C$ tends to result in the almost reversible production of peroxide, 24 which can be decomposed with $\mathrm{O}_{2}$ recycle by appropriate catalysts. The catalytic properties of $\mathrm{C}$, its porosity, and the variation of its activity on various crystal planes, are all of great research interest. This area has been clarified by Appleby, 39 McBreen et al, 40 and Morcos and Yeager. 41 The principal objective of the se studies has been the definition of the mechaniem of $C$ degradation. Rolls 42 and, particularly, Petrova 43 tried to correlate degradation with the formation of $\mathrm{H}_{2} \mathrm{O}_{2}$, but no connection was 
Table 3.4-2. Relation of oxygen coverages to the number of unpaired d-electrons per atom for the noble metals. 36

\begin{tabular}{c|c|c|c|c}
\hline Metal & $\begin{array}{c}\text { Observed } \\
\text { oxygen } \\
\text { coverage, } \\
\text { uC-cm-2 }\end{array}$ & $\begin{array}{c}\text { Calculated oxygen } \\
\text { coverage of a } \\
\text { monolayer, } \\
\text { uC-cm-2 }\end{array}$ & $\begin{array}{c}\text { Fraction of } \\
\text { surface covered } \\
\text { by oxygen (O) }\end{array}$ & $\begin{array}{c}\text { Number of } \\
\text { unpaired d- } \\
\text { electrons } \\
\text { per atom }\end{array}$ \\
\hline Pd & 110 & 510 & 0.22 & 0.55 \\
Pt & 110 & 500 & 0.22 & $0.55-0.6$ \\
Pt & 135 & 500 & 0.27 & $0.55-0.6$ \\
Rh & 480 & 530 & 0.90 & 1.7 \\
Ir & 440 & 525 & 0.84 & 1.7 \\
Ru & 500 & 530 & 0.95 & 2.2 \\
Au & $<15$ & 500 & $<0.03$ & 0 \\
\hline
\end{tabular}

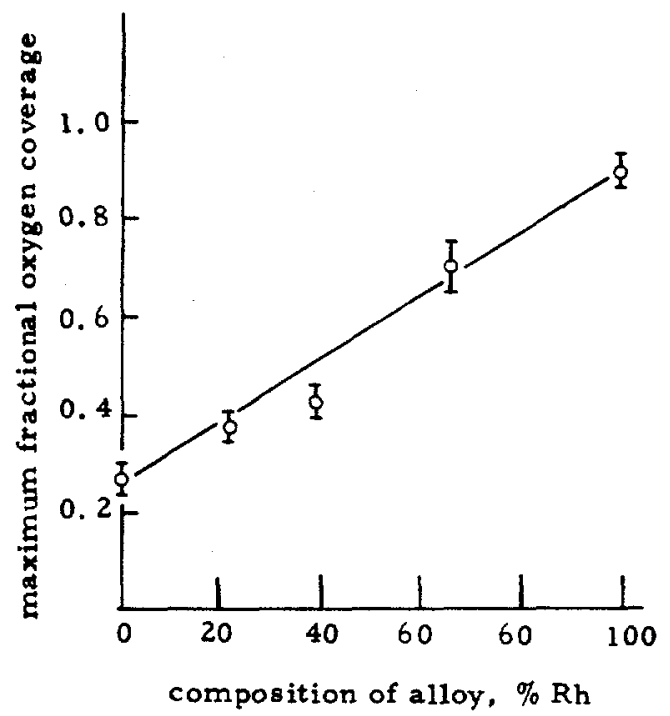

Fig. 3. 4-7. Plot of maximum degree of coverage of adsorbed oxygen on Pt, $R h$, and their alloys vs the composition of the alloy.

Table 3.4-3. Mass-transport-corrected current densities for oxygen reduction in $0.1 \mathrm{~N} \mathrm{KOH}$ at $25^{\circ} \mathrm{C}$ on various substrates from RDE studies, 37

\begin{tabular}{|c|c|c|c|}
\hline Electrocatalyst & $\begin{array}{l}\text { Over potential, } \\
\text { mV vs RHE }\end{array}$ & $\begin{array}{l}\text { Limiting-current } \\
\text { density } \\
i_{1}\left(\mathrm{~mA} / \mathrm{cm}^{2}\right) \\
\text { at the specified } \\
\text { rotation rate }\end{array}$ & $\begin{array}{c}\text { Mass-transport- } \\
\text { corrected current } \\
\text { density } \\
i \times i_{1} /\left(i_{1}-i\right), \mathrm{mA} / \mathrm{cm}^{2}\end{array}$ \\
\hline $\mathbf{R h}$ & 700 & $8.83(3800 \mathrm{r} \mathrm{pm})$ & 5.87 \\
\hline $\mathbf{R} \mathbf{u}$ & 700 & $9.18(4020 \mathrm{r} \mathrm{pm})$ & 12.60 \\
\hline $\mathrm{Ag}$ & 700 & $7.55(3000 \mathrm{rpm})$ & 2.90 \\
\hline Pt & 700 & $\cdots$ & 21.10 \\
\hline $\mathbf{N i}$ & 500 & $7.55(4050 \mathrm{Ipm})$ & 0.32 \\
\hline
\end{tabular}


found. 39 However, the se workers found that the effect of residual $\mathrm{CO}_{2}$ was important. The model which evolved involves first precipitation of solid carbonates and then of bicarbonates in the electrode microstructure. The solid pore contents expand when the bicarbonates were formed, and this caused cracking of the support.

The effects of various $\mathrm{C}$ at constant $\mathrm{pH}$ in alkaline solution on electrode kinetics have been examined, particularly by Appleby and Marie, 48 using the ultra-thin electrode method of Lundquist and Vogel. 44 Tafel slopes (b) were found to be around 2RT/3F to 2RT/F. A change in mechanism therefore arises as a function of the change in properties of the supporting $C$, which may perhaps be connected with the prominence of different crystal faces in defects involving carbon structures that vary with the type of carbon used. These changes are important because, in practice, the Tafel slope as well as $i_{0}$ determine effective cell performance. If a lower slope can be engineered by using electrodes of greater specific area or by other means, then a marked improvement in performance will occur at practical overpotentials. For example, for the same exchange current at an overpotential of $0.3 \mathrm{~V}$, a $T_{a}$ fel slope of $2 R T / F$ will give a current density five orders of magnitude less than a Tafel slope of $3 R T / 2 F$. A suitable change in mechanism and hence a lowering of the Tafel slope are therefore worth much more in practical terms than changes in $i_{0}$ caused by electrocatalysis. These matters have been thoroughly analyzed using various pore sizes, wetting properties, diffusion coefficients, concentrations, and values of $i_{0} 45,46$ The vital $b$-value depends not only on the mechanism and rate-determining step but also on the properties of the electrode, including (for porous electrodes) pore length and diameter, solubility of reactants in the solution, diffusion coefficient, etc. Although b is always related to and affected by the mechanism (in general, the later the rate-determining step in the mechanism, the lower the slope, Refs. 28, 39), its precise value is not easily determined when high surface area electrodes are used. Useful results (i.e., smaller Tafel slopes) should be obtainable by studying the influence of porous electrode parameters on $b$. The area of maximum activity and the dependence of $b$ on contact angle are important considerations, particularly when referring to the amount of catalyst needed (see Figs. 3.5-1 and 3.5-2). This problem has been viewed from the engineering point of view 47 in terms of diffusion alone, and it was concluded that, for pores of $20 \AA$ radius, the accessible length is about 0.4 um or $\sim 20 \%$ of the typical pore length in active carbons.

Bockris and Cahan 45 have shown that the amount of the pore which is active can be calculated if the electrode kinetic parameters of the reaction concerned are known. For example, it depends markedly on $i_{0}$ and, if $i_{0}$ is sufficiently small, the entire pore may be active; but, at higher $i_{0}$, the activity may be only in the region of the meniscus. Other factors which affect the degree of pore activity are the conductance of the solid material, the conductivity of the electrolyte, the solubility of reactants in the solution, etc. One of the factors influencing the degree of pore activity is the influence of the meniscus and its structure in creating an extended layer of electrolyte. Appleby and Marie ${ }^{48}$ extended $\mathrm{O}_{2}$ electrode kinetics to cases involving the thickness of the double layer and concluded that $25 \AA$ is about the minimum radius of an effective pore because of interaction between double layers on opposite sides of the pore walls. This is an interesting result because the majority of the pores in a porous, active carbon electrode is amaller than $25 \AA$ in diameter and, therefore useless from the viewpoint of oxygen reduction.

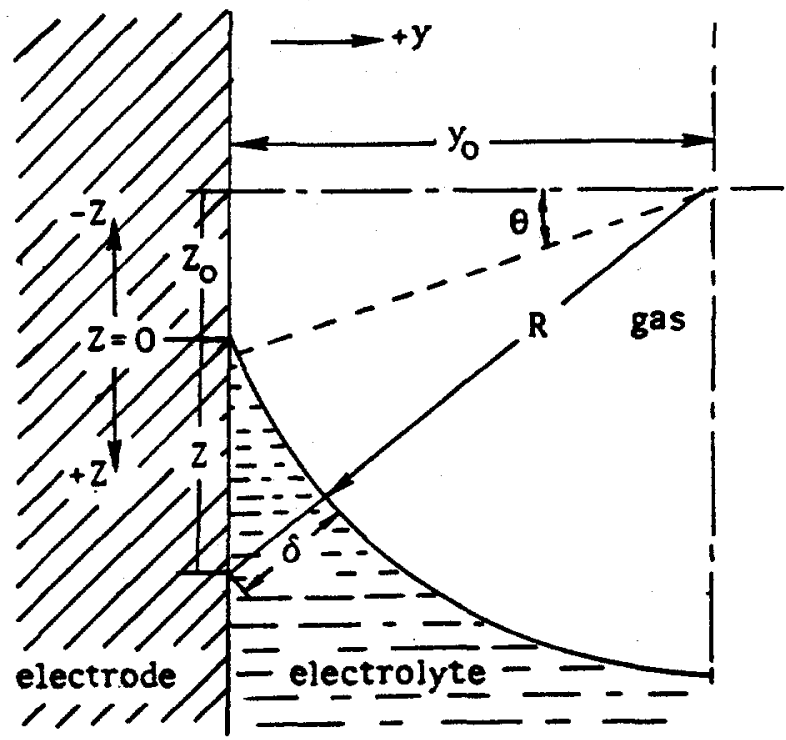

Fig. 3.5-1. Finite-contact-angle model for a porous gas-diffusion electrode. The coordinate system used for theoretical calculations is shown. 45,46 


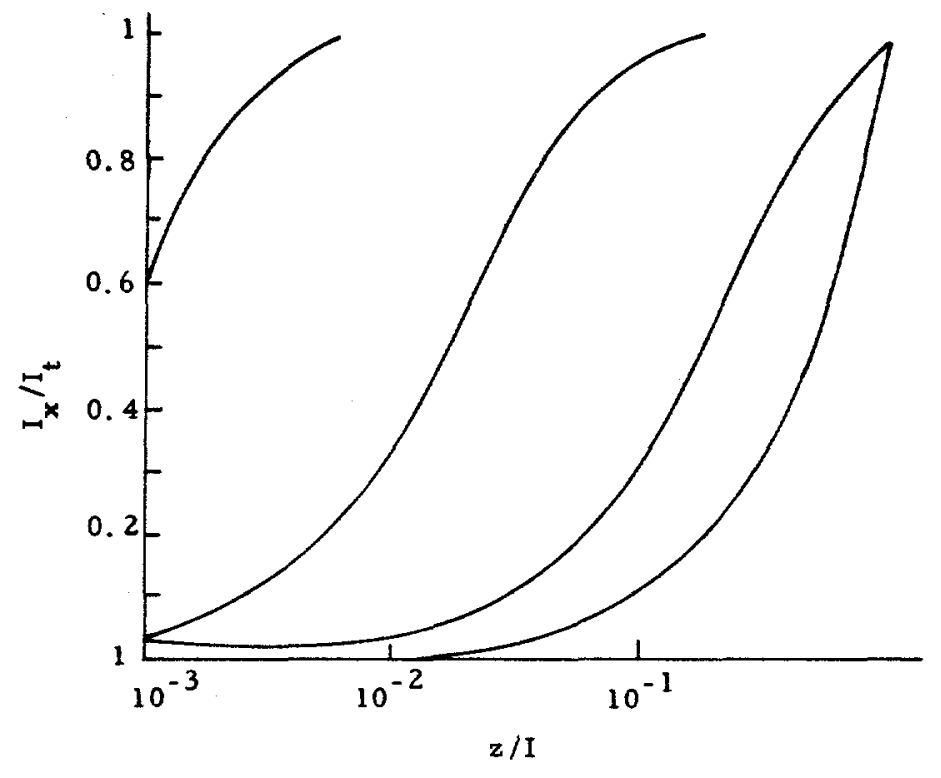

Fig. 3.5-2. Current-distribution relations for the case where all forms of over potential are considered, using the simple-pore model for a porous gas-diffusion electrode as a function of $\mathrm{z} / 1$. $D_{\mathrm{n}} F_{\mathrm{c}}=10^{-7} \mathrm{~A}-\mathrm{cm}^{-1} ; \mathrm{k}=1 \mathrm{ohm}^{-1}-\mathrm{cm}^{-1} .45,46$

The kinetics of oxygen reduction on carbon was also shown to be $\mathrm{pH}$-independent, even when 2RT/3F Tavel slopes were seen. 48 On many carbons, the slope changes from $2 R T / 3 F$ at $\mathrm{pH} 14-15$ to $2 \mathrm{RT} / \mathrm{F}$ at $\mathrm{pH} 12,48$ thus adding a further dimension to the complexity of the $\mathrm{O}_{2-}$ reduction proces on $\mathrm{C}$ in alkaline solution.

In conclusion, we emphasize two points: (1) The only known practical method of avoiding long-term physical deterioration of high surface area carbon-teflon electrodes is to limit $\mathrm{CO}_{2}$ access to less than $10 \mathrm{ppm}$. The development of support materials other than carbon which are less affected by $\mathrm{CO}_{2}$ would therefore be of great significance for AFCs. (2) Useful information may be derived from theoretical analysis of mechanism and rate in practical electrode structures. This information has not yet been introduced into design equations for gas-diffusion electrodes.

\subsection{Engineering Designs}

Recently, Kordesch, Jahangir and Schantz ${ }^{49}$ have made important generalizations concerning electrode engineering. Their four major conclusions are: (1) The selection of the type of carbon material is of prime importance; (2) polytetrafluorethylene (PTFE, teflon) is a suitable binder and wet-proofing agent, whether as a dry powder or in colloidal aqueous suspension; (3) bipolar cell construction is optimal to give the lowest IR drop, uniform current density and, hence, the best cell performance; (4) conducting plastics have become a low-cost alternative construction material for $F_{B}$. These improvements have been incorporated into the Occidental Petroleum-A1sthom AFC, ${ }^{50}$ which is described in Sec. 3.7-6.

\subsection{Recent Contributions to AFC Technology}

We now briefly summarize recent developments in AFC technology.

\section{7-1. AFC Development at UTC}

As was discussed in Sec. 3.2, the Bacon cell was modified at the Pratt \& Whitney Division of UTC for use aboard the Apollo service module to provide primary electric power and potable water. One of the major original reasons for Bacon's use of high pressure at $200^{\circ} \mathrm{C}$ was to prevent boiling of the electrolyte. When Pratt \& Whitney lowered the pressure, it was necessary to use a more concentrated electrolyte, which did not allow sufficient performance at $200^{\circ} \mathrm{C} .51$ A reasonably high performance was maintained at low pressure by increasing the system temperature from about 200 to $260^{\circ} \mathrm{C}$, which required the use of $75 \mathrm{wt} \%$ of $\mathrm{KOH}$ (i.e., molten reagent grade $\mathrm{KOH}$ pellets) to maintain the appropriate water vapor pressure equilibrium. This electrolyte required a complex start-up procedure. The correct three-phase boundary in the 
dual-porosity, sintered nickel (anode)/sintered lithiated nickel oxide (cathode) was maintained by a nitrogen blanket-gas presaure of 3.6 bar. Reactant gas pressures were 0.6 bax above this value. A cell cross-section is shown in Fig. 3.7-1.

Each unit contained a bipolar atack of 31 circular cells, with an active area of $365 \mathrm{~cm}^{2}$. Performance under typical conditions was $0.85 \mathrm{~V}$ at $150 \mathrm{~mA} / \mathrm{cm}^{2}$, which may be compared with the Bacon cell data at more than 10 times higher pressure in Fig. 3.2-1 that gave $400 \mathrm{~mA} / \mathrm{cm}^{2}$ under the same conditions.7,8

Unlike Bacon, Pratt \& Whitney used a stationary electrolyte in each cell, surrounded by a circular diaphragm which was sufficiently flexible to accommodate volume changes as a function of temperature and load.

Heat and water vapor removal were carried out using closed-loop hydrogen recirculation. The Apollo service module was equipped with this AFC system, which was designated PC3A-2. The diameter of each was $57 \mathrm{~cm}$, with a height of $112 \mathrm{~cm}$. Each unit delivered $1.42 \mathrm{~kW}$ at $27-31 \mathrm{~V}$, with a mission a verage power of $0.6 \mathrm{~kW}$. Module life was limited by the slow corrosion of the sintered, lithiated nickel oxide cathode structure to a design life of only about $500 \mathrm{~h}$. No PC3A-2 units were made after the Apollo program ended.

A lighter and much higher performance $\mathrm{H}_{2}-\mathrm{O}_{2}$ alkaline system was developed as the primary power supply of the space shuttle orbiter. 52 Three units (designated PC-17C) were used in the orbiter: each is $35 \mathrm{~cm}$ high, $38 \mathrm{~cm}$ wide and $101 \mathrm{~cm}$ long. The initial units consisted of two parallel-connected stacks, each containing 32 rectangular cells with an active area of $465 \mathrm{~cm}^{2}$ that gave together a nominal power of $12 \mathrm{~kW}$ at $27.5 \mathrm{~V}\left(0.86 \mathrm{~V} / \mathrm{cell}\right.$ at $\left.470 \mathrm{~mA} / \mathrm{cm}^{2}\right)$ and $18 \mathrm{~kW}$ $(25.6 \mathrm{~V})$ at maximum power, corresponding to $750 \mathrm{~mA} / \mathrm{cm}^{2}$.

Most recent space shuttle units contain a third parallel-connected stack, giving a total nominal power output of $18 \mathrm{~kW}$. This modification raises the total system weight from $90 \mathrm{~kg}$ to about $120 \mathrm{~kg}$ at a specific power of $150 \mathrm{~W} / \mathrm{kg}$ or 12 times that for the Apollo cell. This output is a remarkable achievement.

The space shuttle system operates in about the same pressure range as the Apollo cell (4.0 - 4. 4 bar) but at a lower temperature $\left(82^{\circ} \mathrm{C}\right.$ gas inlet, $89^{\circ} \mathrm{C}$ gas outlet). This lower operating temperature allows long life (service life of $2000 \mathrm{~h}$, with refurbishment between missions; 3 stacks have been bench-tested to $10,000 \mathrm{~h}$ ). The high performance at low temperature is achieved by the use of teflon-bonded electrodes with pure, high-loading noble metal catalysts that are similar in structure to those used in early PAFCs (Sec. 2.5). The anode consists of $10 \mathrm{mg} / \mathrm{cm}^{2}$ of pure noble metal black $(80 \% \mathrm{Pt}, 20 \% \mathrm{Pd})$ on a Ag-plated Ni screen, while the cathode has $20 \mathrm{mg} / \mathrm{cm}^{2}(90 \% \mathrm{Au}, 10 \% \mathrm{Pt})$ on a Au-plated $\mathrm{Ni}$ screen. In the cathode, the Au appears to be the catalytic surface, the Pt being essentially a sintering inhibitor. These flight-qualified electrodes, while showing high performance, are no longer state-of-the-art, and they probably have relatively low catalyst utilization.

The bipolar space shuttle cells differ from the Apollo cells in that they contain a thin layer of totally immobilized $35-45 \%$ KOH electrolyte absorbed in a reconstituted asbestos separator. The cells must contain an electrolyte reservoir plate (ERP) to compensate for dilution under load. This separator consists of a porous sintered $\mathrm{Ni}$ sheet in contact with the anode, which is pierced with holes to allow passage of $\mathrm{H}_{2}$. The system resembles the anode ribbed substrate reservoir in the UTC and cell stack (Chapter 2, Fig. 2.10-1). The ERP accounts for almost 50\%

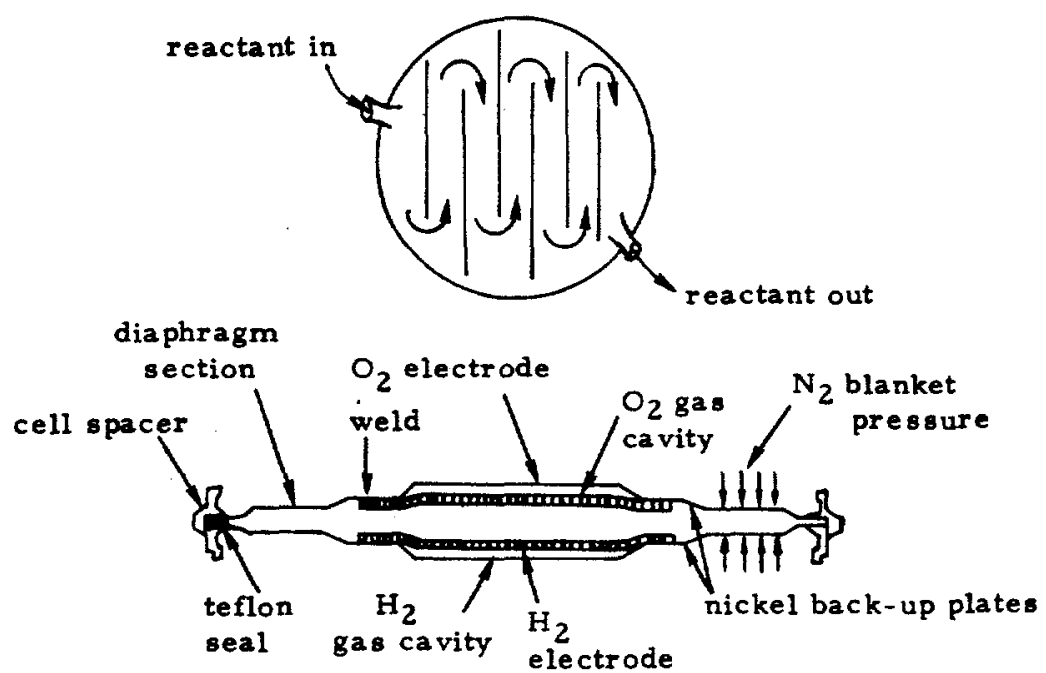

Fig. 3.7-1. Schematic diagram of the UTC AFC (Apollo FC) cell cross-section. 
of the total stack weight, and its replacement by a lighter weight structure would clearly be desirable. In contrast, the bipolar plates are lightweight Au-plated $\mathrm{Mg}$. A schematic view of the stack crosa-section (with $\mathrm{H}_{2}$ and $\mathrm{O}_{2}$ channels rotated $90^{\circ}$ for clarity) showing the arrangement of the main components is given in Chapter 4, Fig. 4.8-3. Like the Apollo cell, the space shuttle orbiter cell uses active water rejection from the hydrogen-feedback loop. Cooling occurs via cooling plates using dielectric liquid circulation within the cell stack, whereas the Apollo system used glycol cooling via a heat-exchanger in the hydrogen loop.

During 1975-79. UTC developed an even more advanced system for specialized aerospace applications requiring a small system of high specific power. The goal was $250 \mathrm{~W} / \mathrm{kg}$ in a $2-\mathrm{kW}$, 30-cell unit, with a cell size of $30 \mathrm{~cm} \times 7.6 \mathrm{~cm}$. The syatem was to have a specific power for the cell stack alone of about $550 \mathrm{~W} / \mathrm{kg}$, compared with about $275 \mathrm{~W} / \mathrm{kg}$ for the space shuttle unit. The system used is, in principle, similar to that of the Allis-Chalmers aerospace cell of the 1960 s and includes passive water rejection via an evaporation assembly in each cell. Like the space-shuttle cell, the cathode has $20 \mathrm{mg}$ (cathode) of noble metal alloy on Au-plated Ni screen. However, the anode is similar to that used in acid cells (Sec. 2. 15C) and consists of teflon-bonded, low-loading $P t\left(0.5-0.75 \mathrm{mg} / \mathrm{cm}^{2}\right)$ supported on $C$. This design gives equivalent performance to the $10 \mathrm{mg} / \mathrm{cm}^{2}$ noble metal space shuttle anode, and there are no corrosion problems with $C$ close to the hydrogen potential in $\mathrm{KOH}$ at elevated temperature.

The electrolyte ( $8 \mathrm{~N} \mathrm{KOH}$ ) is immobilized in a reconstituted asbestos matrix, and an ERP contacts the anode. Unlike the space-shuttle cell, however, the ERP structure is lightweight $\mathrm{Ni}$-plated porous polysulfone. On the opposite side of each anode gas-circulation chamber (which contains a teflon screen) is a passive water-removal assembly consisting successively of an asbestos matrix containing more concentrated electrolyte than that in the DC power generation cell unit, a Ni-plated polysulfone ERP, a porous teflon membrane maintained by an Ag-plated Ni screen, and finally a further teflon screen occupying the space for evaporation of electrolyte. The cathode side of the DC cell contacts a teflon screen for oxygen distribution which, in turn, contacts a $\mathrm{Ni}$-foil separator, on the other side of which is a dielectric liquid cooling flow. DC cell and cooler cell frames are resin-impregnated $\mathrm{ZrO}_{2}$ mats, whereas the water-rejection plate frame is resin-impregnated fiberglass. This complex assembly is shown in cross-section in Fig. 4.8-3, where it is compared with the earlier Allis-Chalmers system. 53 Since the ERPs have poor conductivity, no attempt was made to use a bipolar intercell connection (this is, in any case, impossible because of the use of an asbestos matrix in the water-rejection cell). A monopolar connection along the long edge of each rectangular cell was used instead. The system has been described in Ref. 53.

The system had a target performance of $1.1 \mathrm{~A} / \mathrm{cm}^{2}$ at $0.9 \mathrm{~V}$. To achieve this very ambitious goal, $25-\mathrm{cm}^{2}$ cells were run at various pressures and temperatures to monitor performance up to $149^{\circ} \mathrm{C}$ and 17 bars. Spectacular performances were observed, with very low polarizations (the cell potential was about $1.1 \mathrm{~V}$ at $100 \mathrm{~mA} / \mathrm{cm}^{2}$ at the highest temperatures and pres sures). The target performance goal was achieved at $120+^{\circ} \mathrm{C}$ and 10 bars pressure (Fig. 3.7-2). Performance degradation of about $60-70 \mathrm{mV}$ over $1,000 \mathrm{~h}$ was, however, observed at $120^{\circ} \mathrm{C}$, which was attributed to recrystallization of the cathode catalyst and generalized corrosion problems, including those of the asbestos matrix. It was concluded that the long-term use of asbestos was not possible and potassium hexatitanate and polybenzimidazole were identified as substitutes. The latter proved to be unsatisfactory in further testing.

Platinum supported on carbon (graphitized Vulcan XC-72, see Sec. 2.11, 2. 12), was examined as a cathode catalyst, also with negative results at $120^{\circ} \mathrm{C}$, where catastrophic support corrosion was observed. On the other hand, more recent data 54 have shown acceptable performance for low-loading platinum on carbon at $80^{\circ} \mathrm{C}$. Figure $3.7-2$ shows results for $0.2 \mathrm{mg} / \mathrm{cm}^{2}$ electrodes on pure oxygen. In an optimized electrode structure with $1.0 \mathrm{mg} / \mathrm{cm}^{2}$ of $\mathrm{Pt}$ or with a suitable pyrolyzed macrocyclic, the same performance curve can be obtained on scrubbed air.

The objective of NASA's contract with UTC is to provide a highly reliable power plant for the future space station, combined with an SPE electrolyzer (Chapter 4, Sec. 4.6) in a solarphotovoltaic-hybrid $\mathrm{H}_{2} / \mathrm{O}_{2}$ storage system. However, one may speculate that a cheap terrestrial version of the NASA-UTC lightweight technology, perhaps using conducting plastics in a bipolar or pseudo-bipolar array, combined with porous graphite ERPs, could be used in a $80^{\circ} \mathrm{C} s y s t e m$ with cheap macrocyclic cathodes allowing perhaps $0.8 \mathrm{~V}$ at $300 \mathrm{~mA} / \mathrm{cm}^{2}$. Such a cell would weigh about $7 \mathrm{~kg}$ per nominal $\mathrm{kW}$, with $12 \mathrm{~kg} / \mathrm{kW}$ for the complete power unit. Weights per peak $\mathrm{kW}$ would be about half of these values. This performance is adequate for a future transportation power source using stored hydrogen as fuel.

\section{7-2 Work at BNI}

During the 19708 , Kordesch continued to contribute to the design of porous electrodes and alkaline cells. 55 He designed a cell with circulating electrolyte and with a dual-porosity Ni layer placed adjacent to the $\mathrm{H}_{2}$ plenum to give added structural strength to the electrode, which consisted of a catalyzed $0.04-\mathrm{cm}$ thick Pt-C layer on the electrolyte side of the sintered Ni. The standard Pt loading was $0.2 \mathrm{mg} / \mathrm{cm}^{2}$. Bicell units consisted of two single cells in series 


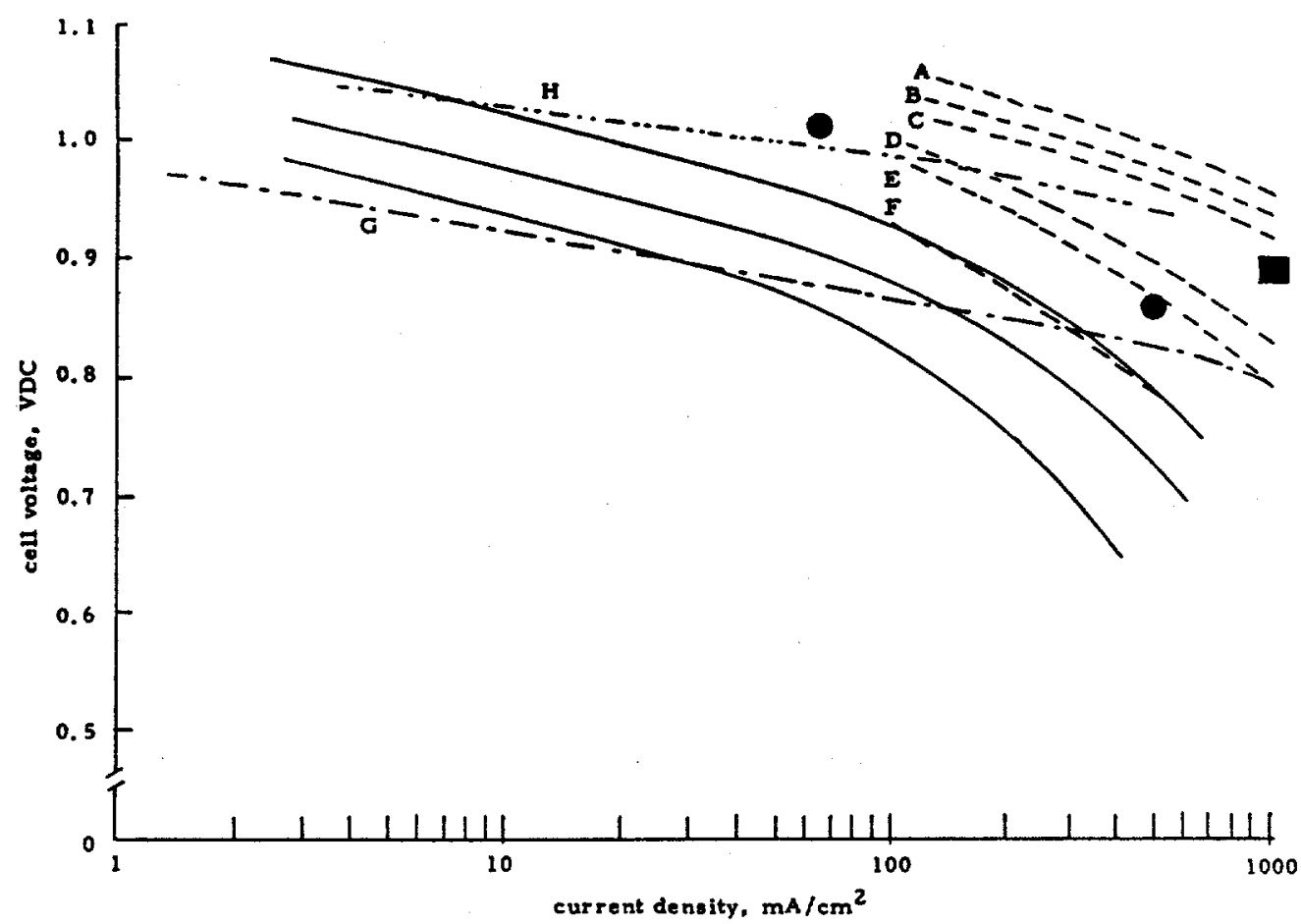

Fig. 3.7-2. Performance of advanced lightweight pressurized AFCs. The dashed lines show initial advanced $A F C$ cell results; F, $82^{\circ} \mathrm{C}, 1$ bar; $E, 82^{\circ} \mathrm{C}, 4$ bar; $\mathrm{D}, 110^{\circ} \mathrm{C}, 4$ bar; $\mathrm{C}, 127^{\circ} \mathrm{C}, 10 \mathrm{bar} ; \mathrm{B}, 127^{\circ} \mathrm{C}, 17$ bar; $\mathrm{A}, 149^{\circ} \mathrm{C}, 17$ bar; the electrolyte was $8+\mathrm{N} \mathrm{KOH}$. Curve G refers to $0.2 \mathrm{Mg} \mathrm{Pt} / \mathrm{C}$ and the same conditions as $F$ (IR-free). Curve $H$ refers to $10 \mathrm{mg} / \mathrm{cm}^{2} \mathrm{Au} / \mathrm{Pt}$, $127^{\circ} \mathrm{C}, 1$ bar (IR-free). - , space-shuttle cell nominal performance $(1000 \mathrm{~h})$; a UTC target goal $(1000 \mathrm{~h})$. Solid lines show SPE cells for comparison under different pressure and temperature conditions (see Figs, 4.6-3,4.8-1).

(Figs. 3.7-3 and 3.7-4), thus allowing an edge-collected circulating electrolyte structure. Kordesch has more recently discussed more advantageous bipolar designs with lower IR drop. 56,57

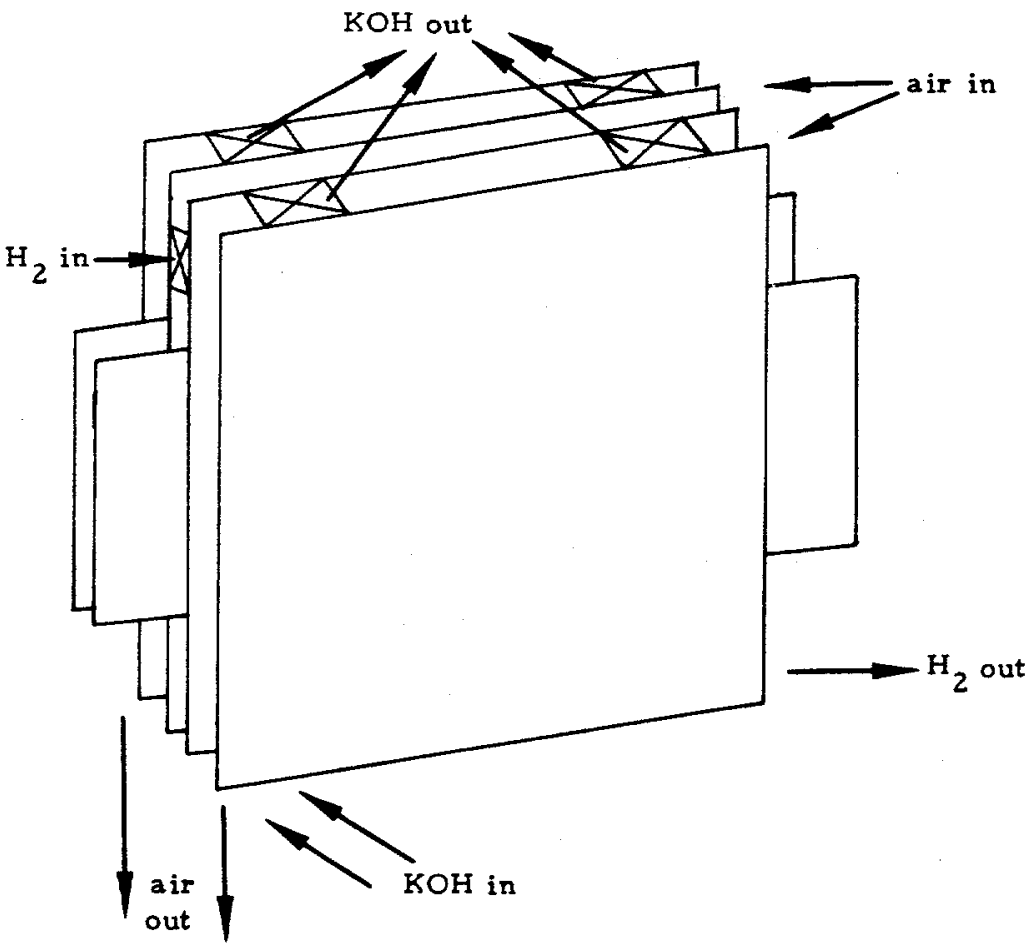

Fig. 3.7-3. Schematic of the KordeBch-BNL alkaline bicell unit. 55 


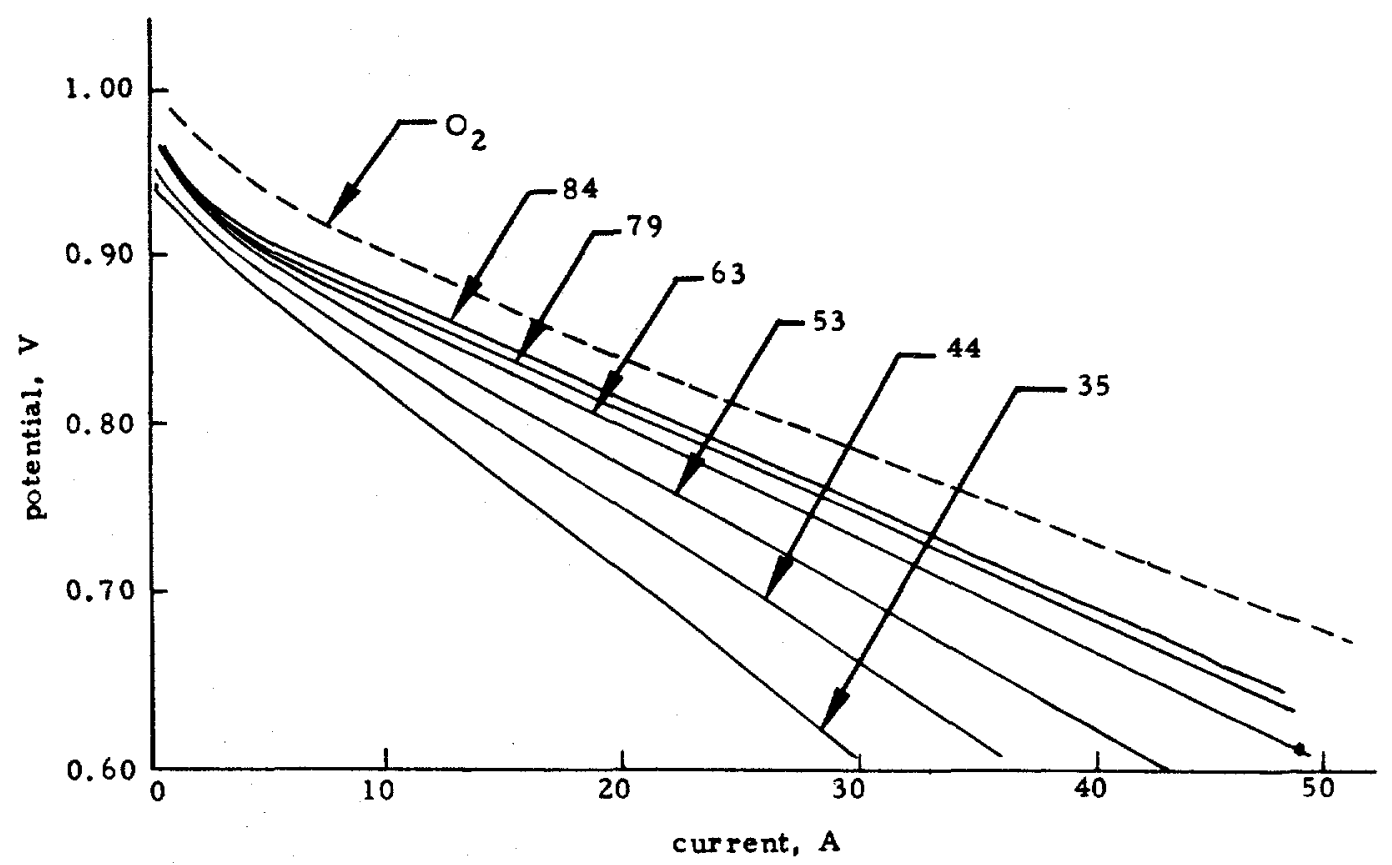

Fig. 3.7-4, Cell potential vs current relation for the Kordesch-BNL AFC at various temperatures $\left({ }^{\circ} \mathrm{C}\right)$ in air and at $70^{\circ} \mathrm{C}$ in oxyger. 55

\section{$3.7-3 \quad A F C s$ at Siemens 56}

At Siemens, Justi and Winsel have initiated many improvements during the last 20 years. In spite of their monjpolar, high-resistance design, the performance of Siemens' AFCs has been outstanding, both from the systems viewpoint and for proven cell lifetime. The main difference between the Siemens AFC and the cells of most other developers involves the use of Raney catalysts $\left(60 \mathrm{mg} / \mathrm{cm}^{2} \mathrm{Ag}\right.$, containing $\mathrm{Ni}, \mathrm{Bi}$ and $\mathrm{Ti}$ as sintering inhibitors at the cathode; $120 \mathrm{mg} /$ $\mathrm{cm}^{2} \mathrm{Ni}$, containing $\mathrm{T}_{i}$ at the anode). These are applied to a sintered carbonyl-supported Ni layer as a mixture with teflon and asbestos fibers, to give a structure resembling Justi's original DSK concept. The typical catalyst particle size is $\sim 50 \mathrm{um}$, with a BET area of $\sim 80 \mathrm{~m}^{2} / \mathrm{g}$. The very high loading $A g$ electrode yields the expected excellent performance, at a cost corresponding to about $\$ 45 / \mathrm{kW}$ for the catalyst alone. For example, at $90^{\circ} \mathrm{C}$ on pure oxygen at $1.95 \mathrm{bar}$, the cathode polarization is only about $75 \mathrm{mV}$ (compared with the practical, not theoretical, OCV) at $600 \mathrm{~mA} / \mathrm{cm}^{2}$. The $\mathrm{H}_{2}$ partial pressure is $2.0 \mathrm{bar}$.

The anode and cathode losses are nearly the same and greater stress has been placed on reducing the severe ohmic losses in the system than in other approaches to improve performance. A typical cell resistance is $0.2 \Omega-\mathrm{cm}^{2}$ compared with only $0.06 \Omega-\mathrm{cm}^{2}$ for a system with immobilized electrolyte of the space-shuttle type. The distribution of potential losses is shown in Fig. 3.7-5, and the 2-kW stack characteriatics are given in Table 3.7-1.

The latest Siemens design was a $7-\mathrm{kW}$ e system, originally developed in 1978 . It is shown schematically in Fig. 3.7-6. The compact unit block has dimensions of $24.5 \times 24.0 \times 102.5 \mathrm{~cm}^{3}$ (volume is $60 \mathrm{l}$ and weight is $85 \mathrm{~kg}$ at a nominal power, $82 \mathrm{~W} / \mathrm{kg}$, and $117 \mathrm{~W} / \mathrm{l}$ for the $2-\mathrm{bar}_{2} \mathrm{O}_{2}$ Bystem). It contains 70 monopolar cells of $34 \mathrm{~cm}^{2}$ active area that are electrically connected in series and hydraulically in parallel for electrolyte circulation. The cell design is very differ ent from that developed by other manufacturers, who are likely to consider it to be unnecessarily complex and difficult to manufacture. The Siemens AFC is non-optimized for minimum IR drop.

The main characteristic of the cells is the use of pressure cushions that are built into the space between each cell to ensure good contact between the various components. These are shown in the cell cross-section in Fig. 3.7-7. They consist of welded pure Ni sheets, which incorporate gas-flow channels contacting the cathode of one cell and the anode of the next. The unit forms a flat balloon uniformly pressing the surface of the plate with flow channels against the electrode which, in turn, presses against the components of the electrolyte compartment. The pressure cushion, which is pressurized to 2.4 to 3 bar, depending on the stack temperature, is in effect a bipolar place which possesses no electronic conductivity on the inside, so that all the current must be conducted around the edges. IR considerations limit the size of the electrodes to a linear dimension of about $20 \mathrm{~cm}$, which makes the system impractical for large power units. The result is rather high IR drop in the current collectors and non-uniform current density. The IR drop across the electrolyte is also not negligible, since the space contains an asbestos diaphragm, a support screen, a coarse separator screen, a further support screen, 


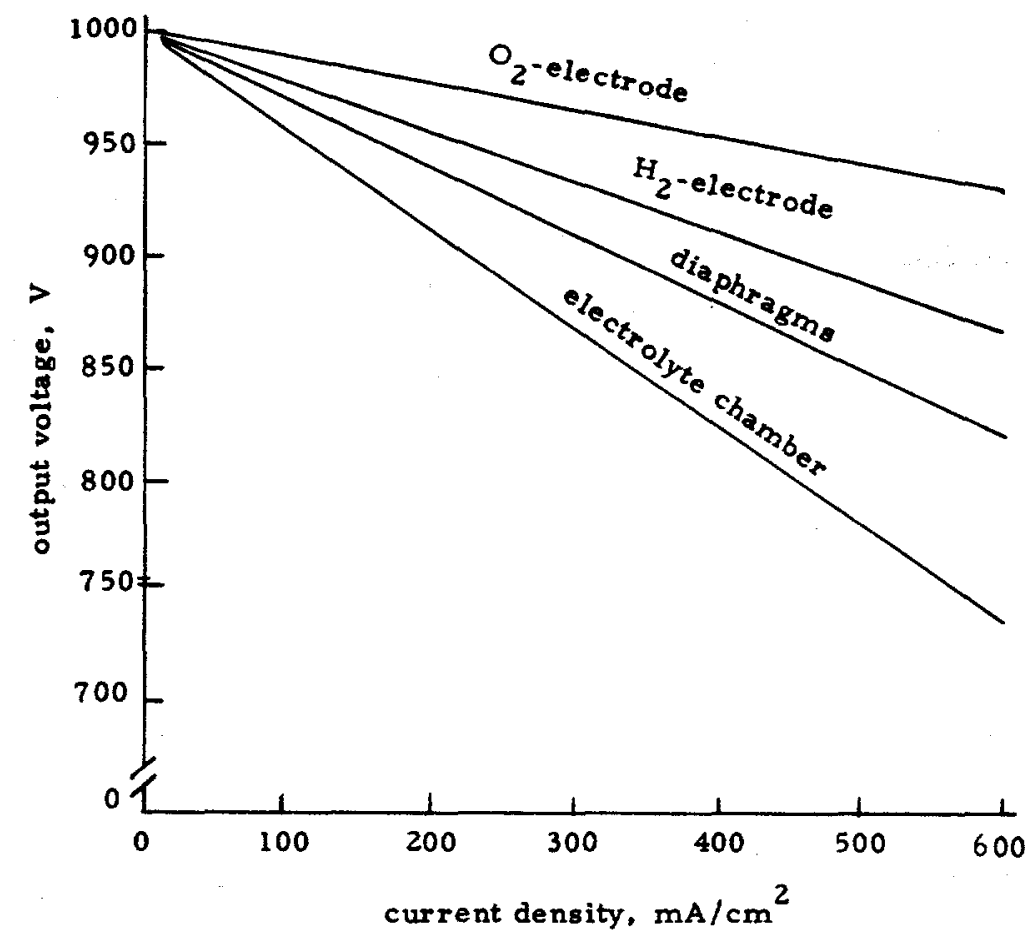

Fig. 3.7-5. Distribution of over potential losses for the Siemens single AFC. 58

Table 3.7-1. Characteristics of the Siemens 2-kW $\mathrm{e}_{2}-\mathrm{O}_{2} \mathrm{AFC}^{55}$

number of cells $=33$, stack voltage $=24$, electrolyte $=6 \mathrm{~N} \mathrm{KOH}, T=80^{\circ} \mathrm{C}$, current density $=300 \mathrm{~mA} / \mathrm{cm}^{2}$, anode electrocatalyst $($ Raney $\mathrm{Ni} / \mathrm{Ti})=$ $120 \mathrm{mg} / \mathrm{cm}^{2}$, cathode electrocatalyst (doped $\left.\mathrm{Ag}\right)=60 \mathrm{mg} / \mathrm{cm}^{2}$, volume = $44 \times 55 \times 32 \mathrm{~cm}^{2}$, weight $=53 \mathrm{~kg}$

and another diaphragm in contact with the other electrode. The asbestos diaphragms, which are sealed to polysulfone frames, are necessary for the correct functioning of the cells, since the electrodes possess such low bubble pressure that the gas bubbles would otherwise leak into the electrolyte. The latter (6-7 $\mathrm{N} \mathrm{KOH})$ circulates in the central space of each cell.

A schematic flow-diagram of the unit is shown in Fig. 3.7-8. After leaving the cell, the electrolyte circulates to the separate water evaporator unit, whose assembly is similar to that of the fuel cell. A schematic evaporator cell cross-section is shown in Fig. 3.7-9. It holds tefloncontaining asbestos diaphragms and incorporates a cooling system. Hydrogen is circulated at 1. 4 bar in the evaporator spaces, and the steam carried out from these is subsequently condensed. The rated performance is $420 \mathrm{~mA} / \mathrm{cm}^{2}$ at about $0.77 \mathrm{~V}$. Under these operating conditions, the performance dispersion between cells is small, as is shown in Fig. 3,7-10. The expected lifetime of the system is about $3,000 \mathrm{~h}$, and the power deterioration is about $5 \%$ per $1,000 \mathrm{~h}$. The system is more complex than that in the space shuttle and probably has commensurate cost. A major disadvantage is the possibility of irreversible oxidation of the Raney $\mathrm{Ni}$ anode resulting from potential excursions at high current density surges outside of the design range.

Siemens cells have undergone steady improvement throughout the years; for example, between 1970 and 1980, the maximum power density increased by $\sim 300 \%$. The system specific power is comparable to that of the space shuttle cell. Doubling the gas pressures to 4 bar (as in the space shuttle cell) would give a somewhat higher potential (about $0.8 \mathrm{~V}$ ) at the same cur rent density. This average potential is, however, about $100 \mathrm{mV}$ less than the 8 pace-shuttle value because of the high IR drop in the Siemens monopolar cells. However, the use of lighter-weight system components and an improved technique for applying uniform mechanical pressure on the cells in a bipolar design should allow Siemens to approach or exceed the space-shuttle power density figure of $125 \mathrm{~W} / \mathrm{kg}$. 


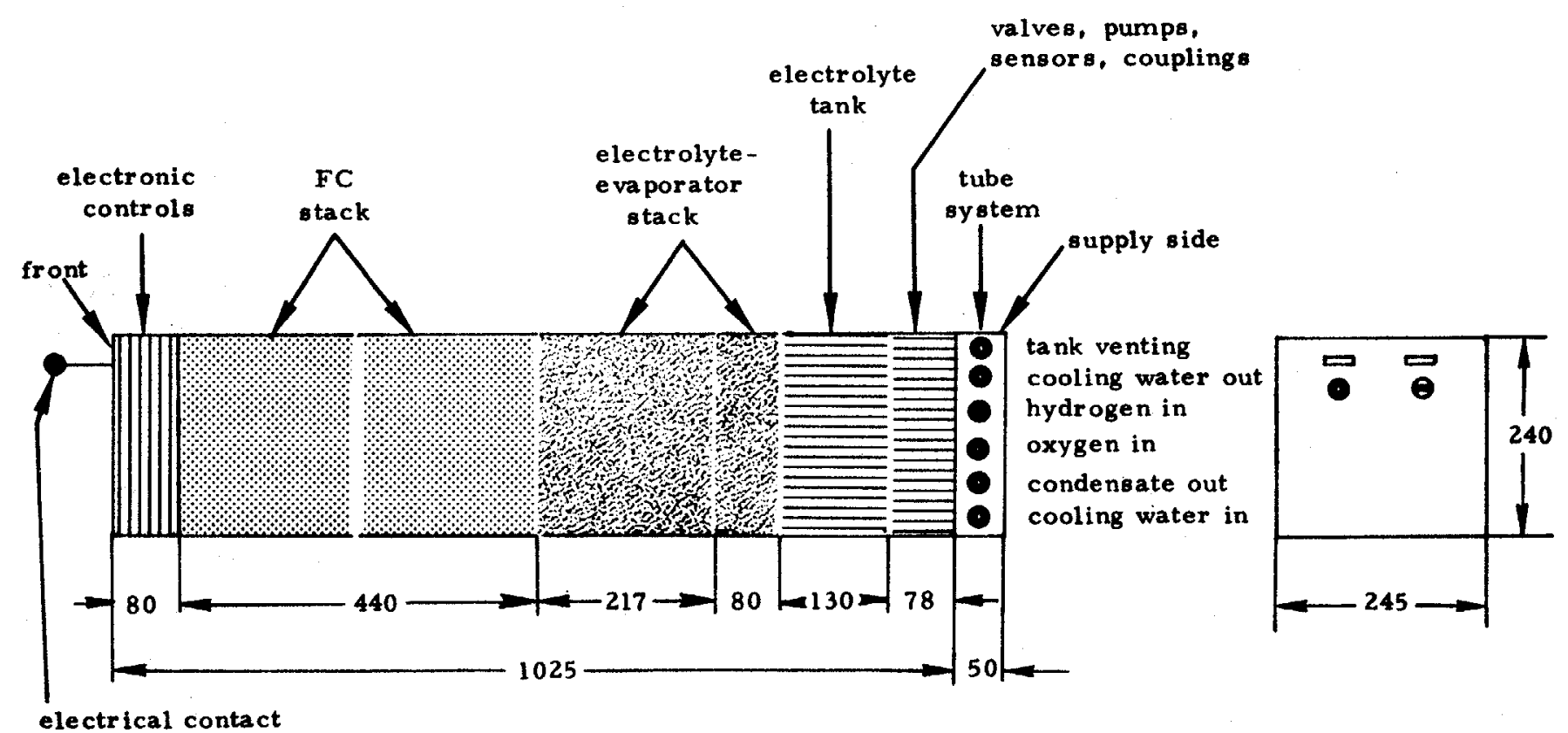

Fig. 3.7-6. Schematic representation of the Siemens $7-\mathrm{kW}_{\mathrm{e}} \mathrm{AFC}$; the dimensions are given in mm; the AFC weighs $85 \mathrm{~kg}$. 58 The mounting position is horizontal. 


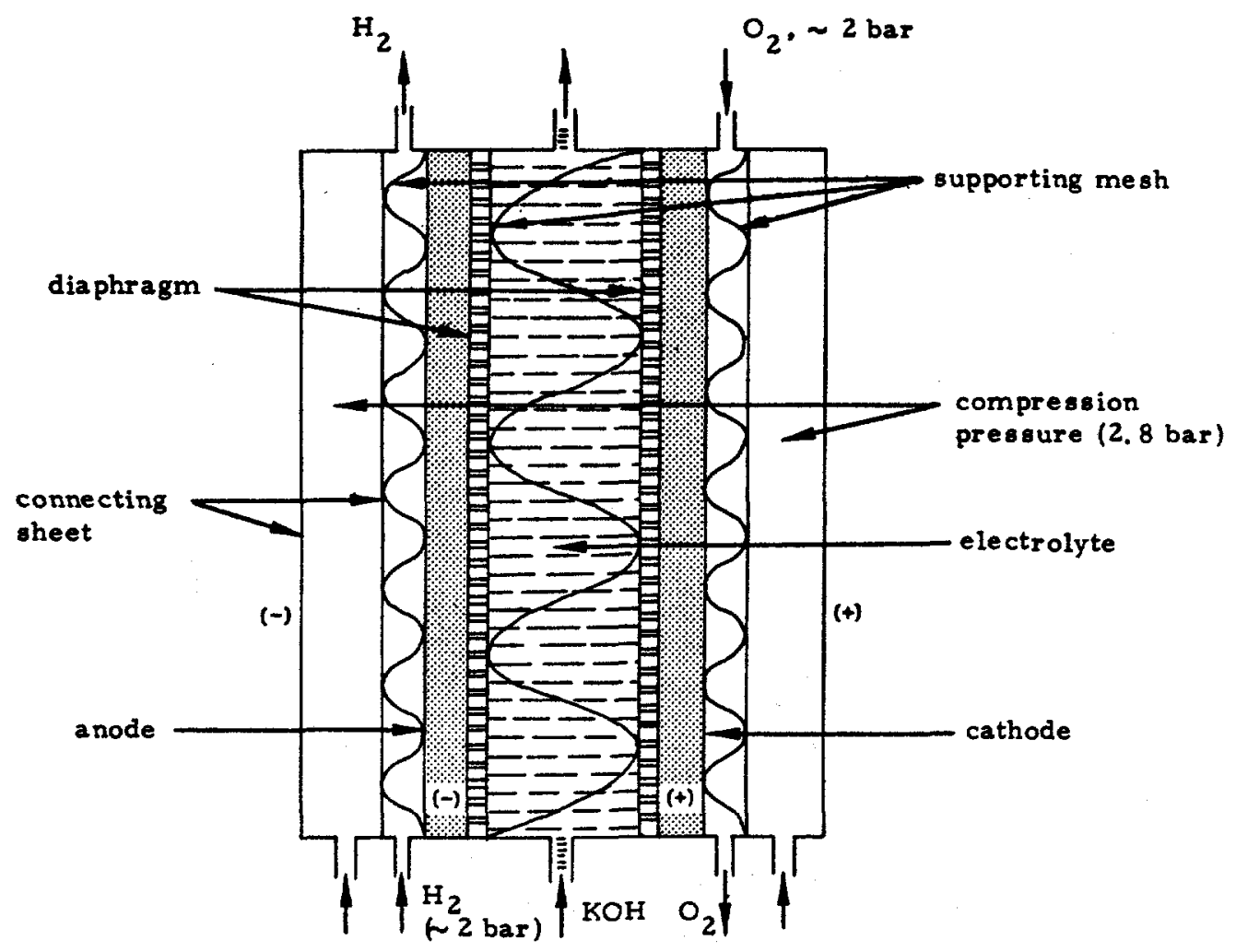

Fig. 3.7-7. An FC with free electrolyte and supported gas-diffusion electrodes. 58

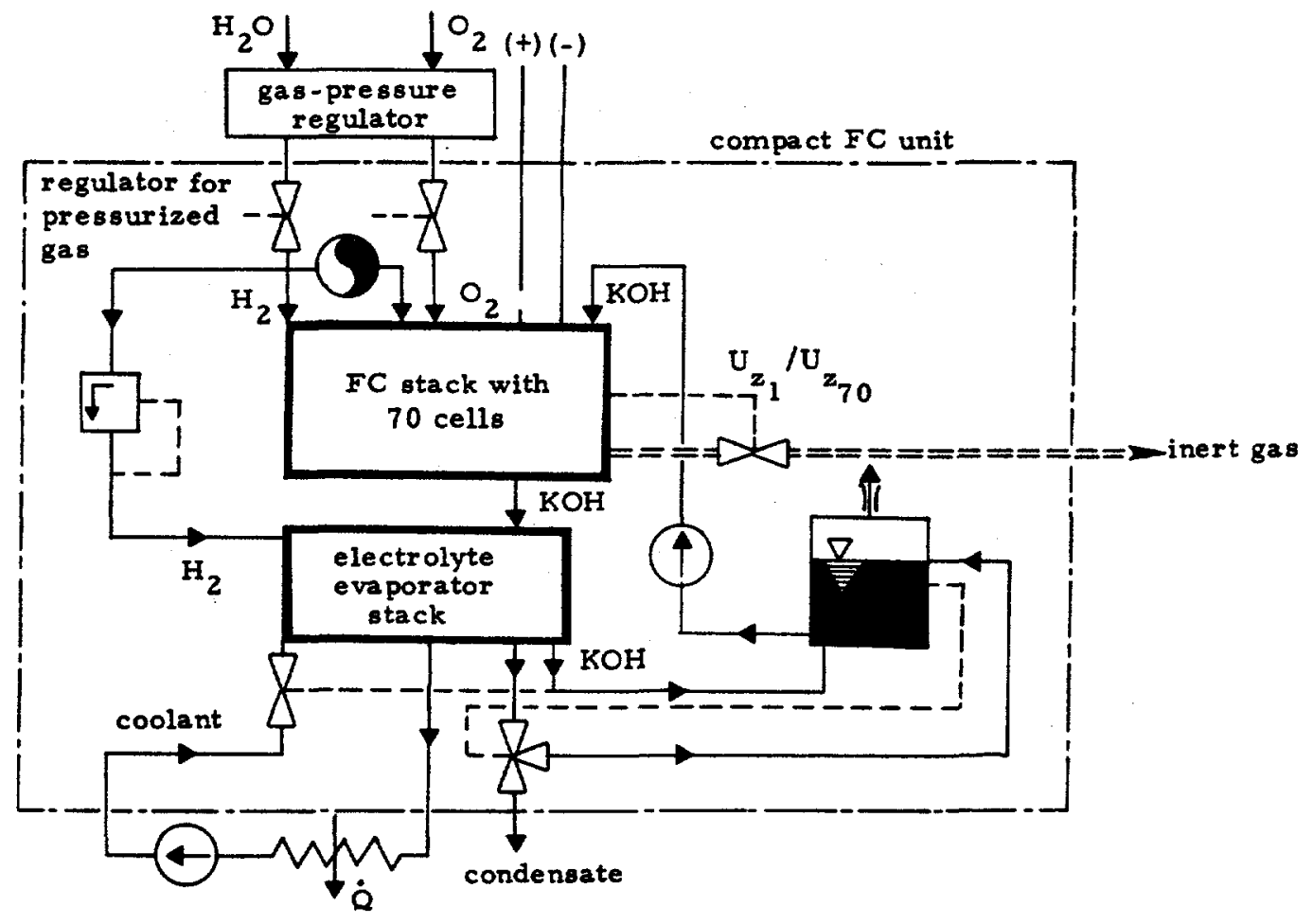

Fig. 3.7-8. Functional schematic of the Siemens AFC unit. 58 


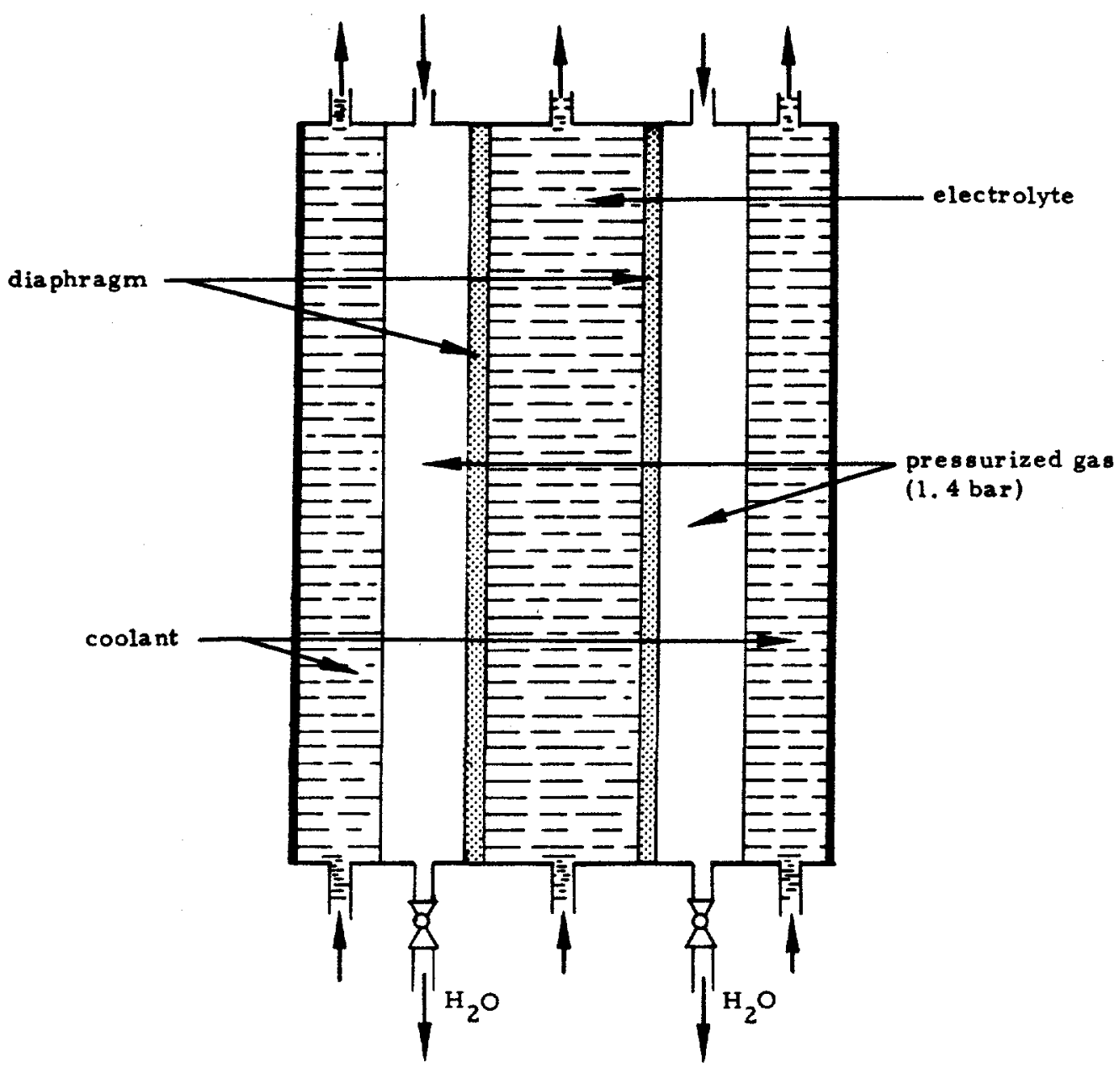

Fig. 3.7-9. The electrolyte-evaporator cell for the Siemens AFC. 58

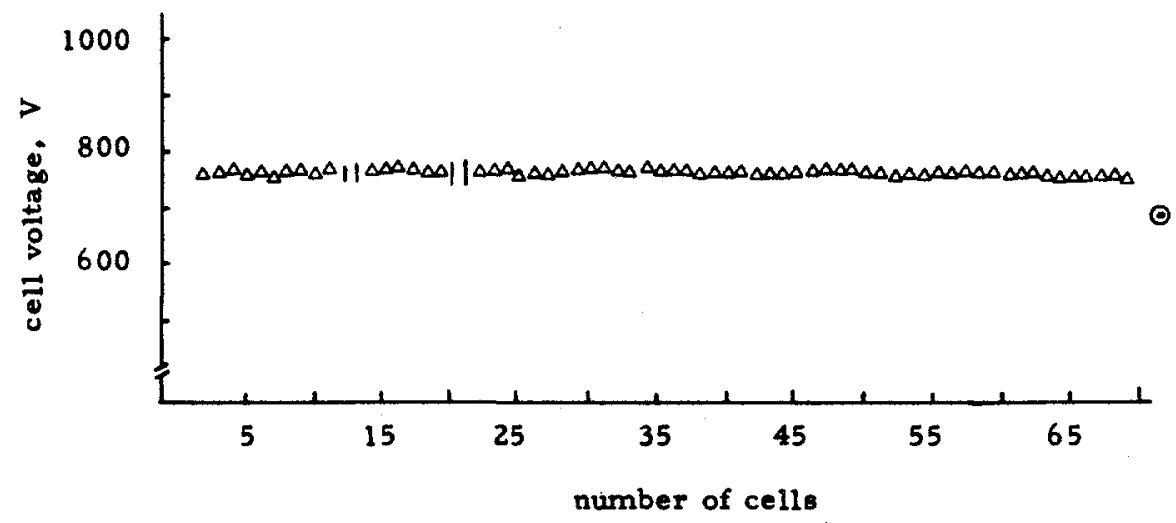

Fig. 3.7-10. Variation of individual cell voltages in the Siemens $7-k W$ AFC stack. 58 The data point $O$ refers to an $\mathrm{O}_{2}$ scavenging cell; the cell voltage depends on the inert-gas concentration. 


\section{7-4 AFC 8 at ELENCO}

ELENCO is a consortium of the Belgium AEC, Dutch State Mines, and the Brussels company Bekaert. This consortium has spent about $\$ 12$ million since the early 1970 s developing $A F C s$ aimed at transportation applications, particularly city buses. 59

The electrodes consist of one or two layers of catalyzed $\mathrm{C}$ on $\mathrm{Ni-screen}$ current collector 8 with hydrophobic backing. While published performance data refer to a combined anode and cathode Pt catalyst loading of $0.7 \mathrm{mg} / \mathrm{cm}^{2}, 60$ it is proposed that the introductory loading will be $0.15 \mathrm{mg} / \mathrm{cm}^{2}$ loading, with the ultimate aim being to reduce this value to $0.05 \mathrm{mg} / \mathrm{cm}^{2}$.

The cells, which operate at $65^{\circ} \mathrm{C}$ have active areas of $17 \times 17 \mathrm{~cm}^{2}$ use circulating $30 \%$ $\mathrm{KOH}$ electrolyte and are monopolar with edge collection. They have injection-molded plastic frames and are arranged in 24-cell modules, with each producing about $0.45 \mathrm{~kW}$ at $0.7 \mathrm{~V}$, $100 \mathrm{~mA} / \mathrm{cm}^{2}$. At the module level, the system-s pecific power is about $100 \mathrm{~W} / \mathrm{kg}$, with a goal of $150 \mathrm{~W} / \mathrm{kg}$. Power degradation is about $4 \% 1,000 \mathrm{~h}, 60$

The present pilot-plant production capacity is about $0.5 \times 10^{6}$ electrodes per year. The latest ELENCO AFC system delivers $10 \mathrm{~kW}$ at $336 \mathrm{~V}$ and consists of 20 of the specified modules. ELENCO plans to use $\mathrm{H}_{2}$ from chlorine plants to operate buses, trucks, and fleet vehicles.

\section{7-5 AFCs at the Compagnie Generale d'Electricité (CGE-France)/} Institut Français des Pétroles (IFP)

This company worked ${ }^{61}$ between 1968 and 1974 on the design of a $5-\mathrm{kW}_{\mathrm{e}}$ AFC, which was intended to be coupled with methanol-reformer $\mathrm{H}_{2}$ unit designed at the Institut Frangais du Pétrole (IFP). The methanol reformer was designed to produce pure $\mathrm{H}_{2}$ from a small unit. Its $\mathrm{CO}_{2-}$ removal system, using temperature-swing absorption with diethanalamine, is of great practical interest. The electrodes had dual porosity, with a fine-pore Ni layer acting as an electrolyte barrier and a coarse-pore layer serving as the active catalyzed structure. Electrodes were prepared by filtration of $\mathrm{Ni}$ powder onto fine-mesh $\mathrm{Ni}$ screens, followed by sintering. The catalysts were described 61 as non-noble metal oxides impregnated in the $\mathrm{Ni}$ sinter (cobalt molybdate at the anode, silver at the cathode, although some experiments were conducted on cobalt oxides).

By the late $1960 \mathrm{~s}$, some single cells had been life-tested for $8,000 \mathrm{~h}$. They were of an interesting monopolar pocket design (Fig. 3.7-11) with an active area of about $450 \mathrm{~cm}^{2}$. Typical IR-corrected performance was $100 \mathrm{~mA} / \mathrm{cm}^{2}$ at $0.6 \mathrm{~V}$ at $70^{\circ} \mathrm{C}$ (Fig. 3.7-12). The system used circulating 6-12 M KOH electrolyte with volume varying with concentration under load 80 that a reserve tank was needed. Accumulation of $\mathrm{CO}_{2}$ in the circulating $\mathrm{KOH}$ required a change of electrolyte every $250 \mathrm{~h}$. Electrodialysis cells were considered as a device for $\mathrm{CO}_{2}$ removal, with a predicted $14 \%$ parasitic power requirement. 61

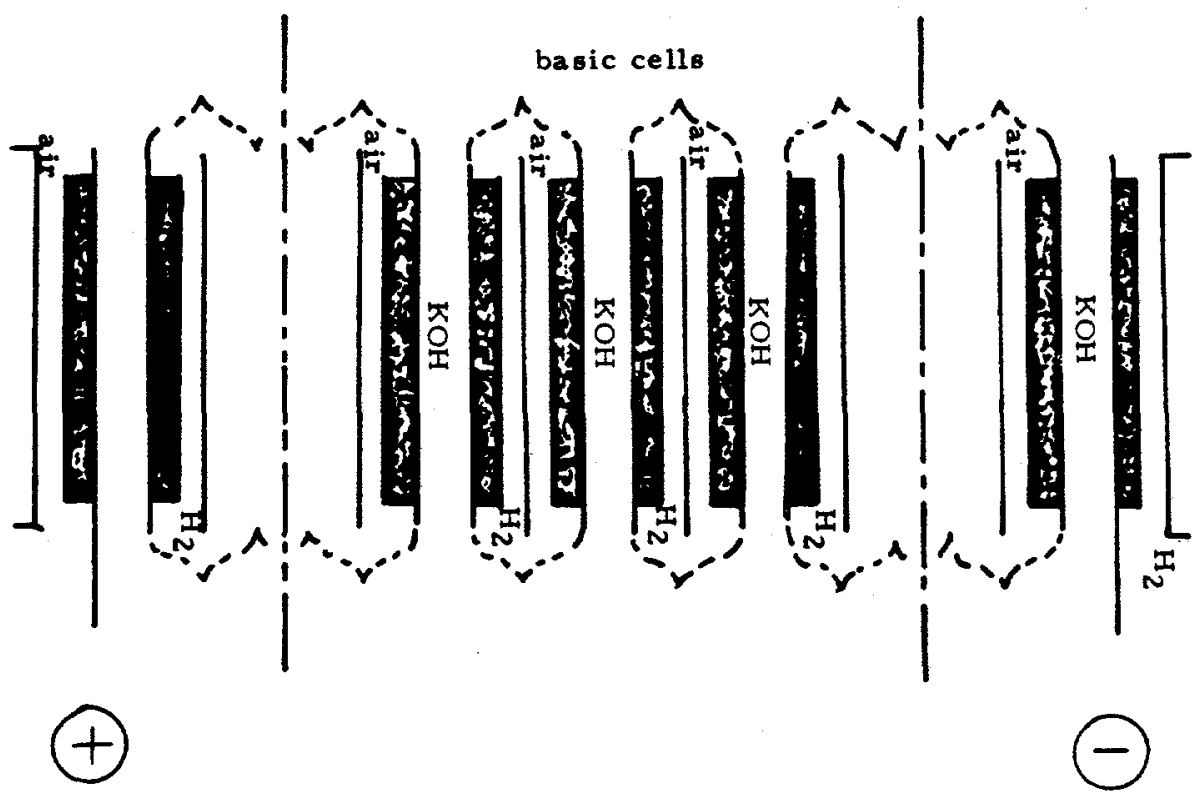

Fig. 3.7-11. Schematic of the Compagnie Génerale d'Electricité AFC.61 


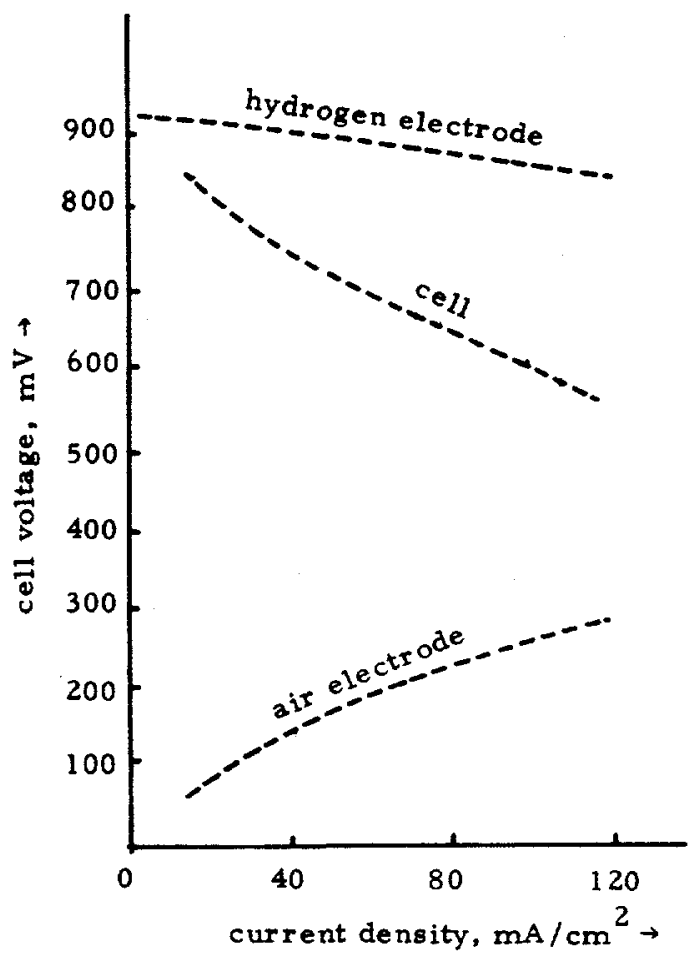

Fig. 3.7-12. Potential vs current-density characteristics of the Compagnie Génerale d'Electricité single cell $\mathrm{AFC}$ at $70^{\circ} \mathrm{C} .61$

The $5-\mathrm{kW}$ system was a conceptual design, and the largest working system constructed was a 500-W (nominal), $28-\mathrm{V}$ unit with a maximum power output of about $1 \mathrm{~kW} .62 \mathrm{This}$ used $\mathrm{Pd}$ (anode) and Ag (cathode) catalysts. The unit was intended to operate as a military Ni-Cd battery charger. All FC work at the CGE was completed by 1972 , except for military acceptance testing, which was completed by 1974 .

IF $P$ work on FACs started in the early 1960 s, the objective being to produce a system suitable for transportation applications. A summary of this work has been published. 63 Studies

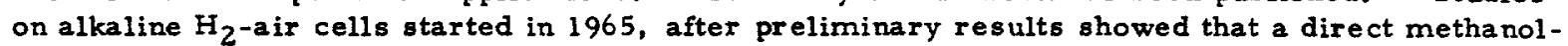
air acid-electrolyte system would require excessive amounts of precious metal catalysts. A consortium was formed at that time between IFP and Renault, CSF, Carbone Lorraine, UgineCarbone, and the battery company CIPEL. A 500-W prototype using multi-layer electrodes was built and tested. The electrodes consisted of a conducting layer (sintered $\mathrm{Ni}$ ) facing the electrolyte, a catalytic layer and, finally, a teflon barrier layer. The a node catalyst combination was Pt $\left(3 \mathrm{mg} / \mathrm{cm}^{2}\right)-\mathrm{Pd}\left(5 \mathrm{mg} / \mathrm{cm}^{2}\right)$, with $\mathrm{Ag}\left(1.8 \mathrm{mg} / \mathrm{cm}^{2}\right)$ at the cathode. The cells used again circulating electrolyte and were monopolar with edge collection. Like the CGE stack, the whole was molded in epoxy to prevent leakage. A 16 -cell 500-W (nominal) unit was constructed in 1968. This was tested for $600 \mathrm{~h}$. The cell thickness was $3.6 \mathrm{~mm}$, which was reduced to $2.8 \mathrm{~mm}$ by 1972. The cell-active area was $200 \mathrm{~cm}^{2}$ in 1968 , which was progressively increased $\left(500 \mathrm{~cm}^{2}\right.$ in $1972,1400 \mathrm{~cm}^{2}$ in 1978 ).

A further 500-W (nominal) 1.5-kW (peak) system was constructed in 1973 for the same purpose as the 500-W CGE system. The stack of this system was capable of delivering $150 \mathrm{~W} / \mathrm{kg}$ at maximum power, but the system as a whole $(1.5 \mathrm{~kW}$ peak power) weighed $55 \mathrm{~kg}$, of which $15 \mathrm{~kg}$ was for the electronic controls. The practical peak-power density was, therefore, only $27 \mathrm{~W} / \mathrm{kg}$. The system was tested over a total elapsed time of $2328 \mathrm{~h}$, which included about $1125 \mathrm{~h}$ of actual operation $h$. Other units were constructed in 1979, including a 2.2-kW, 24-V package.

It was estimated that large units (tens of $\mathrm{kW}$ ) would have $67 \mathrm{~W} / \mathrm{kg}$ (nominal) and $180 \mathrm{~W} /$ $\mathrm{kg}$ (peak). Prototy pe units were estimated to cost (1979) $\$ 10,000 / \mathrm{kW}$. This would be expected to decline considerably in series production, leading to the conclusion that the stack cost would be about (1975) $\$ 120 / \mathrm{kW}$ or about (1985) $\$ 250 / \mathrm{kW}$ for a production rate of $500 \mathrm{MW} / \mathrm{y}$ (about 20,000 small passenger vehicles/y). This cost implies total elimination of noble-metal catalysts. For traction applications, a six-fold decrease in stack cost or increase in power density would be necessary.

The final IFP work 64 on AFCs was conducted in 1980-1981 with a system uging their standard electrodes and catalyst loadings. The electrode-active a rea was $770 \mathrm{~cm}^{2}$, with $75 \mathrm{cells}$ in a series-parallel array and two stacks. The cell thickness was $3.3 \mathrm{~mm}$. Air was scrubbed by using a lithia-containing filter, which gave about 1 residual $\mathrm{ppm}$ of $\mathrm{CO}_{2}$. The stack weight 
(empty) was $35 \mathrm{~kg}$ or $43 \mathrm{~kg}$ with electrolyte (KOH, $8 \mathrm{~N}-11.5 \mathrm{~N}$ ). The power at $65^{\circ} \mathrm{C}$ was $5.3 \mathrm{~kW}$ at $144 \mathrm{~mA} / \mathrm{cm}^{2}, 0.67 \mathrm{~V}(123 \mathrm{~W} / \mathrm{kg})$. All work was terminated on further development of AFCs at the end of 1982 , when the French gover nment contract ended.

\section{7-6 Alsthom/Occidental and Other Developers of AFCs}

Breelle ${ }^{64}$ refers to the future European Hermes mini-s pace shuttle, which will require an $\mathrm{H}_{2}-\mathrm{O}_{2} \mathrm{FC}$ power supply in the $2-3 \mathrm{~kW}$ (maximum power) class. It is understood that work is presently proceeding on the use of high-loading, teflon-bonded, noble-metal electrodes for an $A F C$ for this application, along the lines of those used in the U.S. space shuttle orbiter power plant.

The Alsthom program, under the design direction of B. Warshawski, was always aimed at the production of a cheap, lightweight, bipolar system containing no metallic construction components. The first cell packages constructed (about 1968) used flowing alkaline electrolyte in ultra-thin cells with goffered (i.e., a finely ribbed structure in a herringbone pattern) 50stainless steel bipolar plates for electrolyte and reactant distribution, with a central diaphragm in each cell. At this time, the fuel and oxidant were hydrazine and hydrogen peroxide, respectively, and the catalysts were silver and cobalt-tungsten oxides applied to the bipolar plates with an epoxy adhesive. ${ }^{8}$ The performance of such stacks of thin, lightweight cells sealed in epoxy was spectacular (about $1,000 \mathrm{~W} / \mathrm{kg}, 1 \mathrm{~kW} / \mathrm{l}$ for the stack alone, and about $250 \mathrm{~W} / \mathrm{kg}$ for the complete system at $25^{\circ} \mathrm{C}$ ), but they could only be used for specialized application, because of the unusual fuels.

In 1968, Alsthom entered into an agreement with Peugeot to develop vehicle FCs, but this was superseded in 1970 by an agreement with Exxon (then Es so) to use the lightweight bipolar cell technology in methanol-air cells for stationary applications. In view of the catalytic difficulties encountered, this project was abandoned in 1976-77. Since that time, Alsthom has modified its technology for use on $\mathrm{H}_{2}$-scrubbed air, most recently in partnership with Occidental Petroleum.

Mass-transport considerations have forced Alsthom to abandon the original flow-by electrode arrangement. However, the goffered bipolar plate, which is now made from $30 \%$ conductive carbon black and $70 \%$ polypropylene, still remains for gas distribution. The goffering in the plate is about $0.4 \mathrm{~mm}$ wide and $0.5 \mathrm{~mm}$ deep, with a web thickness of $0.35 \mathrm{~mm}$. The active area of the cell is $400 \mathrm{~cm}^{2}$. Each bipolar plate is incorporated in a $40 \%$ talc-filled polypropylene frame, which has an external area of $900 \mathrm{~cm}^{2}$ and incorporates internal manifolding for gas and electrolyte distribution. The whole bipolar frame-conducting plate unit is manufactured in one piece in a remarkable injection-molding operation using 12 injection points. The remainder of the cell consists of calendared film $\mathrm{Pt}-\mathrm{C} / \mathrm{teflon}$ anodes and cathodes and a spacer frame. Each complete cell is $3.3 \mathrm{~mm}$ thick.

The stacks are process-air cooled, and they are assembled in subgroups of 100 cells to keep shunt currents to a minimum. The end plates of the stack incorporate metal inserts to give uniform current density throughout. All components (including the electrodes) are cemented together with epoxy after suitable surface treatment. An exploded view of the Alsthom stack 50 components is shown in Fig. 3.7-13. The major advantage of this type of structure is its very low potential cost, which is estimated to be about $\$ 30 / \mathrm{m}^{2}$ in mass production or less than $\$ 30 /$ $\mathrm{kW}$ without catalyst.

The present Pt loading is $0.3 \mathrm{mg} / \mathrm{cm}^{2}$, the goal being one half of this value. In the interests of simplicity, the anode and cathode are identical, but specialized electrodes would probably give better performance, for example, if heat-treated macrocycles are used at the cathode. Present pilat-line production volume is 400 cells or $16-20 \mathrm{~kW} /$ day. The performance using $4 \mathrm{~N} \mathrm{KOH}$ at $65^{\circ}$ is $0.72 \mathrm{~V}$ at $150 \mathrm{~mA} / \mathrm{cm}^{2}$ with a goal of $0.8 \mathrm{~V}$. This goal should be easily attainable with specialized electrodes and by IR drop reduction, for example, by improving the bipolar plate conductivity. A component lifetime of $8,000-10,000 \mathrm{~h}$ has been demonstrated. A $4 \mathrm{~N}$ sulfuric acid version of the system also exists, which operates at $100 \mathrm{mV}$ less than the AFCs, as would be expected because of the greater slope of the Tafel line in the acid system.

The AFC air electrode for specialized purposes, which are essentially based on state-ofthe-art PAFC technology, have been developed by organizations other than Alsthom in recent years. In the U.S., the driving forces for these developments have not normally been the AFC (with the notable exception of NASA cells), but rather the energy-conserving air-depolarized cathode for the chlorine-caustic process 65 and the development of the Al-air battery. 35 The organizations involved have been Prototech and Giner, Inc. (low-loading Pt on C) and EltechElectromedia (most recently using pyrolyzed macrocyclics on C). These electrodes possess excellent lifetime and activity for the AFC cathode.

\section{7-7 AFCs at the Royal Institute of Technology (Sweden)}

Major developments have been carried out for the removal of $\mathrm{CO}_{2}$ using $\mathrm{NaOH}$ scrubbing in a counter-current tower using contact elements. 66 The NaOH is regenerated by electrodialysis. 67 In Sweden, $\mathrm{H}_{2}$ will be generated from biomass, special plantations having been 


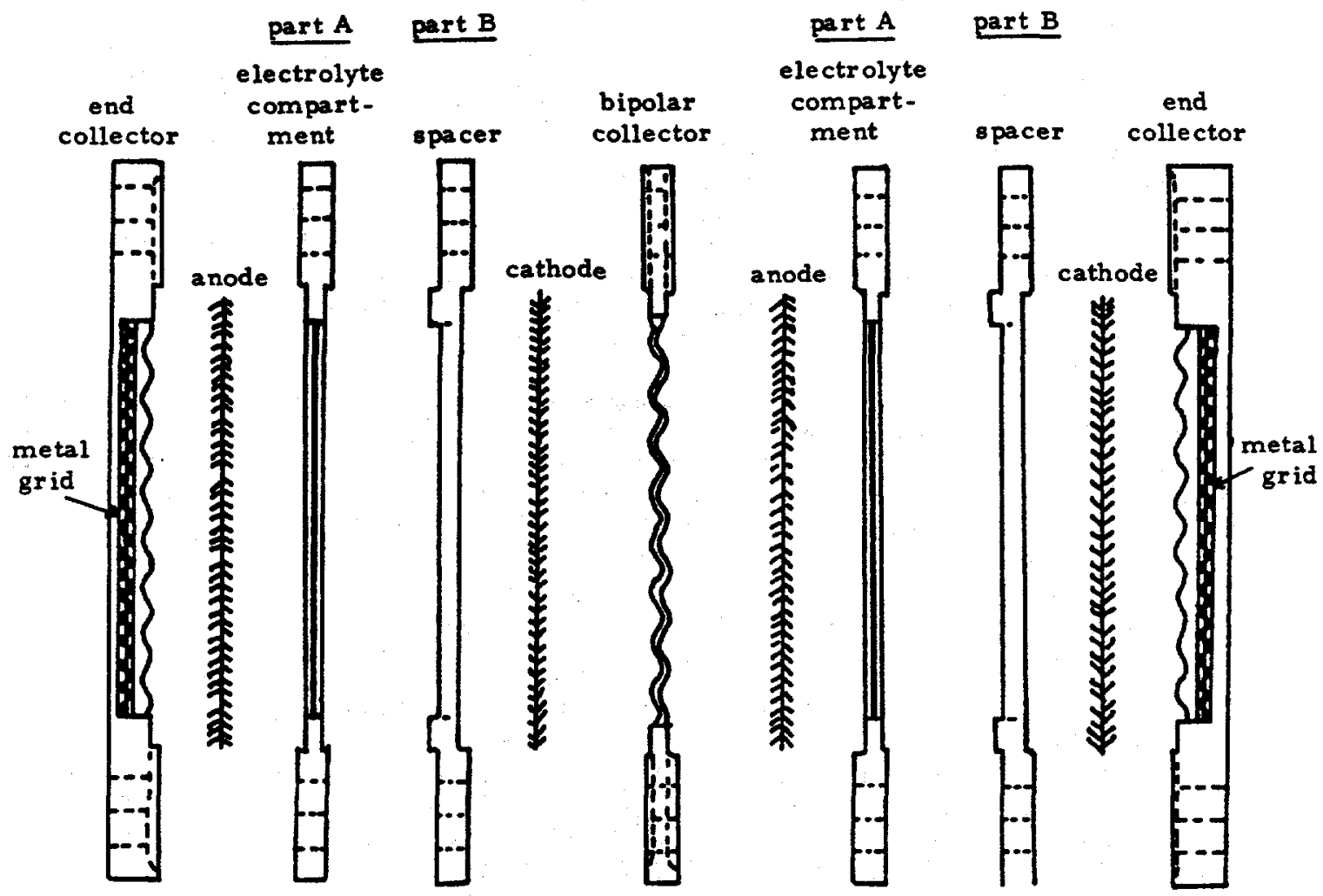

Fig. 3.7-13. The Alsthom-Occidental Petroleum stack components.

initiated to grow trees for this purpose. Either anaerobic pyrolysis or an Fe-steam-wood process will be used to produce $\mathrm{H}_{2}$. Proposed $\mathrm{AFC}$ anodes are $\mathrm{Ag}$ on $\mathrm{C}$ with an active $\mathrm{C}$ cathode on an $\mathrm{Ni}$ support; Raney electrodes have also been developed. The design of the monopolar cell is shown schematically in Fig. 3.7-14.68 The aim is FC use for transportation and industry, with biomass as the primary fuel source. FC development in Sweden is especially important in view

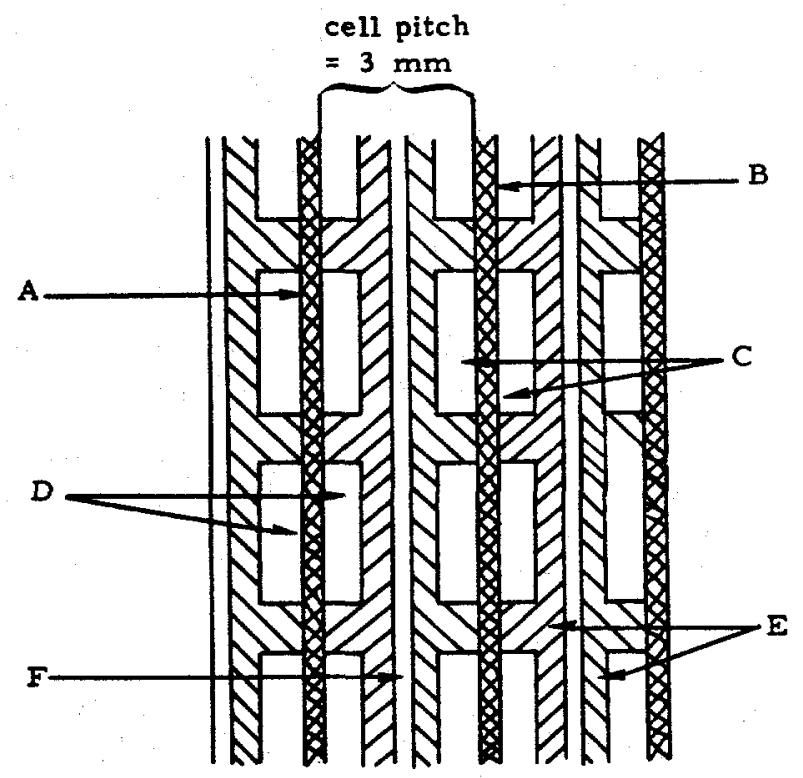

Fig. 3.7-14. Schematic of the 041 cell design in the Royal Institute of Technology FC; A, air electrode; B, hydrogen electrode; C, hydrogen space; $D$, air space; E, ribbed separators; $F$, flat separators, Hydrogen and air flows are per pendicular to each other. 68 
of passage of a referendum that has led to a parliamentary law according to which all nuclear power plants are to phased out in Sweden.

\subsection{Methods for $\mathrm{CO}_{2}$ Removal}

As noted, AFCs are more efficient than PAFCs when pure $\mathrm{H}_{2}$ is used as fuel and their cell atacks will also be less costiy by perhaps a factor of one half. ${ }^{\dagger}$ The preference for PAFCs is, therefore, not entirely clear. PAFC developers believe that FC operations with air require the use of non-alkaline electrolytea to avoid carbonation problems from atmospheric $\mathrm{CO}_{2}$. Acid, molten carbonate, and solid-oxide electrolytes are all $\mathrm{CO}_{2}$-rejecting. At present, phosphoric acid is the preferred acid electrolyte, despite relatively low conductivity $10.6 \Omega^{-1}-\mathrm{cm}^{-1}$ under operating conditions vs $1.0 \Omega^{-1}-\mathrm{cm}^{-1}$ for concentrated KOH) and highly corrosive properties toward metals.

The opinion that the PAFC is superior to the AFC seems inconsistent with the vigorous development of $A F C_{B}$ by German, French, Swedish, and Russian groups. Lindstrom (1984) has expressed the view that the stress laid upon the acid cell in the U.S. has commercial rather than technological foundation. He has pointed out that the cost of $\mathrm{CO}_{2}$ removal, e.g., by the Selexol proposed for sulfur removal from coal-gas process in a $600-\mathrm{MW}$ e plant, would be $\sim 10 \mathrm{mil} / \mathrm{kWh}$. Giner and Swette, 64 however, provide the following estimates: $3.3 \mathrm{mil}(1975) / \mathrm{kWh}, 7 \mathrm{mil}$

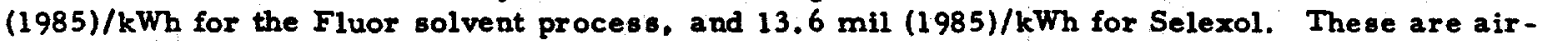
stripped solvent processes and, therefore, residual $\mathrm{CO}_{2}$ will be $-500 \mathrm{ppm}$, which will require removal by alkali absorption.

For reformed carbonaceous fuels, the initial $\mathrm{CO}_{2}$ concentration is about $25 \%$, which must be reduced to a few ppm before introduction into the AFC. If $\mathrm{H}_{2}$ is to be produced from coal and not from biomass, it will be necessary to remove $\mathrm{H}_{2} \mathrm{~S}$ as well as $\mathrm{CO}_{2}$. This operation can be performed efficiently by using the Selexol process.69,70 Replacement of the final absorption medium (e.g., zinc oxide) is a relatively important cost.

The authors in Ref. 64 atate that there are 22 different commercial processes available for $\mathrm{CO}_{2}$ removal. A few of these will now be briefly mentioned.

\section{8-1 Transfer with Liquid-Adsorption Towers}

$\mathrm{CO}_{2}$-containing gases are sent through a solvent which is regenerated by a temperatureswing. Hot KOH may be used, sometimes with organic additives, in non-thermally regenerable processes; $15 \% \mathrm{KOH}+10 \%$ amine borate are typically used for this application. A single pass can reduce $\mathrm{CO}_{2}$ levels from 25 to $0.5 \%$, three passes being necessary to reduce $\mathrm{CO}_{2}$ to $100 \mathrm{ppm}$.

Another version involves ethanolamine and similar compounds, which are thermally regenerable. For the process $2 \mathrm{RNH}_{2}+\mathrm{CO}_{2} \rightarrow \mathrm{RNHCONHR}+\mathrm{H}_{2} \mathrm{O}$ absorption with ethanolamine takes place at $27^{\circ} \mathrm{C}$ and regeneration is done at $115^{\circ} \mathrm{C}$. Reduction of the $\mathrm{CO}_{2}$ level to $50 \mathrm{ppm}$ occurs in one pass. This technique was successfully examined for a small methanol reformer. $61-64$

\section{8-2 Physical Adsorption}

The Selexol process ${ }^{69}$ uses dimethyl ether polyethylene glycol. Adsorption occurs at high pressure (100 psi), and regeneration is accomplished by pressure reduction. The removal per pass is typically around 250 times.

\section{8-3 Solid Beds}

Carbon dioxide may be removed by adsorption in molecular sieves, which are regenerable by temperature rises. The residual $\mathrm{CO}_{2}$ level in the exit gas is about $1 \mathrm{ppm}$, with an estimated aystem cost of about $\$(1985) 40 / \mathrm{kW}$.

Several different types of membranes (e.g., silicon rubber) show good permeability to $\mathrm{CO}_{2}$, which can thereby be separated from $\mathrm{H}_{2}$ under pressure. A classical method of separation of pure $\mathrm{H}_{2}$ is by the use of a $\mathrm{Pd}-\mathrm{Ag}$ membrane under pressure. This technique is used in the Johnson-Matthey man-portable methanol reformer for the supply of $\mathrm{H}_{2}$ to small military FCs.

\section{8-4 Electrochemical Processes}

Several possibilities exist for separating $\mathrm{CO}$ and $\mathrm{H}_{2}$, e.g. . $\mathrm{H}_{2}$ can be oxidized at a porous acid FC anode and regenerated at a porous cathode (completely free of $\mathrm{CO}_{2}$ ), with a total cell potential of about $0.1 \mathrm{~V} .69$ Some data for this process are shown in Fig. 3.8-1.

\footnotetext{
A smaller factor applies of the Alsthom-Occidental cost estimates are accepted.
} 


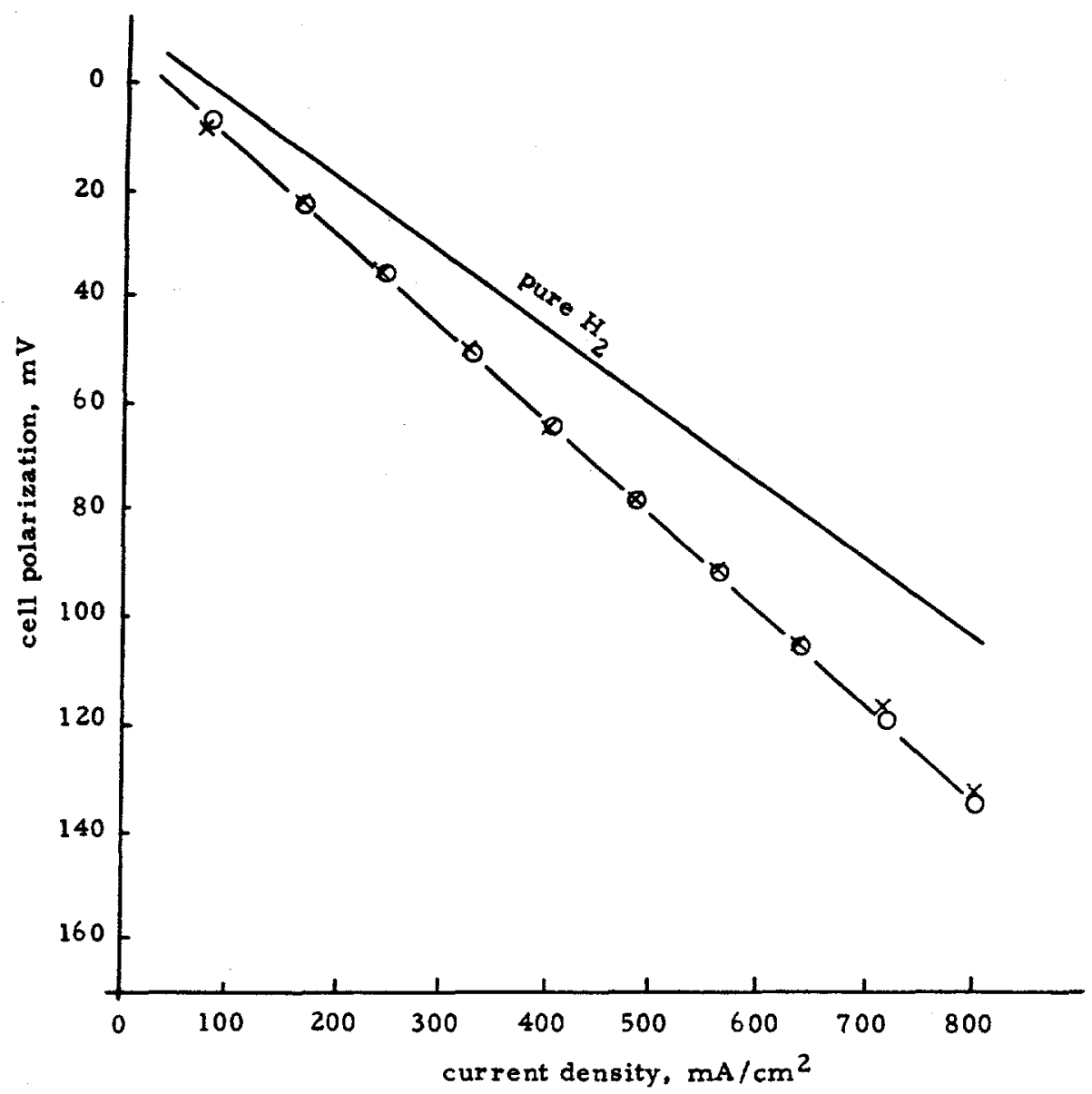

Fig. 3.8-1. Preliminary polarization curves are shown for an electrolytic hydrogen pump. 69 The electrodes were Pt/C/TFE on graphite paper; an Astrel separator was used with $93 \% \mathrm{H}_{3} \mathrm{PO}_{4}$ at $150^{\circ} \mathrm{C}$. The anode feed-gas showed $50 \%$ utilization; $x, 66 \% \mathrm{H}_{2}$ and $34 \% \mathrm{~N}_{2} ; 0,66 \% \mathrm{H}_{2}$ and $34 \% \mathrm{CO}_{2}$.

\section{8-5 Use of Available Heat}

The use of available heat from a fuel cell was examined in Ref. 69. For low-temperature FC, $\mathrm{CO}_{2}$ removal must be very efficient if the cell waste heat is to be sufficient.

The methods for separating $\mathrm{CO}_{2}$ from $\mathrm{H}_{2}$ given in Ref. 69 support the view that $\mathrm{CO}_{2}$ is relatively easily removable at acceptable cost. ${ }^{\dagger}$

\subsection{FCs for Automotive Use}

In assessing the value of $\mathrm{FC}_{s}$ for use as transportation power sources, two observations are appropriate. The amount of time and money that has been historically spent on research in electric motor-FC combinations is a very small fraction of that spent on internal combustion systems. FC research in the U.S. dates from around 1955 (the $20-\mathrm{kW}$ Allis-Chalmers tractor was tested in 1959), but much of the early work was largely oriented towards space applications. Isolated pioneers elsewhere (Bacon, Davtyan, Bauer, Justi, Broers) were the principal earlier eontributors. 7 In fact, the modern phase of research on FCs began about 75 y after the successful development of the IC engine.

Many of the assessments on FCs for transportation have been made by employees of organizations that have no investment in FC technology, as compared with the billions they have spent on IC engine R\& D. The usual viewpoint is that FCs can only be introduced when they are economically competitive. However, introduction of FCs into the transportation sector will be a vast economic undertaking that will require more than 20 y for implementation at significant

'If $\mathrm{AFC}$ s are used with coal, some coal must be burned to provide heat for steam generation. Heat supplied by partial oxidation is also needed to drive the steam-coal reaction. In the future, it will be clearly desirable to make use of well-established technologies without environmental or corrosion problems. 
levels. Inadequate attention is normally paid to the significant reduction of automotive pollution which will occur when FCs replace internal combustion engines. Table 3.9-1 lists the advantages and drawbacks of a battery-FC electrochemical drive process when compared to an IC (chemical) drive for automotive transportation. The weighted trend is clear. In addition, there is no fundamental reason why the cost of the FC-battery-electric motor should be greater than that of the more complex chemical engine for comparable degrees of development and quantity production.

A real difficulty with the FC-battery combination, if this is needed, is less power per unit weight compared with the IC engine. The power-to-weight ratio can be greatly increased by replacing construction materials in the electrochemical devices with conducting plastics, for example, for bipolar plates. The use of printed circuit motors will also be advantageous. These have been shown to give (at $20,000 \mathrm{rpm}$ ) a power-to-weight ratio that is about the same as that of a chemical engine. AFCs without hybrid batteries are now projected to provide acceptable powerto-weight ratios. Using a combination of Alsthom and NASA advanced lightweight AFC technology with macrocyclic cathodes and lightweight bipolar graphite ERs, a weight-to-power ratio of $8 \mathrm{~kg} /$ $\mathrm{kW}$ seems a reasonable prospect at $0.7 \mathrm{~V}, 300 \mathrm{~mA} / \mathrm{cm}^{2}$. Compressed $\mathrm{H}_{2}$ storage is now available in fiber-wound metal cylinders that are capable of more than 12,000 pressure cycles and store $1 \mathrm{~kg}$ of $\mathrm{H}_{2}$ per $30 \mathrm{~kg} i^{63} 1.5 \mathrm{~kg}$ of $\mathrm{H}_{2}$ will yield a $150-\mathrm{km}$ range in a 1 metric ton AFC vehicle (Fig. 3.9-1, Ref. 65). Hence, an AFC stack and $\mathrm{H}_{2}$ storage for a $20-\mathrm{kW}$ (nominal) power unit with a range of $300 \mathrm{~km}$ would weigh about $240 \mathrm{~kg}$. Recently, cracked ammonia has been suggested as fuel for such a unit. 70,71 Its safety from the viewpoint of toxicity may, however, be a problem.

The performance of various power sources is shown in the form of Ragone plots in Fig. 3.9-2. It is seen that an $\mathrm{Li}_{-} \mathrm{FeS}_{2}$ battery hybridized with $1985 \mathrm{AFC}$ technology yields a power and energy of $1 / 2-2 / 3$ that of the inter nal combustion engine, but at about double the efficiency (the typical AFC efficiency at $0.8 \mathrm{~V}$ nominal with $\mathrm{H}_{2}$ is slightly over $50 \%$ based on HHV). If a zero-battery option is decided upon, a fast cold start-up can still be achieved, based on experience with the high power-density alkaline circulating zinc-air battery. 72

Table 3.9-1. Advantages and disadvantages of electrochemical and chemical energy conversion in automotive transportation.

\begin{tabular}{|c|c|c|c|}
\hline \multicolumn{2}{|c|}{ Electrochemical Systems } & \multicolumn{2}{|c|}{ Chemical Systems } \\
\hline Negative & Positive & Negative & Positive \\
\hline $\begin{array}{l}\text { low power/wt ratio } \\
\text { present costs, } \\
\text { without mass pro- } \\
\text { duction, are } \\
\text { greater than for } \\
\text { chemical engines } \\
\text { in mass production }\end{array}$ & $\begin{array}{l}\text { a large increase in } \\
\text { efficiency results } \\
\text { compatible with future } \\
\text { no pollution, including } \\
\mathrm{CO}_{2} \text { emission } \\
\text { low noise } \\
\text { energy-regenerative } \\
\text { better acceleration than } \\
\text { IC engines from } \\
0-35 \text { mph } \\
\text { absence of vibration and } \\
\text { perhaps longer life than } \\
\text { better-driven commer - } \\
\text { cial vehicles, which } \\
\text { usually last } 20 \text { y } \\
\text { electric motors used in } \\
\text { railway traction have } \\
\text { maintenance intervals } \\
\text { of } \sim 10^{6} \text { miles }\end{array}$ & $\begin{array}{l}\text { cause pollution } \\
\text { cause } \mathrm{CO}_{2} \text { increase in } \\
\text { the atmosphere and } \\
\text { perhaps a significant } \\
\text { sea-level rise by } 2060 \\
\text { vibration causes low } \\
\text { vehicle life ( } 5-10 \text { y) } \\
\text { the use of a complex } \\
\text { mechanism results in } \\
\text { a higher frequency of } \\
\text { repair than would } \\
\text { probably be required } \\
\text { for electric motors }\end{array}$ & high power / wt ratio \\
\hline
\end{tabular}




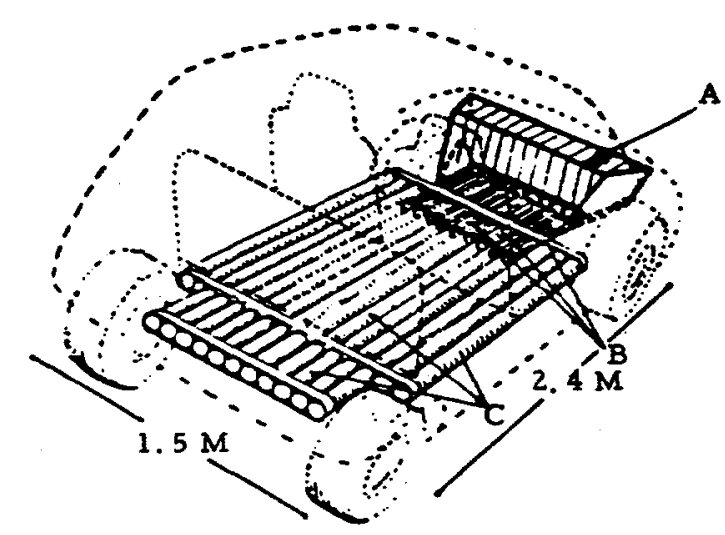

A: $10 \mathrm{~kW}$ fuel cell; $\mathrm{B}: \mathrm{Ni}-\mathrm{H}_{2}$ peaking batteries; $\mathrm{C}$ : chassis tubes (diam $=10 \mathrm{~cm}$, wall thicknes $2 \mathrm{~mm}$ ) storing $1.6 \mathrm{~kg}$ of $\mathrm{H}_{2}$ at 68 bar (1000 psi).

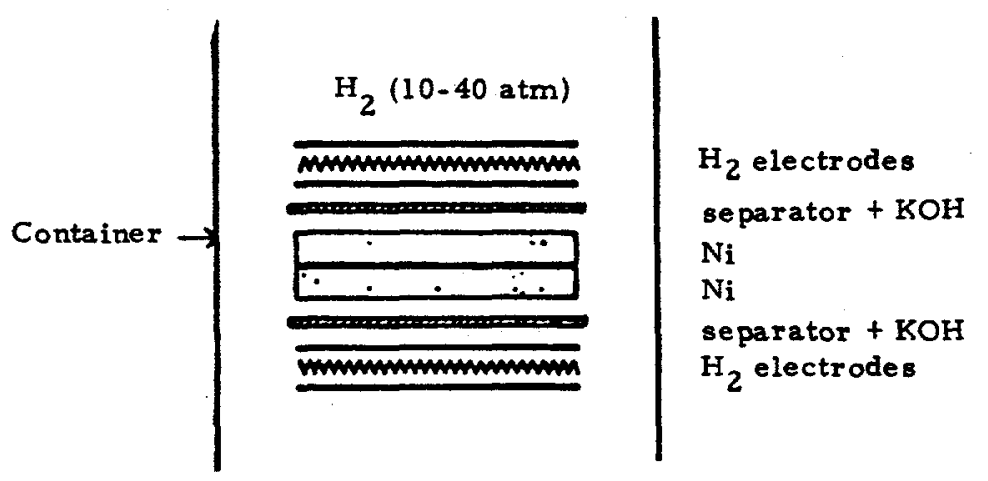

Fig. 3.9-1. $\mathrm{H}_{2}-\mathrm{FC}$ car concept (original design by A. J. Appleby).

Since 1980, Siemens has introduced $7-\mathrm{kW}$ e FCs into Volkswagen vans, while ELENCO equipment has been tested in several buses. The $\mathrm{H}_{2}$-powered $A F C$ should be useful for future transportation because it is efficient, can be made cheaply in mass production, and requires no noble metal catalysts with limited future availability. Development of a cheap, bipolar, noncirculating electrolyte AFC will be required. A step has already been taken in this direction. 73

A thorough examination of optimal $\mathrm{AFC}$ and $\mathrm{H}_{2}$-storage system combinations for vehicles has been carried out by computer data simulation, 74 as suming conversion efficiency to be $45 \%$. Hydrogen storage as liquid ammonia ${ }^{1}$ was considered to be the most effective method.

\section{10 Hydrogen Sources}

Hydrogen is always represented as an expensive (and heavy) fuel because it is normally only a vailable in steel cylinders. Delivered hydrogen in cylinders costs many times the actual gas cost.

A 1978 DoE committee ${ }^{75}$ estimated the price of $\mathrm{H}_{2}$ from coal as $\$ 3-5 M M M B U$. Using the conventional construction cost escalator, its average cost might now be about $\$ 7 / M M B T U$, which is equivalent to gasoline at $\$ 1$ per gallon. However, taking into account the high AFC efficiency compared with that of the IC engine, cost by mile would be only about $40 \%$ of that for gasoline.

Hydrogen from peat and biomas 8 is an attractive option because of the low sulfur content of these fuels (Table 3.10-1). Either anaerobic combustion can be used to produce hydrogen, or steam can be reacted with iron, and the resulting $\mathrm{Fe}_{3} \mathrm{O}_{4}$ reduced using biomass, as has been proposed in Sweden. Very large sources of peat exist in the U.S.

In some countries, particularly Canada, there is more than enough hydroelectric power to supply the entire automotive load. Another often-quoted example is Greenland, which has proven hydroelectric resources of about $15-39 \mathrm{GW}_{\mathrm{e}}(100 \mathrm{~m}$ head) plus $7 \mathrm{GW}$ (200 m head), with a reasonable potential of $25-60 \mathrm{GW}_{e}$, up to a maximum possible of $100 \mathrm{GW}_{e}$. Greenland alone has a reasonable potential for providing hydrogen for 30-40 million AFC-powered EVs if its resources were developed. Hydroelectric power is a vailable in Canada at $1-2 \mathrm{c} / \mathrm{kWh}$; at $2 \mathrm{c}$ / $\mathrm{kWh}$, a simple calculation shows that the $\mathrm{H}_{2}$ cost would be $\sim 10 \mathrm{MMBTU}$, which is equivalent to gasoline at $\$ 1.40$ per gallon. By comparison, current commodity prices for unleaded gasoline (May 1985) are $\$ 0.86 /$ gallon (leaded $\$ 0.77 /$ gallon). 


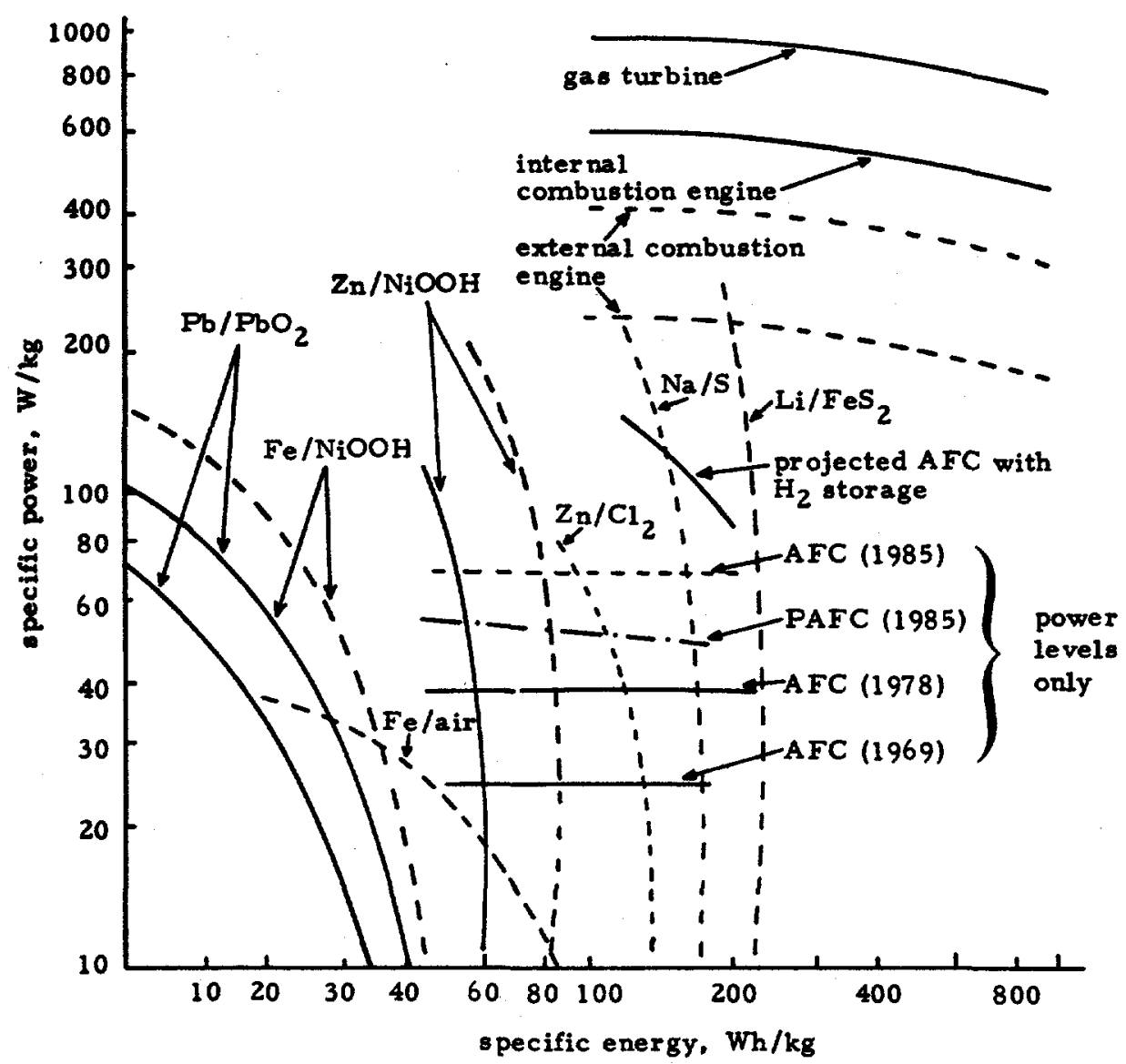

Fig. 3.9-2. Ragone plots for different power sources; the logarithm of specific power is shown as a function of the logarithm of specific weight; achieved performance; - - --- projected; - - 1985 PAFCs.

Table 3.10-1. Sources of $\mathrm{H}_{2}$

\begin{tabular}{|c|c|c|}
\hline Primary Fuel & Positive & Negative \\
\hline coal & $\begin{array}{l}\text { costs of perhaps } \$ 1.10 / \text { gallon of gasoline } \\
\text { equivalent if the plant is large }\end{array}$ & $\mathrm{CO}_{2}, \mathrm{~s}$, fly-ash \\
\hline peat and biomass & $\begin{array}{l}\text { costs of }<\$ 1 / \text { gallon of gasoline equivalent } \\
\text { no net } \mathrm{CO}_{2} \text { or } \mathrm{S} \text { production }\end{array}$ & $\begin{array}{l}\text { no technological } \\
\text { development yet }\end{array}$ \\
\hline nuclesr energy & $\begin{array}{l}\text { in Canada, the CANDU Th-cycle reactor } \\
\text { would yield } \mathrm{H}_{2} \text { equivalent to } \$ 1.20 / \text { gallon } \\
\text { of gasoline }\end{array}$ & $\begin{array}{l}\text { limited to countries } \\
\text { which buy Canadian } \\
\text { technology }\end{array}$ \\
\hline hydroelectric power & $\begin{array}{l}\text { equivalent to around } \$ 1.50 \text { per gallon of } \\
\text { gasoline }\end{array}$ & $\begin{array}{l}\text { restricted to certain } \\
\text { countries, e.g., } \\
\text { Canada }\end{array}$ \\
\hline
\end{tabular}

Although U.S. nuclear electricity is expensive, the Canadian CANDU system yields electricity at $1.5 \mathrm{c} / \mathrm{kWh} .76$ The corresponding hydrogen cost would be $\$ 8.50 / \mathrm{MMBTU}$, which is equivalent to gaooline at $\$ 1.18$ per gallon. Canada has a large excess of nuclear power, as well as hydroelectric power, and it might be that such low use-period electricity could be sold at a lower cost than those stated.

In summary, if the necessary commitments are made by government and industry, it appears that $\mathrm{H}_{2}$ could become a vailable at between $16 \%$ and $50 \%$ more than the wholesale beforetax cost of unleaded gasoline. Assuming equal efficiencies of conversion to mechanical power, 
correction for the much larger conversion efficiency when FC-powered transportation is used would result in costs per mile equal to about $50 \%$ of those for gasoline, if the moment we disregard the capital costs for setting up the appropriate infrastructure for hydrogen delivery. Finally, we should note that if $\mathrm{H}_{2}$ becomes avalable, and if it is burned in IC engines instead of $F C_{8}$, its tremendous potential for energy conversion will be much less used than with fuel cells. ${ }^{\dagger}$ This is because the efficiency of $\mathrm{H}_{2}$ energy conversion in an $\mathrm{IC}$ engine is only 1.5 times, on a BTU basis, that of gasoline or methanol (about $25 \%$ average efficiency, much less under part load). In contrast, the $\mathrm{H}_{2}$ AFC will possess $50 \%$ efficiency at nominal load, and even higher values at part load. Unlike electric utility $P A F C_{8}$, acid $F C_{8}$ for automotive transportation cannot use unleaded gasoline fuel due to reforming problems in small, compact units (Chapter 2), and AFCs are unlikely to perform effectively on methanol, which can be used in acid FC systems, but only with weight, performance, and cost penalties in small units, which in any case presently require noble metal catalysts. In contrast, the high performance AFC requires no noble metals and will give by far the highest efficiency of all conversion process on $\mathrm{H}_{2}$. When the latter material becomes widely available as we approach a Hydrogen Economy, the high capital cost required to generate $\mathrm{H}_{2}$ will make it too valuable to simply burn.

A summary of $\mathrm{H}_{2}$ sources and their merits is presented in Table 3.10-1.

\section{11 Storage of $\mathrm{H}_{2}$ in Transportation}

It is usual to reject cylinders as storage for this application because a steel cylinder holds only $\sim 1 \%$ weight of $\mathrm{H}_{2}$. However, there are possibilities in the use of lightweight materials such as $\mathrm{Mg}$ alloys. 77 In this case, about $4-5 \% \mathrm{H}_{2}$ can be contained in the cylinders. Allen and $Z_{\text {weig }} 78$ have converted a half-ton truck to operate for 150 miles by using a single aluminum cylinder that contains $\mathrm{H}_{2}$ at 150 bar. They tested the cylinder to the bursting point at 600 bars.

Hydrogen storage in fiber-wound aluminum cylinders $\left(3.3 \% \mathrm{H}_{2}\right)$ has already been suggested. 63 Storage as metallic hybrides is clearly a possibility, although FeTiMn is too heavy to be a serious candidate for this application. In the $\mathrm{Daimler}-\mathrm{Benz}$ storage system, $\mathrm{H}_{2}$ is obtained for a cold start from a small quantity of FeTi hydride, and when hot exhaust gases are available, they are passed over $\mathrm{Mg}$ hydride, which is the major source of $\mathrm{H}_{2 .} .79 \mathrm{Mg}$ stores about $10 \%$ by weight of $\mathrm{H}_{2}$; a compressor is not necessary because cooling occurs when $\mathrm{Mg}_{\mathrm{gds}}$ orbs $\mathrm{H}_{2}$. According to Daimler-Benz, their IC-powered vehicles can travel up to $150-250 \mathrm{~km}$ on one recharge.

If the cost of hydrogen in 1985 dollars from coal is $\$ 8 / \mathrm{MMBTU}$ and the cost of liquefaction is $\$ 5 / \mathrm{MMBTU}$, liquid $\mathrm{H}_{2}$ would cost about $\$ 13 / \mathrm{MMBTU}$ (equivalent to $\sim 1.80 /$ gallon gasoline). This may be an acceptable cost taking into account the much greater efficiency of conversion in FCs, i. e., the net reduction in the costs of transportation which would be provided by hydrogen from coal used in AFCs.

According to Lockheed, 80 aircraft would run more cheaply on $\mathrm{H}_{2}$ than on conventional fuel. The difficulty is the large initial investment, not only to invest in a plant which would give the required amounts of liquid hydrogen, but also to de velop and manufacture a new generation of aircraft containing suitable storage for the relatively bulky $\mathrm{LH}_{2}$. $^{\ddagger}$

Sooner or later, however, this will be necessary, ${ }^{83}$ as production increases, and hydrogen cost will come down a learning curve similar to those for the PAFC in Figs. 2.4-1 and 2.4-2 in Chapter 2. Figure 3.11-1 shows a learning curve of cost reduction of $\mathrm{H}_{2}$ as the daily demand is increased.

Methanol from NG is predicted to cost about the same per MMBTU as $\mathrm{LH}_{2}$ from coal, but does not require cryogenic tankage. The incremental cost of $\mathrm{CO}_{2}$ removal must be weighed against the costs and energy $\mathrm{los}_{\mathrm{s}} \mathrm{s}$ involved in liquefaction and cryogenic storage of $\mathrm{LH}_{2}$.

Podgorgny et al 81 have recently published test results on different types of $\mathrm{H}_{2}$ storage for vehicles, and they conclude that hydrides appear to represent the best alternative.

\section{12 Safety Aspects of $\mathrm{H}_{2}{ }^{82}$}

Hydrogen forms combustible and explosive mixtures with air, as do NG, gasoline, and other fuels. Due to its lower critical energy for reaction, detonations occur more easily with $\mathrm{H}_{2}$; therefore, flash arrestors may be needed in pipelines carrying $\mathrm{H}_{2}$. However, although it is easier to ignite an $\mathrm{H}_{2}$-air mixture compared with NG-air or gasoline-air, the energy of a hydrogen explosion is only one-quarter of that of an explosion involving hydrocarbons on an equal volume basis.

'In fact, in IC engines for urban transportation, hydrogen burns about 1.5 times more efficiently than does gasoline. By contrast, $\mathrm{H}_{2}$ in an $\mathrm{FC}$ would have an average efficiency for urban driving of about three times that of gasoline in an IC engine.

F Several meeting of the major airlines of the world took place in the 1980s in search of progress towards converting aircraft to burn hydrogen fuel. A research program has been suggested. 


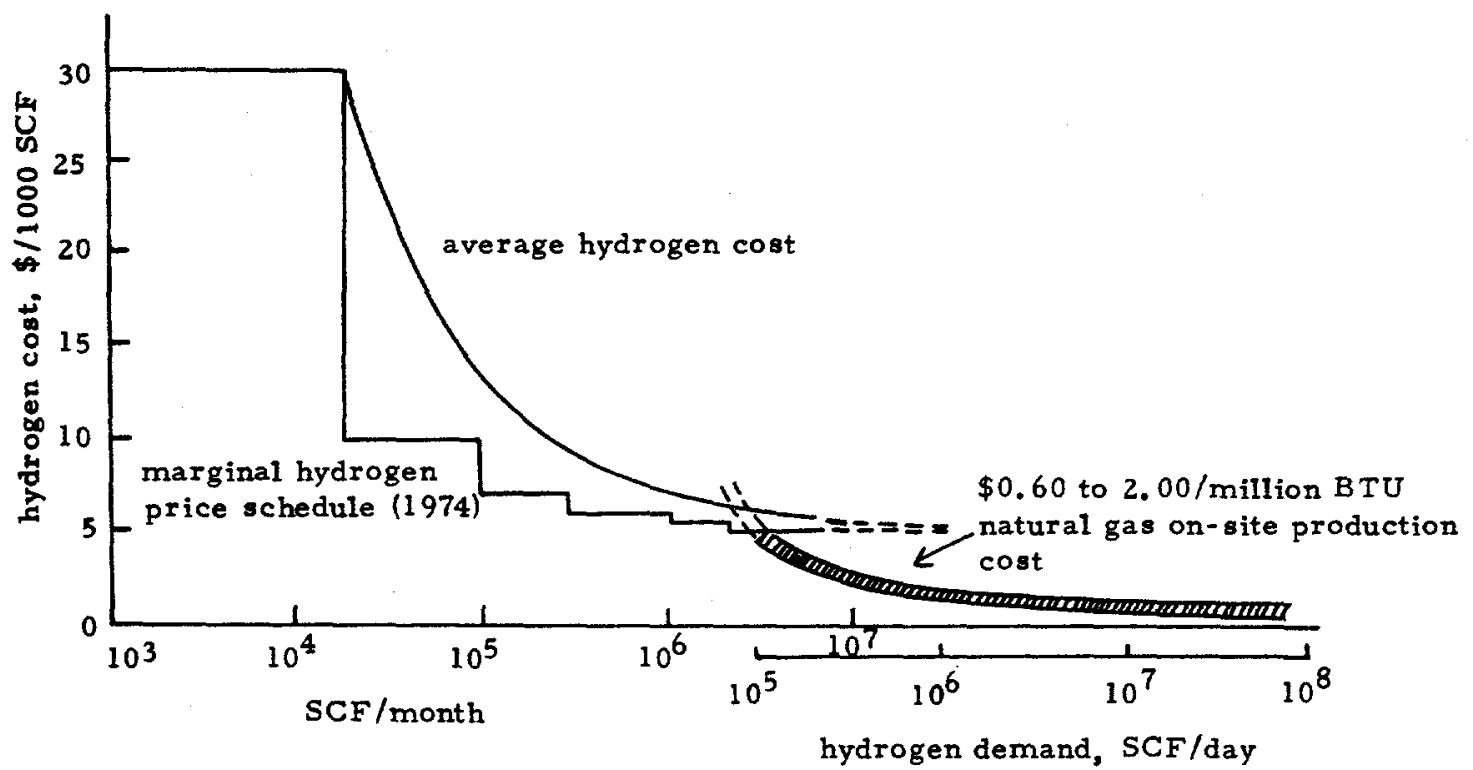

Fig. 3.11-1. Learning curve for $\mathrm{H}_{2}$ cost: dependence of price on demand (SCF/ day), and on supply (SCF/month). 86

Conversely, it is easier to start an $\mathrm{H}_{2}$ fire than one with gasoline or NG. However, the damage which arises from such fires is far less with $\mathrm{H}_{2}$ than with gasoline. The reasons for this are that gasoline fires burn 5-10 times longer than do $\mathrm{H}_{2}$ fires, ( $\mathrm{LH}_{2}$ evaporates and dissipates rapidly, so that very little of the $\mathrm{H}_{2}$ actually burns) and radiate much more heat. In contrast, the pale blue $\mathrm{H}_{2}$ flame radiates little energy. Another important matter is that $\mathrm{H}_{2}$ fires create no toxic chemicals, whereas hydrocarbon fires certainly produce $C O$ and other toxic materials. (Aircraft accident fires usually kill people by the breathing in of gases from decomposing plastic materiala rather than by incineration.)

Hydrogen lacks bdor. The refore, leak detection requires addition of small traces of odorants. Hydrogen diffuses 2.8 times faster than methane, which lowers the probability of catastrophic fires or explosions, although it increases the leak rate from joints.

Thus, if one looks at the whole picture, $\mathrm{H}_{2}(\mathrm{~g})$ and $\mathrm{H}_{2}(\mathrm{~L})$ are not more dangerous to use than NG or gasoline. Table 3.12-1 summarizes some comparative safety information.

\subsection{An Estimate of the Economic Prospects of the Alkaline Fuel Cell}

The economics of a fuel cell depend on its capital costs, the cost of money, the efficiency of the lifetime, conversion at the given capital cost, the cost of any fuel pre-preparation process, and the cost of the fuel.

The efficiency and the capital cost are related, and one such relation (for a projected 600-MW system using coal) is shown in Fig. 3.13-1. 
Table 3.12-1. Summary of the comparative safety situation in respect to hydrogen fuels. 86

\begin{tabular}{|c|c|c|c|c|c|}
\hline & Aspect & Hydrogen & Methane & Gasoline & Remarks \\
\hline \multicolumn{2}{|c|}{$\begin{array}{l}\text { Confinement of } \\
\text { liquid }\end{array}$} & Relatively difficult & $\begin{array}{l}\text { Less } \\
\text { diffic ult }\end{array}$ & Standard & $\begin{array}{l}\text { All done routinely } \\
\text { in the space } \\
\text { program }\end{array}$ \\
\hline \multicolumn{2}{|c|}{$\begin{array}{l}\text { Containment of vapor } \\
\text { at NTP }\end{array}$} & Ea sy & Easy & $\begin{array}{l}\text { Relatively } \\
\text { difficult }\end{array}$ & \\
\hline \multicolumn{6}{|c|}{ Danger in fuel spill: } \\
\hline (i) & $\begin{array}{l}\text { Ease of start- } \\
\text { ing fire }\end{array}$ & Greatest & Intermediate & Less great & \\
\hline (ii) & $\begin{array}{l}\text { Lastingness } \\
\text { of fire once } \\
\text { started }\end{array}$ & Shortest & Intermediate & Greatest & \\
\hline (iii) & $\begin{array}{l}\text { Ignitibility } \\
\text { in presence of } \\
\text { weak sources, } \\
\text { e.g. matches }\end{array}$ & $\begin{array}{l}\text { Most easily } \\
\text { ignited }\end{array}$ & $\begin{array}{l}\text { Same as } \\
\text { gasoline }\end{array}$ & $\begin{array}{l}\text { Same as } \\
\text { methane }\end{array}$ & \\
\hline (iv) & $\begin{array}{l}\text { Detectability } \\
\text { of fire }\end{array}$ & $\begin{array}{l}\text { More difficult but } \\
\text { can be done }\end{array}$ & O.K. & O. K. & \\
\hline (v) & $\begin{array}{l}\text { Fire can be } \\
\text { fought with? }\end{array}$ & Water & Water & Water & $\begin{array}{l}\text { But gasoline } \\
\text { difficult to } \\
\text { fight with } \\
\text { water }\end{array}$ \\
\hline (vi) & $\begin{array}{l}\text { Health hazards } \\
\text { by smoke } \\
\text { inhalation }\end{array}$ & Least bad & $\begin{array}{l}\text { Less bad } \\
\text { than } \\
\text { gasoline }\end{array}$ & Worst & \\
\hline Ease & of explosion & Greatest & Less bad & $\begin{array}{l}\text { Least, but } \\
\text { still con- } \\
\text { sider able }\end{array}$ & $\begin{array}{l}\text { Greater breadth } \\
\text { of explosion } \\
\text { limits for } \mathrm{H}_{2}\end{array}$ \\
\hline $\begin{array}{l}\text { Dang } \\
\text { explc } \\
\text { occu }\end{array}$ & $\begin{array}{l}\text { from an } \\
\text { sion, once } \\
\text { red }\end{array}$ & $\begin{array}{l}\text { Small if unconfined, } \\
\text { devastating if con- } \\
\text { fined, but less so } \\
\text { for same volume } \\
\text { than LNG or gaso- } \\
\text { line }\end{array}$ & $\begin{array}{l}\text { See } \\
\text { hydrogen }\end{array}$ & $\begin{array}{l}\text { See } \\
\text { hydrogen }\end{array}$ & \\
\hline $\begin{array}{l}\text { Safet } \\
\text { and }\end{array}$ & $\begin{array}{l}\text { in commercial } \\
\text { adustrial use }\end{array}$ & No special problems & $?$ & Routine & \\
\hline $\begin{array}{l}\text { Safet } \\
\text { and } \mathrm{r}\end{array}$ & $\begin{array}{l}\text { in transport } \\
\text { e sidential }\end{array}$ & $\begin{array}{l}\text { At present greater } \\
\text { risk than } \mathrm{CH}_{4} \text { of } \\
\text { gas because routine } \\
\text { methods for dealing } \\
\text { with have not been } \\
\text { worked out }\end{array}$ & & & $\begin{array}{l}\text { Long-term past } \\
\text { use of } 50 \% \text { hydro- } \\
\text { gen gas in } \\
\text { European house- } \\
\text { holds supports } \\
\text { safety }\end{array}$ \\
\hline
\end{tabular}




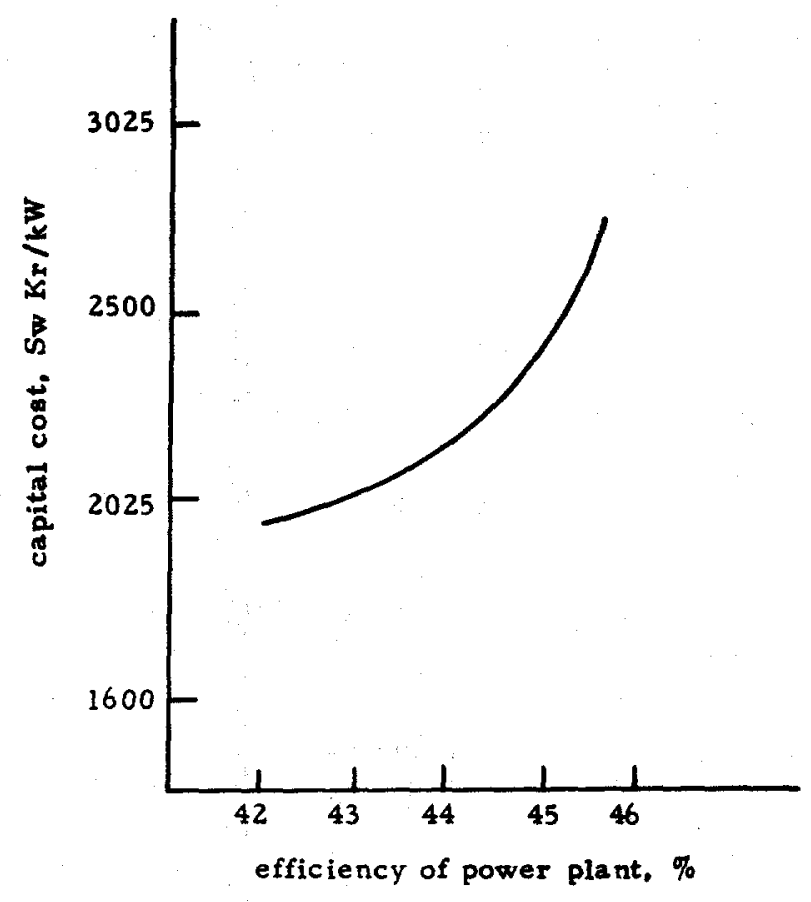

Fig. 3,13-1. Variation of total capital costs in Swedish Kroner (including $\mathrm{CO}_{2}$ removal, reforming, etc.) in a Swedish $600-\mathrm{MW}$ e plant with efficiency. 68

The following rough estimate of electricity costs can be made:

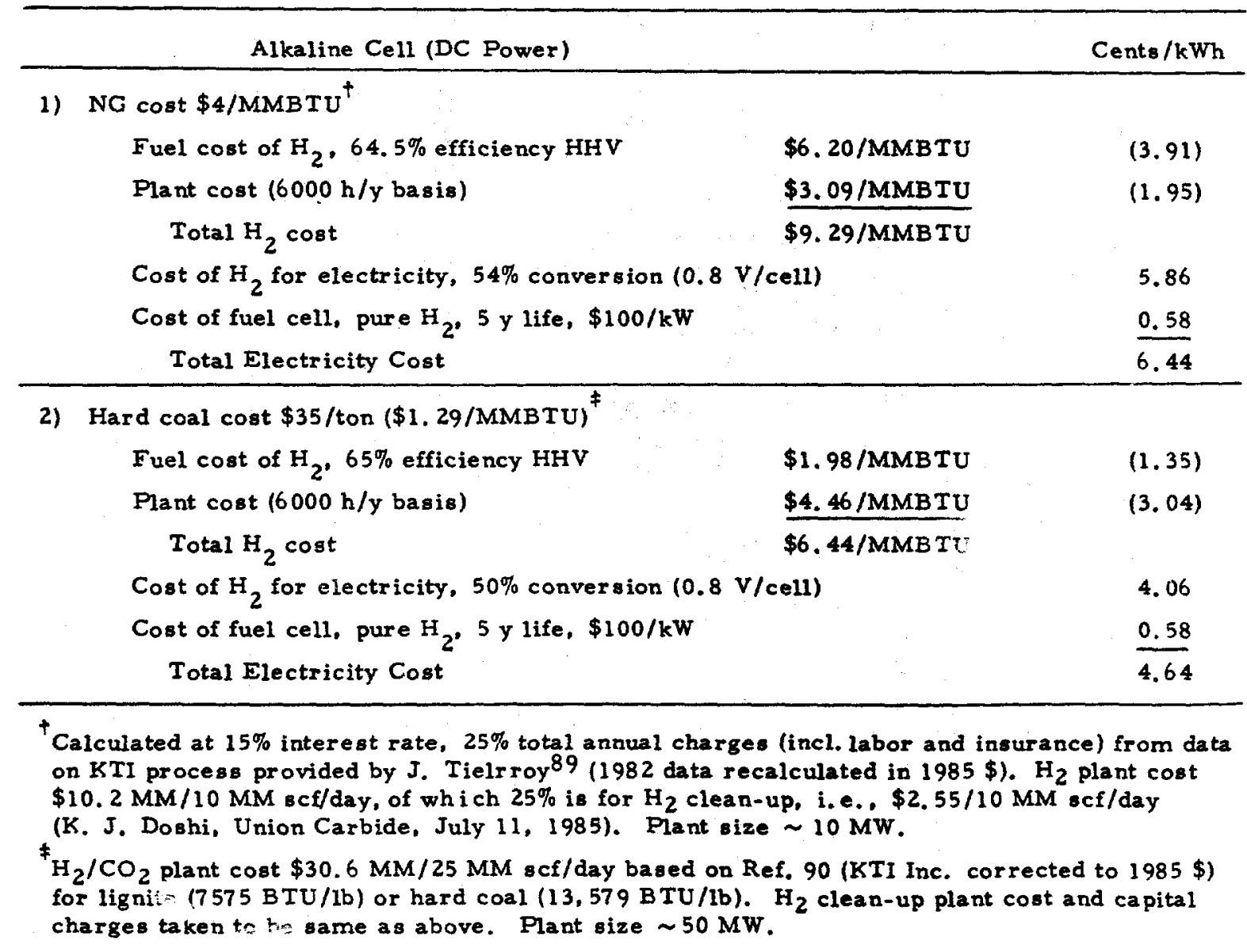


These are rough estimates. They suggest that an alkaline cell working on $\mathrm{H}_{2}$ from natural gas or coal could probably be developed to produce electricity at between 5 and $7 \mathrm{c} / \mathrm{kWh}$, respectively (1985 data).

The corresponding cost of the acid cell is increased because the cost of the cell and fuel processor will be $\sim \$ 30 / \mathrm{kW}$ compared with $\sim \$ 100 / \mathrm{kW}$ for the Alsthom-Occidental cell for pure $\mathrm{H}_{2}$, but the cost is decreased because of the absence of the need for the plant producing pure $\mathrm{H}_{2}$.

Thus, the corresponding acid cell costs may be estimated as follows:

\begin{tabular}{lc}
\hline Acid Cell (DC Power) & Cents $/ \mathrm{kWh}$ \\
\hline
\end{tabular}

Cost of installation (integrated cell - fuel processor)

$\$ 730 / \mathrm{kW}$ without inverter, $6000 \mathrm{~h} / \mathrm{y}, 25 \%$ annual charges

3.04

Cost of $\mathrm{Ng}, \$ 4.00 / \mathrm{MMBTU}, 42 \%$ electrical conversion efficiency

3. 25

Cost of cell stack replacement, 5 y life, $\$ 200 / \mathrm{kW}$

0.67

Total Electrical Cost

6.96

Thus, if AFCs can be developed to show consistently lifetimes of $40,000 \mathrm{~h}$, the cost of the electricity which they produce might be less than that from the acid cell. There are corresponding advantages of no limitation as to catalyst if a massive building program became desirable.

These are two uncertain matter $s$ which can only be dealt with at this time in terms of estimates.

Cell Lifetime: This affects the economics because of replacement cost considerations. The established lifetime of AFCs is $15,000 \mathrm{~h}$ (working on $\mathrm{H}_{2}$ ), and the fact that the PAFC has a lifetime of $40,000 \mathrm{~h}$ appears to favor the latter. However, inquiry 84 shows that the breakdown of alkaline cells after $15,000 \mathrm{~h}$ is connected neither with the deactivation of the catalyst electrodes or with attacks on the carbon. It is connected with the deterioration of polyethylene frames. 84 Since the cause of the breakdown is now known, changes can be made. However, it may prove to be unnecessary, because it has been reported that if "vigorous electrolytic action" (application of an outside potential for a brief period to evolve oxygen) is used after every $15,000 \mathrm{~h}$, the cell can be resuscitated to last for more than $40,000 \mathrm{~h}$. No attack on the carbon or catalyst has been observed within $40,000 \mathrm{~h} .84$ It therefore seems likely that the projected lifetime of future $A_{F C}$ should not be less than that of PAFCs. In fact, having regard to the milder conditions in the AFC (compared with those of the PAFC), one might well expect that the eventual lifetime of the AFC would be greater than that of the PAFC. 85

One reservation must be made here. 86 It is necessary to remove $\mathrm{CO}_{2}$ in the AFC. Most of the methods suggested (see Sec. 3,8 ) remove $\mathrm{CO}_{2}$ to the $1-10 \mathrm{ppm}$ range. A certain amount of carbonate would remain, and the possible effect of catalyst contamination and support destruction by transformation to bicarbonate (and the concomitant expansion and cracking of the support) have to be kept in mind. It is noteworthy that the feasibility of the removal of $\mathrm{CO}_{2}$ to 1 ppm is not in doubt. 88

$A_{8}$ to the economics of the removal, this cannot be established until appropriate experiments have been done. The following estimates exist (recalculated for 1985): Lindstrom (Selexol), 10 mil; Giner (Fluor solvent process), 7 mil; Giner (Selexol), 13.6 mil; mean estimate, $10.6 \mathrm{mil}$. A reasonable cost is, therefore, $1 \mathrm{c} / \mathrm{kWh}$ for the $\mathrm{CO}_{2}$ removal of a $\mathrm{H}_{2}-\mathrm{CO}_{2}$ mixture from coal to $1 \mathrm{ppm}$.

The choice of PAFC for the main emphasis in FC development may be questioned. It was based on three assumptions: (a) Sufficient removal of $\mathrm{CO}_{2}$ from the gas mixture could not be accomplished economically. This now seems to have less credibility. (b) "No steam" would be available from this AFC for use in the reformer. Thus, after the $\mathrm{CO}_{2}-\mathrm{H}_{2}$ mixture enters the acid cell, some $15 \%$ of the $\mathrm{H}_{2}$ remains unconsumed, and it is mainly from this that the reformer heat is obtained. 87 The AFC can afford to use extra $\mathrm{CH}_{4}$ for steam-raising and for providing the heat of reforming. (c) The AFC lifetime was regarded as being only about $10,000 \mathrm{~h}$. This objection was rational and is not entirely superseded. However, the cause of the breakdown has been identified, and a process has been found by which the life 84 of an AFC working on $\mathrm{H}_{2}$ can be extended to $c .40,000 \mathrm{~h}$. The eventual attainment of this life, without intermediate procedures, seems a reasonable prospect.

Thus, the AFC need not be limited to being used with pristine hydrogen but could be run on $\mathrm{H}_{2}$ from natural gas or coal with appropriate pressure-swing absorption of $\mathrm{CO}_{2}$. 
Table 3.13-1. Estimates of mass production fuel cell costs in $1985 \$ / \mathrm{kW}$ (D. C.).

\begin{tabular}{c|c|c|c}
\hline FC Type & Material Cost & $\begin{array}{c}\text { Cell Stack Cost with } \\
\text { Catalyst }\end{array}$ & Installed FC Generator \\
\hline AFC & 10 (without catalyst) & 70 (with catalyst) & 100 (hydrogen fuel) \\
\hline MCFC & 50 & $150-300$ & $500-1000$ (methane fuel) \\
\hline SOFC & 120 & $300(?)$ & $\begin{array}{l}1000 \text { (?) (methane } \\
\text { fuel) }\end{array}$ \\
\hline PAFC & 50 (graphite parts) & 200 & $\sim 750$ (methane fuel) \\
\hline
\end{tabular}

\subsection{Research Priorities for the AFC}

At present, interest in the U.S. in the AFC is obscured by the prevailing opinion that the uses of this cell are restricted to situations where pristine hydrogen (e.g., from electrolysis) is available. The most important priority in research is to establish firmly the economics of sufficient removal. It will evidently be desirable to investigate several approaches.

The second priority is again principally an economic matter: What amount of fuel will be necessary for working the reformer (e.g., by the use of natural gas instead of hydrogen and available steam as with the PAFC).

The third priority is to investigate the cause of the anomalously lower lifetime which this cell has (anomalous because the conditions are much milder than with the PAFC - or indeed, the MCFC). There is evidence 84 to suggest that a specific component, not the carbon or catalyst, causes breakdown. Table 3.14-1 gives a number of items which demand research attention.

\subsection{Concluding Remarks}

The commercial development of the PAFC now taking place has tended to diminish the potentialities of the AFC, the first practical fuel cell. The AFC has certain advantages not present with the acid cell. One is the quicker start-up time. Even at cold, $A F C_{s}$ have about $1 / 4$ of fuel power. Another advantage is the possibility of operating with non-noble metal catalysts. A third advantage is the somewhat higher efficiency of the AFC run on pure hydrogen.

\subsection{Acknowledgments}

The authors are grateful for an initial collaboration with $H$. Wroblowa in discussions which led to the writing of this chapter. She also provided them with several important documents which were used in the chapter's preparation, as well as a list of criticisms of the paper which (for the most part) have been taken into account in the revision of the chapter. The paper by Taylor and Srinivasan 85 was used frequently in the preparation of the present chapter.

Karl Kordesch is to be thanked for an informative discussion of $\mathrm{CO}_{2}$ removal in July 1984.

Particular thanks is to be given to Professor Olle Lindstrom for information giving during a visit to Stockholm in October 1984, as well as extensive correspondence, the giving of documents, tables, etc., and information regarding the lifetimes of alkaline cells given in May 1985.

S. Srinivasan provided early support and gave several important documents. 
Table 3.14-1. Research priorities for the AFC; original table by J. O'M. Bockris

\begin{tabular}{|c|c|c|}
\hline Field & Comment & Priority \\
\hline $\begin{array}{l}\text { Economics of competing } \mathrm{CO}_{2} \\
\text { removal methods }\end{array}$ & $\begin{array}{l}\text { Must include examination of the effects } \\
\text { of trails of } \mathrm{CO}_{3}^{-} \text {; circulation; filtration }\end{array}$ & AA \\
\hline Material decay & $\begin{array}{l}\text { Must identify components in AFC which } \\
\text { fail. Regenerative methods? New } \\
\text { materials }\end{array}$ & $\mathbf{A A}$ \\
\hline $\begin{array}{l}\text { Economics of reformer } \\
\text { heating }\end{array}$ & $\begin{array}{l}\text { Reformer may need more than the } \\
\text { calculated thermodynamical heat }\end{array}$ & AA \\
\hline Non-noble catalysts & $\begin{array}{l}\text { Particularly transition metal oxide } \\
\text { and pyrolyzed "macrocycles." } \\
\text { Not only anodic but also cathodic } \\
\text { studies. Efficiency aim: } 60 \%\end{array}$ & A \\
\hline $\begin{array}{l}\text { Materials of construction: } \\
\text { conducting plastics, plastics }\end{array}$ & $\begin{array}{l}\text { Weight as well as economics is the } \\
\text { concern }\end{array}$ & $\mathbf{A}$ \\
\hline $\begin{array}{l}\text { Circulation of electrolyte } \\
\text { technique }\end{array}$ & Needed if $\mathrm{H}_{2}$ is not pristine & $\mathbf{A}$ \\
\hline $\begin{array}{l}\text { High access porous electrode } \\
\text { design }\end{array}$ & $\begin{array}{l}\text { Current densities of several } \mathrm{A} / \mathrm{cm}^{2} \\
\text { and } \mathrm{kW} / \mathrm{kg} \text { are aims }\end{array}$ & A \\
\hline
\end{tabular}


1. A. M. Adams, F. T. Bacon and R. G. H. Watson, in Fuel Cells, p. 129, W. Mitchell, Jr., ed., Academic Press, NY (1963).

2. M. A. Habib and J. O'M. Bockris, J. Electrochem. Soc. 132, 108 (1985).

3. M. Pourbaix, Atlas D'Equilibres Electrochimiques, p. 2, Gautier-Villars, Paris (1963).

4. J. O'M. Bockris, Energy Options, p. 205, Halstead Pres s, NY (1980).

5. H. Nurnberg, personal communication, December 1984.

6. O. Murphy and F. Gutmann, Modern Aspects of Electrochemistry, Plenum Press, NY, vol. 15, p. 1 (1983).

7. H. A. Liebhafsky and E. J. Cairns, Fuel Cells and Fuel Batteries, Wiley, NY (1968).

8. B. J. Crowe, "Fuel Cells, A Survey," NASA Report No. SP-5115, Washington, D. C. (1973).

9. K. V. Kordesch, Abstract No. 10, Ext. Abs. Fall Electrochem. Soc. Meeting, October 1970 .

10. E. A. Justi, M. Pilkuhn, W. Scheibe and A. Winsel, Akad. Wiss. Lit. Mainz Abhandl. Math. -Nat. K1. 8 (1959); E. W. Justi and A. W. Winsel, Cold Combustion Fuel Cells, Steiner Verlag, Wiesbaden (1962).

11. H. Dahms and J. O'M. Bockris, J. Electrochem. Soc. 111, 728 (1964).

12. E. J. Cairns and D. I. MacDonald, Electrochem. Tech. $\frac{2}{2}, 65$ (1964); E. J. Cairns and D. C. Bartosik, J. Electrochem. Soc. 111,1205 (1964); $\bar{E}$. J. Cairns and A. M. Breitenstein, J. Electrochem. Soc. 114, 349 (1967).

13. O. Lindstrom, Power Sources 7, p. 419, Academic Pres8, NY (1979).

14. E. Justi, Hydrogen As An Energy Medium, Plenum Press, NY (1985).

15. H. Uwe, E. Justi and H. Selbach, Energy Conversion and Management 23, 245 (1983).

16. J. O'M. Bockris and T. Ottagawa, J. Electrochem. Soc. 131, 290 (1984).

17. J. O'M. Bockris and T. Ottagawa, J. Phys. Chem. 87, 2960 (1983).

18. A. Damjanovic, M, A. Genshaw and J. O'M, Bockris, J. Chem. Phys. 45, 4057 (1966).

19. V. G. Levich, Physiochemical Hydrodynamics, pp. 69, 327, Prentice Hall Inc., Englewood Cliffs, NJ (1962).

20. A. Damjanovic, M. A. Genshaw and J. O'M. Bockris, J. Electrochem. Soc. 114, 1108 (1967).

21. V. S. Bagotskii, M. R. Tarasevich and V. Yu. Filiniovskii, Elektrokhimiya 8,84 (1972).

22. H. S. Wroblowa, Y. C. Pan and G. Razumney, J. Electroanal. Chem. 69, 195 (1976).

23. A. J. Appleby and M. Savy, ibid. 92, 15 (1978).

24. W. G. Berl, Trans. Electrochem. Soc. 83, 253 (1963).

25. A. I. Krasilchikov, Proc. Electrochemical Conference Akad. Nauk, Vol, 71 (1953).

26. A. Dajanovic and V. Brusic, Electrochemica Acta 13,615 (1967).

27. A. J. Appleby and M. Appel, C. R. Acad, Sci. Paris 280C, 591 (1975).

28. A. J. Appleby, Modern Aspects of Electrochemistry, Vol. 9, p. 369, J. O'M. Bockris and B. E. Conway, eds., Plenum, NY (1973).

29. F. C. Anson, A. P. Brown and C. Koval, J. Electroanal. Chem. 72, 379 (1976).

30. A. J. Appleby, M. Savy, and P. Caro, J. Electroanal. Chem. 111, 91 (1980).

31. J. P. Collman, M. Morroco, P. Demisevich, C. Koval and F. C. Anson, J. Electroanal. Chem. 111, 117 (1979).

32. J. Zagal, P. Bindra and E. Yeager, J. Electrochem. Soc. 127, 1506 (1980).

33. A. J. Appleby, Comprehensive Treatise of Electrochemistry, Vol. 7, B. E. Conway,

J. O'M. Bockris, E. Yeager, S. U. M. Khan and R. E. White, eds,. Plenum, NY (1983), p. 173.

34. A. C. C. Iseung, J. Elctrochem. Soc. 125, 1660 (1978).

35. J. F. Cooper, "Aluminum-Air Power Cell Research and Development Progress Report," Lawrence Livermore National Laboratory, UCLR-53536, NTIS, Springfield, VA (1985).

36. M. L. B. Rao, A. Damjanovic and J. O'M. Bockris, J. Phys. Chem. 67, 2508 (1963).

37. O. Lindstrom, "Utility Fuel Cells for Sweden," Power Sources, 7 , p. 419-436, J. Thompson, ed. , Academic Press, NY (1979).

38. W. Vielstich, The Electrocatalysis of Fuel Cell Reactions, p. 67, W. E. O'Grady. S. Srinivasan and R. F. Dudley, eds., The Electrochem. Soc., Princeton, NJ (1979).

39. A. J. Appleby, G. Crepy and G. Feuillade, Power Sources 6, p. 549, D. H. Collins, ed., Academic Press, London (1977).

40. J. McBreen, H. Olender, S. Srinivasan and K. V. Kordesch, J. Appl. Electrochem. 11. 8787 (1981).

41. I. Morcos and E. Yeager, Electrochem. Acta 15, 953 (1970).

42. A. Rolla, A. Sadkowski, J. Wild and P. Zoltowski, J. Power Sources 5, $189(1980)$.

43. S. A. Petrova and O. S. Kasenzhek, Elektrokhimiya ㅇ, 1261 (1972).

44. W. N. Vogel and J. T. Lundquist, J. Electrochem. Soc. 117, 1512 (1970).

45. J. O'M. Bockris and B. D. Cahan, J. Chem. Phys. 50, $1 \overline{307}$ (1967).

46. S. Srinivasan, H. D. Horowitz and J. O'M. Bockris, J. Chem. Phys. 46, 3108 (1967).

47. J. Marie, Thesis, Univ. of Paris, Paris, France VI, 1974.

48. A. J. Appleby and J. Marie, Electrochem. Acta 124, 195 (1979). 
49. K. Kordesch, S. Jahangir and M. Schautz, Electrochem. Acta 29, 1589 (1984).

50. A. T.Emery, Ext. Abstract, 1983 Inter natl. Fuel Cell Seminar, Nov.13-16, 1983, Orlando, FL, EPRI, Palo Alto, CA, p. 98; a non, Commercial Brochure, Occidental Petroleum (1985).

51. R. B. Ferguson, "Apollo Fuel Cell Power System, "Proc. 23rd Power Sources Conf. (1969).

52. D. Linden, Handbook of Fuel Cells and Fuel Batteries, P. 42-1, D. Linden, ed., McGrawHil, NY (1984).

53. R.E. Martin, "Advanced Technology Lightweight Fuel Cell Program;" Scientific and Technical Aer os pace Report 17, 3228.

54. D. W. Sheibley (NASA Lewis Research Center, Cleveland, OH), private communication, The American Institute of Aeronautics and Astronautics, January 1985.

55. J. McBreen, G. Kissel, K. V. Kordesch, F. Kulesa, E. J. Taylor, E. Gannon and S. Srinivasan, Proc. 15th IECEC, p. 886, Seattle, WA (1980).

56. K. Kordesch and J. Gsellman, Hydrogen Energy Progress, p. 1657, V. T. N. Veziroglu and J. B. Taylor, eds., Proc. 5th World Hydrogen Energy Conf., Toronto, Ont., 1984, Pergamon, NY (1984).

57. K. V. Kordesch and Ch. Fabjan, Power Sources for Electric Vehicles, p. 901 ,

B. McNichol and D. A. J. Rand, eds., Elsevier, Amsterdam (1984).

58. K. Strasser, H. Gruner and H. B. Gutbier, Power Sources 6, p. 569, K. Strasser, J. Electrochem. Soc. $127,2172(1980) ; H$. Gutbier, H. Mittelmeyer, K. Strasser and B. Stuve, Report BFMT-FB-T 83-113, Siemens, AG, Zentralbereich Technik, Postfach 3240, Erlangen, 8520, F. R. G. (June 1983).

59. H. van der Broek, M. Alfenaar, G. Hovestreydt, A. Blanchard, G. van Bogaert, M. Bomke and L. van Poucke, Hydrogen Energy System, T. N. Veziroglu and W. Seifritz, eds., Proc. 2nd World Hydrogen Energy Conf., Zurich, Switzerland, 1978, Pergamon, NY (1978).

60. H. van der Broek, I. Adriensen, M. Alfenaar, A. Beekman, A. Blanchart, G, van Bogaert and G. Vanneste, Ref. 56, P. 1669.

61. J. Jacquelin and J. P. Pompon, Power Sources 3, p. 391, D. H. Collins, ed., Oriel Press, Newcastle-upon-Tyne, England (1971).

62. A. J. Appleby, J. Jacquelin and J. P. Pompon, Extended Abstracts, 4th Int. Symposium on Fuel Cells, Antwerp, Belgium (October 1972 ).

63. Y. Breelle, J. Cheron, P. Degobert, E. Goldenberg and A. Grehier, Rev. Inst. Français du Pétrole 36(4), 485 (1981).

64. Y. Breelle, "Performance d'un Bloc de Pile Hydrogene-Air," Institut Français du Pétrole, Report No. 29,889 (1982).

65. A. J. Appleby, Electrochemistry, the Past Thirty Years and the Next Thirty Years, p. 384, H. Bloom and F. Gutmann, eds., Plenum, NY (1977).

66. O. Lindstrom, Swedish patent application $7602575,1976$.

67. O. Lindstrom, Swedish patent application $780245,1978$.

68. O. Lindstrom, T. Nilsson, M. Bursell, C. Hornell, G. Karlsson, C. Swylwan and B. Ahgren, Proc. 13th IECEC, p. 1178, Society of Automotive Engineers, Warrendale, PA; San Diego, CA (1978).

69. J. Giner and L. Swette, "Evolution of the Feasibility of Low Cost Carbon Dioxide Transfer/Removal Methods for Fuel Cell Applications," EPRI EM-391, Electric Power Research Institute, Palo Alto, CA (1975).

70. T. L. Bonds, M. H. Dawes, A. W. Schnacke and L. W. Spradin, "Fuel Cell Power Plant Integrated Systems Evaluation," EM-1670, EPRI, Palo Alto, CA (1981).

71. D. Bloomfield, E. Behrin and P. N. Ross, "Ammonia-Air Fuel Cell Power Plant Systems Analysis," LBL-14500, UC 94-cb, University of California, Lawrence Berkeley Laboratory, Berkeley, CA (1982).

72. A. J. Appleby and M. Jacquier, J. Power Sources 1, 17 (1976); A. J. Appleby, M. Jacquier and J. P. Pompon, Soc. Automot. Engineers, Paper No. 77-381, SAE, Detroit, MI (1981).

73. D. Staschewski, Ref. 56, p. 1677.

74. D. Lynn, "Analysis of Vehicle Systems Using Hydrogen Storage/Alkaline Fuel Cell Technology," Los Alamos Scientific Laboratory, LA-UR-80-3094, Los Alamos, NM (1980).

75. R. E. Billings, pp. 397 of Synthetic Fuels Processing Comparative Economics, A. H. Pelofsky, ed., Marcel Dekker, NY (1977).

76. W. Brown, P. Britton, Z. Rucker and A. Scriven, Report prepared for the Ontario $\mathrm{H}_{2}$ Energy Task Force, Vol. 2, Ontario Government, Publication Services, 880 Bay St., Ontario ISBN-7743-7005 (1982).

77. F. J. Plenard, Ref. 59, pp. 2323.

78. N. Allen and R. Zweig, private communication, 1985.

79. H. Buchner, O. Bernauer and W. Strauss, Ref. 59, pp. 1677.

80. G. D. Brewer, pp. 1191, 17th IECEC Proceedings, Los Angeles, California, published by Institute of Electrical and Electronics Engineers, Piscataway, NJ (1982).

81. A. N. Podgorgny, A. I. Mischenko and V. V. Solovey, Int. J. Hydrogen Energy 9,773 (1984). 
82. J.Hord, NBS Technical Notes, 690 U.S. Department of Commerce/National Bureau of Standards, Washington, D. C. 20234, October (1976).

83. E. W. Schmidt, Future Automobile Fuels, pp. 320-341, J. M. Colucci and N. E. Gallpolous, eds., Plenum, NY (1977).

84. O. Lindstrom, "Fuel Cells for Sweden," unpublished report (1983), Royal Institute of Technology, Department of Chemical Technology, S-100 44, Stockholm, Sweden.

85. E. J. Taylor and S. Srinivasan, Ref. 57, Pp. 839.

86. J. O'M. Bockris, Energy Options, pp. 295, Halstead Presa, NY (1980).

87. J. O'M. Bockris and S. Srinivasan, Fuel Cells: Their Electrochemistry, Pp. 205, McGraw-Hill, NY (1969).

88. K. Kordesch, private communication, July, 1984.

89. J. Tielrooy, private communication, July 16, 1985.

90. P. G. Cronin, A. J. Murphy, R. J. Newton and E. S. Wagner, "Assessment of a Coal Gasification Fuel Cell System for Utility Application," EPRI EM-2387, EPRI, Palo A1to, CA (1982). 


\section{SOLID POLYMER ELECTROLYTE FUEL CELLS (SPEFCS) ${ }^{\dagger}$}

\subsection{Background}

Synthetic cation-exchange resins, in the form of polymerized organic sulfonic acids and as small beads, became available in 1945.1 These, in combination with anion-exchange resins containing amine groups, allowed deionization of water and also various preparative separations. Pore-free cation-exchange resin membranes became available 2 in the early 19508 and permitted, for the first time, ion-B pecific electrodialysis.

Grubb at GE first suggested that cation-exchange membranea might be useful as acid FC electrolytes. ${ }^{3}$ At that time (1955-1959), acid FCs, whether with free or matrix-immobilized electrolyte, were limited to the laboratory because of materials problems, particularly corrosion of cathode components and cell hardware. Until the mid 1960s, the only satisfactory electrode materials for SPEFCs were noble metals and noble-metal-plated materials such as tantalum. An advantage perceived in the use of a cation-exchange-membrane as solid polymer electrolyte (or SPE) was that free acid would not be leached from the immobilized acid groups in the polymer. Hence, the only reaction product would be pure water produced entirely at the cathode. The acid form of the anionic membrane possesses a hydrogen-ion transport number of essentially unity. The absence of any other electrolyte component than pure water in the $\mathrm{H}_{2}$ and $\mathrm{O}_{2}$ supplies and water-removal subsystem was expected to allow the use of inexpensive, lightweight construction materials. The complex engineering of the electrolyte circulation loops required for electrolyte and thermal management and water removal in alkaline cells of the later $1950 \mathrm{~s}$ could therefore be eliminated. In addition, the membrane itself could be very thin and light, so that rugged, reliable lightweight systems with low IR drop became possible. Since acidmembrane-based systems, unlike $A F C_{s}$, are intrinsically $\mathrm{CO}_{2}$-rejecting, this $\mathrm{FC}$ system might ultimately be used for $\mathrm{HC}_{\mathrm{B}}$ or other carbon-containing fuels.

\section{2 Current Work}

Reviews of the development of the SPEFC up to the mid-60s have been given by Niedrach and Grubb, 4 Maget, 5 and Liebhavsky and Cairns. 6 Since that period, literature coverage has been restricted to a small number of papers, extended abstracts, and reports that are usually lacking in detail. The patent literature omits many design features such as the description of the important technology of the electrode-membrane interfaces. Most of the early development work was conducted at the GE Research Laboratory (since 1955) and was followed by parallel work on power-plant design and manufacture at the GE Direct Energy Conversion Operation in Lynn, MA, since about 1960 . The successor program was the GE Electrochemical Energy Conversion Program at Wilmington, MA (since the late 1970s). This technology was acquired by UTC as Hamilton-Standard Electrochem, Inc., in August 1984, when the work on the solid polymer electrolyte systems at GE was terminated. Most of the business at the Wilmington facility of GE dealt with SPE electrolyzers. Some FC studies were conducted for the DoE transportation program at Los Alamos. 7 Work on the latest GE version of the system, using Dupont's Nafion $R$ fuorinated sulfonic acid membrane, is being conducted under license by Siemens in the FRG. 8 Other work on SPEFCs is in progress at Engelhard in the US and in Japan (e.g., at the Government Industrial Research Institute, Osaka, again especially for electrolyzer applications.)

\section{3 Early Objectives and Development}

When research on the SPEFC system started at GE in the 1950s, the aim was to produce an electrolyte membrane with the following properties: 6 (a) good hydrogen-ion conductivity with a transport number of unity for hydrogen, requiring the absence of other mobile ions; (b) zero solubility of the polymer in water but high solubility of water in the polymer; (c) low $\mathrm{O}_{2} / \mathrm{H}_{2}$ permeability; (d) rapid water transport; (e) reversible hydration with accompanying dimensional stability; (f) adequate chemical and electrochemical stability in the presence of catalysts; (g) Buitability for bonding electrodes to the membrane surfaces. Early cells used many types of membranes, including sulfonated phenolformaldehyde and polyvinyl compounds such as the lonics 61 series, some reinforced with glass fiber, Dynel or Saran. Another early favorite was divinyl benzene-crosslinked polystyrene sulfonic acid embedded in a polymer (Kel-F) film. The Kel-F film material was obtained from Allied Chemical $\mathrm{Co}_{0}$. and was impregnated with styrene

This chapter has been written by A. J. Appleby and E. B. Yeager. The authors are indebted to their AFCWG colleagues for helpful suggestions and to A. Fickett of EPRI for technical information concerning solid polymer electrolyte FCs. 
containing $2-4 \%$ by weight of divinyl benzene. After polymerization, the film was sulfonated with chlorosulfonic acid to produce a material referred to as P(S-DVB)SA. 6 This composite membrane was initially supplied by American Machine and Foundry (AMF) to GE's specifications, but was later prepared in-house by GE.

After initial attempts to use catalyst-modified foils or screens, 3,9 catalyst layers were integrally bonded by mixing catalyst and a condensation polymer such as sulfonated phenolformaldehyde resin. The mixture was applied to the sulfonated phenol formaldehyde membrane and the whole was heat-treated under pressure to form the correct catalyst-electrolyte interface. 10 The same procedure could not be employed when a free-radical polymer such as P(S-DVB)SA was used, since the polymerization conditions then could not be accurately controlled. This problem was solved by directly bonding the catalyst into the Kel-F layer with the $P(S-D V B) S A$ in the green state. Initially used catalysts were palladium and platinum blacks, but the less expensive palladium showed corrosion at FC cathodes. Platinum thus became the favored catalyst for use in the primary FC application, though not in water electrolysis. Typical loading $8^{6}$ were $3.9 \mathrm{mg} / \mathrm{cm}^{2}$ or greater. For example, for the Gemini mission, the loading was $35 \mathrm{mg} / \mathrm{cm}^{2}$, the catalyst layer being bonded to a gold screen using teflon.

\subsection{The FC for the Gemini Space Mission}

GE was awarded the contract for the FC power plant for the Gemini space mission in 1962, when it became apparent that an SPEFC would be the lightest and most compact unit for this application. FC weight and volume were primary considerations before large boosters became available for the Apollo program. Another advantage was the feasibility of using the SPE system to produce potable water by means of a simple water-collection subsystem using capillary wicking devices (see Fig. 4.4-1, which is reproduced from Ref. 6).

The Gemini capsule carried two of the se SPEFC modules, each rated at $1 \mathrm{~kW}$ nominal power output. These were ejected with the service package before reentry into the atmosphere. Work commenced on system design in March 1962 at DECO. The system was qualified to use only the AMP P(S-DVB)SA membrane, although parallel experiments on alter native membranes and technologies were in progress. A schematic cross-section of the Gemini $F C$ is shown in Fig. 4. 4-2.6 Fuel and oxidant aboard the capsule were supercritical $\mathrm{H}_{2}$ and $\mathrm{O}_{2}$. The operating pressure and temperature of the cell stacks were about 2 atm and $50^{\circ} \mathrm{C}$, respectively. A typical $V-i$ curve for the system is shown in Fig. 4.4-3. Each $1-k W$ module contained three 32-cell stacks operating nominally at $0.78 \mathrm{~V}$ and $37 \mathrm{~mA} / \mathrm{cm}^{2}$ (25 V and $40 \mathrm{~A}$ total for the $3 \mathrm{stacks}$ ). Specific weight and volume of the system were $31 \mathrm{~kg} / \mathrm{kW}$ and $271 / \mathrm{kW}$, respectively. By contrast, the corresponding figures for the UTC Apollo alkaline system, which required circulating electrolyte, were $77 \mathrm{~kg} / \mathrm{kW}$ and $2011 / \mathrm{kW}$, whereas the initial version of the Space Shuttle Orbiter immobilized-electrolyte alkaline system, which operated at much higher current density, was rated at $7.6 \mathrm{~kg} / \mathrm{kW}, 11 \mathrm{l} / \mathrm{kW}, 11$

A major problem with the Gemini type SPEFCs of the early $1960 \mathrm{~s}$ was the development of hot spots in the cells, which resulted in a localized melting of the membrane. A flame front then developed at the se locations within the cells but the flame front did not propagate and there were no explosions. This problem may have been caused by the deterioration of the membrane under chemical attack by free radicals $\left(\mathrm{HO}_{2}\right)$ produced by a minor side reaction at the $\mathrm{O}_{2}$ cathode. It has been solved with improved membranes and cell designs and, particularly, by the incorporation of platinum particles in the membrane phase to decompose the free radicals and the hydrogen peroxide that is also produced in minor quantities at the cathode, which may also lead to the formation of free radicals.

\section{5 Later Work at GE}

Many technological improvements were made at GE after the cell design (see Fig. 4.5-1) and materials for the Gemini system had been frozen. Figure 4.5-1 is adapted from Ref. 6 and updated by using data from Ref. 7. The Gemini cell technology is approximately represented by 1960 data for $\mathrm{H}_{2}-\mathrm{O}_{2} \mathrm{FC}_{8}$. Major performance improvements were introduced about 1963 (see

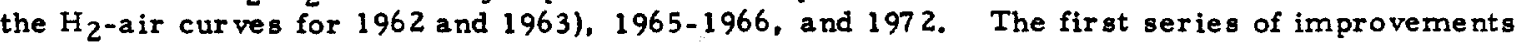
resulted from use of a modified cathode structure incorporating teflon in the catalyst layer directly adjacent to the electrolyte (as in the electrodes developed for PAFCs around the same time at GE). The second series of advancements involved the use of an improved $P(S-D V B) S A$ membrane in which the polystyrene sulfonic acid sidechain was fluorinated, which yielded a more electrochemically stable membrane structure that should have allowed cells to operate at temperatures in excess of $50^{\circ} \mathrm{C} .12$ Unfortunately, the physical properties of membranes derived from trifluorostyrene sulfonic acid made them unsuitable for use in practical FCs. Finally, from the early 19708 onwards, the fully fluorinated $\mathrm{Nafion} \mathrm{R}^{\mathrm{m}}$ membrane, which is chemically and electrochemically stable to more than $200^{\circ} \mathrm{C}$, became available from DuPont. This material removed the stability constraints of the earlier membranes and allowed operation at temperatures up to about $100^{\circ} \mathrm{C}$. This temperature constraint is set by dehydration of the membrane above this temperature and the attending loss of ionic conductivity. In addition, the Nafion ${ }^{R}$ 


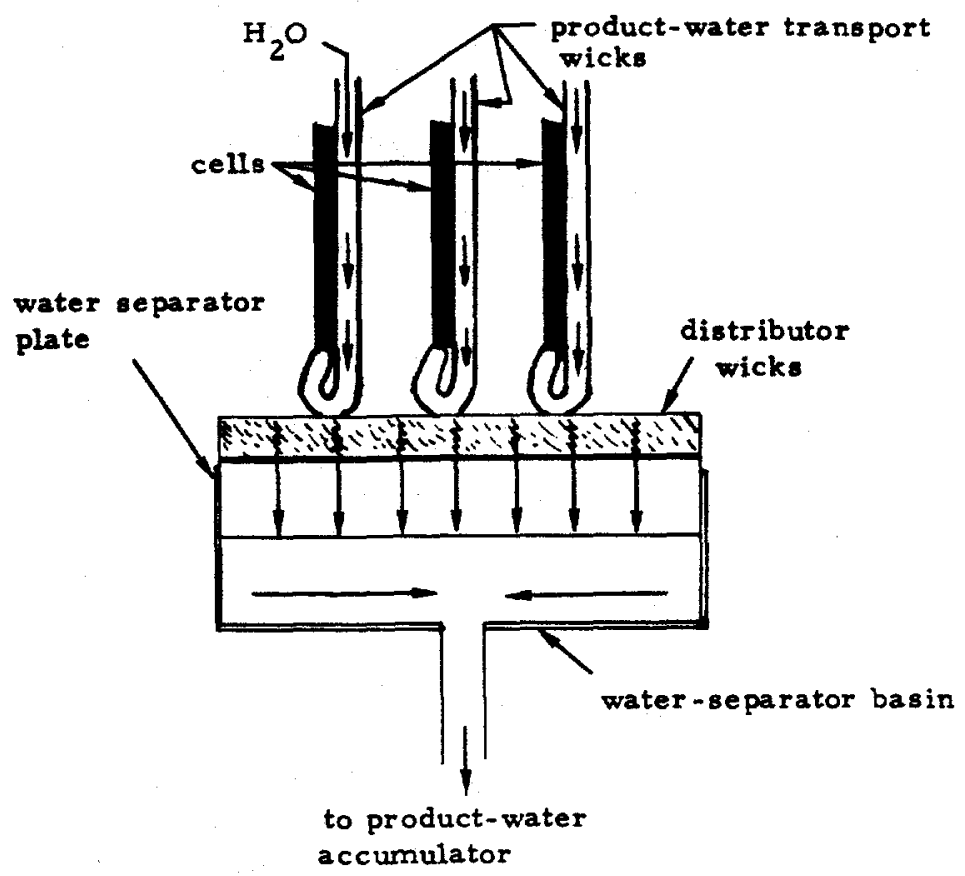

Fig. 4.4-1. Schematic diagram of the GE wicking system for liquid water removal in the Project Gemini FC. 6 Each cell stack contained a separate sys tem in which water removal took place by differential oxygen pressure.

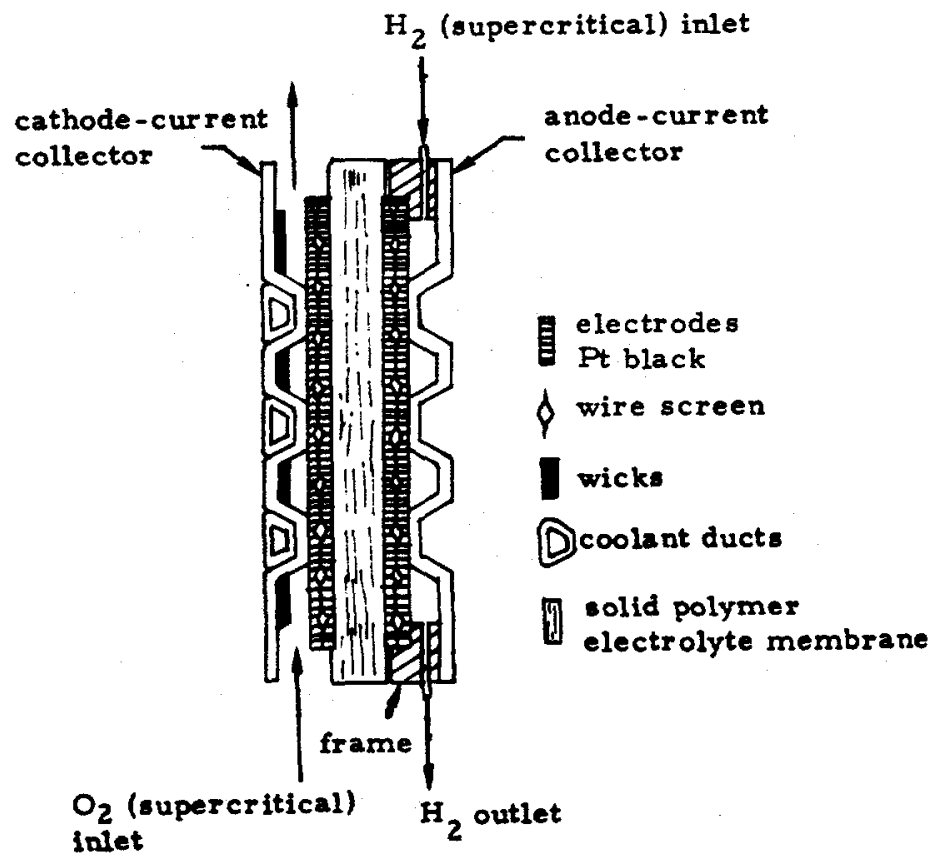

Fig. 4.4-2. Schematic cross-section of the Gemini FC. ${ }^{6}$ In the cell, the $\mathrm{H}_{2}$ and $\mathrm{O}_{2}$ supply channels were rotated $90^{\circ}$ to produce a cross-flow configuration. The system used an AMP P(S-DVB)SA membrane. 


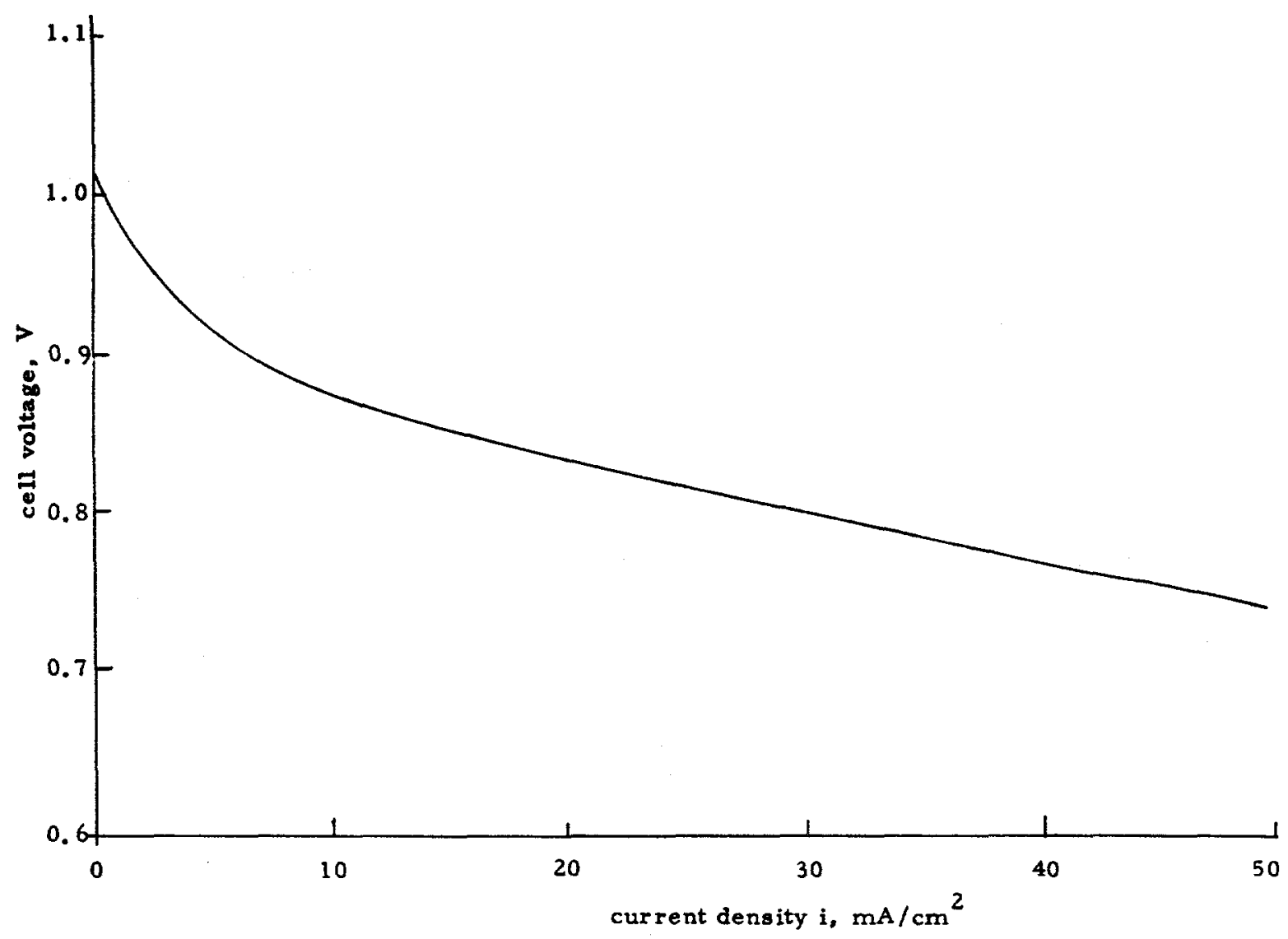

Fig. 4, 4-3. The cell voltage va current density curve for the Gemini FC (adapted from Ref. 6).

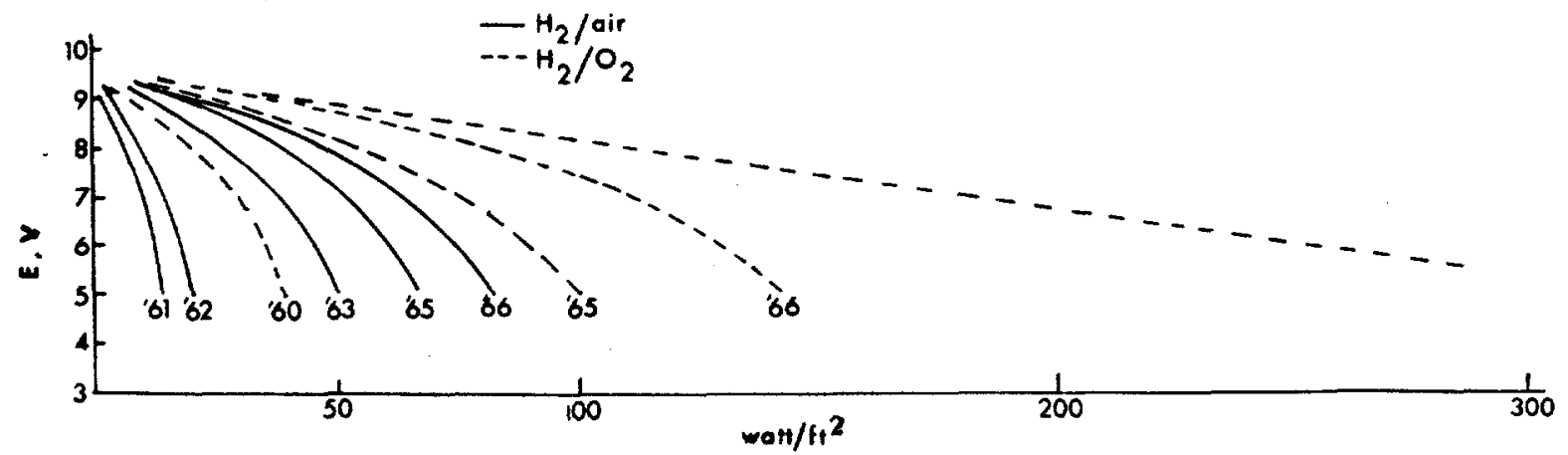

Fig. 4.5-1. Improvements are shown in the SPE single-cell atmospheric pressure performance from 1961-1980. These data were adapted from Refs. 6 and 7. 
flim affords a lower intrinsic oxygen-reduction over potential on platinum than the earlier electrolytes, which results in performance improvements under all temperature conditions. Together with progressive improvements in cathode structure, the FC performance at $80^{\circ} \mathrm{C}$ was about $50 \mathrm{mV}$ better than that of a PAFC at $190^{\circ} \mathrm{C}$ operating at the same current density on pure $\mathrm{H}_{2}$. However, state-of-the-art catalyst loadings in the SPEFC system is far higher than in the PAFC system, as is discussed in Sec. 4.6.

As already noted, a major improvement in current density at constant potential was obtained in the early 1960 s at GE when teflon-containing electrodes were first used. This development appears to have been serendipitous: teflon was substituted for ion-exchange resin in the mixture of catalyst and bonding material applied to the surface of the membrane. This innovation was carried out even before the first tests were conducted on membranes of the P(S-DVB)SA type with a perfluorinated styrene sidechain. The teflon was not aintered in this initial work. It quickly became obvious why the improvement occurred: in the SPE system, with unity hydrogen ion-transport number, all of the water in the cell is produced at the cathode and, in addition, each hydrogen ion migrating through the membrane must be accompanied by at least four water molecules $\left(\mathrm{H}_{9} \mathrm{O}_{4}^{+}\right)$. As a result, the risk of flooding at the hydrophilic cathode by the large amount of transferred water is high in cells operating at temperatures at which liquid water is the product, which was the case with all of the early SPEFCs. Such flooding interferes with the transport of $\mathrm{O}_{2}$ to the platinum catalyst-electrolyte interface. A hydrophobic, teflon-containing layer with the correct structure prevents this flooding. This type of non-wetting structure is not essential at high-performance anodes that do, however, require highly humidified hydrogen with low utilization per pass. It is also unnecessary if the cell is used for electrolyzer applications.

Initial experiments were conducted with inks of colloidal teflon-suspension-catalyst mixtures applied to plastic (e.g.. mylar) films, which were then pressed into the surface of the membrane. The technique is particularly suitable for use with lower catalyst loadings ( $\sim 4 \mathrm{mg} /$ $\mathrm{cm}^{2}$ ) of pure noble metal black. This layer could then be pressed against a current-collector screen. For the Gemini program, much heavier loadings were used $\left(35 \mathrm{mg} / \mathrm{cm}^{2}\right)$, and the catalyst layer was directly teflon-bonded to a gold-screen current-collector before being pressed onto the P(S-DVB)SA membrane. The method of cell construction is robust but does not appear to have yielded an optimum catalyst-electrolyte interface with respect to catalyst-activity loading.

When the very stable Nafion ${ }^{R}$ membrane became available, bonding techniques could be improved, and cathodes of better structure and higher performance were prepared. It also proved to be possible to pressure-bond the teflon directly to the electrode structure at about $200^{\circ} \mathrm{C}$ and moderate pressure. Nafion ${ }^{\mathrm{R}}$ will withstand short-term exposure to these conditions, provided that the membrane material is not allowed to dry out excessively; otherwise, it will be cooked into material resembling potato chips. The favored technique for applying the catalyst with $\sim 15$ wt $\%$ of teflon was to sandwich the membrane between two inked, normally presintered, catalyst-teflon layers on mylar films for both the required anode and cathode and then pressing the combination at about $200^{\circ} \mathrm{C}$ and moderate pressure $(6 \mathrm{~atm})$ for a short time. The die, in which this operation is carried out, is preheated and it must remain well sealed during the pressing operation in order to minimize water loss from the Nafion $R$ during pressing. An alternative technique for preparing the catalyst-teflon mixture was developed at GE-DECO but was apparently rarely used to manufacture practical FC components because of its relative complexity. It consisted of grinding a catalyst-dry teflon powder mixture with dry ice to form a voluminous mix, which could be spread onto a sheet in a thin layer. The $\mathrm{CO}_{2}$ was then allowed to evaporate and the layer again pressed into the Nafion ${ }^{R}$ membrane. In all cases, the applied pressure is a critical parameter. If it is too great, the catalyst particles are pushed into the interior of the membrane surface, which leads to poor reactant-diffusion characteristics and poor electronic contact to the current collector. In contrast, too low a pressure results in inadequate catalyst-to-electrolyte contact.

\subsection{State-of-the-Art SPE Technology}

\section{A. The SPE Membrane}

The baseline SPE membrane in GE's FC work up to its termination in 1984 has been the Nafion ${ }^{R} 1170$ membrane, which is typically $0.175 \mathrm{~mm}$ thick in the dry state. Earlier nonfluorinated membranes were about $0.75 \mathrm{~mm}$ thick and exhibited correspondingly higher IR drop. The structure of this material and an outline of its synthesis are given in Fig. 4.6-1, which is adapted from Ref. 13a. Nafion ${ }^{R} 1170$ has an equivalent weight of 1100 and is one of a family of Dupont polymers with code numbers referring to their equivalent weight $(\mathrm{g})$ added to their thickness in mils $\times 10$. Chemically, all of these polymers possess a modified teflon skeleton. The weak links in the structure are the ether bonds that are stable to strong acids and bases and to chlorine, hydrogen and oxygen at temperatures at least up to $150^{\circ} \mathrm{C}$. Typical equivalent weights (based on $\mathrm{H}^{+}$) are in the range 1000-1500 and their structural formulas approximate $\left(\mathrm{CF}_{2}\right)_{18-28} \mathrm{SO}_{3} \mathrm{H}$. The hydrophilic hydrated $-\mathrm{SO}_{3} \mathrm{H}$ groups tend to be attracted towards each other to form a micellular structure within the teflon-type backbone ${ }^{13 b}$ so that lines of such 
Tetrafluorethylene reacts with $\mathrm{SO}_{3}$ to form a cyclic aultone. After rearrangement, the sultone can then be reacted with hexafluoropropylene epoxide to produce sulfonyl fluoride adducts, where

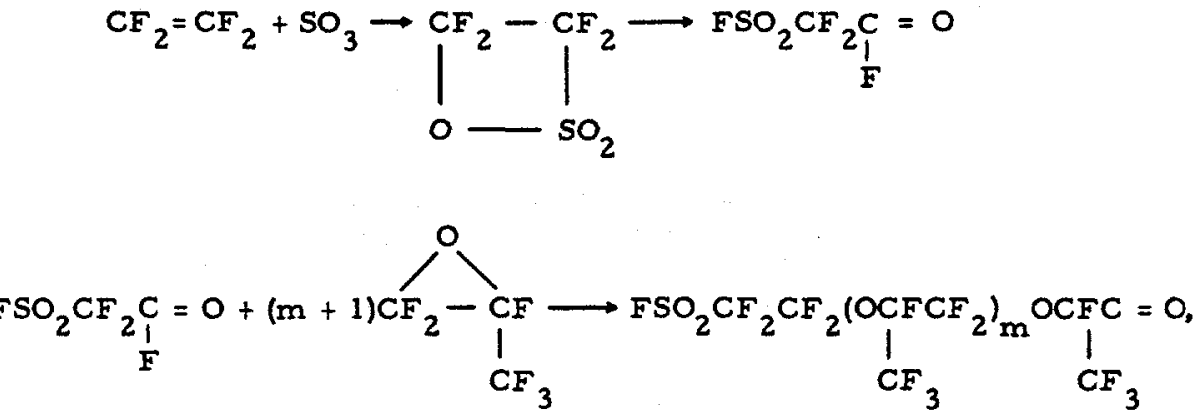

$m \geq 1$. When these adducts are heated with sodium carbonate, a sulfonyl fluoride vinyl ether is formed. This vinyl ether is then copolymerized with tetrafluoroethylene to form XR resin:

$$
\begin{aligned}
& \mathrm{FSO}_{2} \mathrm{CF}_{2} \mathrm{CF}_{2}{ }_{\mathrm{CF}} \mathrm{CFCF}_{2} \mathrm{I}_{\mathrm{m}} \mathrm{OCF}=\mathrm{CF}_{2}+\mathrm{CF}_{2}=C F_{2} \longrightarrow
\end{aligned}
$$

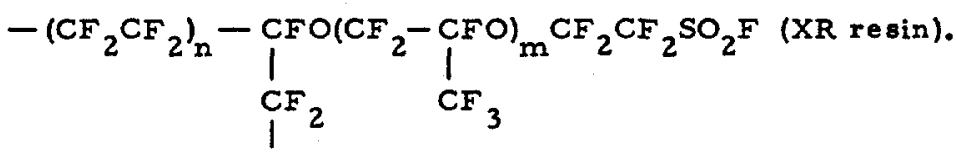

This high molecular weight polymer may be fabricated by melting and can be processed into various forms, such as sheets or tubes, by using standard methods. On base hydrolysis, this resin is converted into Nafion ${ }^{R}$ perfluorosulfonate polymer:

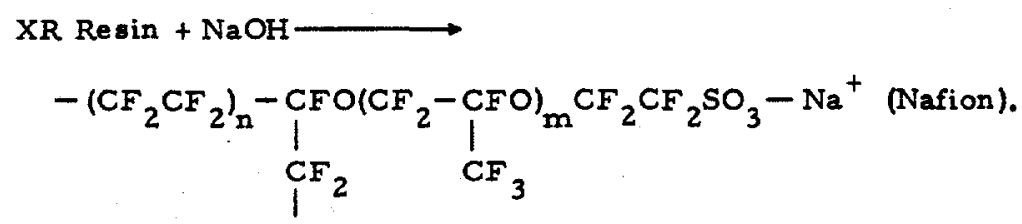

\footnotetext{
Fig. 4, 6-1. Nafion ${ }^{\mathrm{R}}$ synthesis and structure. ${ }^{13 a}$
} 
micelles create ion channels that appear to exist in the material. $13 \mathrm{c}$ This feature is illustrated in Fig. 4.6-2. As would be expected, the materials are, at least briefly, thermally stable even to teflon sintering temperatures $\left(360^{\circ} \mathrm{C}\right)$.

Other fully fluorinated membranes are now commercially available. $13 d-f$ These are, respectively, a membrane range produced by the Asahi Chemical Industry Company, Neosepta-F (Tokuyama Soda Co., Ltd.) and Flemion ${ }^{R}$ (Asahi Glass Co., Ltd.). All of these membranes were developed for maximum selectivity in the chlorine-caustic process. In general, the Japanese membranes are of polycarboxylic acid type, with less stability than sulfonic acid membranes under extreme conditions. They are usually not applicable to SPEFC since the proton versions of the membranes are poor ionic conductors because of the low ionization constants of the carboxylic groups.

\section{B. Catalysts}

All successful SPE cells have been made with pure, unsupported noble-metal catalysts. It is, however, clear that catalysts of this type are used less efficiently in these cells than in PAFC electrodes. As is described in Sec. 2.7, the initial PAFC electrodes of the late 1960s were pure noble-metal (typically platinum)-teflon systems with 3 to $5 \mathrm{mg} / \mathrm{cm}^{2} \mathrm{Pt}$ added to 30 $40 \mathrm{wt} \%$ of teflon to provide the required bulk for typical electrode layers. In general, most pure Pt blacks have a specific surface BET area of about $20-25 \mathrm{~m}^{2} / \mathrm{g}$, whereas Pt prepared on suitable supports may have BET areas on the order of $100 \mathrm{~m}^{2} / \mathrm{g}$ or greater. While there is some evidence that an increase of the $\mathrm{Pt}$ surface area may somewhat degrade activity on a unit area basis at constant potential, ${ }^{14}$ it is clear that increasing the $P t$ surface area by the use of supported catalysts will be advantageous in raising the available mA/mg of $\mathrm{Pt}$ at a given cell voltage. This approach was therefore used by the developers of the prototype PAFC $s$ (both in the US and Japan), who typically use $10 \mathrm{wt} \%$ of Pt supported on a suitable corrosion-resistant carbon, at a final Pt loading of $0.25-0.5 \mathrm{mg} / \mathrm{cm}^{2}$ for the air cathode. These electrodes give equivalent activity to pure $\mathrm{Pt}$-black electrodes with loadings in the $3-5 \mathrm{mg} / \mathrm{cm}^{2}$ range, indicating ten-fold improvement in catalyst utilization for the carbon-supported material. It appears that GE had attempted to reduce the $\mathrm{Pt}$ loading in Nafion ${ }^{R}$-based cells in order to reduce the total system capital cost for specific applications. For military and space use, these cost reductions were clearly not of overriding importance. However, for civil-terrestrial applications, the SPE system would be at a considerable disadvantage with respect to competitors if its Pt or other noblemetal loadings are in the several $\mathrm{mg} / \mathrm{cm}^{2}$ range. This fact became apparent in the mid $1970 \mathrm{~s}$ when the SPE system was being promoted in the electrolysis mode as a competitor to such established commercial alkaline pressure electrolyzers as those produced by Lurgi. 15

\section{Cell Costs}

If we assume a baseline figure of $8 \mathrm{mg} / \mathrm{cm}^{2}$ of cell cross section for the noble-metal catalyst in the cathode and anode, then, for an FC operating at $0.8 \mathrm{~V}$ and $200 \mathrm{~mA} / \mathrm{cm}^{2}$, there would be a capital cost of $\$ 450 / \mathrm{kW}$ at the current depressed Pt prices. The platinum requirement is $1 e s 8$ and the cost would be about $\$ 50 / \mathrm{kW}$ in an idealized electrolyzer operating at $1.5 \mathrm{~V}$ and $1 \mathrm{~A} / \mathrm{cm}^{2}$. Both of these catalyst costs are unacceptable fractions of total systems cost, which may be estimated at about $\$ 800 / \mathrm{kW}$ and $\$ 150 / \mathrm{kW}$ for the $F C$ and electrolyzer applications, respectively. Even more telling is the cost of the Nafion ${ }^{R}$ membrane, which cost about $\$ 250 / \mathrm{m}^{2}$ in the mid-1970s and is now quoted at about $\$ 400 / \mathrm{m}^{2}$. The present membrane cost corresponds to $\sim 250 / \mathrm{kW}$. The costs of typical PAFC components are estimated to be about $\$ 70 / \mathrm{kW}$ without the catalyst, while the catalyst costs about $\$ 50 / \mathrm{kW}$ at present loadings $\left(0.75 \mathrm{mg} / \mathrm{cm}^{2}\right.$ total) and Pt prices. These estimates serve to underline the significance of the cost problem for the SPEFC. Unless both SPE membrane and catalyst costs per $\mathrm{kW}$ can be significantly reduced, the SPE system is only likely to be used in specialized applications, despite its other attractive features.

\section{Current Cell Performance}

The current state-of-the-art baseline performance of the Nafion ${ }^{R}$ SPEFC with traditional catalyst layers (4 mg/ $\mathrm{cm}^{2}$ of Pt black at the anode and cathode) is shown in Fig. 4.6-3 for pure $\mathrm{H}_{2} / \mathrm{O}_{2}$ at $82^{\circ} \mathrm{C}$ and 1 or $2 \mathrm{~atm}$ pressure and at $104^{\circ} \mathrm{C}$ with 7.1 atm pressure. These estimated data are derived from results quoted in Ref. 7 . They indicate excellent performance, but it is difficult to establish what the catalyst utilization is in the high-loading electrodes. There do not appear to be any usable data on the intrinsic rate of $O_{2}$ reduction on $\mathrm{Pt}\left(\mathrm{mA} / \mathrm{real} \mathrm{cm}^{2}\right.$ ) as a function of potential and temperature with a Nafion ${ }^{R}$ electrolyte. Here is then a clear need for future research. Reference 7 also includes a description of a first attempt at GE to reduce the cost of the electrolyte membrane by utilizing commercially available substitutes for Nafion $R$, which may currently or potentially have lower prices. The membranes are not identified; one vendor has been quoted as being RAI, Inc. It seems highly probable that the membranes in question are partially fluorinated materials with sulfonic acid groups inserted by radiation 


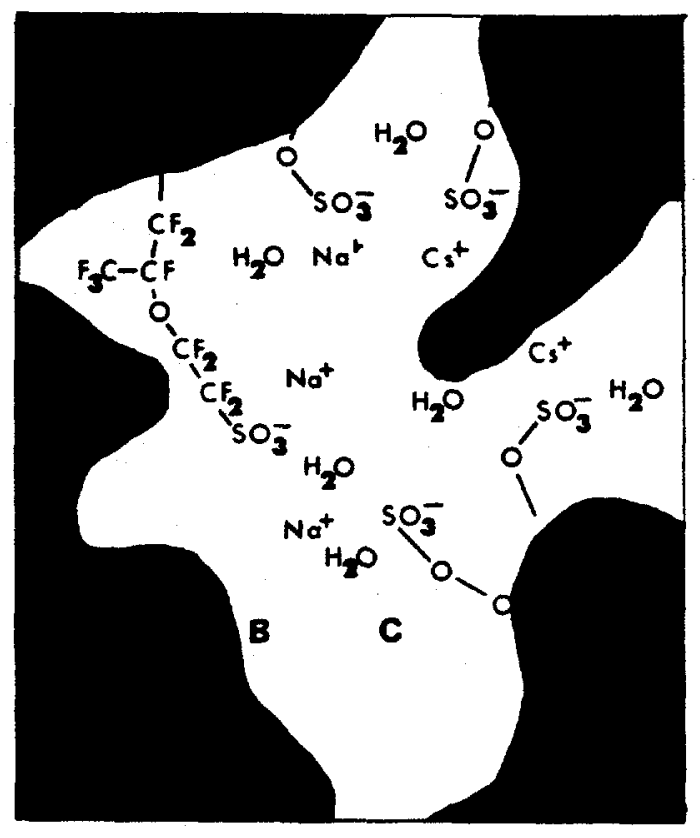

The three-region structural model is shown for Nafion $A$; fluorocarbon, B; interfacial zone, C.
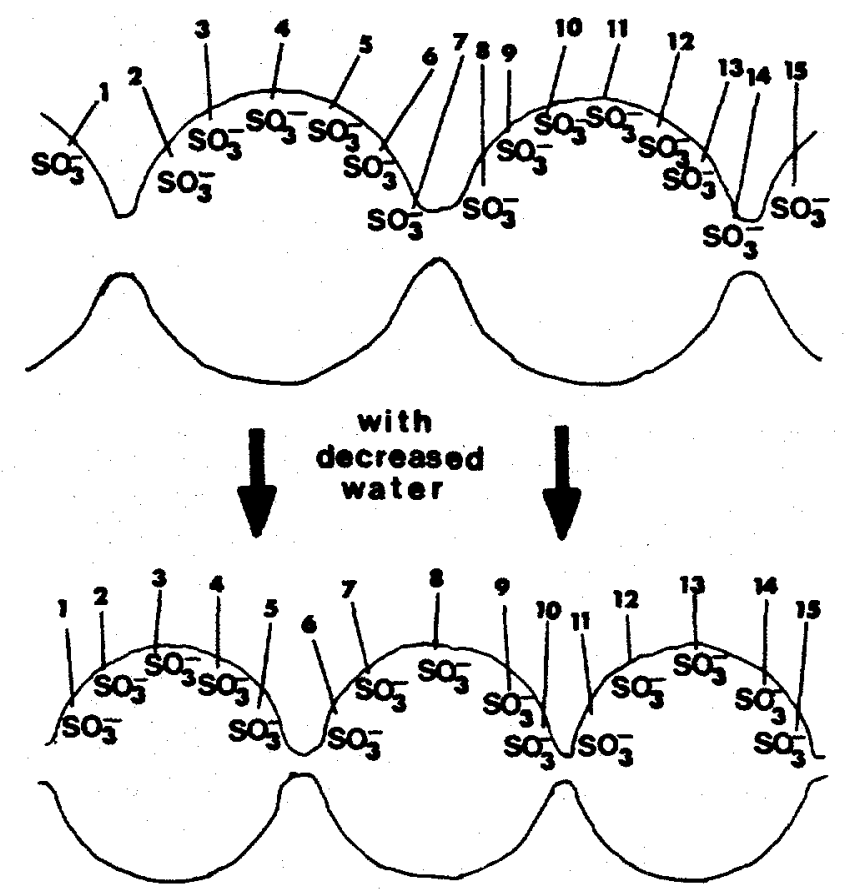

A representation is shown of redistribution of ion-exchange sites that occurs on dehydration of polymers.

Fig. 4,6-2. Micellar and ion-channel structures of $\mathrm{Nafion}^{R}$. 13b,c 


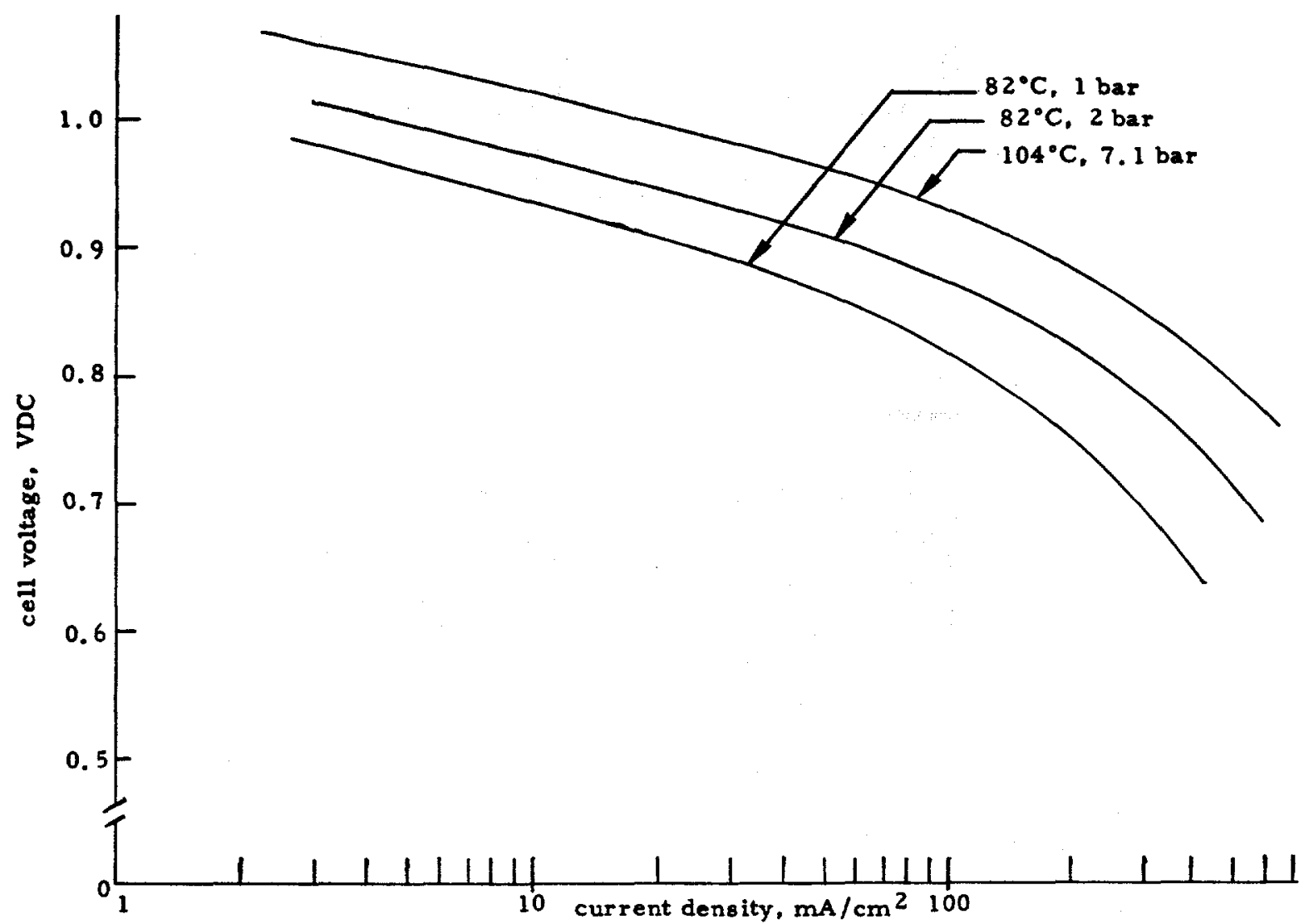

Fig. 4.6-3. Cell-voltage vs current density curves are shown for the baseline SPEFC at various temperatures and pressures on pure $\mathrm{H}_{2}$ and $\mathrm{O}_{2}$; the $\mathrm{Pt}$-black loading $s$ were about $4 \mathrm{mg} / \mathrm{cm}^{2}$ at both the anode and cathode. The SPE baseline refers to $\mathrm{H}_{2} / \mathrm{O}_{2}$ at the same pressure.

grafting. Because a small number of weak linkages (CH groups) is introduced by this method of preparation, the materials are likely to show some degradation as a function of time and will have an upper useful operational temperature well below that of Nafion $R$. Since all C-H compounds are thermodynamically unstable under oxygen cathode conditions, even at room temperature, reliance is being placed on kinetic factors such as steric hindrance in assuring oxidative stability of the polymer. In addition, the oxygen cathode is, to some extent, a producer of free radicals from the decomposition of $\mathrm{H}_{2} \mathrm{O}_{2}\left(\mathrm{e} . \mathrm{g} . . \mathrm{HO}_{2}\right)$ and perhaps also directly. The $\mathrm{HO}_{2}^{\circ}$ is known to be an effective agent for the depolymerization of CH-containing polymers. As mentioned earlier, this problem with Nafion $R_{\text {has been addressed at GE by incorporating a } 8 \mathrm{mall}}$ quantity of highly dispersed Pt prepared by ion-exchange into the polymer in order to decompose traces of peroxide produced at the FC cathode.

\section{7 Current SPE Development Problems}

While some progress has been made in reducing membrane costs for wider application of the SPEFC, no information could be found in the published literature concerning efforts to reduce the catalyst loadings to acceptable values. Much of the following is surmised or else based on recollections by people working in the field a number of years ago.

The catalyst-electrode-gas interface in an SPEFC is much more two-dimensional than in a teflon-bonded three-phase-boundary electrode of the classical variety used in liquid acid and alkaline FCs. The solid electrolyte cannot creep by surface tension to produce the most convoluted possible gas-liquid-solid surface, except during the moment of bonding of the catalyst layer to the electrode. This bonding operation is not well understood and it is clearly different in character and mechanism when condensation polymers are used, as in the early GE work, or when teflon is used to cement the catalyst particles to the surface of the fluorocarbon polymer ion-exchange membrane. In the latter case, the catalyst-electrolyte combination may approach in operation an efficacious three-dimensional structure. The $\mathrm{O}_{2}$ and $\mathrm{H}_{2} 8$ olubilities are expected to be much greater in the fluoropolymer membranes, compared with the values in concentrated aqueous electrolytes such as PA or KOH. However, the diffusion coefficients are expected to be lower in the membranes and this feature may in good part offset the favorable effects of the higher solubility on the transport of $\mathrm{O}_{2}$ and $\mathrm{H}_{2}$ to the platinum-electrolyte interface. 
A major problem in modelling the electrode-polymer interface in the SPEFC has been the lack of published data for $\mathrm{O}_{2}$ solubilities and diffusion coefficients for $\mathrm{H}^{+}$forms of Nafion-type membranes. Recently published data ${ }^{20}$ for $\mathrm{O}_{2}$ in $\mathrm{Na}{ }^{+}$on Nafion peroxide are only an approximate indication of the values to be expected for the $\mathrm{H}^{+}$form. The permeability coefficients of $\mathrm{O}_{2}$ and $\mathrm{H}_{2}$ have been reported to be quite dependent on the water content and cation form, as well as ion. exchange capacity, 21 and the situation is the same for solubilities and diffusion coefficients. Kinetic data for $\mathrm{O}_{2}$ reduction and $\mathrm{H}_{2}$ oxidation on platinum in the SPE are generally lacking. This fact makes it very difficult to use mathematical modelling for the determination of the opti mum electrode structures. Furthermore, little definitive research on the structure of the interfaces involved in state-of-the-art electrodes has been reported ${ }^{24}$ and it is not known how far from ideal it is.

Activation and diffusion polarization are usually not a problem with $F C$ anodes using $P t$ as the catalyst and operating on pure $\mathrm{H}_{2}$. The SPEFC anodes, however, use an order of magnitude more Pt than the PAFC. Part of the reason for this unusually large requirement for Pt may involve an offset of the effects of impurities in the $\mathrm{H}_{2}$ (e.g., CO). At the lower operating temperatures of the SPEFC, even a few parts per million of $\mathrm{CO}$ are very deleterious to the $\mathrm{H}_{2}$-oxidation reaction kinetics. This fact makes it difficult to use $\mathrm{H}_{2}$ derived in a reformer from a carboncontaining source, even when a shift reactor is used to depress the CO content. Alternative catalysts, which are more tolerant of CO, such as Pt-Ru, need to be considered. Workers at GE apparently ${ }^{7}$ did examine such mixed or alloyed catalysts for the SPEFCs, since they have used $\mathrm{Pt}$-Ir materials for $\mathrm{O}_{2}$-evolution anodes in SPE electrolyzers. 16

An alternate solution to the low tolerance of $\mathrm{Pt}$ catalysts to $\mathrm{CO}$ in the $\mathrm{H}_{2}$ supply is to raise the operating temperature. The upper limit on the operating temperature with proton-conducting solid polymer membranes is set by the high. vapor pressure of water in the membranes and their dehydration, which results in $10 \mathrm{~B} s$ of conductivity.

An interesting possibility is to infuse the present Nafion-type membranes with a concentrated electrolyte such as $\mathrm{H}_{3} \mathrm{PO}_{4}$, which ties up the free water in the micellar phase within the membrane and thus permits operation at much higher temperatures without $10 \mathrm{ss}$ of proton conduction. 22 The ability of $\mathrm{H}_{3} \mathrm{PO}_{4}$ as well as $\mathrm{H}_{2} \mathrm{O}$ to solvate the proton 23 from the sulfonic acid groups is an important feature of the use of $\mathrm{H}_{3} \mathrm{PO}_{4}$ rather than of another stronger acid.

A second problem area concerns the more efficient use of the Pt electrocatalyst in the SPEFC. There is no doubt that the use of pure Pt black is wasteful and that it would be better to use a supported catalyst with higher surface area to obtain improved utilization. This problem has appar ently been examined unsuccessfully at GE. It has been verbally reported that catalyst layers of this type were too voluminous (presumably when the low temperature dry-ice method was used). Other verbal reports suggest that Nafion $R$ does not appear to wet carbon supports. Clearly, reexamination is required. Perhaps different, improved methods of creating the opti mal catalyst-electrolyte interface are needed.

A general problem area with Nafion-type membranes concerns the properties of the presently available materials at temperatures exceeding about $100^{\circ} \mathrm{C}$. The membrane material is chemically stable up to temperatures of approximately $200^{\circ} \mathrm{C}$ or greater, even under oxidizing conditions in the presence of Ft catalysts, at least in the absence of free-radical depolymerization agents. It is stable in protected environments up to sintering temperatures of teflon $\left(360^{\circ} \mathrm{C}\right)$, at least for short times. However, it is only suitable for use as an electrolyte at temperatures up to about $85^{\circ} \mathrm{C}$ with atmospheric pressure reactants (water vapor pressure $\approx$ $0.25 \mathrm{~atm}$ ) and up to temperatures slightly over $100^{\circ} \mathrm{C}$ at elevated pressure (7 atm, with water vapor pressure above $1 \mathrm{~atm}$ ). Beyond these limits, maintenance of the membrane environment at $100 \%$ relative humi dity becomes very difficult, even with highly humidified cathode gas, because of the large volume of air that must pass over the cathode catalyst. Presaturation of this air at elevated temperature and pressure is not easy in practical systems. If the membrane dries out, it becomes brittle, cracks and shows very poor conductivity. This last property appears to be a characteristic of the whole fluorinated sulfonic acid polymer family 17 and per. haps of normal strong acid-containing polymers in general. It is, however, not characteristic of $P A$ and Its condensed species, which show abnormally high conductivities in concentrated solutions at high temperature. The difference between the two types of acids is caused by the types of ionic species which are present at high temperatures. The sulfonic acid and similax acidtype membrane systems depend on the solvated hydronium ion $\mathrm{H}_{9} \mathrm{O}_{4}^{+}$as the conducting species, with both ionic migration and Grotthus-type conduction involved, the latter being strongly dependent on the availability of sufficient water for this type of chain conduction to occur. When the water content becomes low in this membrane, both conduction mechanisms become ineffective. In the absence of water, the sulfonic acid groups are not ionized. On the other hand, in concentrated $P A$, the proton is solvated with $\mathrm{H}_{3} \mathrm{PO}_{4}$ to yield $\mathrm{H}_{4} \mathrm{PO}_{4}^{+}$, even when there is no free water. Furthermore, both $\mathrm{H}_{3} \mathrm{O}^{+}$and $\mathrm{H}_{4} \mathrm{PO}_{4}^{+}$, together with $\mathrm{H}_{3} \mathrm{PO}_{4}$ and $\mathrm{H}_{2} \mathrm{O}$, participate in very effec. tive, three-dimensional Grotthus-type chain conduction and ion migration. In very concentrated $\mathrm{H}_{3} \mathrm{PO}_{4}$ solutions ( $285 \%$ by weight), the chain-transfer mechanism is predominant and accounts for the surprisingly high conductivity of concentrated PA. 
Work is clearly required on additives and/or acceptor groups in fluorosulfonic polymers to promote a proton chain-hopping mechanism and thus enhance conductivity at the high cell temperatures required to raise steam efficiently for HC or methanol reforming in utility or transportation FC systems. Some success has been realized with infusion of concentrated $\mathrm{H}_{3} \mathrm{PO}_{4}$ into Nafion ${ }^{R} .22$ Workers at Case Western Reserve University have operated $\mathrm{H}_{3} \mathrm{PO}_{4}$-impregnated Nafion membranes at temperatures in excess of $140^{\circ} \mathrm{C}$ and at a total pressure of 1 atm, with quite acceptable conductivities. The fluorophosphoric and fluorophos phinic polymers and other promising families of compounds need to be explored.

\section{8 Future Prospects for SPEFCs}

Cells with solid polymer electrolytes will continue to show practical advantages over those with immobilized liquid electrolytes, namely, essentially total non-volatility of the electrolyte material, drastic reduction of corrosion and materials problems, production of pure water only and hence negligible electrolyte leaching, integrity of the thin electrolyte layer, and ease in edge sealing of cells. Since fluoropolymer electrolytes have excellent oxygen solubilitydiffusivity characteriatics, they may be expected to yield higher performance than conventional acid cells under equivalent conditions. For use with reformate fuels, they must operate in such a manner that they how reasonable CO tolerance while raising steam for efficiently integrated reforming. A cell temperature well in excess of $100^{\circ} \mathrm{C}$ is required. Present candidate com. pounds such as the Nafion $R$ family have poor-to-negligible proton conductivity under these conditions. This problem must be corrected by research on the design and construction of improved membranes.

The conductivity problem at temperatures above $100^{\circ} \mathrm{C}$ can be avoided if pure $\mathrm{H}_{2}$ is available since the cell may then operate efficiently at temperatures of about $80^{\circ} \mathrm{C}$. At these temperatures, the catalytic activity of $\mathrm{Pt}$ for the $\mathrm{O}_{2}$ electrode and the conductivity of the polymer in the SPEFC are much greater than those in concentrated PA, although they are inferior, with regard to voltage vs current density, to alkaline systems with equivalent effective electrode catalyst loadings; cf. Fig. 4,8-1 for a comparison of state-of-the-art SPEFCs with the advanced AFCs of Ref. 18. However, it may be possible to achieve thinner structures with the SPEFC, and this development may offset the current-density advantage of the AFC. SPEFCs, as well as AFCs, will function on pure $\mathrm{H}_{2}$, even at temperatures below ambient, whereas PAFCs then become non-operational. This feature may make SPEFCs future candidates for transportation applications, for remote site use, for military applications, and even as replacements for portable primary and secondary battery-power sources. Small hydride cylinders in portable sys tems would provide instant recharge capability with an energy and power density of the complete package in excess of $100 \mathrm{Wh} / \mathrm{kg}$ at a $1-\mathrm{h}$ rate. Compared with alkaline systems, they would possess the indicated performance advantages and would be structurally lighter, simpler (compare Figs. 4.8-2 and 4.8-3) and probably more reliable, particularly compared with AFCs utilizing circulating electrolyte.

Early work at GE emphasized the use of a solid-electrolyte membrane on which all of the other cell components were hung. Twenty years of development on the immobilized acid cell have demonstrated its advantages. The PAFC system is built around the bipolar plate and the electrode substrates. There seems to be no particulat reason why this approach cannot be used also for a polymer electrolyte cell, in which the polymer is no longer solid but rather gel-like. The only requirement is that the material is non-leachable and non-volatile. A gel (Nafion soup, Ref. 19) rather than a solid membrane may help to ameliorate interfacial incompatibilities be tween the catalyst layer and the electrolyte. It may also allow the use of supported and ultimately non-noble catalysts (see Sec. 2.15 for a discussion of these catalysts for PAFCs). It is possible that selected gel electrolytes will be found that are more corrosion-resistant than concentrated PA. Composite electrolyte systems (Sec. 2.15) should also not be discounted.

Much polymer research will be required to develop satisfactory, low-cost systems with the correct physical, chemical and electrochemical properties. This research will be a highriak area, which, if succesaful, will yield high pay-offs, perhaps even the development of an effective anion-exchange (alkaline) polymer, which might represent the best of all worlds. In summary, the development of polymer electrolyte systems is expected to complement the functions of acid and alkaline systems.

\subsection{Research Recommendations}

If the shortcoming that have been discussed can be overcome, the SPEFC system should be competitive with the PAFC system dispersed for electric utility power-plant applications and superior for vehicular applications, including the personal transportation vehicle. The SPEFC system as a whole is probably about a decade behind the PAFC in development for commercial use. of SPEFCs.

Research is needed in the following areas to support the development of a new generation 


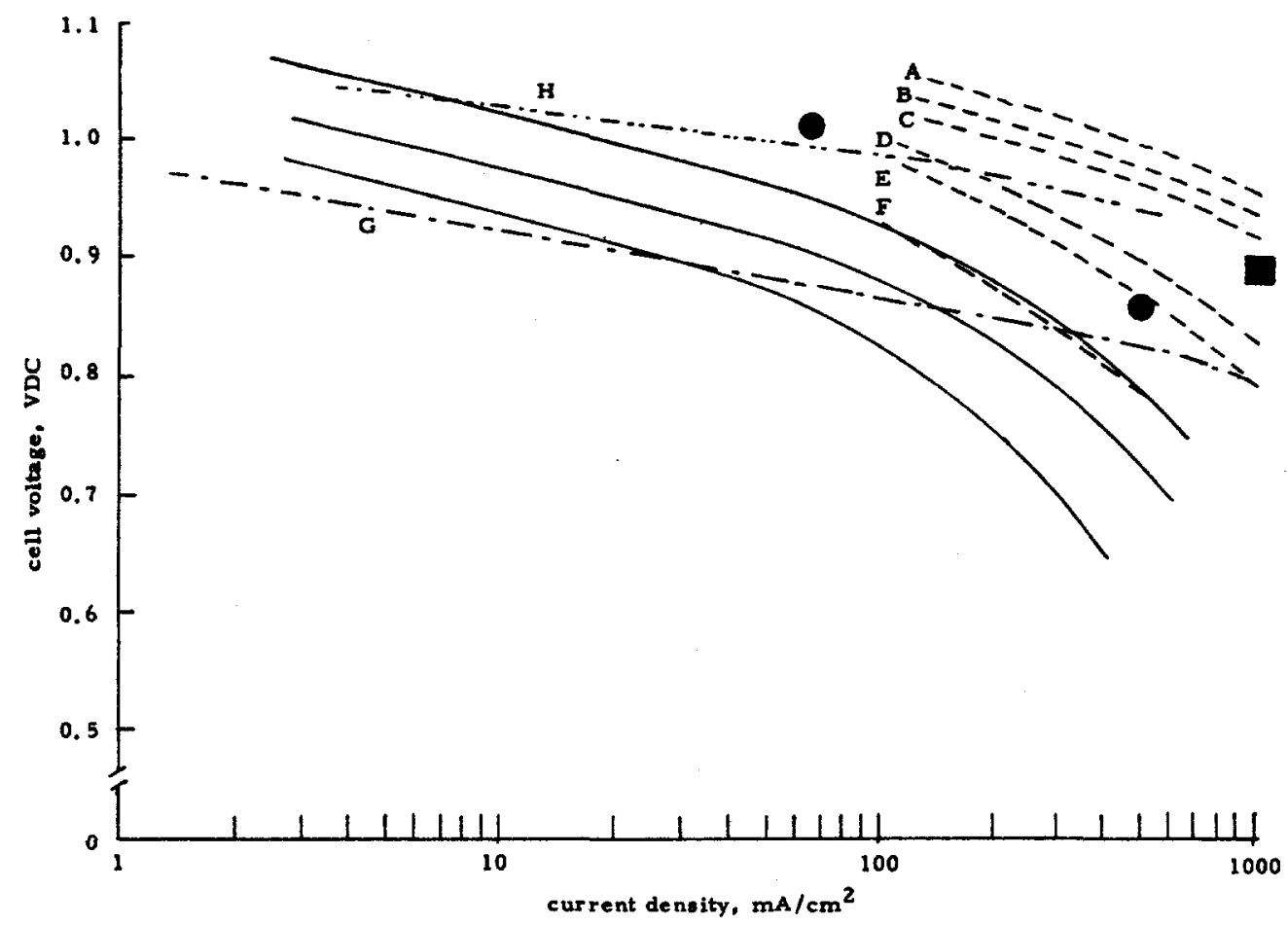

Fig. 4.8-1. The SPEFC performance data are shown and compared with the performance of advanced $A F C s ;$ solid lines: SPE cells $\left(82^{\circ} \mathrm{C} ; 1.2\right.$ bar; $104^{\circ} \mathrm{C}, 7.1$ bar $)$; dashed lines: initial advanced alkaline cell ( $\mathrm{KOH}$ electrolyte, $8 \mathrm{~N}$ ) results at $82^{\circ} \mathrm{C}$, 1 bar (F), $82^{\circ} \mathrm{C}, 4$ bar (E), $110^{\circ} \mathrm{C}, 4$ bar (D), $127^{\circ} \mathrm{C}, 10$ bar (C), $127^{\circ} \mathrm{C}$, $17 \mathrm{bar}(\mathrm{B}), 149^{\circ} \mathrm{C}, 17 \mathrm{bar}$ (A); $0.2 \mathrm{mg} \mathrm{Pt} / \mathrm{C}, 1 \mathrm{bar}, 82^{\circ} \mathrm{C}$, IR free (G), $10 \mathrm{mg} / \mathrm{cm}^{2} \mathrm{Au} / \mathrm{Pt}, 127^{\circ} \mathrm{C}, 1 \mathrm{bar}, \mathrm{IR}$ free (H); 0 , space-shuttle cell nominal performance $(1000 \mathrm{~h}) ; 0$, UTC target coal $(1000 \mathrm{~h})$.

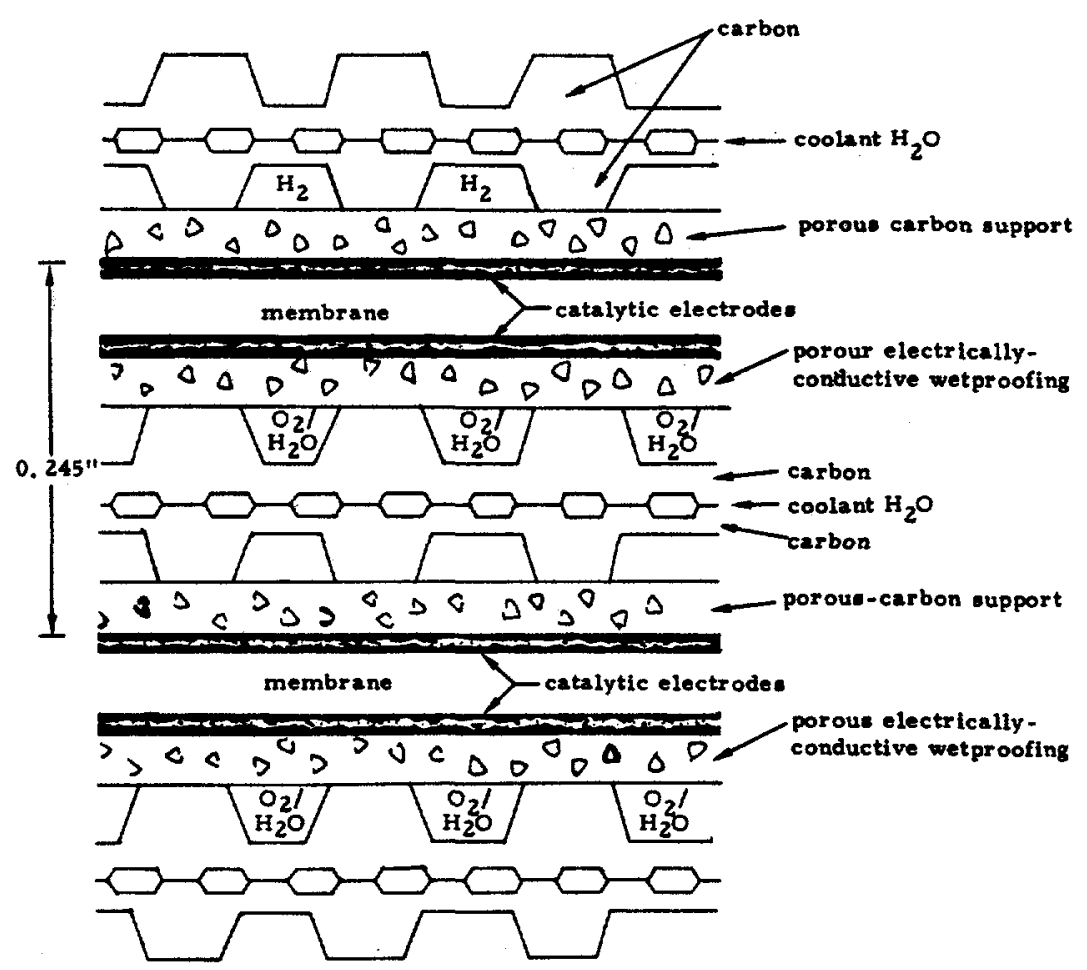

Fig. 4.8-2. The cross-sectional structure is shown for a typical SPEFC. 


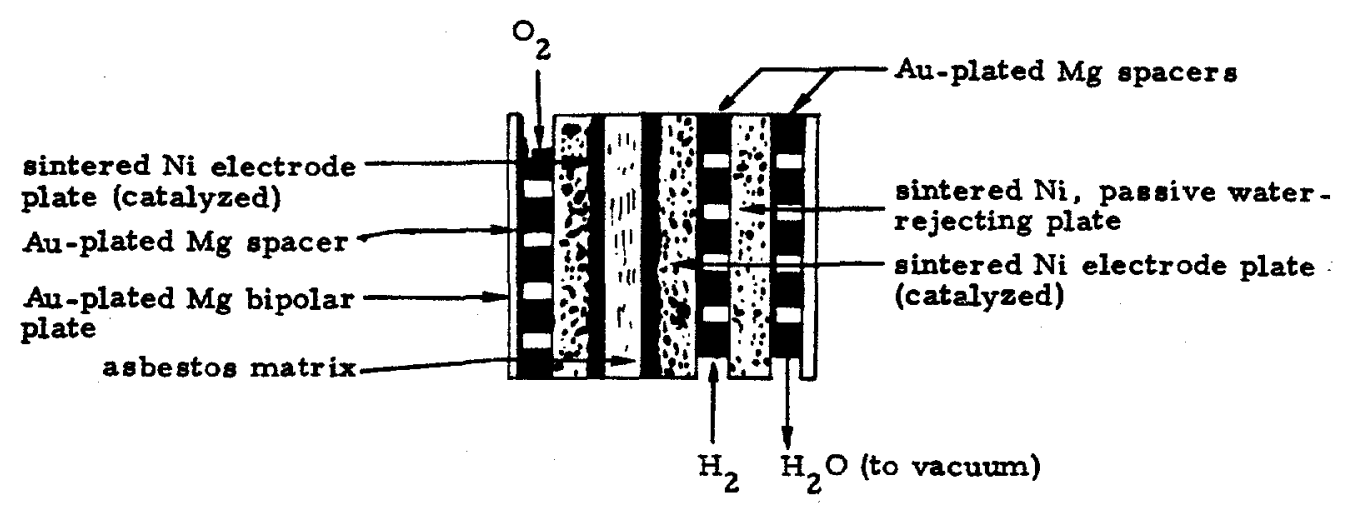

A. Allis-Chalmers cell with passive water rejection (late 1960s, bipolar structure).

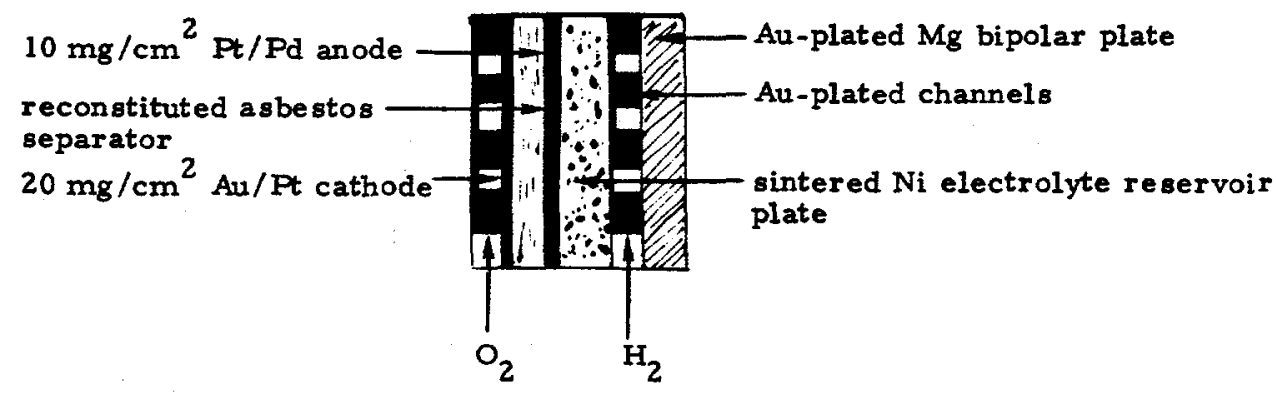

B. UTC orbiter cell with active water rejection from the $\mathrm{H}_{2}$ feedback loop (bipolar).

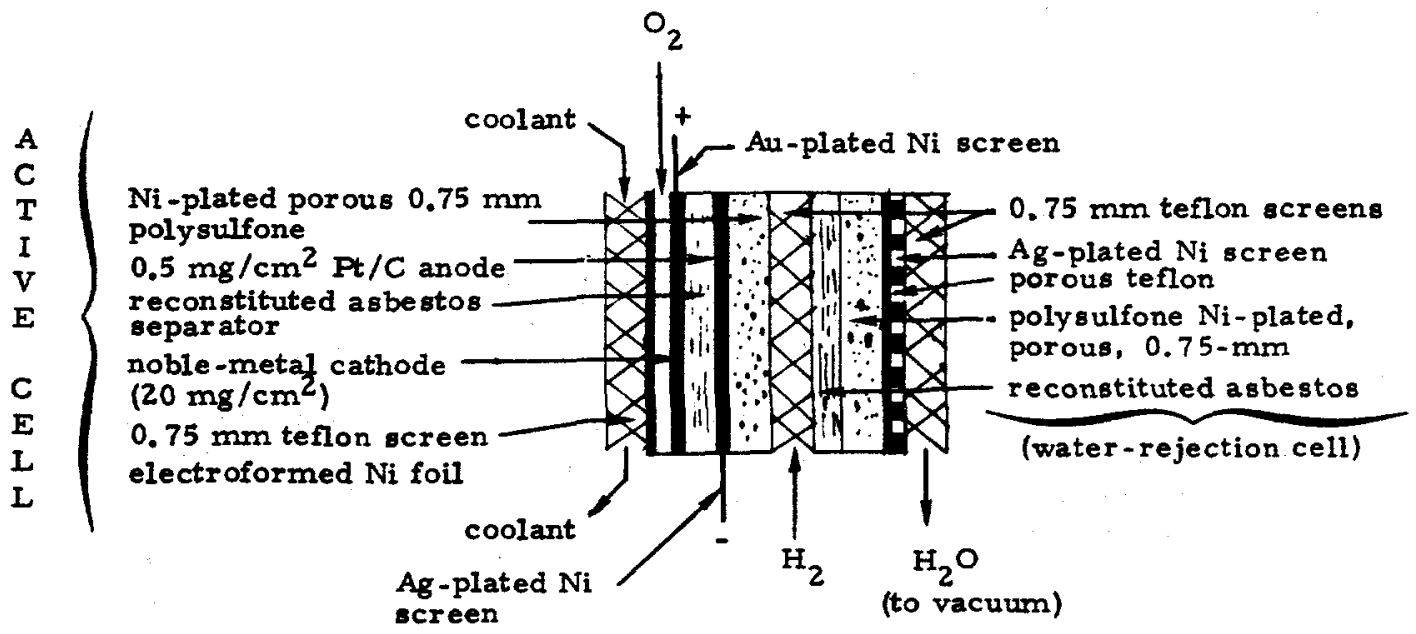

C. UTC advanced lightweight cell with passive water rejection and cell-edge collection via electrode screens (late 1970s).

Fig. 4.8-3. The complex atructures of advanced AFCs with non-circulatingelectrolyte are shown. 


\section{A. Polymer Membrane Electrolyte Research (Priority I)}

New and modified cation-exchange membrane (proton conductor 8 ) should be developed, offering the promise of lower cost and operation at temperatures $2150^{\circ} \mathrm{C}$ with good conductivities and conducive to fast kinetics for the $\mathrm{O}_{2}$-reduction and $\mathrm{H}_{2}$-oxidation reactions with available electrocatalysts. This research should include the synthesis of new membrane materials and their electrochemical evaluation. A relatively large industrial effort is already in progress to find new, lower-cost membranes for the chlor-alkali industry and other applications. This industrial effort may become a source of promising new membrane atructures for the SPEFC. Careful electrochemical evaluation will be necessary under conditions that are suitable for use in advanced SPEFC.

For stability reasons, the most promising new polymeric materials are likely to involve fluorinated organic structures. Inorganic proton conductors, however, should also be considered. Specific types of materials which are possible candidates for electrolytes include: (a) fluorinated organic proton-conducting polymers with replacement acid groups for the presently used sulfonic acids (e.g., phosphoric, phosphonic, phosphinic, silicic acids); (b) Nafion-type membranes (eulfonic acid groups), impregnated with very concentrated ( $85 \%)$ PA or other highly conducting acids to suppress the vapor pressure of water in the inverse micellar structure, while still retaining sufficient conductivity and atability at temperatures $2140^{\circ} \mathrm{C}$; (c) gelled ionexchange type proton-conducting polymeric materials; (d) gelled concentrated acid electrolytes with good conductivities and stabilities at elevated temperatures (e.g., borophosphates, borofluorosulfonic acids); (e) multilayer polymeric proton-conducting membranes (e.g., polymer membranes with different anolyte, bulle and catholyte layers, optimized for low electrode polarization while retaining high conductivity and low leakage of $\mathrm{H}_{2}$ and $\mathrm{O}_{2}$ between the anodes and cathodes); (f) proton-conducting inorganic solid membrane materials such as heteropolyacids (e.g., polymolybdates and tungstates), including dispersions of these in teflon and other fluorinated polymer matrices.

The inorganic materials may be suitable for operations at much higher temperature 8 than organic polymeric materials and offer the possibility of direct oxidation of methanol and other fuels. These are an extension of the solid oxide FC concept but involve froton conductors rather than oxide-ion conductor 8 .

\section{B. Electrocatalysts for SPEFCs (Priority I)}

i. Cathode 프stmonatalysts (Air Electrode)

In contrast to oxygen cathodes in conventional iiquia slect.rolytes, little information is available concerning the factors controlling the polarization of Ft-cataly $\bar{z} \geq \mathrm{d}$ ai $\bar{x}$ cariodes. Th.... studies are necessary as a first step in achieving more effective utilization of Pt and finding effective, lower-cost alternative catalysts to Pt (e.g., oxides, transition metal complexes including macrocycles and non-precious metals). Some catalysts may be stable in the polymeric electrolytes, while lacking adequate stability in concentrated PA or KOH. This fact may make it possible to use some of the highly active macrocycles which catalyze the 4-electron reduction of $\mathrm{O}_{2}$. Kinetic mechanistic studies will be required with these promising electrocatalysts in order to optimize them with respect to activity and long-term stability. Research on electrocatalysts for SPEFCs should include supported Pt and Pt-alloy catalysts on various stable substrates.

\section{Anode Electrocatalysts}

The recommendations for the $\mathrm{H}_{2}$ oxidation anode catalysta are similar to those for the cathode, with special emphasis on new catalyste tolerating significant $C O$ concentrations in the $\mathrm{H}_{2}$. In addition, xesearsh on electrocatalysts for the direct oxidation of methanol and other fitels should be initiated, provides proni aing new eystems are propos cu to accompli ta the oxidation at reasonable potentisls and current densities. Platinunis not sufficiently active at tomperatures below $200^{\circ} \mathrm{C}$ and $n \in w$ ideas are needed before further research is initiated.

\section{Electrode Structures (Priority II)}

The electrode structures presently used in SPEFCs do not appear to be near-optimal designs. Relatively little research has been reported on these electrodes and the relation of transport processes to structure is not clear. It is questionable if substantial fractions of the catalysts are accessible to the chemical reactants and electrolyte while making electronic contact in the present electrode structures. Specific research recommendation include: (a) characterization of the structures of existing SPEFC electrodes; (b) studies of $\mathrm{O}_{2}$ and $\mathrm{H}_{2}$ transport and electrolyte access to the catalyst 8 in exi ating SPEFCs, including modelling; (c) modelling studies; (d) development of more effective electrode structures with emphasic on innovation. 


\section{Innovations in Cell Design (Priority II)}

If improved membranes and electrocatalysts become available for the SPEFC, then it would be desirable to consider innovations in overall cell deaign. As an example, the monolithic structure proposed for the solid oxide FC might be applicable also to the SPEFC. It is conceivable that a monolithic, all-polymer FC can be developed with polymer anodes, polymer cathodes and polymer electrolyte. Progress in this approach is clearly contingent on new and innovative ideas and new materials. 
1. G. F. Alelio, U.S. Patent 2,366,007 (1945).

2. W. Juda and W. A. McRae, U.S. Patent 2,636,851 (1953); G. Bodamer, U.S. Patents $2,681,319,2,681,320(1954)$.

3. W T. Grubb, in Proc. Ilth Ann. Battery Res. Dev. Conf. PSC. PSC Publications Committee, p. 5, Red Bank, N.J., 1957; U.S. Patent 2, 913,511 (1959).

4. L. W. Niedrach and W. T. Grubb, in Fuel Cells, p. 253, W Mitchell, Jr., ed., Academic Press, NY (1963).

5. H. J. R. Maget, in Handbook of Fuel Cell Technology, pp. 425-491, C. Berger, ed., Prentice-Hall, Englewood Cliffs, NJ (1967).

6. H. A. Liebhavsky and E. J. Cairns, Fuel Cells and Fuel Batteries, pp. 587-619. Wiley, NY (1968).

7. R. J. Lawrence, Interim Report, LANL-29, "New Membrane Catalyst Concept for Solid Polymer Electrolyte Systems," P.O. No. 9-X53-D6272-1, University of California, Los Alamos National Laboratory, Los Alamos, NM (1984).

8. J. F. McElroy, Est. Abs. Nat. Fuel Cell Seminar, p. 123, 1983.

9. W. T. Grubb and L. W. Niedrach, J. Electrochem. Soc, 107, 131 (1960).

10. L. W. Niedrach, in Proc. 13th Ann. Power Sources Conf., p. 120, P.S. C. Publications Committee, Red Bank, NJ (1959); U.S. Patent 3, 134,697 (1964).

11. D. Linden, in Handbook of Batteries and Fuel Cells, p. 42-1, D. Linden, ed, McGrawHill. NY (1983). A good early review on FCs for s pace applications may be found in B. J. Crow, "Fuel Cells, A Survey," NASW-2173, National Aeronautics and Space Administration, Washington, D. C. (1973).

12. J. H. Russell, in Proc. 19th Ann. Power Sources Conf., p. 35, P. S. C. Publications Committee, Red Bank, NJ (1965).

13a. H. L. Yeager and A. Eisenberg, in Perfluorinated Ionomer Membranes, p. 1, ACS Symposium Series, American Chemical Society, Washington, D. C. (1982).

13b. H. L. Yeager, ibid., p. 41.

13c. T. D. Gierke and W. Y. Hsu, ibid., p. 283.

13d. M. Seke, S. Ogawa and K. Kimoto, ibid., p. 365 .

13e. T. Sata and Y. Onone, ibid., p. 411.

13f. H. Ukihashi and M. Yamabe, ibid., p. 428.

$13 \mathrm{~g}$. R. L. Dotson and K. E. Woodward, ibid., p. 428.

14. L. Bregoli, J, Electroanal. Chem. 23, 489 (1978).

15. D. P. Gregory, "A Hydrogen Energy System," American Gas Association, Washington, D. C. $(1973)$.

16. A. Damjanovic, A. Dey and J. O'M. Bockris, J. Electrochem. Soc. 113, 739 (1966).

17. P. N. Ross, Est. Abs. Nat. Fuel Cell Seminar, p. 141 (1983).

18. R. E. Martin, Final Report, Advanced Technology Lightweight Fuel Cell Program, N79033591, NASA CR-159653, National Aeronatics and Space Administration, Washington, D.C. (1979).

19. A. J. Appleby, Workshop on Proc. Renewable Fuels and Advanced Power Sources for Transportation, p. 55, Boulder, CO, June 1982, Solar Energy Research Institute; Golden, CO; H. L. Chum and S. Srinivasan, ibid., Executive Summary, p. v.

20. Z. Ogumi, Z. Takehara and S. Yosaizawa, J. Electrochem. Soc. 131, 769 (1984).

21. T. Sakai, H. Takenaka, N. Wakabayashi, Y. Kawami and E. Torikai, J. Electrochem. Soc. 132,1318 (1983).

22. E. O'Sullivan, M. Enayetullah, and E. Yeager, Monthly Report No. VI-7 to the Electric Power Research Institute, EPRI/CWRU Contract RP1200-7, "Oxygen Reduction on Acid Electrolytes, " July 1-31, 1984.

23. R. Kotz, S. Clouser, S. Sarangapani, and E. Yeager, J. Electrochem. Soc. 131, 1097 (1984).

24. J. McBreen, "The Solid Polymer Electrolyte (SPE)/Electrocatalyst Interface," Paper 131, 164th National Meeting. The Electrochemistry Society, Washington, D. C. , Oct. 9-14, 1983; extended abstracts 83-2, 205. 


\section{MOLTEN CARBONATE FUEL CELLS (MCFCs) ${ }^{\dagger}$}

\subsection{Introduction}

The MCFC, operating at a temperature near $650^{\circ} \mathrm{C}$, has been under intensive development for the last decade as a second-generation FC. This label suggests both the considerable advantages of the MCFC and the fact that it still needs substantial development before it can be succes sfully commercialized.

The melt chemistry and electrochemistry involved in the MCFC are unique. At the anode of the MCFC, the net reaction is

$$
\mathrm{H}_{2}+\mathrm{CO}_{3}^{=} \rightarrow \mathrm{H}_{2} \mathrm{O}+\mathrm{CO}_{2}+2 \mathrm{e}^{-} \text {. }
$$

whereas at the cathode

$$
\frac{1}{2} \mathrm{O}_{2}+\mathrm{CO}_{2}+2 \mathrm{e}^{-} \rightarrow \mathrm{CO}_{3}=
$$

is the net process. The $\mathrm{CO}_{2}$ has a function similar to Lewis acids (for example, the proton) in low-temperature cells such as the PAFC; however, protons are transferred in the PAFC via ion conduction in the electrolyte phase, whereas in the carbonate cell another shuttle mechanism is required.

In the present concepts, this normally will be conducted by taking the $\mathrm{CO}_{2}$-rich product gas at the anode, burning it to produce $\mathrm{CO}_{2}$ and $\mathrm{H}_{2} \mathrm{O}$, condensing out the water, and adding the $\mathrm{CO}_{2}$ to the cathode inlet air. However, in the future, the use of a $\mathrm{CO}_{2}$ separative device or, in general, a product exchange device may be preferred, which will allow higher hydrogen utilization in the cell and hence greater syatem efficiency.

The system efficiency and cost of the MCFC appear very attractive, even without a $\mathrm{CO}_{2}$ separation device, in comparison with low-temperature cells. Although phos phoric acid fuel cell (PAFC) power-plant systems are expected to gain commercial acceptance during the next $5 \mathrm{y}$, they will be characterized by relatively high material costs and heat rates. A major cost component will be associated with the noble metal catalysts that are needed to reduce voltage losses at the cathode in acid cells. Another major cost component is the chemical plant necessary for conditioning the fuel by reforming $\mathrm{HCs}$ and largely removing $\mathrm{CO}$ from the feed gas.

In high-temperature systems, these shortcomings can be avoided because elevated tem. peratures accelerate the chemical and physical processes to such an extent that polarization losses are less than with aqueous $F_{B}$, and less expensive materials can be used as electrocatalysts. Although the ideal efficiencies $(\Delta \mathrm{G} / \Delta \mathrm{H})$ of the overall cell reaction loxidation of $\mathrm{H}_{2}$ and $(O)$ decrease at increasing operating temperatures, the higher operating temperature reduces polarization losses to such an extent that the actual cell efficiency is increased.

The MCFC operating temperature $\left(600-650^{\circ} \mathrm{C}\right)$ is high enough to produce valuable waste heat, yet it is sufficiently low so as not to incur too great a free energy penalty (the theoretical open circuit is $100 \mathrm{mV}$ higher than that of the $1000^{\circ} \mathrm{C}$ SOFC). The waste heat can be used to provide compression work (to improve cell performance, particularly cell potential, and hence efficiency), to supply heat for bottoming cycles, and/or for cogeneration purposes (process or space heat). The most important process heat use will be for the reforming of methane.

Assessing the potential use of FCs for electric power generation, the energy-conversion alternatives study 1 (ECAS) of 1976 identified the MCFC as a major contender for base-load power generation from coal. Provided required performance, cost and endurance goals could be attained, ECAS estimated that it would be possible to attain an efficiency close to $50 \%$ (coal-ac) for a plant using a coal gasifier with pressurized MCFCs and appropriate bottoming cycles. This estimate was confirmed in a GE study for EPRI. 2 This study established that such a plant would cost $\$ 1000(1979) / \mathrm{kW}$ and would essentially have zero environmental emissions.

The ECAS study ${ }^{3}$ specified a design-point performance of $160 \mathrm{~mA} / \mathrm{cm}^{2}$ at $0.85 \mathrm{~V}$ per cell when operated on low-BTU gas from air-blown coal gasifiers (the dry composition in mol\% was $\left.22 \mathrm{H}_{2}, 10 \mathrm{CO}_{2}, 19 \mathrm{CO}, 1.4 \mathrm{CH}_{4}, 47.6 \mathrm{~N}_{2}\right)$ with a fuel utilization of $75 \%$. The lifetime design goal was 40,000 operating $\mathrm{h}(4.5 \mathrm{y})$.

this chapter has been written by J. R. Selman with some use of an earlier version by S. S. Penner and E. Williams. Useful comments and suggeations were made by A. J. A ppleby (who contributed greatly to Secs. 5.2 and 5.8B), L. G. Marianowski, and H. Maru, as well as other AFCWG members. 
For single-cell operation, the se goals have now been attained and exceeded (Fig. 5. 1-1). Cells operating at $650^{\circ} \mathrm{C}$ and 7 atm pressure yield voltages of $0.82 \mathrm{~V}$ at $160 \mathrm{~mA} / \mathrm{cm}^{2}$. Single laboratory-scale cells have been operated for well over $40,000 \mathrm{~h}$, without excessive decline in performance. Larger cells, with $94-\mathrm{cm}^{2}$ electrode area, have operated in excess of $10,000 \mathrm{~h}$ with an average decline rate of $10 \mathrm{mV} / 1000 \mathrm{~h}$.

Recent developments in the U.S. are focused on stack design and operation. Although there are as yet no stacks in operation of the size envisioned for commercial applications (e.g. , 100 cells with $10 \mathrm{ft}^{2} / \mathrm{cell}$ ) and lifetimes of minimally $25,000 \mathrm{~h}$ will be required, results on 10-20 cell stacks with $1 \mathrm{ft}^{2} /$ cell are very encouraging. 5,6 These cell stacks in pressurized operation over $3000 \mathrm{~h}$ are approaching the single-cell performance standards (Fig. 5.1-2).

The MCFC therefore appear 8 to have a bright future for 21 st century coal-fired baseload electric utility plants. These, however, will be large (GW scale) and user confidence will be required before the MCFC is built in units of this size. Such user confidence will be best obtained if reasonably small MCFC units can be first built and field-tested under conditions where they can generate power economically. Dispersed units (a few $M W$ in size) are unlikely to be coal-fueled. However, the high temperature waste heat available in the MCFC can be used to advantage in two types of installation, notably in natural-gas units and in applications where high quality waste heat is needed in industrial cogeneration. In both of these, the MCFC may be markedly superior to other FCs.

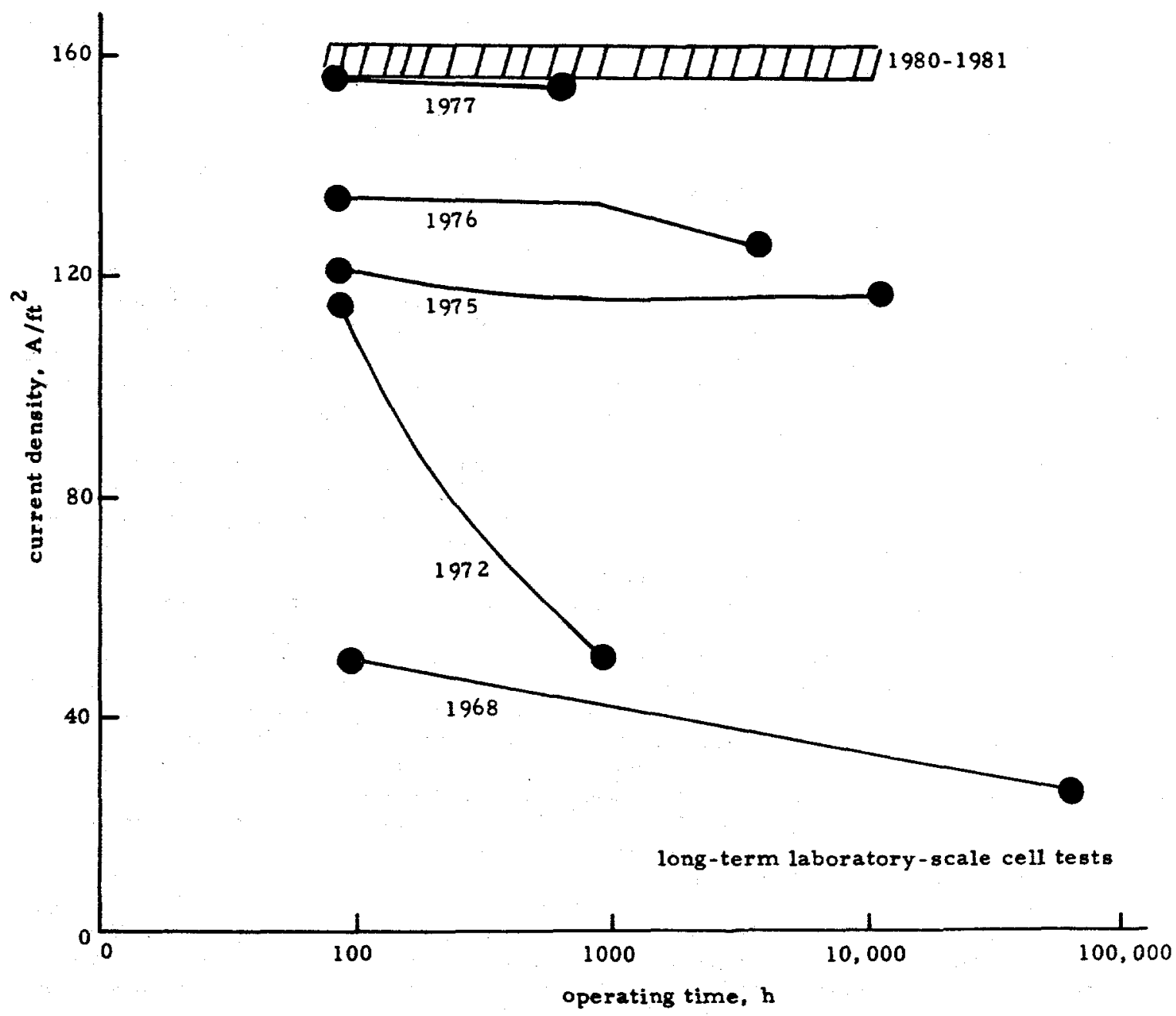

Fig. 5.1-1. Molten carbonate FC performance and endurance history. ${ }^{8}$ The current density refers to the design voltage for a heat $r$ ate of $7500 \mathrm{BTU} / \mathrm{kWh}$.

The 1972 tests showed the necessity to (i) control anode sintering, (ii) improve $\mathrm{LiAlO}_{2}$ and cycling, (iii) introduce $\mathrm{Ni}$ anode current collector, (iv) reduce wet-seal corrosion, and (v) allow carbonate addition during operation. The 1975 tests failed because of wet-seal corrosion. The 1980-81 tests indicated that remaining requirements are to (i) control wet-seal corrosion, (ii) improve the electrolyte tile, and (iii) improve understanding of carbonate distribution. 


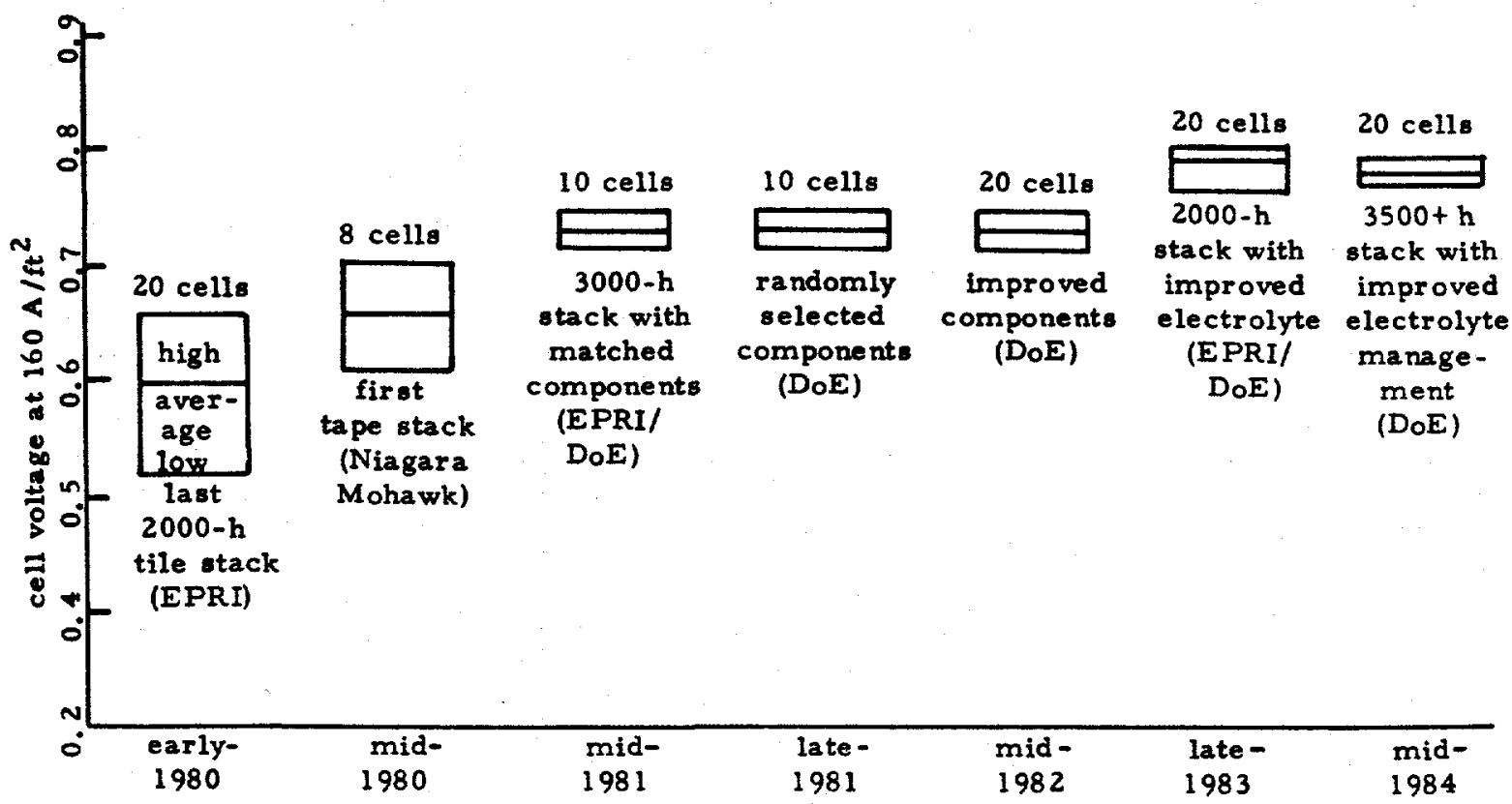

Fig. 5.1-2. MCFC stack performance comparisons at UTC. ${ }^{6}$ A low-BTU fuel $\left(17 \% \mathrm{H}_{2}+\mathrm{CO}\right) \mathrm{w}=\mathrm{Z}=2 \mathrm{~A}$, at $1200^{\circ} \mathrm{F}$ and 65 psia. The organization listed in parentheses were the progam sponsors.

The major advantage of the MCFC over the acid celi is that it can use intarnal reforming, i. e. its waste heat is directly a vailable within the cell for the conversion of desulfurized methane directly to $\mathrm{H}_{2}$ in a driven reforming reaction in the cell anode chamber. In the internal reforming molten carbonate cell (IRMCFC), the space velocities are relatively low and reforming rates are quite adequate, provided that the reforming catalyst is protected from catalyst poisons (particularly $S$ and traces of carbonate). Means to accomplish this protection for short periods of time have been worked out in the laboratory.

If the ongoing development of an inter nally-reforming anode is successful, modular dispersed units should be feasible with a $6000-6500 \mathrm{BTU} / \mathrm{kWh}$ heat rate $(52.5$ to $56.8 \%$ efficiency) on the basis of NG. Provided the installed cost is acceptable, such units should prove to be very attractive for cogeneration. The economics of MCFCs in this application, which are strongly dependent on technical advances, are discussed in Sec. 5.2.

Summarizing the status of its technology, the MCFC may be expected to enter commercial markets approximately 5 to $7 \mathrm{y}$ behind the PAFC, provided that remaining technical problems can be overcome. The time lag is also important to produce general consumer acceptance for FCB. While the MCFC has a considerable number of technical and economic advantages over the PAFC and other low-temperature FCs, its development has been restrained by problems of perception, as well as a lack of research effort. The high-temperature FCs, MCFC 8 and SOFCs, are perceived to have more disadvantages (corrosion and sealing problems) than advantages (rapid kinetics) resulting from the high operating temperature.

Amoig the next-geiseration technologies, the MCFC. has reseived more attention than the SOFC because it prezents a compremise between advantoges and disadvantages in operating at high temperature. The relatively modest resez=ch effert spent on the MCFC has becu very effective. As will be discussed in this chapter, the major practical problems involved in longterm MCFC operation have been solved satisfactorily, without comprehensive optimization. Thus, the performance levels illustrated in Figs, 5.1-1 and 5.1-2 are not merely impressive in themselves but suggest a large potential for further improvement of the state-of-the-art, based on a better fundamental under standing of MCFC operation.

This factor is what makes MCFC technology especially attractive as a research investment, even if the return does not arrive until the medium to long term. The Japane se apparently believe that MCFC technology will replace PAFC technology very early and they are therefore working intensively to commercialize MCFCs. 7 The fundamental research component of the MCFC development is likely to be essential for innovation and long-term commercial success. 
As has been shown in Chapter 2, Secs. 2.4 and 2.6, the probable market in the U.S. for electric utility FCs as a proportion of total added capacity will be about $7 \%$ for a modular, dispersed system with a capital cost of ca $\$ 800(1982) / \mathrm{kWh}$ and a heat-rate of about $8300 \mathrm{BTU} / \mathrm{kWh}$. This market may amount to a total of $3,000 \mathrm{MW}$ per year after the year 2005.9

The on-site cogeneration market in the U.S. may be a further $1,000 \mathrm{MW}, 10$ The industrial cogeneration market may be at least as large as the on-site market, since total 1985 industrial cogeneration capacity is about $50,000 \mathrm{MW}$ and $F C_{8}$ could be attractive for a substantial percentage of the replacement part of this market as the year 2000 approaches. This penetration would be especially true of FCs which offer waste heat at high temperatures, which can be used to raise medium-to high-pressure steam. The PAFC is not suitable for this purpose, since the cell stack operates at only about $200^{\circ} \mathrm{C}$ and its waste heat a vallable as low-pressure steam is very small (in the proposed UTC FCG-1 system, about 10\% of the HHV of the fuel) because of the large steam requirement of the reforming operation. 11 About $30 \%$ of the HHV of the fuel will be a vailable as $70^{\circ} \mathrm{C}$ water, which can be used for space-heating. In the utility WE system, 12 no cogeneration energy at all is available, since all waste heat is used in the reformer and turbocompressor.

The MCFC therefore appears to have a useful future in the industrial cogeneration market. Since it is a dc device, it may be particularly suitable for use with electrochemical processes, for example, in the chlorine-caustic and aluminum industries. In the former, it has the further advantage of being usable for efficient consumption of any $\mathrm{H}_{2}$ produced in the process along with some clean fossil fuel (NG or ayngas from coal). For the industrial application, a heat-rate of about $8000 \mathrm{BTU} / \mathrm{kWh}$ may be most desirable, which will produce about $3500 \mathrm{BTU} /$ $k W h_{e}$ of steam.

It is instructive to consider the response of market projections to the heat rate, as well as the capital cost, of the MCFC. The IRMCFC, if successfully realized, allows a vast extension of the electric utility market by lowering the heat-rate at the expense of cogeneration capability.

The authors of a recent market study ${ }^{9}$ concluded that if the HHV efficiency of the NGfueled FC system could be improved by $10 \%$ (from 8300 to $7500 \mathrm{BTU} / \mathrm{kWh}$ ) for a similar capital cost, the U.S. electric utility market share may triple to $17 \%$ of total added capacity, giving $10000 \mathrm{MW} / \mathrm{y}$ over the decade 1995-2005. The world market might be at least three times this figure or $30000 \mathrm{MW} / \mathrm{y}$. Finally, if the capital cost of the FC could be one-half of that assumed in Ref. 9, or about $\$ 400(1982) / \mathrm{kW}$, the market might be expected to double again to about 60000 $\mathrm{MW} / \mathrm{y}$, provided enough NG remains available for this market. This result is demonstrated in Fig. 2.6-3.

There seems to be little question that the NG-fueled MCFC will fit into the required capital cost range to reach a large part of this market. There is also little question that it will exhibit the required heat-rate qualification and may even exceed it. As a stand-alone, dispersed generator operating on NG, the MCFC will be capable of a heat-rate of $6500 \mathrm{BTU} / \mathrm{kWh}$, with little or no cogeneration capability (see Sec. 5.8).

The IRMCFC promises to have a very bright future for both electric utility and on-site applications, using NG, provided that the installed cost is economically competitive. As is discussed in Sec. 5.7, installed-cost estimates are at present in the $\$ 1200-1300 / \mathrm{kW}$ range.

The higher fuel efficiency would certainly make the IRMCFC somewhat more attractive economically than the PAFC. If it is commercially avalable, it might therefore be preferred by the utility over a PAFC offered at the same price. However, this scenario implies that the PAFC could be made obsolete by the MCFC, which is unlikely to happen in view of the difference in the periods of commercial introduction between the two systems: since the MCFC is technically about 5-7 y behind the PAFC, the latter should be well down the learning curve and probably a vailable at less than $\$ 1000 / \mathrm{kW}$ installed when the IRMCFC goes into pilot production. A second factor may be flexibility of use: the very efficient IRMCFC stack may not be utilizable over.the same wide range of current density (say from 25 to $100 \%$ load) as in the PAFC. This estimate results from the MCFC cell voltage-current characteristics, which are roughly linear, giving a high cell potential at part load, i.e., a disproportionately high electrical efficiency, which will substantially cut down the avalable sensible heat for reforming and stack-temperature maintenance. The IRMCFC stack, therefore, may not be operable adiabatically at low load unless parasitic fuel is burned to maintain its temperature. External reforming is also a possibility, but this will result in about 4 percentage points lower efficiency. 13

The consensus in the utility industry is therefore that the PAFC and the IRMCFC will both find their place in the generation mix. The PAFC will be introduced earlier and will fill the lower efficiency end of the market $(8000 \mathrm{BTU} / \mathrm{kWh})$ at the right-hand side of Fig. 2.6-2. Because of its early introduction, its production cost will be relatively mature when a premium cost IRMCFC is first introduced to start to fill the higher-efficiency market (left-hand side of Fig. 2.6-2). Finally, the market for the PAFC may eventually be limited by Pt supply.

Production of the MCFC should rapidly bring its cost down as it descends the learning curve. In general, a 0.9 learning curve has been assumed in FC studies, i.e., every doubling 
in production results in a cost reduction to $90 \%$ of the previous value. This curve has been shown to be widely applicable to many other technological areas. If pilot production (Iess contingencies) is assumed to give an installed cost of about $\$ 950 / \mathrm{kW}$, then increasing cumulative production by 2 order s of magnitude (to a total of $18,000 \mathrm{MW}$ ) over a period of 12 y will reduce cost to less than $\$ 500(1983) / \mathrm{kW}$. This estimate represents an average yearly production increase of $35 \%$, which is not unreasonable for a growth industry.

If the problems (see Sec. 5.6E) can be overcome, the potential world market for the IRMCFC would be very large, for example, the $60,000 \mathrm{MW} / \mathrm{y}$ quoted above. This estimate depends, however, on the future availability of NG. Finally, the potential base-load market, using syngas from coal, must also be considered, especially with gasifiers that produce some methane. 14 In this case, the internal-reforming capability of the cell will be advantageous and lead to higher system efficiencies. The total incremental base-load market might be about 30,000 $\mathrm{MW} / \mathrm{y}$ in the US and $100,000 \mathrm{MW} / \mathrm{y}$ or more world-wide, which will be shared between nuclear energy and coal. MCFCs give very low environmental impact and potentially large fuel-cost saving 8 , and 80 may take a large percentage of this market.

\subsection{Historical Perspectives}

The present MCFC is the product of a conceptual development that started with the search for an FC capable of converting coal directly to electricity. 15 By 1945 , this search had led, on the one hand, to solid-oxide electrolytes such as stabilized $\mathrm{ZrO}_{2}$, operating at $1000^{\circ} \mathrm{C}$; on the other hand, to cells with semi-solid electrolytes operating at $600-800^{\circ} \mathrm{C}$ (Davtyan). The pioneering work of Broers 16 and co-workers 17 defined the essential role of the $\mathrm{CO}_{3}$ ion when carbonaceous gas is used as fuel. These authors also perfected the electrolyte matrix so that it could effectively prevent contact of fuel and oxidant.

During the 19608 , extensive work on MCFCs was carried out in the US (IGT, Texas Instruments, GE), as well as in the Netherlands, England and France. The US gas industry has supported MCFC development continuously since 1959. This effort, carried out largely at IGT, was first supported by the American Gas Association. Since 1967, the TARGET program, through a subcontract of UTC to IGT, supported MCFC development as a back-up to the PAFC system.

MCFC development up to 1970 was largely Edisonian in character. The importance of the melt chemistry in reaction kinetics (peroxide and superoxide mechanisms) was first realized in 1970. After 1970, technological development was accelerated by extensive use of standardized small-scale cells, rigorous control of component quality, and application of mathematical models in design and scale-up.

After 1975, MCFC research, until then concentrated at IGT and UTC, expanded with support from the US DoE and EPRI. Simultaneously, performance criteria were established, as discussed in Sec. 5.1 .

Fundamental research related to MCFC operation has also been expanded in the last $10 \mathrm{y}$; however, in many respects, it has remained very limited in view of the dominant role envisaged for $\mathrm{MCFC}_{B}$ in the future.

Several overviews of recent MCFC technology and related research and development are available. These present the state of technology as of 1978 (Appleby and Ackerman 18 ) and as of 1982 (Selman and Marianowski, 19 Selman and Claar 20). Comprehensive reviews of basic reBearch relating to MCFC technology are also available (Selman and Maru, 21 Maru22). FC power-plant systems in general have been reviewed by Warshay. 23

\subsection{Cell Reactions and Principles of Operation}

Current cell deaigns are based on a planar square geometrical configuration, with cells stacked to build voltage. Each cell forms a repeating unit consisting of an anode current collector, porous anode, electrolyte matrix, porous cathode, and cathode collector. One cell is separated from the next by means of a separator (bipolar) plate that serves both as gas separator and series current connector.

In this section, the cell reactions and key components are discussed. Special attention is given to the control of electrolyte distribution by capillary equilibrium. Discussions of stack design and associated issues are deferred to Sec. 5.8 .

\section{A. Cell Reactions and Key Components}

In an operating MCFC, negative charge is conducted by electrons from an anode through an external circuit to a cathode, where they participate in reduction reactions. Negative charges are conducted by carbonate anions $\left(\mathrm{CO}_{3}\right)$ from the cathode through the molten electrolyte to an anode. At the anode, electrons are produced by oxidation. Figure 5.4A-1 shows the essential features of an MCFC cell which uses reformate (e.g., a gaseous $\mathrm{H}_{2}$ and $\mathrm{CO}$ mixture) as fuel. The electrode reactions are listed on the next page. 


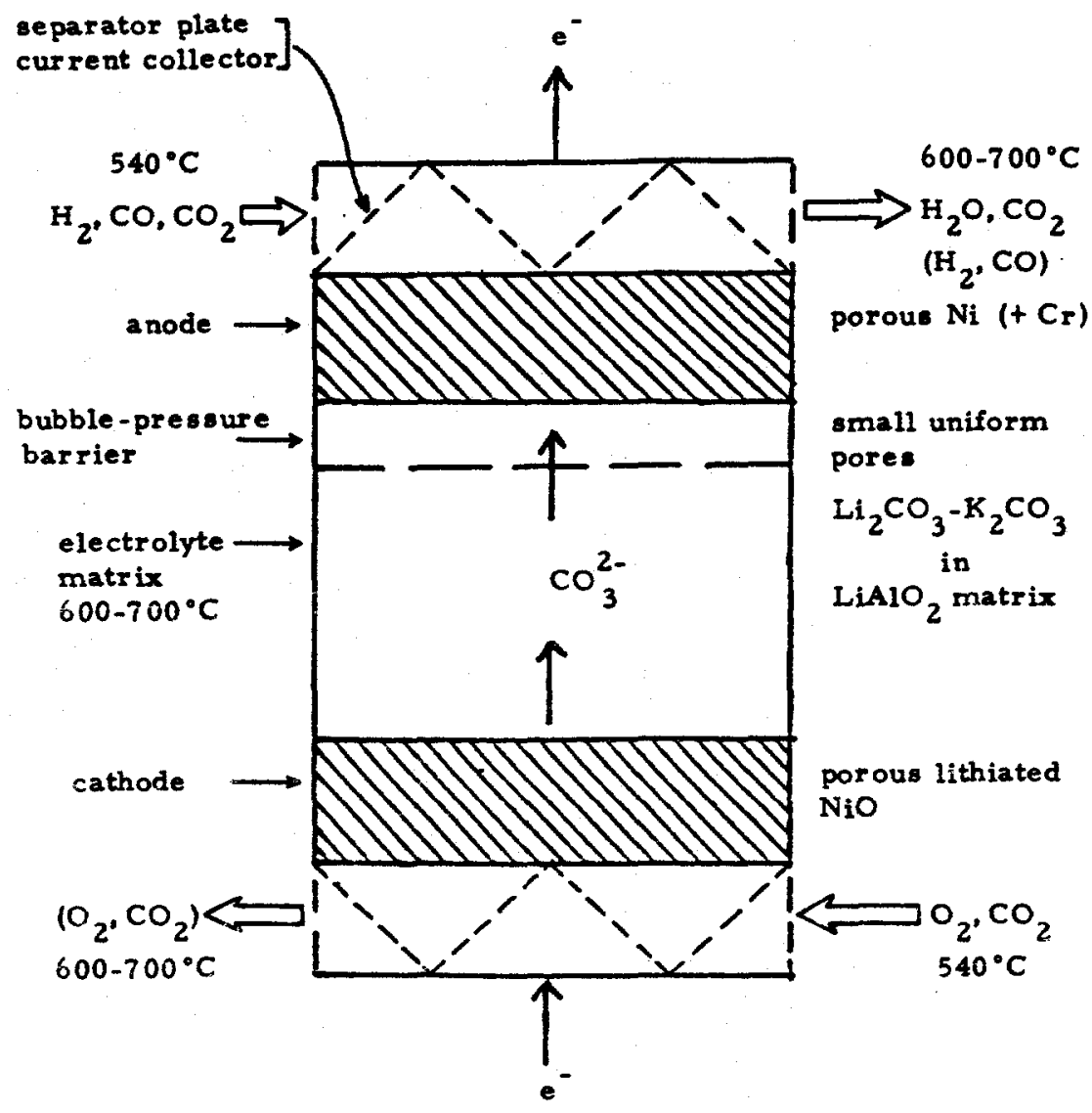

Fig. 5. 4A-1. Schematic of a single cell in an MCFC cell stack, showing cell components, material flows, and current direction.

$$
\begin{array}{ll}
\text { anode reactions: } & \mathrm{H}_{2}+\mathrm{CO}_{3}^{=}=\mathrm{H}_{2} \mathrm{O}+\mathrm{CO}_{2}+2 \mathrm{e}^{-}, \\
& \mathrm{CO}+\mathrm{CO}_{2}=2 \mathrm{CO}_{2}+2 \mathrm{e}^{-} \text {(minor); } \\
& \mathrm{CO}+\mathrm{H}_{2} \mathrm{O}=\mathrm{H}_{2}+\mathrm{CO}_{2} ; \\
\text { shift reaction: } & \frac{1}{2} \mathrm{O}_{2}+\mathrm{CO}_{2}+2 \mathrm{e}^{-}=\mathrm{CO}_{3} .
\end{array}
$$

Thus, two Faradays of electricity generated involve the use of 1 mole of $\mathrm{CO}_{2}$ at the cathode and the production of 1 mole of $\mathrm{CO}_{2}$ at the anode. It is therefore desirable to recycle $\mathrm{CO}_{2}$ in order to maintain the electrolyte composition invariant. This concept has led to a search for a practical product-exchange-device (PED), which still continues. In practice; it has been found adequate tu withdraw part of the anode exit gas after complete combustion and mix it with the cathode-gaz gtream.

The elestrolyte is an alkali-carbonate mixture and is a liquid at $650^{\circ} \mathrm{C}$. The liquid electrolyte is suspended in a porous, insulating and chemically inert, ceramic $\left(\mathrm{LiAlO}_{2}\right) \mathrm{matrix}$. ' $1 \mathrm{he}$ assemblage consisting of the liquid electrolyte and the ceramic matrix is called the electrolyte the. Iile compositions vary among manufacturers, but they typically cuntaic $40-50 \%$ ceramic by weight. Almost every manufacturer uses a mixture of $62 \mathrm{~mol} \% \mathrm{Li}_{2} \mathrm{CO}_{3}$ and $38 \mathrm{~mol}_{2} \mathrm{~K}_{2} \mathrm{CO}_{3}$, or $50 \mathrm{~mol} \%$ each of $\mathrm{Li}_{2} \mathrm{CO}_{3}$ and $\mathrm{K}_{2} \mathrm{CO}_{3}$, in the liquid electrolyte. This composition is a historical evolution and may not yield optimal performance (see Secs. 5.5 and 5.7 ).

The electrodes are porous and facilitate occurrence of the electrode reactions [Eqs. (5. 4A-1) through (5.4A-4)] by means of three-phase coritact or film-diffusion processes. Ar electrode pore containing electrochemically active aites may be completely or partially flooded with liquid electrolyte or else may be covered by a thin film of electrolyte.

The interface processes at the electrodes may be described qualitatively as follows.

(i) The supply (at the cathode) or removal (at the a node) of electrons occurs at the reaction sites. (ii) The electrolyte serves as a sink (at the cathode) or a source (at the anode) of carbonate ions $\left(\mathrm{CO}_{3}\right)$. The carbonate ions travel from the cathode to the anode through the tile, thereby completing the electrical current circuit. (iii) The fuel gas at the anode and the oxidant gas at the 
cathode function as primary reactants. They are supplied to the electrode surface as dissolved species via the electrolyte. For $\mathrm{H}_{2}$, supply to the electrode/electrolyte interface can also occur via the electrode, since $\mathrm{H}_{2}$ is appreciably soluble in many metals. (iv) The anodic products $\left(\mathrm{H}_{2} \mathrm{O}, \mathrm{CO}_{2}\right)$ are transported away from the electrode surface to the electrolyte/gas interface and are desorbed into the bulk gas stream. Conversely, the anodic and cathodic reactants are absorbed from the bulk gas and transported to the electrode surface, where they react (see iii). The reaction mechanisms of oxidation and reduction, as well as the indicated transport steps, are far from completely understood, as will be discussed in Secs. 5.6 and 5.7.

Since 1976, state-of-the-art anodes have been made of nickel-chromium alloys. These show good performance in single cells but they are relatively expensive. Initially, sintering problems impeded the endurance of pure nickel anodes. 24 This problem was successfully overcome by alloying with chromium. However, the tendency of the a node to creep under compression remains a cause of performance degradation in stacks. Hence, the search for alternative anode materials and fabrication procedures continues (see Sec. 5.6).

In addition to its electrocatalytic function, the anode may be required to perform other tasks. First, it may function as an electrolyte reservoir and gas cross-over barrier. As will be discussed in greater detail below, the polarization of the porous anode is relatively insensitive to its degree of filling by electrolyte, while that of the porous cathode must be maintained within a fairly narrow range. Therefore, the anode, but not the cathode, can function as a reservoir of electrolyte for the tile. The latter needs to be replenished, as a result of $8 \mathrm{mall}$ but continuous electrolyte loss during long-term cell operation (see Sec. 5.5C). However, care is needed to prevent too rapid an electrolyte flow from the anode, which is ensured by a thin layer of small, uniform porosity fabricated into the anode at the anode-electrolyte interface; this layer is called the bubble-pressure barrier (BPB). Its primary purpose is that of preventing gas cross-over. The larger capillary-retention ability associated with smaller pores increases the capability of the liquid electrolyte to resist pressure differentials and therefore prevents direct mixing of reactant gases (cross-over) through the electrolyte in the event of matrix failure. Furthermore, the electrolyte resides preferentially in small pores. Thus, proper electrolyte distribution between the anode and the tile may be achieved and the rate of electrolyte loss from the anode is reduced.

In combination with the BPB, the anode is expected to provide major structural cell support in cell stacks, where the cathode and the very thin tile require this support.

The anode may also be required to function as a catalytic site for reforming HC fuels into usable $\mathrm{H}_{2}$ and $\mathrm{CO}$ within the anode chamber. Internal reforming would noticeably reduce the capital cost and heat rate in the overall power-plant system. To achieve this goal, one strives to have the following equilibrium reactions occur in or near the anode:

$$
\begin{aligned}
& \mathrm{CO}+3 \mathrm{H}_{2}=\mathrm{CH}_{4}+\mathrm{H}_{2} \mathrm{O}, \\
& \mathrm{CO}_{2}+4 \mathrm{H}_{2}=\mathrm{CH}_{2}+2 \mathrm{H}_{2} \mathrm{O} .
\end{aligned}
$$

(methanation or methane-steam reforming reactions)

Steam-reforming of methane and light HCs always takes place, to some degree, in a state-of-the-art anode since nickel is a catalyst for reactions (5,4A-5), (5.4A-6). Howe ver, in practice, it is necessary to reform $\mathrm{HC}$ fuel completely since $\mathrm{CH}_{4}$ is electrochemically inert. An external reformer may be used, with appreciable heat exchange to fire the strongly endothermic reforming reaction. It would be very desirable to find an electrocatalyst for $\mathrm{CH}_{4}$ ("direct fuel cell"). Alternatively, an internal reforming cell design may be adopted, in which the HC is completely reformed within the anode chamber. Considerable difficulties are still experienced in realizing such a design without gradual performance decay (Sec, 5,6E).

The MCFC cathode, which in the earliest cells was made of silver or copper, has since the late $1960 \mathrm{~s}$ been a porous nickel mass which, in the first hours of operation, is oxidized to nickel oxide and becomes lithiated. During this in situ oxidation and lithiation, the original structure changes drastically. The cathode mass acquires many very mall pores in addition to the original 5-10 $\mathrm{km}$ size pores, which causes a characteristic wetting pattern, in which very small pores are preferentially filled but all of the internal surface area is easily wetted.

Cathodes show optimum performance when the electrolyte fills the small pores such that 20-25\% of cathode-pore volume is occupied. Larger, gas-filled, pores are then wetted by thin films. Even under optimum design conditions, cathode polarization is much higher than that of the anode, indicating that oxygen reduction has slow and complicated kinetics even at $650^{\circ} \mathrm{C}$. Especially troublesome is the rapid increase in polarization at lower temperatures (Sec. 5.7A). This is an obstacle in attempts to alleviate corrosion and electrolyte problems by a lower design temperature.

Another problem associated with most cathode materials is their tendency to dissolve. Silver and copper, although kinetically very satisfactory, had to be abandoned for this reason. Lithiated nickel oxide cathodes, though far more stable than either, are not expected to last more than $10,000 \mathrm{~h}$ in pressurized operation with low-BTU coal gas as a fuel. This problem is the result of the small, but not negligible, solubility of nickel oxide in the electrolyte. A search 
for alternative cathode materials is under way. In atmospheric operation, nickel oxide cathodes may prove to be satisfactorily stable for periods approaching the $40,000 \mathrm{~h}$ goal. Therefore, in natural-gas fed MCFCB, the nickel oxide cathode is believed to be adequate.

Table 5.4A-1 provides an overview of the most important structural characteristics and dimensions of presently used cell components. In Sec. 5. 4B, the rationale for these structural characteristics will be discussed. A more detailed discussion of electrolyte tile, anode and cathode is given in Secs. 5.5-5.7.

Table 5. 4A-1. Characteristics of presently used single-cell components.

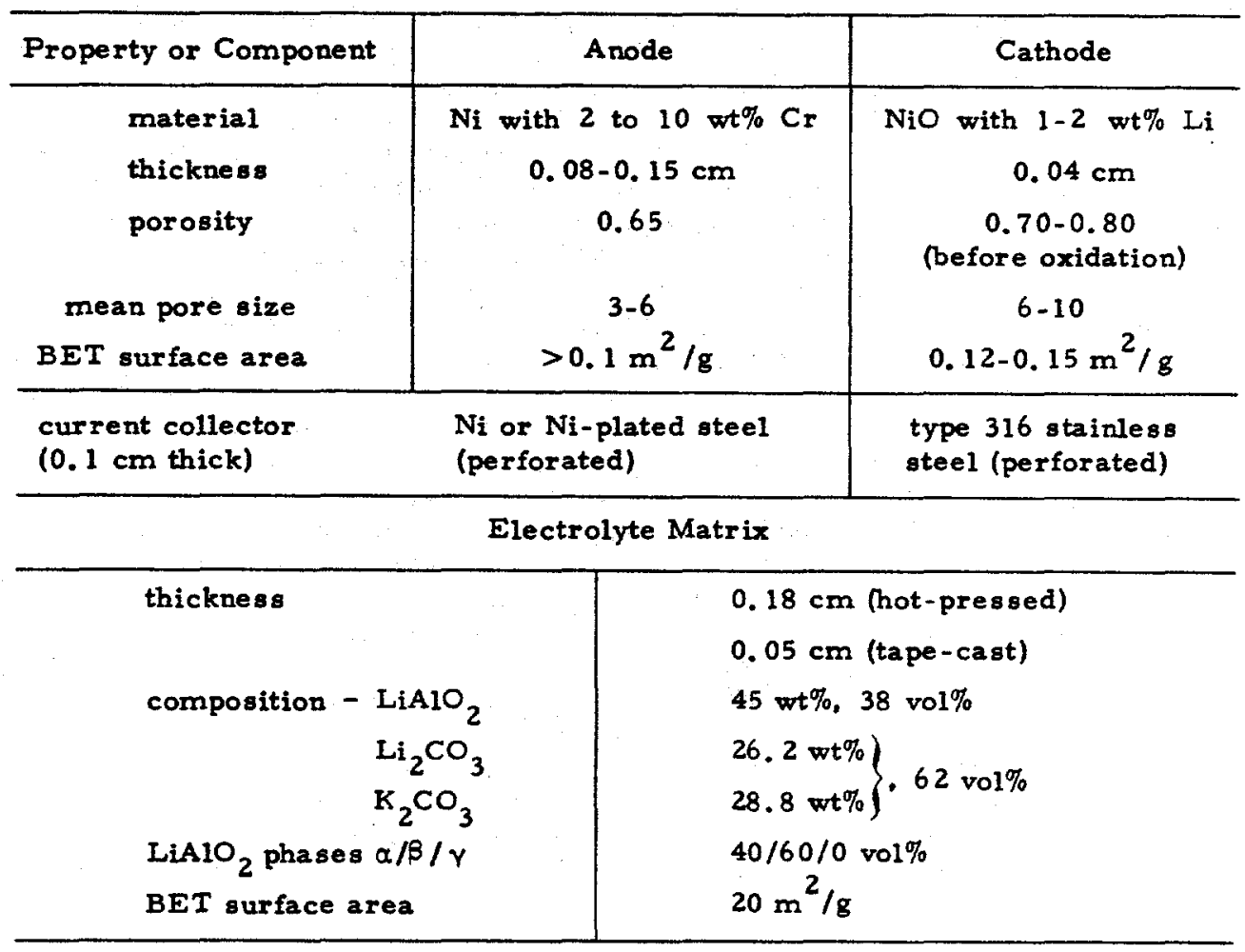

\section{B. Capillary Control of Electrolyte Distribution}

The electrode structure is one of the principal factors determining cell performance in FCs. As already noted, the electrode reaction takes place mainly near the meniscus (threephase boundary), where mass transport resistance is least for gas diffusing through the liquid to the reaction surface. Alternatively, an electrolyte film may cover the pore wall through which gas diffuses to react at the electrode (wall). Flooding of the electrode is usually deleterious to its performance, particularly for the MCFC cathode, which shows optimal performance at 20 $25 \%$ filling. The MCFC a node is less sensitive to flooding and can, in fact, be used as an electrolyte reservoir (Secs, 5.6 and 5.7).

In low-temperature $F C_{B}$, electrodes are fabricated with special provisions for gas-liquid interface control. They may have a dual-porosity structure on a hydrophobic inert binder, which forms a gas-permeable boundary for the electrolyte, as in PAFC electrodes. For the MCFC, no such electrolyte - phobic materials are known, in spite of extensive exploration. Moreover, a pre-fabricated dual-porosity structure of the Bacon FC type is not stable enough to survive the continual corrosion and sintering under operating conditions at $650^{\circ} \mathrm{C}$, especially at the cathode.

In practice, the pre-designed anode and cathode structures each evolve within a few tens of cycles toward quasi-steady-state pore-size distributions and electrolyte filling or wetting characteristics. These may be markedly different from the initial values. The tendency toward capillary equilibrium is exploited to control the electrolyte distribution by carefully matching the initial pore-size spectra of the electrodes and the electrolyte matrix.

The component porosity is adjusted in order to ensure proper electrolyte diatribution. Small pores retain liquid by capillary pressure, while larger pores are gas-filled, even though their walls may be fully or partly wetted by thin liquid films. The mean pore radius (m. $\mathrm{p}$. $r$.) of components is graded such that $r_{A}>r_{B}>r_{T}$ and $I_{C}>r_{T}$, where the subscripts $A, B, T, a n d$ $C$ refer to the gas-supplying layer of the anode, the bubble-pressure barrier, the tile, and the cathode, respectively. The m. p.r. of the cathode is usually larger than that of the anodes, although this is not a mandatory requirement. Liquid electrolyte wets chromium-stabilized nickel 
anodes to variable extents. The lithiated-NiO cathode and ceramic matrix are almost completely wetted $(\theta \sim 0)$ by the carbonate electrolyte.

Maru and Marianowski ${ }^{25}$ first applied the pore-spectrum matching technique. It is based on a composite model of the anode-tile-cathode pore structures. For each of these components, pores of all sizes are assumed to be randomly distributed and interconnected so that they are uniformly accessible. Capillary equilibrium (Fig. 5.4B-1) then dictates that

$$
p_{1}-p_{2}+h p_{g}=\left(4 \sigma_{1} \cos \theta_{1} / d_{1}\right)-\left(4 \sigma_{2} \cos \theta_{2} / d_{2}\right)
$$

If Eq. (5, 4B-1) is not satisfied, the electrolyte column will move in the direction of lower pressure until equilibrium is established. In practice, the terms on the 1.h.s. are negligibly small. Equation (5.4B-1) may be used to predict the amount of electrolyte in each of the various cell components by accounting for the pore-aize distribution in each component and maintaining a balance on the total volume of electrolyte (fixed-electrolyte capillary equilibrium model).

In Fig. 5.4B-2, the cumulative pore-volume distributions of the anode, cathode, and matrix are plotted. If the wetting angles of all three componento are $0^{\circ}$ (complete wetting), the volumes contained in all three components may be summed, as is indicated in Fig. 5.4B-2, and this sum curve intersected with the total volume of electrolyte. This procedure yields the equilibrium or limiting effective pore size, i.e., the maximum size filled by electrolyte for each component, as well as the volume of electrolyte held by each component.

The fixed-electrolyte capillary equilibrium model has been found useful in establishing a quantitative link between structural changes and performance decay. Such changes and, in particular, anode sintering, are illustrated in Figs. 5.4B-3 and 4, where the electrolyte volume fractions sum to $100 \%$ at the equilibrium or limiting effective pore size.

Arendt 26 has recast the analysis of electrolyte equilibrium distribution in terms of freeenergy minimization and included the effect of bubble-pressure barriers at the electrode-electrolyte boundary. The gas pressure pushes the liquid out of matrix pores adjacent to the electrode if they have sufficiently large effective diameters. Large interstices in the interior of the tile are shielded from the immediate effects of gas pressure by intervening small pore. However, if there is a pathway under stress with sufficiently large radii or if a crack propagates across the electrolyte structure, then the reactant gases will mix and the cell will become inoperable. Thin layers of uniform porosity are provided by the BPB and counteract gas crossover (Fig. 5, 4B-5).

If the wetting angle is greater than $0^{\circ}$ for any component, a correction must be made by using the effective pore diameter $d / \cos \theta$. Therefore, information about the wetting angle on various materials, under cell-operating conditions, is essential to predict electrolyte

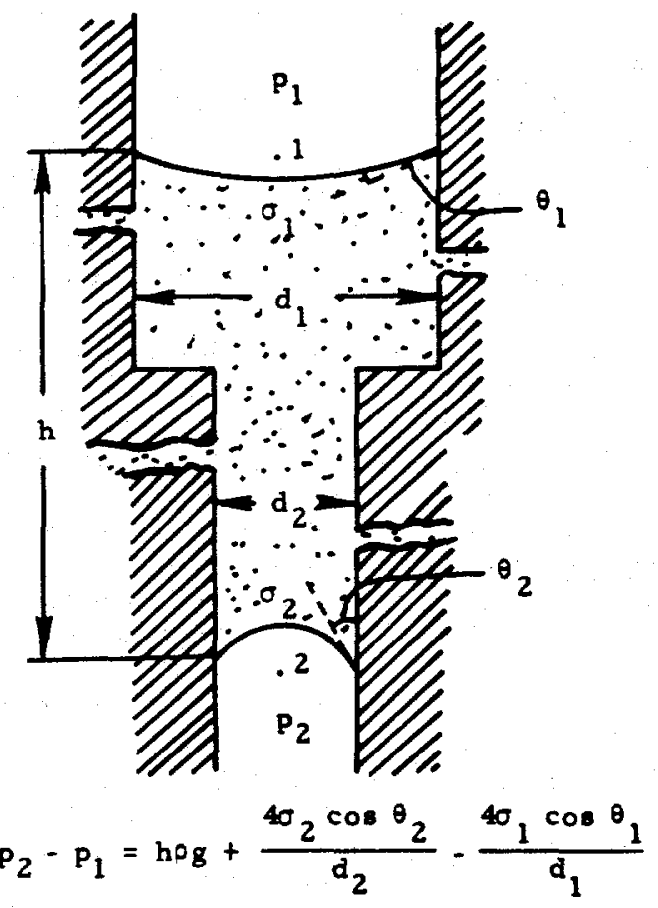

Fig. 5. 4B-1. Schematic of capillary electrolyte diatribution in the electrodes and matrix of an MCFC (from Ref. 25). 


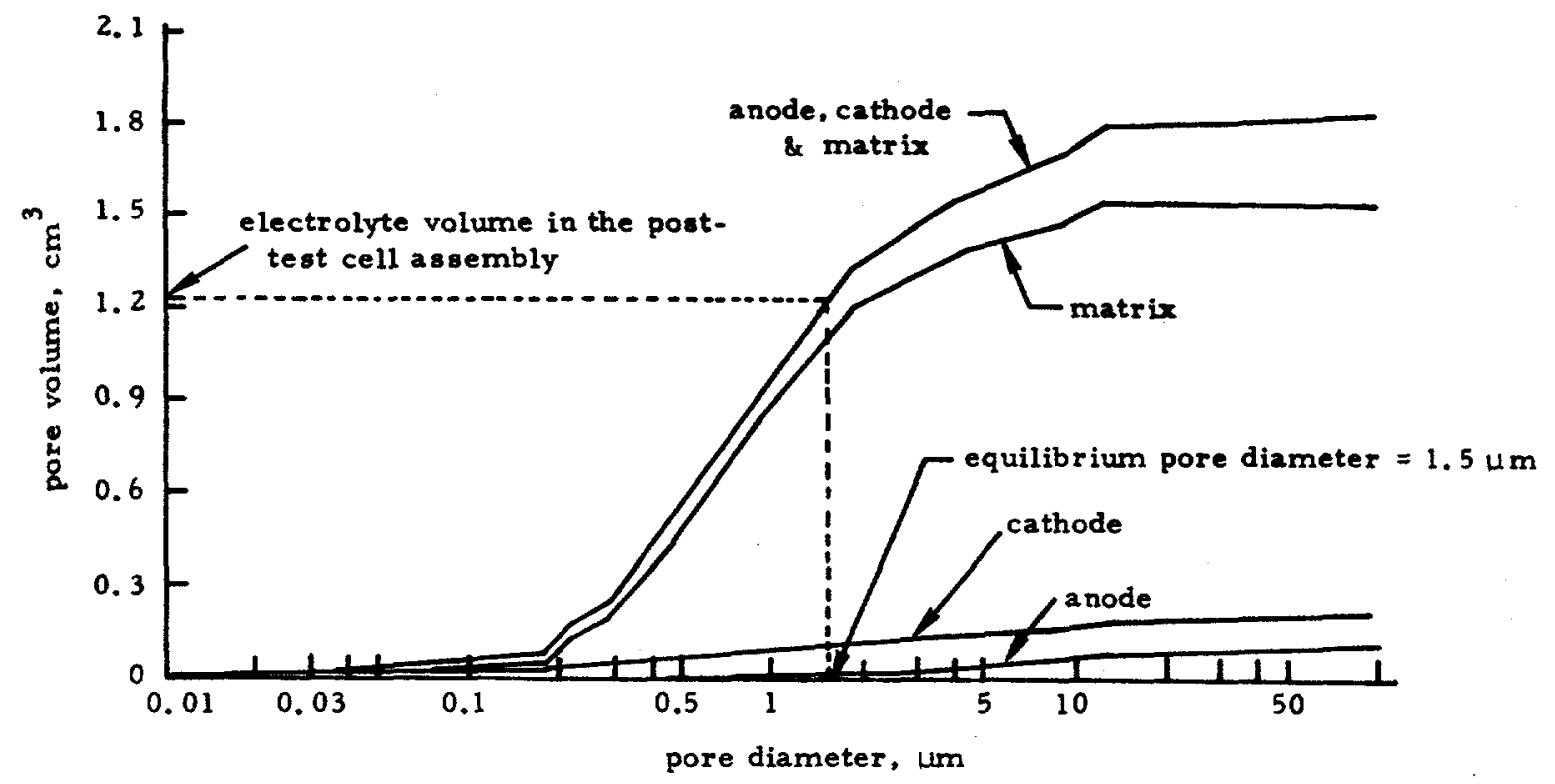

Fig. 5.4B-2. Total pore-volume distribution from the fixed-electrolyte capillary equilibrium model (from Ref. 27 ).

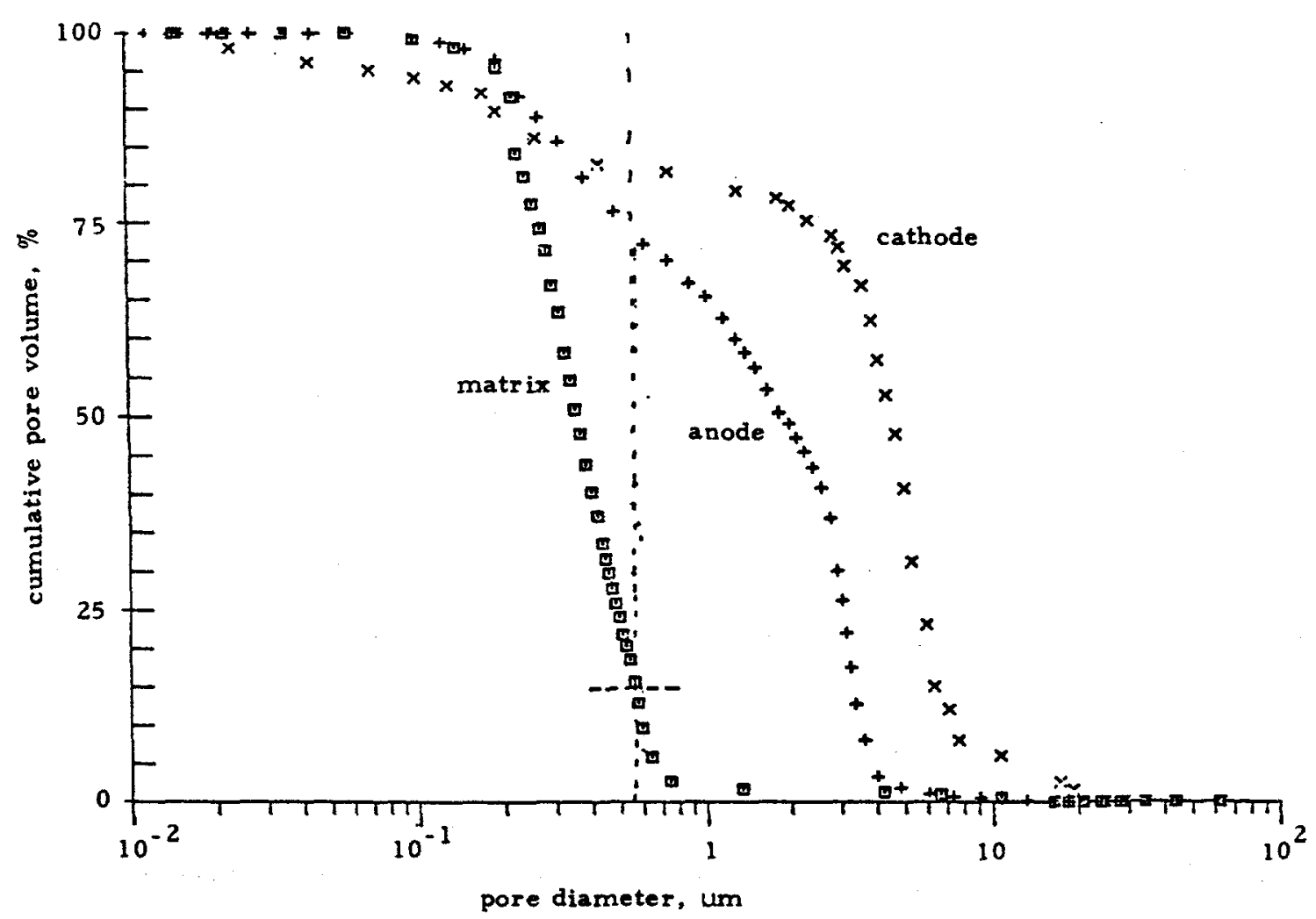

Fig. 5. 4B-3. The pore-size distributions are shown, after $360 \mathrm{~h}$ of operation, for various components of an MCFC utilizing $\mathrm{Ni}-10 \% \mathrm{Cr}$ anodes. The dotted vertical line indicates the limiting effective pore diameter. 28 


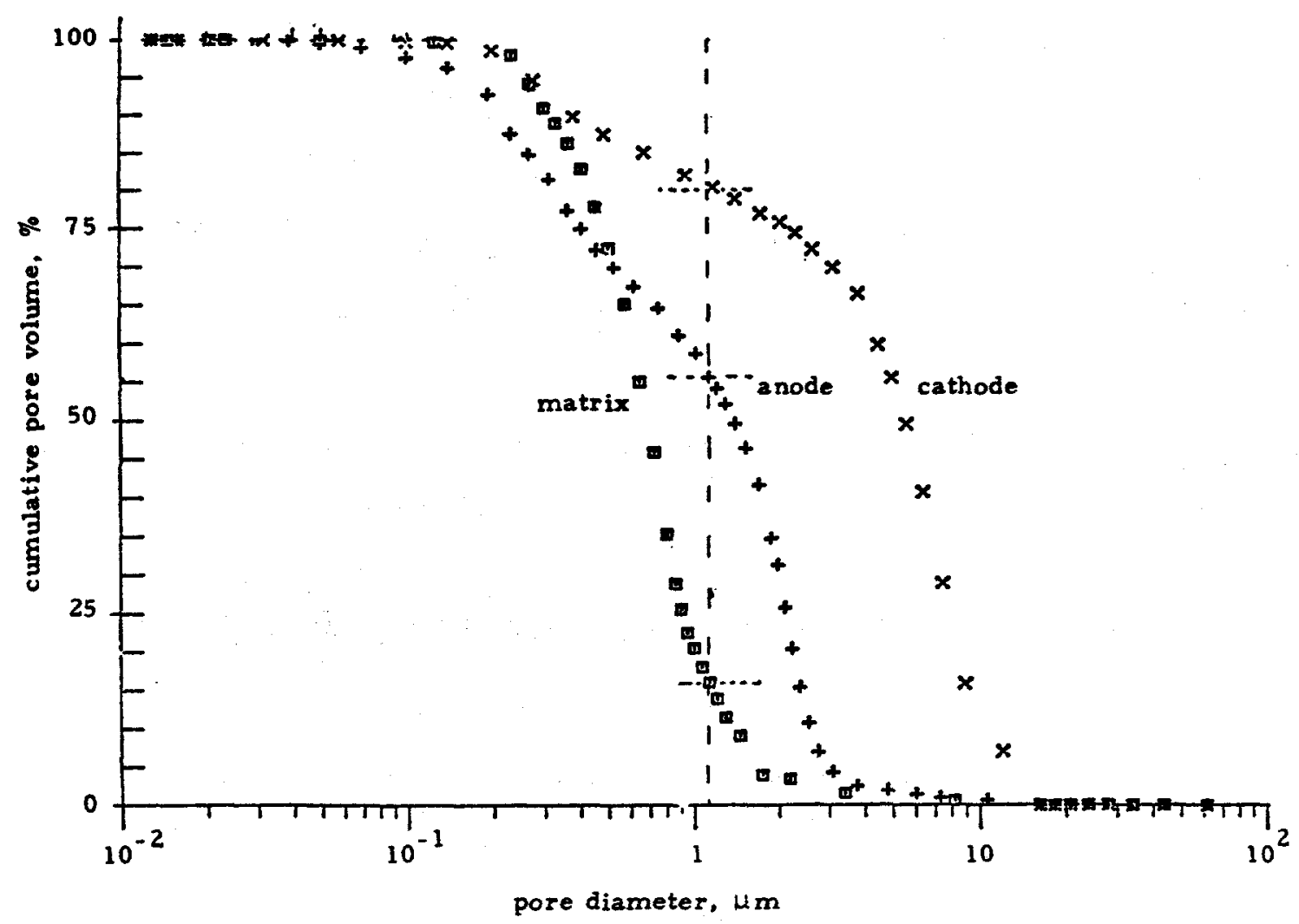

Fig. 5. 4B-4. The pore-size distributions are shown, after $1766 \mathrm{~h}$ of operation, for various components of an MCFC utilizing $\mathrm{Ni}-10 \% \mathrm{Cr}$ anodes. 28

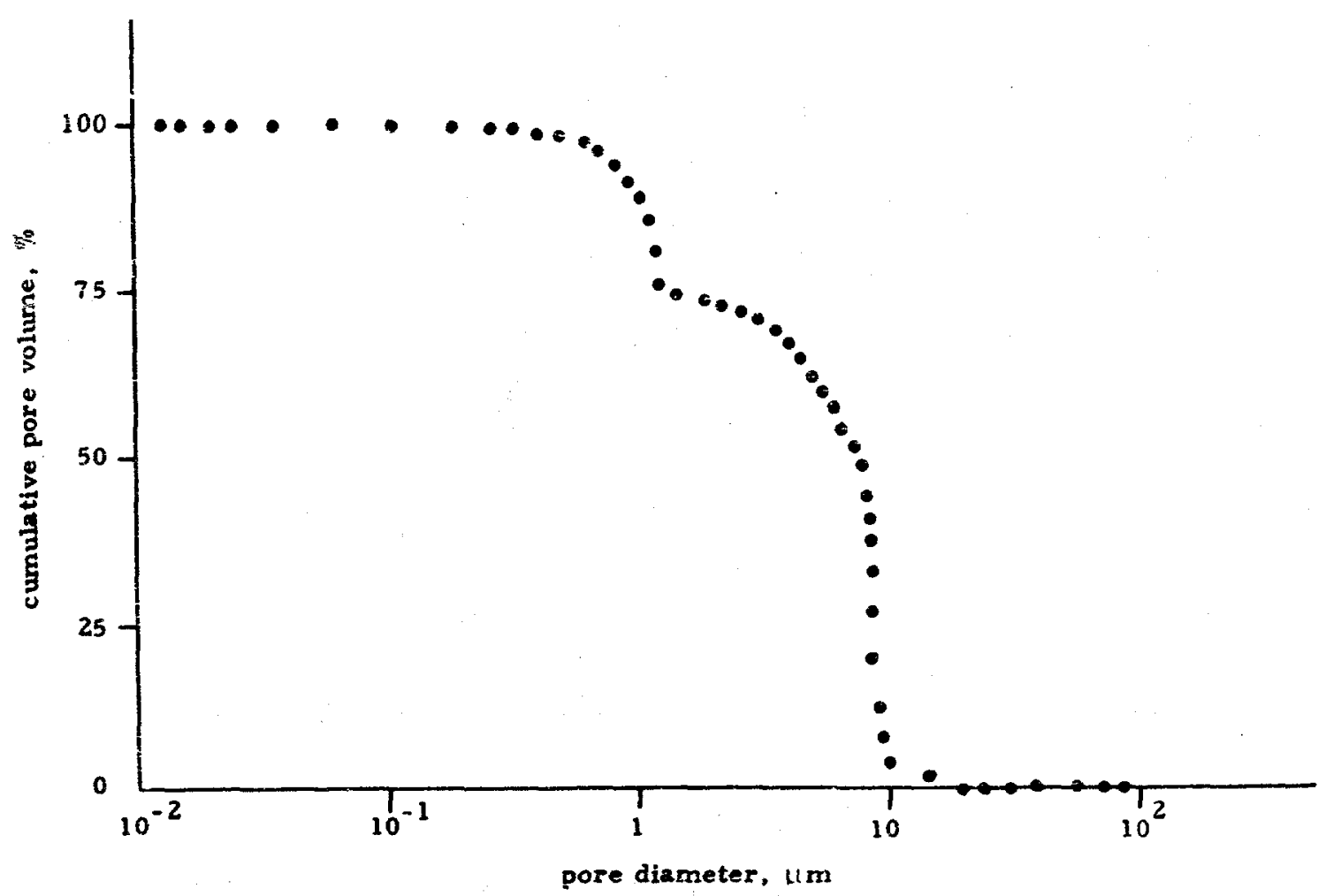

Fig. 5. 4B-5. The pore-size distribution is shown for a plated $\mathrm{SrTiO}_{3}$ anodes, which has a BPB. ${ }^{28}$ 
distribution accurately. This information is at present largely missing (see Sec. 5.5A). Effective wetting-angle estimates are being used, which are poorly reproducible fron one cell to the next. It is clearly essential to obtain better insight into the conditions which control interface movements, auch as impurity content of the electrolyte and gradients of concentration or potential.

\subsection{Electrolyte Tile (or Matrix)}

\section{A. Function and Composition}

The electrolyte tile or matrix has a dual function: (1) to conduct ionic current $\left(\mathrm{CO}_{3}=\right.$ ions) with a minimum of resistance from cathode to anode; (2) to separate the fuel and oxidant gases with a minimum of cross-over through voids or by diffusion of the dissolved gases. These goals are, to some extent, contradictory since the separation function requires thickness and $s$ tiffness, while ionic conduction is optimal in thin, fluid tiles. The thickness and characteristics listed in Table 5. 4A-1 represent a compromise for the currently used $\mathrm{Li}_{2} \mathrm{CO}_{3}-\mathrm{K}_{2} \mathrm{CO}_{3}$ electrolyte with $\mathrm{LiAlO}_{2}$ ceramic.

This electrolyte composition, although certainly favorable, is by no means optimized for electrode performance. If other compositions are selected, different tile (matrix) formulations and thicknesses may be required. Of principal concern is the behavior of the electrolyte that is in contact with the porous electrodes or other porous cell and stack components. Capillary equilibrium, as described in Sec. 5.4B, requires a redistribution of electrolyte in situ at the cell-operating temperature, with consequent changes in the degree of filling of the tile, as well as of the electrodes.

The composition and distribution of liquid electrolyte are of critical importance in determining MCFC cell and stack performance. Optimum cell output is obtained only if the electrolyte is properly distributed and if the composition is carefully tailored to yield the highe at performance.

\section{B. Electrolyte Optimization}

The electrolyte composition affects cell performance in various ways: (1) via tile resistance, which depends on the ionic conductivity; (2) via the polarization of the electrodes, which depends primarily on the electrode kinetics of the reactions (discussed in Secs. 5.6A and 5.7 A), as well as the solubility and diffusivity of the dissolved gases $\left(\mathrm{H}_{2}, \mathrm{CO}_{1} \mathrm{CO}_{2}, \mathrm{O}_{2}\right)$ in the electrolyte; (3) via the distribution of electrolyte among tile and electrodes, which depenis, as discussed in Sec. 5.4B, on its capillary properties such as surface tension and, especially, the wetting angles of the electrolyte with the electrode substrates and other cell materials.

The actual interaction of these various factors in determining the cell IR-drop and polarization is quite complicated. Attempts have been made to analyze this problem quantitatively, starting from available information on physical and transport properties, electrode kinetics, etc. 29,30 These attempts have had only partial success. For example, from conductivity and gas solubility trends, which are relatively well-known, qualitative predictions could be made about favorable composition ranges.

$A s$ is shown in $\mathrm{Fig} .5 .5 \mathrm{~B}-1$, high $\mathrm{Li}^{+}$and $\mathrm{Na}^{+}$contents increase conductivity while high $\mathrm{K}^{+}$content promotes gas solubility. These trends at $600^{\circ} \mathrm{C}$ are expected to be maintained at $650^{\circ} \mathrm{C}$. In fact, measurements with small $3-\mathrm{cm}^{2}$ cells of standardized dimensions and electrode structur $\mathrm{e}^{31}$ have suggested that higher $\mathrm{Li}^{+}$content may be desirable and that $\mathrm{Na}^{+}$addition would not necessarily decrease the performance (Fig. 5.5B-2). These results are not completely under stood and underline the fact that more fundamental information is needed for a rational optimization of the electrolyte composition. Additives other than alkali carbonates may very well be beneficial.

A large part of the information needed concerns the primary physicochemical properties mentioned at the beginning: ionic conductivity, gas solubility and diffusivity, surface tension and wetting angles on various substrates.

Selman and Maru 21 reviewed the availability of these and other properties. There are serious gaps, because of absent or contradictory data, in our knowledge of dissolved gas solubility and diffusivity and in the wetting of metals and ceramics by carbonate melts (especially as affected by gas composition, contaminants, and applied potentials). Ionic mobilities and viscosities, which correlate with dissolved gas diffusivities, are also very poorly known. In general, even when reliable data for a limited number of compositions are available, there is a complete absence of tested rules to predict properties at other compositions or with different components. It is, therefore, necessary to pay increased attention to measurement and prediction of transport and capillary properties of molten carbonates and, by extension, of molten salt mixtures in general. This procedure is an essential condition for optimization of the electrolyte with respect to cell performance. 


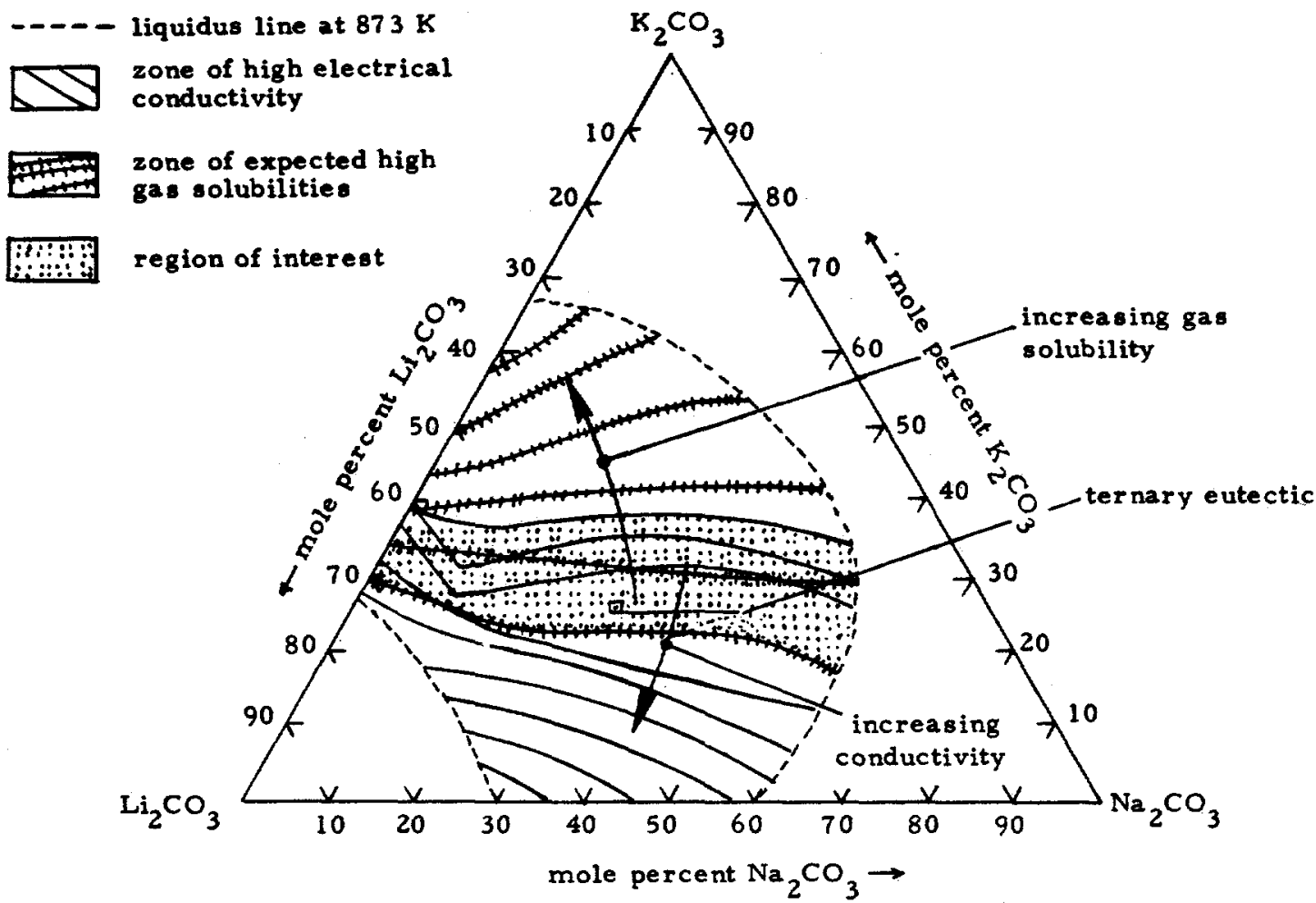

Fig. 5,5B-1. Ionic conductivity and gas solubility trends are shown for various alkali-carbonate mixtures at $600^{\circ} \mathrm{C} .29$

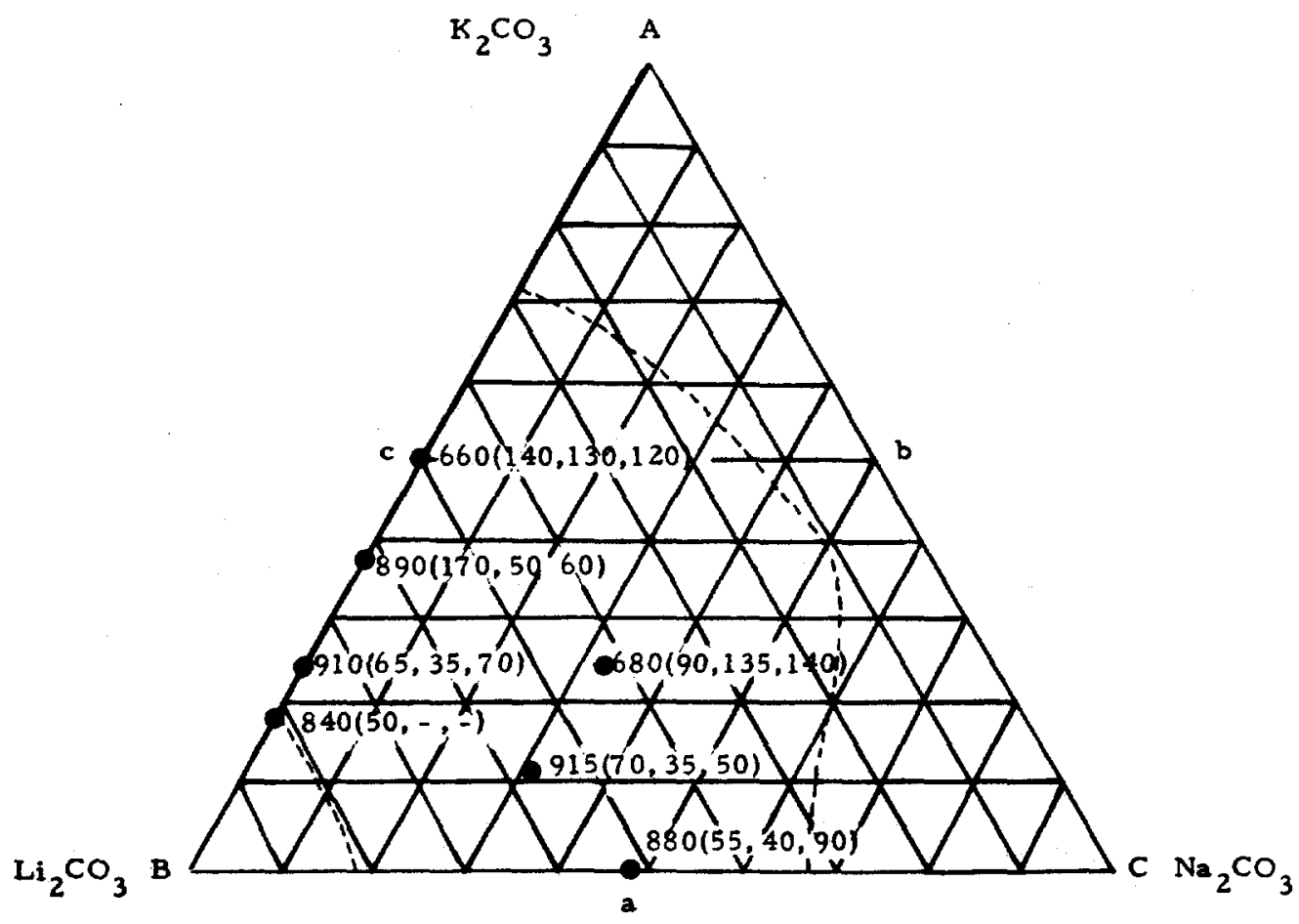

Fig. 5.5B-2. Performance data are shown for cells utilizing various electrolyte compositions and operating at $160 \mathrm{~mA} / \mathrm{cm}^{2}, 650^{\circ} \mathrm{C}$. The numerical entries in $\mathrm{mV}$ refer, respectively, to cell potential (resistance drop, anode polarization, cathode polarization). 31 
Electrolyte optimization is also a key to MCFC lifetime. The long-term performance decay or endurance depends also on electrolyte properties. These do not coincide with the indicated, polarization-controlling, properties.

The following properties are involved: (1) the hydroxide vapor pressure in the presence of exit fuel gas, containing large amounts of $\mathrm{H}_{2} \mathrm{O}$. This has been shown to be the primary factor in electrolyte 1088 by volatilization (see Sec. $5.5 \mathrm{D}$ ). (2) The corrosion rate of cell materials by interaction with electrolytes in the presence of $\mathrm{H}_{2} \mathrm{O}$ and $\mathrm{CO}_{2}$, in particular by formation of lithium compounds. This is another mechanism of electrolyte loss. The corrosion rate is strongly dependent on electrolyte composition via hydrolysis equilibria and oxygen reduction kinetics. (3) The ionic mobilities of the cations as a function of electrolyte composition. The relative mobilities determine the migrational separation of cations in a cell under load, 32 i. e., the degree to which, for example, $\mathrm{Li}^{+}$accumulates preferentially near the positive electrode and $\mathrm{K}^{+}$ near the negative electrode. In single cells, these migration effects are slight; howe ver, in cell stacks, they lead to overall electrolyte displacement and accelerate loss by volatilization, as will be discussed in Sec. 5.8 in more datail. (4) The solubility and diffusivity of nickel and other, alternative, cathode materials in the electrolyte. These properties, which are strongly composition-dependent, determine the slow deterioration of cell performance caused by dissolution of cathode material and its reprecipitation at some point in the electrolyte near the anode (see Sec. 5.7B). Also related to this is cross-over by diffusion of dissolved gas. At present, there is no theory which predicts reliably how the solubilities and diffusivities of such minor solutes depend on composition and temperature.

Electrolyte optimization is, therefore, not a simple matter and may require different compositions, depending on stack-as well as cell-design. In any case, expanded research of fundamental physico-chemical properties on molten carbonate mixtures and related melts is clearly imperative.

\section{Tile (Matrix) Fabrication}

Early fuel cells used tiles constructed by hot-pressing the proper amounts of alkalicarbonate and $\mathrm{LiAlO}_{2}$ at temperatures just below the liquidus point of the carbonate. Hot-pressing is a discontinuous and expensive process and the need for thinner, stronger tiles meant searching for a better fabrication process. Hot-pressed tiles are typically $\sim 70-80$ mils thick; they can be made thinner ( $35 \mathrm{mil})$, but at augmented costs. Also, conductivity studies on hotpressed matrices have st: $n$ that gas-filled pores exist within the tilf itructure.

Rscuic work at IGI, ERC and UTC has demonstrated the advartiges of tape-cast $\mathrm{LiAlO}_{2}$ matrices. Very thin matrices can be made continuously and economically. Typically, the slip composition contains one part of $\gamma$-LiAlO 2 particles $\left(0.2-0.5 \mu \mathrm{m}\right.$ dia 3 ter, $10 \mathrm{~m}^{2} / \mathrm{g}$ surface area) for each 1 to 1.5 parta of binder. 33 This procedure produces $\pi$ rices that are 0.03 $0.07 \mathrm{sm}$ thici and $45-55 \%$ porous, with a mean pore size of $0.5 \mathrm{um}$. porosity is sufficiently uniform to lessen the need ior gas barriers such as BPBs, even when trin matrices are used. However, graded matrix or matrix-anode structures are still desirable to add strength. Coarse particles may be added to minimize crack propagation (crack arrestor . .

Tape-cast tiles have performed very well, both under constant-semperature load tests and in thermal cycling. Data from IGI ${ }^{34}$ show that ohmic losses in tape-cast matrices for various liquid electrolyte loadings ars much smaller than for hot-pressed tiles (see Fig. 5.5C-1). The cell performance shown in Fig. $5.5 \mathrm{C}-2$ for tape-cast matrices is mucl. better, over a fair range of currents and voltages, than for hot-pressed tiles. 34

The performance of matrices deposited by electrophoresis is similar to that of the tapezast matrices, except that cell design becomes some what more difficult with electrophoretically deposited layers. Also, adhesion to the electrodes may be poor, which in tape casting can be overcome by graded tape-casting of the composite structure.

Cells with tape-cast matrices of $10 \mathrm{~cm} \times 10 \mathrm{~cm}$, which included $\mathrm{BPBs}$ of small $\mathrm{LiAlO}_{2}$ particles on the anode side, were tested for thermal-cycling stability and did not produce cracke during five cycles in $850 \mathrm{~h}, 35$ However, 28 mentioned, BPBs have more recently been found unuecessary in tape-cast structures if sufficient crack-arresting features are included.

Before fuel-cell research and development were discontinued at GE, a hot-roll milling process was under development, which did not use solvents but included high binder content. 28 in the GE coated-particle, hot-roll milling procedure, the electrolyte mixture (typicaliy, $45 \%$ by weignt of $\mathrm{LiAlO}_{2}$ particles and $55 \%$ by weight of a $62 / 38 \mathrm{~mole}-\mathrm{ratio}$ of a lithium/potassium carbonate blend) is heated $\left(550^{\circ} \mathrm{C}\right)$ and a binder is added; the mixture is then run through rollers with adjustable spacings at $110^{\circ} \mathrm{C}$. Figure $5.5 \mathrm{C}-3$ shows that, under certain conditions, hot-rollmilled, coated tiles perform better than hot-pressed tiles. However, it appears that this procedure has not been adopted by other deveiopers, possibly because complete binder burn-out may be difficult to accomplish. Tape-casting seems to emerge 23 the most efficient method of tile and cell fabrication for stacks. 


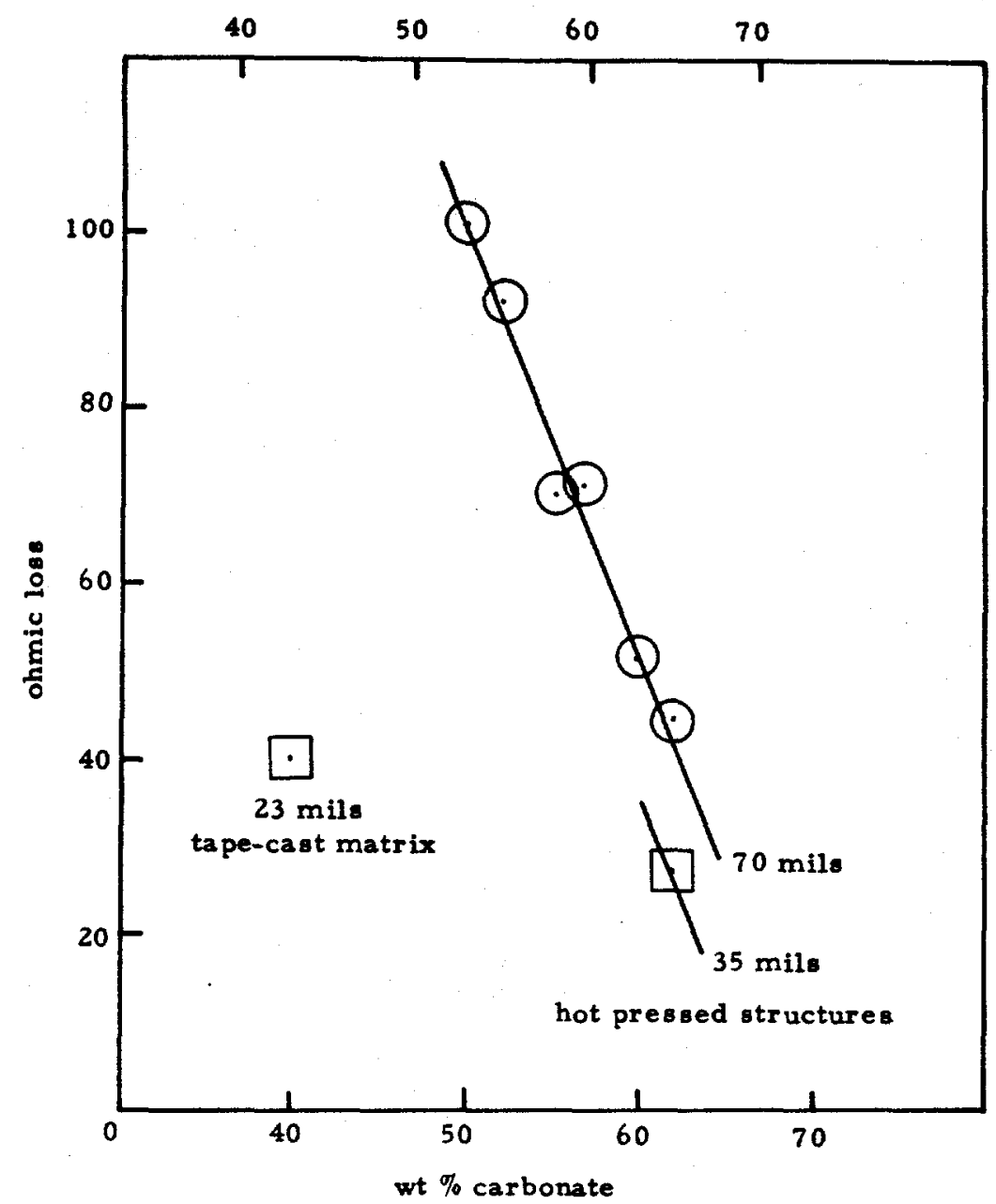

Fig. 5.5C-1. The ohmic losses are shown, as a function of electrolyte loading, for cells utilizing matrices fabricated by tape casting and hot pressing; $650^{\circ} \mathrm{C}, 160 \mathrm{~mA} / \mathrm{cm}^{2} .34$

\section{Electrolyte Management}

Long-term stability of cell and stack performance is, to a large extent, dependent on limiting electrolyte $108 \mathrm{~s}$ by corrosion and volatilization. Since this connection was recognized early on, it has been investigated fairly thoroughly. $31,36,37$

Corrosion $108 \mathrm{~s}$ is largely limited to the first $2000 \mathrm{~h}$ of operation. This is illustrated in Fig. 5.5D-1, which also shows that loss by vaporization is a slow but continuing process. Its rate is relatively independent of the particular fabrication process used in preparing the electrolyte matrix.

The continuous electrolyte 1088 in ambient-pressure cells is of the order of $10^{-9} \mathrm{~g}$ of electrolyte per $\mathrm{cm}^{3} \mathrm{gas}$ flowing. In cells without storage, it causes an overall performance decay of $8-10 \mathrm{mV} / 1000 \mathrm{~h}$. Most of this decay is due to increase of ohmic resistance of the tile. Typically, the resistance increases $10 \%$ per $1000 \mathrm{~h}$, but eventually both ohmic resistance and polarization increase very rapidly as the electrolyte becomes maldistributed among tile and electrodes. For most cells operating at atmospheric pressure, this fact limits useful life to $10,000 \mathrm{~h}$, unless excess electrolyte is stored or added. Electrolyte storage is a solution to the electrolyte-10ss problem; however, the stored electrolyte may cause flooding of the electrodes or additional creepage and corrosion, as discussed below.

Electrolyte loss by volatilization takes place in two steps: (1) electrolyte in the porous electrodes forms volatile hydroxides upon contact with fuel gas and, to a much lesser extent, oxidant gas; (2) the loss electrolyte is replaced by electrolyte wicking from the tile, which thereby may become deficient in electrolyte. Step (1), in particular, has been investigated, both theoretically and experimentally, by in-cell and out-of-cell measurements.

Thermogravimetric studies at IGT 38 have established the dominant loss mechanisms and loss rates as functions of temperature, pressure, gas composition, and carbonate composition. 


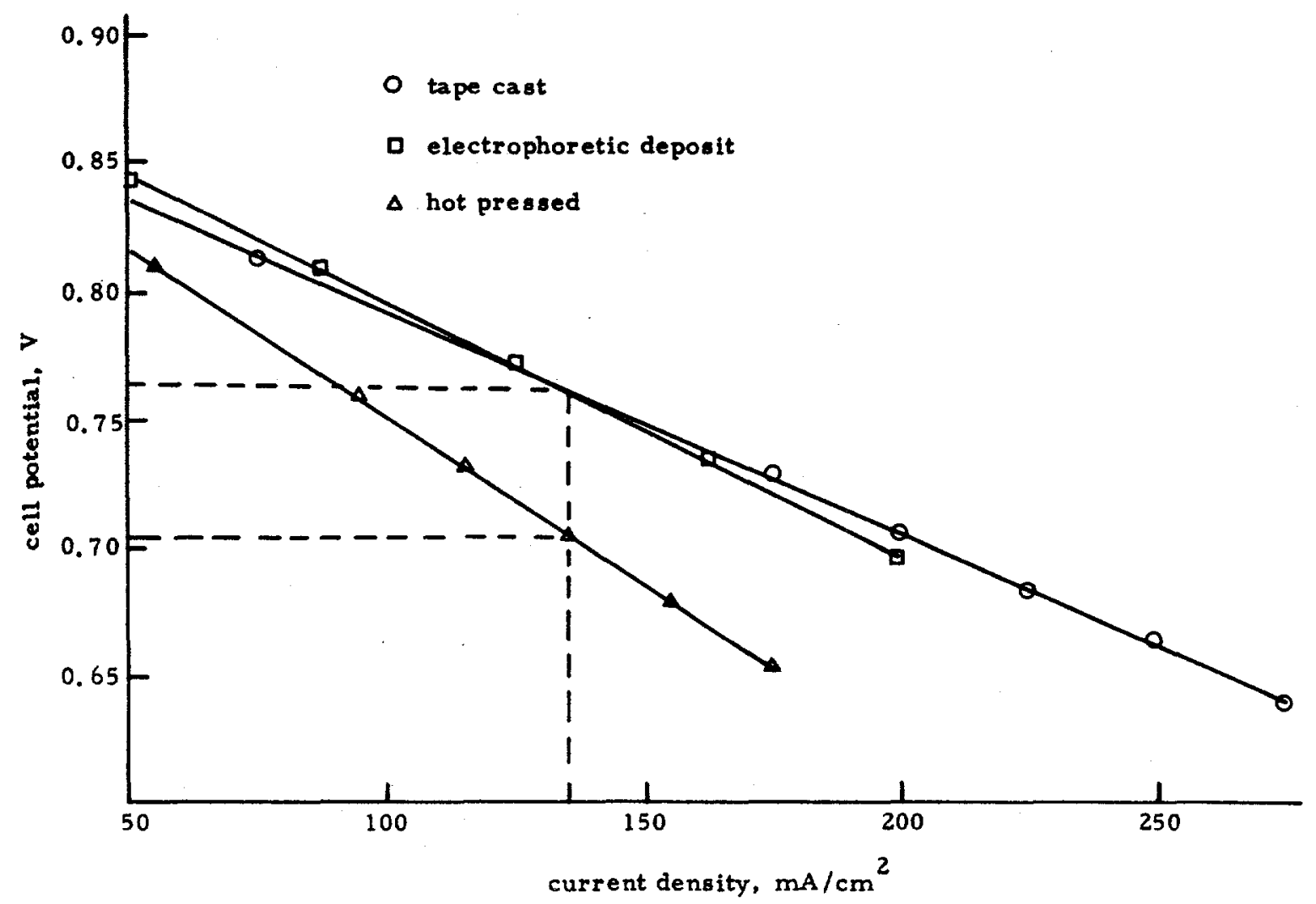

Fig. 5.5C-2. Cell performances (mV) are plotted vs cell current density for cells using tiles fabricated by various techniques. The cells operated at $650^{\circ} \mathrm{C} ; 75 \%$ of the fuel gas $\left(62 \% \mathrm{H}_{2}, 11 \% \mathrm{CO}, 7 \% \mathrm{CO}_{2}, 20 \% \mathrm{H}_{2} \mathrm{O}\right)$ was utilized and $50 \%$ of the oxidant gas $\left(75 \%\right.$ air, $\left.25 \% \mathrm{CO}_{2}\right)$ was utilized. 33

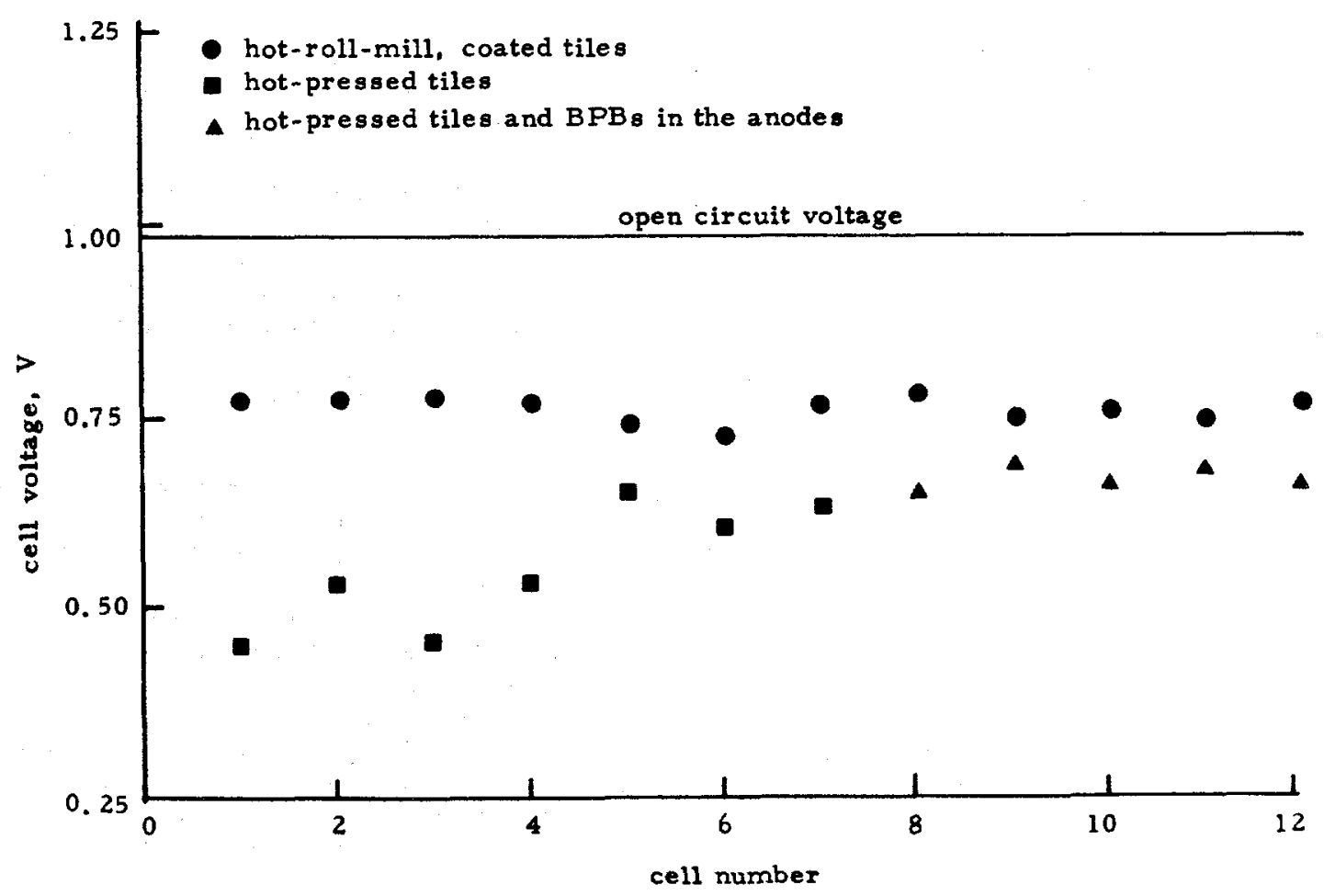

Fig. 5.5C-3. Cell performances (V) at $160 \mathrm{~A} / \mathrm{ft}^{2}$ are shown for cells with hot-roll-milled, coated tiles, hot-pressed tiles, and for cells with hot-pressed tiles with BPBs in the anodes; $75 \%$ of the fuel and $50 \%$ of the oxidant are utilized. 28 


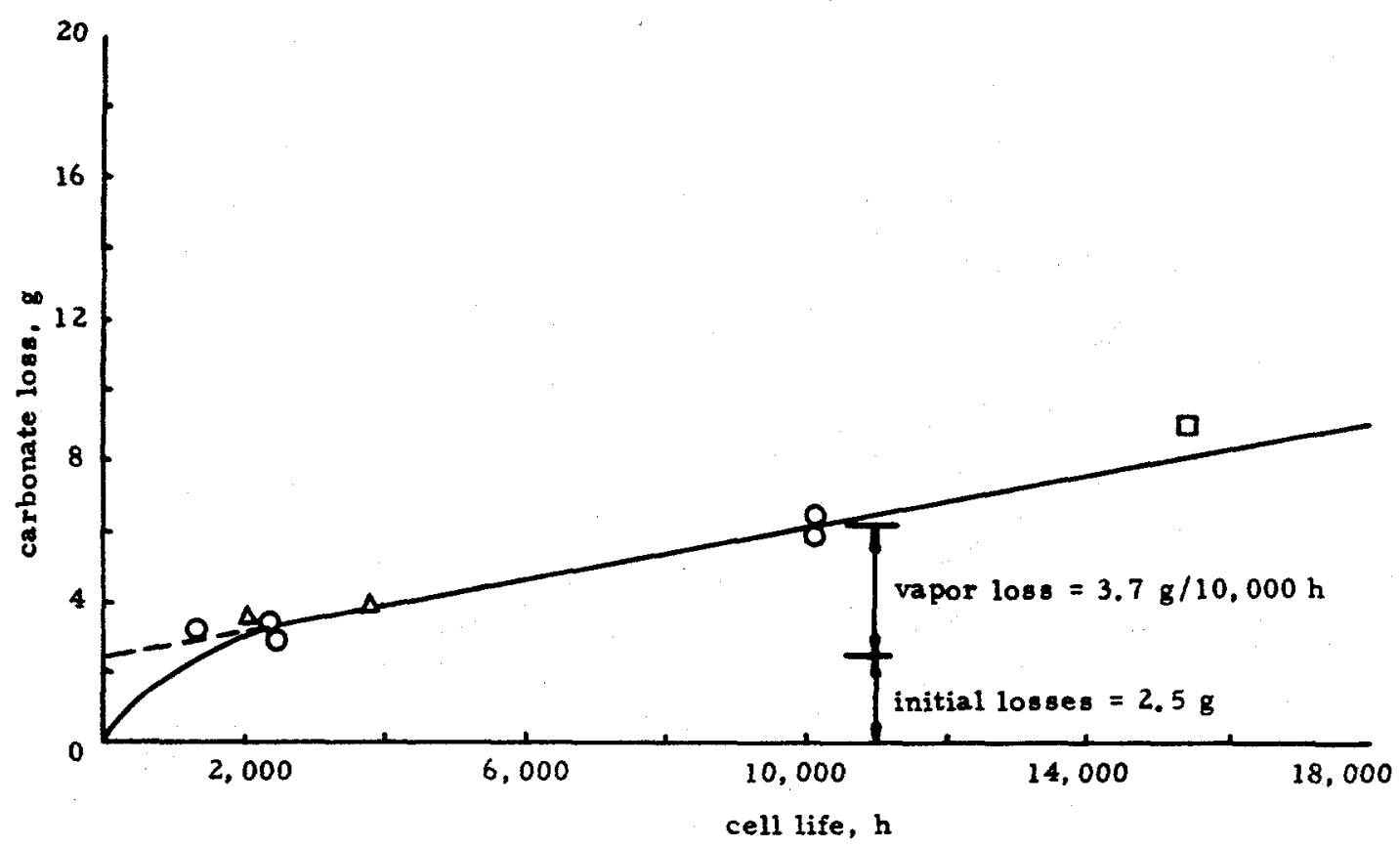

Fig. 5.5D-1. Carbonate loss from a $94-\mathrm{cm}^{2}$ cell at atmospheric pressure for various matrix fabrication procedures. 42 The symbols have the following meaning: $O, K$-free aqueous slurry process; $\Delta$, spraydrying process; $\square$, high-temperature multifiring process.

The experimental results for oxidant gas conditions agree well with thermodynamic equilibrium calculations for the vaporization of $\mathrm{KOH}$ formed by reaction of $\mathrm{K}_{2} \mathrm{CO}_{3}$ in the electrolyte with water vapor in the gas. The measured vapor losses in a fuel-gas environment were significantly greater than predicted by $\mathrm{KOH}$ equilibrium vaporization. The out-of-cell vaporization los rates agreed quite well with in-cell $108 s e s$ determined in $10,000-\mathrm{h}$ cell tests.

Vapor $108 \mathrm{~s}$ measurements at pressures of $1-10 \mathrm{~atm}^{38}$ have shown that pressurized operation has a significant effect on reducing vaporization rates (Fig. 5.5-D2). Under oxidant gas conditions, $108 s$ rates decrease approximately according to a $P^{-1}$ relation, in agreement with a $K O H$ vaporization mechanism. An even greater decrease with pressure was found for the loss rate in fuel-gas environment. These findings are encouraging, for they suggest that, in pressurized stack operation, electrolyte storage need be only a fraction of that necessary in ambient cells.

Theoretical studies have also led to the conclusion that a partially $\mathrm{Na}^{+}$-containing composition would reduce volatilization. 38 However, out-of-cell and in-cell data are required to confirm this statement.

As indicated above, agreement between theory and out-of-cell data is unsatisfactory for electrolyte los 8 under fuel-gas conditions. Other contradictory observations need to be explained: workers at UTC found that the exit gases were not saturated with alkali compounds, as would be expected if evaporation were a rate-limiting otep. 39 The role of $\mathrm{Li}^{+}$is not clear; $\mathrm{LiOH}$ should be volatilized preferentially, according to some in-cell data, but this is not confirmed by thermodynamic analyses.

Quantitative analysis of $\mathrm{Li}_{2} \mathrm{CO}_{3} \mathrm{los} 8$ is complicated by the initial electrolyte 1088 , which appears to be due largely to corrosion of stainless steel parts, with the formation of $\mathrm{LiCr}_{2}$ or of $\mathrm{LiAlO}_{2}$ where aluminized steel is used, 28 in wet-seal areas. The mechanism of wet-seal corroaion is now fairly well underatood. 40 It may lead not only to a large initial $\mathrm{Li}_{2} \mathrm{CO}_{3}$ los $\mathrm{s}$ but also a slow continuing loss by creepage if the wet seal (Fig. 5.5D-3) contacts an oxidizing ambient while an electrolyte film spreads on the cell exterior. Electrolyte leakage through the wet seal occurs because the ambient $\mathrm{O}_{2}$ which surrounds the cell is reduced to form $\operatorname{CO} \overline{3}$ ions on the outer surface of the cell (Fig. 5. 5D-4). More $\mathrm{CO}_{3}^{=}$ions are produced on the anode than on the cathode because the anode is at a lower potential. ${ }^{3}$ Some of the $\mathrm{CO}_{3}=$ ions migrate and react at the anode. The remainder of the charge necessary for neutrality is transferred by the migration of alkali cations to the outer surface of the cell. The area for volatilization thereby may become much larger and a continuous loss of electrolyte may develop. In stacks, this process is driven by large potential gradients (see Sec. 5.8C).

The mechanism of corrosion of stainless steel by molten carbonate, in general, has come under systematic investigation 41 because of its importance for separator material selection in stacks. Nevertheless, the role of the electrolyte chemistry in corrosion, for example, the effect 


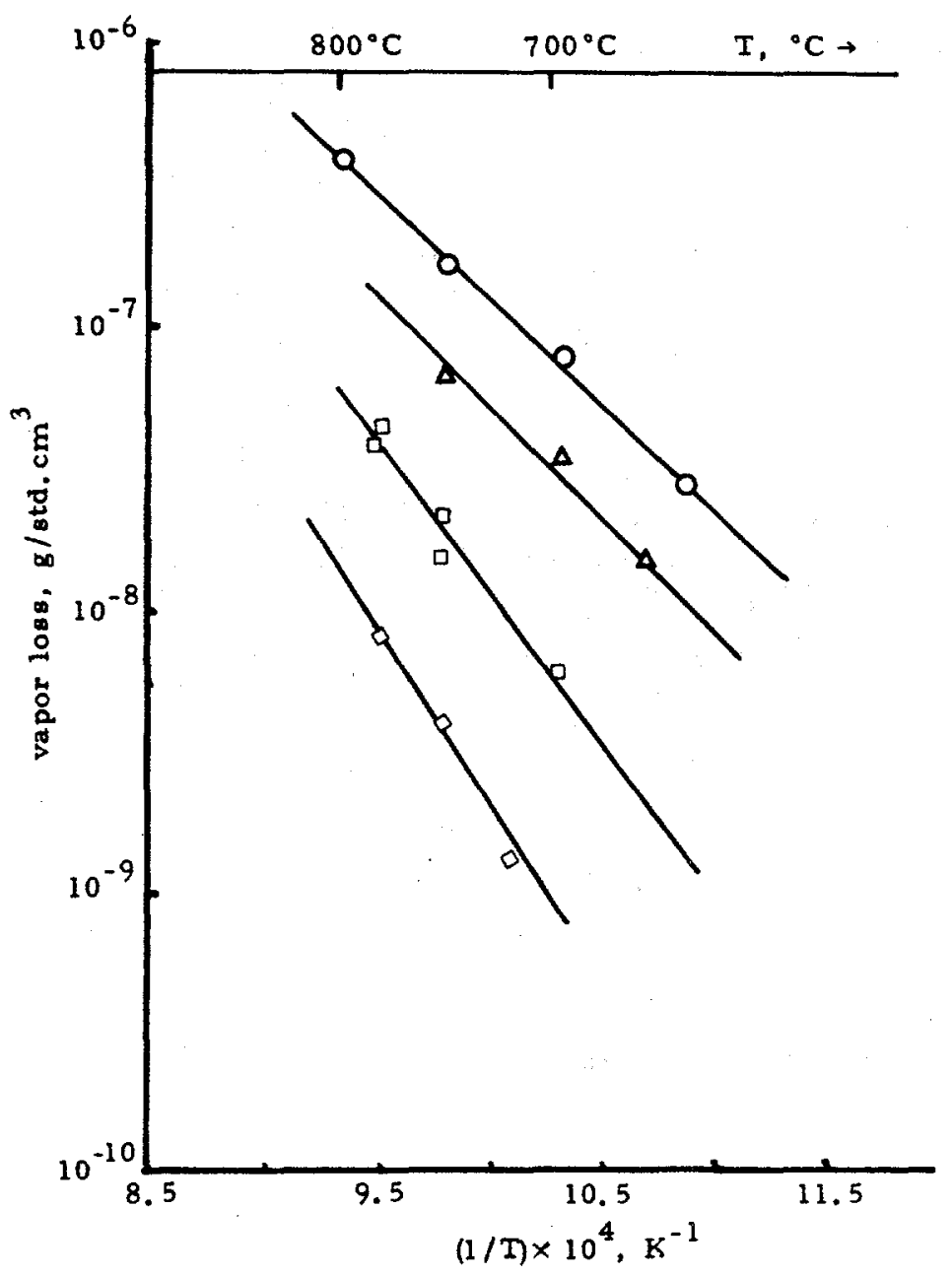

Fig. 5.5D-2. Vapor 1088 for $62 \% \mathrm{Li}_{2} \mathrm{CO}_{3} / 38 \% \mathrm{~K}_{2} \mathrm{CO}_{3}$ under fuel environment at different pressures. 38 The nominal gas composition at $1.0 \mathrm{~atm}$ is $22 \% \mathrm{H}_{2}, 13 \% \mathrm{H}_{2} \mathrm{O}$, $5 \% \mathrm{CO}_{2}, 4 \% \mathrm{CO}, 56 \% \mathrm{~N}_{2}$; at elevated pressure, it is $19 \% \mathrm{H}_{2}, 12 \% \mathrm{H}_{2} \mathrm{O}, 4 \% \mathrm{CO}_{2}, 4 \% \mathrm{CO}, 61 \% \mathrm{~N}_{2}$. The numbers on the curves show the pressures in atm.

of its cationic composition and hydroxide activity, is virtually unexplored. Workers at UTC found that cells constructed from corrosion-resistant material showed relatively smaller increases in cell resistance. This favorable characteristic might be due to decreased electrolyte los $\mathrm{s}$ by corrosion, as well as recurrence of corrosion products with more favorable properties. It is clear that fundamental investigation of alloy corrosion by molten carbonates is necessary to develop a better understanding of cell performance and endurance.

Continuous electrolyte loss by volatilization involves the wicking of electrolyte from the tile to the electrodes or to the wet seal. This process is made possible by changes in the $\mathrm{LiAlO}_{2}$ matrix structure during long-term operation. Recrystallization of $\mathrm{LiAlO}_{2}$ occurs as the result of its low but finite solubility in the electrolyte. This recrystallization is often accompanied by phase transformation if the initial, high-surface area, matrix consisted of an $\alpha$ or $\beta$ phase rather than the stable $Y$ phase (Ref. 21, pp. $352 \mathrm{ff}$.). This Ostwald ripening process leads to a continual decrease in internal surface area (Fig. 5.5D-5) and capillary tension (holding powe $r$ ) of the tile and thus causes voids (Fig. 5.5D-6) and increased IR drop, as well as polarization. 42,44

Electrolyte 1088 is also responsible for tile shrinkage. 44 As gas replaces liquid, gaps may be created between the electrodes and the matrix of internal bubbles may form. As more electrolyte is lost, the matrix becomes more extensively compacted and less effective. At UTC, gaps between components were shown to exiat when the cells were analyzed by scanning electron microscope. The growth of these gaps was caused by electrolyte loss via corrosion and was responsible for increasing cell resistance. 44

By starting from a sufficiently high initial tile area in the stable $\gamma$ phase, it appears now possible to guarantee enough carbonate retention that lifetimes of $25,000-40,000 \mathrm{~h}$ are possible for pressurized cell stacks. Of course, this statement also presupposes absence of micro-cracks 


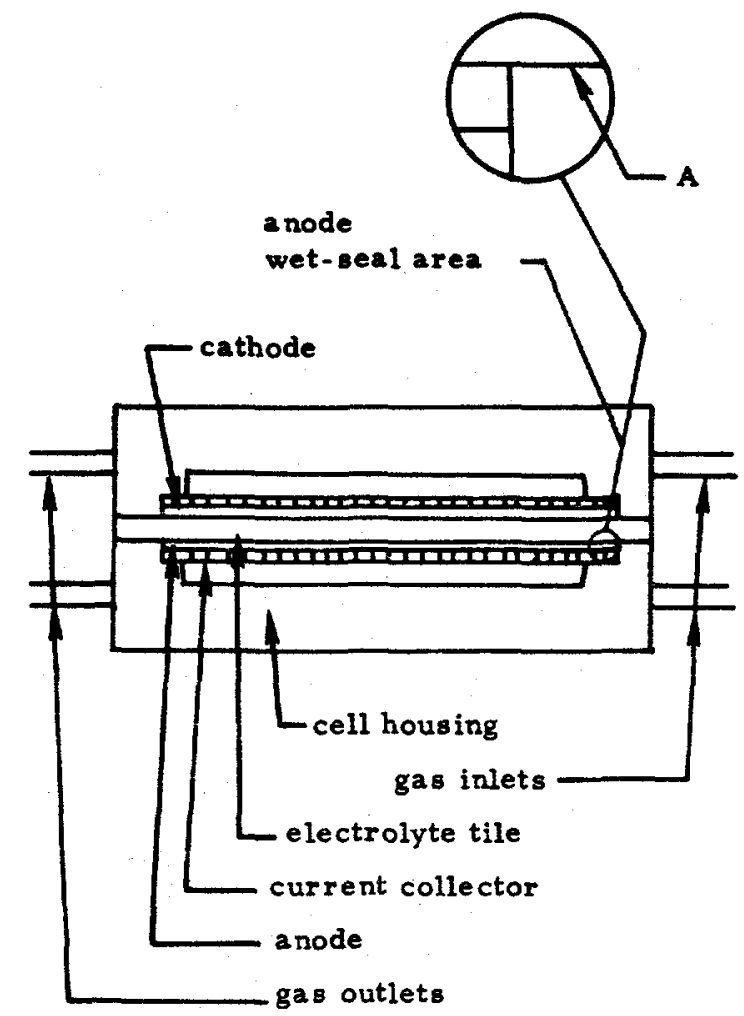

Fig. 5.5D-3. Bench-scale cell showing the anodic wet-seal area (A) in close-up. 43

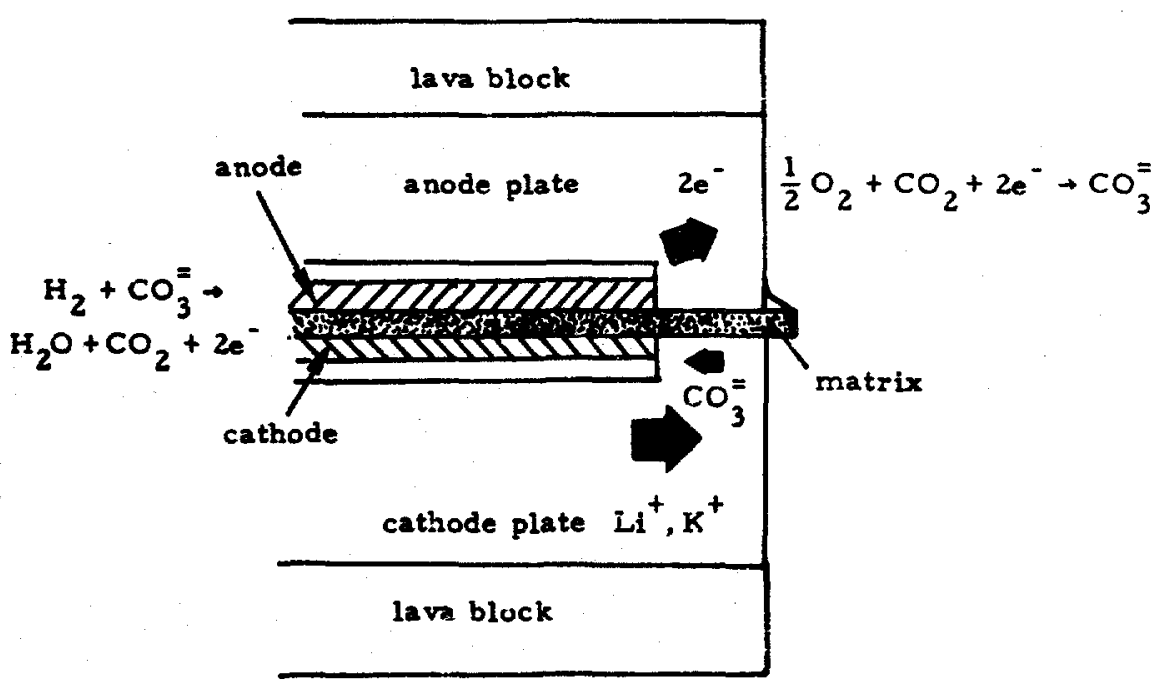

Fig. 5.5D-4, Mechanism for electrolyte leakags through the wet * veal of a bench-scale MCFC. 


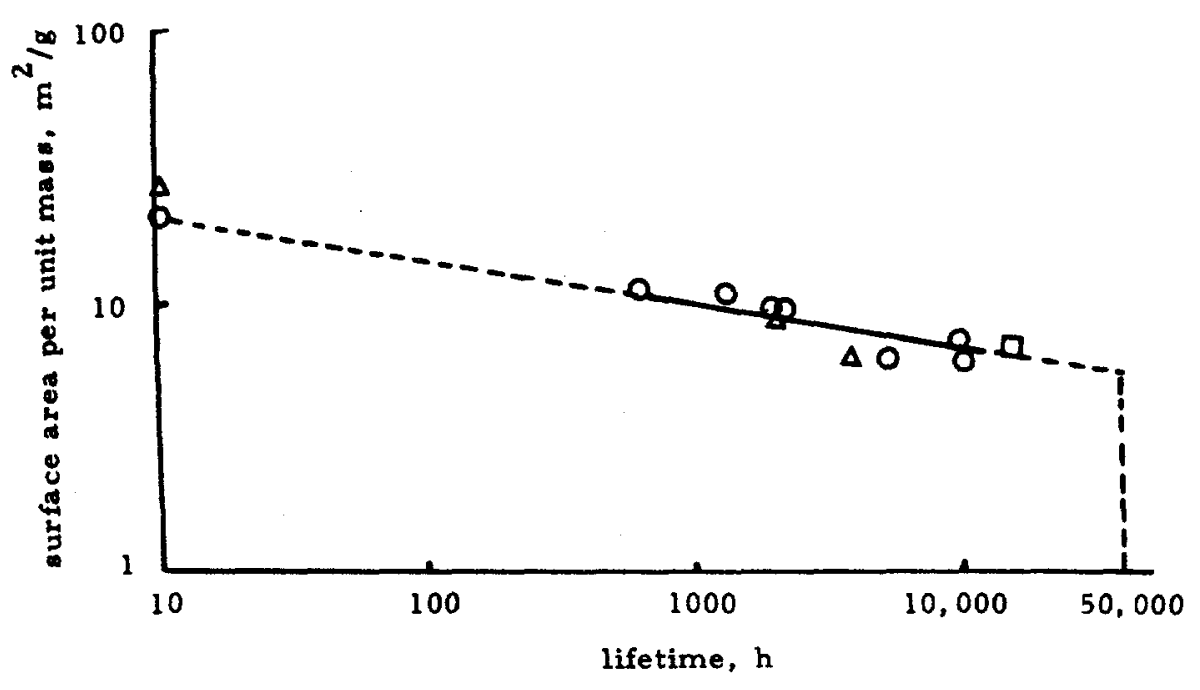

Fig. 5.5D-5. $\mathrm{LiAlO}_{2}$ surface area 1088 in $94-\mathrm{cm}^{2}$ cells. 42 The symbols have the following meaning: $O, K$-free aqueous slurry process; $A$, spray-drying process; $\square$, high-temperature multifiring process.

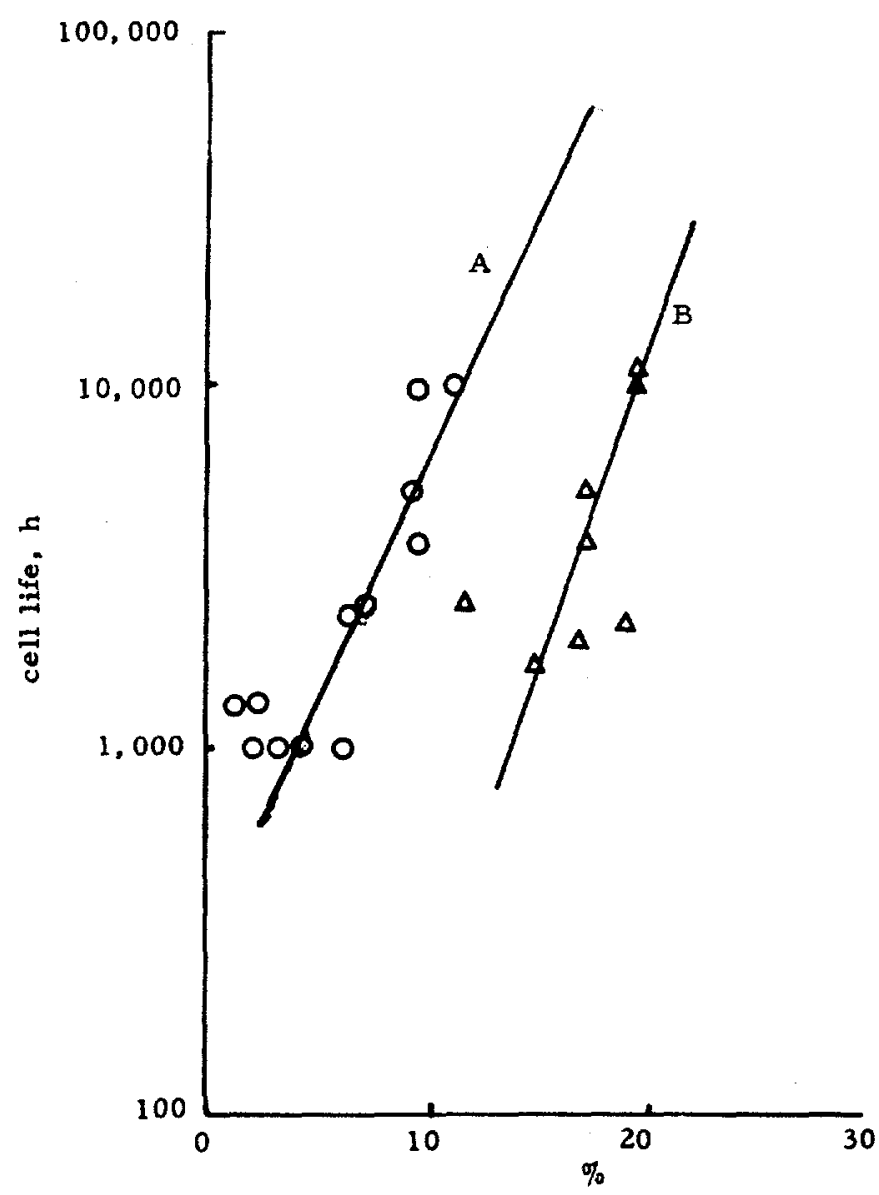

Fig. 5.5D-6. Reduction in matrix thickness (in \%,A) and development of void pores (in vol\%, B) in the electrolyte structure. 34 
and voids caused during tile fabrication, and hence emphasizes the importance of an all-round satisfactory fabrication procedure. Tape casting, as discussed in Sec. 5.5C, appears to yield excellent strength when starting from spray-dried $\mathrm{LiAlO}_{2}$ (Fig. 5.5D-7).

Because some of the problems related to electrolyte loss are caused by structural changes in the $\mathrm{LiAlO}_{2}$ matrix aupport, alternative support materials have been investigated. GE, before termination of its $\mathrm{FC}$ program, experimented with $\mathrm{SrTiO}_{3}$ as a support material since it is single-phase and less subject to recrystallization with loss of area. It was also expected to be compatible with (i.e.. inert with respect to) alternative cathode materials. Tile-fabrication procedures were developed and nickel-coating procedures for $\mathrm{Sr} \mathrm{TiO}_{3}$ particles, to be used in anode structures, were worked out. 28 However, it appears that $\mathrm{LiAlO}_{2}$ will remain the generally accepted aupport material.

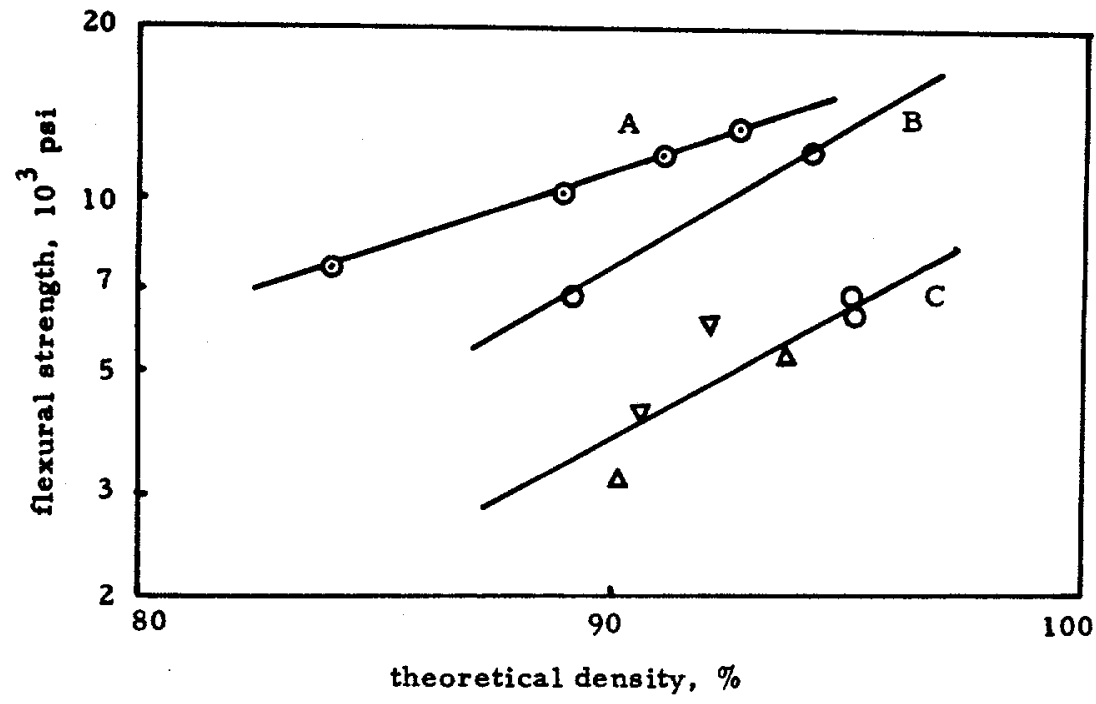

Fig. 5,5D-7. Room-temperature strength as a function of density for various tiles (four-point bending). Curve A refers to a spray-dried tile, $B$ to a ball-milled and $\mathrm{K}$-free tile, and $C$ to a K-free tile. 34

\subsection{Anode}

\section{A. Characteristics and Operation}

The anode is, overall, a less troublesome component than either the tile or the cathode. In contrast to the cathode, it has a reducing environment which protects the metal to such an extent that, with due precautions, the original structure may be preserved. Also, the polarization of the anode is small relative to that of the cathode and compared to the IR loss (Fig. 5.6A-1), and it is much less sensitive to temperature than the cathode polarization. Finally, the anode performance is relatively unaffected by over-filling of electrolyte; it may even be used as a reservoir. This characteristic, like the low polarization, is probably the result of very rapid kinetics of fuel oxidation.

Because the anode has a fairly stable and well-known pore structure, its operation is relatively easy to analyze by means of a porous electrode model. This statement presupposes, of courae, that the electrode kinetics of fuel oxidation are known (see Sec. 5.6B). Recent analyses 45,46 assume either a simple pore model (pores with uniform diameter, partially filled with electrolyte) or an agglomerate model (gas-filled macropores, electrolyte-filled micropores). In either case, it can be shown that only a very small part of the electrode-electrolyte interface, near the meniscus formed with the gas phase, is active. This feature is a very significant difference with the functioning of the cathode and will be discussed further in Sec. 5.7A. It explains the relative insensitivity of anode performance to the electrolyte level in the porous structure, i.e., the degree of filling.

Reactions (5. 4A-1) through (5.4A-3) are the primary reactions taking place at the anode. Their mechanism is overall fairly well understood. In porous anodes, the long-term changes caused by sintering and creep are of great practical importance. The search for alternative anode materials is, in part, an attempt to minimize these long-term structural changes. Another objective is that of developing an anode that resists degradation by contaminants, in particular sulfur. However, the most important objective of current work on the anode is the development of a stable, $\mathrm{CH}_{4}$-reforming a node structure. 


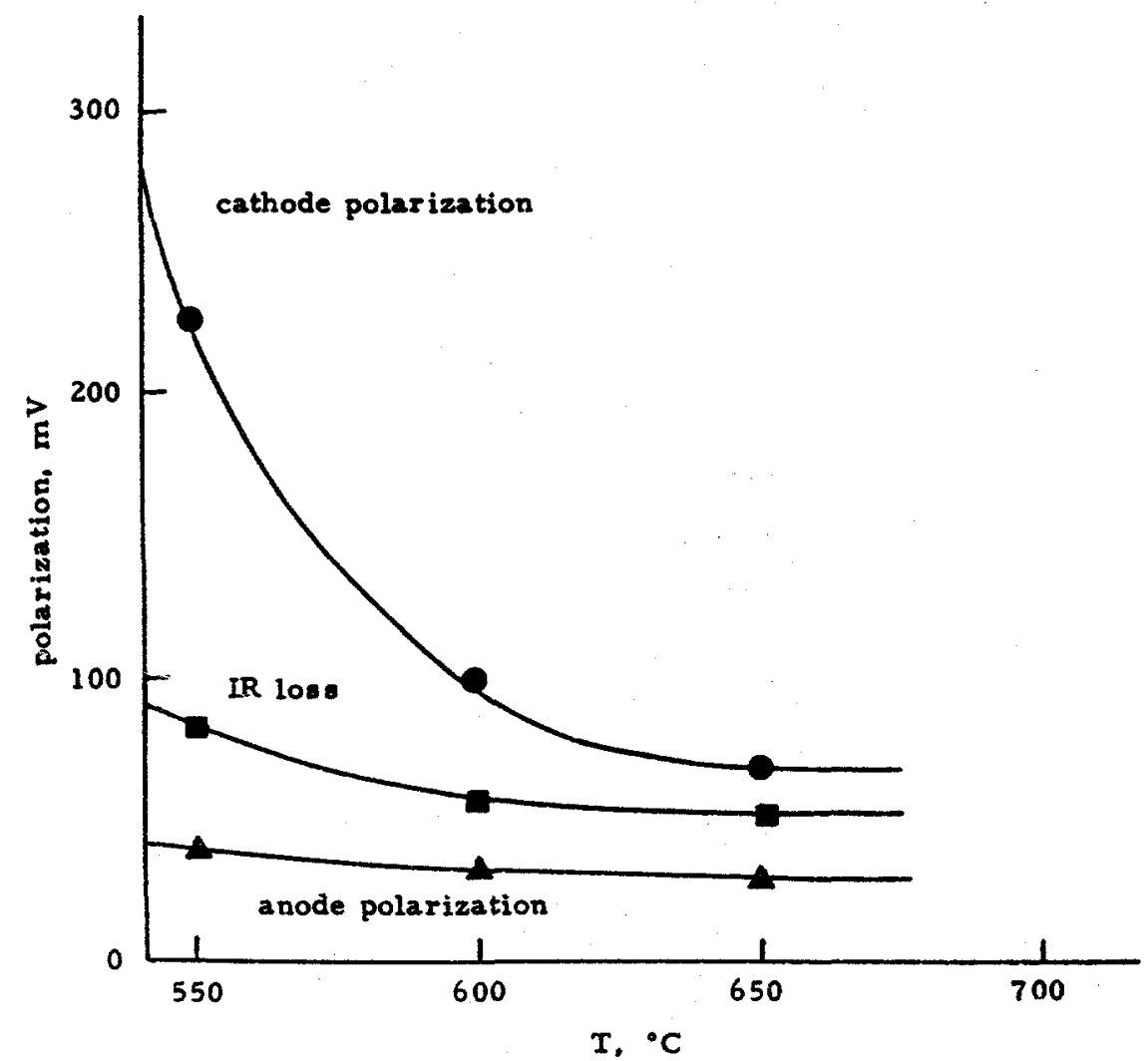

Fig. 5.6A-1. The electrode polarizations and cell-resistance loss are shown at $160 \mathrm{~mA} /$ $\mathrm{cm}^{2}$ for $3 \mathrm{~cm}^{2}$ cells over a range of operating temperatures. The anode is made from $\mathrm{Co}+10 \% \mathrm{Cr}$, the cathode is $\mathrm{NiO}$, and the electrolyte is $55 \mathrm{wt} \%$ of eutectic (the eutectic has 57 wt\% of $\mathrm{Li}_{2} \mathrm{CO}_{3}, 31 \mathrm{wt} \%$ of $\mathrm{Na}_{2} \mathrm{CO}_{3}$, and 12 wt $\%$ of $\mathrm{K}_{2} \mathrm{CO}_{3}$ ) with $45 \mathrm{wt} \%$ of $\mathrm{LiAlO}_{2}$. The fuel consists of $80 \mathrm{~mol} \% \mathrm{H}_{2}$ and $20 \mathrm{~mol} \% \mathrm{CO}_{2}$. The oxidant is $30 \mathrm{~mol} \% \mathrm{CO}_{2}$ and $70 \mathrm{~mol} \%$ air. 59

B. Mechanism of the Electrode Reactions

Hydrogen oxidation is the dominant anode reaction, since it is generally assumed that the other primary reaction, CO oxidation, is kinetically very slow.

The following mechanism has been proposed for reaction $(5.4 \mathrm{~A}-1) \mathrm{s}^{47}$

$$
\begin{gathered}
\mathrm{H}_{2}+2 \mathrm{M} \rightleftharpoons 2 \mathrm{MH}, \\
\mathrm{MH}+\mathrm{CO}_{3}^{=} \rightarrow \mathrm{OH}^{-}+\mathrm{CO}_{2}+\mathrm{M}+e^{-}, \\
\mathrm{MH}+\mathrm{OHI}^{-} \rightleftharpoons \mathrm{I}_{2} \mathrm{O}+\mathrm{M}+\mathrm{e}^{-} .
\end{gathered}
$$

where the anode substrate $M$ is Ni. 47 Equation (5.6B-2) is the rate-determining atep (RDS).

Ang and Sammells, 47 asuming a Langmuir isotherm and low coverage for MH, showed that the theoretical exchange current density muat then be dependent on partial pressures as

$$
i_{0} \sim\left(\mathrm{H}_{2}\right)^{0.25}\left(\mathrm{CO}_{2}\right)^{0.25}\left(\mathrm{H}_{2} \mathrm{O}\right)^{0.25}
$$

On the other hand, their experimental results for $\mathrm{Ni}$ electrodes at $923 \mathrm{~K}\left(650^{\circ} \mathrm{C}\right)$ in an Li-K eutectic melt led to the correlation 47,48

$$
i_{0}\left(\mathrm{~mA} / \mathrm{cm}^{2}\right)=141\left(\mathrm{H}_{2}\right)^{0.258}\left(\mathrm{CO}_{2}\right)^{0.275}\left(\mathrm{H}_{2} \mathrm{O}\right)^{0.178}
$$

with an activation energy for $i_{0}$ of $28 \mathrm{~kJ} / \mathrm{mol}$.

The exchange-current density is the main kinetic parameter in the Butler-Volmer equation 


$$
i=i_{0}\left\{\left[\exp \left(\alpha_{a} F \eta / R T\right)\right]-\left[\exp \left(-\alpha_{c} F \eta / R T\right)\right]\right\}
$$

where $\eta$ is the overpotential, $F$ is Faraday's constant, $R$ the gas constant, $T$ the absolute temperature; $\alpha_{a}$ and $\alpha_{c}$ are the two other kinetic parametera (anodic and cathodic transfer coefficients). At $923 \mathrm{~K}\left(650^{\circ} \mathrm{C}\right), \alpha_{2}$ was found to be 0.70 on nickel and cobalt and 0.50 on gold, in fair agreement with the proposed mechanism described by Eqs. (5.6B-1) to (5.6B-3).

Parallel electrochemical steps have also been proposed ${ }^{49}$ as a possible mechaniam for electrochemical $\mathrm{H}_{2}$ oxidation:

$$
\begin{gathered}
\mathrm{H}_{2}+2 \mathrm{M}=2 \mathrm{MH}, \\
\mathrm{MH}+\mathrm{CO}_{3}^{=} \rightarrow \mathrm{OH}^{-}+\mathrm{CO}_{2}+\mathrm{M}+\mathrm{e}^{-}, \\
2 \mathrm{OH}^{-}+\mathrm{CO}_{2}=\mathrm{H}_{2} \mathrm{O}+\mathrm{CO}_{3}^{=},
\end{gathered}
$$

with reaction (5.6B-2) again as the RDS. Concurrently, CO was assumed to make $\mathrm{H}_{2}$ available at the nickel electrode via the shift reaction (5.4A-3) taking place in the melt, viz.,

$$
\mathrm{CO}+2 \mathrm{OH}^{-}=\mathrm{CO}_{3}^{=}+\mathrm{H}_{2}
$$

Lu and Selman ${ }^{48}$ determined the hydrogen oxidation kinetics at copper and found also good agreement with Eq. (5.6B-4). However, the standard value of $i_{0}$ was somewhat lower:

$$
i_{0}\left(\mathrm{~mA} / \mathrm{cm}^{2}\right)=71.1\left(\mathrm{H}_{2}\right)^{0.33}\left(\mathrm{CO}_{2}\right)^{0.14}\left(\mathrm{H}_{2}\right)^{0.33} \text {. }
$$

while $a_{a}=0.70$ for nickel at $923 \mathrm{~K}\left(650^{\circ} \mathrm{C}\right)$ in $\mathrm{Li}-\mathrm{K}$ eutectic melt.

In a further study, Lu ${ }^{50}$ demonstrated, by analysis of potential sweep and step results, that the mechanism of Suski et al ${ }^{49}$ with parallel electrochemical steps $(5.6 \mathrm{~B}-2)$, followed by (5.6B-7), is not plausible. He concluded that the Ang-Sammells mechanism is most likely and that $C O$ participates in the reaction only via a gas-phase shift equilibrium. However, some uncertainty remains about the role of $\mathrm{H}_{2} \mathrm{O}$ or $\mathrm{OH}^{-}$ions in the reaction mechanism; specifically, if $\mathrm{OH}^{-}$ions are involved in the RDS, one might expect relatively complicated mass-transfer effects, in part dependent on the cationic composition of the melt. This problem should be further investigated in connection with the kinetics of internally reforming $\mathrm{CH}_{4}$.

The electrode kinetics of $\mathrm{CO}$ oxidation have received more attention than those of $\mathrm{H}_{2}$ oxidation but are more controversial. It is generally believed that the direct electrochemical reaction is quite slow, for example, $i_{0}=0.042 \mathrm{~mA} / \mathrm{cm}^{2}$ at $923 \mathrm{~K}$ in ternary eutectic at gold. 51,52 Borucka and Appleby 53 concluded that only $60-70 \%$ of this reaction represents oxidation of physically dissolved $C O$, the remainder being due to oxidation of chemically bound CO (as a hypothetical species $\mathrm{CO}_{2}^{2-}$ ). This interpretation is independently supported by CO-solubility measurements and $\mathrm{Li}_{2} \mathrm{O}$ addition. The second wave oxidation was also observed by workers at UTC, 54 who gave it a different interpretation. 55 Although the relatively slow kinetics of the direct $\mathrm{CO}$ oxidation do not appear to promise much in the way of cell performance, more insight into its reaction mechanism and the related melt chemistry may lead to progress in direct $\mathrm{CH}_{4}$ oxidation or internal reforming. Spectroscopic observations, although very difficult to perform because of the attack of carbonate on silica and silicates, may assist in identifying actual species in the melt.

\section{Sintering and Creep}

Following the introduction of fine-pore nickel electrodes in the early $1970 \mathrm{~s}$, sintering of the anode in long-term operation soon emerged as the most serious impediment to stability (Fig. 5.1-1, 1972 data). . Sintering is spontaneous in all extended-area solids at high temperature. Its effect on performance has been reviewed thoroughly by Kinoshita, 56 who also discussed various mechanisms to inhibit sintering.

Since the mid-1970s, anode sintering in the MCFC has been greatly reduced by the use of a nickel-chromium alloy (2-10 wt\% chromium). Chromium is oxidized in situ to $\mathrm{LiCrO}_{2}$, which collects in grain boundaries and forms a barrier to metal diffusion. Other oxides may be added directly and function similarly. Figure 5,6C-1 illustrates the long-term stability of porous nickel or cobalt when stabilized with chromium or zironia. This stability demonstrates that it is possible and desirable to optimize the choice of sintering inhibitor, based on matching with basemetal structure and melt chemistry.

Addition of ceramic components is also instrumental in reducing anode creep, i.e., change in thickness and tendency to flow under compression. The creeping of currently used 
nickel-chromium anodes is illustrated in Fig. 5.6C-2. In long-term operation of MCFC stacks', the slight shrinkages indicated here may cause severe performance decay by redistribution of the electrolyte, which is especially serious since the anode will probably function as an electrolyte reservoir in some or all cells of an MCFC stack.

Creep can be minimized by using cermets ( $\mathrm{Ni}$ or $\mathrm{Ni}-\mathrm{Cu}$ in combination with $\mathrm{LiAlO}_{2}$ or $\mathrm{SrTiO}_{3}$ ) as anode material; 57 this is an application of dispersed-oxide strengthening. 58 Figures 5.6C-3 and 5.6C-4 show that the porosities of cermet anodes remain es sentially invariant with time.

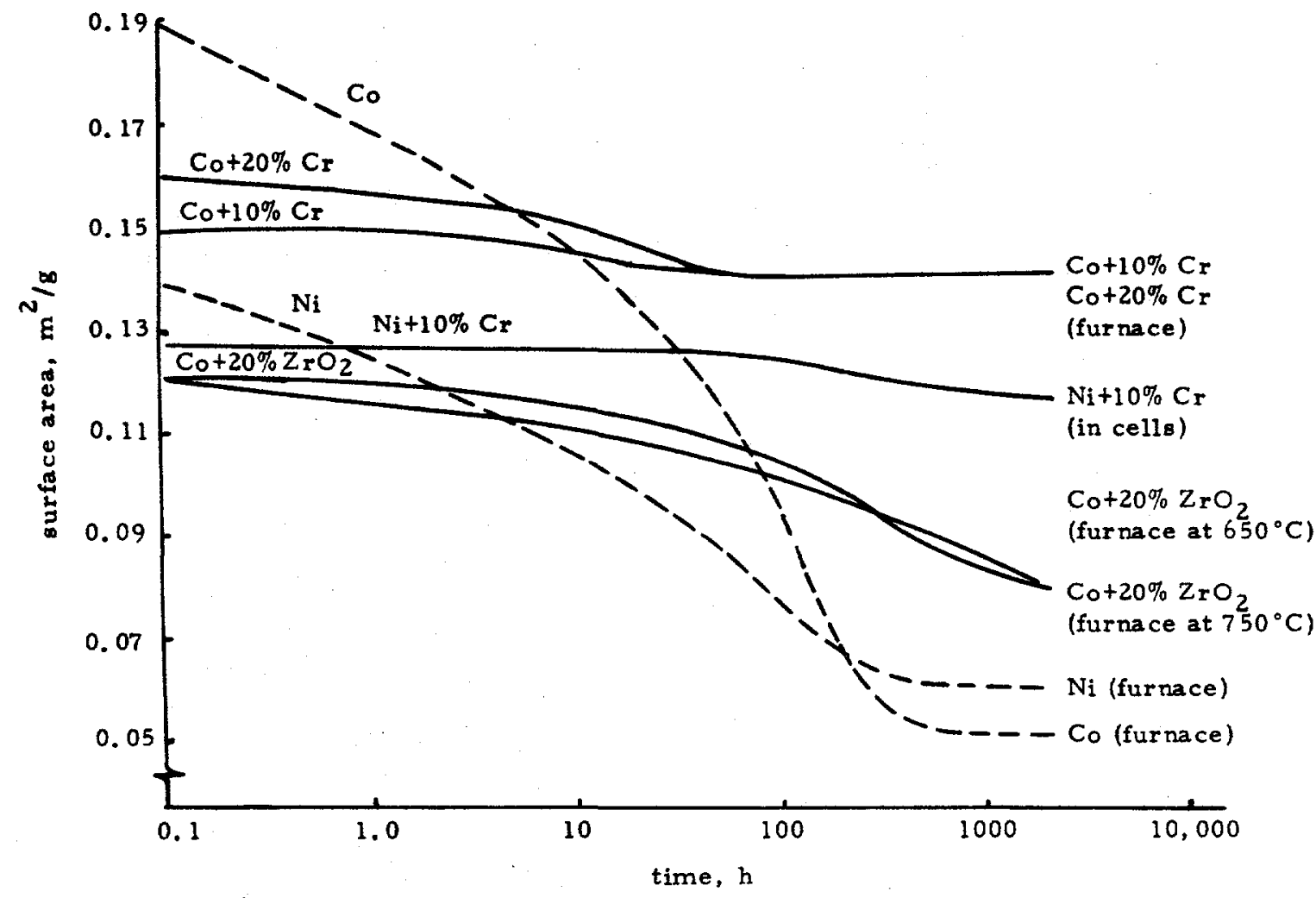

Fig. 5,6C-1. Changes in surface area are shown as a function of time for a variety of testing conditions for stabilized anode materials. 72

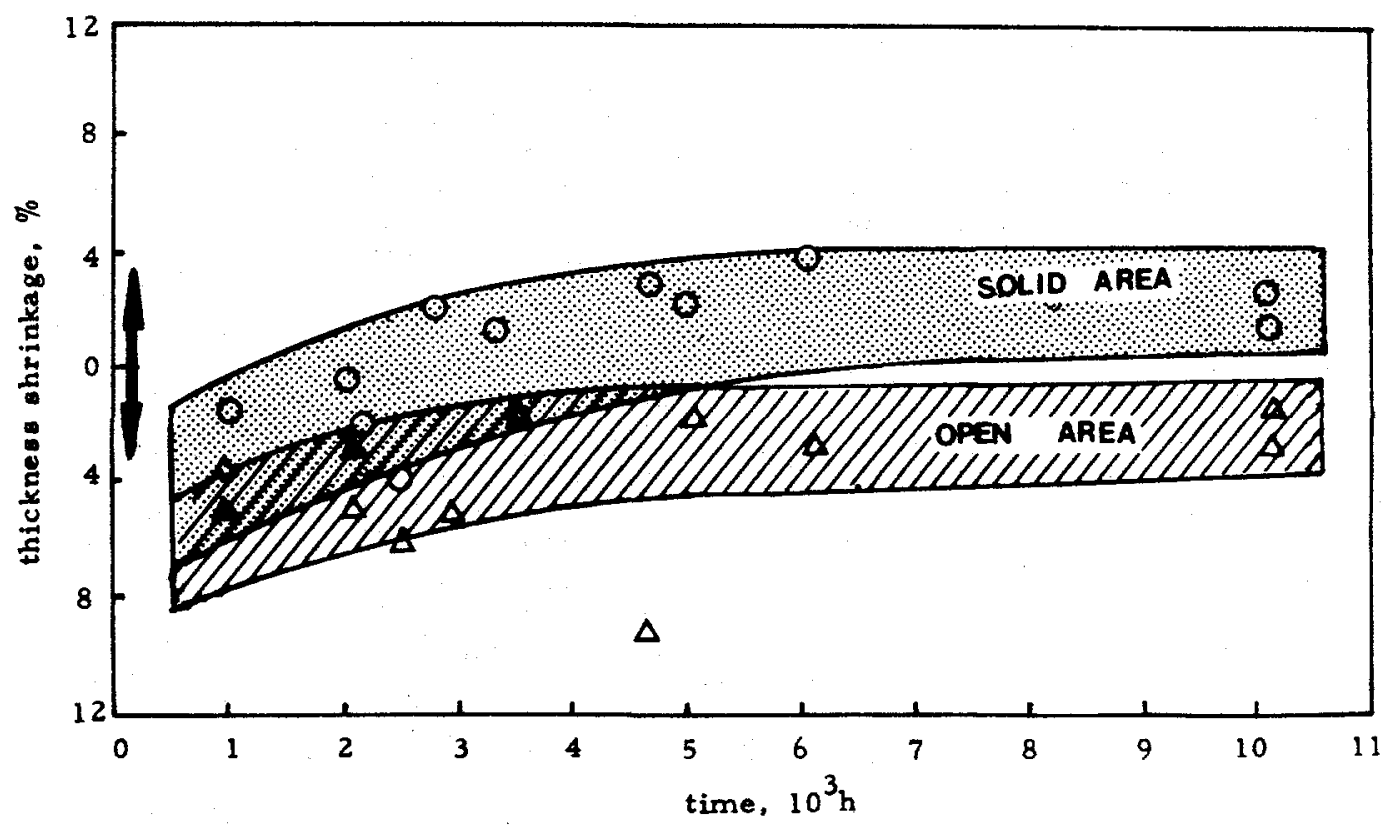

Fig. 5.6C-2. Changes in the thickness are shown for the $\mathrm{Ni}+10 \% \mathrm{Cr}$ anode in boiler plate tests at $650^{\circ} \mathrm{C}$ with 70 mil tiles of $55-60$ wt $\%$ carbonate. 59 


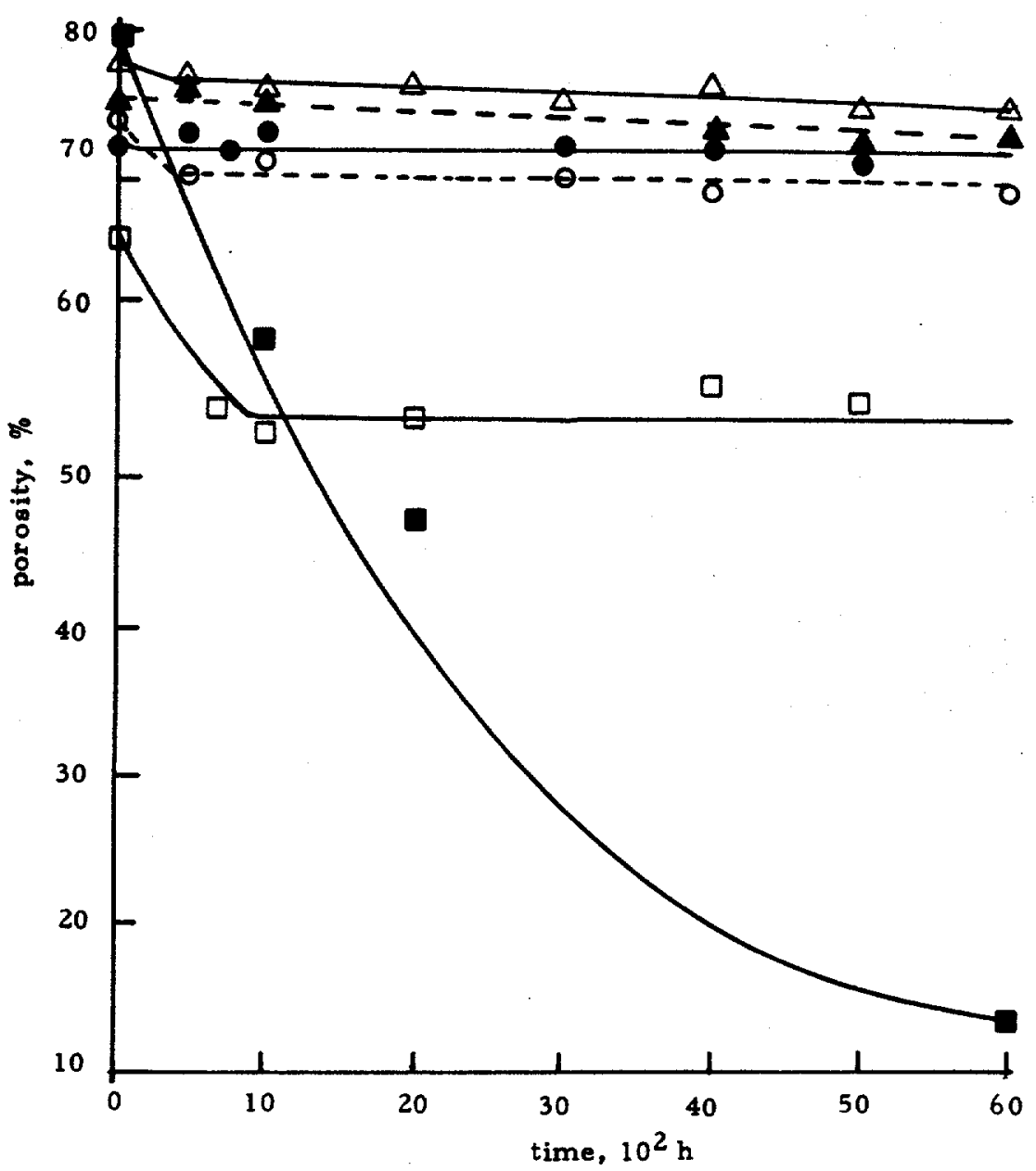

Fig. 5.6C-3. The porosities of anodes made from $\mathrm{Ni}, \mathrm{Ni} / \mathrm{Cr}$ alloys, and $\mathrm{Ni}$ cermets are shown as functions of time. Anode sintering was responsible for the porosity shifts. 57 The data points refer to different materials as follows: $\square$, nickel; $\triangle$, gold; $\triangle, \mathrm{Ni}-3 \mathrm{Cr}_{r}$; - $\mathrm{Ni}-10 \mathrm{Cr}(2 \mathrm{um}) ; \square, \mathrm{Ni}-\mathrm{Al}_{2} \mathrm{O}_{3} ; 0, \mathrm{Ni}$-plated $\mathrm{Sr}_{\mathrm{TiO}}$.

\section{Alternative Anode Materials}

Nickel shows good performance 28 an anode material. However, nickel anodes are relatively expensive. Furthermore, they must be stabilized to prevent sintering and strengthened to minimize creep (see Sec. 5.6C). Although sintering has been practically eliminated by alloying with chromium or other inhibitors, the development of a satisfactory strengthening mechanism is not yet completely successful. It has prompted a search for alternatives to nickel-chromium.

Many factors must be considered in choosing suitable replacement anodes: ${ }^{28}$ (i) they must be electronically conductive, (ii) they must have suitable wettability, porosity and endurance to assure long-term effectiveness as electrochemical and internally reforming catalytic sites,

(iii) they must be thermally compatible with other components, (iv) fatigue caused by creep must be minimal, (v) chemical stability and compatibility with other components in the electrolyte and fuel-stream environments is required under both operating and non-operating conditions.

As a base metal for the anode, copper would be a suitable replacement for nickel. Its kinetic properties are only slightly less favorable than those of nickel. 48 Suitable sintering inhibitors have been developed. 59 Copper also has a more positive potential than nickel and is therefore more stable under accidental current overload. However, it is not as good a prospect as nickel for internal-reforming catalysis and its application may therefore be more restricted.

Other materials have been extensively screened by $\mathrm{GE}, 28 \mathrm{e.g} ., \mathrm{LiFe}_{5} \mathrm{O}_{8}, \mathrm{LaCrO}_{3}, \mathrm{MnO}_{\text {, }}$ and $\mathrm{Nb}$ - or $\mathrm{Ta}$-doped $\mathrm{TiO}_{2}$, of which only $\mathrm{LiFe}_{5} \mathrm{O}_{8}$ and doped $\mathrm{TiO}_{2}$ looked promising. Howe ver, fabrication methods for these materials were not further developed. Instead, the $\mathrm{Ni}-$ or $\mathrm{Cu}$ plated ceramic anode was pursued as a solution to both creeping problems and high cost of nickel. The ceramics were $\mathrm{LiAlO}_{2}$ or $\mathrm{Al}_{2} \mathrm{O}_{3}$ and $\mathrm{SrTiO}_{3}$. Since the se materials do not contain chromium, the initial $\mathrm{Li}_{2} \mathrm{CO}_{3} \mathrm{los} 8$ is also minimized. The sintering and creep resiatance of 


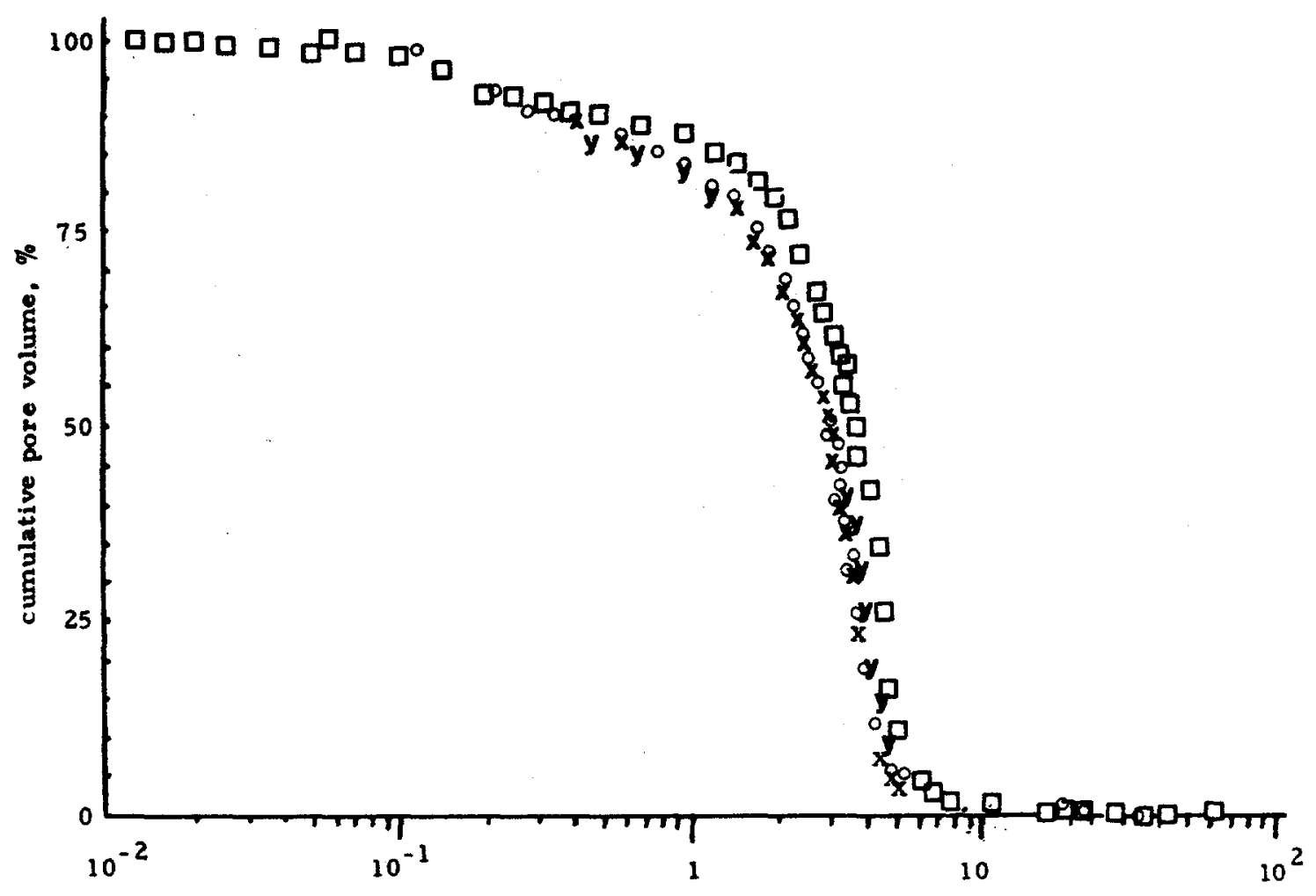

pore diameter, $\mathrm{um}$

Fig. 5.6C-4. The pore-size distributions of $\mathrm{Ni}$-plated $\mathrm{SrTiO}_{3}$ anodes are plotted as functions of time. 57 The data points have the following meaning: $x, 3000 \mathrm{~h}$ for $69 \% ; D, 4000 \mathrm{~h}$ for $69 \% ; 0,5000 \mathrm{~h}$ for $71 \%, y$, $\ldots h$ for ... \% .

plated-ceramic anodes was demonstrated but in-cell tests in combination with BPB anodes were not conclusive as to electrolyte retention.

Some version of ceramic-metal composite as an alternative anode is at present being pursued by all major developers. Though metal-plated ceramic particles may be ideal, the fabrication process is relatively complicated. Oxide additions to bulk metal also appear to be effective and are simpler to fabricate. 60

Basic research has much to contribute to a better understanding of the sintering and creep processes of metals in contact with molten (or solid) electrolyte, as well as fuel gas. Most fundamental studies are carried out on metal surfaces in contact with an inert gas; howe ver, the potential gradients driving solid diffusion are certainly affected by the presence of a metal/ electrolyte interface where faradaic reactions (dissolution, fuel oxidation), a s well as adsorption, can take place. This problem area is virtually terra incognita in electrochemistry as well as materials cience.

\section{E. Internal Reforming Anode}

Internal reforming of $\mathrm{CH}_{4}$ and light $\mathrm{HC}$ is a compromise between direct oxidation and external reforming. Direct conversion (anodic oxidation) of $\mathrm{CH}_{4}$ at the presently used anode structure is practically nil. This fact has been ascribed to the extremely low solubility of $\mathrm{CH}_{4}$ in molten carbonate, which is two orders of magnitude smaller than that of $\mathrm{H}_{2}, \mathrm{CO}_{0}, \mathrm{or} \mathrm{CO}_{2 .} 61$ In fact, humidification of methane is necessary to prevent carbon deposition, as the thermodynamic C-H-O diagram (Fig. 5, 6E-1) shows.

At a suitable catalyst, $\mathrm{CH}_{4}-\mathrm{H}_{2} \mathrm{O}$ mixtures are converted to $\mathrm{CO}$ or $\mathrm{CO}_{2}$ and $\mathrm{H}_{2} \mathrm{O}$ (steamreforming), under absorption of heat, by the equilibria

$$
\begin{aligned}
& \mathrm{CO}+3 \mathrm{H}_{2}=\mathrm{CH}_{4}+\mathrm{H}_{2} \mathrm{O}, \\
& \mathrm{CO}_{2}+4 \mathrm{H}_{2}=\mathrm{CH}_{4}+2 \mathrm{H}_{2} \mathrm{O} .
\end{aligned}
$$

In external reforming, reaction is accomplished by a separate catalytic reformer (fuel processor), under transfer of heat (and $\mathrm{H}_{2} \mathrm{O}$ ) from the $\mathrm{FC}$ to the processor, as is indicated in Fig. 5, 6E-2(a). 


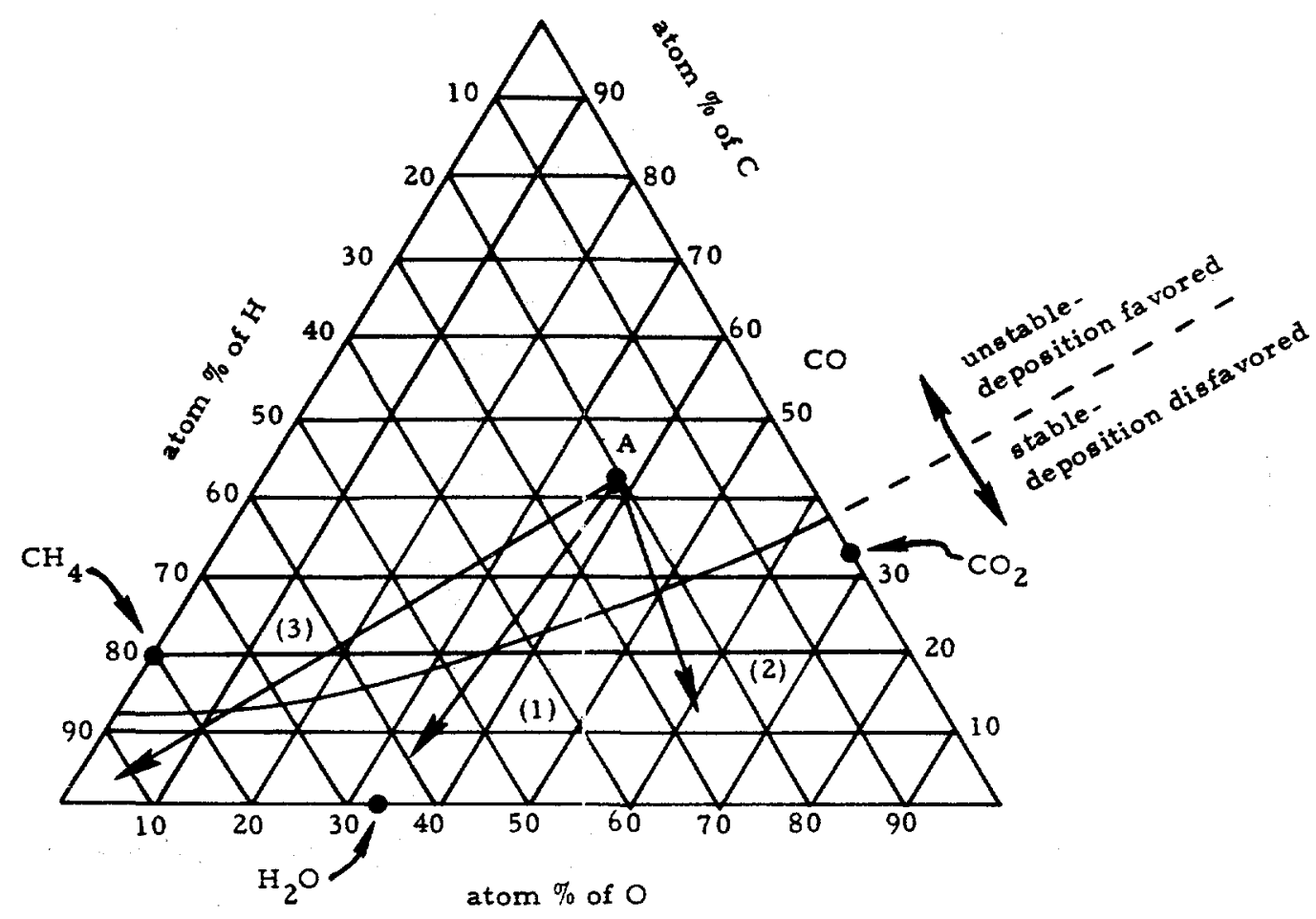

Fig. 5.6E-1. A C-H-O ternary ciagram is shown with stability limits and the effects on fuel-mixture stability of steam injection (1), a node-gas recycling (2), and anode-gas reprocessing (3); $T=1300^{\circ} \mathrm{F}, \mathrm{p}=150 \mathrm{psia}$, amorphous $\mathrm{C} .62$

Internally reforming $\mathrm{MCFC} s$ have an anode structure which enables the equilibria (5. 4A-5) and (5.4A-6) to be established catalytically in the gas phase adjacent to the electrocatalyst, i.e., within the anode structure or its :manifolding. This is schematically indicated in Fig. 5.6E-2(b). The heat and $\mathrm{H}_{2} \mathrm{O}$ exchange now take place within the overall anode structure. The reactant flows are detailed in Fig. 5.6E-3. The reforming reaction is endothermic, with heat a upplied for reforming by the exothermic electrochemical reaction $(5,4 A-1)$.

HYOROCARBON

FUEL

(a)

(b)

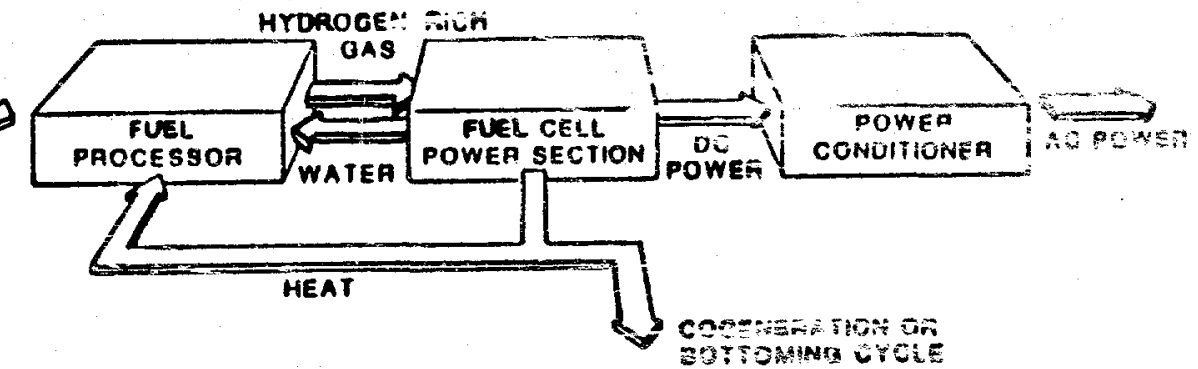

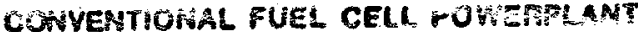

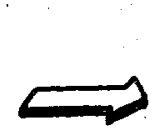

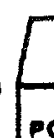

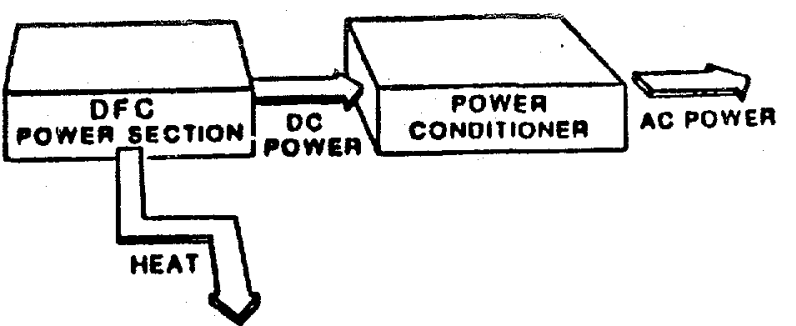

Fig. 5.6E-2. An IRMCFC power plant is shown schematically. The IRMCFC plant is simpler than a conventional FC power plant because it does not require a full-reprocessing system. 63 


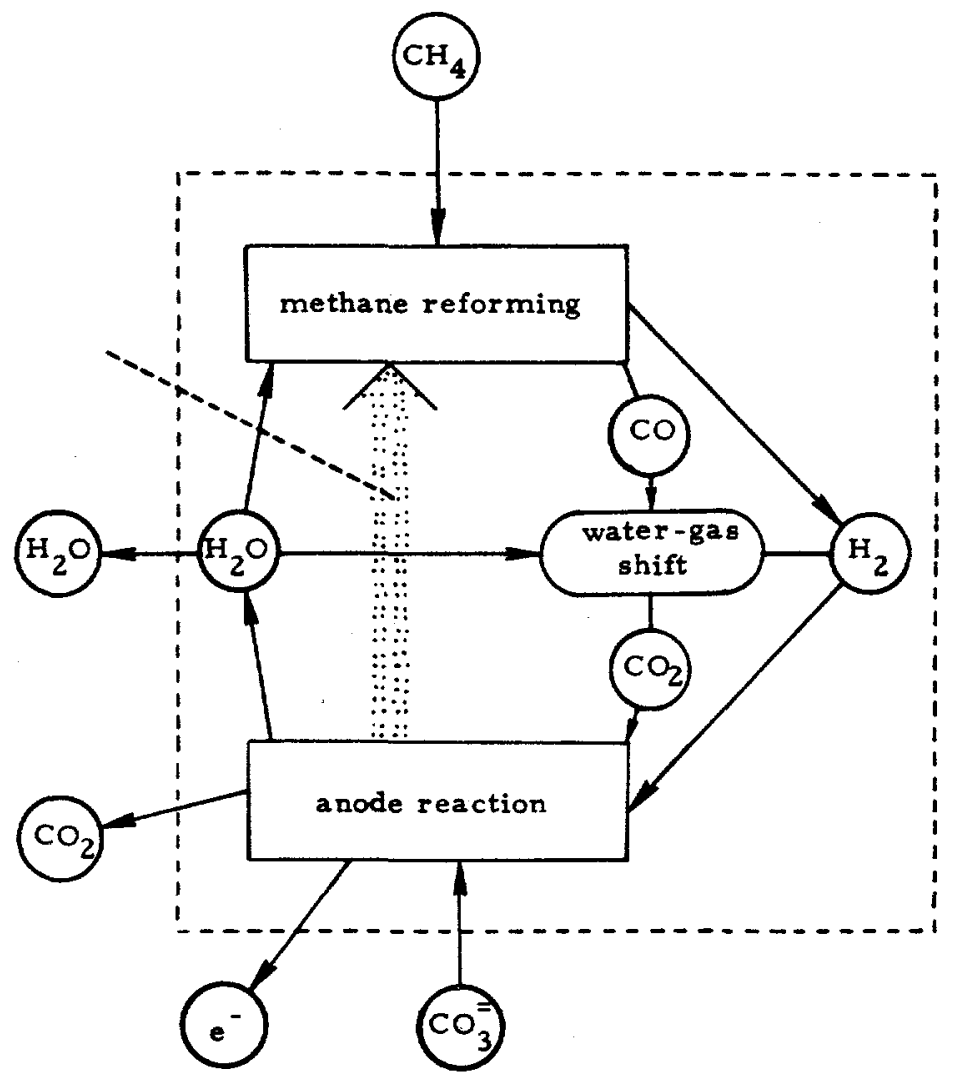

Fig. 5.6E-3. This schematic represents the reactions which occur on the anode or in its manifolding during internal reforming. 64

The IRMCFC can, from the thermodynamic viewpoint, be simply considered as a black box which consumes methane. Its electrical efficiency is then ( $/ H H V) U_{H_{2}}$, where $V$ is the unit cell voltage at the operating current density, HHV is the higher-heating-value of methane in $\mathrm{eV}$ (electron-volts or volts per equivalent; $23.06 \mathrm{kcal}=\mathrm{l} \mathrm{eV}$ ), and $\mathrm{U}_{\mathrm{H}_{2}}$ is the hydrogen utilization (i.e., the methane utilization) in the cell.

Since the equilibria (5.4A-5) and (5.4A-6) are favorable at low pressure, the IRMCFC should operate at atmospheric pressure. Performance modeling of the cell (see Sec. 5.8B) shows that stacks of atmospheric pressure cells operating at $0.70-0.73 \mathrm{~V}$ and $160 \mathrm{~mA} / \mathrm{cm}^{2}$, with a $\mathrm{CH}_{4}$ utilization of $85-90 \%$, should be capable of system efficiencies on the order of $52-57.6 \%$. Parasitic power and dc/ac (power conditioning) losses should decrease the performance by $2-3 \%$.

The indicated very attractive values may be improved even further by employing a bottoming cycle and an efficient $\mathrm{CO}_{2}$-transfer device (to increase utilization), as is discussed in Sec. 5.8B. Consequently, development of a successful internal-reforming a node would greatly increase the market potential for MCFCs (see Sec. 5.2). The technical barriers are considerable, but much progress has already been made.

Maru 63,64 has discussed requirements for internal reforming catalysts. The activity of the catalyst should be $\sim 500 \mathrm{\mu mol}$ of $\mathrm{CH}_{4} / \mathrm{g}-\mathrm{sec}$-atm. The catalyst degradation should not be $>50 \%$ after $25,000 \mathrm{~h}$. Both the catalyst and its ceramic support should be physically stable. The support must also be chemically stable. Finally, the catalyst must be tolerant of the electrolyte and fuel-gas contaminants.

Nickel supported on nonwetting ceramics is currently being used as a reforming cata lyst. It functions quite satisfactorily, 28 is shown by Fig. 5.6E-4, which also illustrates that the rate of steam-reforming drops considerably when the fuel cell is on open circuit. As will be discussed in Sec. 5.6F, sulfur-contaminant levels in the fuel gas when using nickel must be no more than 0.1 ppm.

Figure 5.6E-5 shows the activity of $\mathrm{Ni}$ supported on $\mathrm{MgO}$ after $450 \mathrm{~h}$ and on $\mathrm{Y}-\mathrm{LiAlO}_{2}$ after $1000 \mathrm{~h}$ of operation when used as methane-reforming catalysts at various temperatures. The $\mathrm{MgO} / \mathrm{Ni}$ catalyat has slightly higher activity at higher temperatures but $\gamma-\mathrm{LiAlO} 2 / \mathrm{Ni}_{2}$ performs somewhat better at lower temperatures, where catalytic function is more urgently required. The deficlencies (e.g., crystal growth, solid-phase changes, etc.) noted for $\mathrm{LiAlO}_{2}$ as a matrix material also present problems in its use as a reforming-catalyst support. 64,65 


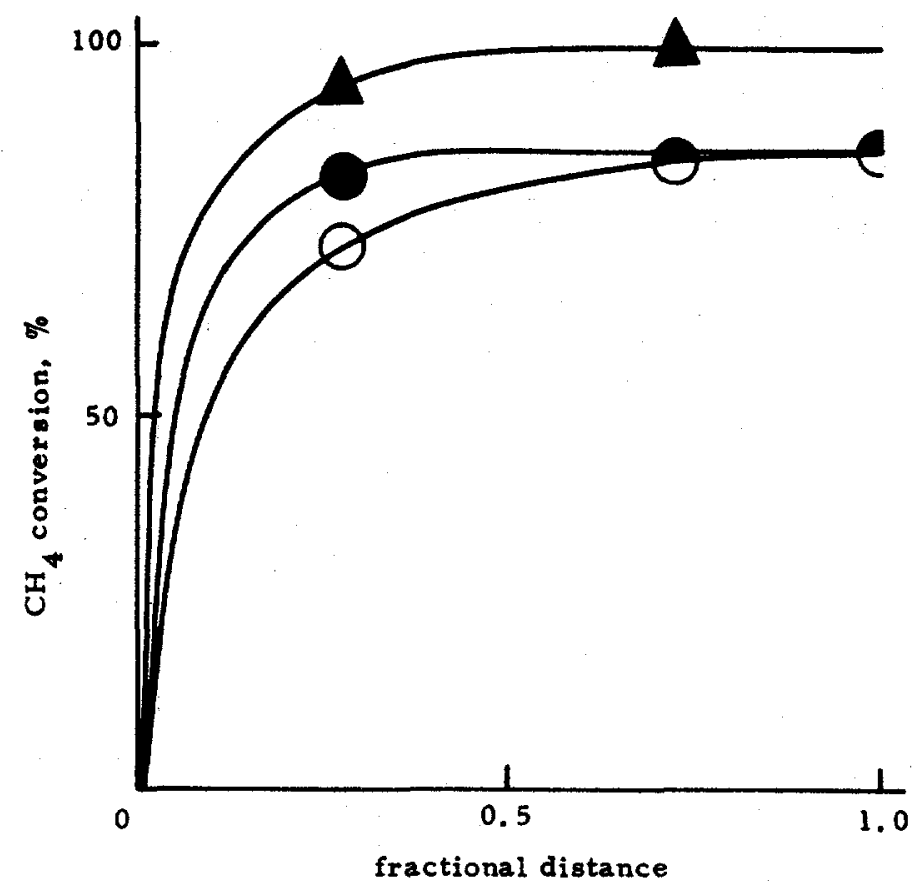

Fig. 5.6E-4. The percentage of $\mathrm{CH}_{4}$ converted into usable $\mathrm{H}_{2}(\mathrm{~g})$ and $\mathrm{CO}(\mathrm{g})$ is plotted vs the fractional cell length for operating and non-operating cells. 65 The ymbols have the following meaning: $O \mathrm{OCV}$, initial performance; $O, O \mathrm{CV}$, after $4000 \mathrm{~h}$ of operation; 1 , operating at $4000 \mathrm{~h}$.

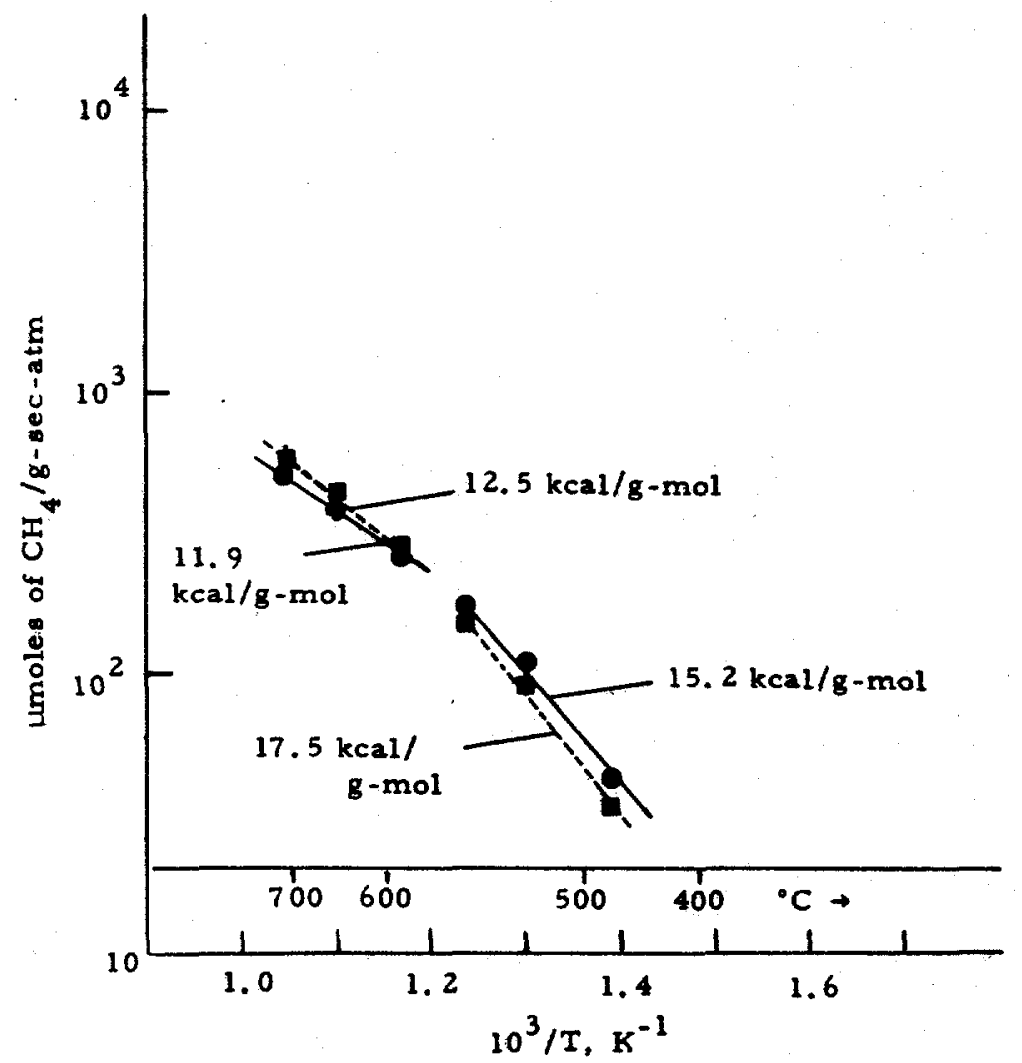

Fig. 5.6E-5. The methane-reforming activities of $\mathrm{Ni}$ supported on $\mathrm{MgO}$ and on $\gamma-\mathrm{LiAlO}_{2}$ are shown at various temperatures $\left(<0.1 \mathrm{ppm}\right.$ of $\mathrm{H}_{2} \mathrm{~S}$ ). 64 The oolid curve refers to $Y-\mathrm{LiAlO}_{2} / \mathrm{Ni}$ at $450 \mathrm{~h}$ and the dotted curve to $\mathrm{MgO} / \mathrm{Ni}$ at $1,000 \mathrm{~h}$. 
The reforming catalyst is degraded in time because of contamination by molten carbonate $(\sim 4000 \mathrm{~h}$, cf. Fig. 5.6E-6). The support structure, as well as pure Ni, are lyophobic to carbonate, but protection is nevertheless needed against capillary wetting. Ni-cermet a nodes with BPBs may be a suitable remedy for capillary wetting. Nickel-chromium alloys are highly wettable and should not be used in conjunction with reforming catalysts.

It is noteworthy that no thorough study has been made of the suitability of available reforming catalysts besides nickel. Such a study is desirable; it should include systematic investigation of the wetting behavior of these substances with respect to molten carbonate. As is dis cussed in Sec. 5.5B, there is an urgent need for a fundamental understanding of what determines wetting behavior in molten salts at a given substrate.

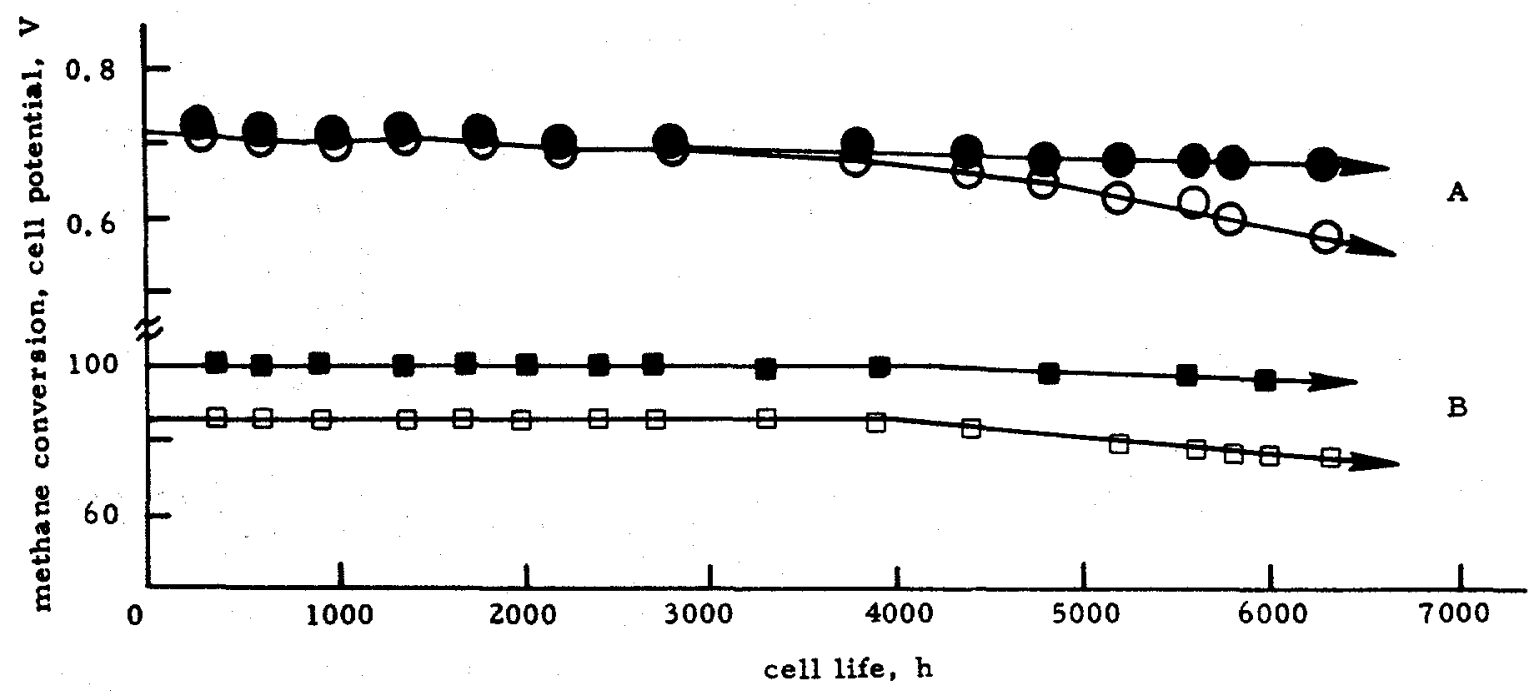

Fig. 5.6E-6. The performance of a cell using $\mathrm{MgO} / \mathrm{Ni}$ as a methane-reforming catalyst is compared, as a function of time, with the performance of a cell which was fed fuels that did not require reforming (top curve). The bottom curve shows the percentage of methane conversion under standard load and opencircuit conditions as a function of time; $T=650^{\circ} \mathrm{C}$; cell area $=300 \mathrm{~cm}^{2} .64$ For $A$, the current was $160 \mathrm{~mA} / \mathrm{cm}^{2}$ at $50 \%$ oxidant utilization; $\bullet, 74 \% \mathrm{H}_{2}$, $19 \% \mathrm{CO}_{2}, 7 \% \mathrm{H}_{2} \mathrm{O} ; 0,30 \% \mathrm{CH}_{4}, 70 \% \mathrm{H}_{2} \mathrm{O}$. For $\mathrm{B}, \mathrm{D}$, standard load conditions; $\square, \mathrm{OCV}$.

\section{F. Effect of Fuel-Gas Contaminants}

The performance of currently a vailable MCFCs is degraded when small amounts of contaminants, such as $\mathrm{H}_{2} \mathrm{~S}$ or COS, are present in the fuel stream. Chlorine contaminants such as $\mathrm{HCl}$ can also exacerbate the corrosion of current collectors. The $\mathrm{HCl}$ is easily scrubbed out with water but only if low-temperature gas clean-up is utilized (Ref. 28, p. 4-347).

Sulfur, which is the major contaminant, can be removed from the entering fuel stream by using the Selexol liquid-adsorption process. This and various other clean-up processes suitable for coal gas have been reviewed by Anderson and Garrigan, 66 who also discuss the economics of gas clean-up.

Nearly complete removal (below $100 \mathrm{ppb}$ ) of $\mathbf{S}$ from the fuel stream eliminates degradation caused by $S$. The delivered electricity cost would obviously be lower if less complete $S$ scrubbing could be tolerated. However, the performance of presently available anode materials is substantially reduced by small amounts $(\sim 1 \mathrm{ppm})$ of $\mathrm{S}$ in the fuel streams. 67 This degradation is almost completely reversible when clean fuel is reintroduced (see Fig. 5.6F-1). The reason for this behavior will be discussed below. Operation at hi gher temperatures $\left(\sim 700^{\circ} \mathrm{C}\right)$ can offset some of the performance losses associated with sulfur contaminants. Howe ver, the utilization of high temperatures reduces cell endurance to such an extent that the use of high temperatures is not a 8 uitable long-term solution. 67

Besides its direct action on the nickel anode, sulfur contaminant acts also via the cathode. If $\mathrm{S}$ occurs in the fuel stream, $\mathrm{SO}_{2}$ is introduced into the cathode stream as the result of $\mathrm{CO}_{2}$ recycling. Corrosion of $\mathrm{Ni}$ in $\mathrm{SO}_{2}$ or $\mathrm{SO}_{2} / \mathrm{O}_{2}$ mixtures results in the formation of $\mathrm{NiO}$ in the external surfaces and the formation of internal $\mathrm{Ni}_{3} \mathrm{~S}_{2}$ scales. 68 Sulfur dioxide reacts with the liquid electrolyte as follows:

$$
\mathrm{M}_{2} \mathrm{CO}_{3}+\mathrm{SO}_{2}+\frac{1}{2} \mathrm{O}_{2} \rightarrow \mathrm{M}_{2} \mathrm{SO}_{4}+\mathrm{CO}_{2} \text {, }
$$




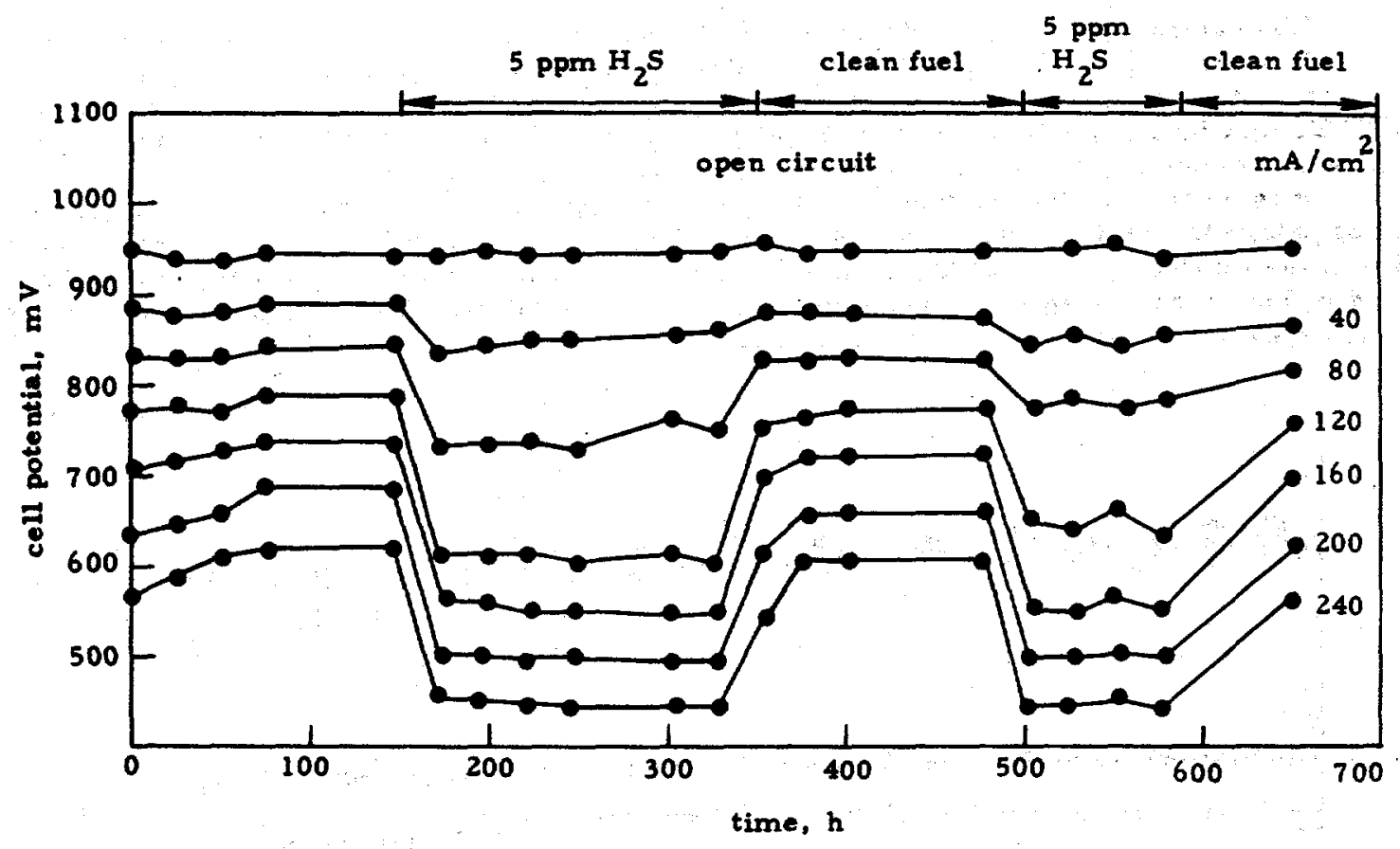

Fig. 5.6F-1. The cell potential is shown vs operating time for various current densities as $\mathrm{H}_{2} \mathrm{~S}(5 \mathrm{ppm})$ is introduced into the fuel stream $\left(10 \% \mathrm{H}_{2}, 5 \% \mathrm{CO}_{2}, 10 \%\right.$ $\left.\mathrm{H}_{2} \mathrm{O}, 75 \% \mathrm{He}\right) .71$

where $M$ is a monovalent alkali metal. Reaction (5.6F-1) is strongly favored to go to completion. Sulfate is transported to the anode where it is reduced and acts like $\mathrm{H}_{2} \mathrm{~S}$ coming from the fuel stream. The sulfur will also accumulate in the tile to some extent as sulfide and sulfate.

The rapid onset of degradation when $\mathrm{H}_{2} \mathrm{~S}$ is introduced in the fuel gas suggeats changes in wettability, as well as poisoning by chemisorption or reaction. If $\mathrm{Ni}-10 \% \mathrm{Cr}$ anodes are used, the wetting angle $\theta$ is increased, which may produce isolated pockets of electrolyte at locations where previously connected thin films occurred.

Although $S$ may poison the internal reforming reactions, it is believed by some to interfere primarily with adsorption during the electrochemical process. Thus, Vogel and Smith 69,70 believe that the area of hydrogen chemisorption, Eq. (5.6B-1), becomes the limiting factor in fuel oxidation, due to chemisorption of $S$. They found that the current at a submerged rotating electrode remained diffusion limited but at a lower level. It appears also from other work that the hydrogen-oxidation mechanism itself remains unchanged. 68

While most earlier work thus points to chemisorption of sulfur as the key step in performance degradation, it does not explain the reveraibility at low contaminant levels. Recent work at IGT, however, has shown that the layer of sulfur deposited on a submerged wire electrode at maximum sulfur coverage is many times greater than that required for monolayer coverage. Remick et $a 1^{71}$ have proposed an alternative $\mathrm{H}_{2}$ oxidation mechanism mediated by 8 ulfide ions in the electrolyte that is operative only at high current densities in a cell poisoned with sulfide:

$$
\begin{aligned}
& \mathrm{H}_{2} \mathrm{~S}+\mathrm{CO}_{3}=\mathrm{S}+\mathrm{H}_{2} \mathrm{O}+\mathrm{CO}_{2} \text {, } \\
& \mathrm{Ni}+\mathrm{xS}^{=} \neq \mathrm{NiS}_{x}+2 x \mathrm{e}^{-} \text {. } \\
& \mathrm{NiS}_{x}+x \mathrm{H}_{2}=x \mathrm{H}_{2} \mathrm{~S}+\mathrm{Ni}
\end{aligned}
$$

the overall process is then

$$
\mathrm{H}_{2}+\mathrm{CO}_{3}^{=} \rightarrow \mathrm{H}_{2} \mathrm{O}+\mathrm{CO}_{2}+2 e^{-}
$$

Although this mechanism can explain how a poisoned nickel electrode could remain active for the oxidation of $\mathrm{H}_{2}$, the kinetic of this mechanism would be expected to be different from that of the Tafel-Volmer mechanism.

Thus far, little consistent attention has been given to the development of a sulfurresistant anode. It is obvious that this is a very challenging task, especially if the anode must 
also be capable of internal reforming. Part of the problem is a lack of agreement about the mechanism of sulfur poisoning: does this primarily affect the catalyst aurface or the melt chemistry and properties? More fundamental approaches (surface characterization techniques), combined with continued electrochemical work of the kind discussed here, may lead to development of moderately sulfur-tolerant electrode materials.

\subsection{Cathode}

\section{A. Characteristics and Operation}

The MCFC cathode, which in pre-1970 cells was made of silver or copper, is in most of the present-day cells a porous nickel mass. which in the first hours of operation is oxidized to nickel oxide and becomes lithiated.

Nickel oxide is a p-type semiconductor, which must be made conducting in order to serve as a useful cathode. In the fuel cell, the conductivity of $\mathrm{NiO}$ is enhanced by doping with lithium provided by $\mathrm{Li}_{2} \mathrm{CO}_{3}$ in the molten carbonate electrolyte. The incorporation of $\mathrm{Li}$ into $\mathrm{NiO}$ is accompanied by the creation of an electron hole. where $\mathrm{Ni}^{2+}$ is replaced by $\mathrm{Ni}^{3+}$ :

$$
(x / 2) \mathrm{Li}_{2} \mathrm{O}+(1-\mathrm{x}) \mathrm{NiO}+(x / 4) \mathrm{O}_{2} \rightarrow \mathrm{Li}_{x}^{2+} \mathrm{Ni}_{1-2 x}^{2+} \mathrm{Ni}_{x}^{3+} \mathrm{O}
$$

Typically, the conductivity increases from $0.034 \mathrm{ohm}^{-1}-\mathrm{cm}^{-1}$ to approximately $40 \mathrm{ohm}^{-1} \mathrm{~cm}^{-1}$ after doping with 2 wt\% LI at $973 \mathrm{~K} .73$ In the nickel oxide layer, an equilibrium is set up between vacancies of charge $(-2)$ in the $\mathrm{Ni}^{2+}$ lattice $\left(\mathrm{V}_{\mathrm{Ni}} \mathrm{2+}\right)$ and twice that number of $\mathrm{Ni}^{3+}$ ions (or electron vacancies):

$$
\mathrm{O}_{2}+4 \mathrm{Ni}^{2+}+4 \mathrm{e}^{-}=2 \mathrm{O}^{2-}+4 \mathrm{Ni}^{3+}+2 \mathrm{~V}_{\mathrm{Ni}}{ }^{2+}
$$

In this type of equilibrium, the concentration of vacancies, $\mathrm{V}\left(\mathrm{Ni}^{2+}\right)$, is proportional to the $1 / 6$ power of the oxygen partial pressure. Since the oxygen partial pressure is very low at the metal surface, vacancies diffuse toward this surface, where they react with the metal. This vacancy diffusion is equivalent to diffusion of $\mathrm{Ni}^{2+}$ ions and electrons from the metal to the $\mathrm{NiO} / \mathrm{melt} \mathrm{sur}-$ face. Passivation is therefore not achieved. However, in an $\mathrm{Li}^{+}$-containing melt (or aqueous solution), the corrosion rate is lower because $\mathrm{Li}^{+}$ions take the place of $\mathrm{Ni}^{2+}$ ions, whereby the vacancy concentration is decreased. "If continued, this penetration would ultimately lead to the formation of an $\mathrm{Li}_{2} \mathrm{NiO}_{2}$ or $\mathrm{LiNiO}_{2}$ layer. However, in the $\mathrm{FC}, 1-2$ atom\% of $\mathrm{Li}_{i}$ is in dynamic equilibrium with melt and oxidant gas.

During the in situ oxidation and lithiation, the original structure changes drastically. The cathode mass acquires many very small pores, in addition to the original 5-10 um size pores. This process causes a characteristic wetting patter $n$, in which very small pores are preferentially filled and larger pores remain open as gas channels. However, the walls of the larger, gas-filled, pores are wetted by electrolyte.

The performance of cathodes is, in fact, very sensitive to the degree of filling of the pore volume by electrolyte, as illustrated in Fig. 5.7A-1. The optimum filling is $20 \%$ and even then the polarization is relatively high compared to that of the anode (Table 5.4A-2).

Another remarkable feature of cathode performance is its atrong dependence on temperature (Fig. 5.6A-1). The difference in temperature dependence at various current levels is especially clear in Fig. 5.7A-2.

Polarization can be analyzed by a porous electrode model, provided the electrode kinetics of the electrode reaction are adequately known. Although insight in the reaction mechanism is still far from satisfactory, modeling has elucidated some aspects of cathode polarization. For example, the agglomerate model, which was found satisfactory to predict anode performance (see Sec. 5.6A-1), is also adequate for the cathode. 75,76 However, the cathode structure is better represented by film-covered agglomerates. The gas-channel walls are covered by electrolyte, while in the anode the electrolyte does not wet the outside of the agglomerate (see Fig. 5.7A-3).

The polarization of the cathode can become very large at very small $\mathrm{O}_{2}$ or $\mathrm{CO}_{2}$ partial pressures. 77 This behavior can, in part, be explained by the porous electrode model. Figure 5. 7A-4 illustrates the polarization predicted by this model using the peroxide-mechanism of oxygen-reduction kinetics (Sec, 5, 7B). The polarization at low $\mathrm{O}_{2}$ partial pressures is not linear; 77 such apparent limiting-current behavior is not observed at the anode (Fig. 5, 7A-5). In general, the reaction distribution in the cathode is controlled roughly equally by kinetic and mas 8 -transfer resistance. Thus, the entire film-covered area is uniformly active, which is unlike the situation in the anode, where reaction is concentrated near the meniscus (Sec. 5.6A). This fact explains the sensitivity of the cathode to flooding (over-filling).

The cathode thickness plays an important role in the polarization. Ohmic losses in the liquid and solid phases and diffusional lossee in the gas phase increase as the cathode thickness 


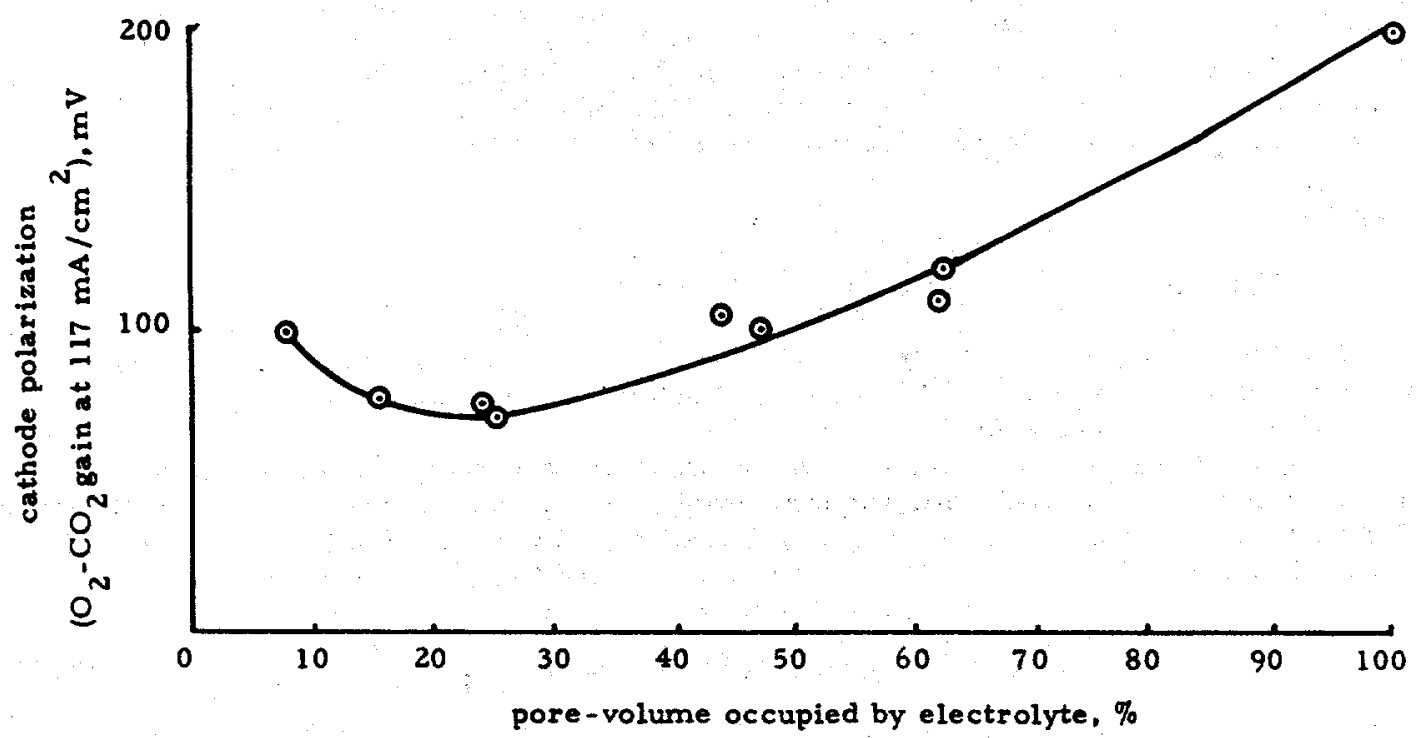

Fig. 5.7A-1. Effect of degree of pore-volume filling on cathode performance; cell temperature $=650^{\circ} \mathrm{C} .74$

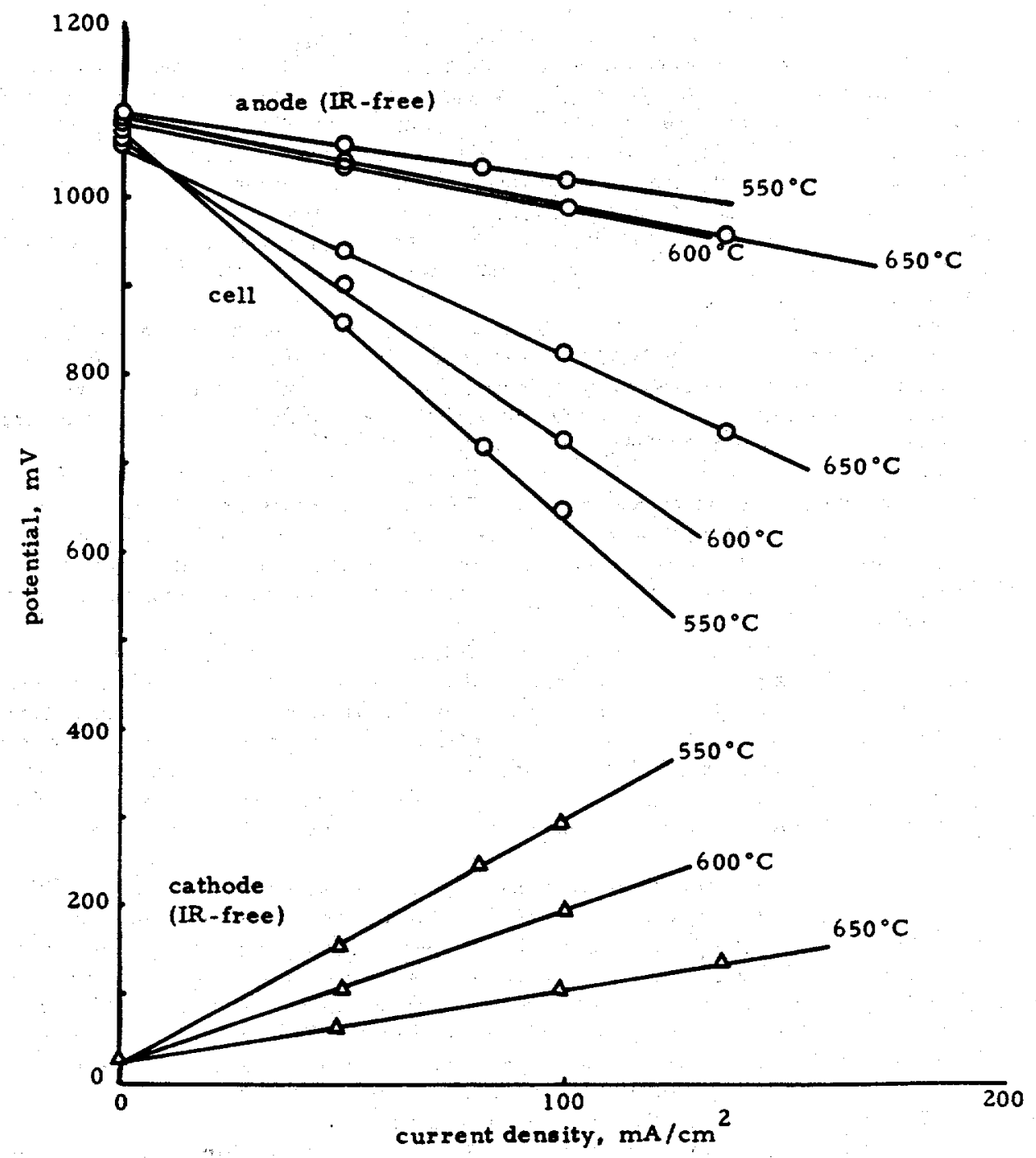

Fig. 5.7A-2. Temperature effect on components of polarization. 31 


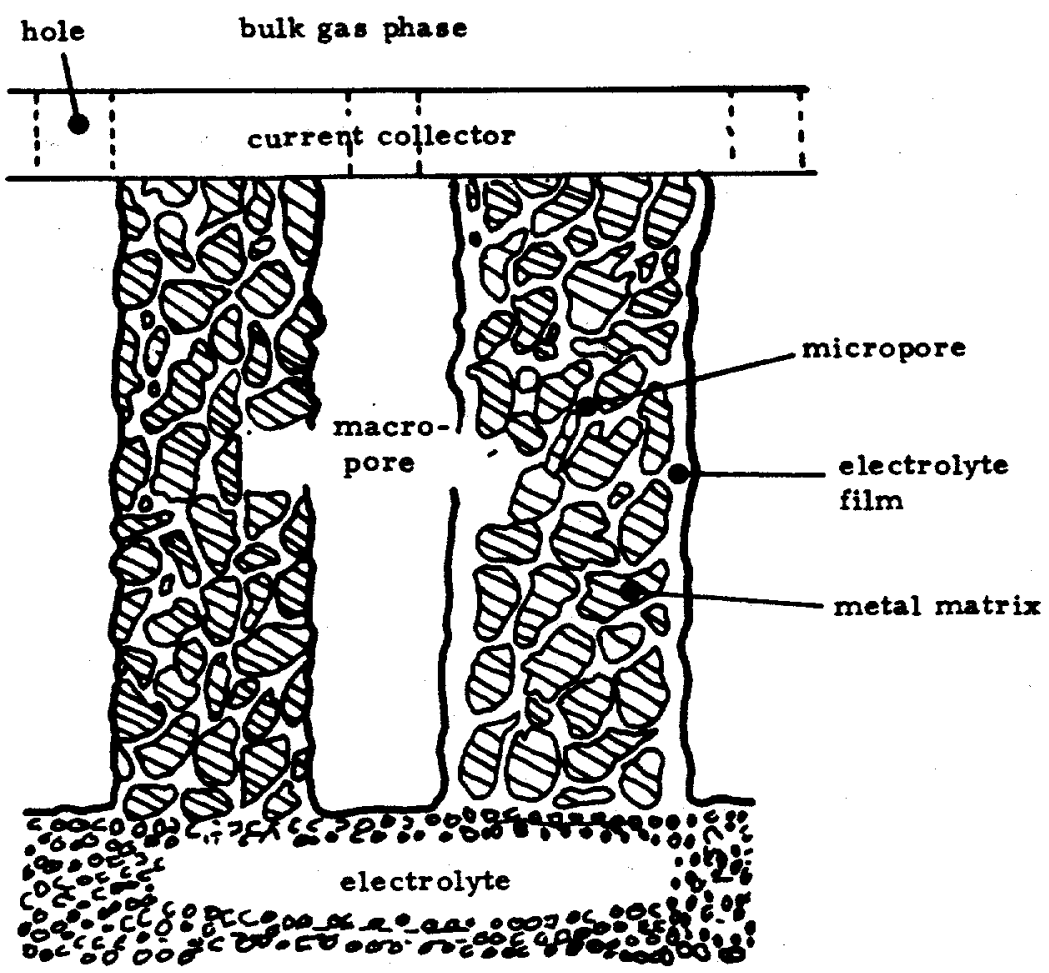

Fig. 5.7A-3. Schematic showing the agglomerate structure of the MCFC a node (left) and cathode (right). 75

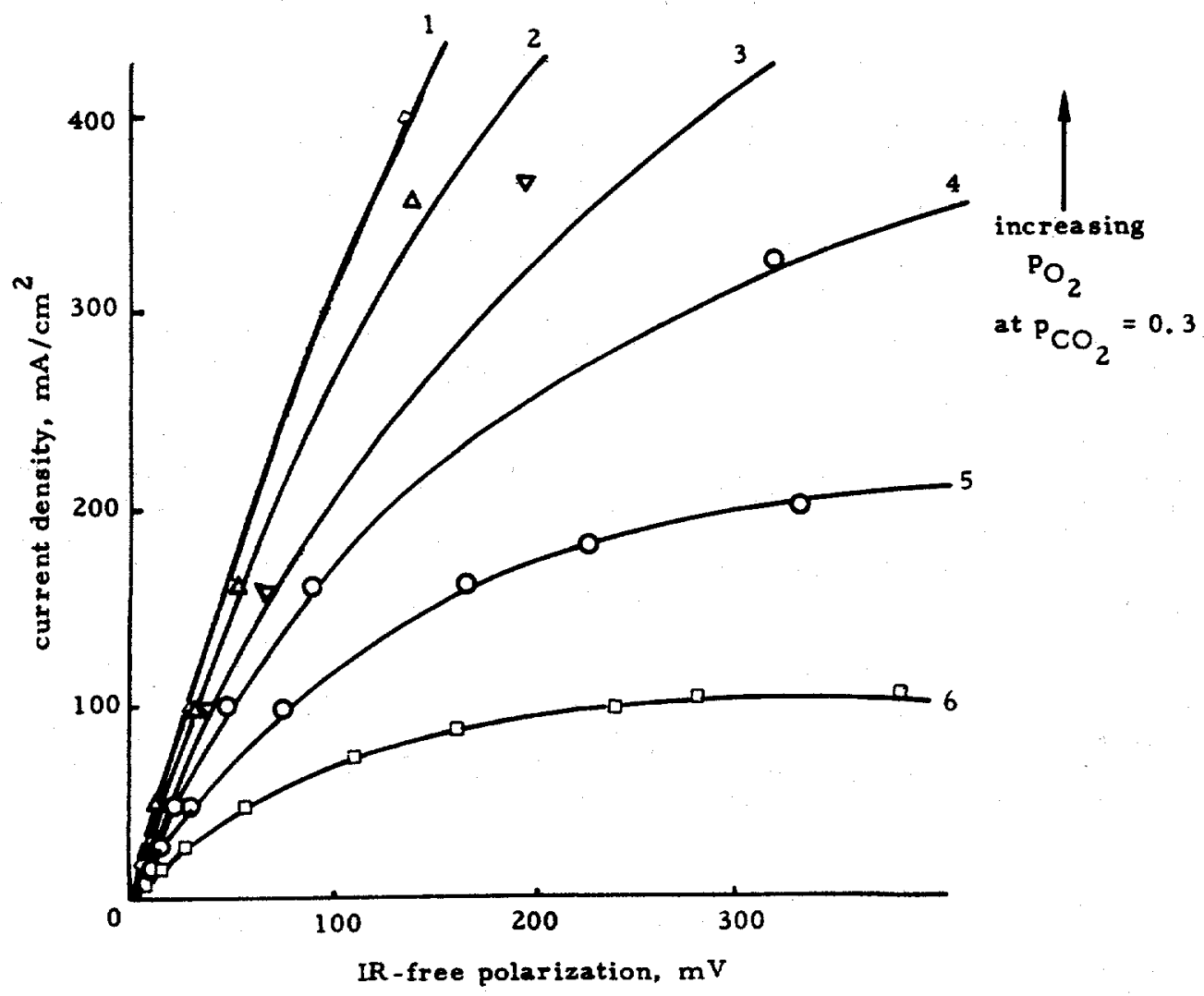

Fig. 5.7A-4. Steady-state polarization characteristics of a laboratory-scale MCFC NiO cathode at various oxygen partial pressures (increasing from 0.016 to $0.15 \mathrm{~atm}) .75$ The solid lines are model predictions according to the peroxide mechanism; $i_{0}^{0}=1.91 \mathrm{~mA} / \mathrm{cm}^{2}$. 


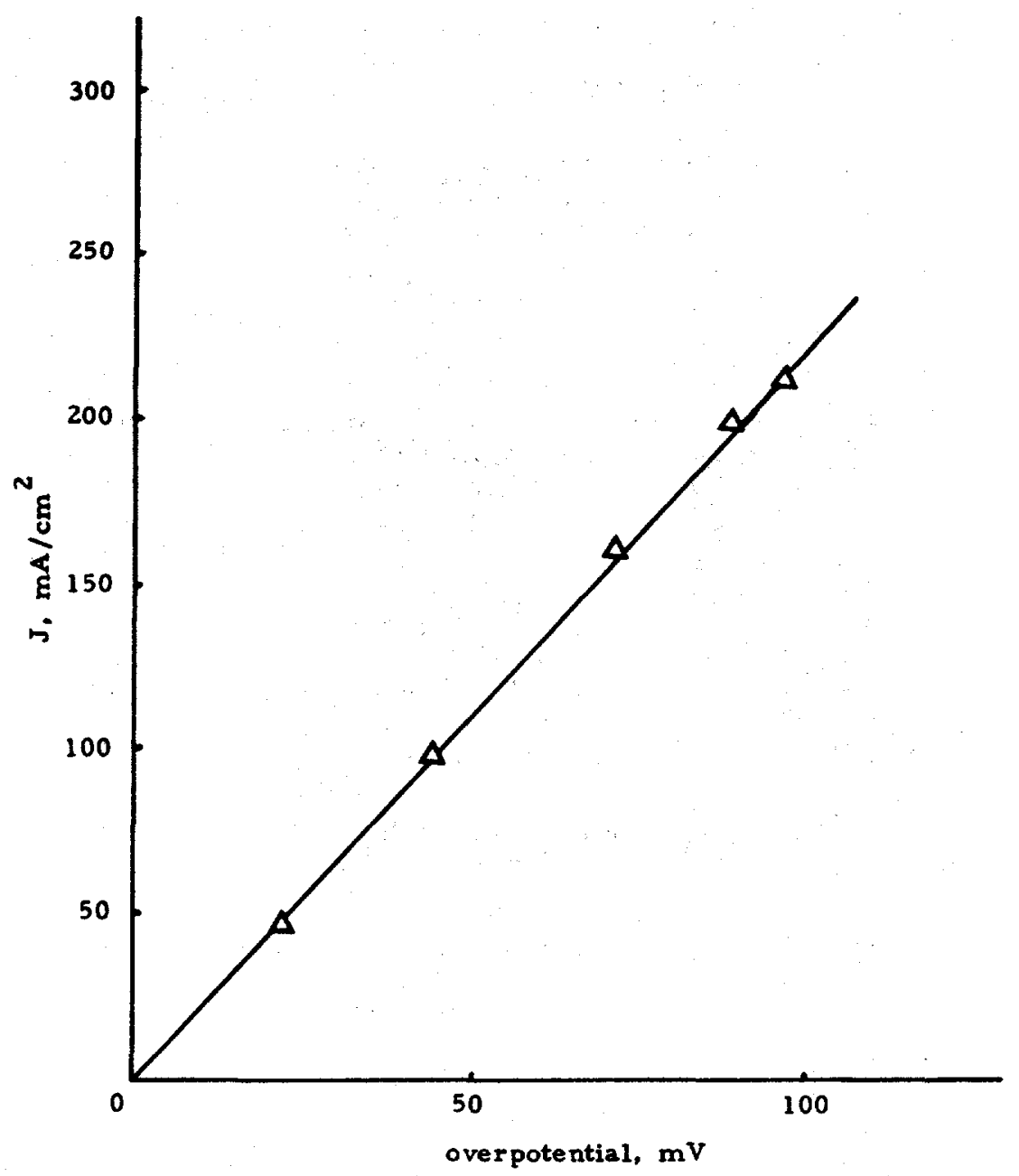

Fig. 5.7A-5. Steady-state polarization characteristics of a laboratory-scale MCFC copper anode. 75 The solid lines are model predictions according to the fuel oxidation kinetics of $\mathrm{Eq}_{\mathrm{q}}(\mathbf{2} .6 \mathrm{~B}-9)$. The experimental data $(\Delta)$ refer to $\mathrm{Cu}(\mathrm{RD}-1), i_{0}^{0}=72 \mathrm{~mA} / \mathrm{cm}^{2}, A=1500 \mathrm{~cm}^{2} / \mathrm{cm}^{3}$.

increases. Liquid-phase diffusion and activation losses decrease as the cathode thickness is increased. The optimum cathode thickness is attained when the total losses are at a minimum. Workers at UTC found that NiO cathodes perform optimally at a thickness of $0.84 \mathrm{~cm} .78$

Apart from the complicated interactions which determine its performance, the nickel oxide cathode has some drawbacks in long-term performance. One of these is strength and the other stability. The morphology changes on oxidation leave a porous masa without inherent structural strength. In stack operation, this mass is likely to be compressed excessively, with negative consequences for electrolyte distribution and performance. However, reinforcement by passivating materials, such as $316 \mathrm{s.s.}$ screens, shows promise in preventing compression. 79

Nickel dissolution is also a serious problem in long-term performance. Although nickel has a very small solubility, lithiated nickel oxide cathodes are not expected to last more than $10,000 \mathrm{~h}$ in pressurized operation with low-BTU coal gas as fuel (Sec. 5.7C). Dissolution has prompted a search for alternative cathode materials (Sec. 5.7D). In atmospheric operation, nickel oxide cathodes may prove to be satisfactorily stable for periods approaching $10,000 \mathrm{~h}$ (e.g. . in internally-reforming MCFCs).

\section{B. Mechanism of Electrode Reactions}

The rate of $\mathrm{O}_{2}$ reduction per unit area has been found to be a weak function of the electrode material and catalytic surface area. 78 However, the reduction rate depends strongly on electrolyte chemistry. Oxygen has a smaller solubility than any other MCFC reactant or product gas (except $\left.\mathrm{CH}_{4}\right) .17,61$ Its aolubility is almost completely chemical, by formation of peroxide $\left(\mathrm{O}_{2}^{-}\right)$and ouperoxide $\left(\mathrm{O}_{2}^{-}\right)$. Solubilities have been determined by Broer and co-workers 17,61 and by Appleby and van Drunen, 80 and reviewed by Selman and Maru (Ref, 21, p. 300). 
The chemical solubility of oxygen implies a strong dependence of the reacting species on the cationic composition of the melt, which has important conaeguences for the reaction mechanism, as was pointed out and demonstrated by Appleby and Nicholson, 81 Uchida, 82 and Lu. 50 ism is:

In $\mathrm{Li}_{2} \mathrm{CO}_{3}$ or Li-rich melts, peroxide ion is predominant, and a likely reaction mechan-

$$
\begin{gathered}
2 \mathrm{CO}_{3}^{=}+\mathrm{O}_{2}=\mathrm{CO}_{2}^{=}+2 \mathrm{CO}_{2} . \\
\text { (peroxide formation) } \\
\mathrm{O}_{2}^{=}+2 e^{-} \rightarrow 2 \mathrm{O}^{=}
\end{gathered}
$$

(the fir at electron transfer is the rate-determining step)

$$
20=2 \mathrm{CO}_{2}=2 \mathrm{CO}_{3}^{=}
$$

The exchange-current density for the peroxide mechanisms is then given by

$$
i_{0} \sim\left(\mathrm{O}_{2}\right)^{0.375}\left(\mathrm{CO}_{2}\right)^{-1.25}
$$
is:

In $\mathrm{K}_{2} \mathrm{CO}_{3}$-rich melts, superoxide is predominant. The proposed superoxide mechanism

$$
\begin{gathered}
2 \mathrm{CO}_{3}^{-}+3 \mathrm{O}_{2}=4 \mathrm{O}_{2}^{-}+2 \mathrm{CO}_{2} . \\
\text { (superoxide formation) }
\end{gathered}
$$

$$
\mathrm{O}_{2}^{-}+\mathrm{e}^{-} \rightarrow \mathrm{O}_{2}^{=},
$$

(rate-determining step)

$$
\begin{gathered}
\mathrm{O}_{2}^{=}+2 \mathrm{e}^{-}=20^{=}, \\
2 \mathrm{O}^{=}+2 \mathrm{CO}_{2}=2 \mathrm{CO}_{3}^{=}
\end{gathered}
$$

in this case,

$$
i_{0} \sim\left(O_{2}\right)^{0.625}\left(\mathrm{CO}_{2}\right)^{-1.25}
$$

As a variant on the se schemes, a mechaniem involving a hypothetical percarbonate ion, $\mathrm{CO}_{4}=$, has been suggested:

$$
\frac{1}{2} \mathrm{O}_{2}+\mathrm{CO}_{3}=\mathrm{CO}_{4}^{=} \text {. }
$$

(percarbonate formation)

$$
\mathrm{CO}_{4}+\mathrm{e}^{-}=\mathrm{CO}_{3}^{=}+\mathrm{O}^{-}
$$

(rate-determining step)

$$
\mathrm{O}^{-}+\mathrm{CO}_{2}+\mathrm{e}^{-}=\mathrm{CO}_{3}^{=}
$$

This mechanism yields

$$
i_{0} \sim\left(O_{2}\right)^{0.375}\left(\mathrm{CO}_{2}\right)^{-0.25}
$$

The effect of cationic composition is especially evident in kinetic measurements employing potential sweep techniques. $81,82,50$ In Fig. $5.7 \mathrm{~B}-1$, the current response is plotted against potential with respect to the $\left(\mathrm{O}_{2} / \mathrm{O}^{\circ}\right)$ reference electrode, i, e. accounting for the relative basicity of the various melts. Figure 5.7B-1 illustrates that $\mathrm{K}$-containing melts have the advantage of almost simultaneous reaction of both peroxide and superoxide within the $100-150 \mathrm{mV}$ polarization range typical for MCFC cathodes.

Thus, the $\mathrm{Ii}_{2} \mathrm{CO}_{3}-\mathrm{K}_{2} \mathrm{CO}_{3}$ melt presently used by most developers appears to be a favorable choice from the kinetic viewpoint. Workers at UTC 83 have measured the equilibrium 


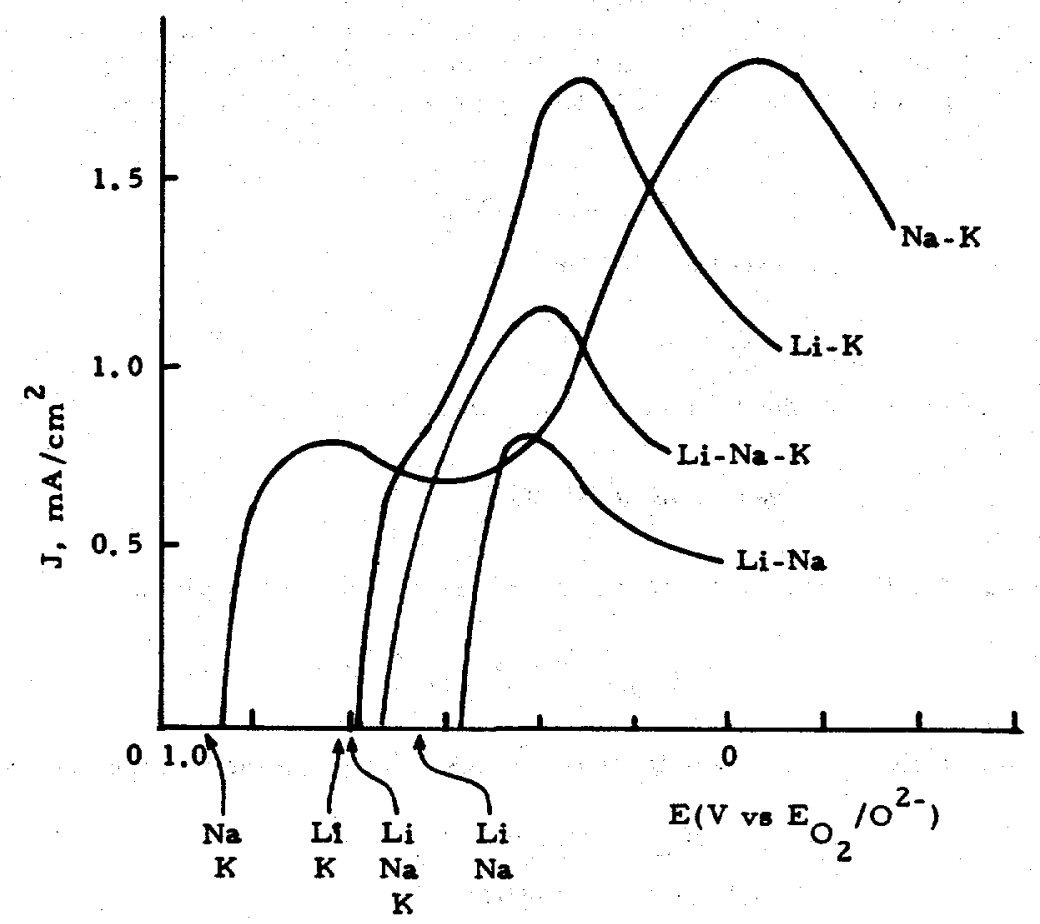

Fig. 5.7B-1. Voltammetric characteristics at $750^{\circ} \mathrm{C}$ of a gold electrode in various carbonate eutectics, under a $9 \mathrm{O}_{2}: 1 \mathrm{CO}_{2}$ atmosphere. 82

solubilities for $\mathrm{O}_{2}, \mathrm{O}_{2}^{-}$, and $\mathrm{O}_{2}^{=}$in a mixture of $62 \mathrm{~mol} \% \mathrm{LiCO}_{3}$ and $39 \mathrm{~mol}_{2} \mathrm{~K}_{2} \mathrm{CO}_{3}$ at $650^{\circ} \mathrm{C}$. The total solubility of oxygen was found to be

$$
s=\left(x_{O_{2}}\right)+\frac{1}{2}\left(x_{O_{2}}\right)+\frac{3}{4}\left(x_{O_{2}^{-}}\right)
$$

where $X_{i}$ is the equivalent mole fraction of species $i$. This relation may be rewritten as

$$
S=K_{H} P_{2}+\frac{1}{2} K_{1} P_{O_{2}}^{1 / 2} / \mathrm{PCO}_{2}+\frac{3}{4} K_{2} P_{O_{2}}^{3 / 4} / P_{C O} O_{2}^{1 / 2}, \quad(5.7 B-17)^{1 / 4}
$$

where $K_{H}$ is Henry's constant and $K_{1}$ and $K_{2}$ are appropriate equilibrium constants. The UTC data did not allow determination of $\mathrm{K}_{1}$ and $\mathrm{K}_{2}$. The dominant oxygen component was found to be $O_{2}^{-}$while $O_{2}^{=}$and $O_{2}$ were not found in solution in appreciable amounts.83

While half-cell data at UTC were reported to be consistent with the superoxide mechanism of (5.7A-7) through (5.7A-11),83 other experimental resulti are not 80 unambiguous. In particular, recent kinetic measurements by the potential-step technique 50 indicate exchange-reaction orders which cannot be reconciled with any of the three mechanisms discussed above.

Even more importantly, the polarization of porous nickel oxide cathodes determined experimentally in laboratory-scale cells, using $\mathrm{Li}_{2} \mathrm{CO}_{3}-\mathrm{K}_{2} \mathrm{CO}_{3}$ tiles, 77 cannot be adequately fitted with superoxide kinetics over a large range of $\mathrm{O}_{2}$ and $\mathrm{CO}_{2}$ partial pressures.

Some of these discrepancies may be explained by the slow kinetics of the recombination reaction according to reactions $(5.7 A-5)$ or $(5.7 A-10)$ :

$$
\mathrm{O}=\mathrm{CO}_{2}=\mathrm{CO}_{3}=
$$

That this reaction is slow was demonstrated by $L u, 50$ who measured its rate constant. This fact has important implications for MCFC kinetics.

Much work remains to be done to clarify the oxygen-reduction mechaniam in molten carbonates. In the first place, the kinetic measurements referred to above were limited to the generally used $\mathrm{Li}_{2} \mathrm{CO}_{3}-\mathrm{K}_{2} \mathrm{CO}_{3}$ eutectic; they should be extended to other melt compositions (not necessarily limited to $\mathrm{Li}-\mathrm{Na}-\mathrm{K}$ ) in order to arrive at a comprehensive picture of the melt 
chemistry. From Fig. 5.7B-1, in combination with the performance map of Fig. 5.5B-2, it is clear that the electrolyte composition can be further optimized on a rational basis.

An attempt should also be made to obtain spectroscopic evidence for the various species in the melt and how they participate in the reaction mechanism. These studies are very difficult to perform, aince silicates are rapidly attacked by carbonate melt or vapor; howe ver, the total absence of such techniques in elucidating reaction mechanisms is regrettable and should be corrected.

\section{Cathode Dissolution}

The severe oxidizing conditions at the cathode limit the number of metals that are suitable electrocatalysts. Silver and copper, although stable and kinetically very satisfactory, had to be abandoned because they tended to dissolve in the electrolyte. ${ }^{7}$ Lithiated NiO, adopted as a cathode material in the early 1970s, appeared to solve this problem. Nickel oxide cathodes survived for over $40,000 \mathrm{~h}$ in the early experimental IGT cells with a rather thick, hot-pressed electrolyte. However, work at UTC78 showed that nickel oxide tends to dissolve after all, especially in cells with thin tape-cast matrix layers. The solubility of nickel, although far less than that of silver or copper, is finite (Fig. 5.7 C-1).

The problem here is not cathode thinning, since the total loss of cathode material would be less than $10 \%$ over $40,000 \mathrm{~h}$. Rather, the small amounts of dissolved NiO may cause the electrolyte to become electronically conducting. Metallic nickel granules are deposited throughout the melt upon reduction by dissolved hydrogen diffusing into the melt: 78

$$
\begin{gathered}
\mathrm{NiO}+\mathrm{CO}_{2} \rightleftharpoons \mathrm{NiCO}_{3} \rightleftharpoons \mathrm{Ni}^{++}+\mathrm{CO}_{3}= \\
\mathrm{NiCO}_{3}+\mathrm{H}_{2} \rightarrow \mathrm{Ni}+\mathrm{H}_{2} \mathrm{O}+\mathrm{CO}_{2} .
\end{gathered}
$$

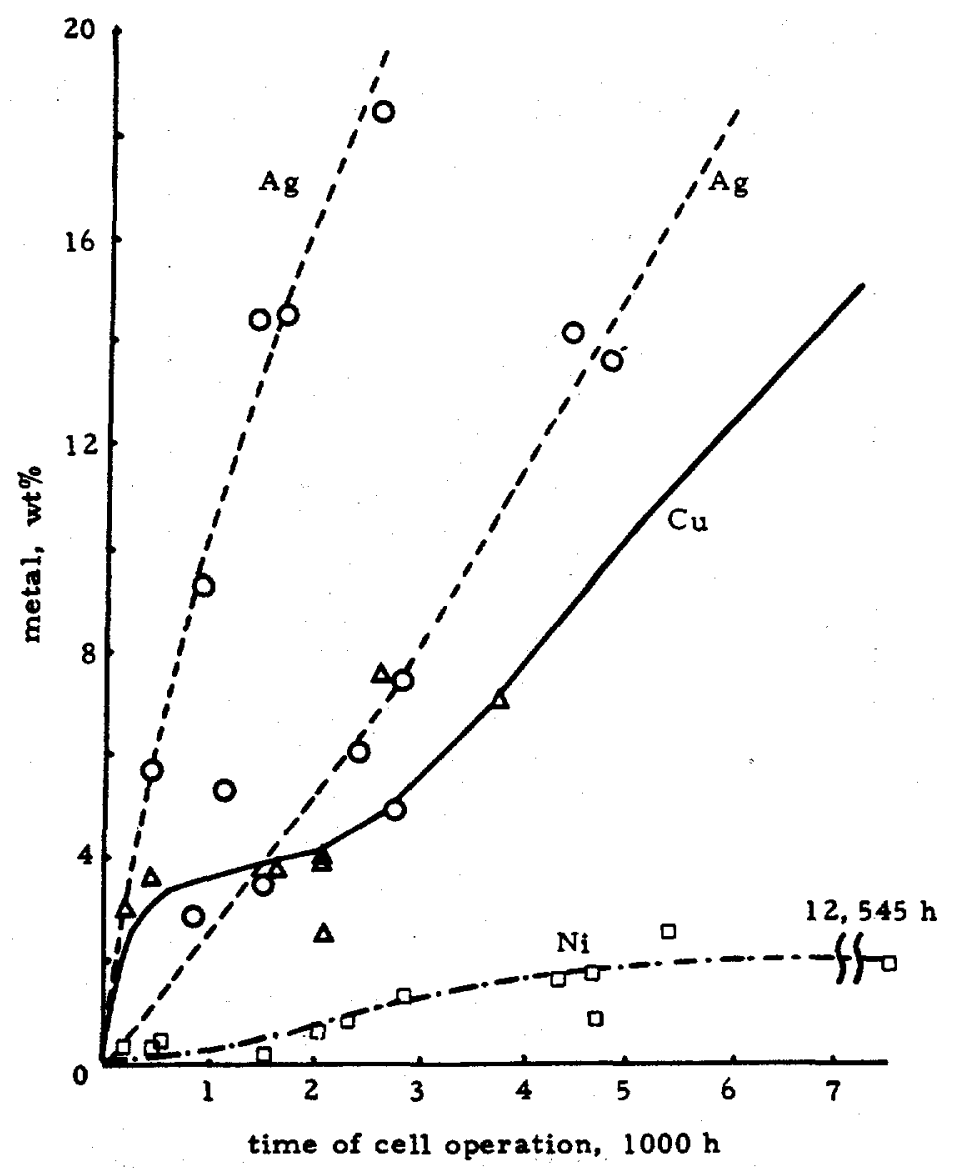

Fig. 5.7C-1. Solubility of metals in carbonate during operation as FC cathodes in ternary electrolyte at $700^{\circ} \mathrm{C}$; the tile was 0.250 inch thick. 84 
Nickel oxide has a markedly increased solubility at increased PCO, (see Fig. 5.7C-2). Lowering $\mathrm{PCO}_{2}$ auficiently $(0.2$ to $0.45 \mathrm{~atm})$ to ensure adequate cathode lifetimes results in a cell voltage drop of $60-90 \mathrm{mV}, 85$ which is not acceptable.

The solubility of NiO increases with temperature (Fig. 5.7C-3) but seems to be a weak function of electrolyte composition. 85,86 The $\mathrm{Ni}^{2+}$ ion saturates the electrolyte $(5-10 \mathrm{ppm}$ at $1 \mathrm{~atm}$ ) near the cathode and is reduced to metal as it approaches the anode. A concentrationdriven flux of $\mathrm{Ni}^{2+}$ from the cathode to the anode defines the distribution of $\mathrm{Ni}^{2+}$ and allows $\mathrm{Ni}$ to deposit throughout the electrolyte. The process is therefore more rapid in thin electrolyte tiles.

There is evidence that the dissolution mechanism is actually more complex than is shown by Eqs. (5.7C-1) and $(5.7 \mathrm{C}-2)$. Elementary steps suggested by workers at UTC 78,87 include

$$
\underline{\mathrm{Ni}}^{2+}+\frac{1}{2} \mathrm{Li}_{2} \mathrm{CO}_{3}+\frac{1}{4} \mathrm{O}_{2} \Rightarrow \underline{\mathrm{Ni}}^{3+}+\underline{\mathrm{Li}}^{+}+\underline{\mathrm{O}}^{2}+\frac{1}{2} \mathrm{CO}_{2}
$$

and

$$
\underline{\mathrm{Ni}}^{2+}+\mathrm{Li}^{+}+\mathrm{CO}_{3}^{=}=\underline{\mathrm{Ni}}^{3+}+\underline{\mathrm{Li}}^{+}+\underline{\mathrm{O}}^{=}+\mathrm{CO}_{2}+\mathrm{e}^{-}
$$

where the underline - identifies species in the solid phase. Reaction (5.7C-4) is a lithiation and oxidation reaction. The electrode material becomes

$$
\mathrm{Ni}_{1-2 x^{2+}}^{2 \mathrm{Ni}_{x}^{3+}} \mathrm{Li}_{x}^{+} \mathrm{O}
$$

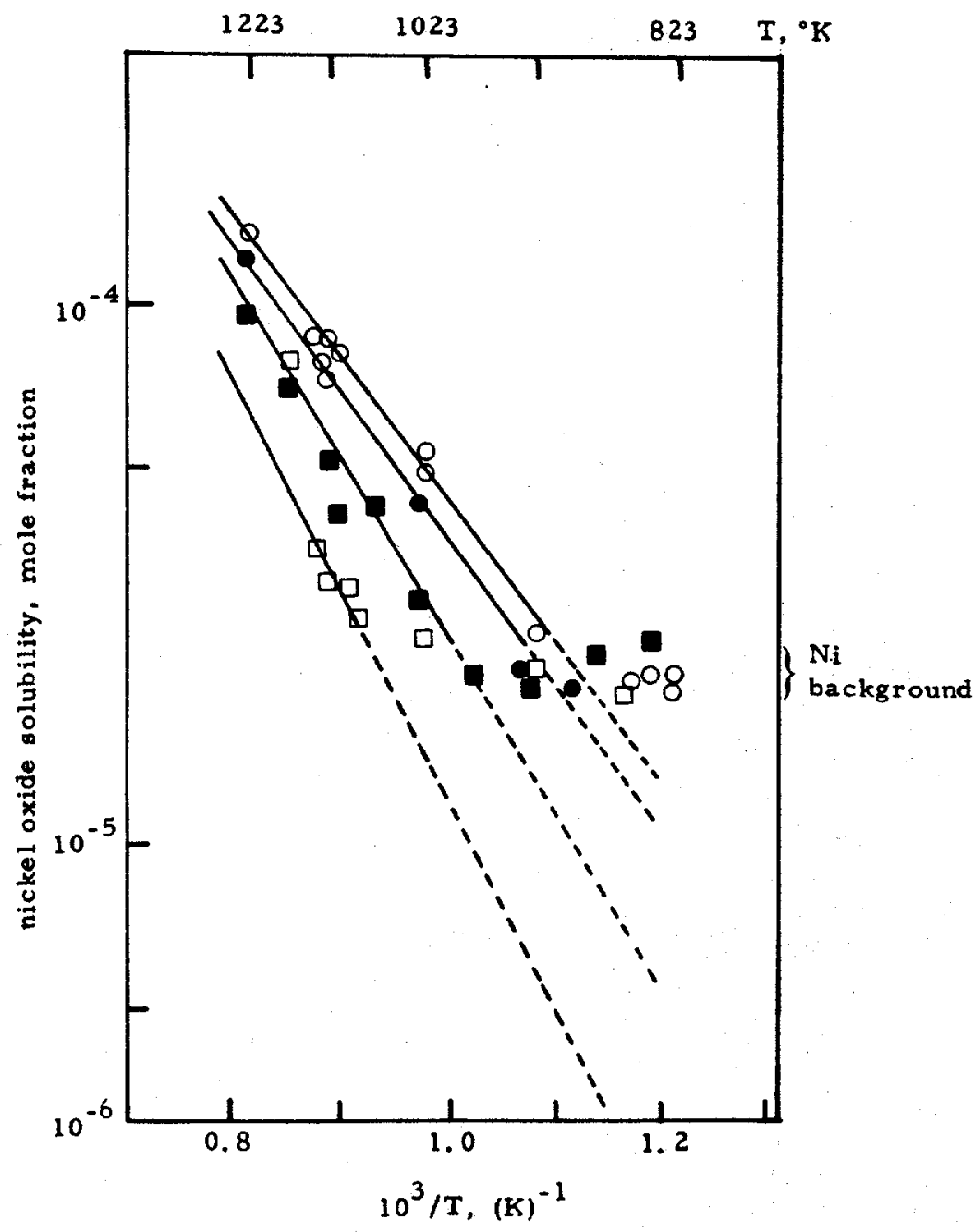

Fig. 5.7C-2. The NiO solubility is plotted 28 a function of temperature for representative cathode-gas compositions. 88 The symbols refer to the following compositions: $\mathrm{O}=64.6 \% \mathrm{CO}_{2}+32.3 \% \mathrm{O}_{2}+3.1 \% \mathrm{H}_{2} \mathrm{O} ;=48.5 \% \mathrm{CO}_{2}+$ $24.2 \% \mathrm{O}_{2}+3.1 \% \mathrm{H}_{2} \mathrm{O}+$ bal $\mathrm{N}_{2} ; \mathbf{I}=29.1 \% \mathrm{CO}_{2}+14.2 \% \mathrm{O}_{2}+3.1 \% \mathrm{H}_{2} \mathrm{O}+$ bal $\mathrm{N}_{2}: \mathrm{O}=5.8 \% \mathrm{CO}_{2}+2.9 \% \mathrm{O}_{2}+3.1 \% \mathrm{H}_{2} \mathrm{O}+$ bal $\mathrm{N}_{2}$. 
as the result of reactions $(5,7 \mathrm{C}-3)$ and $(5,7-4)$. Nickel may dissolve from this material and form $\mathrm{Ni}^{2+}$. $\mathrm{Ni}^{3+}, \mathrm{NiO}_{2}^{-}$, or $\mathrm{NiO}_{2} ; \mathrm{NiO}_{2}^{=}$is not present in appreciable concentration, except at very low $\mathrm{PCO}_{2}$. A dissolution process for $\mathrm{Ni}^{2+}$ is reaction $(5.7 \mathrm{C}-1)$; dissolution processes for
$\mathrm{Ni}{ }^{3}$ are

$$
\underline{\mathrm{Ni}}^{3+}+\underline{\mathrm{Li}}^{+}+2 \mathrm{O}^{=}+2 \mathrm{CO}_{2} \rightleftharpoons \frac{1}{2} \mathrm{Li}_{2} \mathrm{CO}_{3}+\frac{1}{2} \mathrm{Ni}_{2}\left(\mathrm{CO}_{3}\right)_{3}
$$

and

$$
\underline{\mathrm{Ni}}^{3+}+\underline{\mathrm{Li}}^{+}+\underline{2 O}^{=} \neq \mathrm{LiNiO}_{2}
$$

The total solubility of $\mathrm{Ni}$ is the sum of the solubilities of $\mathrm{Ni}^{2+}$ and $\mathrm{Ni}^{3+}$.

The preceding mechanism is by no means definitively established. Recently, some fundamental work on nickel solubility in carbonate has started, stimulated by the cathode-lifetime problem. 86 This work promises to resolve important practical questions, e.g., about the temperature dependence of the solubility: retrograde effects from those of Figs. 5.7C-2 and 5.7C-3 have occasionally been observed. Such effects may be caused by changes in the alkalinity of the carbonate melt, which depends on temperature, water partial pressure and $\mathrm{CO}_{2}$ partial pressure. A systematic investigation of these factors is highly desirable. A theory of solubility of metal oxides in carbonate melt $B$, with adequate predictive capacity, is urgently needed.

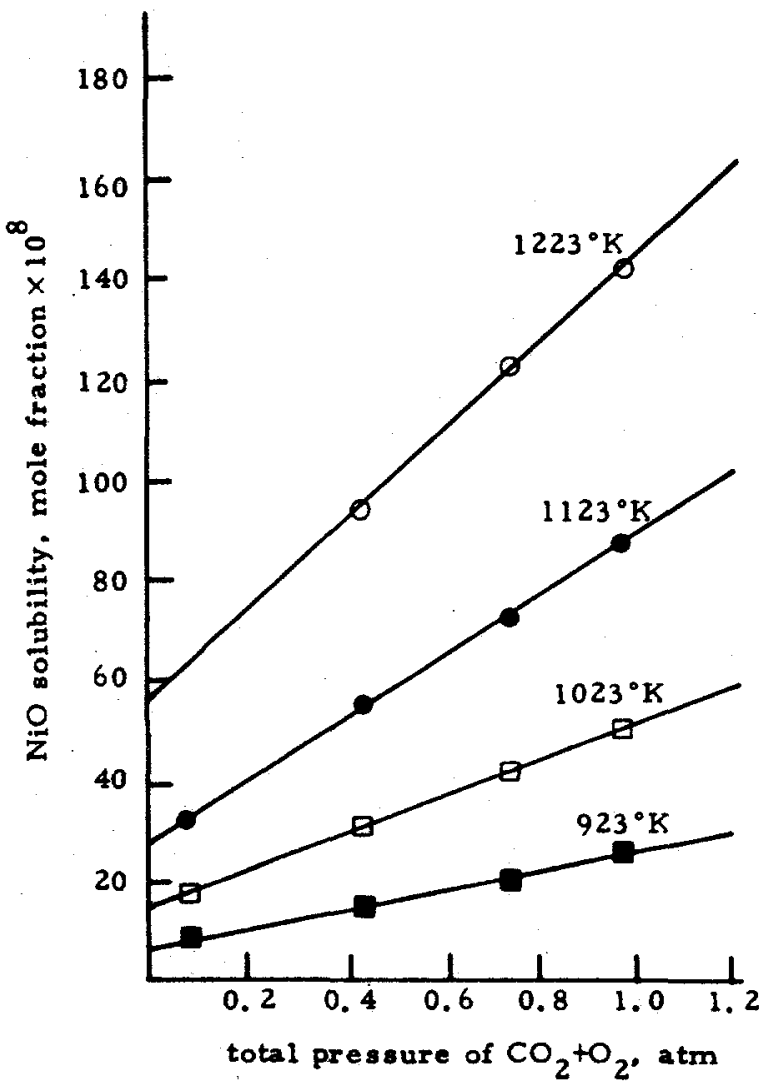

Fig. 5.7C-3. The NiO solubility is plotted as a function of temperature and total pressure of $\mathrm{CO}_{2}$ and $\mathrm{O}_{2}$ with $\mathrm{PCO}_{2} / \mathrm{PO}_{2}=2.88$

\section{Alternative Cathode Materials}

Even before the slow dissolution of nickel oxide was generally accepted as a fact, work was conducted at Ceramatec, Inc., on $\mathrm{NiO}$ substitutes, with the objective of reducing component costs. Materials examined included doped $n$-type perovskites and related compounds such as $\mathrm{CaTiO}_{3}$ and $\mathrm{SrTiO}_{3}$ doped with $\mathrm{Nb}^{5+}$, and $\mathrm{PbZrO}_{3}$ doped with $\mathrm{Nb}^{5+}$ or $\mathrm{Ta}^{5+}$. While these compounds showed good stability, their electronic conductivity at cathode potential was not sufficiently high to give adequate performance. 90

This work is being continued with a study of barium ferrites and yttrium iron garnets, which should not require doping for electronic conduction. These studies appeared to show that 
p-type perovskites may be more promising from the conductivity viewpoint. LanthanumGroup VIII compounds have been examined from this viewpoint. 90 Studies at UTC (Ref, 87, p.6-1) suggest that $\mathrm{LaMnO}_{3}$ is highly conductive and active in $\mathrm{O}_{2}$ reduction; however, it is reduced at cathode potential. The materials $\mathrm{LaNiO}_{3}$ and $\mathrm{LaCoO}_{3}$ were also very active catalysts, but lanthanum appears to be lost from these compounds and reacts with the $\mathrm{LiAlO}_{2}$ matrix support. Requirements for an acceptable cathode material $90^{\circ}$ have been discussed by Pierce and co-workers.79 These are: (1) not too low an electronic conductivity $(>1 \mathrm{mho} / \mathrm{cm}$ according to $\mathrm{GE}^{89}$ ); (ii) chemical and physical stability in both oxidant and electrolyte environments; (iii) low solubility and no tendency to precipitate upon reduction; (iv) proper wettability in the oxidant environment, so that the cathode surface is efficiently used; $(v)$ adequate catalysis of reduction of $O_{2}$; finally, (vi) thermal expansion characteristics compatible with those of other components. Following the NiO cathode studies at UTC, workers at ANL began investigations of cathode dissolution and migration. Emphasis was placed on identification of alternative cathode materials. 91 A spray-drying process was developed to synthesize alternative cathode materials. Figure 5.7D-1 shows the approach devised at ANL to develop alternative cathode materials. Many materials have been assessed or tested for stability in a multitude of anticipated operating environments. Among the metals which dissolve, $\mathrm{Ni}, \mathrm{Cu}$, and $\mathrm{Co}$ should deposit at the anode, while $M n, C r, Z_{n}, Z_{r}, Y, A l, U, T i$ and $F e$ should not. The following materials were found to be stable: $\mathrm{Li}_{2} \mathrm{MnO}_{3}, \mathrm{LiFeO}_{2}, \mathrm{ZnO}, \mathrm{LiTiO}_{3}, \mathrm{Li}_{3} \mathrm{TaO}_{4}, \mathrm{LiCrO}_{2}, \mathrm{MgO}, \mathrm{K}_{2} \mathrm{WO}_{4}, \mathrm{Li}_{3} \mathrm{VO}_{4}$, and $\mathrm{Li}_{2} \mathrm{SnO}_{3} .91$ The stable materials were then prepared for conductivity measurements (see Fig. 5.7D-2). The materials were doped to promote conductivity. Adequate conductivities were achieved in $\mathrm{Mg}-\mathrm{Li}_{2} \mathrm{MnO}_{3}, \mathrm{Mn}-\mathrm{LiFeO}, \mathrm{Zr}-\mathrm{ZnO}$. Solubilities of the doped species were verified over a broad range of operating conditions. Evaluation of migration was determined by post-test examinations of cells using cathodes made from these materials. Table 5.7D-1 shows the ANL results. The compounds $\mathrm{Li}_{2} \mathrm{MnO}_{3}$ and $\mathrm{LiFeO}_{2}$ were selected as promising materials and workers at ANL concentrated on developing these materials for use in cell stacks. A systematic stady of the effect of dopant incorporation on stability and conductivity in materials such as $\mathrm{LiFeO} 2$ and $\mathrm{Li}_{2} \mathrm{MnO}_{3}$ has been going on since February 1984.

IDENTIFY STABLE MATERLALS

(rich cathode-ges eavironnent)

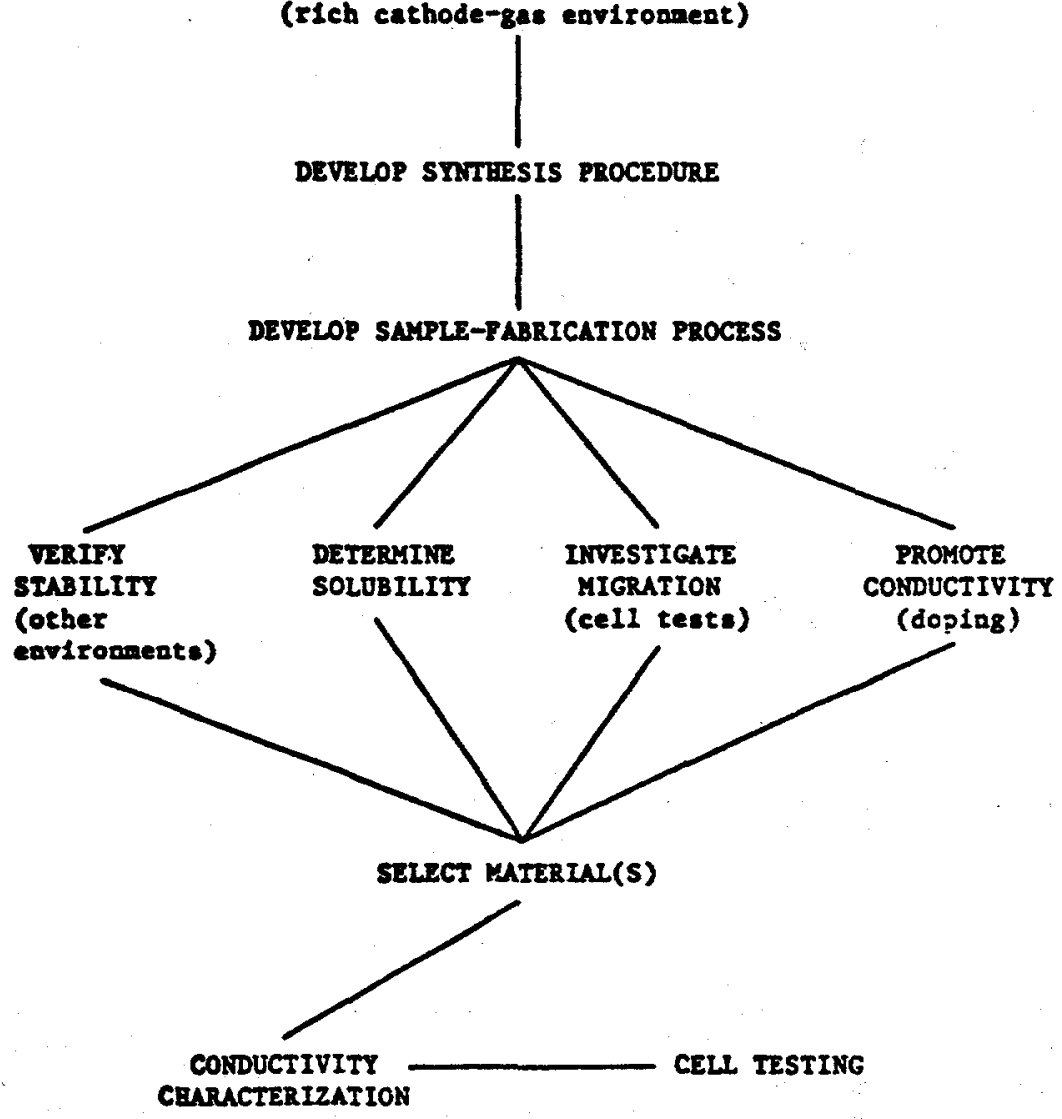

Fig. 5.7D-1. Flow chart of the ANL approach to develop alternative cathode materials. 91 
HOMOGENEOUS MIXTURE OF PARENT/DOPANT OXIDES

(interdiffusion, reaction with molten carbonate under cathode environment)

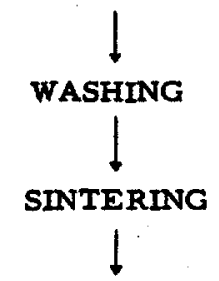

CHEMICAL AND X-RAY DIFFUSIONAL ANALYSIS

MEASUREMENT OF CONDUCTIVITY BY FOUR-PROBE TECHNIQUE

Fig. 5.7D-2, Flow chart to explain the preparation of candidate cathode materials for conductivity measurements at ANL, 91

Table 5.7D-1. Properties of possible alternative cathode materials examined at ANL.

\begin{tabular}{|c|c|c|c|c|}
\hline Material & $\begin{array}{l}\text { Conductivity, } \\
\text { mho/em at } \\
650^{\circ} \mathrm{C}\end{array}$ & Solubility & $\begin{array}{l}\text { Migration } \\
\text { (NiO as ref) }\end{array}$ & Comments \\
\hline $\begin{array}{l}\mathrm{Li}_{2} \mathrm{MnO}_{3} \\
\text { (Mg-doped) }\end{array}$ & 0.2 & $\sim 1 / 10$ of that of $\mathrm{NiO}$ & undetectable & $\begin{array}{l}\text { Conductivity was not as } \\
\text { good as expected; } \mathrm{Mg} \\
\text { dissolves in the electro- } \\
\text { lyte and the removal of } \\
\text { excess } \mathrm{MgO} \text { could be a } \\
\text { problem. }\end{array}$ \\
\hline $\begin{array}{l}\mathrm{LiFeO}_{2} \\
\text { (Mn-doped) }\end{array}$ & 0.2 & $\begin{array}{l}\text { About the same as } \\
\text { NiO, but does not } \\
\text { operate as a reduc- } \\
\text { ing medium. Fe has } \\
\text { high solubility when } \\
\text { humid cathode gases } \\
\text { and Li-rich electro- } \\
\text { lytes are introduced. }\end{array}$ & low & $\begin{array}{l}\text { Conductivity improves } \\
\text { when the material is } \\
\text { synthesized in air. } \\
\text { There is a possibility of } \\
\text { finding better dopants. }\end{array}$ \\
\hline $\begin{array}{l}\mathrm{ZnO} \\
\text { (Zr-doped) }\end{array}$ & 0.33 & $\begin{array}{l}\text { About } 10 \text { times that } \\
\text { of NiO. The high } \mathrm{Z}_{\mathrm{n}} \\
\text { solubility was in- } \\
\text { duced by } \mathrm{Zr} \text {-doping. } \\
\text { Humidity and large } \\
\mathrm{PCO}_{2} \text { in the cathode } \\
\text { gas and Li-rich } \\
\text { electrolytes cause } \\
\text { higher } \mathrm{Z}_{\mathrm{n}} \text { solubility. }\end{array}$ & not tested & $\begin{array}{l}\mathrm{Zn} \text { was found to be in- } \\
\text { corporated into the } \\
\mathrm{LiAlO}_{2} \text { matrix. }\end{array}$ \\
\hline
\end{tabular}

In these recent explorations of alternative cathode materials, an intuitive, Edisonian, approach is used for lack of an adequate theory for predicting the properties of doped compounds such as perovskites and mixed oxides. The intuitive strategy is reasonably helpful in aiming for adequate electronic conductivity. However, it fails completely in assesaing stability and solubility in the carbonate electrolyte. There is an urgen need for systematic and fundamental research in this area. 


\section{A. Stack Design and Operation}

Design and operation of the MCFC stack poses problems over and above those associated with single-cell operation. The key design is that of either external manifolding (schematically shown in Fig. 5.8A-1, with cross-flow gas configuration) or internal manifolding.

The internal manifolding design, adopted by GE (Fig. 5.8A-2), featured counterflow fuel and oxidant gas configuration but also allows coflow. 92 Advantages claimed are: (1) minimal electrolyte paths between cells, which cause shunt currents and electrolyte migration (see below); (2) absence of sliding seals, with negligible gas leaks up to 5 pai pressure differentials; (3) uniform ambient gas contact at the external edges of electrolyte tiles.

In representative tests, the $12^{\prime \prime}$ stack with internal manifolding approached the theoretical value for the open-circuit voltage per cell $(973 \mathrm{mV}$ vs $984 \mathrm{mV})$ but performed poorly under load (550-600 mV at $160 \mathrm{~mA} / \mathrm{cm}^{2}$ ). Problems were encountered with internal manifolding; most manufacturers appear to reject this design option.

Figure 5.8A-1 shows an exploded view of the MCFC stack concept developed at ERC. 93 It is a cross-flow configuration with external manifolding. The electrolyte matrix extends beyond the electrodes to form wet seals, which counteract overboard gas leakage at the edge. The bipolar plate serves 28 a dual channel for reactant and product gases and also as a current jumper.

External manifolding requires an insulator ceramic material that is stable with respect to carbonate and of minimal porosity to avoid shunt currents. 94 In practice, zirconia densified by filling with another ceramic component is used. However, this material still has appreciable capillary capacity for electrolyte. As a result, migration of electrolyte from the positive end to the negative end of the stack takes place when a cell stack is under load.

Migration is caused by the different mobilities of ions in the electric field set up inside the manifold as a result of cell stacking. $\mathrm{Li}^{+}$is transported more rapidly than $\mathrm{K}^{+}$toward the negative end of the stack, where it accumulates, thereby changing the cationic composition locally. 32 Since $\mathrm{CO}_{3}^{=}$migrates to the positive end of the stack, electroneutrality can be maintained only by faradaic reaction of $\mathrm{O}_{2}$ and $\mathrm{CO}_{2}$ to form $\mathrm{CO}_{3}^{=}$at the negative end of the stack, while the opposite electrode reaction occurs at the positive end. Overall, these processes amount to a displacement of electrolyte from the positive to the negative end of the stack. The electrodes at the negative end of the cell stack become flooded, while those at the positive end

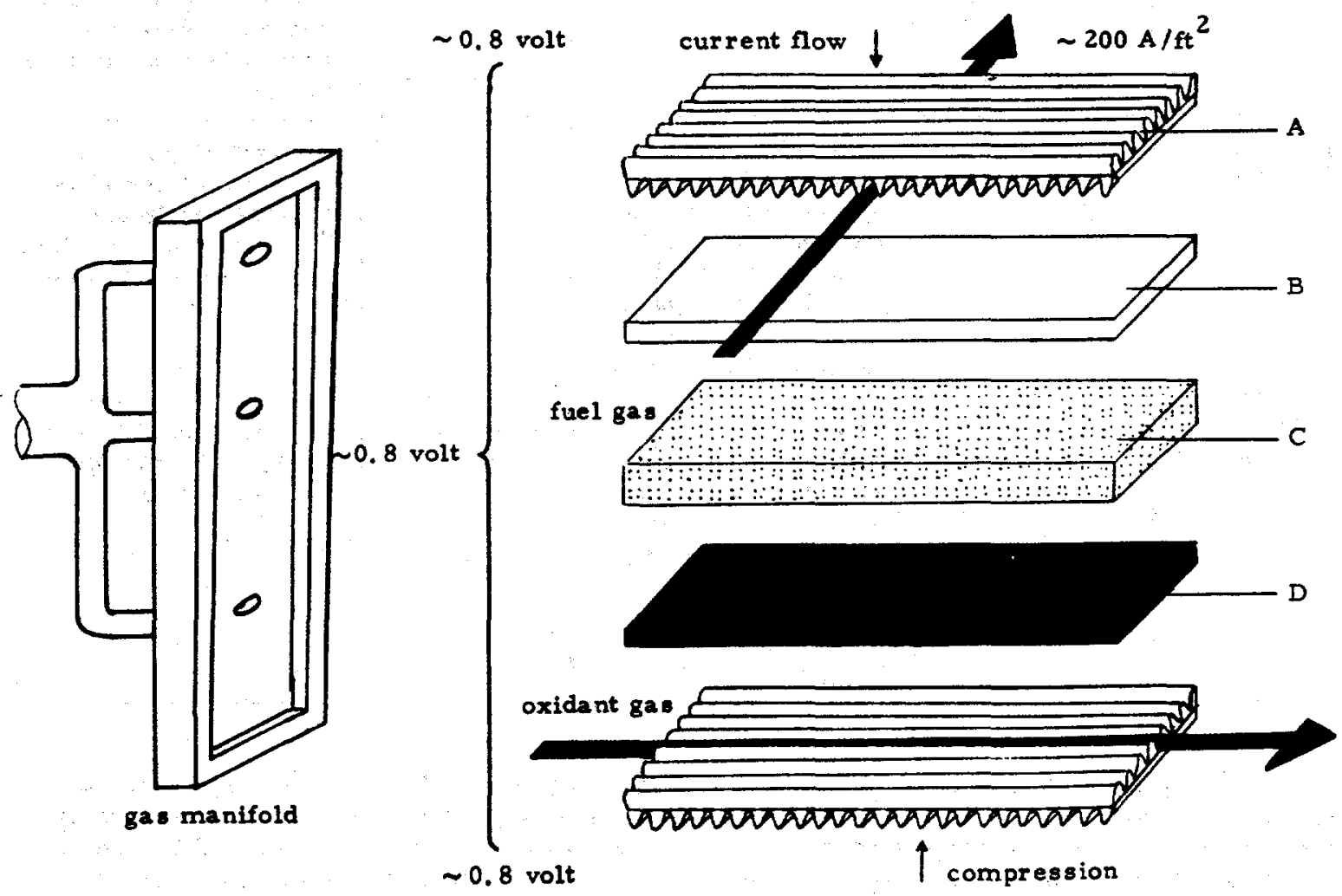

Fig. 5. 8A-1. An exploded view is shown of a cell in the MCFC stack as designed at ERC; 93

A, bipolar plate (including two corrugated gas channel/current collector sheets);

$\mathrm{B}$, porous $\mathrm{Ni}$ anode (pure or alloyed); C, electrolyte tile $(\sim 50 \%$ molten carbonates, $\sim 50 \%$ lithium aluminate); $\mathrm{D}$, porous cathode, NiO (Li-doped). 


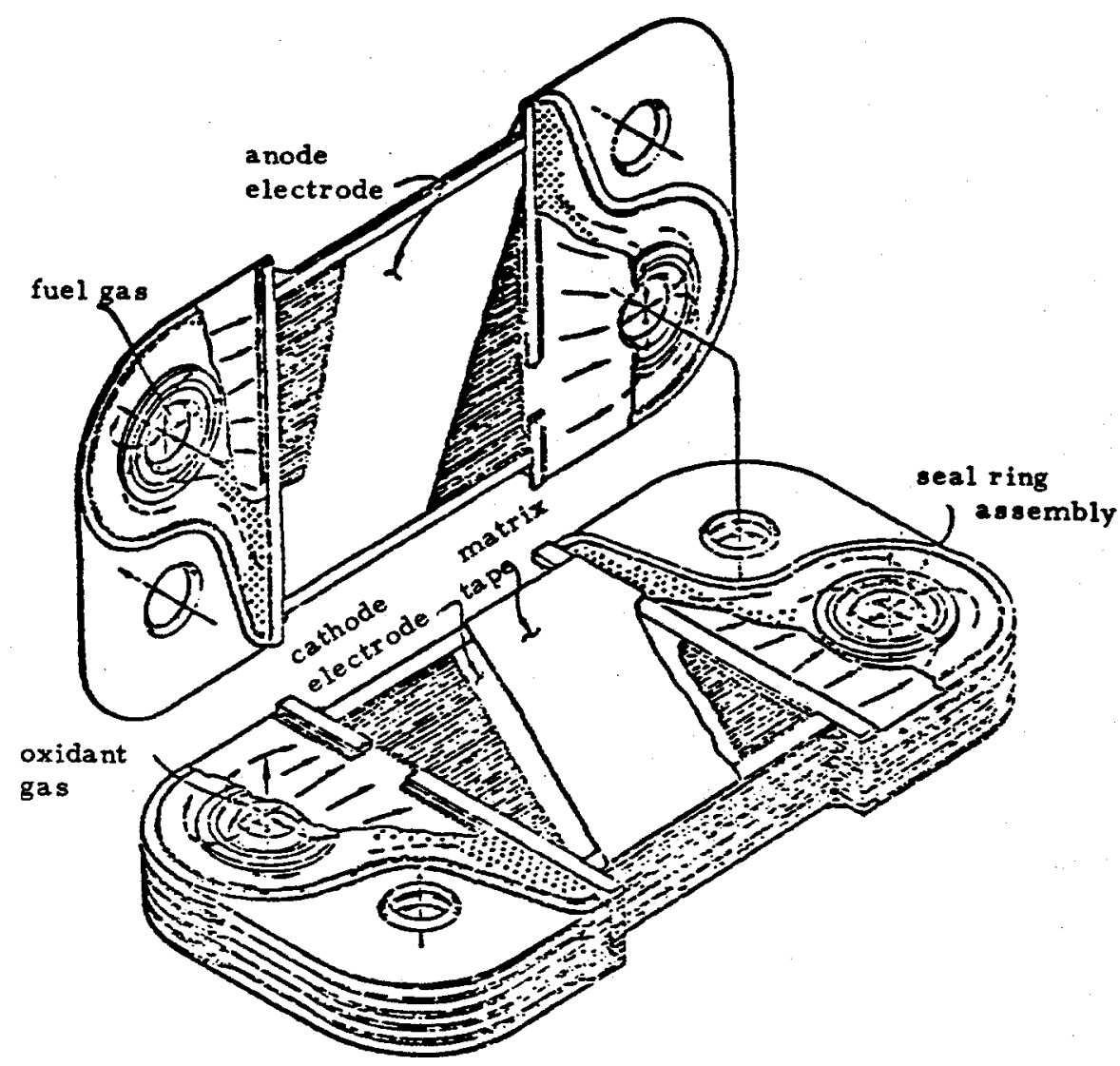

Fig. 5.8A-2. The $12^{\prime \prime}$ MCFC stack designed at GE is shown. Test conditions used for the $12^{\prime \prime}$ cells: $160 \mathrm{~mA} / \mathrm{cm}^{2}$, fuel utilization $=0.75, \mathrm{CO}_{2}$ utilization $=0.50$, the oxidant was $75 \%$ air plus $25 \% \mathrm{CO}_{2}$, both fuel and oxidant were humidified at $60^{\circ} \mathrm{C}$, co-flow, outlet value for the Nernst voltage $=832 \mathrm{mV}$, atmospheric pressure. 92

are greatly depleted in electrolyte. Furthermore, the electrolyte composition changes drastically at both ends due to $\mathrm{Li}^{+}$or $\mathrm{K}^{+}$accumulation. Both factors degrade the performance of the stack. However, only the 5 end cells on each side of the stack are adversely affected by migration. Therefore, this migration has a relatively less important overall effect as the number of stacked cells increases.

An illustration is provided by some data for a UTC 20-cell stack, operated for $600 \mathrm{~h} .94$ It was found that much of the electrolyte was lost. Twenty-five percent of the $\mathrm{Li}_{2} \mathrm{CO}_{3}$ and $11 \%$ of the $\mathrm{K}_{2} \mathrm{CO}_{3}$ were not accounted for when the final composition was determined for all of the components; these los ses probably occurred by evaporation following migration out of the cells. Cell 20 (the positive end) was filled with $116 \%$ of the original amount of electrolyte, while cell 1 had only $27.5 \%$. The electrolyte in cell 20 shifted to $\sim 56 \mathrm{~mol} \%$ of $\mathrm{Li}_{2} \mathrm{CO}_{3}$ and $\sim 44 \mathrm{~mol} \%$ of $\mathrm{K}_{2} \mathrm{CO}_{3}$; in cell 1 , it shifted to $\sim 30 \mathrm{~mol} \%$ of $\mathrm{Li}_{2} \mathrm{CO}_{3}$ and $\sim 70 \mathrm{~mol} \%$ of $\mathrm{K}_{2} \mathrm{CO}_{3}$.

The migration of electrolyte in the manifold seals reinforces the leaking of electrolyte through the wet-seal, as discussed in Sec. 5.5D. Wet-seal leaking and migration in the manifold interact with each other. Together they accelerate the long-term electrolyte loss catastrophically, since the stack manifold provides increased area for volatilization.

Electrolyte migration cannot be stopped completely, but it can be greatly reduced by using well-designed wet seals 95 and manifolds. Furthermore, the utilization of reservoirs at the end of the stacks may immobilize electrolytes, especially when combined with the natural characteristic of electrolyte to undergo pumping to the negative end of the stack.

Besides the rather specific migration problem, there are several other challenges in stack design: (1) the optimization of stack dimensions and operating conditions must be accomplished with respect to current and temperature distribution; (2) fabrication is required of a bipolar plate, or separator, of optimal corrosion resistance (at the positive side) and adequate electronic conductivity.

The design of stack dimensions and optimal operating conditions requires the application of computer models. These models, built up from single-cell models, allow one to a nalyze the effect of pressure, temperature, and various gas-flow configurations on stack performance. 96,97 
The most frequently proposed flow configurations, crossflow and counterflow, each have their own advantages. The crossflow configuration is easier to manifold, but the counterflow stack yields better performance because of better current and temperature distribution characteris tics. Thus, it appears that the crossflow configuration may be adopted in relatively inexpensive, lower-efficiency systems, but that the counterflow configuration will be necessary for central generating stations operating with higher efficiency. 90

To ensure that a stack design is satisfactory for specific fuel conditions and power requirements, it is necessary to predict the current and temperature distribution accurately. Mathematical models of cell and stack performance 96,97 use estimates of local polarization. A central concern here is the need for accurate polarization data as a function of local gas composition over a range of temperatures. These data can be obtained by experimentation but must be supported by a deeper insight into electrode kinetics and mass transfer in the porous MCFC electrodes. 75,77

Figure 5.8A-3 illustrates some of the current and temperature distributions calculated for representative flow configurations and feed compositions in internal reforming. 96 Computer modeling of IRMCFCs shows that atmospheric pressure cells should operate at $0.70-0.73 \mathrm{~V}$ and at $160 \mathrm{~mA} / \mathrm{cm}^{2}$, with a methane utilization on the order of $85-90 \% .98$ The exact voltage depends on system complexity (recycle loops, steam separation, etc.), and it may be improved slightly by pressurization. Since the HHV value of methane is $1.14 \mathrm{eV}$, a system efficiency on the order of $52-57.6 \%$ can be expected before parasitic power and dc-ac requirements are taken into account. Overall efficiencies might therefore be in the $49-55 \%$ range, depending on the $8 y s t e m$ chosen, giving heat-rates in the 6965 to $6200 \mathrm{BTU} / \mathrm{kWh}$ range. These values might be improved even further (by 4-5 absolute percentage points) if $\mathrm{CO}_{2}$ separation is possible, so that fuel utilization can be increased. Finally, a bottoming cycle might add about 7 further absolute percentage points, giving overall potential efficiencies of $67 \%(5100 \mathrm{BTU} / \mathrm{kWh})$ at $160 \mathrm{~mA} / \mathrm{cm}^{2}$. A typical atmospheric pressure system which should be capable of a $6500 \mathrm{BTU} / \mathrm{kWh}$ heat-rate (52.5\% efficiency), is shown in Fig. 5.8A-4.

These projections illustrate the power of predictive models but it should be realized that much information is still missing, for example, information necessary to assess the endurance of pressurized atacks operating on coal gas.

Among the design and operating variables, some, such as the electrolyte composition (Sec. 5.5B), have already been discussed. Pressure and temperature are among the most important variables for both performance and endurance.

Operation at high pressures (10 atm vs $1 \mathrm{~atm}$ ) enhances performance (see Fig. 5.8A-5). At $160 \mathrm{~mA} / \mathrm{cm}^{2}$, performance is predicted thermodynamically to be enhanced by $45 \mathrm{mV}$; the observed enhancement was $\sim 80 \mathrm{mV} .93$ This enhancement result because electrode polarizations are reduced and gas solubilities are increased with increasing operating pressures. At higher pressure, system volume and syatem costs, as well as electrolyte vaporization, would be reduced. However, higher operating pressures increase the risk of reactant-gas crossover, even when BPBs are used. The formation of methane and carbon are strong functions of pressure; for internal reforming, MCFCs with pressurized operation have no advantages (see Sec. 5.6E).

Lowering of the cell-operating temperature from 650 to $615^{\circ} \mathrm{C}$ may provide a generic solution to endurance issues. At the lower temperature, cathodes are more stable, hardware is less extensively corroded, electrolyte $108 \mathrm{~s}$ and anode creep are reduced, while performance is not degraded much, except for increased cathode polarization (Fig. 5.7A-2). However, tolerance to $S$ and overall system performance are reduced at lower operating temperatures (Fig. 5.8A-6).93

Endurance is also a key criterion in the selection of the separator, or bipolar plate, material (see Fig. 5.8A-1). The bipolar separator sheet must satisfy the following complex inter related requirements: 99,100 (i) The corrosion rate must be extremely low to attain a lifetime of $40,000 \mathrm{~h}$ when the separator thickness cannot be more than 0.125 to $0.75 \mathrm{~cm}$ thick because of cost and formability. (ii) The separator material must have sufficient strength so as not to yield or creep under holding forces applied to the stack in order to maintain good component contact. (iii) Any oxidation or corrosion layers formed must be electrically conductive to minimize cell-to-cell resistive losses; on the other hand, they must be stable enough in the gas/ electrolyte environment to provide protection against further consumption of the sheet.

Ideally, a single material is desirable. However, this should satisfy all the above requirements at both sides, in radically different gas environments. In the anode environment, at high carbon activity, carburization may occur and formation of compact protective oxide layers is inhibited. On the oxidizing side, this problem may not occur but trace contaminants may destroy the oxide layers and accelerate corrosion. Therefore, a bimetallic plate would be a possible solution but this would still require elimination of carburization, 41 chromium diffusion, etc. A single separator sheet may be the optimal solution from the viewpoint of fabrication. This would require a careful tailoring of the alloy employed with respect to the two environments, based on a thorough understanding of corrosion mechanisms and rates of alloys in gas/ carbonate environments. This understanding is presently lacking, although considerable progress has been made in the last eight years, in particular through development of corrosionresistant wet seals. 95 

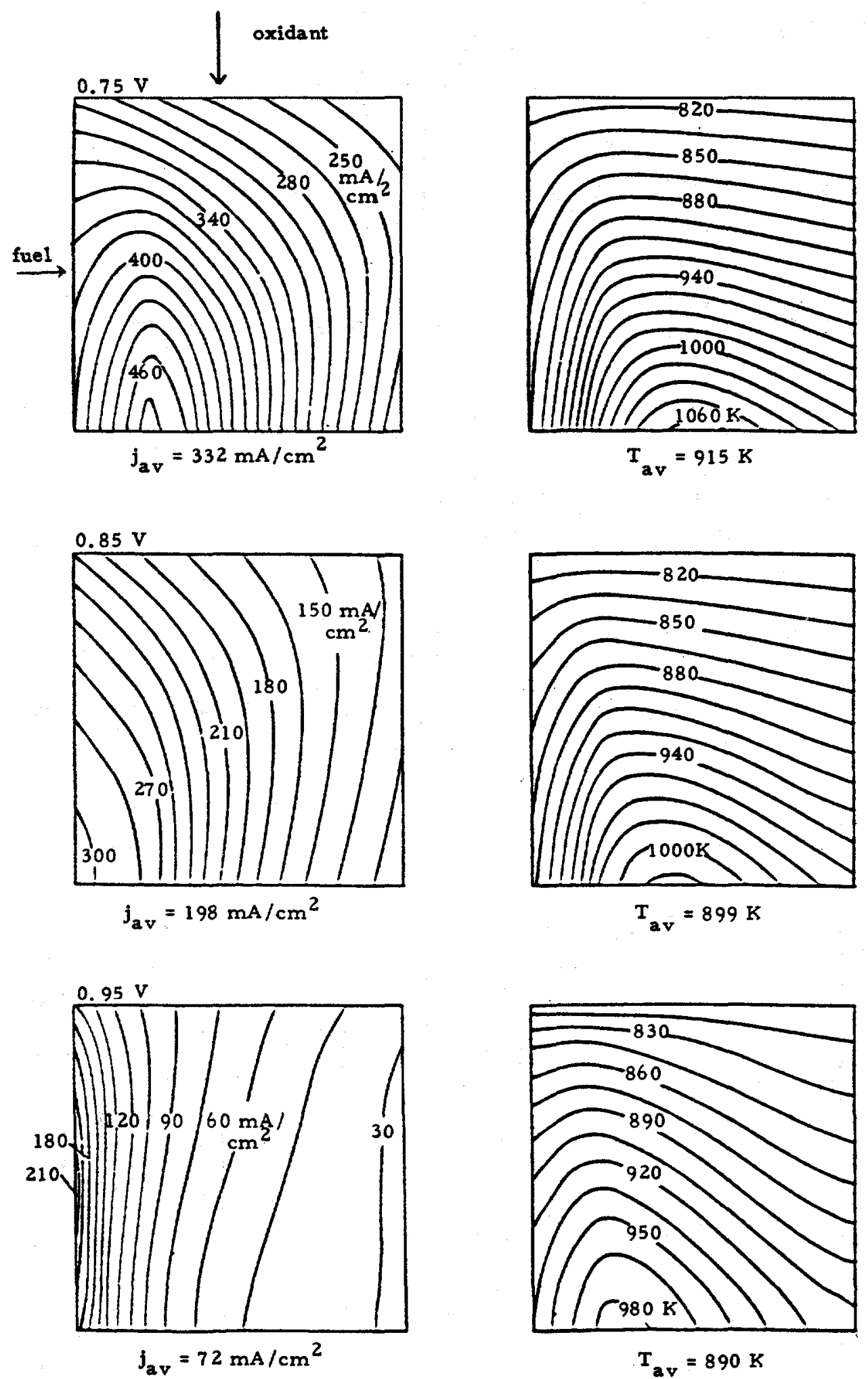

Fig. 5.8A-3. Calculated distributions of current density and cell temperature for $1-\mathrm{m}^{2}$, non-isothermal cells with crossflow geometry corresponding to constant utilization ( $75 \%$ fuel, $25 \%$ oxidant). The fuel is low BTU gas with shift equilibrium; standard oxidant was used. The inlet gas temperatures are $800 \mathrm{~K}, \mathrm{Z}^{923}=0.5 \Omega-\mathrm{cm}^{2} .96$ 
fuel (LHV 11,773,000 BTU/h)

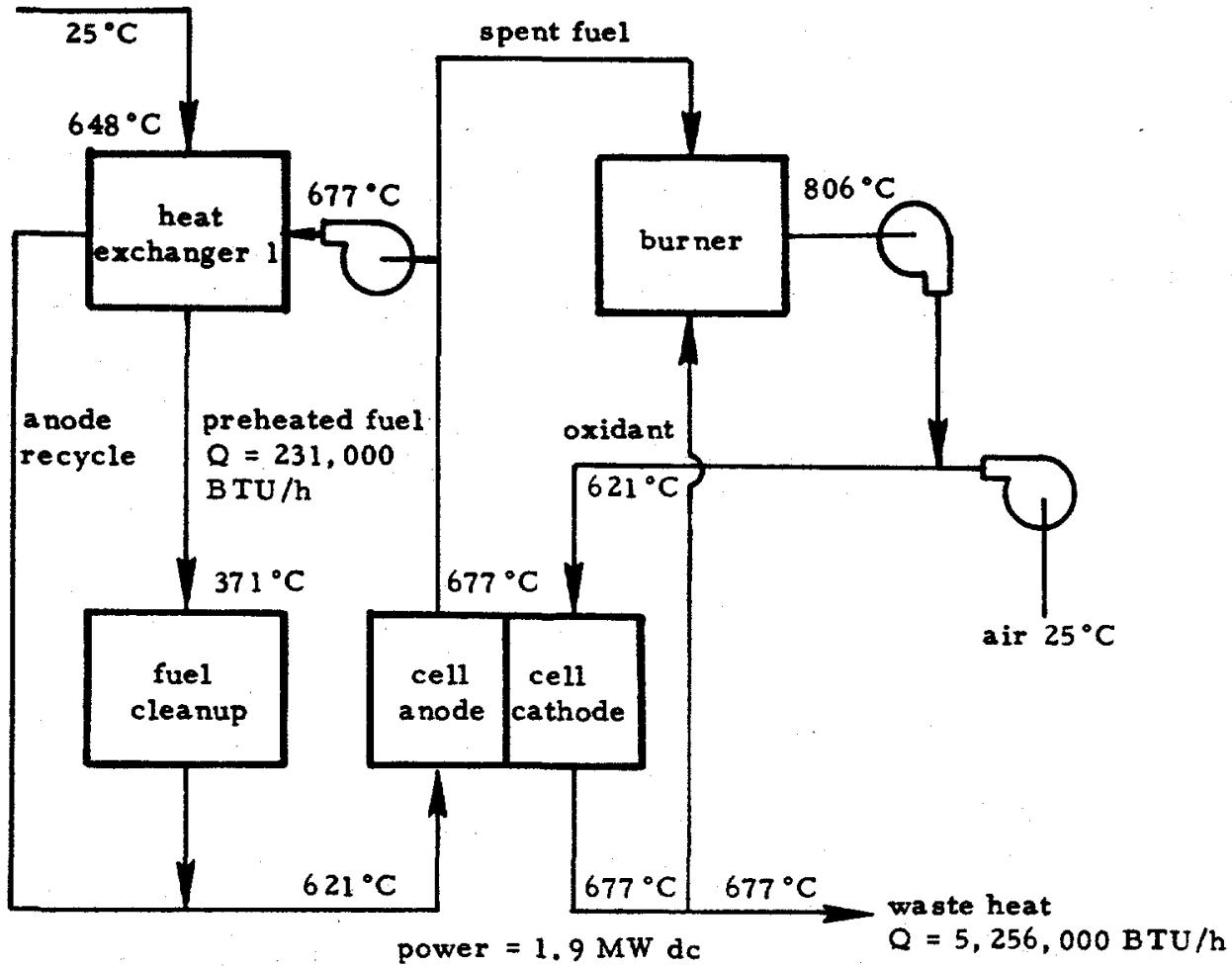

Fig. 5.8A-4. The system configuration is shown for an NG MCFC power plant. 98

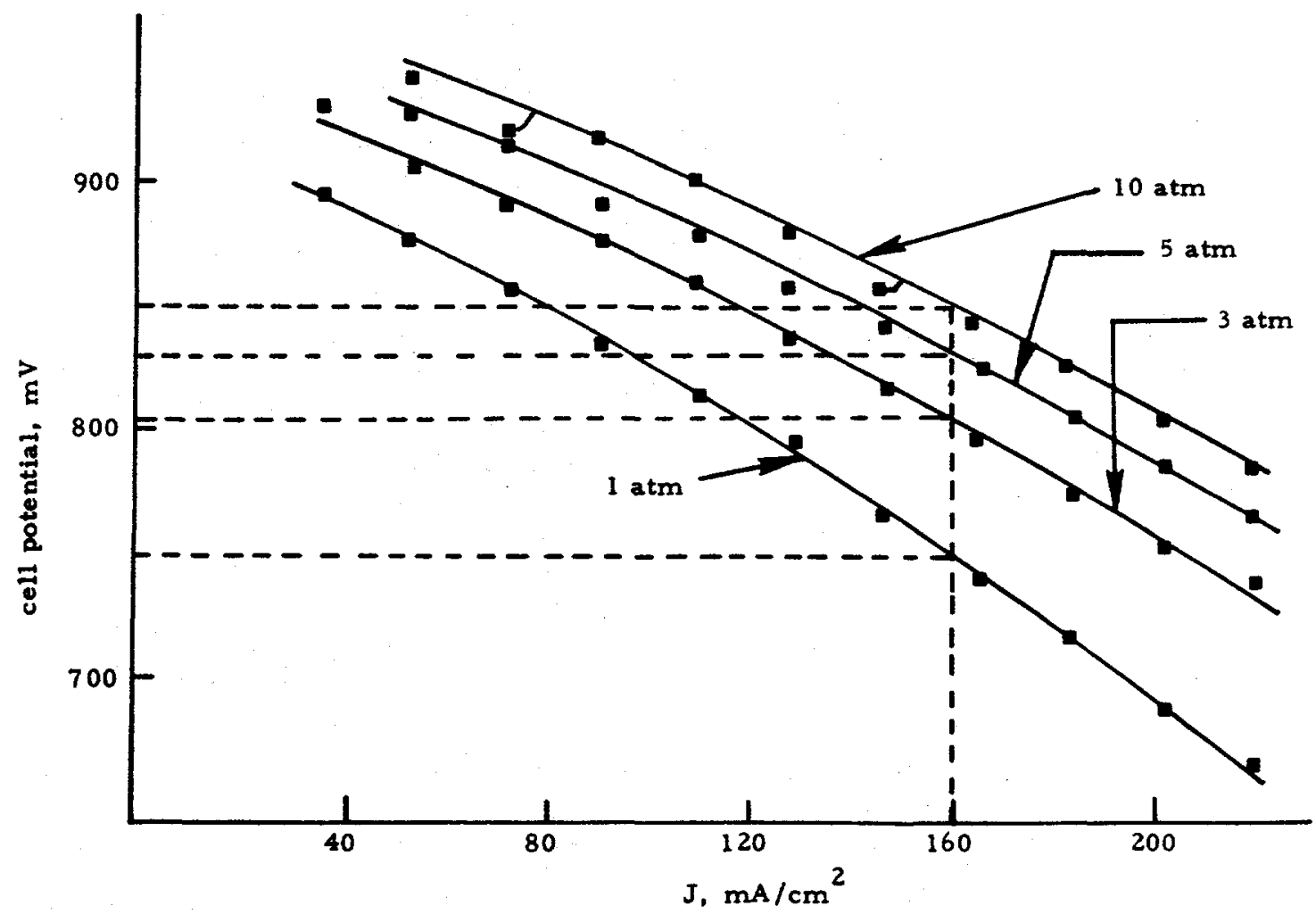

Fig. 5.8A-5. The measured and predicted potential (mV) of MCFCs is plotted as a function of current density $\left(\mathrm{mA} / \mathrm{cm}^{2}\right)$ and of operating pressure (atm). The anode gas $\left(28 \% \mathrm{H}_{2}, 28 \% \mathrm{CO}_{2}, 44 \% \mathrm{H}_{2}\right)$ had $80 \%$ utilization; the cathode gas $\left(15 \% \mathrm{O}_{2}\right.$, $30 \% \mathrm{CO}_{2}, 55 \% \mathrm{~N}_{2}$ ) had $50 \%$ utilization. 


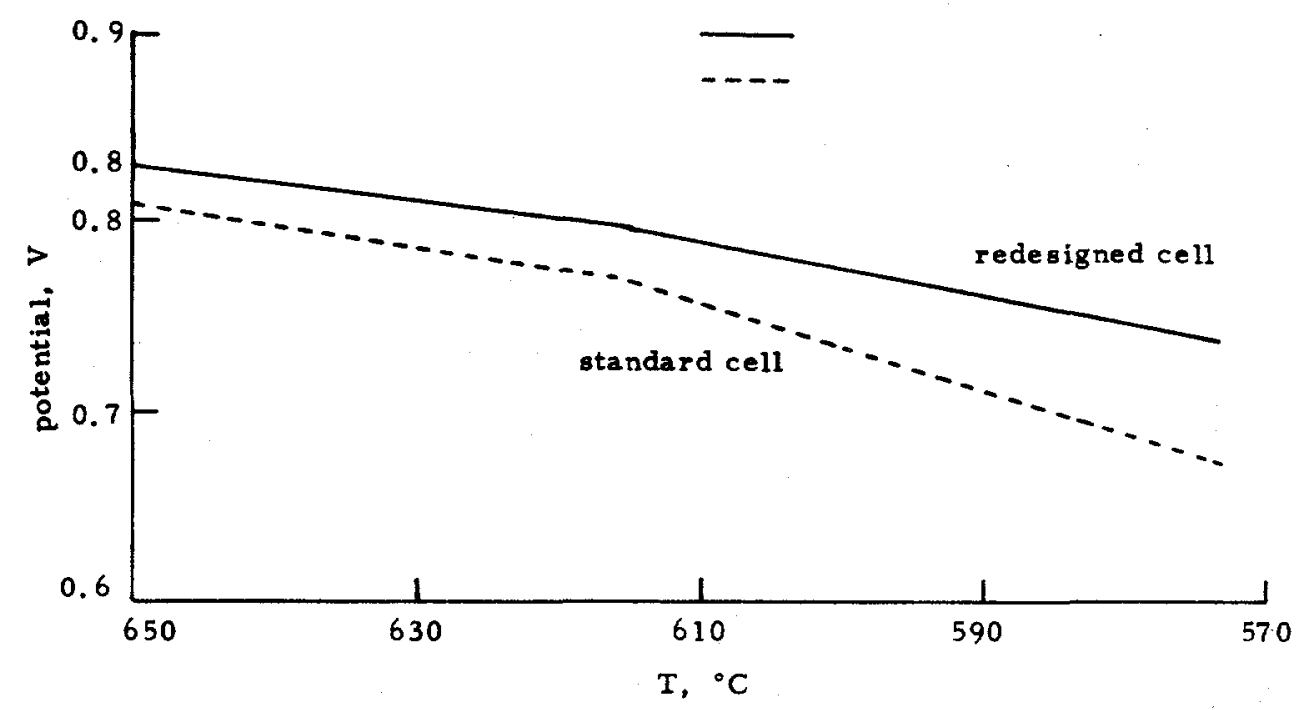

Fig. 5.8A-6. The potential (V) at $120 \mathrm{~mA} / \mathrm{cm}^{2}$ for $\mathrm{MCFCs}$ is plotted as a function of temperature $\left({ }^{\circ} \mathrm{C}\right) .93$

A related corrosion issue, which impacts on separator-material selection, is the corrosion of current collectors. Considerable experience has been gained with various types of stainless steel.

Formation of lithium ferrite on cathode current collectors (CCs, 316-SS) is expected to lead to films of $\sim 4$ mils thickness after $40,000 \mathrm{~h}$ of operation, provided no unexpected corrosion problems (e.g., by contaminants) occur. 90 The corrosion of the cathode CC is given by the approximate relation

$$
y=0.0134 t^{1 / 2}
$$

where $y$ is the thickness of the corroded layer (mils) and $t$ is time in $h$ (see Fig. 5.8A-7). Extrapolations of the curve in Fig. 5.8A-6 to times greater than the test time of $12,000 \mathrm{~h}$ may be optimistic.

Corrosion of pure 316 -SS occurs much more rapidly in anode than cathode environments. Figure 5.8A-8 shows that corrosion rates increase with the water contents of the fuel gas. This result indicates that more extensive corrosion will be observed at the fuel outlet than at the inlet and at points of high rather than low electrochemical activity. The anode CC must be resistant to carburization by the fuel and chemical attack by the electrolyte. Non-corroding Ni, which is presently clad onto the $316-$ SS, eliminates these problems. 90 However, a less expensive solution is desirable.

It is obvious that corrosion of metals and alloys in molten carbonates is a key topic on which fundamental understanding needs to be extended. The same type of need exists for the transport properties and mechanisms in molten carbonate under potential as well as concentration gradients.

An applied research objective of great importance for stack design and system efficiency is the $\mathrm{CO}_{2}$-transfer device or Product Exchange Device (PED), which has been mentioned in Secs, 5.1,5.2 and 5.6. Direct transfer of $\mathrm{CO}_{2}$ from anode to cathode would make close to $100 \%$ fuel utilization possible, with consequent gains in overall efficiency. At present, only some conceptual devices are available or under atudy. 101,102 Even if, in the future, pure $\mathrm{H}_{2}$ is used as a fuel for the MCFC, it seems inevitable that $\mathrm{CO}_{2}$ make-up will be required. However, addition of a few percent fossil fuel (methane, biogas, etc.) to pure $\mathrm{H}_{2}$ may be sufficient to accomplish a continuous $\mathrm{CO}_{2}$ mass-balance for the system as a whole, provided a $\mathrm{CO}_{2}$ transfer device is used. The MCFC may then be a viable power source for stationary applications, even in the era of the hydrogen economy, though other types of cell, particularly the AFC, would be more suitable.

It appears that fundamental solid-state and ceramic research on gas solubility and transport (with the exception of $\mathrm{H}_{2}$ in metals) has not addressed this issue. There is a clear need for ideas and development of concepts here.

\section{B. Cost and Commercialization}

Material costs may be increased considerably by efforts to improve performance or endurance. For example, a great deal of stainles steel has been traditionally used in experimental bench-scale cells. This material helped to maintain dimensional stability and optimal 


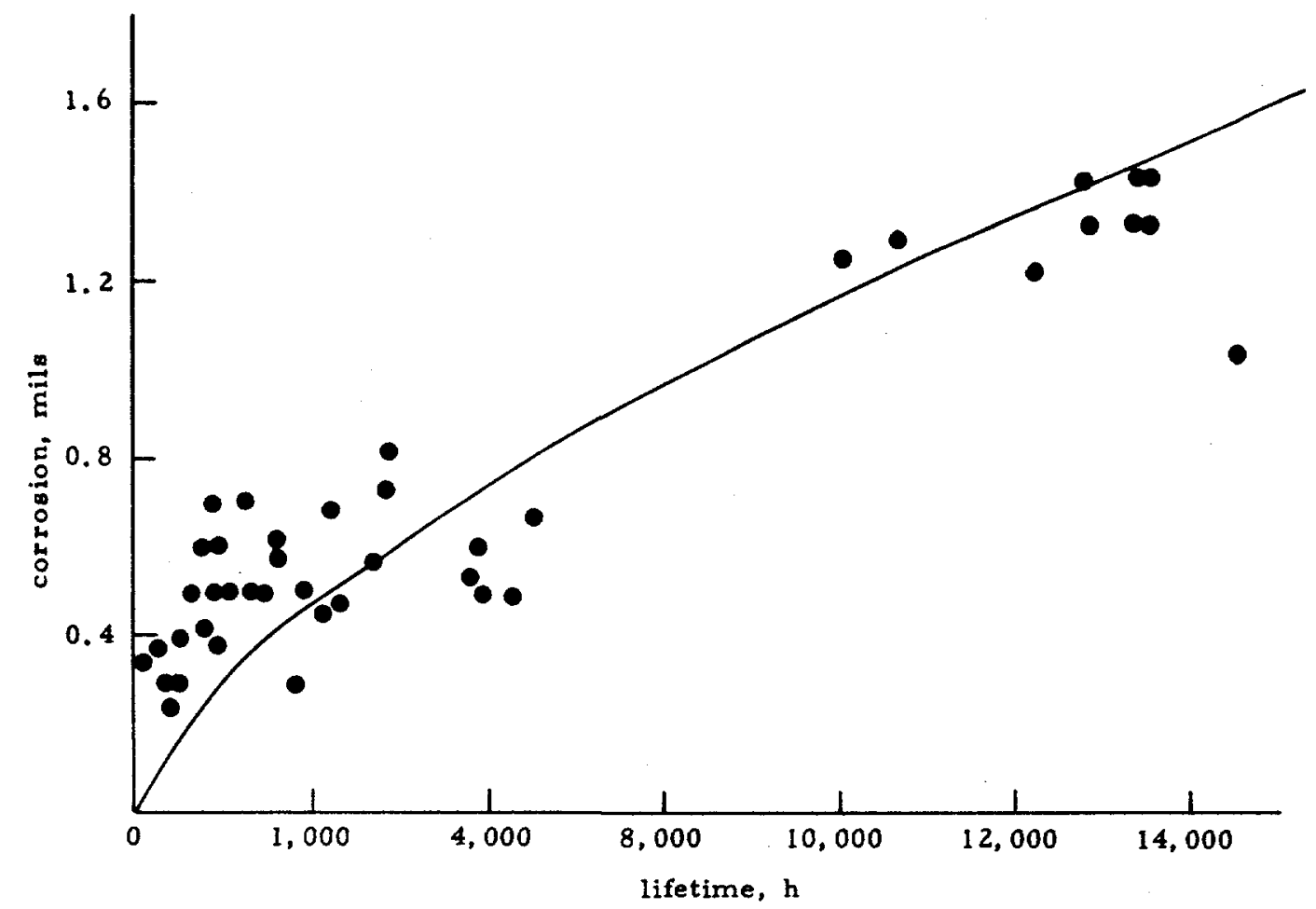

Fig. 5.8A-7. The corrosion of a cathode CC (316-SS) is plotted as a function of time. The curve is represented by $\mathrm{y}=0.0134 \mathrm{t}^{\mathrm{l} / 2}$ for $62 \mathrm{~mol} \%$ of $\mathrm{Li}_{2} \mathrm{CO}_{3}$ and $38 \mathrm{~mol} \%$ of $\mathrm{K}_{2} \mathrm{CO}_{3} ; \mathrm{T}=650^{\circ} \mathrm{C} .99$

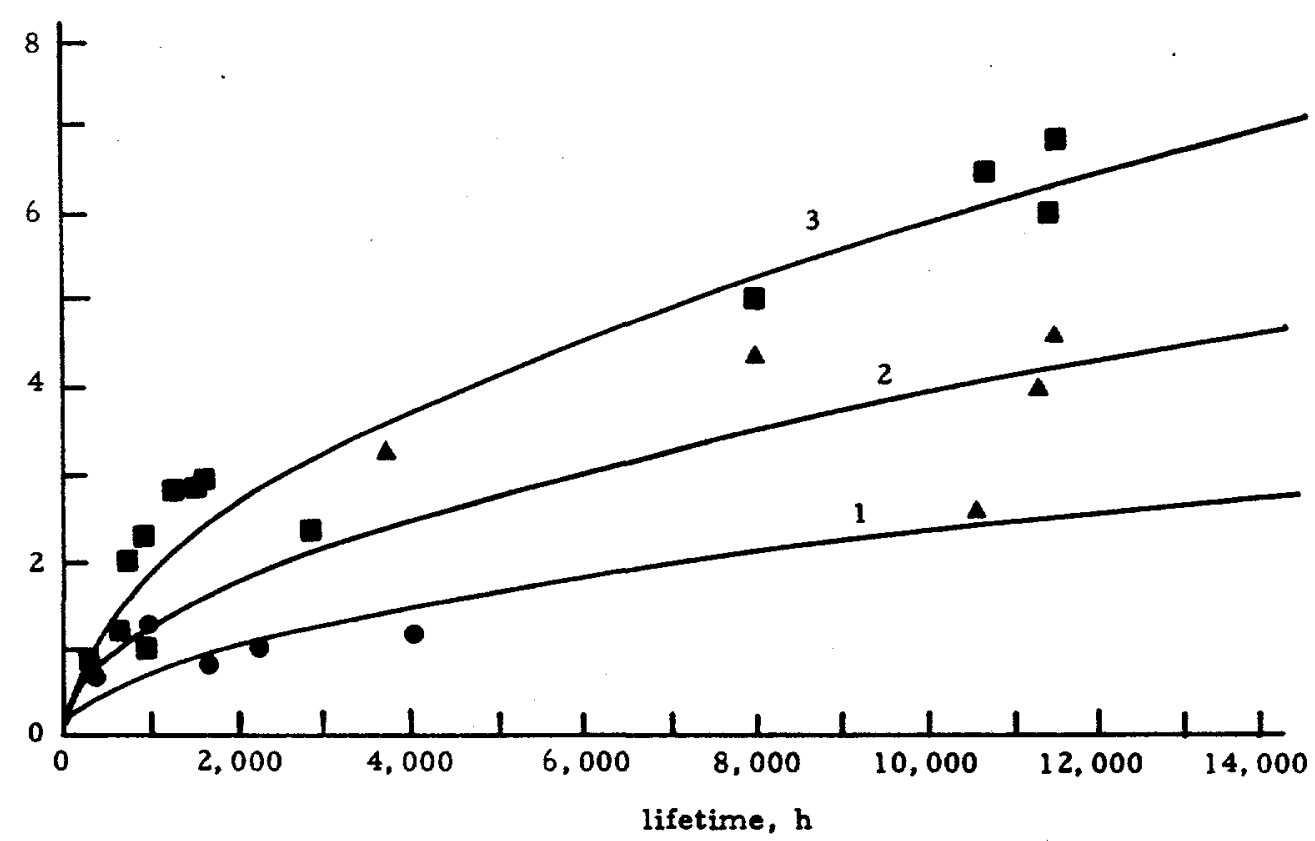

Fig. 5.8A-8. The corrosion of an anode CC (316-SS) is plotted as a function of time and of $\mathrm{H}_{2} \mathrm{O}$-content of the fuel. The electrolyte is $62 \mathrm{~mol} \%$ of $\mathrm{Li}_{2} \mathrm{CO}_{3}$ with $38 \mathrm{~mol} \%$ of $\mathrm{K}_{2} \mathrm{CO}_{3} ; \mathrm{T}=650^{\circ} \mathrm{C} .99$ For curve $1(0), \mathrm{C}=0.0229 \pm 0.0027,16 \% \mathrm{H}_{2} \mathrm{O}$; curve $2(\Delta), C=0.0387 \pm 0.0044,28 \% \mathrm{H}_{2} \mathrm{O}$; curve $3(\mathrm{D}), \mathrm{C}=0.0583 \pm 0.0026$, $43 \% \mathrm{H}_{2} \mathrm{O}$. A general equation for the curves is $y=c t / / 2$. 
performance but increased costs. It also has unintentionally led to large lithium losses due tó corrosion of the cathodic components (Secs. 5.5D and 5.8A), which decreases cell lifetime and impacts negatively on cost: MCFC market costs are strongly dependent on endurance.

Material costs could be reduced if replacements were available for the expensive nickel and stainless steel now used. For example, dispersion-hardened copper might be used at the anode. The use of nickel may decline if cermet anodes and alternative cathode materials become a vailable. However, these substitutes will decrease cost only when their long-term viability has been proved. Fabrication costs also introduce uncertainty. The bipolar plate is an expensive component of the MCFC. Fabrication costs could be reduced if the bipolar plate were less corrugated. The frequency of corrugation is determined by electrode strength.

Materials cost estimates, therefore, must always be considered as only part of the picture. For the IRMCFC, which promises to be very marketable (Sec. 5.2), there are quite reliable estimates of materials costs (nickel and sheet metal parts, lithium aluminate, molten salt electrolyte, oxide cathode material). Materials can be estimated to weigh a total of about $10 \mathrm{~kg} /$ $\mathrm{kW}$, at an average cost of $\$ 8 / \mathrm{kg}$, giving $\$ 80 / \mathrm{kW}$ overall. In Ref. 98 , where all costs are given in January $1983 \$$, this procedure was assumed to lead to a stack cost of about $\$ 300 / \mathrm{kW}$ based on stack dc output or $\$ 333 / \mathrm{kW}$ for system ac output. Stack costs of $\$ 200-300 / \mathrm{kW}$ are considered acceptable to maintain overall costs in agreement with market projections. The total cost of the IRMCFC plant, using largely off-the-shelf items, is estimated to be about $\$ 900 / \mathrm{kW}$ (uninstalled) or $\$ 1230 / \mathrm{kW}$ (installed). 98

A complete cost breakdown for the proposed dispersed IRMCFC plant is given in Table 5.8-1, which refers to the final cost of a complete production $r$ un of $180 \mathrm{MW}$, i. e. . a pilot line. 98 Such a pilot plant would have a similar capital cost to that of an early PAFC used in a cogeneration mode, about half-way down the learning curve shown in Fig. 2.4-1. This would correspond to a total cumulative production of about $5211-\mathrm{MW}$ class PAFC plants, or $570 \mathrm{MW}$, compared with only $180 \mathrm{MW}$ for the MCFC system with its much simpler chemical engineering.

To illustrate the impact of design factors on costs, the IRMCFC design discussed in Sec. 5.8A is not necesssarily optimal for all applications. Even higher efficiencies than the 49-55\% (overall) values quoted there could be obtained, should this be considered necessary, by running cells at lower current density, which will involve a corresponding increase in cell area and, therefore, in capital cost. More likely, however, the initial tendency will be to design very low-cost systems, using the simplest chemical engineering and operating at fairly high current densities to have the lowest possible capital cost and promote integration as soon as possible into the energy economy. An initial heat-rate of $7000 \mathrm{BTU} / \mathrm{kWh}$ would be a good goal, with an expected capital cost for the simple system of about $\$ 400-500 / \mathrm{kW}$ in volume production.

\subsection{Fundamental Research Needs}

Table 5.9-1 presents an overview of the most important challenges to MCFC developers in the final stage before commercialization.

Most of the research needs listed in Table 5.9-1 are applied-research needs but with important fundamental components. Two fundamental research areas impact strongly on performance and endurance, but are not listed as such in Table 5.9-1. These are (not in order of priority): (1)Electrode kinetics (electrocatalysis) of fuel oxidation and oxygen reduction at various materials and as a function of composition. Work in this area may eventually lead to development of a direct electrocatalyst for $\mathrm{CH}_{4}$, as well as sulfur-and $\mathrm{HCl}$-tolerant electrodes.

(2) Solid-state electrochemistry of conductive ceramics, at temperatures between 500 and $700^{\circ} \mathrm{C}$, either in contact with molten carbonates or related electrolyte or as solid electrolytes in their own right. This work may eventually lead to development of a tailored alternative cathode for the MCFC or to a solid-electrolyte cell which is itself an improved alternative to the MCFC.

Additional fundamental research needs are: (3) Corrosion mechanisms and control of corrosion rates under molten carbonate/solid/gas contact conditions, without and with applied polarization. Studies should include quantitative modeling of corrosion processes. (4) Fundamental physicochemical property and engineering studies, pertaining to (a) the determination and theoretical prediction of (i) gas and solid solubilities, (ii) gas and ionic transport properties, and (iii) capillary behavior of the electrolyte as a function of composition and temperature; (b) the development of novel performance and performance-decay models, in conjunction with experimental data to verify model validity; (c) the development of in situ diagnostic techniques for applications to cells and cell stacks. (5) Densification of porous or particulate materials. Experimental and theoretical analyses should be performed on the densification of porous materials that are in contact with gases and molten salts. (6) Solubility and transport of gases in metals and ceramics, with emphasis on $\mathrm{CO}_{2}$ separation at $500-700^{\circ} \mathrm{C}$. (7) Spectroscopic techniques applied to molten salts, and carbonate melts, in particular, to confirm the melt chemistry assumed in electrolyte optimization to minimize polarization and corrosion. 
Table 5.8B-1. Total plant investment (in January $1983 \$$ ) for a $1.8 \mathrm{MW}$ IRMCFC power plant.98

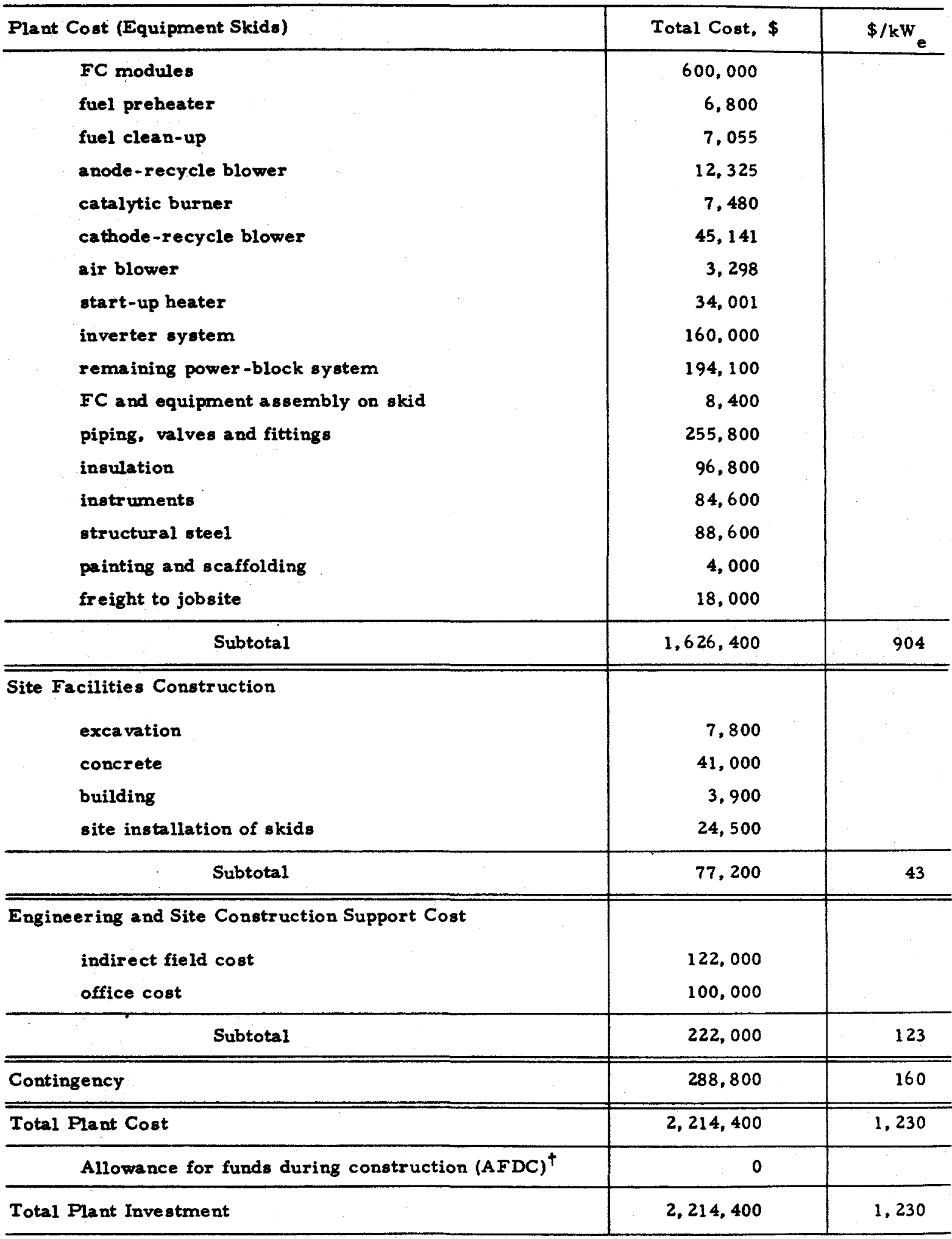

AFDC is assumed to be negligible for projects with a construction period of less than one year. 
Table 5.9-1. A summary of MCFC is sues and their proposed resolution.

\begin{tabular}{|c|c|c|}
\hline Issue & Problem Status & Research Need \\
\hline Anode creep & $\begin{array}{l}\mathrm{Ni} \text { or } \mathrm{Ni} / \mathrm{Cu}_{\mathrm{u}} \text { with } \mathrm{LiAlO}_{2} \text { or } \mathrm{Sr}_{\mathrm{TiO}} \\
\text { cermet structures have initially } \\
\text { eliminated anode creep. }\end{array}$ & $\begin{array}{l}\text { Sintering and creep of cermets } \\
\text { in three-phase contact (gas, } \\
\text { molten salt). }\end{array}$ \\
\hline Cathode dissolution & $\begin{array}{l}\text { Lithiated-NiO is probably unsuit- } \\
\text { able for pressurized stacks. } \\
\text { Nickel deposits occur in the } \\
\text { matrix after } 10,000 \mathrm{~h} \text { of opera- } \\
\text { tion but may be adequate for } \\
\text { IRMCFC to } 25,000 \mathrm{~h} \text {. Few alter- } \\
\text { natives are a vailable }\left(\mathrm{Mg}-\mathrm{Li}_{2} \mathrm{MnO}_{3} \text {, }\right. \\
\left.\mathrm{Mn}-\mathrm{LiFeO}_{2}, \mathrm{LaMnO}_{3}\right) .\end{array}$ & $\begin{array}{l}\text { Solubility and transport of dis- } \\
\text { Bolved metals-oxides in molten } \\
\text { salt, conductivity and corrosion } \\
\text { (in carbonates) of mixed oxides } \\
\text { and doped perovskites. }\end{array}$ \\
\hline $\begin{array}{l}\text { Internal-reforming } \\
\text { a node }\end{array}$ & $\begin{array}{l}\text { Nickel supported on } \mathrm{MgO} \text { and } \\
\mathrm{LiAlO}_{2} \text { has been tested. Catalyst } \\
\text { degradation has occurred in } \\
\text { about } 4,000 \mathrm{~h} .\end{array}$ & $\begin{array}{l}\text { Available catalysts need sys- } \\
\text { tematic testing. Wetting and } \\
\text { corrosion of ceramics is not } \\
\text { fundamentally understood. The } \\
\text { effects of } \mathrm{H}_{2} \mathrm{O} \text { on fuel-oxidation } \\
\text { mechanism should be studied. }\end{array}$ \\
\hline $\begin{array}{l}\text { Corrosion of the } \\
\text { cathode-current }\end{array}$ & $\begin{array}{l}\text { Formation of lithium-ferrite films } \\
\text { will not cause significant cathode } \\
\text { CC }(316 \text {-SS) degradation before } \\
40,000 \mathrm{~h} \text {. }\end{array}$ & $\begin{array}{l}\text { Mechanism of alloy corrosion, } \\
\text { effect of electrolyte composition } \\
\text { on mechanism and rates. }\end{array}$ \\
\hline $\begin{array}{l}\text { Corrosion of the anode- } \\
\text { current collector }\end{array}$ & $\begin{array}{l}\text { Corrosion of the } 316 \text {-SS is not a } \\
\text { problem because of cladding with } \\
\text { non-corroding Ni but it is expen- } \\
\text { sive. }\end{array}$ & As for the cathode. \\
\hline $\begin{array}{l}\text { Electrolyte inventory } \\
\text { and distribution, } \\
\text { optimization of } \\
\text { electrolyte composi- } \\
\text { tion }\end{array}$ & $\begin{array}{l}\text { Failure of end cells in test stacks } \\
\text { has occurred within } 500 \mathrm{~h} \text { due to } \\
\text { high or low inventory. Wet-seal } \\
\text { aluminizing of the correct type } \\
\text { may reduce seal leakage greatly. } \\
\text { Reservoirs at the ends of the stack } \\
\text { may immobilize electrolytes, when } \\
\text { combined with electrolyte pumping } \\
\text { to the negative end of the stack. }\end{array}$ & $\begin{array}{l}\text { Effect of electrolyte composition } \\
\text { on ion transport mechanisms and } \\
\text { on wet-seal corrosion; quanti- } \\
\text { tative analysis of wet-seal cor- } \\
\text { rosion rates; electrolyte opti- } \\
\text { mization with respect to elec- } \\
\text { trode polarization, migration and } \\
\text { corrosion. }\end{array}$ \\
\hline $\begin{array}{l}\text { Matrix cracking and } \\
\text { gas crossover }\end{array}$ & $\begin{array}{l}\text { Reactant gas-crossover has been } \\
\text { reduced by using tape-cast or hot- } \\
\text { rolled matrices and by bubble- } \\
\text { pressure barriers. Crack arrest- } \\
\text { ors and reinforcing screens } \\
\text { appear promising for strength. }\end{array}$ & $\begin{array}{l}\text { Capillary behavior of different } \\
\text { compositions of electrolyte, in } \\
\text { contact with support material and } \\
\text { electrodes; dis solved-gas } \\
\text { transport mechanisms and rates. }\end{array}$ \\
\hline Sulfur tolerance & $\begin{array}{l}\text { The performance of present anode } \\
\text { materials is degraded (reversibly) } \\
\text { by small amounts ( } 1 \text { ppm) of } S \text { in } \\
\text { the fuel or oxidant streams. Sulfur } \\
\text { can be removed from the fuel by } \\
\text { the Selexol liquid-adsorption } \\
\text { process. }\end{array}$ & $\begin{array}{l}\text { Development of sulfur-tolerant } \\
\text { electrode material; mechanism } \\
\text { and controlling rate processes } \\
\text { of performance decay; effect of } \\
\text { electrolyte composition on } \\
\text { poisoning kinetics. }\end{array}$ \\
\hline $\begin{array}{l}\text { Product exchange } \\
\text { device }\left(\mathrm{CO}_{2} \text { transfer }\right. \\
\text { device) }\end{array}$ & $\begin{array}{l}\text { Various electrochemical or diffu- } \\
\text { sion concepta have been proposed, } \\
\text { but experimental testing in an } \\
\text { operating MCFC system is re- } \\
\text { quired. Ceramic type membranes } \\
\text { for } \mathrm{CO}_{2} \text { transfer would be desir- } \\
\text { able. }\end{array}$ & $\begin{array}{l}\text { Gas solubility and transport in } \\
\text { oxide-type ceramics. Effect of } \\
\text { fabrication techniques on trans- } \\
\text { port properties of ceramics. }\end{array}$ \\
\hline
\end{tabular}


1. NASA Lewis Research Center, "Comparative Evaluation of Phase I Results from the Energy Conversion Alternatives Study (ECAS), "NASA TM-X718S5, NASA, Washington, D. C. $(1976)$.

2. T. L. Bonds, M. H. Dawes, A. W. Schnake, and L. W. Spradin, "Fuel Cell Power Plant Integrated Systems Evaluation," EM-1670, EPRI, Palo Alto, CA (1981).

3. 'Energy Conversion Alternatives Study: Integrated Coal Gasifier/Molten Carbonate Fuel Cell Power Plant Conceptual Design and Implementation Assessment, " NASA-PB-270016, UTC, S. Windsor, CT (1976).

4. A. J. Appleby, "Fuel Cell Developments in the United States," presented at the New Energy Development Organization, Tokyo, Japan, March 1, 1983.

5. A. P. Meyer and C. A. Reiser, "The Status of Molten Carbonate Fuel Cells at United Technologies Corporation," 1985 Fuel Cell Seminar, p. 170.

6. "Molten Carbonate Fuel Cell System Verification and Scale-Up, "EPRI EM-4129, Project 1273-1, Final Report, UTC, July 1985.

7. J. P. Ackerman and H. Shimotake, "High Temperature Fuel Cells in Japan, "Argonne National Laboratory (ANL), Argonne, IL, ANL-CMTI-8715, June 12, 1984.

8. T. G. Benjamin, E. H. Camara, and L. G. Marianowski, "Handbook of Fuel Cell Performance," Project 61012 Final Report, IGT, Chicago, IL (1980).

9. C. K. Pang, S. T. Lee, K. Lee, and D. T. Imamura, "Application of Fuel Cells on Utility Systems," EM-3205, EPRI, Palo Alto, CA (1983).

10. D. T. Hooie and E. H. Camara, Proc. 1985 Fuel Cell Seminar, p. 182, Tucson, AZ. EPRI, Palo Alto, CA (1985).

11. R. D. Breault, J. V. Congdon, R. D. Coykendall, and W. L. Luoma, "Improved FCG-1 Cell Technology," EM-1566, EPRI, Palo Alto, CA (1980); L. M. Handley, "Description of a Generic 11-MW Fuel Cell Power Plant for Utility Applications, "EM-3161, EPRI, Palo Alto, CA (1983).

12. J. M. Feret, "Gas Cooled Fuel Cell Systems Technology Development," DoE/NASA/ 0290-1 (NASA CR-174732), NTIS, Springfield, VA (1983).

13. A. J. Appleby, "Advanced Fuel Cell Technology," EPRI J. , p. 63, EPRI, Palo Alto, CA, December 1984.

14. D. K. Fleming, "Systems Analysis of Electricity Production from Coal using Fuel Cells," IGT Project 65060 Final Report, IGT, Chicago, IL, Nov. 1983.

15. H. A. Liebhafsky and E. J. Cairns, Fuel Cells and Fuel Batteries, Chaps. 2 and 12 , McGraw-Hill, NY (1968).

16. G. H. J. Broers, 'High Temperature Galvanic Fuel Cells," Thesis, University of Amsterdam, Amsterdam, The Netherlands (1958).

17. G. H. J. Broers, "Survey of Fundamental Research on Molten Carbonate Fuel Cells." Int. Report No. 69-0667/1272-7211, Centraal Technisch Instituut INO, Apeldoorn, Netherlands (1969); translation in ORNL-tr-4663, Oak Ridge National Laboratory.

18. A. J. Appleby and J. P. Ackerman (eds.), Proc. DoE/EPRI Workshop on Molten Carbonate Fuel Cells, EPRI WS-78-135 (Nov. 1979).

19. J. R. Selman and L. G. Marianowski, in Molten Salt Technology, p. 323 (D. G. Lovering, ed.), Plenum, NY (1982).

20. J. R. Selman and T. D. Claar, eds., Molten Carbonate Fuel Cell Technology, PV 84-13, The Electrochemical Society, Pennington, NJ (1984).

21. J. R. Selman and H. C. Maru, Adv. Molten Salt Technology (G. Mamantov and J.Braunstein, eds.), 4, 159 (1981).

22. H. C. Maru, in Molten Salt Technologies, Vol. 2, p. 15 (R. J. Gale and D. G. Lovering, eds.), Plenum, NY (1984).

23. M. Warshay, in The Science and Technology of Coal and Coal Utilization (B. R. Cooper and W. A. Ellingson, eds.), Plenum, NY (1984).

24. K. Kinoshita, "Critical Survey of Electrode Aging in Molten Carbonate Fuel Cells," ANL-79-55, Argonne, IL (Dec. 1979).

25. H. C. Maru and L. G. Marianowski, Ext. Abstr. 76-2, p. 82, The Electrochemical Society, Princeton, NJ (1976).

26. R. H. Arendt, J. Electrochem. Soc. 129,942 (1982).

27. "Development of Molten Carbonate Fuel Cell Technology," Project 11304, Interim Technical Progress Report, March 1976-January 1980, SAN/11304-15, ERC, Danbury, CT.

28. "Development of Molten Carbonate Fuel Cell Power Plant," DoE/ET/1701920, Final Report Contract DE-AC02-80ET17019 (2 volumes), GE, March 1985.

29. "Fuel Cell Research on Second-Generation Molten Carbonate Systems," Project 8984. Quarterly and Final Status Reports, July 1, 1976-Sept. 30, 1977, Vol. 2, IGT, Chicago, IL.

30. H. C. Maru, D. Patel, and L. Paetsch, "Electrolyte Distribution and Performance Modeling for MCFC Electrodes," Ext. Abstr. 81-1, p. 27, Electrochemical Society,

Pennington, NJ (1981). 
31. "Fuel Cell Research on Second-Generation Molten Carbonate Systems," Project 9105 , Final Technical Report, Oct. 1, 1977 -Sept. 30, 1978, SAN-1735-4, IGT, Chicago, IL.

32. J. Braunstein and C. W. Vallet, 'Electromigrational Composition Gradients in Molten Carbonate 8, a Review," in Molten Carbonate Fuel Cell Technology, p. 175 (J. R. Selman and T. D. Claar, eds. ), PV 84-13, Electrochemical Socity, Pennington, NJ (1984).

33. H. C. Maru, ERC, private communication (1985).

34. L. G. Marianowski, IGT, private communication (1985).

35. D. L. Maricle, "Identification of Improved Electrolyte Configuration," EPRI RP-1085-4 Report Task 1 (July 1983), UTC, S. Windsor, CT.

36. "Fuel Cell Research on Second-Generation Molten Carbonate Systems," Project 8984, Final Status Report, July 1, 1976 -Sept. 30, 1977, Vol. II, IGT, Chicago, IL.

37. "Advanced Technology Fuel Cell Program," Project 114, EPRI Reports, EPRI EM-576 (Nov. 1977) and EPRI EM-1328 (Jan. 1980), UTC, S. Windsor, CT.

38. E. T. Ong and T. D. Claar, in Molten Carbonate Fuel Cell Technology, P. 54 (J. R, Selman and T. D. Claar, eds.), PV 84-13, Electrochemical Society, Pennington, NJ (1984).

39. A. J. Appleby, EPRI, private communication (1984).

40. R. A. Donado, L. G. Marianowski, H. C. Maru, and J. R. Selman, J. Electrochem. Soc. 131, 2535 and 2541 (1984).

41. D. A. Shores and P. Singh, in Molten Carbonate Fuel Cell Technology, p. 271 (J. R. Selman and T. D. Claar, eds.), PV 84-13, The Electrochemical Society, Pennington, NJ (1984).

42. L. G. Marianowski and J. B. O'Sullivan, "Status of Molten Carbonate Fuel Cell Technology," paper presented at the Eighth Annual Energy Technology Conference and Exposition, Washington, D. C., March 9-11, 1981.

43. R. W. Swaroop, J. W. Sim, and K. Kinoshita, J. Electrochem. Soc. 125, 1799 (1978).

44. C. L. Bushnell and R. C. Nickols, "Analysis of Endurance Cell Teardown, " prepared by UTC, South Windsor, CT, for EPRI, RP-1085-4, Task 4, July 1983.

45. G. Wilemski, J. Electrochem. Soc. 130, 117 (1983).

46. C. Y. Yuh and J. R. Selman, J. Electrochem. Soc. 131, 2062 (1984).

47. P. G. P. Ang and A. F. Sammells, J. Electrochem. Soc. 127, 1287 (1980).

48. S. H. Lu and J. R. Selman, J. Electrochem. Soc. 131, (1984).

49. J. Jewulski and L. Suski, J. Appl. Electrochem. 14, 135 (1984); also, L. Suski, $\mathrm{J}$. Jewulski and J. Wyrwa, in Molten Carbonate Fuel Cell Technology, p. 113 (J. R. Selman and T. D. Claar, eds.), PV 84-13. The Electrochemical Society, Pennington, NJ (1984).

50. S. H. Lu, Ph. D. Thesis, Illinois Institute of Technology, Chicago, IL (1985).

51. A. Borucka, in Fuel Cell Systems-II. Adv. in Chemsitry Series 90, 242-268 (1969).

52. S. E. Chuck, M. S. Thesis, Illinois Institute of Technology, Chicago, IL (1966).

53. A. Borucka and A. J. Appleby, J. Chem. Soc. (Far. Trans. 1) 73, 1420 (1977).

54. W. M. Vogel, L. Bregoli, and S. W. Smith, J. Electrochem. Soc. 127, 833 (1980).

55. Ref. 21, p. $295 \mathrm{ff}$.

56. K. Kinoshita, "Critical Survey of Electrode Aging in Molten Carbonate Fuel Cells," ANL-79-55, Argonne, IL (Dec, 1979).

57. C. D. Iacovangelo, GE, private communication (1975).

58. D. L. Johnson, "Influence of Dispersed Refractory Oxides in Retarding Catalyst Sinter ing," EPRI EM-624, Project 371-1 Report, Palo Alto, CA, March 1978.

59. L. G. Marianowski, IGT, private communication (1984).

60. L. G. Maria nowski, IGT, private communication (1985).

61. G. H. J. Broers, M. Schenke, and H. J. J. van Ballegoy, Ext. Abstr. No. 77, 28th Meeting Internat. Soc. Electrochemistry, Varna, Bulgaria, Vol. 2, pp. 313-316, (Sept. 1977).

62. R. M. Reinstrom, "Carbonate Fuel Cell Power Plant Systems," IEEE Trans. Power Apparatus and Systems PAS-100, 4752 (1980).

63. H, C. Maru, ERC, private communication (1984).

64. H. C. Maru, Quarterly Progress Report, DoE Project DEAC03-76 (ET-11304), ERC, Da nbury. CT (1985).

65. H. C. Maru and B. S. Baker, in Progr. Batt. Solar Cells, JEC Press, Vol. 5 (1984).

66. G. L. Anderson and P. C. Garrigan, in Molten Carbonate Fuel Cell Technology, p. 297 (J. R. Selman and T. D. Claar, eds.), PV 84-13, The Electrochemical Society, Pennington, NJ (1984).

67. "Fuel Cell Research on Second-Generation Molten Carbonate Systems," Project 8984 Final Status Report, July 1, 1976 -Sept. 30, 1977, Vo1. I, IGT, Chicago, IL.

68. A. F. Sammells, S. B. Nicholson, and P. G. P. Ang, J. Electrochem. Soc. 127,350 (1980).

69. W. M. Vogel and S. W. Smith, J. Electrochem. Soc. 129, 1441 (1982).

70. S. W. Smith, H. R. Kunz, W. M. Vogel, and S. J. Szymanski, "Effects of Sulfur on Molten Carbonate Fuel Cells - A Survey," in Molten Carbonate Fuel Cell Technology, 
p. 246 (J. R. Selman and T. D. Claar, eds.), PV 84-13, The Electrochemical Society, Pennington, NJ (1984).

71. R. J. Remick, "Effects of $\mathrm{H}_{2} \mathrm{~S}$ on Molten Carbonate Fuel Cells," DoE/MC/20212-1739 (February 1985); R. J. Remick and D. R. Vasil, in Fuel Cell Seminar Abstracts 1985. p. 173.

72. L. G. Marianowski, R. A. Donado, and H. C. Maru, U.S. Pat. 4, 247,604 (Jan. 27,1981 ).

73. J. B. Goodenough, in Progr. Solid-State Chem. H. Reia 8, ed. , 5,271 (1971).

74. H. C. Maru, D. Patel, and L. Paetsch, Ext. Abstr. 81-1, Electrochemical Society, Pennington, NJ (1981).

75. C. Y. Yuh and J. R. Selman, J. Electrochem. Soc. 131, 2062 (1984).

76. L. J. Bregoli and H. R. Kunz, J. Electrochem. Soc. 129, 2711 (1982).

77. C. Y. Yuh, Ph. D. Thesis, Mlinois Institute of Technology, Chicago, IL (1985).

78. L. J. Bregoli, H. R. Kunz, S. W. Smith, and W. M. Vogel, "Development of Improved Cathodes: Kinetics," Report EPRI RP-1085-4, Task 3A, UTC, S. Windsor, CT (July 1983).

79. R. D. Pierce, J. L. Smith, and R. B. Poeppel, in Molten Carbonate Fuel Cell Technology. p. 147 (J. R. Selman and T. D. Claar, ede.), PV 84-13, The Electrochemical Society, Pennington, NJ.

80. A. J. Appleby and C. van Drunen, J. Electrochem. Soc. 127, 1655 (1980).

81. A. J. Appleby and S. B. Nicholson, J. Electroanal. Chem. 53, 105 (1974); 83, 309 (1977), 112, 71 (1980); J. Electrochem. Soc. 127, 759 (1980).

82. N. I. Uchida, "Gas Electrode Reaction in Molten Carbonate," Abstr. 50th Mtg. Jap. Electrochem. Soc. Meeting, March 1983, Tokyo.

83. S. W. Smith, W. M. Vogel, and S. Kapelner, J. Electrochem. Soc. 129, 1668 (1982).

84. L. G. Maria nowski, IGT, private communication (1984).

85. C. Reiser, UTC, private communication (1985).

86. M. K. Orrfield and D. A. Shores, Ext. Abstr. 84-2, p. 74, Electrochemical Soc., Pennington, NJ (1984).

87. "Development of Improved Molten Carbonate Fuel Cell Technology," p. 5-1, Project RP 1085-4, EPRI Final Report, UTC, S. Windsor, CT, July 1983.

88. C. D. Iacovangelo, GE, private communication (1984).

89. "Development of Molten Carbonate Fuel Cell Power Plant," Final Report DoE/ET/1701920, Vol. 1, p. 4-138 ff, GE, March 1985.

90. A. J. Appleby, "Advanced Fuel Cell Technology," EPRI J., p. 63, Dec. 1984.

91. "Advanced Fuel Cell Development," Progr. Report July-Sept. 1984, ANL-85-7, Argonne, IL (June 1985).

92. Ref. 89, Vol. II, p. 5-9.

93. H. C. Maru, ERC, private communication (1984).

94. 'Molten Carbonate Fuel Cell System Verification and Scale-Up, " EPRI EM-4129, Project 1273-1 Final Report, UTC, S. Windsor, CT (July 1985).

95. E. H. Camara, L. G. Marianowski, and R. A. Donado, U. S. Patent 4, 160,067 (July 3 , 1979).

96. G. Wilemski, A. Gelb, and T. L. Wolf, in Molten Carbonate Fuel Cell Technology, p. 218 (J. R. Selman and T. D. Claar, eds.), PV 84-13, The Electrochemical Society, Pennington, NJ (1984).

97. T. L. Wolf and G. Wilemski, J, Electrochem. Soc. 130, 48 (1983).

98. P. S. Patel, "Assessment of a 6500 BTU/kWh Heat Rate Dispersed Generator," EM-3307, EPRI, Palo Alto, CA (1983).

99. L. G. Marianowski, IGT, private communication (1984).

100. P. Singh and H. C. Maru, "Stability of Iron and Nickel Base Alloys in Molten Carbonate Fuel Cells," NACE (1985).

101. B. S Baker and H. G. Ghezel-Ayagh, U.S. Pat. 4,532, 192 (July 30, 1985).

102. M. P. Kang and J. Winnick, J. Appl. Electrochem. 15, 431 (1985). 


\section{HIGH-TEMPERATURE SOLID-OXIDE FUEL CELIS (SOFCs) ${ }^{\dagger}$}

\subsection{Introduction}

Several years ago, it was believed that the solid-oxide fuel cell was such an advanced technical concept that many years of diligent research would be required to bring the technology to a commercial state. At that time, it was believed by many authorities that the solid-oxide fuel cell offered the widest potential range of application with the promise of highest achievable system efficiency. This technical evaluation was made because the technology offered an all solid-state power system operating at a sufficiently high temperature $\left(1000^{\circ} \mathrm{C}\right)$ to provide highquality waste heat for cogeneration applications and bottoming cycles utilizing conventional steam turbines. System electrical efficiencies of about $60 \%$ can be shown to be readily obtainable.

Within the past 24 months, a series of technical accomplishments have led to improved materials and processing that have resulted in stable cell performance at high power density. The se developments have motivated a complete reassessment of the future of the SOFC. It now appears that this technology, with an aggressive development effort, can reach commercial status by about 1990. If this goal is met, the solid-oxide fuel cell will be of value to a broad segment of the U.S. economy 10 to 15 years earlier than was believed to be the case just two or three years ago.

In the past, SOFC technologies have not received as much financial support from the U.S. DOE as PAFCs or MCFCs. In 1984, Congress mandated, for Department of Energy expenditures, $\$ 28.8$ and $\$ 9.2 \mathrm{M}$ for $R \& D$ on $P A F C s$ and MCFCs, respectively, while support for SOFC was $\$ 2.0 \mathrm{M} .1$ These funds were mostly spent in industry and not-for-profit organizations. However, significantly increased funding is available in FY85 and plans to continue increasing funds in FY86 and beyond are being formulated.

Westinghouse Electric (WE) is the only U.S. manufacturer pursuing the commercial production of SOFCs. Management at WE foresees a significant potential for combined heat and electric power plants for the industrial and commercial markets and their aim is penetration of this market beginning in 1990.

\section{IA Advantagee of SOFC Power-Plant Systems}

Use of the solid-phase electrolyte reduces corrosion and eliminates electrolyte-manage ment problems. High-temperature SOFCs have been shown to be effective in supporting electrode kinetics. Therefore, cataly sts may not be needed to reduce activation overpotentials dur ing operation. 1 However, in 1981 , workers at the Brookhaven National Laboratory (BNL) showed that $39 \%$ of the yoltage $1088 e s$ for operating, state-of-the-art SOFCs were caused by electrode overpotentials. 2 This value could be a $10 \mathrm{w}$ estimate because $26 \%$ of the $108 \mathrm{ses}$ were then caused by electrolyte resistance, which has been reduced to $\sim 4 \%$ of total losses by successful implementation of electrochemical vapor deposition (EVD) to fabricate much thinner electrolytes. Therefore, there is evidence that work on electrocatalysis may be beneficial to the performance of SOFCs.

Recycling of $\mathrm{CO}_{2}(\mathrm{~g})$ and the complex apparatus needed to perform this task are not re quired with SOFCs in the manner used with MCFCs. Moreover, the fuel stream in SOFCs does not need to be as extensively reformed and scrubbed free of impurities as in other FC aystems. Internal reforming of methane or of other hydrocarbons to $\mathrm{CO}$ and $\mathrm{H}_{2}$ is virtually assured at the high prevailing operating temperatures. Both MCFCs and SOFCs are designed to use CO directly as fuel, whereas anodes in PAFCs are poisoned by CO. SOFCs are thermodynamically more tolerant to $S$-containing contaminants, such as $\mathrm{H}_{2} \mathrm{~S}$ or COS, than are carbonate or acid systems. Thermodynamic limits are not accurate gauges for occurrences of sulfidation in flow systems. 3 However, SOFCs fed with $50 \mathrm{ppm}$ of $\mathrm{H}_{2} \mathrm{~S}$ in the fuel stream for $800 \mathrm{~h}$ showed only a $5 \%$ decrease in performance, and this degradation was reversible once the $\mathrm{H}_{2} \mathrm{~S}$ was removed from the fuel stream.

The power densities of FCs are currently much lower than those of competitive electricity-generating technologies. 4 With the successful implementation of EVD to produce thinner electrolytes, the power densities of SOFCs have been increased. The development of monolithic SOFCs will lead to further increases in power densities and will hopefully make these cells superior to other electricity-generating technologies. Workers at the Argonne National Laboratory (ANL) eatimate a power density of $4 \times 10^{3} \mathrm{~kW} / \mathrm{m}^{3}$ and a specific power of $8.08 \times 10^{3} \mathrm{~W} / \mathrm{kg}(\mathrm{cell}$

This chapter has been prepared by J. T. Brown using, in part, an earlier manuscript supplied by S. S. Penner and E. Williams. The author is indebted to many experts at WE, as well as to his AFCWG colleagues, for helpful advice. 
only) for a monolithic SOFC, as compared with $140 \mathrm{~kW} / \mathrm{m}^{3}$ and $97 \mathrm{~W} / \mathrm{kg}$ for a state-of -the-art design FC. 4 Tape-casting is a promising procedure for the fabrication of monolithic SOFCs. Figure 6. $1 \mathrm{~A}-1$ shows a cross-sectional schematic of the monolithic cell proposed at ANL. 5

SOFCs are intrinsically simple systems. By applying an external potential, the cell can be run reversibly as an electrolyzer. If water is present at the cathode, $\mathrm{H}_{2}$ is evolved $\left[\mathrm{H}_{2} \mathrm{O}(\mathrm{g})+\right.$ $\left.2 \mathrm{e}^{-} \rightarrow \mathrm{H}_{2}(\mathrm{~g})+\mathrm{O}^{=}\right]$while $\mathrm{O}_{2}$ is evolved at the anode $\left[\mathrm{O}^{=} \rightarrow 2 \mathrm{e}^{-}+(1 / 2) \mathrm{O}_{2}(\mathrm{~g})\right]$. If only oxygen is present at the cathode and $a$ sufficiently large potential is applied, the cell will act as an oxygen pump (the rever se of the reaction in an oxygen-concentration cell occura), i.e. , oxygen gas is consumed at the cathode $\left[(1 / 2) \mathrm{O}_{2}(\mathrm{~g})+2 \mathrm{e}^{-} \rightarrow \mathrm{O}^{=}\right]$and evolved at the anode $\left[\mathrm{O}^{-} \rightarrow 2 \mathrm{e}^{-}+(1 / 2) \mathrm{O}_{2}(\mathrm{~g})\right]$.

\section{1B Disadvantages of SOFCB}

The ideal efficiencies $[\Delta G(T) / \Delta H(T)]$ of the overall cell redox reactions $\left[H_{2}(g)+\right.$ $\left.(1 / 2) \mathrm{O}_{2}(\mathrm{~g}) \rightarrow \mathrm{H}_{2} \mathrm{O}(\mathrm{g}), \mathrm{CO}(\mathrm{g})+(1 / 2) \mathrm{O}_{2}(\mathrm{~g}) \rightarrow \mathrm{CO}_{2}(\mathrm{~g})\right]$ are reduced at $1000^{\circ} \mathrm{C}$ below those occurring at lower operating temperatures used in other cells. On the other hand, the higher operating temperature should reduce cell overpotentials by reducing component resistivities and increasing electrode reaction rates. These advantages have been demonstrated in currently available SOFCs, and continued research is directed at reducing the se overpotentials still further. Since the reaction products are discharged at higher temperatures than from other cells, $108 \mathrm{~s}$ in ideal efficiency may be compensated for, at least in part, by improved Carnot efficiencies of associated bottoming cycles.

Cells operating at $1000^{\circ} \mathrm{C}$ could pose safety problems and may be unsuitable for some applications. However, since the cells operate well above the ignition temperature, any $\mathrm{H}_{2}$ that comes in contact with air will immediately react. This process prevents accumulation of explosive mixtures, which is the real safety hazard with gases.

It is obvious that high-temperature cells must either be maintained at operating temperatures or else must be heated before start-up. Significant energy losses will be associated with some intermittent applications. The required heating times for start-up and the need for heating pose additional problem areas for SOFCs in transportation applications. Studies at WE have shown that a 3-cell stack endured 24 thermal cycles without performance degradation. After thermal cycling was terminated, the stack continued to operate satisfactorily. 6 Far more than 24 cycles will be needed for FCs in transportation applications. It is also important that monolithic FCs be developed successfully if we are to obtain acceptable power densities for utilization in most transportation applications. WE has demonstrated start-up, stand-by, and load following in a 24-cell generator of their design, but design limits that could cause system damage have not as yet been described.

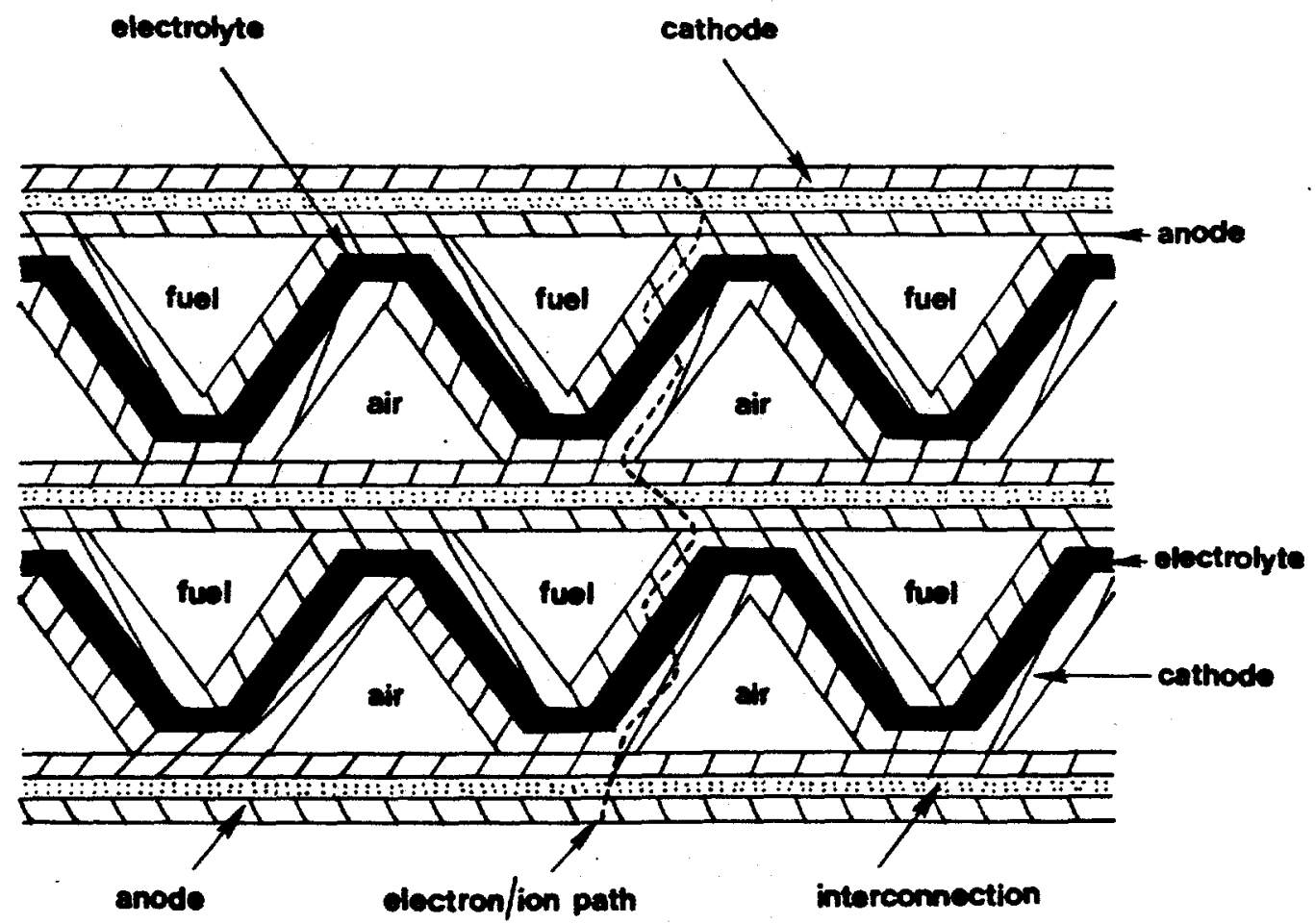

Fig. 6. 1A-1. A schematic cross section is shown of the monolithic SOFC proposed by workers at ANL. The interconnection is an electronic conductor; the channels are 1 to $2 \mathrm{~mm}$ thick, while the electrodes and electrolyte are about 1 to 2 mils in thickness. 5 
As with other fuel cells, adequate endurance for SOFCs remains to be demonstrated. Current endurance tests at WE have shown favorable progress ( $>8000 \mathrm{~h}$ of testing has been accomplished without performance degradation). Currently available SOFCs have an efficiency of $45 \%$ at typical usable system power outputs. Fee of ANL claims that a pressurized SOFC could yield $60 \%$ efficiency. 7 A successful technique for pressurizing SOFCs remains to be developed. 3 The economics of SOFC systems have not been adequately addressed to date but analysis of process and materials costs shows these to be 1 ess than $\$ 100 / \mathrm{kW}$. High costs for SOFC $\mathrm{s}$ could result from the required use of sophisticated high-temper ature fabrication procedures for cells. Sales prices for cell-bundle assemblies are estimated to be $\$ 300$ to $\$ 500$ per $\mathrm{kW}$. Systems industrial and commercial applications in sizes up to several $M W$ are expected to range from $\$ 700-\$ 1500$ per $\mathrm{kW}(\$ 1985)$. These cost estimates were provided by WE.

\section{1C Reactions Occurring in Operating SOFCs}

In an operating SOFC, negative charge is conducted by electrons from an anode through an external circuit to a cathode; it is conducted by negative ions $\left(O^{\prime}\right)$ from a cathode through the electrolyte to an anode.

Figure 6.1C-1 shows the operating principle, the anode and cathode reactions, and the overall reactions for an SOFC operating on a reformate fuel (mixture of $\mathrm{CO}$ and $\mathrm{H}_{2}$ ). The SOFCs typically operate at $1000^{\circ} \mathrm{C}$; at this temperature, the electrolyte is an oxygen-ion conductor, and

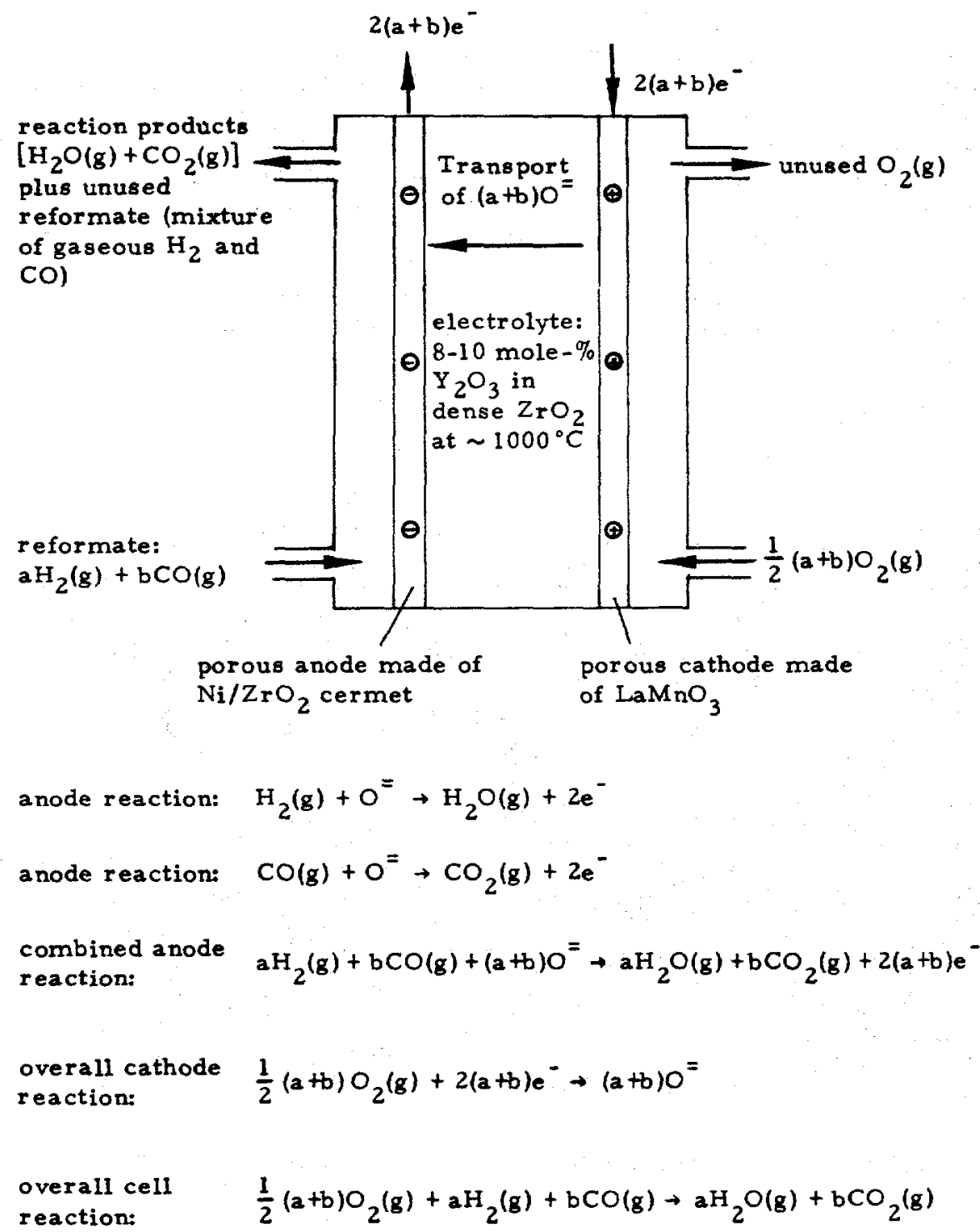

Fig. 6.1C-1. A schematic is shown of the essential features and the overall electrode reactions of an SOFC unit. 
the free energies (and therefore the associated Nerst potential) of the overall reactions are substantially lower than at lower temperatures $(e .8 ., 43.3 \mathrm{kcal} / \mathrm{mole}$ at $1200 \mathrm{~K} \mathrm{vs} 54.617 \mathrm{kcal} /$ mole at $300 \mathrm{~K}$ for $\mathrm{H}_{2}$ ). Since the heat of reaction is nearly independent of temperature, the po. tential (ideal) efficiency is reduced by the high-temperature operation. The actual efficiency and electrical energy produced will, of course, be lower if the voltage is reduced by IR losses or polarization. Maintaining the temperature level requires cooling of the cell to remove a quantity of heat equal to the difference between the heat of reaction and the electricity produced. The heat removed is the difference in enthalpy between the products of the reaction leaving the generator at high temperature and the cooler reactants supplied to the generator. Since the heat is available at high temperature $\left(\sim 1000^{\circ} \mathrm{C}\right)$, it can be used in an efficient bottoming cycle to produce more electricity or in applications requiring high grade heat.

The electrolyte is $\mathrm{ZrO}_{2}$ (zirconia) doped with $8-10$ mole-\% of $\mathrm{Y}_{2} \mathrm{O}_{3}$ (yttria). Pure zir conia is an in aulator. When yttria is mixed with zixconia, some $\mathrm{Y}^{+3}$ replaces $\mathrm{Zr}^{+4}$ in the fluo. rite-type crystal structure 8 A number of oxide-ion sites become vacant because three $O=$ replace four $\mathrm{O}^{=}$when two $\mathrm{Zr}^{+4}$ are replaced by two $\mathrm{Y}^{+3}$ in the lattice. At high temperatures, the $O=$ move across the electrolyte via vacant lattice sites, thus conducting charge ionically.

Porous gas-diffusion electrodes are used because diffusion of reactant species through solid plate electrodes becomes the limiting rate process at low currents and sufficient power outputs are attainable only with porous electrodes. The processes occurring at the electrodes are not well understood. There are no quantitative models for the effects of electrode structure and composition on electrode performance for solid electrolyte systems. For liquid electrolyte systems, the simple-pore, thin-layer, and hybrid models are useful in describing the influence of electrode morphology on electrode performance, while variants of the double-layer theory describe the effects of the electrolyte-electrode interface on electrode performance. Studies of the mechanism and $r$ ate-determining step for electrode reactions should be useful in future electrode development.

Some attempts have been made to understand electrode reaction mechanisms. Isaacs has proposed that oxygen adsorption on the electrode surface is the rate-controlling step for the electro-reduction of $\mathrm{O}_{2}(\mathrm{~g})$ at the cathode. $9-11$ This is at variance with the more frequently proposed mechanism expressed as $\mathrm{O}_{2}+4 e^{-} \rightarrow 20^{=}$. The WE state-of-the-art cathode is made from $\mathrm{LaMnO}_{3}$. Although the evolution of oxygen $\left[\mathrm{O}^{=} \rightarrow(1 / 2) \mathrm{O}_{2}(\mathrm{~g})+2 \mathrm{e}^{-}\right]$, followed by the formation of water $\left[\mathrm{H}_{2}(\mathrm{~g})+(1 / 2) \mathrm{O}_{2}(\mathrm{~g}) \rightarrow \mathrm{H}_{2} \mathrm{O}(\mathrm{g})\right]$, is a possible anodic reaction mechanism, the direct electro-oxidation of $\mathrm{H}_{2}$ is more likely to occur $\left[\mathrm{H}_{2}(\mathrm{~g})+2 \mathrm{M} \rightarrow 2 \mathrm{MH}, 2 \mathrm{MH}+\mathrm{O}^{=} \rightarrow 2 \mathrm{M}+\mathrm{H}_{2} \mathrm{O}+2 \mathrm{e}^{-}\right.$, where $M$ stands for an electrode molecule]. 12 The WE state-of-the-art anode is a nickelzirconia cermet. Cell stacking is required in order to obtain usable voltages. In the past, problems were encountered in the search for suitable interconnection material. Workers at WE are currently using $\mathrm{Mg}$-doped $\mathrm{LaCrO}_{3}$.

A derivation from first principles of the open circuit potential of the SOFC follows. For a reversible cell, the free energy change $(\Delta G)$ of the cell reaction is related to the EMF (E). i. e. , the open circuit voltage of the cell, by the expression

$$
\Delta G=-n F E \text {, }
$$

where $n$ is the number of electrons involved in the cell reaction and $F$ is Faraday's constant. For the SOFC, it is generally accepted that the cathode reaction is

$$
\mathrm{O}_{2}+4 \mathrm{e}^{-} \rightarrow 2 \mathrm{O}^{=}
$$

and the anode reaction with $\mathrm{H}_{2}$ as fuel is

$$
2 \mathrm{O}^{=}+2 \mathrm{H}_{2} \rightarrow 2 \mathrm{H}_{2} \mathrm{O}+4 \mathrm{e}^{-} \text {. }
$$

Thus, by combining these two half-cell reactions, the overall cell reaction is obtained as

$$
2 \mathrm{H}_{2} \text { (anode) }+\mathrm{O}_{2} \text { (cathode) }=2 \mathrm{H}_{2} \mathrm{O} \text { (anode) }
$$

When the reactants and products do not occur in their standard states, $\Delta G$ for the reaction may be related to the standard state free energy change $\left(\Delta G^{\circ}\right)$ and the various gas partial pressures by

$$
\Delta G=\Delta G^{\circ}+R T \text { en }\left[\mathrm{P}_{\mathrm{H}_{2}}^{2} \mathrm{O} \text {, anode } / \mathrm{P}_{\mathrm{H}_{2}}^{2} \text {, anode }{ }^{\left.\mathrm{P}_{\mathrm{O}_{2}}, \text { cathode }\right]}\right. \text {. (6.1C-5) }
$$

If equilibrium is assumed at the anode, the ratio in Eq. (5) becomes

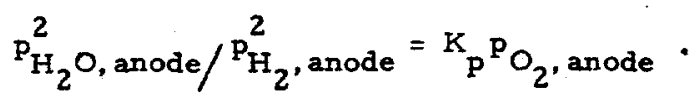


Since the equilibrium constant $K_{p}$ is related to $\Delta G^{\circ}$ through the expression

$$
\Delta G^{\circ}=-R T \text { en } K_{p} \text {, }
$$

$\Delta G$ may be written in terms of the two oxygen partial pressures by combining Eqs. (6.1C-5) to (6. 1C-7) as

$$
\Delta G=\operatorname{RT} \text { en }\left[P_{\mathrm{O}_{2}} \text {, anode } / \mathrm{P}_{2} \text {, cathode }\right] \cdot \quad \text { (6.1C-8) }
$$

From Eqs. (6.1C-1) and (6.1C-8), it follows that the open circuit voltage of an SOFC is

$$
E=\frac{R T}{4 F} \text { in }\left[P_{O_{2}} \text {, cathode } / P_{O_{2}} \text {, anode }\right] \text {. }
$$

Although Eq. (6.1C-9) has the form of $E$ found for a concentration cell, the SOFC is not a concentration cell because the net reaction is given by Eq. (6.1C-4) and cannot occur in a concentration cell.

\subsection{The Westinghouse SOFC $7, \dagger, \ddagger$}

Westinghouse plans to build a pilot plant to produce units of $25-200 \mathrm{~kW}$ for field testing by 1987. The plant will have a production capacity of $1000 \mathrm{~kW} / \mathrm{y}$ of various size power plants in the specified size range. The units will be distributed to selected customers. WE will design fuel cell systems for specific applications. Field testing will be done to establish user acceptance and provide good estimates of SOFC production cost $B$, allowable market price, and size of the demand for SOFCs. This program is expected to lead to improved under standing of technical difficulties that may be encountered in large-scale commercial applications. These WE SOFCs will be fueled by natural gas, $\mathrm{H}_{2}(\mathrm{~g})$, or process gas rich in $\mathrm{CO}$ and $\mathrm{H}_{2}$. Presently estimated material costs for SOFCs will have to be refined. Consi stent methodology for cost comparisons among $F C_{s}$ does not exist as yet. Fabrication costs for SOFCs have not been determined but depend largely on effective mechanization of the thin-film deposition processes.

WE is also working on a DOE-supported program to develop a 5-kW unit by mid-1985. A model of a 324 -cell stack $\left(\sim 5 \mathrm{~W}_{e} /\right.$ cell) has been constructed. The stack will be compact $\left(2.5^{\prime} \times\right.$ $\left.2.5^{\prime} \times 4^{\prime}\right)$ and will include an envelope of $6^{\prime \prime}$ of insulation.?

\section{2A. Design of the Closed-End Tubular SOFC $6,7, T^{1}$}

Since about 1981, workers at WE have used a closed-end tubular support upon which the cathode, interconnection, electrolyte, and anode are equentially deposited. Figure 6.2A-1 shows that air is fed to the tube via a concentric alumina tube in a co-flow configuration. The central air-delivery tube is integrated into a heat exchanger to allow maintenance of proper cell operating temperatures at various cell power outputs. Figure 6.2A-2 shows how the cella will be stacked. The series-parallel stack design protects the syatem against complete stack failure in the event of individual cell failure. Accommodation of stacked cells by nickel felt pads inhibits the development of destructive stresses in the cells. Workers at WE are optimistic that the large number of individual repeating cells will not cause problems in operating stacks.

The support tube is made of calcia-stabilized zirconia and accounts for $50 \%$ of the material cost in this design. The support tube provides structural support and allows the use of very thin structures for the other components. Each tibe has an inside diameter of $\sim 1 / 2$ inch, $\sim 1 / 16$ wall thickness, 15 inches length (with $\sim 12$ inches of active length), an active surface area of $100 \mathrm{~cm}^{2}$, and about $30 \%$ porosity. The support tube is porous to facilitate air flows to the cathode for reaction at the $\mathrm{LaMnO}_{3}$ cathode. The tube is made of stabilized zirconia so that its thermal expansion properties match those of the cathode, electrolyte, anode, and interconnection. The electrolyte is $<50 \mathrm{\mu m}$ thick, made of the dense yttria-stabilized zirconia (YSZ), and eliminates gas crossover. The anode is a nickel-zirconia cermet. The interconnection is also $<50 \mathrm{\mu m}$ thick and is made of $\mathrm{LaCrO}_{3}$.

Each tube has a design point power output of $18 \mathrm{~W}_{\mathrm{e}}$. The current density it higher at the closed than at the open end of the cell. The efficiency of this design is currently $44 \%$ but is 800 n

'Discussions in this section are based on material presented to the AFCWG by WE workers, Refs. 6, 7, 13-16.

\footnotetext{
F Introductory comments are based on a presentation made by G. W. Wiener to AFCWG, Ref. 7.

This discussion is based on presentations by J. T. Brown to AFCWG, Ref. 6.
} 


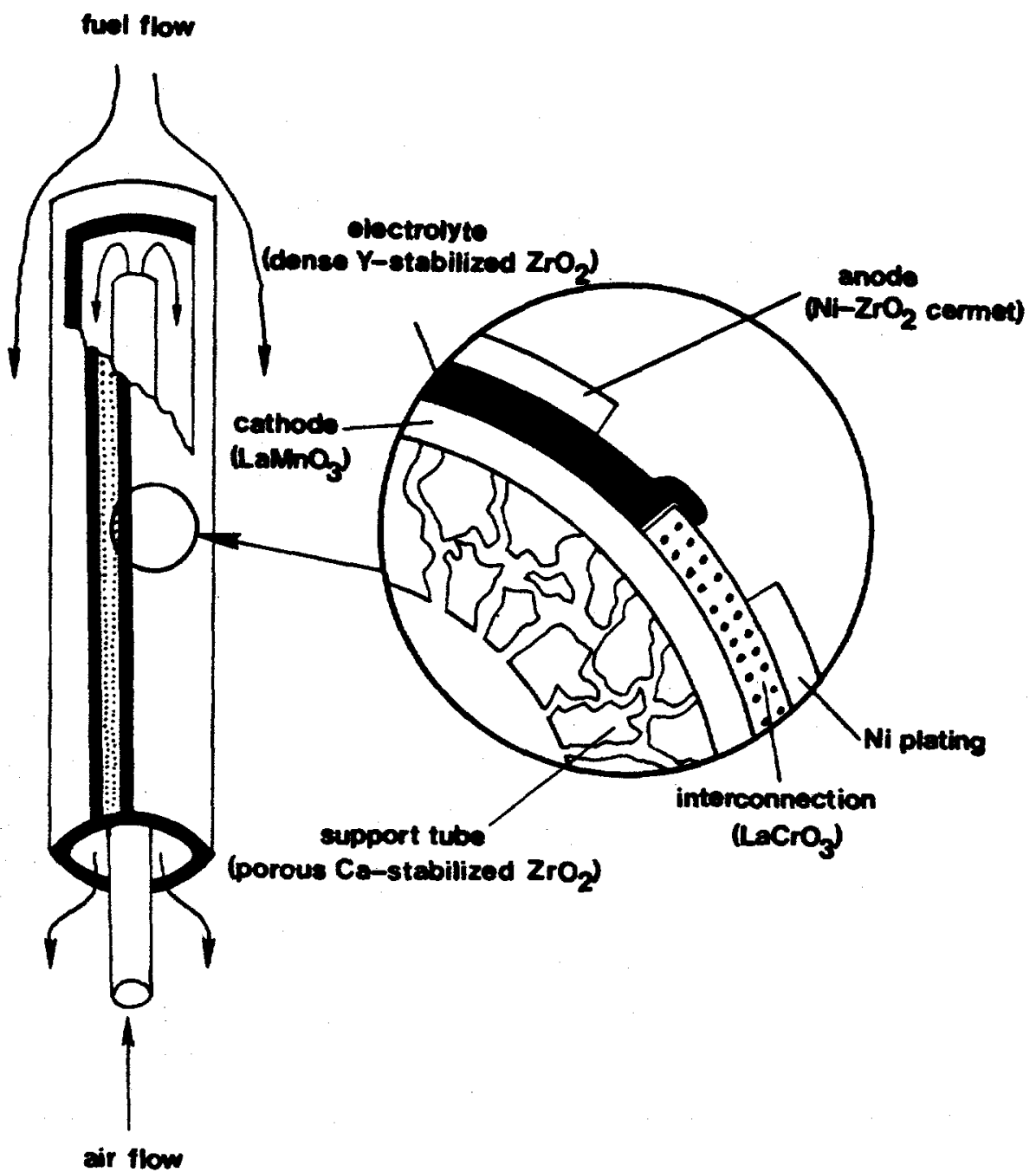

Fig. 6.2A-1. A schematic is shown of the WE closed-end, tubular SOFC design. 6

expected to increase to $50 \%$ when thinner components with less resistance are used. These cells have a designed fuel utilization of $85 \%$; the unused $15 \%$ of fuel is subsequently burned with excess oxygen.

More than $8000 \mathrm{~h}$ of testing of 3-cell stacks have been successfully completed. The development goal was $1.71 \mathrm{~V}$ at $160 \mathrm{~mA} / \mathrm{cm}^{2}$ and 1 atm for a fuel mixture corresponding to 1.5 moles of $\mathrm{H}_{2} \mathrm{O}$ per mole of $\mathrm{CH}_{4}\left(67 \% \mathrm{H}_{2}, 22 \% \mathrm{CO}, 11 \% \mathrm{H}_{2} \mathrm{O}\right)$. Potentials from 1.85 to $1.93 \mathrm{~V}$ were achieved at $176 \mathrm{~mA} / \mathrm{cm}^{2}$ during the first $3900 \mathrm{~h}$ of testing. Fuel utilization was $85 \%$. Four times the stoichiometric amount of air was used. After $3900 \mathrm{~h}$, the current density was increased to $252 \mathrm{~mA} / \mathrm{cm}^{2}$; at this average current density, the voltage decreased to 0.60 volt per cell and the terminal voltage remained constant for another $4000 \mathrm{~h}$ of testing. When pure $\mathrm{O}_{2}$ was used intermittently instead of air during the tests, the voltage increased by $\sim 70 \mathrm{mV} .7$

Operational characteristics of the WE SOFC include rapid response to load variation, tolerance to fuel-feed inter ruption and toler ance to load interruption, with or without continuing fuel flow. Stack performance is always better after fuel-flow inter ruption while under load. Marianowski believes that the anode must sinter under normal operating conditions and, when the fuel-stream is shut off, the anode is oxidized; it is then reduced when the fuel is reintroduced to a larger active surface area. 3

Worker 8 at WE state that all of the SOFC components for their closed-end tubular design have been thoroughly investigated and have been proved to describe a potentially commercial state-of-the-art performance. They consider their fabrication processes, when appropriately mechanized, to be suitable for mass production. Multiple-cell testing is being used to obtain statistical evaluations of cell performance. The WE 3-cell stack has shown stable and acceptable performance under realistic air - and fuel-flow conditions. 


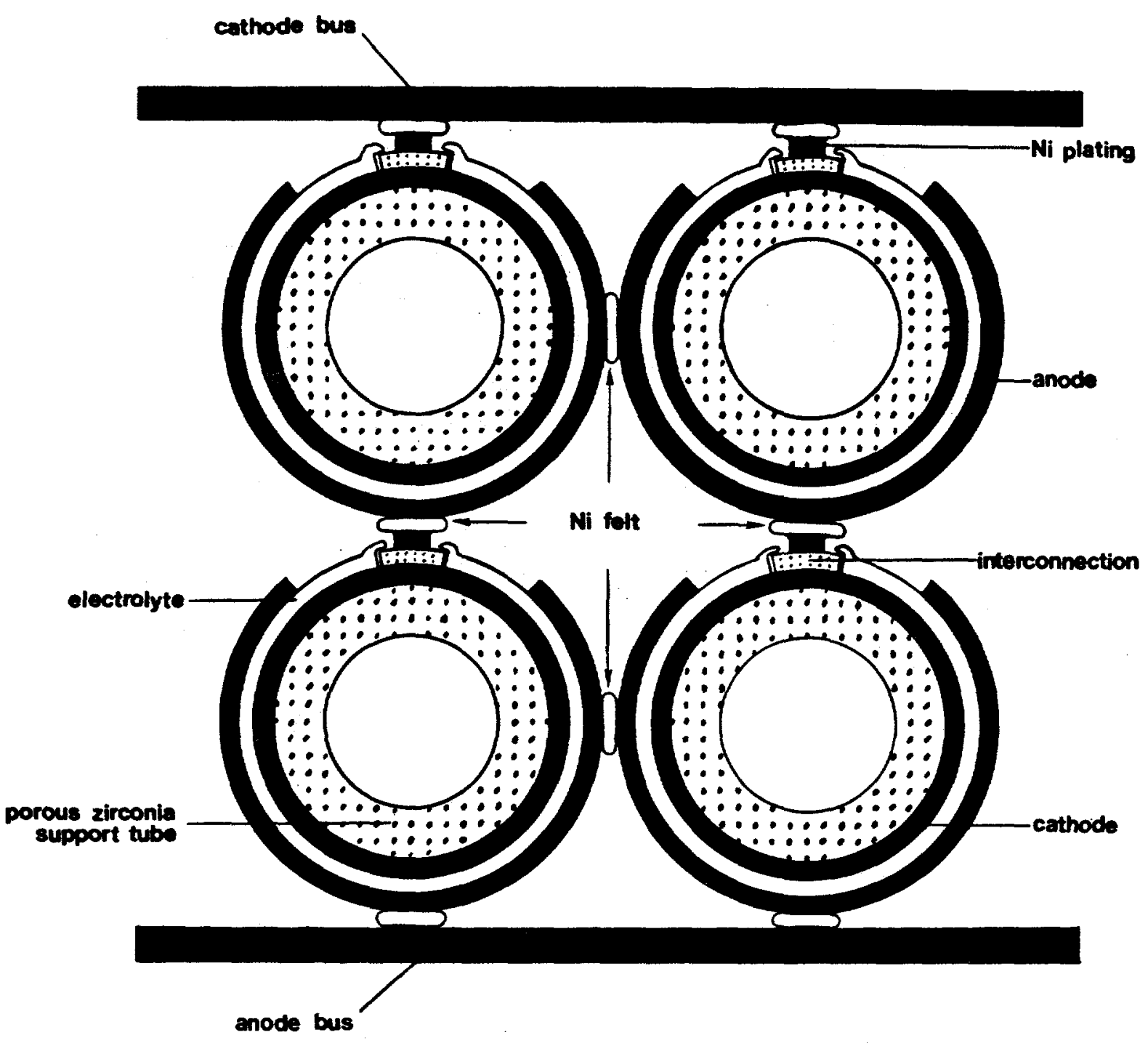

Fig. 6.2A-2. The schematic cross section shows the method that WE uses to stack their closed-end, tubular SOFCs.6

\section{2B Material Requirements for SOFC Components ${ }^{13,+}$}

The electrolyte has been successfully fabricated into a thin, low-resistance layer. Other SOFC components can be improved by utilizing better materials, geometries, or fabrication processes that reduce manufacturing costs and increase cell performance and endurance. Ruka has reviewed component-selection criteria. He considers two stages. The first stage refers to individual characteristics such as chemical stability at high temperatures, appropriate electrical properties, low cost, and plentiful supply. The second stage involves considerations of intercomponent-compatibility characteristics such as minimum chemical interactions, matching thermal expansion behavior, and suitability for fabrication without damage to other cell components.

\section{Support Tube}

The use of an improved porous support-tube material or structure will increase the cellpower density, improve mechanical strength and reduce material cost. Specific requirements for the support tube, which depend on the material used, are thermal-expansion matching and chemical stability. Properties relating to both fabrication and material selection are mechanical strength, porosity, dimensional perfection (all cells must be symmetric to permit a stressfree stacking arrangement), and low cost.

\footnotetext{
This discussion is based on the presentations by R. J. Ruka to AFCWG, Ref. 13.
} 
Gaseous oxygen must diffuse through the pores of the support tube to reach the cathode: An estimate of the diffusion-limited current density $j_{\text {lim }}$ for cells with new support-tube mate$r i a l s$ or atructures is desirable. This may be calculated from the equation $j_{\lim }=(4 F / R T) \times$ $\left(D_{\text {eff, }} T / 1\right) \mathrm{PO}_{2}$ if the effective diffusion coefficient $D_{\text {eff, }} T$ for oxygen in the pores is known at $T$, and $l=$ effective diffusion distance. For sufficiently large pores $(>5 \mathrm{~mm})$, molecular diffusion will predominate, and $D_{\text {eff }}, T$ may be estimated for the porous material by measuring the diffusion at room temperature, $D_{e f f}, T_{0}$, and using the relation

$$
D_{\text {eff, } T}=D_{\text {eff, } T}\left(T / T_{0}\right)^{1.75}
$$

\section{Anode}

Nickel-zirconia cermet is a suitable anode material. Specific requirements for the anode, which may be met by proper material selection, are sufficient electronic conductivity, high tolerance to sulfur contaminants in the fuel stream, and chemical stability in fuel environments. Fabrication-related requirements involve adequate mechanical stability and low cost.

\section{Cathode}

The primary design problems relate to the cathode. Much of the cell-resistance 10s8, as well as significant electrode-reaction overpotentials, occur at the cathode. Therefore, new and improved cathode materials are being sought. Performance requirements include high electronic conductivity, suitable thermal expansion behavior, chemical stability in operating environments, suitable catalytic properties, and moderate cost; the cathode material must be chemically and dimensionally stable during EVD of the electrolyte and interconnection since a part of the cathode is coated with these materials (see Fig. 6.2A-1). 13

After hundreds of perovskite-type materials had been tested, materials such as $\mathrm{La}_{1-x} \mathrm{Sr}_{x} \mathrm{MnO}_{3}(0 \leq x \leq 0.5)$ or $\mathrm{La}_{1-\mathrm{y}} \mathrm{Ca}_{y} \mathrm{MnO}_{3}(0 \leq \mathrm{y} \leq 0.6)$ were selected for resistivity tests. At $1000^{\circ} \mathrm{C}$, La. ${ }_{5} \mathrm{Sr}_{0} . \mathrm{MnO}_{3}$ has the lowest resistivity $(0.0034 \Omega-\mathrm{cm})$ and is followed by $\mathrm{La}_{0.4} \mathrm{Ca}_{0.6} \mathrm{MnO}_{3}(0.0039 \Omega-\mathrm{cm})$ and $\mathrm{La}_{0.5} \mathrm{Ca}_{0.5} \mathrm{MnO}_{3}(0.0041 \Omega-\mathrm{cm})$. A mathematical model for the conduction mechanism in compounds of these types agrees well with experimental results. 13 According to this model.

$$
\log \sigma T=\log C^{\prime}-E_{h} /(2.303 k T),
$$

where

$$
C^{\prime}=n e\left[f(e / k) a^{2} v\right]
$$

Here, $T$ is the absolute temperature $(K), \sigma$ the conductivity $(\Omega-\mathrm{cm})^{-1}, E_{h}$ the hopping energy, $k$ the Boltzmann constant, $e=1.602 \times 10^{-19} \mathrm{C}$ is the elementary charge, $n$ is the number density of elementary charges conducted with each hop, $f$ the fraction of unoccupied hopping, sites, a the jump distance $(\mathrm{cm})$, and $v$ the longitudinal optical phonon frequency $\left(\mathrm{sec}^{-1}\right) .13$

Non-electrochemical requirements are the most difficult to meet for the cathode. The use of high fabrication temperatures $\left(\sim 1500^{\circ} \mathrm{C}\right)$ may lead to interactions with other cell components, excessive sintering, material vaporization, cell cracking due to thermal expansion mismatch, or oxygen loss from the cathode material which could cause dimensional changes. For La $0.5 \mathrm{Ca}_{0 .} \mathrm{MnO}_{3}, \mathrm{O}$ vacancies can be produced by oxygen loss if the material is in an atmosphere with low $\mathrm{PO}_{2}$. The loss mechanism is 13

$$
2 \mathrm{Mn}^{3+}+\left(\frac{1}{2}\right) \mathrm{O}_{2}(\mathrm{~g})+\square_{0}=0^{=}+2 \mathrm{Mn}^{4+}
$$

where $\square_{0}$ is a vacant $O^{=}$site within the molecular lattice. Ionic equilibria will be maintained if the temperatures are sufficiently high or if the time is sufficiently long. At equilibrium, 13

$$
K=\left\{\left[O^{=}\right]\left[\mathrm{Mn}^{4+}\right]^{2}\right\} /\left\{\left[\mathrm{Mn}^{3+}\right]^{2} \mathrm{p}_{\mathrm{O}_{2}}^{1 / 2}\left[\mathrm{a}_{0}\right]\right\}
$$

or

$$
\log \mathrm{PO}_{2}=2 \log \left\{\frac{\left[\mathrm{O}^{=}\right]\left[\mathrm{Mn}^{4+}\right]^{2}}{\left[\mathrm{Mn}^{3+}\right]^{2}\left[\square_{0}\right]}\right\}-2 \log \mathrm{K}
$$


where $K$ is the equilibrium constant. It is apparent from Eq. (6.2B-6) that the concentration of oxygen vacancies increases as $\mathrm{PO}_{2}$ decreases. An experimental oxygen-loss vs. $10 g$ PO curve was constructed by utilizing controlled, high-temperature micro-weighing at atmospheric pressure. The resulte agree well with the equilibrium analysis if $\log K^{2}$ is taken to be 8.45 at $1000^{\circ} \mathrm{C}$. Equilibrium is obtained within the highly porous air electrode materials within an hour at this temperature.

\section{Inter connection}

The interconnection is fabricated on a part of the cathode (see Fig, 6, 2A-2). It is an integral cell component with atringent material requirements for performance and fabrication. The inter connection material must be stable in air or fuel environmente, conduct nearly $100 \%$ electronically with a resistivity $\leq 20 \mathrm{ohm}-\mathrm{cm}$, have thermal expansion transformations that match other components between room temperature and $1000^{\circ} \mathrm{C}$, be chemically non-reactive with other cell components, be producible as thin and gas-impervious layers, have low volatility at $1000^{\circ} \mathrm{C}$, have low ion mobility, maintain these properties for the cell life $(240,000 \mathrm{~h})$, and have moderate cost. 13

Pure $\mathrm{LaCrO}_{3}$ has a small oxygen-vacancy concentration. Its electrical conductivity increases approximately linearly with the $\mathrm{Mg}$-doping level, but doping allows for higher oxygenvacancy concentrations in atmospheres with low $\mathrm{PO}_{2}$. The electrical conductivity decreases non-linearly as the oxygen-veancy concentration is increased because positive holes $\left(\mathrm{h}^{+}\right)$are eliminated. In fuel-cell atmospheres, the $\mathrm{Mg}$-doped $\mathrm{LaCrO}_{3}$ interconnection has an oxygenvacancy diffusion gradient. This gradient is not desirable because oxygen permeation through $\mathrm{LaCrO}_{3}$ increases with the oxygen-vacancy concentration. 13

\section{2C Fundamental Measurements in SOFCs ${ }^{14, t}$}

Figure 6.2C-1 shows an SOFC unit in a stack configuration. The lines indicate average current-flow paths. The interconnection area is not an active cell area. In operating cells, the current density varies ouch that the largest current densities are near the interconnection. The active area is therefore divided into small elements for calculations when the current density is non-uniform. Testing of segments (Fig. 6.2C-2), at various currents and in different environments, yields data for modeling the non-uniform case. Using an ac square-wave technique, the resistance of the electrolyte and the product of resistance and contact area of the anodes were observed to decrease linearly with temperature. These studies also show that the sheet resistance of the cathode decreases with temperature and (weakly) with the $\mathrm{O}_{2}$ contents of the oxidant.

Cathode quallty is determined by using inductive-10si measurements. Currents circulating circumferentially, similar to those occurring in cell operation, can be induced in the cathode. The induced currents increase the effective resistance of the current-inducing coil. The resistance 1088 can be measured with a Q-meter to obtain the cathode-sheet resistance. A profile of the cathode-sheet resistance is then obtained by moving the coil along the length of the tubular cell. These proflles are employed to monitor chemical changes in the cathode arising during various stages of fabrication. They may also be used to detect lengthwise cracks in the cathode.

\section{2D Suggested Research Areas for SOFCs 15,}

Brown reviepred technical problem areas of the SOFCs and has indicated how research should be performed in order to obtain improved cell performance.

\section{Hydrogen and Oxygen Diffusion in LaCr $1-\mathrm{Mg}_{x} \mathrm{O}_{3}$ at High Temperatures}

Magnesium-doped lanthanum chromite is a perovskite-type materials with good electronic conductivity at high temperatures. The bulk-diffusion rates of $\mathrm{H}_{2}$ and $\mathrm{O}_{2}$ through $\mathrm{LaCr}_{1-\mathrm{x}} \mathrm{Mg}_{\mathrm{x}} \mathrm{O}_{3}$ at $1000^{\circ} \mathrm{C}$ must be under stood in terms of the effects of doping on conduction. Better understanding of ite behavior under oxygen-activity gradients will hopefully make improved interconnection materials possible. Such efforts are currently in progress at WE under DOE contracta.

\section{Kinetice of Sulfidation Reactions}

The primary fuels which SOFC systems will utilize are NG and fuels derived from coal. These fuels contain $\mathrm{H}_{2} \mathrm{~S}, \mathrm{COS}$, or organically bound sulfur. In the $700-1100^{\circ} \mathrm{C}$ range,

\footnotetext{
This discussion is based on a presentation by J. E. Bauerle to AFCWG, Ref. 14.

* This diecussion is based on a presentation by J. T. Brown to AFCWG, Ref. 15.
} 


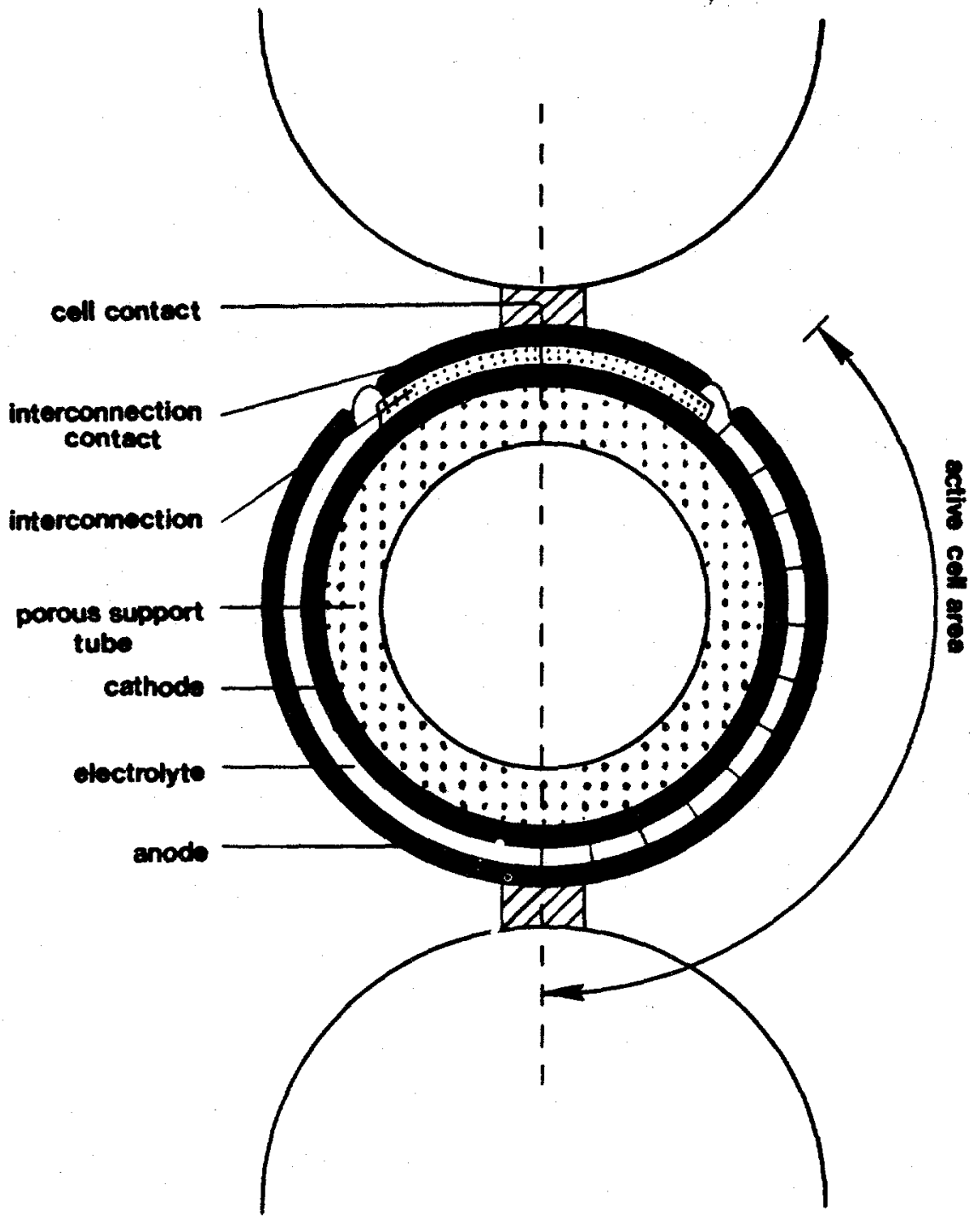

Fig. 6.2C-1. A schematic cross section is shown of a tubular SOFC in a stack. The active cell area is divided into sections for calculations when the current density is not constant. 14

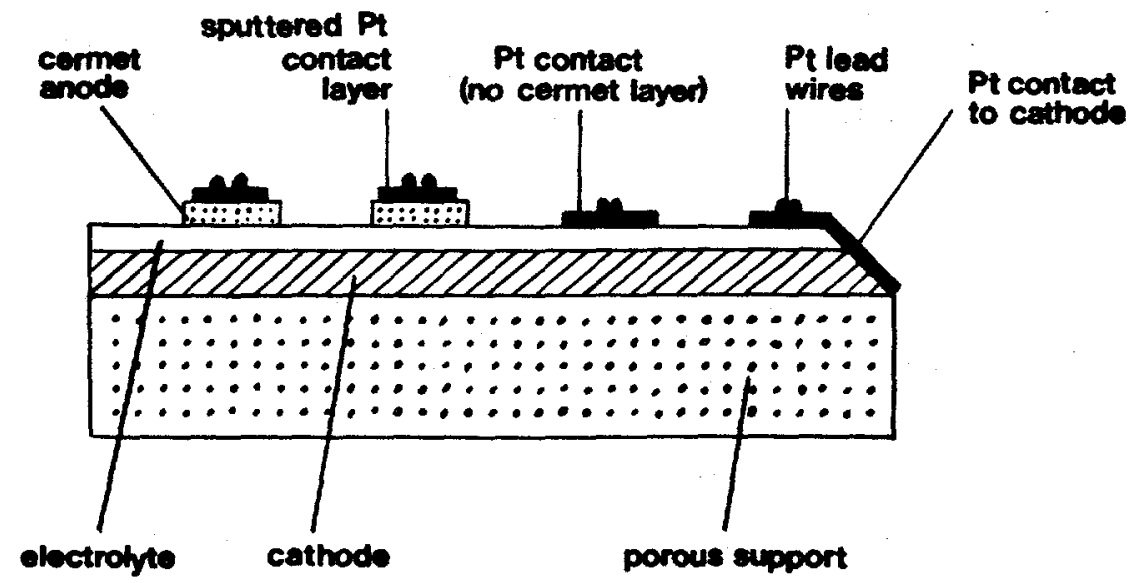

Fig. 6.2C-2. A schematic is shown of the test specimen used to obtain data for models of the finite segments described in Fig, 6. $2 \mathrm{C}-1.14$ 
equilibrium compositions corresponding to $\mathrm{H}_{2} \mathrm{~S} / \mathrm{SO}_{2} / \mathrm{H}_{2} \mathrm{O} / \mathrm{H}_{2}$ and $\mathrm{COS} / \mathrm{SO}_{2} / \mathrm{CO}_{2} / \mathrm{CO}$ may not be attained because of the occurrence of flows and electrochemical oxidation. Thus, sulfidation damage may result at the anode or at other components exposed to the fuel-gas environment. Sulfur tolerance of the anode is the more pressing problem. Better understanding of this problem may be gained if the conversion rates of $\mathrm{H}_{2} \mathrm{~S}$ and $\mathrm{COS}$ to $\mathrm{SO}_{2}\left(700-1000^{\circ} \mathrm{C}\right)$ are measured under conditions when the fuel gas is in contact with (i) largely non-catalytic surfaces, (ii) nickel or cobalt surfaces, and (iii) nickel or cobalt electrodes at which electrochemical oxidation is occurring. Maximum allowable sulfur contents in the fuel gas should be determined for the sulfidation limits of nickel and cobalt electrodes. Also, the tolerance limits of the fuel cell to sulfur, without significant voltage losses, should be measured. Finally, sulfur adsorption isotherms should be established on $\mathrm{Ni}$ - or Co-cermet electrodes in $\mathrm{CO} / \mathrm{H}_{2}$ gas environments for sulfur concentrations below those causing NiS or CoS formation at temperatures that are characteristic of the fuel stream $\left(700-1100^{\circ} \mathrm{C}\right)$.

\section{Diffusion of Cations in Stabilized Zirconia}

Diffusion of cations from the cathode material into the electrolyte may potentially result in performance degradation of the cell. Small concentrations of elements such as $\mathrm{Mn}$ and $\mathrm{La}$ from the air electrode may dope YSZ during cell fabrication or cell operation. Such cation doping may alter ionic conductivity, thermal expansion behavior, and the sintering characteristics of the YSZ electrolyte. Such doping may also alter the interfacial conductivity and the electrontransfer reaction rates of the electrochemical processes.

The $\mathrm{Mn}$ cation is probably the most mobile cation in the air-electrode material of the fuel-cell environment. The diffusion of $\mathrm{Mn}$ cation from the cathode into the electrolyte may change the electronic conductivity of the electrolyte.

Lau and Singhal have determined diffusion coefficients of $\mathrm{Mn}$ in various types of $\mathrm{Z}_{\mathrm{rO}_{2}}-$ 10 mole- $\% \mathrm{Y}_{2} \mathrm{O}_{3}$. These diffusion coefficients are about $3 \times 10^{-13} \mathrm{~cm}^{2} / \mathrm{sec}$ for single-crystal $\mathrm{ZrO}_{2}\left(\mathrm{Y}_{2} \mathrm{O}_{3}\right)$ and about $3 \times 10^{-9} \mathrm{~cm}^{2} / \mathrm{sec}$ for pressed and sintered polycrystalline $\mathrm{ZrO}_{2}\left(\mathrm{Y}_{2} \mathrm{O}_{3}\right)$ at $1400^{\circ} \mathrm{C}$. 3,16 Lau and Singhal expect that future measurements on actual EVD $\mathrm{ZrO}_{2}\left(\mathrm{Y}_{2} \mathrm{O}_{3}\right)$ will yield a diffusion coefficient falling between these two values. Also, the diffusion coefficients at $1000^{\circ} \mathrm{C}$ are about four orders of magnitude lower.

A program to improve our understanding in this area would serve to establish the degree of solubility and rates of diffusion of the se metal ions in YSZ, yield thermal expansion data, provide electronic and ionic conductivities as a function of the doping level in YSZ, and define the nature and degree of cation migration across interfaces into YSZ.

\section{Structural and Compositional Effects of the Cathode-Electrolyte Interface}

Factors that improve cathode-electrolyte interfacial effectiveness should be studied. An understanding of the performance of cathodes covered by EVD electrolytes and analysis of the effect of EVD conditions on cathode properties is desirable. Also, a workable model is needed for high-performance electrode structures that is similar to the simple-pore or thin-film models for gas-diffusion electrodes in liguid electrolyte systems.

\section{Fuel-and Air-Electrode Kinetics}

Electrode performance could be optimized by adjusting electrode materials, porosities, thickness, and interfacial areas if we knew how these properties affect the reaction kinetics. Knowledge of reaction mechanisms may facilitate improvements in interfacial design. Efforts to determine the mechanisms of interfacial reactions are in progress and utilize electron microprobe analysis and scanning electron microscope studies.

Methane is not completely reformed when it reaches the electrode reaction site. The effects of unreformed fuels on electrode kinetics need to be examined.

\section{New Cathode Materials}

About $50 \%$ of the total cell resistance $108 \mathrm{~s}$ occurs at the cathode. Cost and conductivity are therefore important considerations for the development of new cathode materials such as mixed oxides. Theories that predict relations between dopants and their concentrations and the conductivity must be further developed [see Eq. (6. 2B-2)].

\section{Stress Analysis of Thin-Oxide Layer s}

Three-dimensional stress analyses of cell components should be carried out to provide data for failure analysis of stacked SOFCs. Operation at various levels produces changes in stack operating temperatures which, in turn, induces variations in stack stresses. 
6.3 SOFC Research at BNI $2,9,17,18, \uparrow$

Voltage losses in SOFCs are the sums of resistance losses in the electrodes, electrolyte and interconnection and of the diffusional or activation losses associated with the electrochemical reactions occurring at the electrode-electrolyte interfaces. Ohmic losses may be reduced by using improved materials and geometries. Over half of the losses are associated with non-ohmic losses ( $6 \%$ cor responds to deviation from the theoretical open-circuit value). ${ }^{2}$ For known mechanisms, it may be possible to accelerate the rate-determining steps without the use of expensive catalysts by adjusting electrode porosity, tortuosity, composition, surface purity, electrode-electrolyte contact area, particle-size distribution, or the distances between electrodeparticle contacts. For instance, if adsorption on the electrolyte is the rate-determining step, increasing the distance between particle contacts will be advantageous. On the other hand, if adsorption on the electrode surface is rate controlling, decreasing the distances between particle contacts may be advantageous. 10

\section{3A The Polarization Cell}

Most of the experiments at BNL have been done with polarization cells that were retrofitted with single-contact test electrodes (see Fig. 6. 3A-1). Single-contact electrodes are used to separate the influences of electrode composition and electrode morphology on reaction kinetics. The determination of rate-controlling mechanisms is evidently easier when electrode mor phologies do not need to be considered.

The dc polarization curves are obtained by varying the voltages across the polarization cell at constant rates. A reference electrode is used to regulate the potential differences that are applied to the cell and a counter -electrode is used to measure currents induced by potential differences.

Alternating currents are passed between the test and counter electrodes, while the corresponding impedance is monitored. Figure 6.3A-2 show a simple capacitance-resiatance model of an electrode-electrolyte interface. The reaction impedance of this circuit is

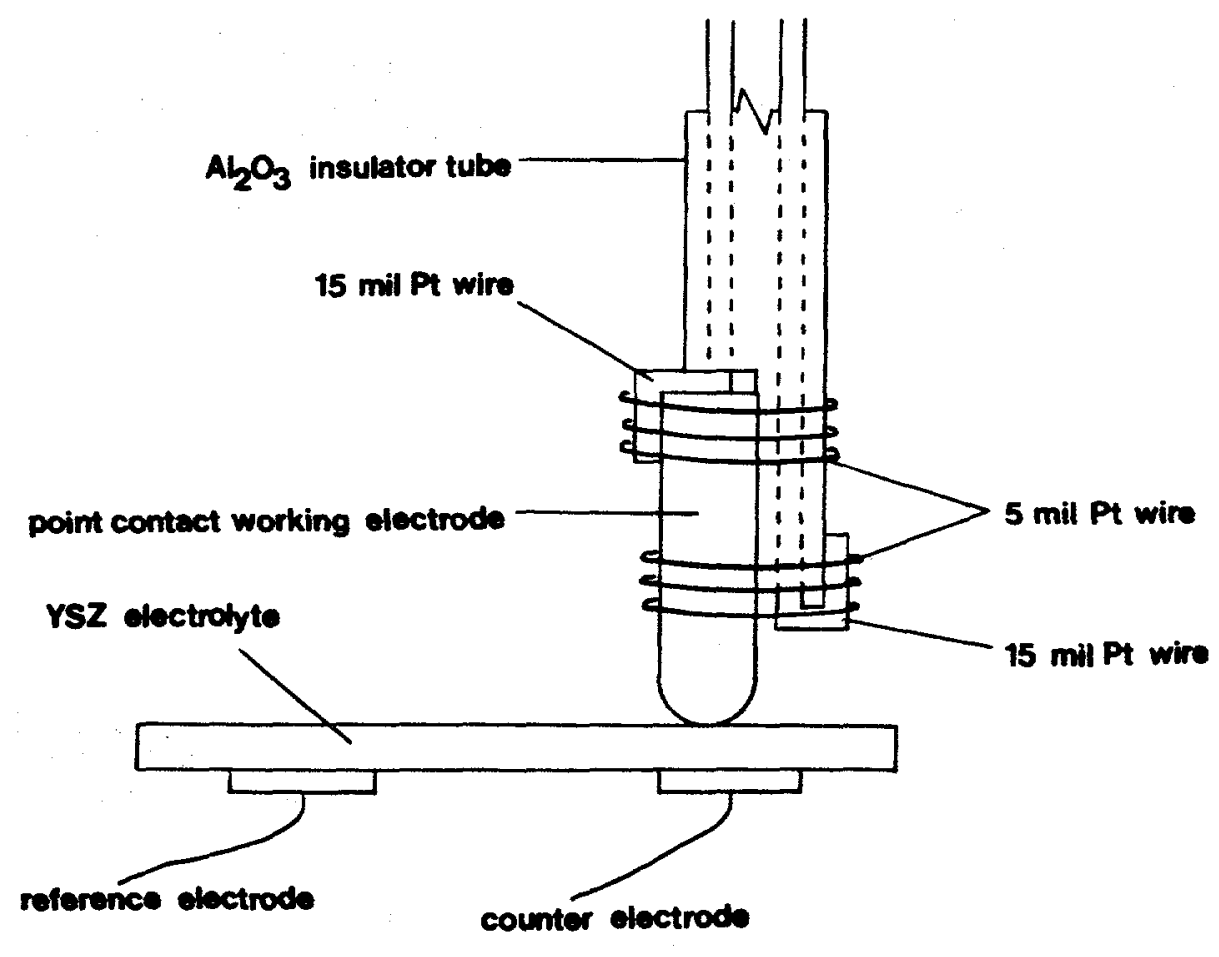

Fig. 6.3A-1. The design is shown of a polarization cell utilizing single-contact test electrodes. The reference electrode is used to apply potential differ ences, while the counter-electrode is used to measure induced currents; both electrodes are made of Pt paste. 17

this discussion is based on a presentation by H. S. Isaacs to AFCWG, Ref. 2. 


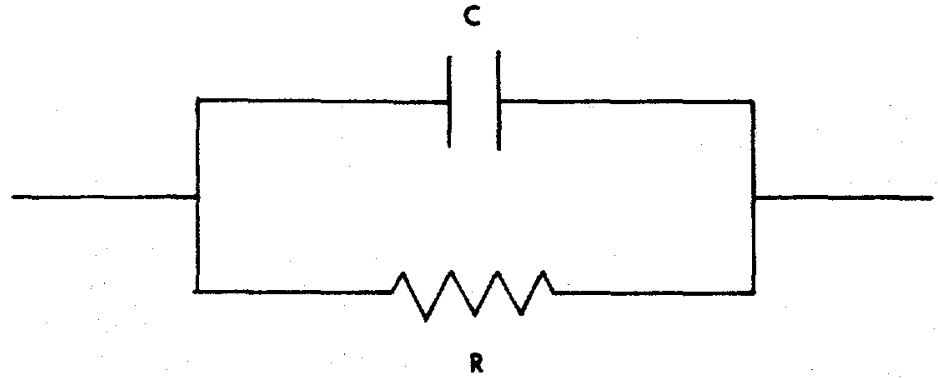

Fig. 6. 3A-2. A simple electric circuit model of an electrodeelectrolyte interface is shown.

$$
1 / Z=(1 / R)+1 /(i / \omega C)
$$

or

$$
Z=[(1 / R)+i \omega C] /\left[(1 / R)^{2}+(\omega C)^{2}\right]
$$

where $R$ is the interfacial resistance, $C$ the interfacial capacitance, $\omega$ the angular frequency of the alternating current, and $i=\sqrt{-1}$. Hence,

$$
\operatorname{Re}(Z)=[1 / R] /\left[(1 / R)^{2}+(\omega C)^{2}\right]
$$

and

$$
\operatorname{Im}(Z)=[w C] /\left[(1 / R)^{2}+(w C)^{2}\right]
$$

Figure 6. 3A-3 shows the impedance curve for a constant overpotential of the circuit shown in Fig. 6.3A-2; it is typical of reaction-impedance plots for a rate-controlling oxygen-adsorption reaction at the cathode. 10 When $C=0, \operatorname{Re}(Z)=R$. When $R=\infty, \operatorname{Im}(Z)=1 / \omega C$. As $\omega \rightarrow 0$, $\operatorname{Re}(Z) \rightarrow R$ and $\operatorname{Im}(Z) \rightarrow 0$. As $w \rightarrow \infty, \operatorname{Re}(Z) \rightarrow 0$ and $\operatorname{Im}(Z) \rightarrow 0$. When the frequency is in creased, the capacitor short-circuits the resistor. As the frequency is decreased, the impedance is increasingly caused by the resistor (dc corresponds to $\omega=0$ ). Thus, the de resistance of the interface is the diameter of the arc (see Fig. 6.3A-2).

Isacs ${ }^{2}$ has performed tests of electrode materials over ranges of values for cathodic and anodic potentials, thereby utilizing the fact that the SOFC can be run reversibly as an electrolyzer.

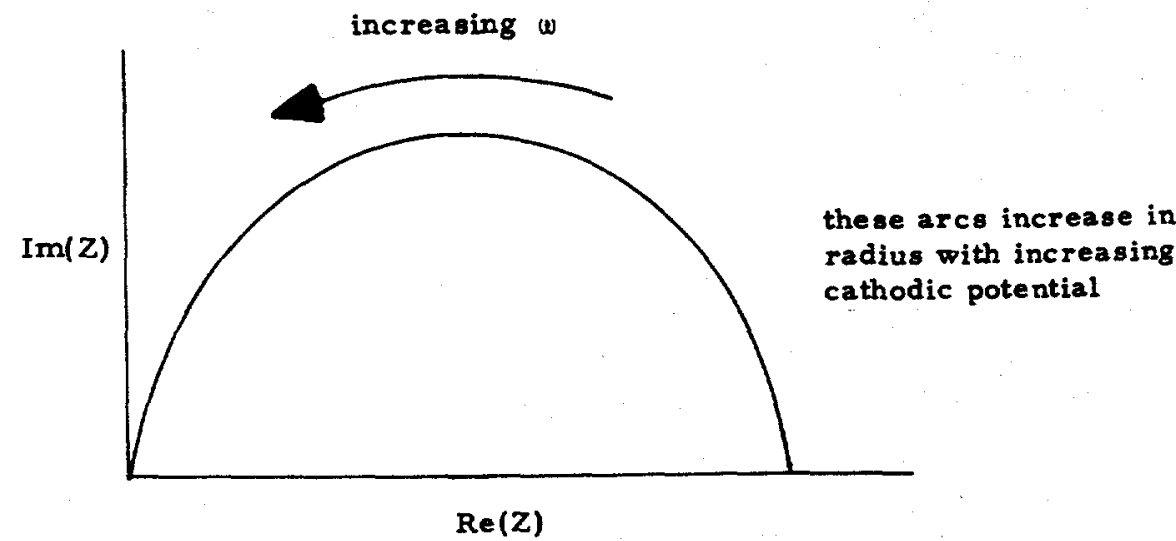

Fig. 6. 3A-3. A typical reaction-impedance plot is shown for a rate-controlling oxygen-adsorption reaction at the cathode. The increasing growths of arcs with potential are indicative of cathodic limiting polarization currents. 10 
Isaacs tested $\mathrm{Ni}, \mathrm{CO}, \mathrm{Fe}, \mathrm{Mo}$, and $\mathrm{LaCrO}_{3}$ using an apparatus similar to that shown in Fig. 6.3A-1. Specific currents $(1, \mathrm{~mA} / \mathrm{cm})$ were measured for listed materials at fixed $\mathrm{PH}_{2} / \mathrm{PH}_{2} \mathrm{O}$ and $-1200 \mathrm{mV}$, with air as reference. The specific current is given by

$$
I=i / T P B L \text {, }
$$

where $i$ is the measured current and the triple-phase boundary-length (TPBL) is the total perimeter of the electrode-electrolyte contact. Determination of the TPBL is necessary to find $I$, where $I=i / 2 \pi r$ and $r$ is the radius of the electrode-electrolyte contact. The quantity $I$ is a measure of the electrocatalytic performance of the material and is a more suitable gauge for contact resistance than the current density because dc current flows around the perimeter of the contact area where electrochemical reactions take place. Newman developed a model which yields $r=1 /\left(4 \sigma R_{e}\right)$, where $\sigma$ is the electrolyte conductivity and $R_{e}$ is the effective electrolyte resistance of an insulated electrode disk in contact with an aqueous electrolyte. 20 This model is not strictly applicable to solid electrolytes. The conductivity $R_{e}$ is determined by performing high-frequency impedance measurements. At high frequencies, the interfacial impedance vanishes and the measured impedance reduces to $R_{e}$ (see Fig. 6.3B-1),

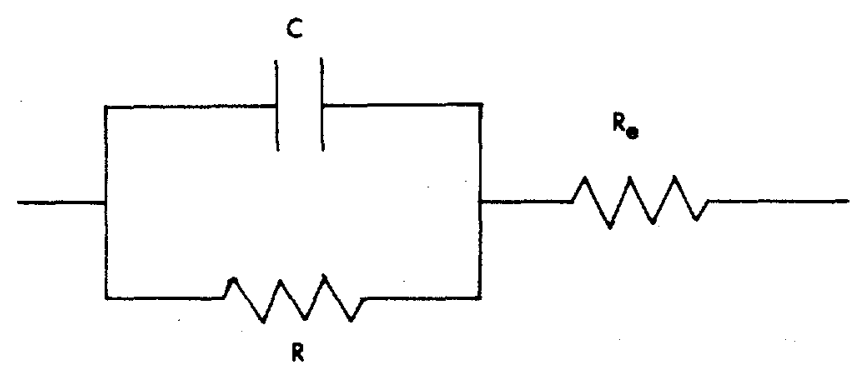

Fig. 6.3B-1. An electric circuit model of an electrode-electrolyte interface is shown in series with an effective electrolyte resistance $R_{e}$.

The value of I was found to decrease for the electrode materials in the order Fe $>$ $\mathrm{Co}>\mathrm{Ni}>\mathrm{Mo}_{\mathrm{O}}>\mathrm{La}\left(\mathrm{Al}, \mathrm{Mg} / \mathrm{CrO}_{3}\right.$. The measured current (i) was found to depend on the concentrations of $\mathrm{H}_{2}$ and $\mathrm{H}_{2} \mathrm{O}$ and also on the extent of electrode oxidation and the evolution of oxygen at the most positive potentials. Figure 6.3B-2 shows this dependence qualitatively. Anodic currents are characterized by electron repellance, i.e, negative charges leaving the cell. There is no current when the potential is such that the oxidation of $\mathrm{H}_{2}(\mathrm{~g})$ is in equilibrium with the reduction of $\mathrm{H}_{2} \mathrm{O}(\mathrm{g})\left[\mathrm{H}_{2}(\mathrm{~g})+\mathrm{O}=\mathrm{H}_{2} \mathrm{O}(\mathrm{g})+2 \mathrm{e}^{-}\right]$. As the anode potential is increased, the number of electrons is reduced, the reaction shifts to the right and $\mathrm{H}_{2}(\mathrm{~g})$ is oxidized. When the potential is further increased, the thermodynamic stability limit of the metal electrode will be reached and the metal oxidizes (i.e., Fe $+\mathrm{O}^{=} \rightleftharpoons \mathrm{FeO}+2 \mathrm{e}^{-}$). Metal oxidation increases rapidly as the potential is raised above the thermodynamic limit but it is self-limiting because the oxide coats the electrode. Finally, at very high potentials, $O_{2}(g)$ is evolved $\left[i . e ., O=(1 / 2) O_{2}(g)\right.$ $+2 \mathrm{e}^{-1} .19$

$\mathrm{LaCrO}_{3}$ was stable against oxidation at all potentials but its defect concentration varied. All of the tested metals had limited stability ranges. The metals can, in fact, form higherorder oxides. Although $\mathrm{Fe}$ and $\mathrm{Co}$ are more active catalytically than $\mathrm{Ni}, \mathrm{Ni}$ is the best choice as electrode material because it has a larger stability range than the other metals. The WE Ni- $\mathrm{Zr}_{\mathrm{r}}$ cermet anodes are well chosen.

The limiting rate of $\mathrm{H}_{2}(\mathrm{~g})$ oxidation on Ni electrodes varies linearly with $\mathrm{PH}_{2} .19$ Thus, $\mathrm{H}_{2}$ adsorption on the $\mathrm{Ni}$ surface is likely to be the rate-controlling step in fuel-cell operation.

\section{3C Air Electrode-Reaction Processes}

Figure 6.3C-1 shows possible pathways for the electro-reduction of $\mathrm{O}_{2}(\mathrm{~g})$ at the cathodeelectrolyte interface. Rate-controlling electrochemical steps are either surface-controlled or bulk-controlled. The concentrations of several surface impurities on single-contact Pt electrodes were varied and the results suggest that adsorption of oxygen on the electrode surface is the rate-controlling mechanism. The impurities may affect adsorption kinetics by either changing adsorption energy or blocking adsorption sites. 9 When the surface concentrations of $\mathrm{Bi}_{2} \mathrm{O}_{3}$ or Au were increased, cathodic currents were increasingly lower than for pure Pt surfaces at the same potential. 9,11 When a coat of praesodymium oxide was placed on the Pt surface, 


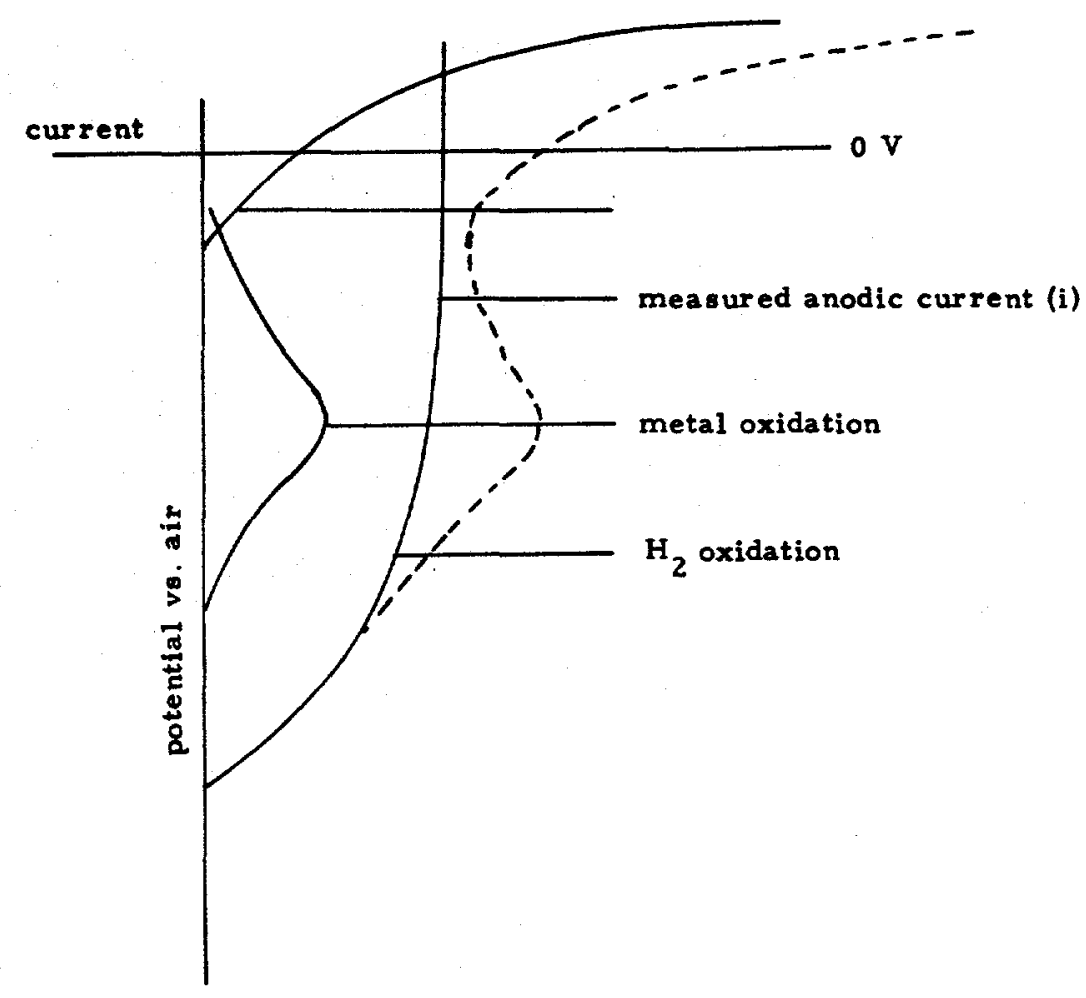

Fig. 6. 3B-2 The qualitative plot shows the measured anodic current for a polarization cell placed in $\mathrm{H}_{2} / \mathrm{H}_{2} \mathrm{O}$ gas vo. the potential difference with air as reference. 17

cathode performance was enhanced. 11 The overall oxygen-reduction reaction is then $O_{2}(g) \rightarrow$ $2 \mathrm{O}_{\mathrm{ads}}$. followed by the relatively rapid process $0_{\mathrm{ads}}+2 \mathrm{e}^{-}=0^{=} .10$ Because the chargetransfer resction is fast, it remains near equilibrium when current flows. The Nernst equation will, of course, apply to this step. Thus, the potential is related to the surface concentration of Oads:

For the cathodic currents, dc-polarization curves showed hysteresis patterns, which further supported the view that urface- or bulk-diffusion rates were not rate-controlling steps. For all of the materials tested, other than $\mathrm{Cr}_{2} \mathrm{O}_{3}$, cathodic currents were observed to be smaller when the magnitude of the potential increased with time than when it decreased. If a diffusion process were rate-controlling, the current would be-expected to be greater when the magnitude of the potential increased with time because reactant concentrations at the electrode are greater for increasing than for decreasing currents. 11

The apecific currents for 12 materials on YSZ were measured at $-100 \mathrm{mV}$. The materials tested, listed in order of decreasing catalytic ability, are: $\mathrm{Rh}>\mathrm{La} 0 . \mathrm{SSr}_{0 .} \mathrm{FeO}_{3}>$ $\mathrm{La}_{0.8} \mathrm{Ba}_{0.2} \mathrm{CoO}_{3}>\mathrm{PrClO}_{3}>\mathrm{Pd}>\mathrm{Cr}_{2} \mathrm{O}_{3}>\mathrm{Pt}>\mathrm{Co}_{3} \mathrm{O}_{4}>\mathrm{La}_{0.95} \mathrm{MgO}_{0.05} \mathrm{Cr}_{0.85} \mathrm{Al}_{0.15} \mathrm{O}_{3}>\mathrm{NiO}$ $>\mathrm{In}_{2} \mathrm{O}_{3}>\mathrm{Au} .11$

Impedance techniques were also used to test different electrodes composed of the listed materials. The frequency was varied from 50 to $10,000 \mathrm{~Hz}$ at $0 \mathrm{~V}$. Figure $6.3 \mathrm{C}-2$ is an impedance diagram of an $\mathrm{Rh}$ single-contact teat-electrode on $\mathrm{YSZ}$ in air at $1000^{\circ} \mathrm{C}$. Extrapolation of the arc to the $\operatorname{Im}(Z)=0$ axis at high frequencles again yields $R_{e}$. Extrapolation to the axis at low frequencies gives $R+R_{e}$. The interfacial resistance $R$ is a measure of cathode polarization, which may be normalized for differences in contact area by utilizing $R_{e}$ defined by $r=$ $1 /\left(4 \circ R_{e}\right)$. A measure for the specific conductance is the ratio of the electrolyte to the inter facial resistance, which is much less sensitive to material propertles than I. 11

Preliminary studies have been performed on the conduction mechanisms in donor-doped, acceptor-doped, or undoped $\mathrm{In}_{2} \mathrm{O}_{3}$. Base-metal oxides are relatively inexpensive and ghow good chemical atability in atr at $1000^{\circ} \mathrm{C}$. Hopefully, information obtained on $\mathrm{In}_{2} \mathrm{O}_{3}$ will prove to be useful for atudies on other cathode semi-conductor materiala if $\mathrm{In}_{2} \mathrm{O}_{3}$ turns out to be unsuitable for electrode use. 21 


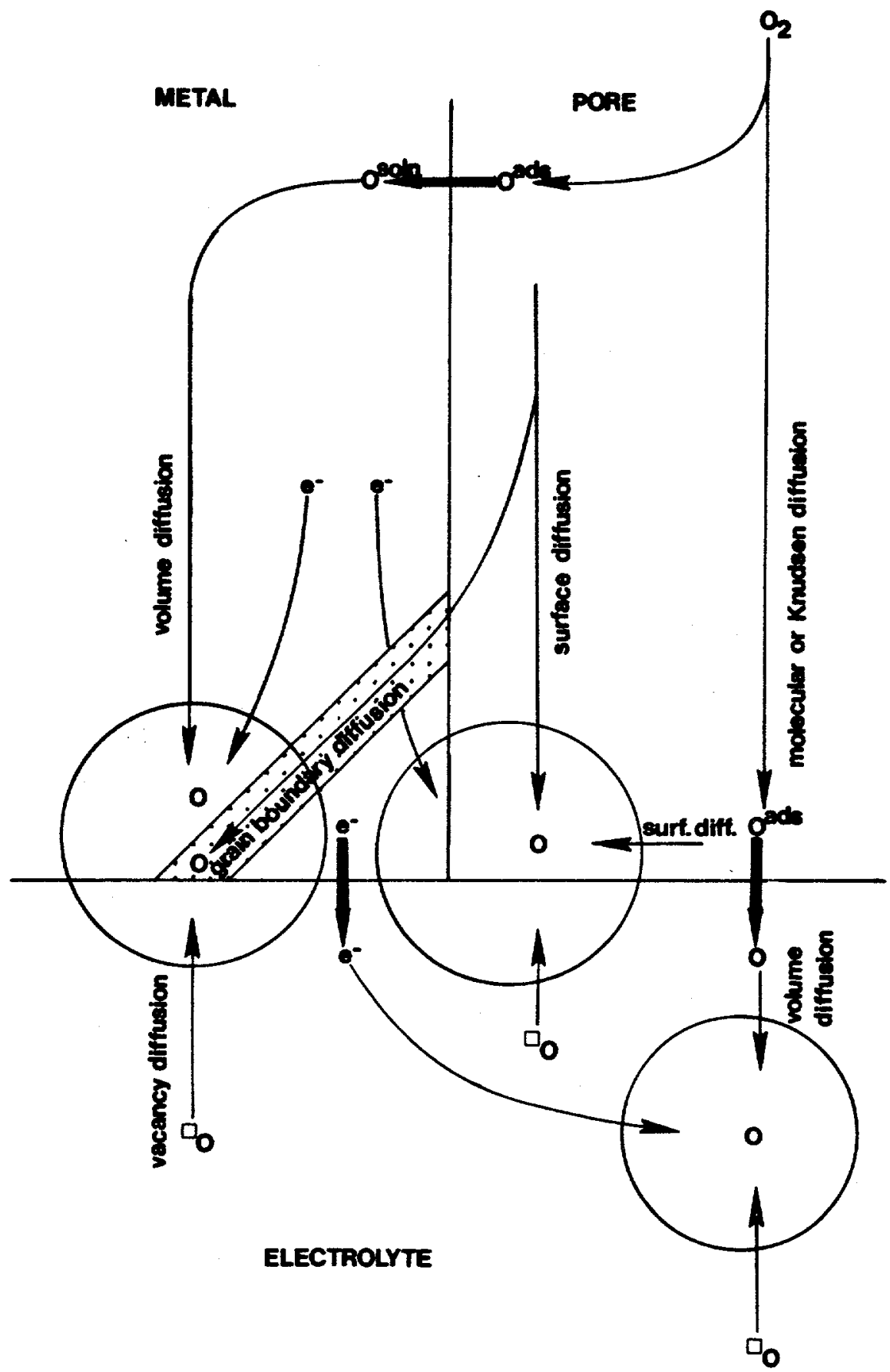

Fig. 6.3C-1. Possible pathways are shown for the reduction of $\mathrm{O}_{2}(\mathrm{~g})$ at the interface of the cathode and of the electrolyte for an SOFC. ${ }^{2}$ 


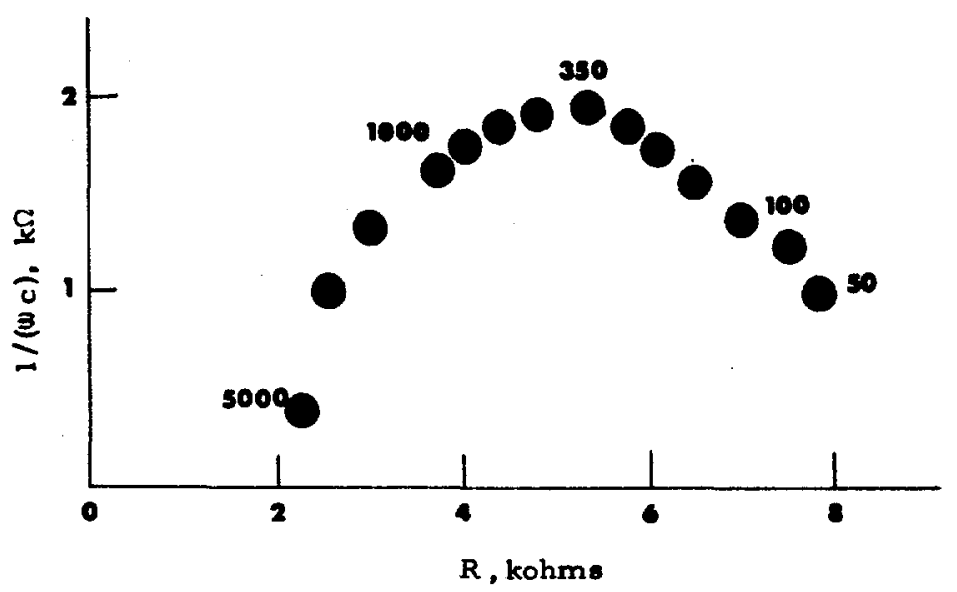

Fig. 6. 3C-2. An impedance diagram is shown for an $\mathrm{Rh}$ single-contact electrode on YSZ in air at $1000^{\circ} \mathrm{C} .11$

\section{SUMMARY OF RESEARCH RECOMMENDATIONS}

Research Areas on Fundamental Electrochemistry

Reaction sites should be studied to determine active dimensions and the effects of mixed ionic/electrode conductivity in the electrode or electrolyte on active-site dimensions. The relative degrees of electron-transfer and mass-transport control of electrode reactions should be investigated to define solid-state and surface diffusion of oxygen to reaction sites and relative contributions of molecular and Knudsen diffusion in small pores of the electrodes. Electrode kinetics should be studied for direct reaction of oxygen at air-electrode/electrolyte interfaces and compared with indirect reaction involving extraction of oxygen from the electrode at high current-flow conditions. The effects on fuel-electrode kinetics of contaminants (e.g. sulfurcontaining gases) in the fuel should be investigated. Electrochemical effects at contact areas of the interconnection and solid electrolyte should be clarified. Effects of multi-component diffusion of reactants and reactant-product gases in porous electrodes require elucidation. Electrochemical permeation of oxygen and hydrogen needs to be studied through the interconnection, with fuel on one side and air (oxygen) on the other side, as functions of the doping level of magnesium in the lanthanum-chromite interconnection and the fuel composition. Investigations are needed of dual atmosphere electrical conductivities of interconnection materials, as well as determinations of details of conductivity mechanisms in the resulting oxygen chemical-potential gradient.

Materials Research

A. Optimization of High-Temperature Stability of Electrode Materials

Studies are needed of: long-term pore volume, grain-size and grain-boundary morphology interrelationships with segregation, sintering. second-phase formation, and liquefaction; intrinsic and extrinsic (fuel gas) trace-element effects on long-term electrode structure and chemical stability; effects of thermochemical and electromechanical stresses on interfacial phase formation, precipitation phenomena, element segregation, pore geometry, and grain-morphology stability; effects of high-temperature, short-time excursions on second-phase formation, electrode-phase decomposition, segregation, and interdiffusion. Research should be directed at enhancing the electrode high-temperature, long-term stability and electrochemical performance.

\section{B. Environmental Effects on Materials for SOFC Systems}

Studies are needed of the effects of: fuel gas $\left(\mathrm{H}_{2}-\mathrm{CO}-\mathrm{H}_{2} \mathrm{O}\right)$ on metal (oxidation, carburization) and ceramic (leaching of $\mathrm{Si}$, alkalis, $\mathrm{Cl}$ ) structural components, as determined by longterm chemical and physical property alterations; fuel-gas (coal-derived gas, natural gas) behavior in terms of carbon deposition, formation and decomposition of metal carbonyls, traceelement contamination, and high-temperature chemical transport or reactions; sulfur reaction rates and reversibility of reaction with fuel electrode, electrical interconnection materials and structural system components; downtime effects of gases, condensates and deposits on the chemical and physical behavior of structural system components. Research should enhance the long-term reliability of components and aid in the selection of materials with the best cost/ benefit ratios. 


\section{Processes in Mixed-Conducting Oxides}

The reaction of $\mathrm{H}_{2}\left[\mathrm{H}_{2}+\mathrm{O}^{=} \rightarrow \mathrm{H}_{2} \mathrm{O}(\mathrm{g})+2 \mathrm{e}^{-}\right]$in $\mathrm{FC}_{8}$ is geometrically restricted to regions near the lines of 3 -phase (gas-electrode-electrolyte) contact. Mixed conducting-oxide materials (electronic-ionic) facilitate reaction over the entire surface area of the electrode-gas interface to enhance current production. It may be useful to search for entirely new, highlyconducting metal oxides.

D. Hydrogen and Oxygen Diffusion in Magnesium-Doped La nthanum Chromite $\left(\mathrm{LaCr}_{1-x^{-}} \mathrm{Mg}_{\mathbf{x}} \mathrm{O}_{3}\right.$ )
at Elevated Temperatures

Modified lanthanum chromite is a perovskite-type material that exhibits good electronic conductivity at elevated temperatures $\left(900-1000^{\circ} \mathrm{C}\right.$ ). Additional understanding of this material would be obtained from a program designed to: establish diffusion coefficients of $\mathrm{H}_{2}$ and $\mathrm{O}_{2}$ in the bulk material over the temperature range 800 to $1100^{\circ} \mathrm{C}$, enhance understanding of the roles of $\mathrm{H}_{2}$ and $\mathrm{O}_{2}$, enhance understanding of the physico-chemical behavior of the compound under oxygen-activity gradients.

E. Interstitial Cations in Stabilized Zirconia

The usual additives to stabilize zirconia, such as $\mathrm{Ca}^{2+}$ or $\mathrm{Y}^{3+}$, replace $\mathrm{Zx}^{4+}$, introduce vacancies at $O=$ sites and considerably alter some of the important physical properties of the oxide (e.g. , electrical conductivity). Another method for modifying material properties is addition of small amounts of 3-d transition elements such as iron, manganese, nickel, or tita nium to the stabilized zirconia structure. In this case, at least some of the added 3-d ions may reside at inter stitial sites, midway between normally occupied metal-ion sites, and the extra charge added to the lattice is then compensated for by oxygen ions at sites which would otherwise be vacant because of the presence of $\mathrm{Ca}^{2+}$ or $\mathrm{Y}^{3+}$ stabilizing ions. These effects can alter the ionic conductivity and, perhaps, result in enhanced metal-ion mobility, which would affect the sintering characteristics of the ceramic that are significant factor 8 in applications utilizing zirconia compositions.

The information obtained would also be of value to other areas of application of zirconia, where changes in thermal expansion and electrical conductivity are important A pertinent program in this area would: establish the degree of solubility of the 3-d transition-metal ions (manganese, iron, nickel, cobalt, and titanium) in yttria-or calcia-stabilized zirconia and yield changes in thermal expansion caused by addition of these interstitial ions; define changes in electrical conductivity (ionic or electronic) as related to composition; elucidate qualitatively the nature and degree of migration of selected 3 -d ions across an interface between a stabilized zirconia interface and an oxide phase containing the $3-d$ ion.

\section{F. Thin-Film Coatings for SOFC-Generator Components}

Improvements may arise from: development of a thin, flexible, adherent coating that can be used to prevent the sintering together of the interconnect loop material; establishment of coating durability by conducting thorough testing under typical operating conditions; improvements in the method of applying the nickel-plated interconnection contact, thereby optimizing the speed of the process and the properties of the plated contact.

\section{Transport Properties}

A. Phase Equilibria in the $\mathrm{ZrO}_{2} / \mathrm{Y}_{2} \mathrm{O}_{3} / \mathrm{La}_{2} \mathrm{O}_{3} / \mathrm{MnO}_{2}$ System

In high-temperature FC applications, inadvertent doping of the zirconia electrolyte by an electrode material containing a 3-d transition element may alter the conductivity of the interface or the electron-transfer reactions of the electrochemical processes. This is another area where information on either direct or interstitial solution of the 3-d elements in the zirconia is of direct interest. Important facts and needs are the following: Interdiffusion may occur between the $\mathrm{ZrO}_{2}\left(\mathrm{Y}_{2} \mathrm{O}_{3}\right)$ electrolyte and $\mathrm{Sr}$-doped $\mathrm{LaMnO}_{3}$ air electrode during long-term cell operation. Mn is the most mobile cation. There is need to understand the effects of $\mathrm{Mn}_{\mathrm{n}}$ dis solution on the stability of $\mathrm{ZrO}_{2} \mathrm{Y}_{2} \mathrm{O}_{3}$. The phase relationship in the $\mathrm{ZrO}_{2} / \mathrm{Y}_{2} \mathrm{O}_{3} / \mathrm{La}_{2} \mathrm{O}_{3} / \mathrm{MnO}_{3}$ system are required.

\section{Electrode Kinetics}

In situ reformation of natural gas on SOFC fuel electrodes has been demonstrated. However, the reformation reaction of natural gas or higher hydrocarbons with steam remains to be investigated as a function of cell geometry. The following is an important fact. Water vapor is formed through anodic oxidation and becomes available as reforming water and the direct 
oxidation of $\mathrm{CH}_{4}$ is a possibility. Measurements should be made to determine the competing anodic reaction steps that occur in combination with the chemical reformation reactions.

\section{Surface Chemistry}

Fuel electrodes are exposed to many different species, including combustibles. Partially-oxidized fuel (NG) or sulfur species (contaminant) can influence electrode structure and stability and the corresponding changes should be studied. Investigations are needed of adsorbed (chemisorbed) species of partially reformed fuel and oxidized sulfur.

Structural changes occur in fuel electrodes during FC operation. Studies are needed of nickel particles in electrodes as they sinter during FC operation at elevated temperature $B$. Porosity may be induced in the electrode by the controlled oxidation/reduction of the nickel and needs to be defined in quantitative terms. Conditioning cycles should be developed to restore electrode porosity/structure.

Other important effects are the long-term changes produced by FC operation on the structure and integrity of the fuel-electrode/electrolyte interface and long-term effects of trace fuel impurities on the structure of the fuel electrode. Measurements should be made of electrode coverage by reactive components (e.g., by using infrared Fourier-transform spectroscopy).

\section{Improved Diagnostics}

Two-terminal RC or other techniques are needed to estimate the effective fuel-electrode coverage, electrolyte quality, inter connect quality, and to detect the presence of interfacial layers between cell components. Flaw detection should be implemented in partial or complete cells by sonic, ultrasonic or other means. Tests should be made of thermal shock resistance of cells, cell segments and cell assemblages. Stresses should be measured in cells as a function of temperature. Tests are needed to determine the degree of diffusion-limited behavior in cells.

\section{Cell-Stack Design}

Developments of cell/stack configuration concepts are needed to increase power density, reduce manufacturing cost, improve efficiency, allow utilization of a broader range of fuels.

These studies should include configuration studies and experimental demonstration of concepts which: assure uniformity of fuel and air-flow distribution within the cell stack; provide the manifolding of fuel, air, and exhaust gas passages; provide cell support and positioning functions with the proper selection of high-temperature materials; demonstrate assembly techniques which are economical and enhance stack reliability for large cell quantities; accommodate non-uniformities in temperature and gas flows during start-up and shut-down transients and during peripheral losses of heat; avoid adverse thermal disturbance of the cell stack with power take-off/lead concepts that also provide maximum electrical efficiency.

\section{A. Cell Electrical Interconnection}

Investigations are needed of long-term changes in the electrical and mechanical properties of fibrous metallic inter connect materials in order to assure adequate life at cell operating temperatures and through start-up/shut-down cycles. Development is desired of new structural configuration of porous metallic inter connection materials to improve electrical conductivity, mechanical flexibility, and cell bond strength. Development is required of advanced inter connect manufacturing and assembly techniques to improve quality and reduce cost.

\section{B. SOFC Modeling and Tests for Verification of Models}

There is need to de velop a detailed, three-dimensional mathematical model of SOFCs, which includes allowance for the following effects: axial variations in current and heat generation, circumferential variations in current and heat generation, axial variations of reactant composition caused by consumption and interspecies reactions, heat losses from the cell periphery, heat transfer by convection, axial conduction, circumferential conduction, inter cell radiation, intracell radiation. This model should be used in studies to determine the effects of cell-performance improvements, cell-operating conditions (including reactant composition, flow rate, temperature, current or voltage), cell size on overall system performance, and economics to guide research and development.

Experiments should be performed to verify the three-dimensional model of the SOFC. These tests should be designed to measure the fundamental features in order to ensure credibility of the model. Examples of determinations to be made are cell-temperature distributions, reactant-temperature distributions, current-density distribution, heat-generation distribution, local reactant compositions. 
Stress analyses should be performed on the five-component SOFC, which will predict its behavior under intended operating conditions. Thus, (1) cell-stress models should be established, (2) a nalyses are needed for transverse and axial cell directions by finite-element techniques under isothermal and operating (non-isothermal) conditions, (3) critical stress problem areas should be defined, (4) model solutions should be developed to reduce stresses in critical areas.

\section{Prototype Development}

The objective is to advance SOFC technology by incorporating timely design and technology advancements into a prototype generator test and evaluation program. Thus, advances should be sought in the technology of cell materials, components and processing area; advancements should be verified by cell and stack testing; prototype generator designs should be developed for applications derived from user/market/economic analyses; prototype generators should be built and evaluated to verify design and operational features for selected applications. 


\section{REFERENCES}

1. "Summary of Presentations Made at the First Meeting of DOE/AFCWG," April 19, 1984, unpublished.

2. H. S. Isaacs, Adv. in Ceramics 3, 406 (1981).

3. L. G. Marianowski, presentation made at a meeting of the DOE/AFCWG, Westinghouse Electric (WE), 1310 Beulah Rd., Pittsburgh, PA, July 27, 1984.

4. R. Steunenberg and D. Fee, "Oxide Fuel Cell Research Laboratory Tour, "presented at a meeting of the DOE/AFCWG, Argonne National Laboratory (ANL), Argonne, IL, April 19, 1984.

5. D. Fee, "Oxide Fuel Cell Research," ibid. , April 19, 1984.

6. J. T. Brown, "Technology Update on Solid Oxide Fuel Cells," presented at a meeting of the DOE/AFCWG, WE, R\& D Center, 1310 Beulah Rd., Pittsburgh, PA, July 27, 1984.

7. "Report of the 3rd Meeting of the DOE/AFCWG at the Research and Development Center. Westinghouse Electric Corporation, "ibid., July 27, 1984, unpublished.

8. J. Weisbart and R. Ruka, J. Electrochem. Soc. 109, 723 (1962).

9. H. S. Isaacs and L. J. Olmer, J. Electrochem. Soc. 129, 345 (1982).

10. H. S. Isaacs, L. J. Olmer, E. J. L. Schouler, and C. Y. Yang, Solid State Ionics $3 / 4$, 503(1981).

11. H. S. Isaacs and L. J. Olmer, J. Electrochem. Soc. 129, 436 (1982).

12. E. Gor in and H. Recht, "High Temperature Fuel Cells" in Chemical Technology, vol. 1, p. 197, Academic Press, New York, NY (1963).

13. R. Ruka, 'Materials Considerations for Solid Oxide Fuel Cells," presented at a meeting of the DOE/AFCWG, WE, R\&D Center, 1310 Beulah Rd., Pittsburgh, PA, July 27, 1984.

14. J. Bauerle, "Component Measurements on Solid Oxide Fuel Cells," ibid., July $27,1984$.

15. J. T. Brown, "Suggested Complementary R\& D Programs on Solid Oxide Fuel Cells," ibid. , July $27,1984$.

16. S. K. Lau and S. C. Singhal, "Reactivity and Diffusion Studies at Solid Oxide Fuel Cell Component Interfaces, " ibid. , July $27,1984$.

17. H. S. Isaacs, "High Temperature Electrolytes," presented at a meeting of the DOE/ AFCWG, Engelhard Corp., 70 Wood Ave., South, Metro Park Plaza, Iselin, NJ, September 13, 1984.

18. H. S. Isaacs and L. J. Olmer, J. Electroanal. Chem. 132, 59 (1982).

19. E. J. L. Schouler and H. S. Isaacs, Solid State Ionics 5, 555 (1981).

20. J. Newman, J. Electrochem. Soc. 113, 501 (1966).

21. G. P. Wirtz and H. S. Isaacs, Solid State Ionics $9 / 10,963$ (1983). 


\section{APPENDIX A}

\section{ORIGINAL STATEMENT OF WORK}

UNIVERSITY OF CALIFORNIA, SAN DIEGO

(ADVANCED FUEL CELL WORKING GROUP)

The Contractor shall furnish all personnel, facilities, equipment, material, supplies, and services (except as may be expressly set forth in this contract as furnished by the Government) and ctherwise do all things necessary for, or incident to, the performance and providing of the following items of work:

Item 1 - Convene Advanced Fuel Cell Working Group (AFCWG) to examine current activities underway in the Technological area of Fuel Cells and to recommend priority research needs and opportunities for DOE support.

Item 2 - Reports in accordance with "Reporting Requirements CheckT ist" FORM DOE 537 and the clause entitled "Reporting Requirements". 